\title{
Synergistic Dinuclear Rhodium Induced Rhodium-Walking Enabling Alkene Terminal Arylation: A Theoretical Study
}

Dan Heng, ${ }^{\dagger}$ Haohua Chen, ${ }^{\dagger}$ Xiaoqian He, ${ }^{\dagger}$ Song Liu, ${ }^{\S}$ Lei Zhu, ${ }^{\dagger}$ Kangbao Zhong, ${ }^{\dagger}$ Tao Zhang, ${ }^{+}$Ruopeng Bai, ${ }^{\dagger}{ }^{*}$ Yu Lan ${ }^{\dagger, t, *}$

${ }^{\dagger}$ School of Chemistry and Chemical Engineering, Chongqing Key Laboratory of

Theoretical and Computational Chemistry, Chongqing University, Chongqing 400030,

China

†reen Catalysis Center, and College of Chemistry, Zhengzhou University,

Zhengzhou, Henan 450001, China

${ }^{\S}$ Chongqing Key Laboratory of Environmental Materials and Remediation

Technologies, Chongqing University of Arts and Sciences, Chongqing 402160, China

$$
\begin{gathered}
\text { ruopeng@cqu.edu.cn } \\
\text { lanyu@cqu.edu.cn }
\end{gathered}
$$

\section{Table of contents}

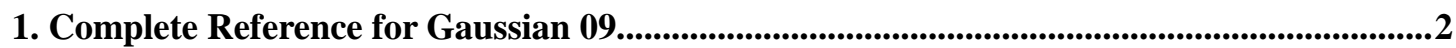

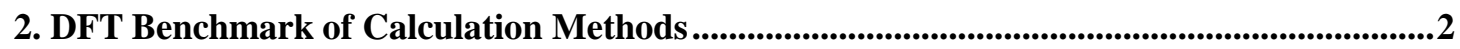

3. Localized Orbital Bonding Analysis (LOBA) Calculation .....................................................3

4. Full Free Energy Profile of the Calculated Dinuclear and Mononuclear Pathway.................4

5. Absolute Calculation Energies, Enthalpies, and Free Energies .................................................14

6. B3-LYP Geometries for All the Optimized Compounds and Transition States ....................20

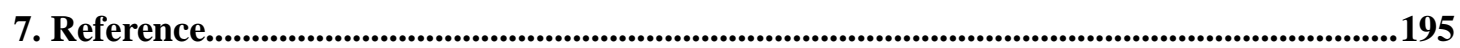




\section{Complete Reference for Gaussian 09}

Gaussian 09, Revision D.01, Frisch, M. J.; Trucks, G. W.; Schlegel, H. B.; Scuseria, G. E.; Robb, M. A.; Cheeseman, J. R.; Scalmani, G.; Barone, V.; Mennucci, B.; Petersson, G. A.; Nakatsuji, H.; Caricato, M.; Li, X.; Hratchian, H. P.; Izmaylov, A. F.; Bloino, J.; Zheng, G.; Sonnenberg, J. L.; Hada, M.; Ehara, M.; Toyota, K.; Fukuda, R.; Hasegawa, J.; Ishida, M.; Nakajima, T.; Honda, Y.; Kitao, O.; Nakai, H.; Vreven, T.; Montgomery, Jr., J. A.; Peralta, J. E.; Ogliaro, F.; Bearpark, M.; Heyd, J. J.; Brothers, E.; Kudin, K. N.; Staroverov, V. N.; Kobayashi, R.; Normand, J.; Raghavachari, K.; Rendell, A.; Burant, J. C.; Iyengar, S. S.; Tomasi, J.; Cossi, M.; Rega, N.; Millam, N. J.; Klene, M.; Knox, J. E.; Cross, J. B.; Bakken, V.; Adamo, C.; Jaramillo, J.; Gomperts, R.; Stratmann, R. E.; Yazyev, O.; Austin, A. J.; Cammi, R.; Pomelli, C.; Ochterski, J. W.; Martin, R. L.; Morokuma, K.; Zakrzewski, V. G.; Voth, G. A.; Salvador, P.; Dannenberg, J. J.; Dapprich, S.; Daniels, A. D.; Farkas, Ö.; Foresman, J. B.; Ortiz, J. V.; Cioslowski, J.; Fox, D. J. Gaussian, Inc., Wallingford CT, 2013.

\section{DFT Benchmark of Calculation Methods}

DFT benchmark of different methods of solvation single point calculations energies for regioselective reductive elimination transition states are shown in Table S1. The results show that in dinuclear pathway the relative free energy of 21-ts is the lowest, indicating that $\delta$-arylation is the thermodynamically favorable product. in mononuclear pathway, the relative free energy of 61-ts is the lowest, directing to the generation of $\beta$-arylation product. The benchmark data shows that calculation methods selection does not affact the main conclusion.

\begin{tabular}{|c|c|c|c|c|c|c|c|c|c|}
\hline \multirow{2}{*}{ Entry } & \multirow{2}{*}{ Method } & \multicolumn{8}{|c|}{$\Delta \mathrm{G}(\mathrm{kcal} / \mathrm{mol})$} \\
\hline & & 18-ts & 19-ts & 20-ts & 21-ts & 60-ts & 61-ts & 62-ts & 63-ts \\
\hline 1 & $\begin{array}{l}\text { M06-L/6-311+G(d,p)/SMD(SDD for Rh) } \\
\text { //M06-L/6-31G(d)/SMD (SDD for Rh) }\end{array}$ & 30.1 & 28.9 & 31.2 & 25.1 & 30.6 & 28.2 & 29.7 & 31.4 \\
\hline 2 & M06-L & 29.0 & 25.8 & 29.4 & 24.5 & 28.9 & 26.8 & 29.0 & 30.5 \\
\hline 3 & B3-LYP-D3 ${ }^{[1]}$ & 25.7 & 22.8 & 25.2 & 20.5 & 28.7 & 25.9 & 27.6 & 28.4 \\
\hline 4 & M06 $6^{[2]}$ & 27.2 & 24.9 & 27.8 & 22.1 & 28.8 & 26.5 & 27.1 & 29.2 \\
\hline 5 & $\mathrm{M} 06-2 \mathrm{X}^{[2]}$ & 28.4 & 26.2 & 28.5 & 23.9 & 31.1 & 26.9 & 27.9 & 29.3 \\
\hline 6 & $\mathrm{M} 11^{[3]}$ & 29.4 & 26.6 & 29.6 & 24.8 & 31.5 & 25.3 & 27.4 & 28.6 \\
\hline 7 & M11-L $\mathrm{L}^{[4]}$ & 27.6 & 25.9 & 28.5 & 22.8 & 24.8 & 23.0 & 24.2 & 26.3 \\
\hline
\end{tabular}


Table S1. DFT benchmark of different methods of solvation single point calculations energies for key reductive elimination transition states. For entry 1, the M06-L method with the standard 6-31G(d) basis set (SDD basis set for Rh) was used for the geometry optimizations in toluene. For entery 2-8, The B3-LYP functional with the standard 6-31G(d) basis set (SDD basis set for Rh) was used for the geometry optimizations in the gas phase. For all single-point energies calculations, the corresponding methods were used with the 6-311+G(d,p) basis set (SDD basis set for $\mathrm{Rh})$ in toluene.

\section{Localized Orbital Bonding Analysis (LOBA) Calculation}

Localized orbital bonding analysis (LOBA) calculation were performed using Multiwfn 3.7 used the Pipek-Mezey orbital localization method on the basis of the check files from geometry optimization to explore the oxidation state of $\mathrm{C}-\mathrm{H}$ activation intermediate. The oxidation state of an atom is calculated from the number of electrons sufficiently localized on that atom, i.e. above the threshold of $60 \%$ (valence electrons which are totally localized $>60 \%$ on the atom). The results show that oxidation state of $\mathrm{Rh} 1$ and $\mathrm{Rh} 2$ are +3 and +1 respectively, indicating that $\mathrm{Rh} 1$ is the catalytic center while $\mathrm{Rh} 2$ is innocent in the bond cleavage and formation process.

$\begin{array}{lcc}\text { 2.0000 electrons in LMO } & 50 \text { are attributed to atom } & 41 \\ 2.0000 \text { electrons in LMO } & 52 \text { are attributed to atom } & 41 \\ 2.0000 \text { electrons in LMO } & 53 \text { are attributed to atom } & 41 \\ 2.0000 \text { electrons in LMO } & 54 \text { are attributed to atom } & 41 \\ 2.0000 \text { electrons in LMO } & 163 \text { are attributed to atom } & 41 \\ 2.0000 \text { electrons in LMO } & 164 \text { are attributed to atom } & 41 \\ \text { 2.0000 electrons in LMO } & 165 \text { are attributed to atom } & 41 \\ \text { Oxidation state of atom } & 41(\mathrm{Rh}): \text { 3 } & \\ \text { 2.0000 electrons in LMO } & 51 \text { are attributed to atom } & 46 \\ 2.0000 \text { electrons in LMO } & 55 \text { are attributed to atom } & 46 \\ \text { 2.0000 electrons in LMO } & 56 \text { are attributed to atom } & 46 \\ \text { 2.0000 electrons in LMO } & 57 \text { are attributed to atom } & 46 \\ \text { 2.0000 electrons in LMO } & 166 \text { are attributed to atom } & 46 \\ 2.0000 \text { electrons in LMO } & 167 \text { are attributed to atom } & 46\end{array}$




\section{Full Free Energy Profile of the Calculated Dinuclear and Mononuclear Pathway.}

Full free energy profile of the dinuclear pathway are shown in Figure S1, S2 and S3.

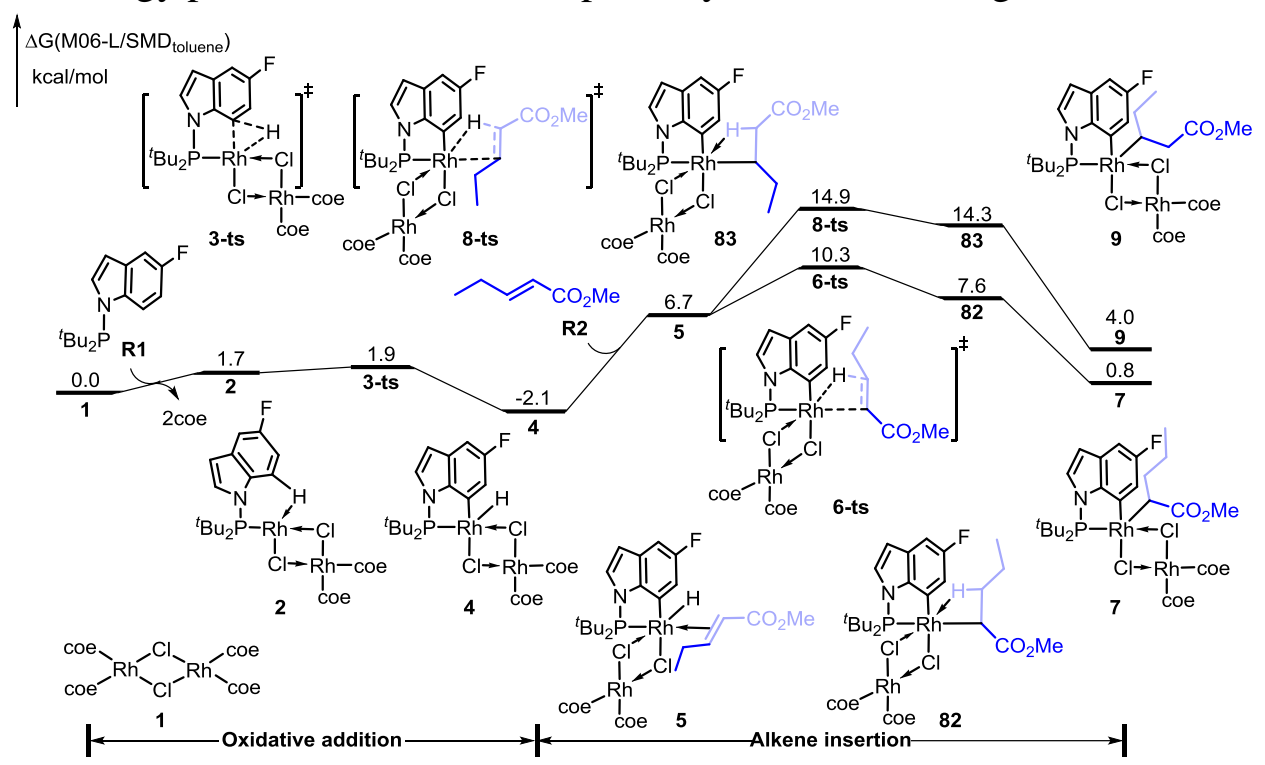

Figure S1. Full free energy profile of the initial oxidative addition and alkene insertion steps for the dinuclear pathway. The energies are in $\mathrm{kcal} / \mathrm{mol}$ and represent the relative free energies calculated by the M06-L/6-311+G(d,p)/SMD//B3-LYP/6-31G(d) (SDD for Rh) method in toluene. The bond distances are in angstrom. 


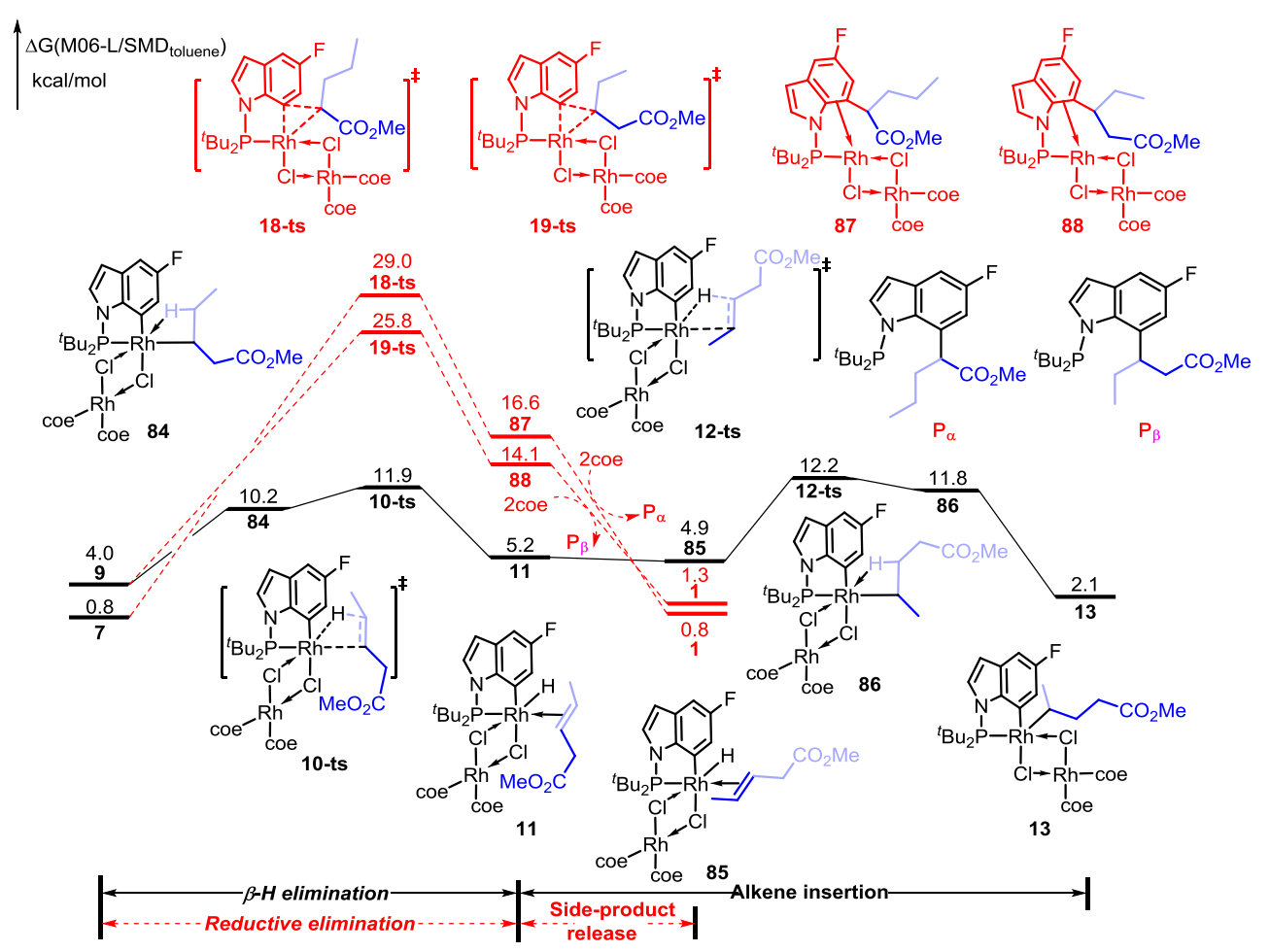

Figure S2. Full free energy profile of Rh-walking for the dinuclear pathway starting from intermediates 7 and 9. The energies are in $\mathrm{kcal} / \mathrm{mol}$ and represent the relative free energies calculated by the M06-L/6-311+G(d,p)/SMD//B3-LYP/6-31G(d) (SDD for Rh) method in toluene.

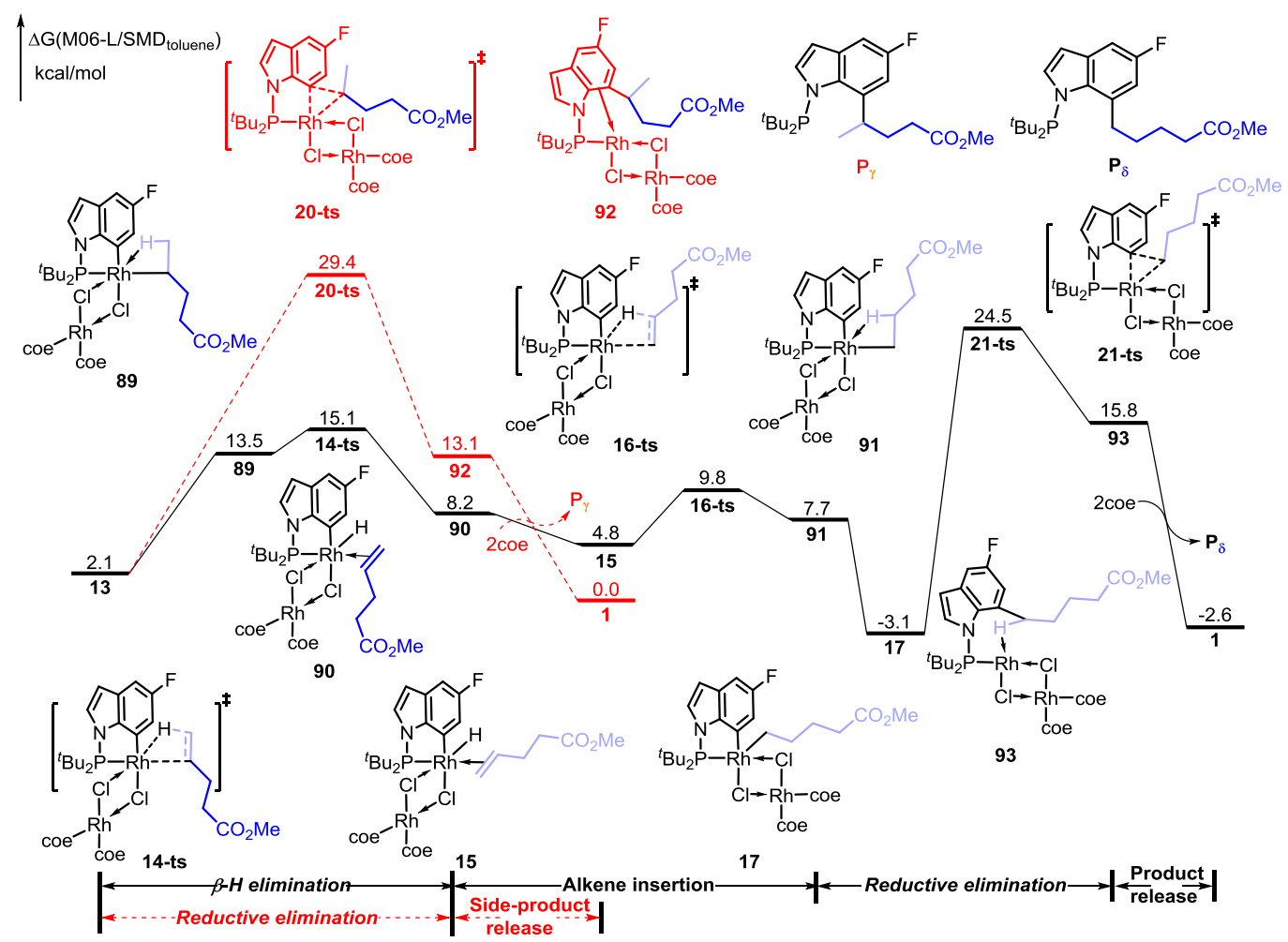

Figure S3. Full free energy profile of further Rh-walking and reductive elimination 
for the dinuclear pathway. The energies are in $\mathrm{kcal} / \mathrm{mol}$ and represent the relative free energies calculated by the M06-L/6-311+G(d,p)/SMD//B3-LYP/6-31G(d) (SDD for $\mathrm{Rh})$ method in toluene.

Full free energy profile of the mononuclear pathway are shown in Figure S4, S5 and S6.

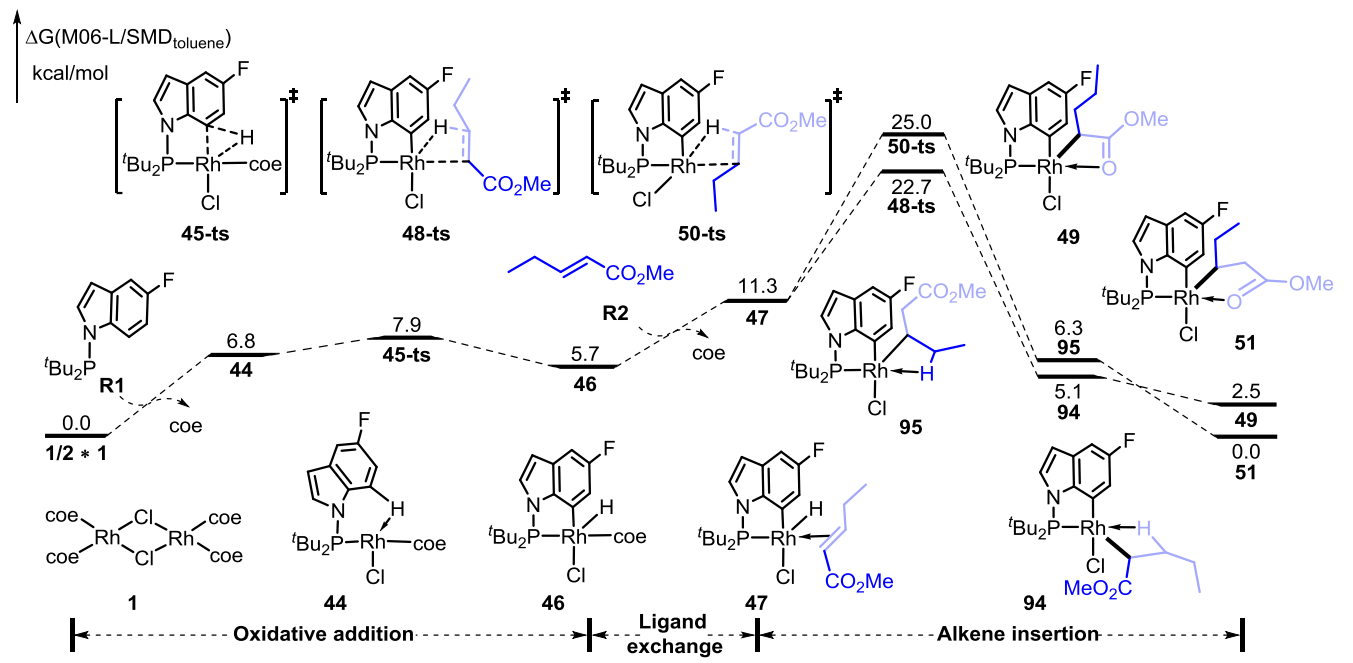

Figure S4. Full free energy profile of the initial oxidative addition and alkene insertion steps for the mononuclear pathway. The energies are in $\mathrm{kcal} / \mathrm{mol}$ and represent the relative free energies calculated by the M06-L/6-311+G(d,p)/SMD//B3-LYP/6-31G(d) (SDD for Rh) method in toluene. The bond distances are in angstrom.

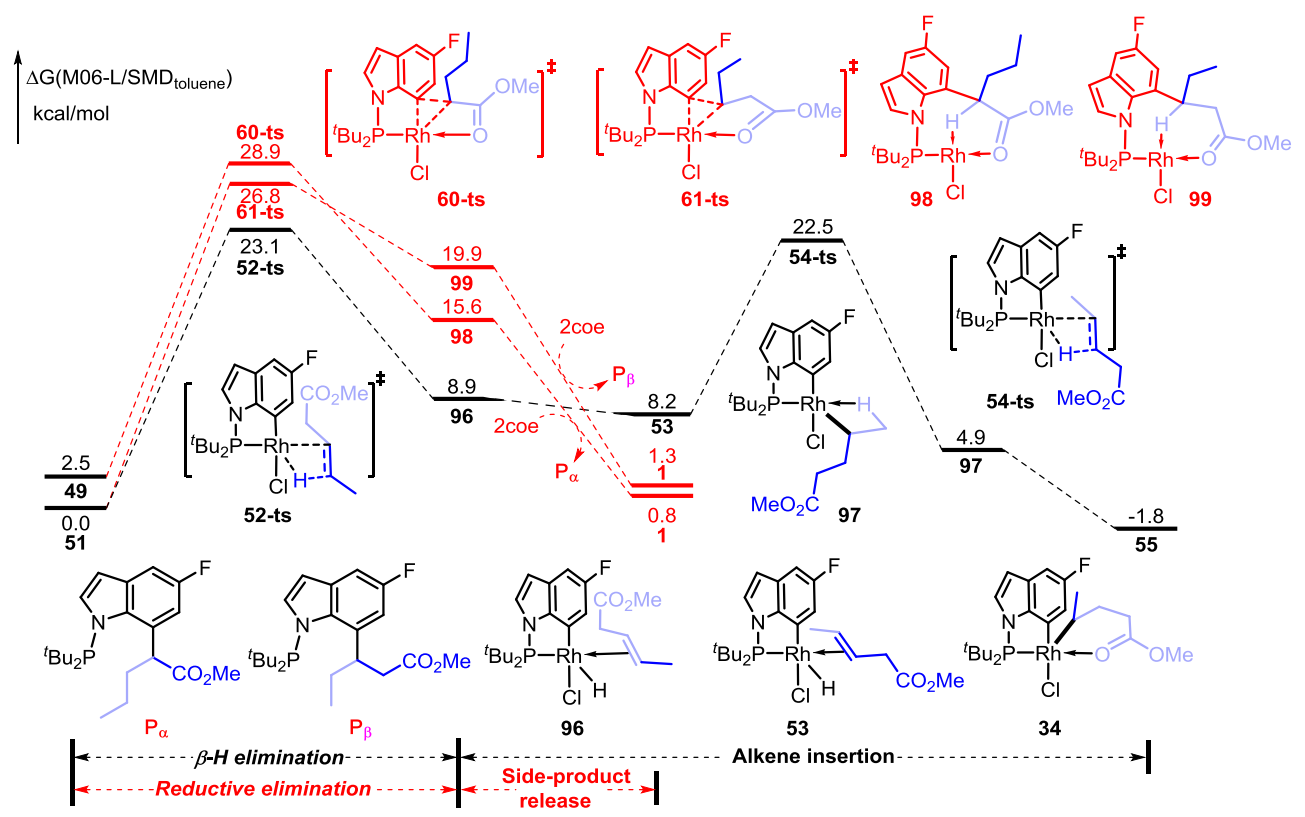

Figure S5. Full free energy profile of Rh-walking for the mononuclear pathway. The energies are in $\mathrm{kcal} / \mathrm{mol}$ and represent the relative free energies calculated by the 
M06-L/6-311+G(d,p)/SMD//B3-LYP/6-31G(d) (SDD for Rh) method in toluene. The bond distances are in angstrom.

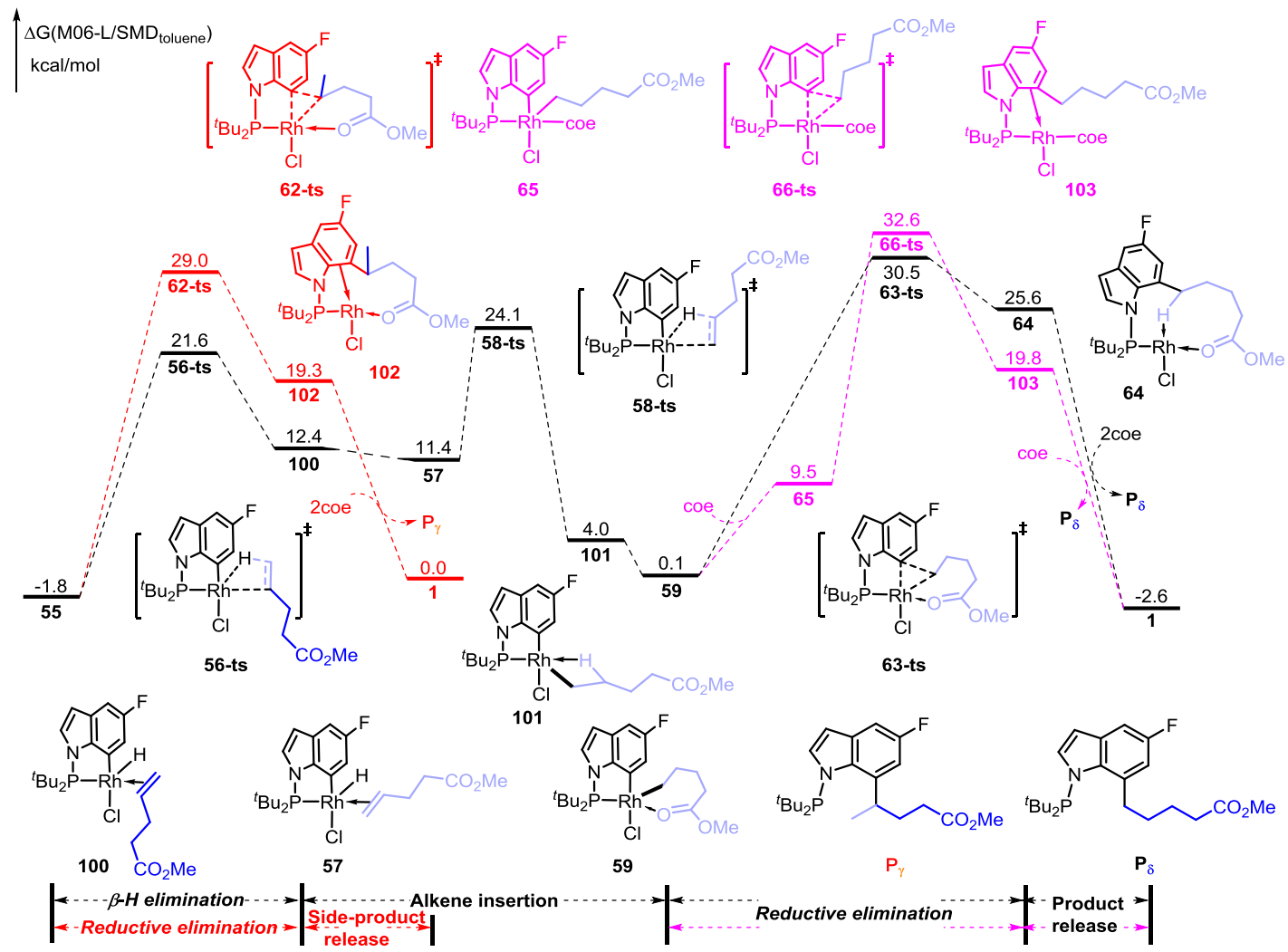

Figure S6. Full free energy profile of further Rh-walking and reductive elimination for the mononuclear pathway. The energies are in $\mathrm{kcal} / \mathrm{mol}$ and represent the relative free energies calculated by the M06-L/6-311+G(d,p)/SMD//B3-LYP/6-31G(d) (SDD for $\mathrm{Rh}$ ) method in toluene. The bond distances are in angstrom.

To further confirm the tether effect, we dissociated the ester group from rhodium center to give the corresponding 14 electron intermediates 94, 95, 97 and 101 calculate the energy barriers of corresponding $\alpha, \beta, \gamma$ and $\delta$-reductive elimination transition states. Free energy profiles are shown in Figure S7. The results show that dissociation of the ester group to give the corresponding 14 electron intermediates are unfavorable in the mononuclear pathway. 


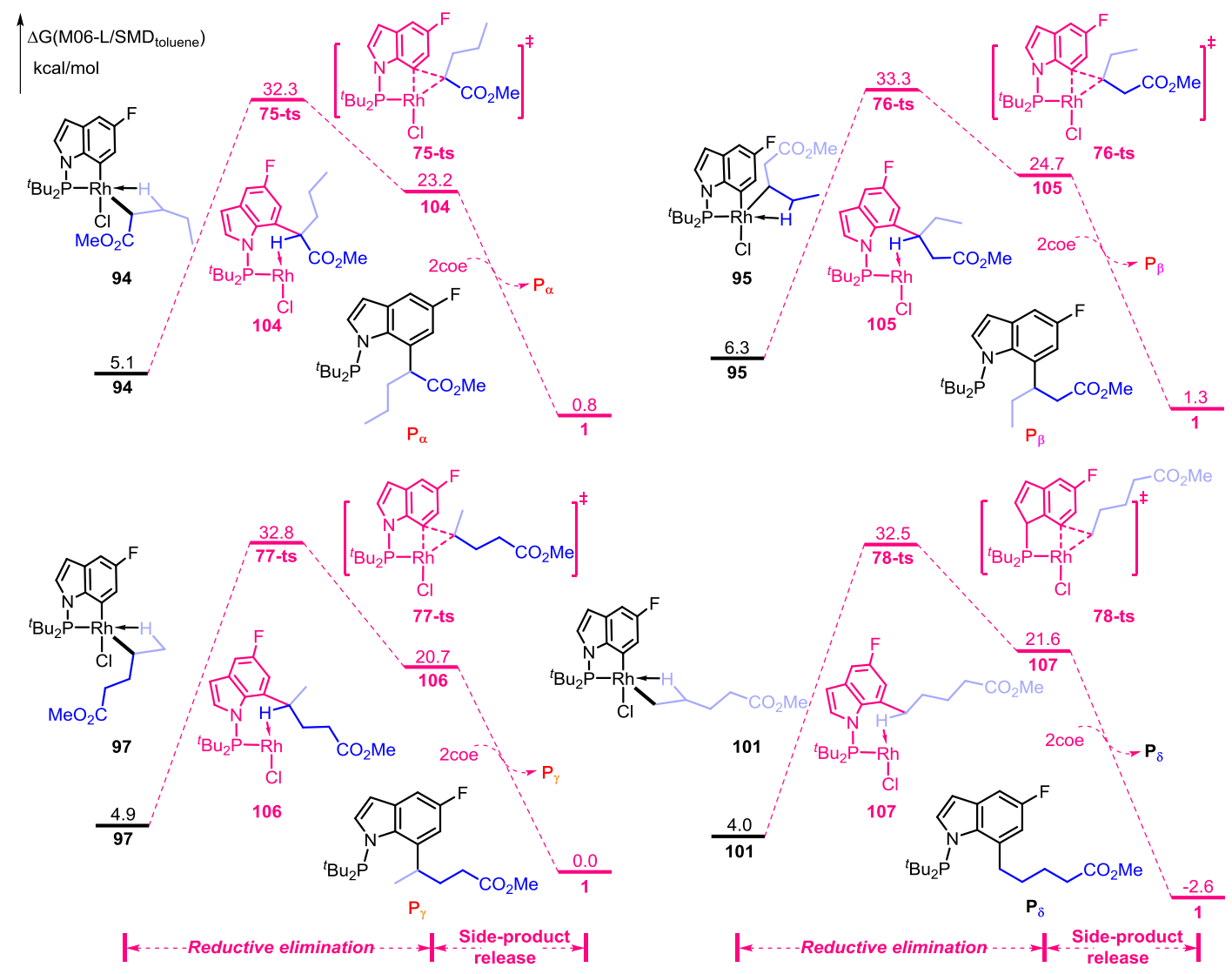

Figure S7. Free energy profile of the mononuclear rhodium catalyzed reductive elimination with a dissociative ester group. The energies are in $\mathrm{kcal} / \mathrm{mol}$ and represent the relative free energies calculated by the M06-L/6-311+G(d,p)/SMD//B3-LYP/6-31G(d) (SDD for Rh) method in toluene.

The dinuclear rhodium catalyzed $\mathrm{C} 2$ alkylation pathway was also considered theoretically. As shown in Figure S8, S9 and S10, the dinuclear pathway for C2 alkylation starts from the same dinuclear rhodium species 1. C2-H oxidative addition to rhodium could occur via transition state 80-ts with an energy barrier of 8.8 $\mathrm{kcal} / \mathrm{mol}$, which is $6.9 \mathrm{kcal} / \mathrm{mol}$ higher than that of $\mathrm{C} 7-\mathrm{H}$ oxidative addition via 3-ts. The relative free energy of the generated four-membered rhodacycle $\mathbf{8 1}$ is 5.7 $\mathrm{kcal} / \mathrm{mol}$, which is $7.8 \mathrm{kcal} / \mathrm{mol}$ higher than that of the corresponding five-membered rhodacycle 4. The corresponding energies of Rh-walking and reductive elimination of $\mathrm{C} 2-\mathrm{H}$ alkylation are also significantly higher than for $\mathrm{C} 7-\mathrm{H}$ alkylation. The corresponding alkene insertion and $\beta$-hydride elimination could provide four-membered rhodacycle alkyl intermediates 111, 114, 121, and 132. However, their relative free energies are significantly higher than those of corresponding five-membered intermediates $\mathbf{7 , 9 , 1 3}$, and $\mathbf{1 7}$. Therefore, the relative free energies for 
the subsequent reductive elimination transition states 122-ts, 124-ts, 135-ts, and 133-ts is also higher. As a result, C2-alkylation of indole is thermodynamically and dynamically unfavorable.

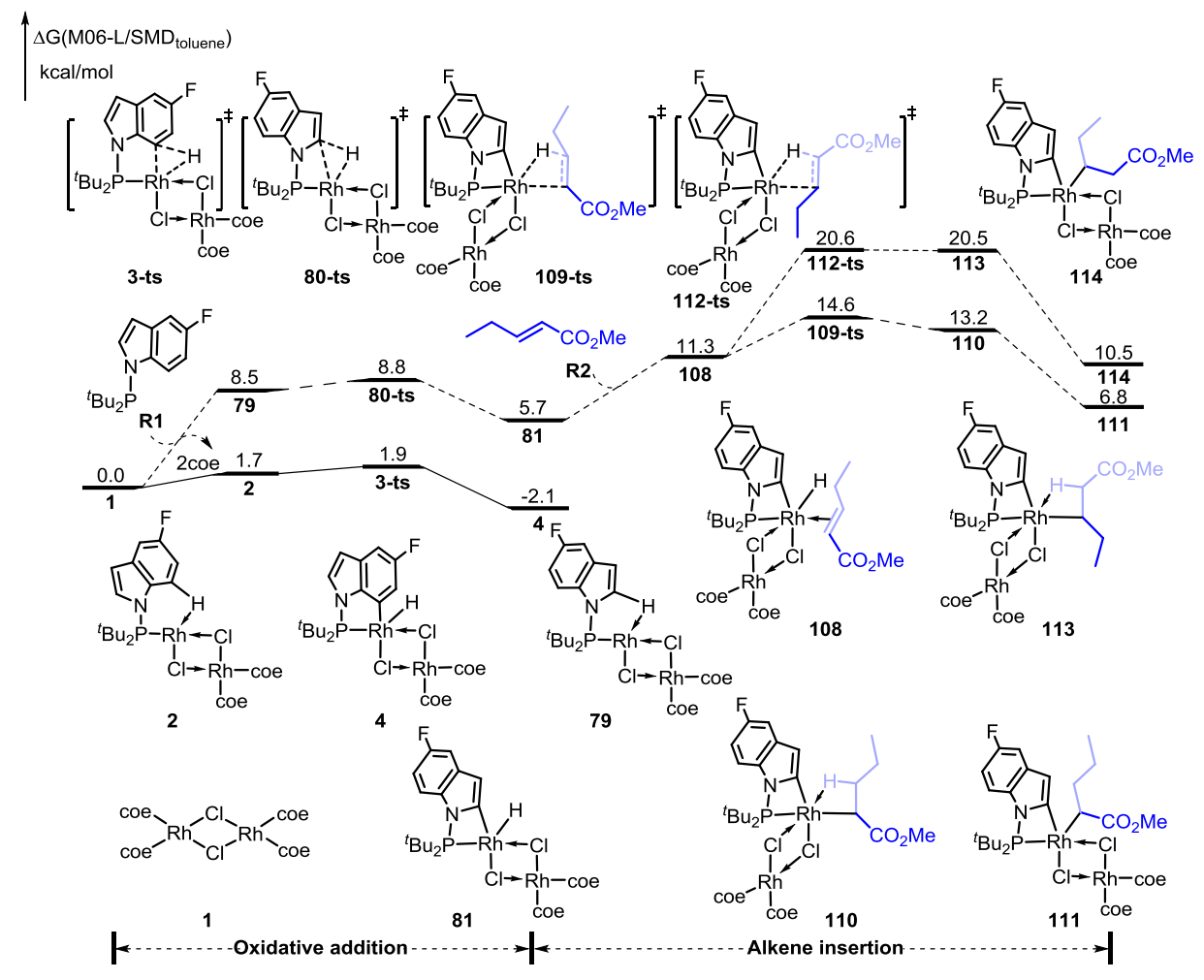

Figure S8. Full free energy profile of the initial oxidative addition and alkene insertion steps for the dinuclear C2-alkylation pathways. The energies are in $\mathrm{kcal} / \mathrm{mol}$ and represent the relative free energies calculated by the M06-L/6-311+G(d,p)/SMD//B3-LYP/6-31G(d) (SDD for Rh) method in toluene. 


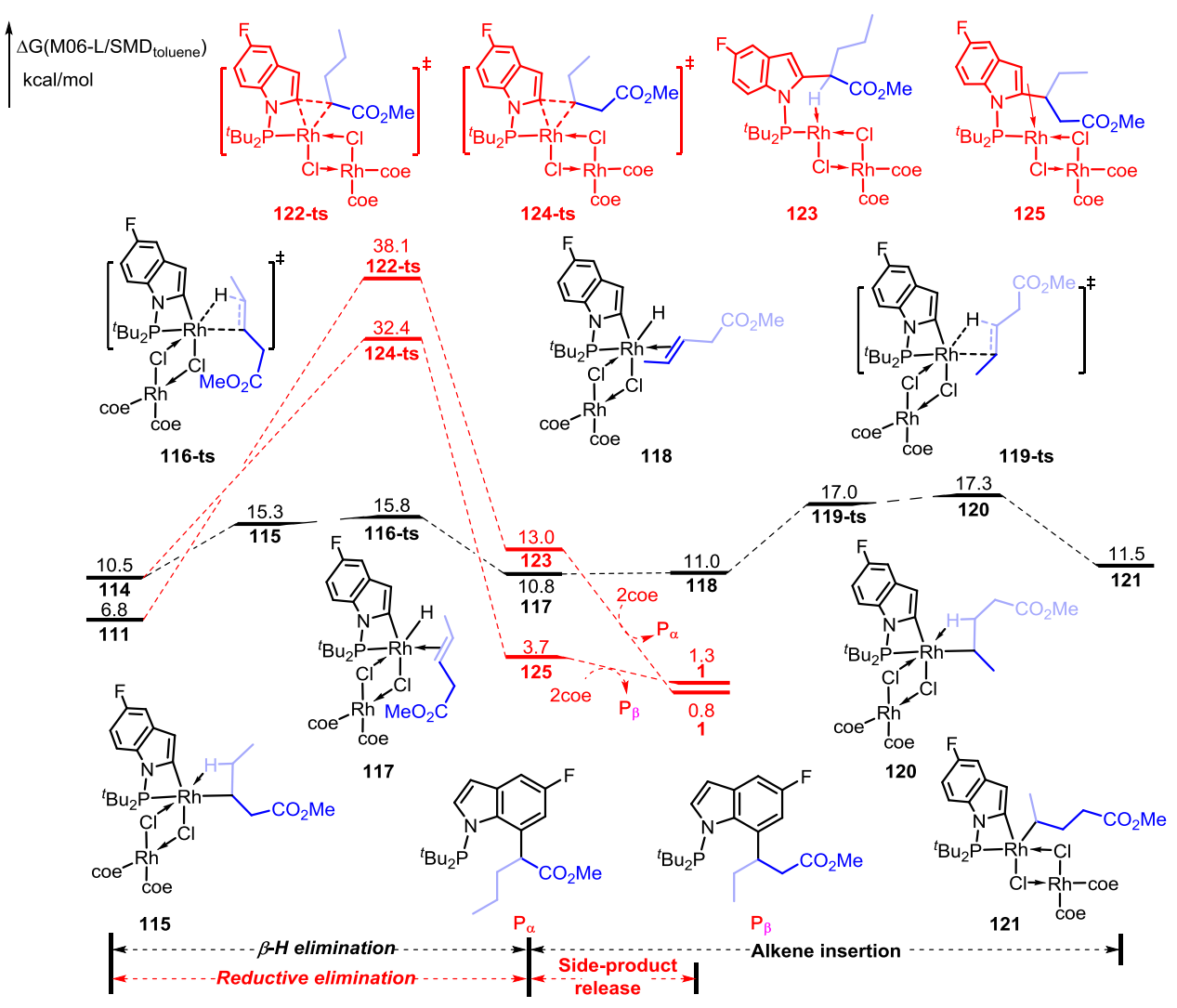

Figure S9. Full free energy profile of Rh-walking for the dinuclear pathway starting from intermediates $\mathbf{1 1 1}$ and 114. The energies are in $\mathrm{kcal} / \mathrm{mol}$ and represent the relative free energies calculated by the M06-L/6-311+G(d,p)/SMD//B3-LYP/6-31G(d) (SDD for Rh) method in toluene. 


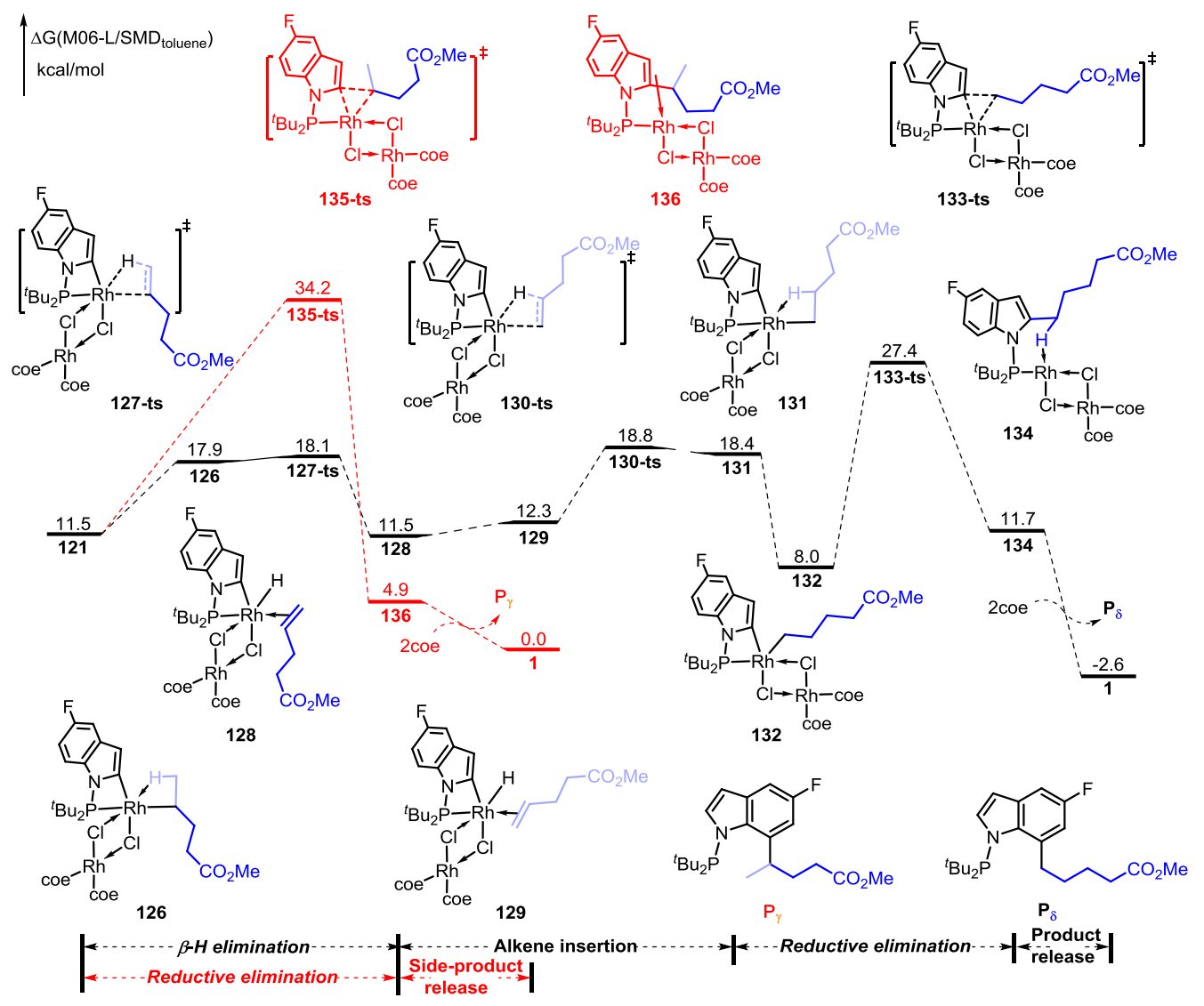

Figure S10. Full free energy profile of further Rh-walking and C2-reductive elimination for the dinuclear pathway. The energies are in $\mathrm{kcal} / \mathrm{mol}$ and represent the relative free energies calculated by the M06-L/6-311+G(d,p)/SMD//B3-LYP/6-31G(d) (SDD for Rh) method in toluene.

The mononuclear rhodium catalyzed C2 alkylation pathway was shown in Figure S11. The mononuclear pathway for $\mathrm{C} 2$ alkylation starts from the same dinuclear rhodium species 1. C2-H oxidative addition to rhodium could occur via transition state 138-ts with an energy barrier of $11.9 \mathrm{kcal} / \mathrm{mol}$, which is $4.0 \mathrm{kcal} / \mathrm{mol}$ higher than that of $\mathrm{C} 7-\mathrm{H}$ oxidative addition via 24-ts. The relative free energy of the generated four-membered rhodacycle 139 is $10.0 \mathrm{kcal} / \mathrm{mol}$, which is $4.3 \mathrm{kcal} / \mathrm{mol}$ higher than that of the corresponding five-membered rhodacycle 25. Similar to the dinuclear mechanism, the strain of the four-membered ring in this case also leads to higher relative free energy than $\mathrm{C} 7-\mathrm{H}$ alkylation for the whole process. 


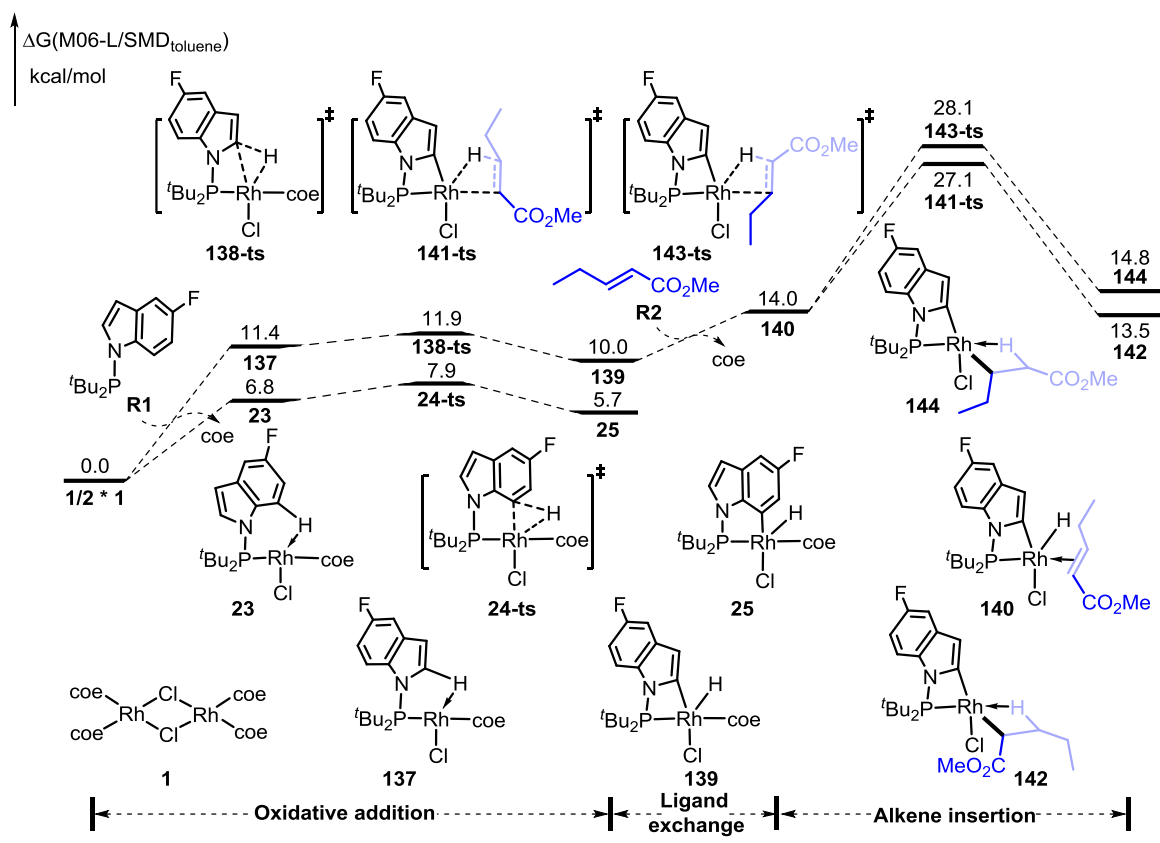

Figure S11. Full free energy profile of the initial oxidative addition and alkene insertion steps for the mononuclear C2-alkylation pathways. The energies are in $\mathrm{kcal} / \mathrm{mol}$ and represent the relative free energies calculated by the M06-L/6-311+G(d,p)/SMD//B3-LYP/6-31G(d) (SDD for Rh) method in toluene.

To further understand the regioselectivity of $\mathrm{C} 7$ and $\mathrm{C} 2 \mathrm{C}-\mathrm{H}$ activation, distortion-interaction analysis was performed, ${ }^{[6]}$ and the results are given in Table S2. Distortion-interaction analysis of the oxidative addition transition states 3-ts(C7) and 80-ts(C2) was performed. The energies required to distort the rhodium moiety and reactant into the geometries of the corresponding transition states are $\Delta E^{*}$ dist(Rh) and $\left.\Delta E^{*}{ }_{\text {dist( }} \mathbf{R}\right)$, respectively. The distortion energy is the energy required to distort the reactants into the geometries of their transitional states. The interaction energy is the energy change upon interaction of the two distorted fragments. The results showed that the total distortion energies involved in the $\mathrm{C} 7-\mathrm{H}$ activation transition state 3-ts and $\mathrm{C} 2-\mathrm{H}$ activation transition state 80-ts are 38.3 and $43.4 \mathrm{kcal} / \mathrm{mol}$, while the interaction energies of 3-ts and 80-ts are -36.5 and $-33.0 \mathrm{kcal} / \mathrm{mol}$, respectively. This indicates that both the total distortion energy and interaction energy involved in transition state 3-ts are lower than those involved in 80-ts. As a result, both the distortion and interaction energies lead to generation of the $\mathrm{C} 7$ functionalization product. 
Table S2. Distortion and interaction energies of the oxidative addition transition states. $^{\mathrm{a}}$

\begin{tabular}{|c|c|c|c|c|c|}
\hline ts & $\Delta E^{\ddagger} \operatorname{dist}(\mathbf{R h})$ & $\Delta E^{\ddagger} \operatorname{dist}(\mathbf{R})$ & $\Delta E^{\ddagger} \operatorname{dist}(\mathbf{R h})+\Delta E^{\ddagger} \operatorname{dist}(\mathbf{R})$ & $\Delta E_{\mathrm{int}}^{\ddagger}$ & $\Delta E^{\ddagger}$ \\
\hline 3-ts(C7) & 0.9 & 37.4 & 38.3 & -36.5 & 1.8 \\
\hline 80-ts(C2) & 0.1 & 43.3 & 43.4 & -33.0 & 10.4 \\
\hline
\end{tabular}

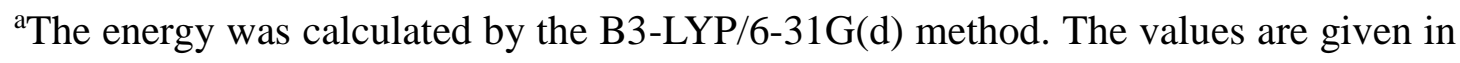
$\mathrm{kcal} / \mathrm{mol}$.

To reveal the origin of the distortion and interaction energy differences, atomic charge analysis and geometrical structure analysis were performed, and the results are shown in Figure S12. Natural bond orbital (NBO) atomic charge analysis of the C7 atom showed that it is more electron rich than the $C 2$ atom $(-0.275$ vs -0.053$){ }^{[7]}$ These results indicate that the higher nucleophilicity of C7 could lead to the lower energy barrier of $\mathrm{C}-\mathrm{H}$ oxidative addition and the corresponding intermediate 4 being more stable than $\mathbf{8 1}$. In addition, the $\mathrm{C}-\mathrm{N}-\mathrm{P}$ bond angle $\left(A_{\mathrm{C}-\mathrm{N}-\mathrm{P}}\right)$ in $N-\mathrm{P}^{t} \mathrm{Bu}_{2}$ indole $\mathbf{R} \mathbf{1}$ is $122.3^{\circ}$. Oxidation addition of the $\mathrm{C}(\operatorname{aryl})-\mathrm{H}$ bond at different positions leads to generation of five-membered rhodacycle intermediate $\mathbf{4}$ or four-membered rhodacycle intermediate 81. The $A_{\mathrm{C}-\mathrm{N}-\mathrm{P}}$ values in intermediates $\mathbf{4}$ and $\mathbf{8 1}$ are $113.2^{\circ}$ and $147.8^{\circ}$, respectively. This indicates larger ring strain in rhodacycle 81. It is worth noting that the ring strain exists in all of the following intermediates until reductive elimination occurs, indicating higher activation energy at the $\mathrm{C} 2$ position. Therefore, $\mathrm{C}-\mathrm{H}$ arylation at the $\mathrm{C} 2$ position is determined to be thermodynamically and dynamically unfavorable.

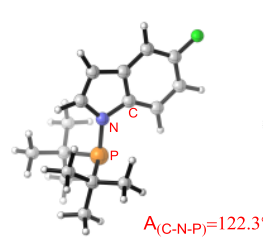

R1

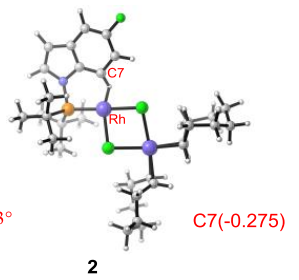

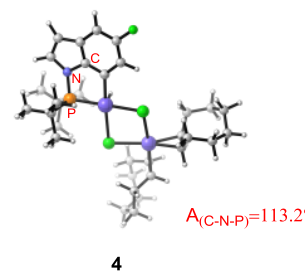

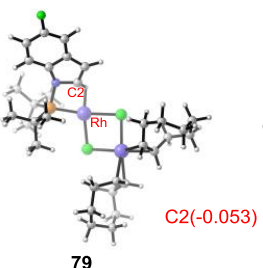

79

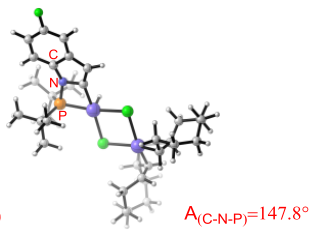

81

Figure S12. Atomic charge analysis and geometrical structure analysis of R1, 2, 4, 79, and $\mathbf{8 1}$. 
5. Absolute Calculation Energies, Enthalpies, and Free Energies

\begin{tabular}{|c|c|c|c|c|c|}
\hline Geometry & $E_{(\text {elec-B3-LYP) }}{ }^{1}$ & $G_{(\text {corr-B3-LYP })^{2}}$ & $H_{\text {(corr-B3-LYP) }}{ }^{3}$ & $E_{(\mathrm{solv}, \mathrm{M} 06-\mathrm{L})}{ }^{4}$ & $\mathrm{IF}^{5}$ \\
\hline $\mathbf{R 1}$ & -1119.486778 & 0.312953 & 0.380488 & -1119.628610 & - \\
\hline $\mathbf{R 2}$ & -385.096392 & 0.117890 & 0.163392 & -385.164008 & - \\
\hline coe & -313.258847 & 0.173669 & 0.213791 & -313.304825 & - \\
\hline $\mathbf{A}$ & -1197.377928 & 0.366319 & 0.436015 & -1197.537300 & - \\
\hline B & -1341.043282 & 0.255052 & 0.334392 & -1341.236585 & - \\
\hline $\mathbf{C}$ & -2809.823058 & 0.649478 & 0.769555 & -2810.189699 & - \\
\hline D & -2003.604765 & 0.507515 & 0.601898 & -2003.862334 & - \\
\hline $\mathbf{E}$ & -2887.742963 & 0.700106 & 0.823654 & -2888.113097 & - \\
\hline $\mathbf{F}$ & -2682.142405 & 0.537075 & 0.673713 & -2682.543543 & - \\
\hline $\mathbf{G}$ & -3380.686866 & 0.642933 & 0.772312 & -3381.104299 & - \\
\hline $\mathbf{H}$ & -3031.416073 & 0.589085 & 0.722949 & -3031.827156 & - \\
\hline 1 & -2394.798250 & 0.760242 & 0.875654 & -2395.122602 & - \\
\hline 2 & -2887.742963 & 0.700106 & 0.823654 & -2888.113097 & - \\
\hline 3-ts & -2887.740023 & 0.698279 & 0.820614 & -2888.111011 & $683.27 i$ \\
\hline 4 & -2887.751451 & 0.698975 & 0.822370 & -2888.118101 & - \\
\hline 5 & -3272.842146 & 0.844157 & 0.989310 & -3273.295309 & - \\
\hline 6-ts & -3272.836355 & 0.843400 & 0.986796 & -3273.288840 & $550.16 i$ \\
\hline 7 & -3272.852317 & 0.844889 & 0.991343 & -3273.305497 & - \\
\hline 8-ts & -3272.826267 & 0.840779 & 0.986262 & -3273.278786 & $591.35 i$ \\
\hline 9 & -3272.848186 & 0.846279 & 0.991677 & -3273.301790 & - \\
\hline 10 -ts & -3272.832243 & 0.841960 & 0.986548 & -3273.284758 & $589.54 i$ \\
\hline 11 & -3272.843390 & 0.842885 & 0.988912 & -3273.296451 & - \\
\hline 12-ts & -3272.830172 & 0.841777 & 0.986219 & -3273.284161 & $625.61 i$ \\
\hline 13 & -3272.851147 & 0.844882 & 0.991926 & -3273.303368 & - \\
\hline 14-ts & -3272.829820 & 0.842843 & 0.987550 & -3273.280647 & $648.88 i$ \\
\hline 15 & -3272.846698 & 0.842303 & 0.989126 & -3273.296446 & - \\
\hline 16-ts & -3272.835722 & 0.839166 & 0.986408 & -3273.285424 & $597.18 i$ \\
\hline
\end{tabular}




\begin{tabular}{|c|c|c|c|c|c|}
\hline 17 & -3272.861499 & 0.841194 & 0.991360 & -3273.308020 & - \\
\hline 18-ts & -3272.806704 & 0.846079 & 0.990051 & -3273.261657 & $328.18 i$ \\
\hline 19-ts & -3272.806976 & 0.844973 & 0.989881 & -3273.265660 & $334.52 i$ \\
\hline 20-ts & -3272.806508 & 0.845899 & 0.990599 & -3273.260858 & $344.66 i$ \\
\hline 21-ts & -3272.816470 & 0.844898 & 0.990544 & -3273.267606 & $373.84 i$ \\
\hline $\mathbf{P}_{a}$ & -1504.601807 & 0.455091 & 0.547711 & -1504.815536 & - \\
\hline $\mathbf{P}_{\beta}$ & -1504.598983 & 0.455087 & 0.547574 & -1504.814853 & - \\
\hline $\mathbf{P}_{\boldsymbol{\delta}}$ & -1504.603378 & 0.454097 & 0.547886 & -1504.820048 & - \\
\hline $\mathbf{P}_{\gamma}$ & -1504.600586 & 0.455974 & 0.547901 & -1504.817739 & - \\
\hline 22 & -3765.808268 & 0.785358 & 0.939948 & -3766.302481 & - \\
\hline 23-ts & -3765.805036 & 0.782464 & 0.936581 & -3766.300095 & $706.38 i$ \\
\hline 24 & -3765.816414 & 0.781746 & 0.938078 & -3766.305827 & - \\
\hline 25 & -4150.896663 & 0.927932 & 1.105322 & -4151.475689 & - \\
\hline 26-ts & -4150.895212 & 0.927423 & 1.102832 & -4151.472910 & $430.67 i$ \\
\hline 27 & -4150.917188 & 0.930264 & 1.107482 & -4151.495950 & - \\
\hline 28-ts & -4150.884999 & 0.928006 & 1.102807 & -4151.462809 & $581.67 i$ \\
\hline 29 & -4150.910418 & 0.926835 & 1.107573 & -4151.488622 & - \\
\hline 30-ts & -4150.892353 & 0.926537 & 1.102569 & -4151.471082 & $525.10 i$ \\
\hline 31 & -4150.898599 & 0.927612 & 1.104917 & -4151.478941 & - \\
\hline 32-ts & -4150.891029 & 0.926974 & 1.102613 & -4151.470490 & $605.72 i$ \\
\hline 33 & -4150.914166 & 0.929095 & 1.107636 & -4151.491542 & - \\
\hline 34-ts & -4150.892117 & 0.928345 & 1.103780 & -4151.468101 & $620.39 i$ \\
\hline 35 & -4150.905752 & 0.926768 & 1.105582 & -4151.479363 & - \\
\hline 36-ts & -4150.897250 & 0.926676 & 1.103183 & -4151.470656 & $566.65 i$ \\
\hline 37 & -4150.925473 & 0.924557 & 1.106872 & -4151.496001 & - \\
\hline 38-ts & -4150.868804 & 0.926571 & 1.105600 & -4151.448680 & $317.63 i$ \\
\hline 39-ts & -4150.870660 & 0.926464 & 1.105612 & -4151.451225 & $334.59 i$ \\
\hline 40-ts & -4150.871664 & 0.930012 & 1.106248 & -4151.449003 & $346.93 i$ \\
\hline 41-ts & -4150.881891 & 0.928072 & 1.106258 & -4151.455626 & $380.12 i$ \\
\hline
\end{tabular}




\begin{tabular}{|c|c|c|c|c|c|}
\hline 42 & -4150.901186 & 0.928013 & 1.108195 & -4151.469119 & - \\
\hline 43-ts & -4150.856256 & 0.929605 & 1.107372 & -4151.428543 & $345.65 i$ \\
\hline 44 & -2003.604765 & 0.507515 & 0.601898 & -2003.862334 & 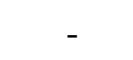 \\
\hline 45-ts & -2003.598741 & 0.504914 & 0.598494 & -2003.857965 & $704.92 i$ \\
\hline 46 & -2003.606117 & 0.505770 & 0.600194 & -2003.862302 & - \\
\hline 47 & -2075.436987 & 0.450738 & 0.549951 & -2075.713329 & - \\
\hline 48-ts & -2075.413420 & 0.448833 & 0.547352 & -2075.693228 & $211.75 i$ \\
\hline 49 & -2075.453306 & 0.454110 & 0.552332 & -2075.730732 & - \\
\hline 50-ts & -2075.410912 & 0.447027 & 0.547059 & -2075.687832 & $644.56 i$ \\
\hline 51 & -2075.456551 & 0.457239 & 0.552594 & -2075.737937 & - \\
\hline 52-ts & -2075.411820 & 0.449034 & 0.547626 & -2075.692874 & $134.22 i$ \\
\hline 53 & -2075.436197 & 0.447449 & 0.549182 & -2075.715015 & - \\
\hline 54-ts & -2075.410459 & 0.448233 & 0.547278 & -2075.692953 & $250.87 i$ \\
\hline 55 & -2075.459206 & 0.457538 & 0.552530 & -2075.741049 & - \\
\hline 56-ts & -2075.410947 & 0.445371 & 0.547456 & -2075.691608 & $849.20 i$ \\
\hline 57 & -2075.435177 & 0.449176 & 0.549900 & -2075.711660 & 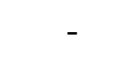 \\
\hline 58-ts & -2075.410629 & 0.449075 & 0.547610 & -2075.691245 & $134.68 i$ \\
\hline 59 & -2075.458106 & 0.456711 & 0.552847 & -2075.737192 & - \\
\hline 60-ts & -2075.404438 & 0.453906 & 0.550592 & -2075.688428 & $282.01 i$ \\
\hline 61-ts & -2075.407411 & 0.457897 & 0.551229 & -2075.695873 & $349.30 i$ \\
\hline 62-ts & -2075.406397 & 0.458331 & 0.551622 & -2075.692730 & $310.67 i$ \\
\hline 63-ts & -2075.404536 & 0.456867 & 0.551757 & -2075.688880 & $357.64 i$ \\
\hline 64 & -2075.417897 & 0.457402 & 0.553858 & -2075.697252 & - \\
\hline 65 & -2388.706346 & 0.649834 & 0.769415 & -2389.046568 & - \\
\hline 66-ts & -2388.667924 & 0.650012 & 0.768045 & -2389.009785 & $361.76 i$ \\
\hline 67 & -2076.654166 & 0.472044 & 0.574883 & -2076.929204 & - \\
\hline 68-ts & -2076.613080 & 0.472766 & 0.573937 & -2076.892070 & $343.38 i$ \\
\hline 69 & -2076.645319 & 0.475559 & 0.575122 & -2076.927055 & 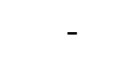 \\
\hline 70-ts & -2076.598753 & 0.474457 & 0.573910 & -2076.884994 & $339.48 i$ \\
\hline
\end{tabular}




\begin{tabular}{|c|c|c|c|c|c|}
\hline 71 & -2076.648912 & 0.473598 & 0.574875 & -2076.929372 & - \\
\hline 72-ts & -2076.601486 & 0.474333 & 0.573951 & -2076.885051 & $326.81 i$ \\
\hline 73 & -2076.657222 & 0.472732 & 0.575256 & -2076.931544 & - \\
\hline 74-ts & -2076.611798 & 0.473092 & 0.573956 & -2076.890447 & $371.41 i$ \\
\hline 75-ts & -2075.392546 & 0.452569 & 0.550740 & -2075.681755 & $249.00 i$ \\
\hline 76-ts & -2075.388690 & 0.451247 & 0.550460 & -2075.678750 & $293.35 i$ \\
\hline 77-ts & -2075.389365 & 0.451762 & 0.550874 & -2075.680194 & $326.93 i$ \\
\hline 78-ts & -2075.391375 & 0.449384 & 0.550866 & -2075.678306 & $357.84 i$ \\
\hline 79 & -2887.730497 & 0.699257 & 0.823481 & -2888.101348 & - \\
\hline 80 -ts & -2887.726440 & 0.696385 & 0.820148 & -2888.098002 & $733.82 i$ \\
\hline 81 & -2887.738387 & 0.696426 & 0.821869 & -2888.102999 & - \\
\hline 82 & -3272.841164 & 0.844235 & 0.989444 & -3273.294007 & - \\
\hline 83 & -3272.829107 & 0.842455 & 0.989323 & -3273.281453 & - \\
\hline 84 & -3272.834958 & 0.841399 & 0.989058 & -3273.286930 & - \\
\hline 85 & -3272.841296 & 0.843190 & 0.988840 & -3273.297183 & - \\
\hline 86 & -3272.833758 & 0.843370 & 0.989252 & -3273.286364 & - \\
\hline 87 & -3272.832706 & 0.845472 & 0.992435 & -3273.280830 & - \\
\hline 88 & -3272.832007 & 0.846119 & 0.992010 & -3273.285413 & - \\
\hline 89 & -3272.832730 & 0.843715 & 0.989901 & -3273.284004 & - \\
\hline 90 & -3272.841637 & 0.843409 & 0.989630 & -3273.292234 & - \\
\hline 91 & -3272.837982 & 0.838319 & 0.988971 & -3273.287912 & - \\
\hline 92 & -3272.830937 & 0.842660 & 0.992480 & -3273.283676 & - \\
\hline 93 & -3272.837221 & 0.847566 & 0.992568 & -3273.284145 & - \\
\hline 94 & -2075.440952 & 0.452844 & 0.551489 & -2075.725371 & - \\
\hline 95 & -2075.438828 & 0.453492 & 0.551858 & -2075.724156 & - \\
\hline 96 & -2075.436892 & 0.448962 & 0.549216 & -2075.715430 & - \\
\hline 97 & -2075.439064 & 0.452273 & 0.551450 & -2075.725165 & - \\
\hline 98 & -2075.429983 & 0.455126 & 0.552038 & -2075.710872 & - \\
\hline 99 & -2075.426827 & 0.457764 & 0.553309 & -2075.706771 & - \\
\hline
\end{tabular}




\begin{tabular}{|c|c|c|c|c|c|}
\hline 100 & -2075.432219 & 0.448193 & 0.549569 & -2075.709002 & - \\
\hline 101 & -2075.440942 & 0.450535 & 0.551683 & -2075.724762 & - \\
\hline 102 & -2075.425480 & 0.457992 & 0.553707 & -2075.707918 & - \\
\hline 103 & -2388.694830 & 0.649094 & 0.769536 & -2389.029301 & - \\
\hline 104 & -2075.416635 & 0.453732 & 0.552551 & -2075.697431 & - \\
\hline 105 & -2075.414089 & 0.455643 & 0.553172 & -2075.696861 & - \\
\hline 106 & -2075.416961 & 0.452459 & 0.552968 & -2075.700138 & - \\
\hline 107 & -2075.416742 & 0.453005 & 0.553320 & -2075.699221 & - \\
\hline 108 & -3272.839321 & 0.842754 & 0.988955 & -3273.286616 & - \\
\hline 109-ts & -3272.831904 & 0.840308 & 0.986373 & -3273.278906 & $595.38 i$ \\
\hline 110 & -3272.835436 & 0.841585 & 0.989349 & -3273.282355 & - \\
\hline 111 & -3272.850365 & 0.844230 & 0.991134 & -3273.295234 & - \\
\hline 112-ts & -3272.820349 & 0.838397 & 0.985873 & -3273.267450 & $575.39 i$ \\
\hline 113 & -3272.823721 & 0.841490 & 0.989189 & -3273.270599 & - \\
\hline 114 & -3272.838403 & 0.842273 & 0.991415 & -3273.287375 & - \\
\hline 115 & -3272.828946 & 0.838136 & 0.988593 & -3273.275646 & - \\
\hline 116-ts & -3272.826509 & 0.837563 & 0.986137 & -3273.274195 & $588.69 i$ \\
\hline 117 & -3272.837938 & 0.840125 & 0.988245 & -3273.284671 & - \\
\hline 118 & -3272.836684 & 0.839958 & 0.988584 & -3273.284246 & - \\
\hline 119-ts & -3272.824062 & 0.837719 & 0.985739 & -3273.272465 & $575.11 i$ \\
\hline 120 & -3272.826526 & 0.840089 & 0.988625 & -3273.274367 & - \\
\hline 121 & -3272.839658 & 0.843519 & 0.991410 & -3273.287015 & - \\
\hline 122-ts & -3272.790928 & 0.843693 & 0.989788 & -3273.244825 & $307.66 i$ \\
\hline 123 & -3272.840959 & 0.844947 & 0.991332 & -3273.286030 & - \\
\hline 124-ts & -3272.799338 & 0.844907 & 0.989778 & -3273.255131 & $298.57 i$ \\
\hline 125 & -3272.848956 & 0.847814 & 0.992357 & -3273.303733 & - \\
\hline 126 & -3272.825672 & 0.839043 & 0.988718 & -3273.272411 & - \\
\hline 127-ts & -3272.823427 & 0.837457 & 0.986353 & -3273.270487 & $627.01 i$ \\
\hline 128 & -3272.835483 & 0.837725 & 0.988509 & -3273.281295 & - \\
\hline
\end{tabular}




\begin{tabular}{cccccc}
$\mathbf{1 2 9}$ & -3272.836507 & 0.838971 & 0.988487 & -3273.281139 & - \\
$\mathbf{1 3 0}-\mathbf{t s}$ & -3272.824917 & 0.837648 & 0.986053 & -3273.269437 & $566.83 i$ \\
$\mathbf{1 3 1}$ & -3272.827022 & 0.839114 & 0.988671 & -3273.271544 & - \\
$\mathbf{1 3 2}$ & -3272.845686 & 0.840130 & 0.991270 & -3273.289170 & - \\
$\mathbf{1 3 3 - t s}$ & -3272.810905 & 0.842427 & 0.989887 & -3273.260536 & $378.05 i$ \\
$\mathbf{1 3 4}$ & -3272.842833 & 0.845307 & 0.992697 & -3273.288428 & - \\
$\mathbf{1 3 5}-\mathbf{t s}$ & -3272.797727 & 0.845408 & 0.989905 & -3273.252687 & $300.48 i$ \\
$\mathbf{1 3 6}$ & -3272.846107 & 0.844119 & 0.992211 & -3273.298178 & - \\
$\mathbf{1 3 7}$ & -2003.593562 & 0.503599 & 0.600854 & -2003.851115 & - \\
$\mathbf{1 3 8 - t s}$ & -2003.589284 & 0.501350 & 0.597662 & -2003.848105 & $675.05 i$ \\
$\mathbf{1 3 9}$ & -2003.597876 & 0.501877 & 0.599340 & -2003.851688 & - \\
$\mathbf{1 4 0}$ & -2075.431542 & 0.447551 & 0.548986 & -2075.705942 & - \\
$\mathbf{1 4 1 - t s}$ & -2075.408341 & 0.448925 & 0.547589 & -2075.686414 & $76.58 i$ \\
$\mathbf{1 4 2}$ & -2075.427154 & 0.451717 & 0.551364 & -2075.710781 & - \\
$\mathbf{1 4 3 - t s}$ & -2075.400606 & 0.444578 & 0.546594 & -2075.680361 & $803.09 i$ \\
$\mathbf{1 4 4}$ & -2075.422099 & 0.449134 & 0.551110 & -2075.706176 & - \\
\hline
\end{tabular}

${ }^{1}$ The electronic energy calculated by B3-LYP in gas phase. ${ }^{2}$ The thermal correction to Gibbs free energy calculated by B3-LYP in gas phase. ${ }^{3}$ The thermal correction to enthalpy calculated by B3-LYP in gas phase. ${ }^{4}$ The electronic energy calculated by M06-L in toluene solvent. ${ }^{5}$ The B3-LYP calculated imaginary frequencies for the transition states. 


\section{B3-LYP Geometries for All the Optimized Compounds and Transition States}

R1<smiles>CCCCPn1ccc2cc(F)ccc21</smiles>$$
\text { C } \quad-2.36170600
$$$$
\text { C }-1.48955500
$$$$
\text { C }-1.98240600
$$$$
\text { C } \quad-3.36097200
$$$$
\text { C }-4.21424700
$$$$
\text { C } \quad-3.75399100
$$$$
\text { C }-1.53118300
$$$$
\text { C }-0.23324500
$$$$
\mathrm{H} \quad-1.31102500
$$$$
\text { H } \quad-3.79647900
$$$$
\text { H } \quad-4.45628700
$$$$
\text { H } \quad-1.85974800
$$$$
\text { H } 0.67132400
$$$$
\mathrm{N}-0.16483100
$$$$
\text { P } \quad 1.22236500
$$$$
\text { C } \quad 2.20620200
$$$$
\text { C } 2.13280900
$$$$
\text { C } \quad 3.02953200
$$$$
\text { H } 2.41234800
$$$$
\text { H } \quad 3.51407300
$$$$
\text { H } 3.82309100
$$$$
\text { C } 1.16759500
$$$$
\text { H } 1.69237100
$$$$
\text { H } 0.50456000
$$$$
\text { H } \quad 0.54435800
$$$$
\text { C } \quad 3.15316500
$$$$
\text { H } 2.60343600
$$$$
\text { H } \quad 3.92838800
$$$$
\text { H } \quad 3.66058600
$$$$
\text { C } \quad 3.48693600
$$$$
\text { H } 4.21029100
$$$$
\text { H } 3.37880700
$$$$
\text { H } \quad 3.92187200
$$$$
\text { C } \quad 1.23913100
$$$$
\mathrm{H}
$$

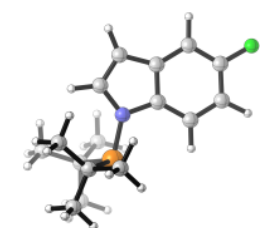

$-0.20005400$ 0.01098400

0.23733400

0.25388600

0.04809300

$-0.17847700$

$-0.40240400$

$-0.30755100$

0.39411500

0.42421000

$-0.33277000$

$-0.59166000$

$-0.40160100$

$-0.05118400$

0.07043900

$-1.54239500$

1.62104200

$-1.64326000$

$-1.54877700$

$-2.62851700$

$-0.89132400$

$-2.68515600$

$-3.64965800$

$-2.66570700$

$-2.64218500$

$-1.71952200$

$-1.69826100$

$-0.94812900$

$-2.69048800$

1.71894200

0.98235400

1.58681100

2.71293900

2.79675300

2.75043700
1.06754600

$-0.03494400$

$-1.32562100$

$-1.50739300$

$-0.41301100$

0.86991100

2.21963100

1.80140500

$-2.16285300$

$-2.48635300$

1.68269200

3.23280000

2.38099600

0.43023200

$-0.65480500$

$-0.30618800$

0.00683900

0.98937800

1.88765500

1.03504800

1.03591800

$-0.34152300$

$-0.34634200$

0.52823500

$-1.24228300$

$-1.51676800$

$-2.46386900$

$-1.55888600$

$-1.44364600$

$-0.72539200$

$-0.36049700$

$-1.80857100$

$-0.55730500$

$-0.45276800$

0.00204800
H 1.69952700

H 1.11517800

3.74762700

$-0.15287000$

C 2.35167200

2.81011600

$-1.54109400$

H 2.87768200

1.74485300

1.52492200

2.68591700

1.73752600

H 1.40226200

1.77599300

2.06696700

H 2.95889700

0.93345400

1.93494300

F $\quad-5.55103800$

0.07426700

$-0.63621600$

R2
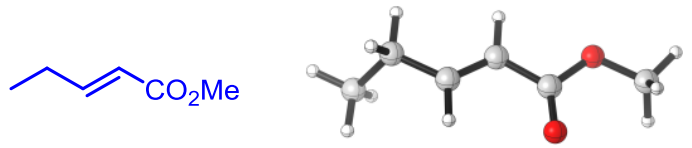

C 1.11830500

0.15949500

$-0.06529400$

O 1.31201000

1.35851200

$-0.13444100$

O 2.11333900

$-0.74434400$

0.11697900

C 3.43010400

$-0.18789700$

0.22438200

H 3.49131600

0.49846900

1.07374000

H 4.09786700

$-1.03794000$

0.36982700

Н 3.69464900

0.35664000

$-0.68657800$

C -0.19305300

$-0.51938800$

$-0.16292200$

H $\quad-0.19567100$

$-1.60312100$

$-0.08160700$

C $\quad-1.31794200$

0.18360200

$-0.34647900$

H $\quad-1.22653700$

1.26827600

$-0.41422400$

C $\quad-2.69810700$

$-0.39248000$

$-0.45198300$

H $\quad-3.11231000$

$-0.13773500$

$-1.43904200$

H $\quad-2.65410600$

$-1.48722200$

$-0.40076500$

C $\quad-3.64548500$

0.15051100

0.63391500

H $\quad-3.71694200$

1.24332800

0.58622500

H $\quad-4.65370300$

$-0.25877900$

0.50782000

H $\quad-3.29029400$

$-0.11831100$

1.63458300

coe
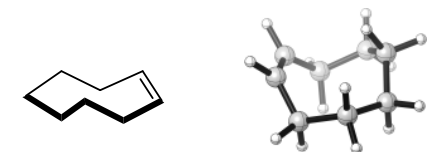

C $\quad-1.73016800$

0.78178500

$-0.04783800$

C -1.65590200

$-0.69962500$

0.39202200

C 1.86599300

0.51255300

0.06011600

C -0.74810900

$-1.57358100$

$-0.44540500$

C $\quad 1.39204600$

$-0.83697700$

0.65049800

C $\quad 0.58219700$

$-1.65336700$

$-0.32108400$ 


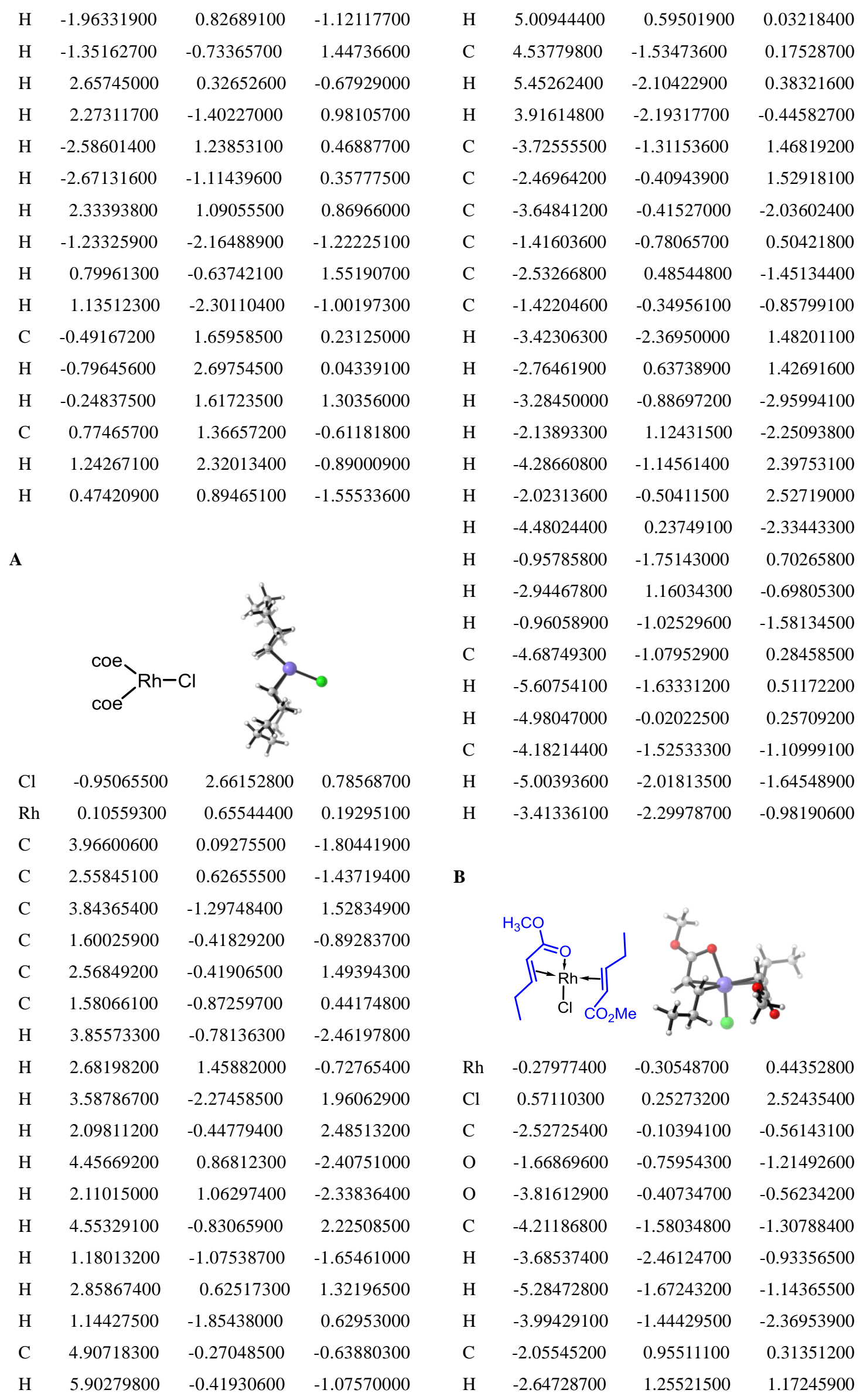




\begin{tabular}{|c|c|c|c|c|c|c|c|}
\hline $\mathrm{C}$ & -0.90170900 & 1.67825500 & -0.10982200 & $\mathrm{H}$ & 0.56576600 & 3.19099700 & -2.02536000 \\
\hline $\mathrm{H}$ & -0.65574500 & 1.66363100 & -1.17291700 & $\mathrm{H}$ & 4.79659800 & 3.58257500 & -2.48711400 \\
\hline $\mathrm{C}$ & -0.43726800 & 2.91207000 & 0.62174500 & $\mathrm{H}$ & 6.28829200 & 1.09017100 & -1.58770600 \\
\hline $\mathrm{H}$ & -0.64538700 & 2.81424500 & 1.69112300 & $\mathrm{H}$ & 5.34610100 & -1.17004100 & -0.52071000 \\
\hline $\mathrm{H}$ & 0.65137000 & 2.99051100 & 0.52307800 & $\mathrm{~N}$ & 3.39755000 & -0.26110600 & -0.67260600 \\
\hline $\mathrm{C}$ & -1.09605100 & 4.18424400 & 0.05476400 & $\mathrm{P}$ & 2.30333000 & -1.24050100 & 0.31662700 \\
\hline $\mathrm{H}$ & -2.18526700 & 4.15548300 & 0.17441500 & $\mathrm{C}$ & 3.17949900 & -1.22492500 & 2.03379500 \\
\hline $\mathrm{H}$ & -0.72234100 & 5.07174600 & 0.57706200 & $\mathrm{C}$ & 2.39086900 & -2.96613100 & -0.52703000 \\
\hline $\mathrm{H}$ & -0.87937300 & 4.30680100 & -1.01336500 & $\mathrm{C}$ & 4.48913400 & -2.03385100 & 2.12636000 \\
\hline $\mathrm{C}$ & 2.47553100 & 0.30886100 & -0.75010900 & $\mathrm{H}$ & 5.26769900 & -1.66399900 & 1.45551700 \\
\hline $\mathrm{O}$ & 3.36457400 & 0.45657200 & 0.06001400 & $\mathrm{H}$ & 4.88025400 & -1.93889200 & 3.14785400 \\
\hline $\mathrm{O}$ & 2.27252900 & 1.15266200 & -1.79786800 & $\mathrm{H}$ & 4.34053600 & -3.10019500 & 1.93747500 \\
\hline $\mathrm{C}$ & 3.19912100 & 2.24561100 & -1.88197200 & $\mathrm{C}$ & 3.48310400 & 0.25765400 & 2.34732100 \\
\hline $\mathrm{H}$ & 4.22372400 & 1.87494800 & -1.97447200 & $\mathrm{H}$ & 3.86025400 & 0.32955800 & 3.37560700 \\
\hline $\mathrm{H}$ & 2.91258600 & 2.80582200 & -2.77273800 & $\mathrm{H}$ & 4.24432000 & 0.67683200 & 1.68238100 \\
\hline $\mathrm{H}$ & 3.13261500 & 2.87714400 & -0.99150100 & $\mathrm{H}$ & 2.58330900 & 0.87687800 & 2.27405600 \\
\hline $\mathrm{C}$ & 1.48504700 & -0.80568400 & -0.78270700 & $\mathrm{C}$ & 2.20507100 & -1.75950400 & 3.10564400 \\
\hline $\mathrm{H}$ & 1.04443400 & -1.00360500 & -1.75841400 & $\mathrm{H}$ & 1.27864300 & -1.18430200 & 3.14001500 \\
\hline $\mathrm{C}$ & 1.41864000 & -1.75495200 & 0.23255900 & $\mathrm{H}$ & 1.93334600 & -2.80532200 & 2.94530300 \\
\hline $\mathrm{H}$ & 2.06299800 & -1.60060000 & 1.09562200 & $\mathrm{H}$ & 2.69876300 & -1.68632300 & 4.08386600 \\
\hline $\mathrm{C}$ & 0.85604400 & -3.14634500 & 0.05177600 & $\mathrm{C}$ & 1.94264300 & -4.07804800 & 0.44533700 \\
\hline $\mathrm{H}$ & 0.25878400 & -3.41948500 & 0.93252800 & $\mathrm{H}$ & 2.66866700 & -4.24700700 & 1.24676300 \\
\hline $\mathrm{H}$ & 0.18462200 & -3.17392900 & -0.81535600 & $\mathrm{H}$ & 0.96859300 & -3.86406500 & 0.88880900 \\
\hline $\mathrm{C}$ & 1.97910400 & -4.18808400 & -0.12471200 & $\mathrm{H}$ & 1.86041300 & -5.01594000 & -0.11944300 \\
\hline $\mathrm{H}$ & 2.65516600 & -4.18784200 & 0.73749800 & $\mathrm{C}$ & 1.39325200 & -2.89829700 & -1.70450300 \\
\hline $\mathrm{H}$ & 1.55848700 & -5.19501700 & -0.22512000 & $\mathrm{H}$ & 1.65172800 & -2.10069500 & -2.41090800 \\
\hline $\mathrm{H}$ & 0 & 0 & 400 & $\mathrm{H}$ & 1.42447600 & -3.85002500 & -2.25117800 \\
\hline & & & & $\mathrm{C}$ & 3.77921800 & -3.32605800 & -1.09351200 \\
\hline C & & & & $\mathrm{H}$ & 3.71217300 & -4.32083700 & -1.55240700 \\
\hline & & & & $\mathrm{H}$ & 4.10047000 & -2.62970800 & -1.87263700 \\
\hline & & & & $\mathrm{H}$ & 4.55594400 & -3.38034400 & -0.32489500 \\
\hline & & & & $\mathrm{F}$ & 2.47170000 & 4.71014000 & -2.77007700 \\
\hline & & & & $\mathrm{Rh}$ & 0.25698900 & -0.07503300 & 0.30749500 \\
\hline & & & & $\mathrm{Cl}$ & -0.73852600 & -1.95499500 & 1.34833600 \\
\hline & & & & $\mathrm{H}$ & 0.37246100 & -2.73476000 & -1.35189900 \\
\hline $\mathrm{C}$ & 4.11704700 & 1.70 & -1.59456300 & $\mathrm{C}$ & -4.77869900 & -1.19216900 & -0.65744200 \\
\hline $\mathrm{C}$ & 2.97732100 & 0.97414900 & -1.16419800 & $\mathrm{C}$ & -3.42191400 & -0.77026300 & -0.64031500 \\
\hline $\mathrm{C}$ & 1.67742100 & 1.481 & -1.28715200 & $\mathrm{C}$ & -2.49943800 & -1.26518000 & -1.56890700 \\
\hline $\mathrm{C}$ & 1.53762700 & 2.75 & -1.84580300 & $\mathrm{C}$ & -2.94055200 & -2.18741100 & -2.51114600 \\
\hline $\mathrm{C}$ & 2.66804700 & 3.48056300 & -2.23967100 & $\mathrm{C}$ & -4.28026500 & -2.60105300 & -2.51447500 \\
\hline $\mathrm{C}$ & 3.95656400 & 2.99001200 & -2.14005700 & $\mathrm{C}$ & -5.21271300 & -2.12936100 & -1.61178100 \\
\hline $\mathrm{C}$ & 5.25493900 & 0.86525600 & -1.36050800 & $\mathrm{C}$ & -5.45162100 & -0.48756700 & 0.39408100 \\
\hline $\mathrm{C}$ & 4.78962500 & -0.29584300 & -0.81212700 & $\mathrm{C}$ & -4.52659300 & 0.31641900 & 0.99264000 \\
\hline $\mathrm{H}$ & 0.76778800 & 0.81347300 & -1.25969500 & $\mathrm{H}$ & -1.45782800 & -0.96131500 & -1.53615100 \\
\hline
\end{tabular}




\begin{tabular}{|c|c|c|c|c|c|c|c|}
\hline $\mathrm{H}$ & -2.26091700 & -2.60383100 & -3.24738000 & $\mathrm{C}$ & -0.18228100 & 4.21558400 & -0.12092900 \\
\hline $\mathrm{H}$ & -6.24000100 & -2.47644200 & -1.65322800 & $\mathrm{C}$ & -1.55650700 & 4.28585300 & 0.04911800 \\
\hline $\mathrm{H}$ & -6.49347600 & -0.57164800 & 0.67241200 & $\mathrm{C}$ & -3.66948100 & 2.72223300 & 0.08851400 \\
\hline $\mathrm{H}$ & -4.66614600 & 1.00133800 & 1.81180900 & $\mathrm{C}$ & -3.74881400 & 1.36466700 & -0.06416000 \\
\hline $\mathrm{N}$ & -3.26759100 & 0.16882900 & 0.40062000 & $\mathrm{H}$ & 1.57371300 & 3.07736200 & -0.57959100 \\
\hline $\mathrm{P}$ & -1.82025300 & 1.11612700 & 0.73578400 & $\mathrm{H}$ & -2.03139900 & 5.24524400 & 0.22603700 \\
\hline $\mathrm{C}$ & -1.90332100 & 1.54065200 & 2.63312800 & $\mathrm{H}$ & -4.50560200 & 3.38906200 & 0.25189300 \\
\hline $\mathrm{C}$ & -2.20183100 & 2.70322300 & -0.28795300 & $\mathrm{H}$ & -4.62356200 & 0.73480500 & -0.05257000 \\
\hline $\mathrm{C}$ & -2.82932500 & 2.70103800 & 3.05728000 & $\mathrm{~N}$ & -2.47861300 & 0.81613500 & -0.28364600 \\
\hline $\mathrm{H}$ & -3.88482400 & 2.52318400 & 2.83625200 & $\mathrm{P}$ & -1.79850000 & -0.80076600 & -0.03201100 \\
\hline $\mathrm{H}$ & -2.74769300 & 2.81353300 & 4.14619300 & $\mathrm{C}$ & -2.58420700 & -1.84202400 & -1.42365700 \\
\hline $\mathrm{H}$ & -2.54448500 & 3.65832900 & 2.61514900 & $\mathrm{C}$ & -2.43351000 & -1.27004700 & 1.72107900 \\
\hline $\mathrm{C}$ & -2.29726200 & 0.28848600 & 3.44705400 & $\mathrm{C}$ & -4.07011900 & -1.53307600 & -1.69069600 \\
\hline $\mathrm{H}$ & -2.17743400 & 0.52706600 & 4.51242900 & $\mathrm{H}$ & -4.21976600 & -0.49703300 & -2.00822900 \\
\hline $\mathrm{H}$ & -3.33761700 & -0.00554800 & 3.28862400 & $\mathrm{H}$ & -4.41733600 & -2.17792400 & -2.50833700 \\
\hline $\mathrm{H}$ & -1.67077000 & -0.57173500 & 3.21071400 & $\mathrm{H}$ & -4.71096000 & -1.73291700 & -0.82753300 \\
\hline $\mathrm{C}$ & -0.45666500 & 1.92972100 & 3.02181100 & $\mathrm{C}$ & -1.77712300 & -1.49698900 & -2.69617300 \\
\hline $\mathrm{H}$ & 0.24361600 & 1.11963300 & 2.79673600 & $\mathrm{H}$ & -2.18673400 & -2.06354700 & -3.54275900 \\
\hline $\mathrm{H}$ & -0.10906300 & 2.82992500 & 2.50527600 & $\mathrm{H}$ & -1.84559700 & -0.43148000 & -2.94426500 \\
\hline $\mathrm{H}$ & -0.42247500 & 2.13153200 & 4.10068000 & $\mathrm{H}$ & -0.72070000 & -1.75710700 & -2.58236100 \\
\hline $\mathrm{C}$ & -1.17638900 & 3.80144300 & 0.06320000 & $\mathrm{C}$ & -2.40665400 & -3.34188000 & -1.10973700 \\
\hline $\mathrm{H}$ & -1.38645800 & 4.26126800 & 1.03291400 & $\mathrm{H}$ & -1.36980700 & -3.58740600 & -0.86182600 \\
\hline $\mathrm{H}$ & -0.14896200 & 3.42931500 & 0.08454700 & $\mathrm{H}$ & -3.05236000 & -3.66893400 & -0.28864800 \\
\hline $\mathrm{H}$ & -1.22623400 & 4.60025600 & -0.68849100 & $\mathrm{H}$ & -2.69111000 & -3.92067500 & -1.99798500 \\
\hline $\mathrm{C}$ & -2.06159700 & 2.29153800 & -1.77250400 & $\mathrm{C}$ & -1.68468200 & -2.52100500 & 2.22840100 \\
\hline $\mathrm{H}$ & -2.85150500 & 1.59482400 & -2.06702400 & $\mathrm{H}$ & -1.90124600 & -3.41283900 & 1.63541200 \\
\hline $\mathrm{H}$ & -2.15331800 & 3.18522500 & -2.40392900 & $\mathrm{H}$ & -0.60274700 & -2.38159800 & 2.22753500 \\
\hline $\mathrm{H}$ & -1.10640000 & 1.81383000 & -1.99940400 & $\mathrm{H}$ & -2.01321300 & -2.71743900 & 3.25759800 \\
\hline $\mathrm{C}$ & -3.62943500 & 3.26634500 & -0.12629300 & $\mathrm{C}$ & -2.06143900 & -0.08124400 & 2.63597200 \\
\hline $\mathrm{H}$ & -3.75100900 & 4.10188800 & -0.82867300 & $\mathrm{H}$ & -2.62140300 & 0.82663000 & 2.39191000 \\
\hline $\mathrm{H}$ & -4.39223000 & 2.52149400 & -0.36817400 & $\mathrm{H}$ & -2.29697000 & -0.35156600 & 3.67313000 \\
\hline $\mathrm{H}$ & -3.83043800 & 3.65512600 & 0.87290200 & $\mathrm{C}$ & -3.94870100 & -1.53604400 & 1.81564700 \\
\hline \multirow[t]{2}{*}{ F } & -4.66713500 & -3.50085000 & -3.45163300 & $\mathrm{H}$ & -4.19759300 & -1.74320100 & 2.86456600 \\
\hline & & & & $\mathrm{H}$ & -4.55297800 & -0.67930700 & 1.50761000 \\
\hline & & & & $\mathrm{H}$ & -4.25516000 & -2.41051700 & 1.23402400 \\
\hline & & & & $\mathrm{F}$ & 0.53873000 & 5.35878100 & -0.05169200 \\
\hline & & & & $\mathrm{Rh}$ & 0.51984500 & -0.36568200 & -0.18922600 \\
\hline & & & & $\mathrm{Cl}$ & 1.02868300 & -2.65703700 & 0.12565300 \\
\hline & & & & $\mathrm{H}$ & -0.99113200 & 0.14458800 & 2.58110300 \\
\hline & & & & $\mathrm{H}$ & 0.22444700 & 1.02112100 & -1.21145400 \\
\hline $\mathrm{C}$ & 100 & 3.0 & -0.02630900 & $\mathrm{C}$ & 4.65601000 & -0.08100600 & 1.91422200 \\
\hline $\mathrm{C}$ & -1.58232300 & 1.8 & -0.27149000 & $\mathrm{C}$ & 3.17968000 & -0.45881000 & 1.64243700 \\
\hline $\mathrm{C}$ & 3600 & 7700 & -0.46825900 & $\mathrm{C}$ & 4.95684700 & -0.46325200 & -1.66593400 \\
\hline $\mathrm{C}$ & 0.50578800 & 3.026 & -0.39545000 & $\mathrm{C}$ & 2.54053300 & 0.37794100 & 0.55547400 \\
\hline
\end{tabular}




\begin{tabular}{|c|c|c|c|c|c|c|c|}
\hline $\mathrm{C}$ & 3.51447800 & -0.95366800 & -1.39563700 & $\mathrm{H}$ & 4.31932700 & -3.46809300 & 2.91651000 \\
\hline $\mathrm{C}$ & 2.66363700 & 0.14291800 & -0.80673700 & $\mathrm{H}$ & 4.99935900 & -3.32964500 & 1.29482800 \\
\hline $\mathrm{H}$ & 4.73851800 & 1.00534900 & 2.06789500 & $\mathrm{C}$ & 2.37245100 & -1.56379600 & 2.75066500 \\
\hline $\mathrm{H}$ & 3.11376600 & -1.52318600 & 1.40615600 & $\mathrm{H}$ & 2.32642100 & -2.21511600 & 3.63367100 \\
\hline $\mathrm{H}$ & 4.95401300 & 0.22400400 & -2.52426800 & $\mathrm{H}$ & 2.94195700 & -0.66869800 & 3.02507800 \\
\hline $\mathrm{H}$ & 3.07870800 & -1.30676900 & -2.33820800 & $\mathrm{H}$ & 1.35662300 & -1.25255300 & 2.48974100 \\
\hline $\mathrm{H}$ & 4.93621500 & -0.54350100 & 2.87045500 & $\mathrm{C}$ & 2.17835100 & -3.56481500 & 1.24629100 \\
\hline $\mathrm{H}$ & 2.60931200 & -0.31288800 & 2.56869400 & $\mathrm{H}$ & 1.19374800 & -3.27644700 & 0.86401100 \\
\hline $\mathrm{H}$ & 5.55072600 & -1.33435300 & -1.97621100 & $\mathrm{H}$ & 2.66063100 & -4.21692500 & 0.51124500 \\
\hline $\mathrm{H}$ & 2.25472700 & 1.37850100 & 0.87606900 & $\mathrm{H}$ & 2.02450900 & -4.15788200 & 2.15696600 \\
\hline $\mathrm{H}$ & 3.53071900 & -1.81637900 & -0.72739400 & $\mathrm{C}$ & 2.48505600 & -2.71812900 & -2.17082400 \\
\hline $\mathrm{H}$ & 2.48440900 & 0.98163000 & -1.48276900 & $\mathrm{H}$ & 2.12459900 & -3.55020300 & -1.55944800 \\
\hline $\mathrm{C}$ & 5.69164200 & -0.51823900 & 0.85713300 & $\mathrm{H}$ & 1.63768500 & -2.06224700 & -2.37922800 \\
\hline $\mathrm{H}$ & 6.68163000 & -0.37937600 & 1.31105100 & $\mathrm{H}$ & 2.83541300 & -3.13656900 & -3.12356400 \\
\hline $\mathrm{H}$ & 5.59754200 & -1.59964600 & 0.68192900 & $\mathrm{C}$ & 4.06008200 & -0.79342400 & -2.45278000 \\
\hline $\mathrm{C}$ & 5.66649500 & 0.24103700 & -0.49270200 & $\mathrm{H}$ & 4.95437200 & -0.26960200 & -2.10225100 \\
\hline $\mathrm{H}$ & 6.69941200 & 0.43034100 & -0.81308000 & $\mathrm{H}$ & 4.27964900 & -1.20474400 & -3.44635500 \\
\hline \multirow[t]{2}{*}{$\mathrm{H}$} & 5.22270900 & 1.23349900 & -0.33637500 & $\mathrm{C}$ & 4.84735900 & -2.92245200 & -1.36242200 \\
\hline & & & & $\mathrm{H}$ & 5.14785000 & -3.26489900 & -2.36131000 \\
\hline \multirow[t]{7}{*}{$\mathbf{E}$} & & & & $\mathrm{H}$ & 5.72391400 & -2.45142300 & -0.90940500 \\
\hline & & & & $\mathrm{H}$ & 4.58814200 & -3.81338600 & -0.78239000 \\
\hline & & & & $\mathrm{F}$ & 3.62814100 & 5.32868600 & -0.31995700 \\
\hline & & & & $\mathrm{Rh}$ & 1.26067000 & 0.24243400 & -0.13043500 \\
\hline & & & & $\mathrm{Cl}$ & -0.41121000 & -1.39296300 & -0.73669500 \\
\hline & coe & & & $\mathrm{H}$ & 3.25008600 & -0.06439300 & -2.55846600 \\
\hline & & & & $\mathrm{H}$ & 1.91441200 & 1.37080300 & 0.99416900 \\
\hline $\mathrm{C}$ & 5.19743700 & 2.10623400 & 0.21757400 & $\mathrm{Cl}$ & -0.60281600 & 1.77564400 & -0.48857600 \\
\hline $\mathrm{C}$ & 4.02328400 & 1.33467700 & 0.33329300 & $\mathrm{Rh}$ & -2.38354500 & 0.05723900 & -0.18355900 \\
\hline $\mathrm{C}$ & 2.71895600 & 1.84939400 & 0.25486400 & $\mathrm{C}$ & -4.48276400 & -3.71914600 & -1.64721400 \\
\hline $\mathrm{C}$ & 2.61186500 & 3.22990200 & 0.03217800 & $\mathrm{C}$ & -3.54577700 & -2.48716500 & -1.65234700 \\
\hline $\mathrm{C}$ & 3.77537900 & 4.00004000 & -0.10266000 & $\mathrm{C}$ & -3.59730200 & -3.83478200 & 1.85789100 \\
\hline $\mathrm{C}$ & 5.06271100 & 3.48979000 & -0.00053200 & $\mathrm{C}$ & -3.87297200 & -1.47780400 & -0.56849600 \\
\hline $\mathrm{C}$ & 6.29117900 & 1.17833600 & 0.35052500 & $\mathrm{C}$ & -2.65544800 & -2.73387800 & 1.31410200 \\
\hline $\mathrm{C}$ & 5.75167900 & -0.07104400 & 0.50828900 & $\mathrm{C}$ & -3.45359000 & -1.56976700 & 0.77358900 \\
\hline $\mathrm{H}$ & 1.64360800 & 3.71515700 & -0.01634700 & $\mathrm{H}$ & -5.53057000 & -3.38342500 & -1.64303000 \\
\hline $\mathrm{H}$ & 5.91501600 & 4.15541000 & -0.08928300 & $\mathrm{H}$ & -2.50973500 & -2.82848600 & -1.57954800 \\
\hline $\mathrm{H}$ & 7.34810400 & 1.40995100 & 0.33810700 & $\mathrm{H}$ & -4.06321800 & -3.48555900 & 2.79039200 \\
\hline $\mathrm{H}$ & 6.25784600 & -1.01392900 & 0.64337600 & $\mathrm{H}$ & -1.99363900 & -2.40293600 & 2.12446100 \\
\hline $\mathrm{N}$ & 4.35179500 & -0.00378700 & 0.50336900 & $\mathrm{H}$ & -4.33716800 & -4.24249000 & -2.60218800 \\
\hline $\mathrm{P}$ & 3.04989100 & -1.13575300 & 0.11047800 & $\mathrm{H}$ & -3.63501900 & -1.98891400 & -2.62652500 \\
\hline $\mathrm{C}$ & 3.04435000 & -2.33647800 & 1.59280900 & $\mathrm{H}$ & -2.97663400 & -4.69938700 & 2.13271000 \\
\hline $\mathrm{C}$ & 3.65761700 & -1.95546500 & -1.51661200 & $\mathrm{H}$ & -4.81794700 & -0.96415700 & -0.74761500 \\
\hline $\mathrm{C}$ & 4.44087800 & -2.78884000 & 2.06255900 & $\mathrm{H}$ & -2.00941000 & -3.15263300 & 0.54081000 \\
\hline $\mathrm{H}$ & 5.04386500 & -1.94296100 & 2.40532200 & $\mathrm{H}$ & -4.09655000 & -1.11946500 & 1.52904800 \\
\hline
\end{tabular}




\begin{tabular}{|c|c|c|c|c|c|c|c|}
\hline $\mathrm{C}$ & -4.28202100 & -4.74427500 & -0.51133600 & $\mathrm{C}$ & 2.79561100 & 0.51290400 & -1.81095300 \\
\hline $\mathrm{H}$ & -4.86152300 & -5.63550200 & -0.78517900 & $\mathrm{H}$ & 2.01724000 & 0.46399700 & -2.56686700 \\
\hline $\mathrm{H}$ & -3.23181300 & -5.07058600 & -0.49669800 & $\mathrm{C}$ & 3.46366500 & -0.66069300 & -1.42465800 \\
\hline $\mathrm{C}$ & -4.71811200 & -4.29858400 & 0.90671200 & $\mathrm{H}$ & 4.40451600 & -0.52910000 & -0.89520600 \\
\hline $\mathrm{H}$ & -5.22890300 & -5.13522500 & 1.40110100 & $\mathrm{C}$ & 3.31969400 & -1.98835100 & -2.13282100 \\
\hline $\mathrm{H}$ & -5.47382900 & -3.50717500 & 0.81564900 & $\mathrm{H}$ & 3.36610000 & -2.80057200 & -1.39588000 \\
\hline $\mathrm{C}$ & -4.21267400 & 3.55093500 & 2.10433800 & $\mathrm{H}$ & 2.34512300 & -2.06066200 & -2.62634600 \\
\hline $\mathrm{C}$ & -3.20661100 & 2.44277500 & 1.70859200 & $\mathrm{C}$ & 4.44890600 & -2.18185000 & -3.16485600 \\
\hline $\mathrm{C}$ & -4.79437400 & 3.71367100 & -1.46400600 & $\mathrm{H}$ & 5.43421200 & -2.13238300 & -2.68767900 \\
\hline $\mathrm{C}$ & -3.80032700 & 1.38590000 & 0.79841800 & $\mathrm{H}$ & 4.35669300 & -3.15788000 & -3.65438000 \\
\hline $\mathrm{C}$ & -3.57899700 & 2.76123300 & -1.36488300 & $\mathrm{H}$ & 4.41094700 & -1.40773600 & -3.93967400 \\
\hline $\mathrm{C}$ & -3.94503600 & 1.51245100 & -0.59725600 & $\mathrm{C}$ & 4.30323100 & -1.34988100 & 1.55226300 \\
\hline $\mathrm{H}$ & -5.13033700 & 3.08951800 & 2.49947000 & $\mathrm{O}$ & 5.34941500 & -1.08902600 & 0.98771800 \\
\hline $\mathrm{H}$ & -2.32347700 & 2.90040100 & 1.25604200 & $\mathrm{O}$ & 4.07627800 & -2.53085100 & 2.17436100 \\
\hline $\mathrm{H}$ & -5.53933700 & 3.28489800 & -2.14945000 & $\mathrm{C}$ & 5.15985800 & -3.47115900 & 2.12877000 \\
\hline $\mathrm{H}$ & -3.24413700 & 2.50576600 & -2.37769200 & $\mathrm{H}$ & 6.05434400 & -3.04943500 & 2.59538300 \\
\hline $\mathrm{H}$ & -3.76942900 & 4.10658700 & 2.94204100 & $\mathrm{H}$ & 4.81229900 & -4.34368800 & 2.68228400 \\
\hline $\mathrm{H}$ & -2.85788000 & 1.95071300 & 2.62625800 & $\mathrm{H}$ & 5.39250700 & -3.74016300 & 1.09468400 \\
\hline $\mathrm{H}$ & -4.44828500 & 4.64497800 & -1.93367700 & $\mathrm{C}$ & 3.12014900 & -0.45751000 & 1.70175600 \\
\hline $\mathrm{H}$ & -4.53182500 & 0.75604200 & 1.30547900 & $\mathrm{H}$ & 2.44987700 & -0.77602200 & 2.49480900 \\
\hline $\mathrm{H}$ & -2.74697900 & 3.28303500 & -0.88970700 & $\mathrm{C}$ & 3.11256600 & 0.88179500 & 1.28101300 \\
\hline $\mathrm{H}$ & -4.75820800 & 0.95867200 & -1.06535300 & $\mathrm{H}$ & 3.98021300 & 1.21616900 & 0.71657500 \\
\hline $\mathrm{C}$ & -4.59721000 & 4.57067600 & 1.01187200 & $\mathrm{C}$ & 2.35094000 & 1.98139300 & 1.98442000 \\
\hline $\mathrm{H}$ & -5.12400200 & 5.39005300 & 1.51810900 & $\mathrm{H}$ & 1.97939300 & 2.69827700 & 1.24071800 \\
\hline $\mathrm{H}$ & -3.68402000 & 5.02150200 & 0.59729200 & $\mathrm{H}$ & 1.47576000 & 1.57472800 & 2.50083200 \\
\hline $\mathrm{C}$ & -5.49922000 & 4.04883900 & -0.13468200 & $\mathrm{C}$ & 3.25801200 & 2.72363400 & 2.98646100 \\
\hline $\mathrm{H}$ & -6.26203600 & 4.80557700 & -0.35973400 & $\mathrm{H}$ & 4.13221100 & 3.15507800 & 2.48581100 \\
\hline \multirow[t]{2}{*}{$\mathrm{H}$} & -6.05485500 & 3.1703 & 600 & $\mathrm{H}$ & 2.70742700 & 3.53777800 & 3.47123800 \\
\hline & & & & $\mathrm{H}$ & 3.61900200 & 2.04551500 & 3.76812100 \\
\hline \multirow[t]{3}{*}{$\mathbf{F}$} & & & & $\mathrm{Rh}$ & -1.81554300 & -0.28898800 & 0.03792500 \\
\hline & & & & $\mathrm{C}$ & -4.30309000 & -1.34977500 & -1.55258600 \\
\hline \multirow{2}{*}{$\begin{array}{l}\mathrm{R} 2 \\
\mathrm{R}\end{array}$} & & & & $\mathrm{O}$ & -5.34934200 & -1.08907500 & -0.98807800 \\
\hline & & & & $\mathrm{O}$ & -4.07595800 & -2.53068800 & -2.17470900 \\
\hline & & & & $\mathrm{C}$ & -5.15938300 & -3.47117700 & -2.12913100 \\
\hline $\mathrm{Cl}$ & -0.02922700 & -0.80460700 & -1.57851600 & $\mathrm{H}$ & -6.05391500 & -3.04963800 & -2.59582300 \\
\hline $\mathrm{Cl}$ & 0.02917200 & -0.80496900 & 1.57841400 & $\mathrm{H}$ & -4.81164500 & -4.34368300 & -2.68256900 \\
\hline $\mathrm{Rh}$ & 1.81551100 & -0.28902700 & -0.03799300 & $\mathrm{H}$ & -5.39204500 & -3.74015900 & -1.09504100 \\
\hline C & 3.40013900 & 1.86813700 & -1.69056400 & $\mathrm{C}$ & -3.12010600 & -0.45727700 & -1.70193800 \\
\hline $\mathrm{O}$ & 4.47258700 & 2.15029000 & -1.18959200 & $\mathrm{H}$ & -2.44977200 & -0.77559800 & -2.49501600 \\
\hline $\mathrm{O}$ & 2.58970700 & 2.79258900 & -2.26138000 & $\mathrm{C}$ & -3.11257100 & 0.88198600 & -1.28104100 \\
\hline $\mathrm{C}$ & 3.08637600 & 4.13920600 & -2.24787300 & $\mathrm{H}$ & -3.98021300 & 1.21626900 & -0.71652300 \\
\hline H & 4.04911300 & 4.19898900 & -2.76270800 & $\mathrm{C}$ & -2.35086100 & 1.98169600 & -1.98414900 \\
\hline 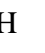 & 2.33464700 & 4.73311700 & -2.76828900 & $\mathrm{H}$ & -1.97842400 & 2.69786600 & -1.24019100 \\
\hline $\mathrm{H}$ & 3.21127700 & 4.49359300 & -1.22079600 & $\mathrm{H}$ & -1.47621500 & 1.57495200 & -2.50141300 \\
\hline
\end{tabular}




\begin{tabular}{|c|c|c|c|c|c|c|c|}
\hline $\mathrm{C}$ & -3.25822300 & 2.72514300 & -2.98503600 & $\mathrm{H}$ & -6.44084800 & -1.20125200 & 1.27853400 \\
\hline $\mathrm{H}$ & -4.13181000 & 3.15672500 & -2.48343900 & $\mathrm{~N}$ & -4.65388900 & -0.09743800 & 0.76746600 \\
\hline $\mathrm{H}$ & -2.70753100 & 3.53930800 & -3.46965200 & $\mathrm{P}$ & -3.33998200 & -1.18660800 & 0.30338400 \\
\hline $\mathrm{H}$ & -3.62017500 & 2.04778200 & -3.76691200 & $\mathrm{C}$ & -4.09826400 & -2.20211700 & -1.13956700 \\
\hline $\mathrm{C}$ & -3.40013100 & 1.86778800 & 1.69077500 & $\mathrm{C}$ & -3.04803000 & -2.23215100 & 1.87349800 \\
\hline $\mathrm{O}$ & -4.47249000 & 2.14999500 & 1.18963200 & $\mathrm{C}$ & -5.16908700 & -3.22987000 & -0.72595500 \\
\hline $\mathrm{O}$ & -2.58980700 & 2.79217300 & 2.26184200 & $\mathrm{H}$ & -6.00914900 & -2.77615800 & -0.19282000 \\
\hline $\mathrm{C}$ & -3.08647100 & 4.13878700 & 2.24841100 & $\mathrm{H}$ & -5.57735300 & -3.69272500 & -1.63402000 \\
\hline $\mathrm{H}$ & -4.04948400 & 4.19845000 & 2.76274000 & $\mathrm{H}$ & -4.75812700 & -4.03592200 & -0.11057400 \\
\hline $\mathrm{H}$ & -2.33501700 & 4.73257800 & 2.76935900 & $\mathrm{C}$ & -4.72537700 & -1.16978700 & -2.10419200 \\
\hline $\mathrm{H}$ & -3.21081800 & 4.49343600 & 1.22135300 & $\mathrm{H}$ & -5.04742300 & -1.69192400 & -3.01435500 \\
\hline $\mathrm{C}$ & -2.79566300 & 0.51253700 & 1.81114000 & $\mathrm{H}$ & -5.60202600 & -0.67748700 & -1.67250100 \\
\hline $\mathrm{H}$ & -2.01724500 & 0.46346700 & 2.56697500 & $\mathrm{H}$ & -4.00287700 & -0.39927200 & -2.39232700 \\
\hline $\mathrm{C}$ & -3.46378500 & -0.66090600 & 1.42459400 & $\mathrm{C}$ & -2.96654500 & -2.93706600 & -1.89019000 \\
\hline $\mathrm{H}$ & -4.40460400 & -0.52918800 & 0.89512200 & $\mathrm{H}$ & -2.22109600 & -2.24167400 & -2.28179700 \\
\hline $\mathrm{C}$ & -3.31981600 & -1.98875700 & 2.13240500 & $\mathrm{H}$ & -2.44893800 & -3.67189400 & -1.26726700 \\
\hline $\mathrm{H}$ & -3.36607800 & -2.80079100 & 1.39524900 & $\mathrm{H}$ & -3.40806400 & -3.47621400 & -2.73860300 \\
\hline $\mathrm{H}$ & -2.34530700 & -2.06113900 & 2.62604300 & $\mathrm{C}$ & -2.16092200 & -3.44154700 & 1.51603800 \\
\hline $\mathrm{C}$ & -4.44915900 & -2.18259200 & 3.16423800 & $\mathrm{H}$ & -2.69731900 & -4.18629400 & 0.91953700 \\
\hline $\mathrm{H}$ & -5.43439700 & -2.13309500 & 2.68692600 & $\mathrm{H}$ & -1.26138200 & -3.14020900 & 0.97003300 \\
\hline $\mathrm{H}$ & -4.35693200 & -3.15872900 & 3.653 & $\mathrm{H}$ & -1.84172500 & -3.93470700 & 2.44321000 \\
\hline \multirow[t]{2}{*}{$\mathrm{H}$} & -4.41137000 & -1.40865700 & 3.93924100 & $\mathrm{C}$ & -2.27556400 & -1.31215500 & 2.84546200 \\
\hline & & & & $\mathrm{H}$ & -2.86471300 & -0.43040600 & 3.12114100 \\
\hline \multirow[t]{5}{*}{ G } & & & & $\mathrm{H}$ & -2.05634400 & -1.86962400 & 3.76587000 \\
\hline & & & & $\mathrm{H}$ & -1.33369700 & -0.96663100 & 2.40990300 \\
\hline & & & & $\mathrm{C}$ & -4.33069200 & -2.70899000 & 2.58175100 \\
\hline & & & & $\mathrm{H}$ & -4.04531400 & -3.28216800 & 3.47360700 \\
\hline & & & & $\mathrm{H}$ & -4.93968900 & -1.86625200 & 2.92127600 \\
\hline $\mathrm{Cl}$ & -0.06123500 & 1.88710900 & -1.13405000 & $\mathrm{H}$ & -4.94852900 & -3.36220500 & 1.96084100 \\
\hline $\mathrm{Cl}$ & 0.01342000 & -1.34702100 & -0.95695700 & $\mathrm{~F}$ & -4.46225900 & 5.13985700 & -0.70536300 \\
\hline $\mathrm{Rh}$ & 1.75929700 & 0.31404600 & -0.63 & $\mathrm{C}$ & 5.42856100 & 2.13073100 & 0.90266200 \\
\hline $\mathrm{Rh}$ & -1.70865100 & 0.25884700 & -0.33169900 & $\mathrm{C}$ & 4.43096300 & 1.38970800 & 0.23779200 \\
\hline $\mathrm{C}$ & -5.68896100 & 1.91055000 & 0.38884200 & $\mathrm{C}$ & 3.21069200 & 1.91841200 & -0.21398700 \\
\hline $\mathrm{C}$ & -4.45407500 & 1.23094100 & 0.41 & $\mathrm{C}$ & 2.99235800 & 3.27927400 & 0.04566100 \\
\hline $\mathrm{C}$ & -3.21641400 & 1.81357500 & 0.09660800 & $\mathrm{C}$ & 3.97167100 & 4.01745200 & 0.72509400 \\
\hline $\mathrm{C}$ & -3.24764000 & 3.16287900 & -0.28468600 & $\mathrm{C}$ & 5.18793000 & 3.49573400 & 1.14557300 \\
\hline $\mathrm{C}$ & -4.47447900 & 3.83916700 & -0.33034000 & $\mathrm{C}$ & 6.49076700 & 1.19770500 & 1.17959800 \\
\hline $\mathrm{C}$ & -5.69241300 & 3.26528800 & 0.00988100 & $\mathrm{C}$ & 6.09292500 & -0.02720000 & 0.71212700 \\
\hline $\mathrm{C}$ & -6.68082700 & 0.93850700 & 0.77152400 & $\mathrm{H}$ & 2.81635400 & 1.51732100 & -1.26534000 \\
\hline $\mathrm{C}$ & -6.03142000 & -0.24953300 & 0.97861700 & $\mathrm{H}$ & 2.08868900 & 3.77383500 & -0.29197300 \\
\hline $\mathrm{H}$ & -2.28163600 & 1.47505000 & 0.75100100 & $\mathrm{H}$ & 5.90822600 & 4.13960700 & 1.63953800 \\
\hline $\mathrm{H}$ & -2.33500200 & 3.69748900 & -0.52227300 & $\mathrm{H}$ & 7.43715500 & 1.41049000 & 1.65916500 \\
\hline $\mathrm{H}$ & -6.59789600 & 3.86214500 & -0.02501400 & $\mathrm{H}$ & 6.62522900 & -0.96515000 & 0.73291300 \\
\hline $\mathrm{H}$ & -7.74434100 & 1.10229000 & 0.88544400 & $\mathrm{~N}$ & 4.82345700 & 0.06271700 & 0.12468500 \\
\hline
\end{tabular}




\begin{tabular}{|c|c|c|c|c|c|c|c|}
\hline $\mathrm{P}$ & 3.49762000 & -1.07072400 & -0.16403300 & $\mathrm{H}$ & -7.18123500 & 1.21264400 & -0.91845600 \\
\hline $\mathrm{C}$ & 3.37269200 & -2.02562400 & 1.50042100 & $\mathrm{H}$ & -6.03036300 & -1.19308000 & -0.71249400 \\
\hline $\mathrm{C}$ & 4.13901400 & -2.15556500 & -1.59718300 & $\mathrm{~N}$ & -4.16745100 & -0.11383900 & -0.51177500 \\
\hline $\mathrm{C}$ & 4.53362800 & -2.99431000 & 1.79523200 & $\mathrm{P}$ & -2.91265000 & -1.13201900 & 0.20774100 \\
\hline $\mathrm{H}$ & 5.50918600 & -2.50101700 & 1.79690200 & $\mathrm{C}$ & -2.71423900 & -2.55447000 & -1.04806600 \\
\hline $\mathrm{H}$ & 4.38669400 & -3.41787300 & 2.79750900 & $\mathrm{C}$ & -3.69440500 & -1.69610200 & 1.86746900 \\
\hline $\mathrm{H}$ & 4.56526300 & -3.83293700 & 1.09340300 & $\mathrm{C}$ & -4.04360600 & -3.08935800 & -1.61672600 \\
\hline $\mathrm{C}$ & 3.31465600 & -0.94705200 & 2.60622900 & $\mathrm{H}$ & -4.58935700 & -2.31518800 & -2.16374100 \\
\hline $\mathrm{H}$ & 3.12446300 & -1.44169700 & 3.56761800 & $\mathrm{H}$ & -3.81965600 & -3.89470300 & -2.32818100 \\
\hline $\mathrm{H}$ & 4.25342800 & -0.39233700 & 2.69628200 & $\mathrm{H}$ & -4.70130000 & -3.50773800 & -0.85050200 \\
\hline $\mathrm{H}$ & 2.50518600 & -0.23183700 & 2.42748500 & $\mathrm{C}$ & -1.88708200 & -1.96656500 & -2.21343600 \\
\hline $\mathrm{C}$ & 2.04935300 & -2.81749400 & 1.53277200 & $\mathrm{H}$ & -1.75751100 & -2.74060900 & -2.98159500 \\
\hline $\mathrm{H}$ & 1.18618000 & -2.16440800 & 1.39400500 & $\mathrm{H}$ & -2.39277400 & -1.11361200 & -2.68031000 \\
\hline $\mathrm{H}$ & 2.00492300 & -3.59995100 & 0.77045100 & $\mathrm{H}$ & -0.89850400 & -1.63799400 & -1.88022000 \\
\hline $\mathrm{H}$ & 1.96077800 & -3.30414000 & 2.51309400 & $\mathrm{C}$ & -1.91629200 & -3.70489800 & -0.40101000 \\
\hline $\mathrm{C}$ & 3.22655800 & -3.39019300 & -1.74502000 & $\mathrm{H}$ & -0.98839500 & -3.35271800 & 0.06059600 \\
\hline $\mathrm{H}$ & 3.36952100 & -4.11234700 & -0.93457900 & $\mathrm{H}$ & -2.50032500 & -4.24194600 & 0.35323500 \\
\hline $\mathrm{H}$ & 2.16732800 & -3.11560300 & -1.78528800 & $\mathrm{H}$ & -1.64627500 & -4.42878100 & -1.18039300 \\
\hline $\mathrm{H}$ & 3.47245200 & -3.90285600 & -2.68391900 & $\mathrm{C}$ & -2.60133200 & -2.32123000 & 2.76158500 \\
\hline $\mathrm{C}$ & 4.01001000 & -1.28433100 & -2.86757000 & $\mathrm{H}$ & -2.16026800 & -3.22425400 & 2.33116300 \\
\hline $\mathrm{H}$ & 4.62098500 & -0.37711900 & -2.80028900 & $\mathrm{H}$ & -1.79345300 & -1.61662300 & 2.96866900 \\
\hline $\mathrm{H}$ & 4.36323800 & -1.86241600 & -3.73180800 & $\mathrm{H}$ & -3.05932100 & -2.60184300 & 3.71902800 \\
\hline $\mathrm{H}$ & 2.97420200 & -0.98232800 & -3.04716900 & $\mathrm{C}$ & -4.20087900 & -0.40874800 & 2.55682200 \\
\hline $\mathrm{C}$ & 5.60976500 & -2.59642900 & -1.46423400 & $\mathrm{H}$ & -5.04187400 & 0.04503700 & 2.02374900 \\
\hline $\mathrm{H}$ & 5.87628400 & -3.19504000 & -2.34501100 & $\mathrm{H}$ & -4.54310600 & -0.66460300 & 3.56774800 \\
\hline $\mathrm{H}$ & 6.28681300 & -1.73749800 & -1.43993800 & $\mathrm{C}$ & -4.85818400 & -2.69699200 & 1.73550500 \\
\hline $\mathrm{H}$ & 5.79640800 & -3.21553300 & -0.58341800 & $\mathrm{H}$ & -5.26167900 & -2.89342600 & 2.73735800 \\
\hline \multirow[t]{2}{*}{$\mathrm{F}$} & 3.72245400 & 5.32760600 & 0.95847100 & $\mathrm{H}$ & -5.68241600 & -2.31189900 & 1.12936800 \\
\hline & & & & $\mathrm{H}$ & -4.53788300 & -3.65843200 & 1.32360500 \\
\hline \multirow[t]{5}{*}{ H } & & & & $\mathrm{F}$ & -3.62300400 & 5.30360700 & -0.45586300 \\
\hline & & & & $\mathrm{Rh}$ & -1.18454900 & 0.32360400 & 0.44404500 \\
\hline & & & & $\mathrm{Cl}$ & 0.42822200 & -1.16925600 & 1.44976300 \\
\hline & & & & $\mathrm{H}$ & -3.40420700 & 0.33710900 & 2.64679400 \\
\hline & & & & $\mathrm{H}$ & -1.70938100 & 1.24872600 & -0.91866500 \\
\hline $\mathrm{C}$ & -5.06978400 & 1.9870 & -0.66073500 & $\mathrm{Cl}$ & 0.61613700 & 1.94568200 & 0.78126200 \\
\hline $\mathrm{C}$ & -3.87958200 & 1.24469100 & -0.51787400 & $\mathrm{Rh}$ & 2.19276500 & 0.12443100 & 0.28792600 \\
\hline $\mathrm{C}$ & -2.60240000 & 1.81012000 & -0.37381500 & $\mathrm{C}$ & 3.27656800 & -2.01328500 & -1.74239500 \\
\hline $\mathrm{C}$ & -2.54121800 & 3.21108200 & -0.35657600 & $\mathrm{O}$ & 4.30286500 & -1.67916900 & -2.30413000 \\
\hline $\mathrm{C}$ & -3.72329300 & 3.95418800 & -0.47847900 & $\mathrm{O}$ & 2.26572000 & -2.65380300 & -2.38484100 \\
\hline $\mathrm{C}$ & -4.98145600 & 3.39091000 & -0.64788000 & $\mathrm{C}$ & 2.49492400 & -2.91709100 & -3.77652600 \\
\hline $\mathrm{C}$ & -6.12749300 & 1.01547500 & -0.77182900 & $\mathrm{H}$ & 3.39068500 & -3.52979500 & -3.91046300 \\
\hline $\mathrm{C}$ & -5.55663700 & -0.22512300 & -0.66825400 & $\mathrm{H}$ & 1.60962600 & -3.45103700 & -4.12333800 \\
\hline $\mathrm{H}$ & -1.59257700 & 3.72919500 & -0.27251700 & $\mathrm{H}$ & 2.61955900 & -1.98264500 & -4.33091400 \\
\hline $\mathrm{H}$ & -5.84793100 & 4.03372300 & -0.76344700 & $\mathrm{C}$ & 2.94080400 & -1.83447600 & -0.30479400 \\
\hline
\end{tabular}




\begin{tabular}{|c|c|c|c|c|c|c|c|}
\hline $\mathrm{H}$ & 2.16283600 & -2.51121900 & 0.03527000 & $\mathrm{H}$ & -4.57234300 & 3.27701000 & 2.2 \\
\hline $\mathrm{C}$ & 3.83089100 & -1.27803400 & 0.62968500 & $\mathrm{H}$ & -1.75554300 & 2.91003300 & 1.06157100 \\
\hline $\mathrm{H}$ & 4.74803000 & -0.85068500 & 0.23055500 & $\mathrm{H}$ & -4.87384300 & 3.17044100 & -2.42749800 \\
\hline $\mathrm{C}$ & 3.91645300 & -1.71320100 & 2.07529200 & $\mathrm{H}$ & -2.61765300 & 2.27960000 & -2.55014000 \\
\hline $\mathrm{H}$ & 4.11839500 & -0.83889400 & 2.70749000 & $\mathrm{H}$ & -3.18489800 & 4.27456400 & 2.63849100 \\
\hline $\mathrm{H}$ & 2.96177700 & -2.13238000 & 2.40955800 & $\mathrm{H}$ & -2.33962900 & 2.07402800 & 2.48516900 \\
\hline $\mathrm{C}$ & 5.04376300 & -2.74615600 & 2.27229500 & $\mathrm{H}$ & -3.73205000 & 4.49688700 & -2.27541300 \\
\hline $\mathrm{H}$ & 6.01255800 & -2.33818600 & 1.96241000 & $\mathrm{H}$ & -4.04130900 & 0.85329000 & 1.22302800 \\
\hline $\mathrm{H}$ & 5.11865500 & -3.03594700 & 3.32660000 & $\mathrm{H}$ & -2.10845800 & 3.13619000 & -1.10998800 \\
\hline $\mathrm{H}$ & 4.85671400 & -3.65131900 & 1.68315100 & $\mathrm{H}$ & -4.21607600 & 0.89229400 & -1.16191000 \\
\hline $\mathrm{C}$ & 4.90012400 & 1.70292900 & 0.51938300 & $\mathrm{C}$ & -3.95622100 & 4.63327000 & 0.66312300 \\
\hline $\mathrm{O}$ & 5.85350400 & 1.01936900 & 0.19392600 & $\mathrm{H}$ & -4.46535600 & 5.50239300 & 1.09963700 \\
\hline $\mathrm{O}$ & 4.93715900 & 2.56709300 & 1.56165400 & $\mathrm{H}$ & -3.01824800 & 5.02374500 & 0.24203200 \\
\hline $\mathrm{C}$ & 6.18326100 & 2.62709500 & 2.27032800 & $\mathrm{C}$ & -4.84886900 & 4.06525900 & -0.46847400 \\
\hline $\mathrm{H}$ & 6.99488300 & 2.91786700 & 1.59765700 & $\mathrm{H}$ & -5.57735100 & 4.83109000 & -0.76502500 \\
\hline $\mathrm{H}$ & 6.04073000 & 3.37753400 & 3.04825400 & $\mathrm{H}$ & -5.44455700 & 3.23308500 & -0.07050900 \\
\hline $\mathrm{H}$ & 6.42113800 & 1.65595800 & 2.71348600 & $\mathrm{C}$ & -4.06865900 & -3.82812600 & -1.45833000 \\
\hline $\mathrm{C}$ & 3.56927600 & 1.76237500 & -0.14574800 & $\mathrm{C}$ & -3.09436100 & -2.62649300 & -1.50571700 \\
\hline $\mathrm{H}$ & 3.00570100 & 2.65093000 & 0.12099000 & $\mathrm{C}$ & -3.32411500 & -3.73861600 & 2.08100400 \\
\hline $\mathrm{C}$ & 3.27842600 & 1.09034500 & -1.34427300 & $\mathrm{C}$ & -3.42860900 & -1.53964300 & -0.50278100 \\
\hline $\mathrm{H}$ & 4.04140500 & 0.40857300 & -1.71263200 & $\mathrm{C}$ & -2.32533900 & -2.70752900 & 1.50325500 \\
\hline $\mathrm{C}$ & 2.34713100 & 1.63204500 & -2.40507400 & $\mathrm{C}$ & -3.06161700 & -1.55671600 & 0.85685100 \\
\hline $\mathrm{H}$ & 1.80384400 & 0.79970200 & -2.87146600 & $\mathrm{H}$ & -5.10526500 & -3.46396300 & -1.51865700 \\
\hline $\mathrm{H}$ & 1.59764000 & 2.29045200 & -1.95498000 & $\mathrm{H}$ & -2.07261500 & -2.99076900 & -1.37169900 \\
\hline $\mathrm{C}$ & 3.13440700 & 2.39021900 & -3.49244000 & $\mathrm{H}$ & -3.81421500 & -3.31305200 & 2.96828900 \\
\hline $\mathrm{H}$ & 3.88126600 & 1.74246400 & -3.96557500 & $\mathrm{H}$ & -1.68108000 & -2.34490500 & 2.31407100 \\
\hline $\mathrm{H}$ & 2.45720900 & 2.75505000 & -4.27316100 & $\mathrm{H}$ & -3.90209600 & -4.41735500 & -2.37049100 \\
\hline \multirow[t]{2}{*}{$\mathrm{H}$} & $3.65 \mathrm{c}$ & 3.25369700 & 0 & $\mathrm{H}$ & -3.13204300 & -2.19021800 & -2.51252000 \\
\hline & & & & $\mathrm{H}$ & -2.74313900 & -4.60070700 & 2.43781600 \\
\hline \multirow[t]{5}{*}{1} & & & & $\mathrm{H}$ & -4.34706400 & -1.00763500 & -0.75161900 \\
\hline & & & & $\mathrm{H}$ & -1.66654000 & -3.19902200 & 0.78553900 \\
\hline & & & & $\mathrm{H}$ & -3.71245300 & -1.03156100 & 1.55470700 \\
\hline & & & & $\mathrm{C}$ & -3.94300300 & -4.78133400 & -0.25087200 \\
\hline & & & & $\mathrm{H}$ & -4.53708500 & -5.67284900 & -0.49047400 \\
\hline $\mathrm{Cl}$ & -0.02557700 & 1.56475500 & -0.52356200 & $\mathrm{H}$ & -2.90414300 & -5.13364500 & -0.17262200 \\
\hline $\mathrm{Cl}$ & 0.02556800 & -1.56477300 & -0.52393300 & $\mathrm{C}$ & -4.42100600 & -4.23262500 & 1.11684200 \\
\hline $\mathrm{Rh}$ & 1.89480100 & 0.03409000 & -0.16603300 & $\mathrm{H}$ & -4.97510600 & -5.02132000 & 1.64238400 \\
\hline $\mathrm{Rh}$ & -1.89481700 & -0.03408800 & -0.16595600 & $\mathrm{H}$ & -5.14906600 & -3.42892100 & 0.94418200 \\
\hline $\mathrm{C}$ & -3.63134800 & 3.67822400 & 1.83089600 & $\mathrm{C}$ & 4.06887600 & 3.82792300 & -1.45843700 \\
\hline $\mathrm{C}$ & -2.65914400 & 2.51239300 & 1.53039600 & $\mathrm{C}$ & 3.09453700 & 2.62631600 & -1.50572600 \\
\hline $\mathrm{C}$ & -4.12678400 & 3.61373800 & -1.75374900 & $\mathrm{C}$ & 3.32409200 & 3.73879600 & 2.08086000 \\
\hline $\mathrm{C}$ & -3.27641300 & 1.41483700 & 0.68634100 & $\mathrm{C}$ & 3.42865600 & 1.53958600 & -0.50261600 \\
\hline $\mathrm{C}$ & -2.95622400 & 2.61966600 & -1.56356600 & $\mathrm{C}$ & 2.32521200 & 2.70778400 & 1.50315200 \\
\hline $\mathrm{C}$ & -3.38833400 & 1.44401200 & -0.71732600 & $\mathrm{C}$ & 3.06139600 & 1.55681300 & 0.85694300 \\
\hline
\end{tabular}




\begin{tabular}{|c|c|c|c|c|c|c|c|}
\hline $\mathrm{H}$ & 5.10547000 & 3.46370900 & -1.51867600 & & & & \\
\hline $\mathrm{H}$ & 2.07279300 & 2.99064600 & -1.37181700 & & & & \\
\hline $\mathrm{H}$ & 3.81410600 & 3.31324000 & 2.96819400 & & & & \\
\hline $\mathrm{H}$ & 1.68084500 & 2.34534200 & 2.31396100 & & & & \\
\hline $\mathrm{H}$ & 3.90238100 & 4.41706100 & -2.37066900 & & & & \\
\hline $\mathrm{H}$ & 3.13226900 & 2.18990200 & -2.51246600 & & & & \\
\hline $\mathrm{H}$ & 2.74319200 & 4.60097300 & 2.43758900 & $\mathrm{C}$ & 5.19743700 & 2.10623400 & 0.21757400 \\
\hline $\mathrm{H}$ & 4.34716100 & 1.00755700 & -0.75123200 & $\mathrm{C}$ & 4.02328400 & 1.33467700 & 0.33329300 \\
\hline $\mathrm{H}$ & 1.66654000 & 3.19930000 & 0.78533700 & $\mathrm{C}$ & 2.71895600 & 1.84939400 & 0.25486400 \\
\hline $\mathrm{H}$ & 3.71202700 & 1.03159400 & 1.55494300 & $\mathrm{C}$ & 2.61186500 & 3.22990200 & 0.03217800 \\
\hline $\mathrm{C}$ & 3.94320100 & 4.78125500 & -0.25108000 & $\mathrm{C}$ & 3.77537900 & 4.00004000 & -0.10266000 \\
\hline $\mathrm{H}$ & 4.53735600 & 5.67271100 & -0.49071800 & $\mathrm{C}$ & 5.06271100 & 3.48979000 & -0.00053200 \\
\hline $\mathrm{H}$ & 2.90435900 & 5.13364000 & -0.17293500 & $\mathrm{C}$ & 6.29117900 & 1.17833600 & 0.35052500 \\
\hline $\mathrm{C}$ & 4.42107600 & 4.23262600 & 1.11671300 & $\mathrm{C}$ & 5.75167900 & -0.07104400 & 0.50828900 \\
\hline $\mathrm{H}$ & 4.97521100 & 5.02132000 & 1.64222000 & $\mathrm{H}$ & 1.64360800 & 3.71515700 & -0.01634700 \\
\hline $\mathrm{H}$ & 5.14908100 & 3.42884900 & 0.94416300 & $\mathrm{H}$ & 5.91501600 & 4.15541000 & -0.08928300 \\
\hline $\mathrm{C}$ & 3.63122300 & -3.67813100 & 1.83102300 & $\mathrm{H}$ & 7.34810400 & 1.40995100 & 0.33810700 \\
\hline $\mathrm{C}$ & 2.65905400 & -2.51229000 & 1.53044400 & $\mathrm{H}$ & 6.25784600 & -1.01392900 & 0.64337600 \\
\hline $\mathrm{C}$ & 4.12683400 & -3.61381900 & -1.75360600 & $\mathrm{~N}$ & 4.35179500 & -0.00378700 & 0.50336900 \\
\hline $\mathrm{C}$ & 3.27637600 & -1.41479500 & 0.68635500 & $\mathrm{P}$ & 3.04989100 & -1.13575300 & 0.11047800 \\
\hline $\mathrm{C}$ & 2.95627900 & -2.61973100 & -1.56349900 & $\mathrm{C}$ & 3.04435000 & -2.33647800 & 1.59280900 \\
\hline $\mathrm{C}$ & 3.38837400 & -1.44404600 & -0.71729500 & $\mathrm{C}$ & 3.65761700 & -1.95546500 & -1.51661200 \\
\hline $\mathrm{H}$ & 4.57221300 & -3.27691500 & 2.23648700 & $\mathrm{C}$ & 4.44087800 & -2.78884000 & 2.06255900 \\
\hline $\mathrm{H}$ & 1.75545500 & -2.90992200 & 1.06160000 & $\mathrm{H}$ & 5.04386500 & -1.94296100 & 2.40532200 \\
\hline $\mathrm{H}$ & 4.87394000 & -3.17055200 & -2.42732200 & $\mathrm{H}$ & 4.31932700 & -3.46809300 & 2.91651000 \\
\hline $\mathrm{H}$ & 2.61772900 & -2.27970600 & -2.55009200 & $\mathrm{H}$ & 4.99935900 & -3.32964500 & 1.29482800 \\
\hline $\mathrm{H}$ & 3.18474000 & -4.27443000 & 2.63862900 & $\mathrm{C}$ & 2.37245100 & -1.56379600 & 2.75066500 \\
\hline $\mathrm{H}$ & 2.33951900 & -2.07387200 & 2.48518500 & $\mathrm{H}$ & 2.32642100 & -2.21511600 & 3.63367100 \\
\hline $\mathrm{H}$ & 3.73212000 & -4.49697800 & -2.27526800 & $\mathrm{H}$ & 2.94195700 & -0.66869800 & 3.02507800 \\
\hline $\mathrm{H}$ & 4.04123100 & -0.85318400 & 1.22304300 & $\mathrm{H}$ & 1.35662300 & -1.25255300 & 2.48974100 \\
\hline $\mathrm{H}$ & 2.10850200 & -3.13623200 & -1.10991600 & $\mathrm{C}$ & 2.17835100 & -3.56481500 & 1.24629100 \\
\hline $\mathrm{H}$ & 4.21612200 & -0.89233400 & -1.16187700 & $\mathrm{H}$ & 1.19374800 & -3.27644700 & 0.86401100 \\
\hline $\mathrm{C}$ & 3.95611300 & -4.63324000 & 0.66330500 & $\mathrm{H}$ & 2.66063100 & -4.21692500 & 0.51124500 \\
\hline $\mathrm{H}$ & 4.46519400 & -5.50236300 & 1.09988600 & $\mathrm{H}$ & 2.02450900 & -4.15788200 & 2.15696600 \\
\hline $\mathrm{H}$ & 3.01814500 & -5.02369900 & 0.24219000 & $\mathrm{C}$ & 2.48505600 & -2.71812900 & -2.17082400 \\
\hline $\mathrm{C}$ & 4.84884000 & -4.06532700 & -0.46827900 & $\mathrm{H}$ & 2.12459900 & -3.55020300 & -1.55944800 \\
\hline $\mathrm{H}$ & 5.57728800 & -4.83121600 & -0.76476600 & $\mathrm{H}$ & 1.63768500 & -2.06224700 & -2.37922800 \\
\hline \multirow[t]{2}{*}{$\mathrm{H}$} & 5.44456200 & -3.23317200 & -0.07032300 & $\mathrm{H}$ & 2.83541300 & -3.13656900 & -3.12356400 \\
\hline & & & & $\mathrm{C}$ & 4.06008200 & -0.79342400 & -2.45278000 \\
\hline & & & & $\mathrm{H}$ & 4.95437200 & -0.26960200 & -2.10225100 \\
\hline & & & & $\mathrm{H}$ & 4.27964900 & -1.20474400 & -3.44635500 \\
\hline & & & & $\mathrm{C}$ & 4.84735900 & -2.92245200 & -1.36242200 \\
\hline & & & & $\mathrm{H}$ & 5.14785000 & -3.26489900 & -2.36131000 \\
\hline & & & & $\mathrm{H}$ & 5.72391400 & -2.45142300 & -0.90940500 \\
\hline
\end{tabular}




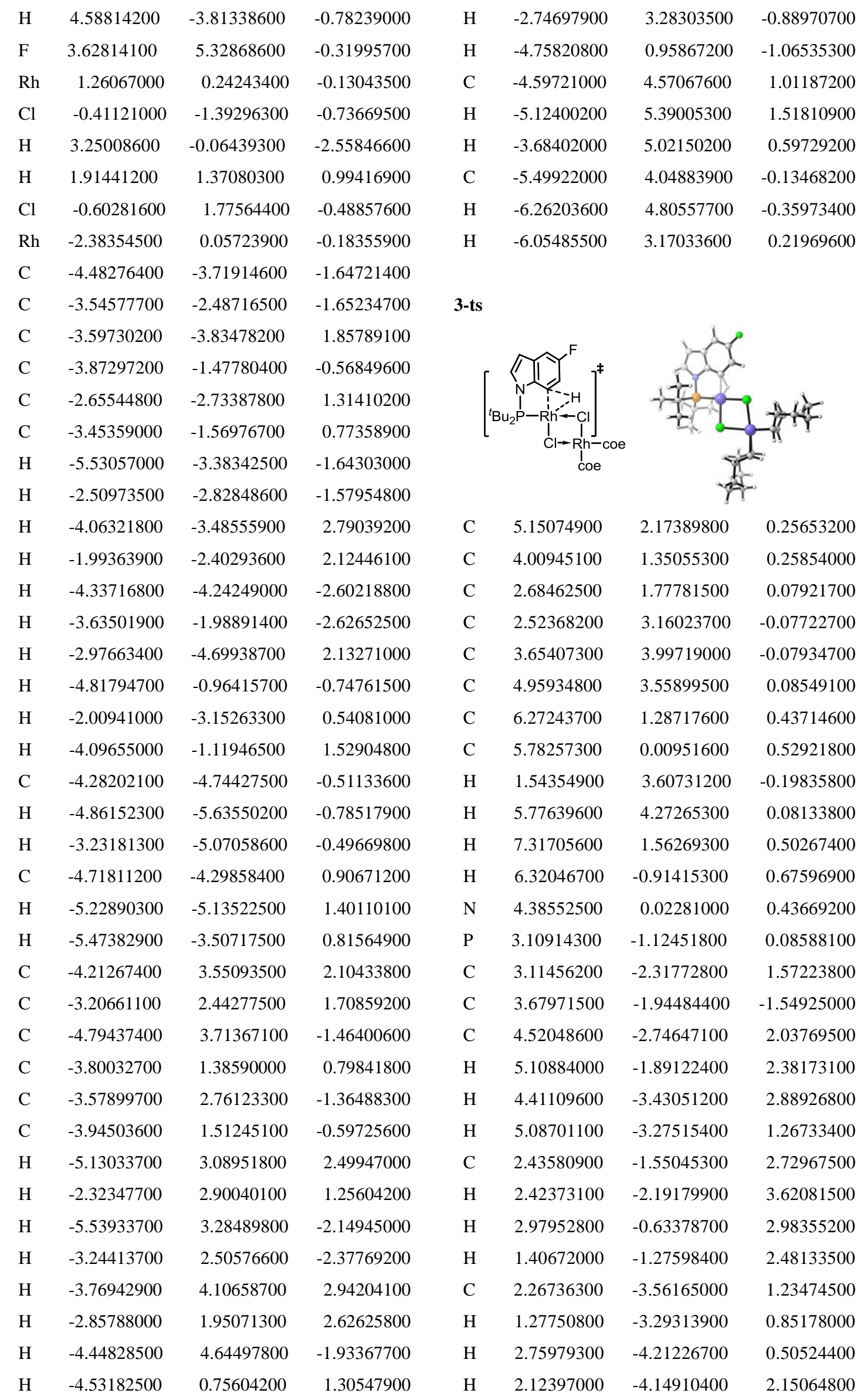




\begin{tabular}{|c|c|c|c|c|c|c|c|}
\hline C & 2.51189500 & -2.73643000 & -2.17548700 & $\mathrm{C}$ & -3.55911800 & 2.73843300 & -1.37905500 \\
\hline $\mathrm{H}$ & 2.18605000 & -3.57677200 & -1.55650400 & $\mathrm{C}$ & -3.94161400 & 1.49422800 & -0.61210800 \\
\hline $\mathrm{H}$ & 1.64251100 & -2.10317300 & -2.36472700 & $\mathrm{H}$ & -5.18385400 & 3.08685800 & 2.45201300 \\
\hline $\mathrm{H}$ & 2.84939200 & -3.14618600 & -3.13646800 & $\mathrm{H}$ & -2.35269000 & 2.89001800 & 1.26697900 \\
\hline C & 4.04516400 & -0.78224400 & -2.50016700 & $\mathrm{H}$ & -5.50352000 & 3.26057300 & -2.20313600 \\
\hline $\mathrm{H}$ & 4.92816500 & -0.23262400 & -2.16099500 & $\mathrm{H}$ & -3.20583700 & 2.47757200 & -2.38424100 \\
\hline $\mathrm{H}$ & 4.26711000 & -1.19885800 & -3.49085000 & $\mathrm{H}$ & -3.83067400 & 4.10402500 & 2.91754700 \\
\hline $\mathrm{C}$ & 4.89684600 & -2.87825100 & -1.40198800 & $\mathrm{H}$ & -2.91493300 & 7900 & 2.62980300 \\
\hline $\mathrm{H}$ & 5.19432600 & -3.22279200 & -2.40099700 & $\mathrm{H}$ & -4.41405000 & 4.61994000 & -1.97470300 \\
\hline $\mathrm{H}$ & 5.76362300 & -2.37395300 & -0.96497800 & $\mathrm{H}$ & -4.56233200 & 0.74573900 & 1.28238500 \\
\hline $\mathrm{H}$ & 4.67115200 & -3.76917300 & -0.80825500 & $\mathrm{H}$ & -2.73578000 & 3.26201200 & -0.89081500 \\
\hline F & 3.44159400 & 5.32610100 & -0.24621200 & $\mathrm{H}$ & -4.74666300 & 0.93903400 & -1.09242100 \\
\hline $\mathrm{Rh}$ & 1.29493300 & 0.25834800 & -0.12209500 & $\mathrm{C}$ & -4.61765500 & 1300 & 0.96864500 \\
\hline $\mathrm{Cl}$ & -0.40976500 & -1.41898600 & -0.69115300 & $\mathrm{H}$ & -5.15078900 & 5.38451800 & 1.46049000 \\
\hline $\mathrm{H}$ & 3.21911000 & -0.07213000 & -2.60876200 & $\mathrm{H}$ & -3.69497500 & 5.00573700 & 0.56939400 \\
\hline $\mathrm{H}$ & 1.81913400 & 1.12206200 & 1.09550900 & $\mathrm{C}$ & -5.49967700 & 4.03635000 & -0.19217400 \\
\hline $\mathrm{Cl}$ & -0.60129200 & 1.75746900 & -0.43334900 & $\mathrm{H}$ & -6.25507300 & 4.79476200 & -0.43567000 \\
\hline $\mathrm{Rh}$ & -2.38933500 & 0.03997300 & -0.16709300 & $\mathrm{H}$ & -6.06568100 & 3.1618 & 0.15566700 \\
\hline $\mathrm{C}$ & -4.47833800 & -3.74326400 & -1.63212700 & & & & \\
\hline $\mathrm{C}$ & -3.54136700 & -2.51113800 & -1.63625100 & 7 & & & \\
\hline $\mathrm{C}$ & -3.62485900 & -3.83964400 & 1.88154000 & & & & \\
\hline $\mathrm{C}$ & -3.87803000 & -1.49568300 & -0.56115300 & & & & \\
\hline $\mathrm{C}$ & -2.67874800 & -2.74124700 & 1.34010900 & & & & \\
\hline $\mathrm{C}$ & -3.47256400 & -1.58111400 & 0.78536400 & & & & \\
\hline $\mathrm{H}$ & -5.52618000 & -3.40775800 & -1.63879900 & & & & \\
\hline $\mathrm{H}$ & -2.50588600 & -2.85132900 & -1.55234200 & $\mathrm{C}$ & 5.08721900 & 2.19301400 & 0.51412900 \\
\hline $\mathrm{H}$ & -4.09934400 & -3.48567600 & 2.80785600 & $\mathrm{C}$ & 3.97508900 & 1.34356800 & 0.36266100 \\
\hline $\mathrm{H}$ & -2.02503400 & -2.40492000 & 2.15486000 & $\mathrm{C}$ & 2.66186600 & 1.73332900 & 0.06806100 \\
\hline $\mathrm{H}$ & -4.32450400 & -4.27179700 & -2.58292900 & $\mathrm{C}$ & 2.47578200 & 3.11447500 & -0.02737000 \\
\hline $\mathrm{H}$ & -3.62254400 & -2.01850800 & -2.61402000 & $\mathrm{C}$ & 3.56985900 & 3.98672600 & 0.14747500 \\
\hline $\mathrm{H}$ & -3.00591600 & -4.70213000 & 2.16662900 & $\mathrm{C}$ & 4.86728600 & 3.58137800 & 0.41253900 \\
\hline $\mathrm{H}$ & -4.82005400 & -0.98094100 & -0.75218800 & $\mathrm{C}$ & 6.22128100 & 1.32732000 & 0.72074600 \\
\hline $\mathrm{H}$ & -2.02507000 & -3.16412900 & 0.57556200 & $\mathrm{C}$ & 5.77397400 & 0.03144800 & 0.68245900 \\
\hline $\mathrm{H}$ & -4.12183700 & -1.12545100 & 1.53206900 & $\mathrm{H}$ & 1.50400600 & 3.54883000 & -0.23587000 \\
\hline $\mathrm{C}$ & -4.28715200 & -4.76218600 & -0.48899300 & $\mathrm{H}$ & 5.65750400 & 4.31643100 & 0.52285900 \\
\hline $\mathrm{H}$ & -4.86343000 & -5.65530200 & -0.76340500 & $\mathrm{H}$ & 7.24800200 & 1.62723800 & 0.88770500 \\
\hline $\mathrm{H}$ & -3.23686500 & -5.08754700 & -0.46311200 & $\mathrm{H}$ & 6.33166100 & -0.88448300 & 0.80246700 \\
\hline $\mathrm{C}$ & -4.73652800 & -4.30954800 & 0.92267200 & $\mathrm{~N}$ & 4.38744300 & 0.01355600 & 0.48788800 \\
\hline $\mathrm{H}$ & -5.25087500 & -5.14409700 & 1.41685100 & $\mathrm{P}$ & 3.16350500 & -1.12873200 & 0.02181900 \\
\hline $\mathrm{H}$ & -5.49225300 & -3.51947700 & 0.82062200 & $\mathrm{C}$ & 3.15284800 & -2.46156200 & 1.38391900 \\
\hline $\mathrm{C}$ & -4.25769300 & 3.54527900 & 2.07359400 & $\mathrm{C}$ & 3.74596000 & -1.79087000 & -1.67917300 \\
\hline C & -3.24492800 & 2.43437100 & 1.70331200 & $\mathrm{C}$ & 4.56542900 & -2.88910400 & 1.83441300 \\
\hline $\mathrm{C}$ & -4.77087700 & 3.69213500 & -1.50628900 & $\mathrm{H}$ & 5.10774900 & -2.06110300 & 2.29850900 \\
\hline $\mathrm{C}$ & -3.82191200 & 1.37400300 & 0.78648800 & $\mathrm{H}$ & 4.46113400 & -3.67605300 & 2.59221900 \\
\hline
\end{tabular}




\begin{tabular}{|c|c|c|c|c|c|c|c|}
\hline $\mathrm{H}$ & 5.17337100 & -3.29886500 & 1.02420100 & $\mathrm{H}$ & -4.99197000 & -5.63199500 & -0.55029200 \\
\hline $\mathrm{C}$ & 2.42983400 & -1.85266000 & 2.60592400 & $\mathrm{H}$ & -3.35711400 & -5.08605800 & -0.25403600 \\
\hline $\mathrm{H}$ & 2.48918600 & -2.56544500 & 3.43856200 & $\mathrm{C}$ & -4.85487700 & -4.22428200 & 1.08388400 \\
\hline $\mathrm{H}$ & 2.89588200 & -0.91655700 & 2.93157200 & $\mathrm{H}$ & -5.39284300 & -5.02784100 & 1.60360900 \\
\hline $\mathrm{H}$ & 1.37470000 & -1.66042600 & 2.39600200 & $\mathrm{H}$ & -5.59202700 & -3.42286700 & 0.94240400 \\
\hline $\mathrm{C}$ & 2.36168500 & -3.69454000 & 0.90032200 & $\mathrm{C}$ & -4.15366500 & 3.66035900 & 1.96556700 \\
\hline $\mathrm{H}$ & 1.37294800 & -3.42509900 & 0.51580500 & $\mathrm{C}$ & -3.16831700 & 2.51668300 & 1.62214400 \\
\hline $\mathrm{H}$ & 2.89879800 & -4.25559700 & 0.12932700 & $\mathrm{C}$ & -4.72153900 & 3.67606400 & -1.60927600 \\
\hline $\mathrm{H}$ & 2.21586600 & -4.37119900 & 1.75197900 & $\mathrm{C}$ & -3.78305700 & 1.43294200 & 0.75869800 \\
\hline $\mathrm{C}$ & 2.59667700 & -2.55911800 & -2.36633400 & $\mathrm{C}$ & -3.52828800 & 2.70163600 & -1.46473300 \\
\hline $\mathrm{H}$ & 2.32990300 & -3.47988300 & -1.84191000 & $\mathrm{C}$ & -3.92278400 & 1.49733800 & -0.64134900 \\
\hline $\mathrm{H}$ & 1.69032600 & -1.95400800 & -2.45646500 & $\mathrm{H}$ & -5.08168000 & 3.23522100 & 2.37672100 \\
\hline $\mathrm{H}$ & 2.92043900 & -2.83657200 & -3.37790200 & $\mathrm{H}$ & -2.27625500 & 2.93928300 & 1.15354200 \\
\hline $\mathrm{C}$ & 4.07470800 & -0.53925000 & -2.52578200 & $\mathrm{H}$ & -5.47449400 & 3.23359400 & -2.27702100 \\
\hline $\mathrm{H}$ & 4.91108900 & 0.03201200 & -2.11296800 & $\mathrm{H}$ & -3.20022300 & 2.39390300 & -2.46532700 \\
\hline $\mathrm{H}$ & 4.35152000 & -0.86463600 & -3.53657200 & $\mathrm{H}$ & -3.70156700 & 4.24305400 & 2.77962800 \\
\hline $\mathrm{C}$ & 4.99707400 & -2.68687400 & -1.61186900 & $\mathrm{H}$ & -2.83057300 & 2.05886300 & 2.56133500 \\
\hline $\mathrm{H}$ & 5.30829100 & -2.93118400 & -2.63585100 & $\mathrm{H}$ & -4.35324800 & 4.57705600 & -2.11929800 \\
\hline $\mathrm{H}$ & 5.84132100 & -2.18640200 & -1.12766000 & $\mathrm{H}$ & -4.52900200 & 0.84441400 & 1.29305500 \\
\hline $\mathrm{H}$ & 4.80582500 & -3.63321900 & -1.09797900 & $\mathrm{H}$ & -2.68500800 & 3.22615900 & -1.01265800 \\
\hline $\mathrm{F}$ & 3.32362900 & 5.31772500 & 0.04130700 & $\mathrm{H}$ & -4.74893000 & 0.94251300 & -1.08477700 \\
\hline $\mathrm{Rh}$ & 1.32867700 & 0.23622800 & -0.18451500 & $\mathrm{C}$ & -4.51236200 & 4.63847800 & 0.82727400 \\
\hline $\mathrm{Cl}$ & -0.45478400 & -1.50196700 & -0.59241900 & $\mathrm{H}$ & -5.02239800 & 5.49090200 & 1.29443200 \\
\hline $\mathrm{H}$ & 3.21620500 & 0.13460800 & -2.61745200 & $\mathrm{H}$ & -3.58776100 & 5.04995800 & 0.39736600 \\
\hline $\mathrm{H}$ & 1.26997500 & 0.28548200 & 1.32688100 & $\mathrm{C}$ & -5.42209800 & 4.08662300 & -0.29884400 \\
\hline $\mathrm{Cl}$ & -0.57653400 & 1.70352400 & -0.52320700 & $\mathrm{H}$ & -6.16603600 & 4.84999600 & -0.56132800 \\
\hline $\mathrm{Rh}$ & -2.39619800 & 0.02540000 & -0.15723400 & $\mathrm{H}$ & 70400 & 3.23807300 & 0.09154700 \\
\hline $\mathrm{C}$ & -4.55768800 & -3.76355500 & -1.48835000 & & & & \\
\hline $\mathrm{C}$ & -3.59381900 & -2.55312400 & -1.52931300 & 5 & & & \\
\hline $\mathrm{C}$ & -3.74336400 & -3.74183500 & 2.03693000 & & & & \\
\hline $\mathrm{C}$ & -3.91724100 & -1.49103700 & -0.49680300 & & & & \\
\hline $\mathrm{C}$ & -2.76444500 & -2.68972200 & 1.46268500 & & & & \\
\hline $\mathrm{C}$ & -3.52314100 & -1.53286200 & 0.85495000 & & & & \\
\hline $\mathrm{H}$ & -5.59811300 & -3.40694400 & -1.52023800 & & & & \\
\hline $\mathrm{H}$ & -2.56688800 & -2.91143200 & -1.42251700 & & & & \\
\hline $\mathrm{H}$ & -4.21957200 & -3.33968600 & 2.94244700 & $\mathrm{C}$ & 5.32066700 & 1.59737100 & -0.00840000 \\
\hline $\mathrm{H}$ & -2.10976700 & -2.33752600 & 2.26987800 & $\mathrm{C}$ & 4.10688800 & 0.88283300 & -0.05028300 \\
\hline $\mathrm{H}$ & -4.40485900 & -4.33185200 & -2.41607300 & $\mathrm{C}$ & 3.07665100 & 0.93947200 & 0.89718100 \\
\hline $\mathrm{H}$ & -3.65558800 & -2.09582900 & -2.52555800 & $\mathrm{C}$ & 3.33894500 & 1.79099600 & 1.97066700 \\
\hline $\mathrm{H}$ & -3.14860700 & -4.60649000 & 2.36390800 & $\mathrm{C}$ & 4.55582800 & 2.50103900 & 2.04549100 \\
\hline $\mathrm{H}$ & -4.84573500 & -0.96345000 & -0.71571500 & $\mathrm{C}$ & 5.56219800 & 2.43192200 & 1.10090800 \\
\hline $\mathrm{H}$ & -2.11451700 & -3.15859200 & 0.72245100 & $\mathrm{C}$ & 6.01627100 & 1.27134900 & -1.22614200 \\
\hline $\mathrm{H}$ & -4.16616200 & -1.03060900 & 1.57644000 & $\mathrm{C}$ & 5.22221000 & 0.41725100 & -1.94586500 \\
\hline $\mathrm{C}$ & -4.40014100 & -4.74079500 & -0.30413300 & $\mathrm{H}$ & 2.62855200 & 1.95416100 & 2.77114100 \\
\hline
\end{tabular}




\begin{tabular}{|c|c|c|c|c|c|c|c|}
\hline $\mathrm{H}$ & 6.47136200 & 3.01122300 & 1.22096900 & $\mathrm{H}$ & -0.53360000 & 0.35604900 & 5.38315000 \\
\hline $\mathrm{H}$ & 6.99475200 & 1.62026500 & -1.53065900 & $\mathrm{H}$ & -1.10619600 & 2.00652600 & 5.07406100 \\
\hline $\mathrm{H}$ & 5.41555900 & -0.04127800 & -2.90227800 & $\mathrm{H}$ & -1.90187100 & 0.62972100 & 4.29065500 \\
\hline $\mathrm{N}$ & 4.04909000 & 0.13935100 & -1.23364100 & $\mathrm{C}$ & 1.42227700 & -0.89734600 & 2.83360000 \\
\hline $\mathrm{P}$ & 2.45128600 & -0.44027800 & -1.61638100 & $\mathrm{H}$ & 2.45204800 & -1.35019400 & 0.72828600 \\
\hline $\mathrm{C}$ & 2.69979000 & -2.24907500 & -2.22530300 & $\mathrm{H}$ & 2.33388600 & -0.46016400 & 3.22832400 \\
\hline $\mathrm{C}$ & 1.87766000 & 0.70673900 & -3.04853600 & $\mathrm{C}$ & 0.03849000 & 1.16623400 & 3.42387100 \\
\hline $\mathrm{C}$ & 3.97558600 & -2.41067500 & -3.08089200 & $\mathrm{H}$ & -0.38543200 & 1.86092500 & 2.69289400 \\
\hline $\mathrm{H}$ & 4.87909000 & -2.22644700 & -2.49371100 & $\mathrm{H}$ & 0.98344700 & 1.58877500 & 3.77738300 \\
\hline $\mathrm{H}$ & 4.01911900 & -3.44928900 & -3.43261600 & $\mathrm{Cl}$ & -0.33931500 & 1.64946800 & 0.07186500 \\
\hline $\mathrm{H}$ & 3.99350600 & -1.77020200 & -3.96536700 & $\mathrm{Rh}$ & -2.36301500 & 0.20881300 & -0.16864000 \\
\hline $\mathrm{C}$ & 2.85217600 & -3.17464800 & -0.99953100 & $\mathrm{C}$ & -4.25879200 & 4.05575400 & 1.44126400 \\
\hline $\mathrm{H}$ & 3.06625600 & -4.18893200 & -1.36206800 & $\mathrm{C}$ & -3.33671300 & 2.81423300 & 1.37325000 \\
\hline $\mathrm{H}$ & 3.68261700 & -2.86965400 & -0.35418000 & $\mathrm{C}$ & -3.76759100 & 4.07998200 & -2.14094100 \\
\hline $\mathrm{H}$ & 1.93769800 & -3.21298000 & -0.40300500 & $\mathrm{C}$ & -3.78649400 & 1.78122600 & 0.35843800 \\
\hline $\mathrm{C}$ & 1.47065500 & -2.71722900 & -3.03243100 & $\mathrm{C}$ & -2.77881300 & 2.98476700 & -1.67722200 \\
\hline $\mathrm{H}$ & 0.53915600 & -2.57176600 & -2.47920000 & $\mathrm{C}$ & -3.51902900 & 1.84132300 & -1.02240200 \\
\hline $\mathrm{H}$ & 1.39087300 & -2.21652600 & -4.00084600 & $\mathrm{H}$ & -5.30280400 & 3.73033800 & 1.56409700 \\
\hline $\mathrm{H}$ & 1.57836100 & -3.79120500 & -3.23104100 & $\mathrm{H}$ & -2.31070400 & 3.13661100 & 1.17757500 \\
\hline $\mathrm{C}$ & 0.36949700 & 0.52193800 & -3.31410900 & $\mathrm{H}$ & -4.33580100 & 3.71445000 & -3.00831900 \\
\hline $\mathrm{H}$ & 0.12966900 & -0.46445900 & -3.71748100 & $\mathrm{H}$ & -2.21472400 & 2.62935800 & -2.54846300 \\
\hline $\mathrm{H}$ & -0.22449300 & 0.68170500 & -2.41407200 & $\mathrm{H}$ & -4.00295000 & 4.60126000 & 2.36013200 \\
\hline $\mathrm{H}$ & 0.06178900 & 1.26689300 & -4.06016700 & $\mathrm{H}$ & -3.32640700 & 2.34097900 & 2.36439500 \\
\hline $\mathrm{C}$ & 2.13558800 & 2.15289200 & -2.56650600 & $\mathrm{H}$ & -3.17430700 & 4.93178700 & -2.50203300 \\
\hline $\mathrm{H}$ & 3.20191900 & 2.35357500 & -2.42516600 & $\mathrm{H}$ & -4.70954200 & 1.27915300 & 0.65034300 \\
\hline $\mathrm{H}$ & 1.76503100 & 2.84064900 & -3.33764300 & $\mathrm{H}$ & -2.04563800 & 3.41591900 & -0.99422600 \\
\hline $\mathrm{C}$ & 2.64887000 & 0.49164700 & -4.36734900 & $\mathrm{H}$ & -4.23937100 & 1.37324200 & -1.69247000 \\
\hline $\mathrm{H}$ & 2.30670700 & 1.24816000 & -5.08534600 & $\mathrm{C}$ & -4.18065200 & 5.05174300 & 0.26553300 \\
\hline $\mathrm{H}$ & 3.72788800 & 0.62670400 & -4.25112500 & $\mathrm{H}$ & -4.72234000 & 5.95302300 & 0.58070900 \\
\hline $\mathrm{H}$ & 2.45897800 & -0.48646500 & -4.81734000 & $\mathrm{H}$ & -3.13718100 & 5.37020500 & 0.12741200 \\
\hline $\mathrm{F}$ & 4.73420400 & 3.30296800 & 3.12710300 & $\mathrm{C}$ & -4.77378100 & 4.57705200 & -1.08436500 \\
\hline $\mathrm{Rh}$ & 1.45132300 & -0.21241100 & 0.49508500 & $\mathrm{H}$ & -5.32902200 & 5.40729500 & -1.54000700 \\
\hline $\mathrm{Cl}$ & -0.61914100 & -1.56365600 & -0.20931100 & $\mathrm{H}$ & -5.52122900 & 3.79589100 & -0.89196200 \\
\hline $\mathrm{H}$ & 1.60658700 & 2.37712900 & -1.63803200 & $\mathrm{C}$ & -4.69537100 & -3.26627500 & -2.02106400 \\
\hline $\mathrm{C}$ & 1.40672500 & -2.39225700 & 2.77743800 & $\mathrm{C}$ & -3.58582300 & -2.20469300 & -1.82967800 \\
\hline $\mathrm{O}$ & 0.42782400 & -3.09495600 & 2.63909700 & $\mathrm{C}$ & -4.63027100 & -3.34848200 & 1.59352200 \\
\hline $\mathrm{O}$ & 2.65400800 & -2.88204100 & 2.98214400 & $\mathrm{C}$ & -3.96481300 & -1.10297600 & -0.85892200 \\
\hline $\mathrm{C}$ & 2.75339400 & -4.31311800 & 3.01226700 & $\mathrm{C}$ & -3.41683400 & -2.44754800 & 1.26111100 \\
\hline $\mathrm{H}$ & 2.47611900 & -4.73720400 & 2.04301400 & $\mathrm{C}$ & -3.86964100 & -1.19780500 & 0.54229100 \\
\hline $\mathrm{H}$ & 3.79798500 & -4.52852300 & 3.23878400 & $\mathrm{H}$ & -5.64595000 & -2.76516700 & -2.25815000 \\
\hline $\mathrm{H}$ & 2.09807300 & -4.72885700 & 3.78236800 & $\mathrm{H}$ & -2.66252800 & -2.70229000 & -1.52189200 \\
\hline $\mathrm{C}$ & 0.25915800 & -0.17805800 & 2.79023100 & $\mathrm{H}$ & -5.22249900 & -2.88162400 & 2.39364200 \\
\hline $\mathrm{H}$ & -0.63737500 & -0.71200700 & 2.48106700 & $\mathrm{H}$ & -2.90005600 & -2.19685900 & 2.19537900 \\
\hline $\mathrm{C}$ & -0.93284100 & 1.02736300 & 4.61443000 & $\mathrm{H}$ & -4.43389100 & -3.85394800 & -2.91203500 \\
\hline
\end{tabular}




\begin{tabular}{|c|c|c|c|c|c|c|c|}
\hline $\mathrm{H}$ & -3.37634500 & -1.74573300 & -2.80541800 & $\mathrm{H}$ & 1.46288800 & -4.10695900 & -2.42336400 \\
\hline $\mathrm{H}$ & -4.23981700 & -4.28697500 & 2.01071400 & $\mathrm{H}$ & 1.58095800 & -5.11682700 & -0.98294300 \\
\hline $\mathrm{H}$ & -4.74939800 & -0.45342600 & -1.24765400 & $\mathrm{C}$ & 0.50597100 & -1.32581200 & -3.13611500 \\
\hline $\mathrm{H}$ & -2.69680200 & -3.00471300 & 0.66113200 & $\mathrm{H}$ & 0.23670700 & -2.37930400 & -3.02860500 \\
\hline $\mathrm{H}$ & -4.56954400 & -0.60565700 & 1.13106300 & $\mathrm{H}$ & -0.09533100 & -0.74538200 & -2.43446900 \\
\hline $\mathrm{C}$ & -4.92736200 & -4.25253900 & -0.85674200 & $\mathrm{H}$ & 0.23123200 & -1.01844800 & -4.15416300 \\
\hline $\mathrm{H}$ & -5.57797600 & -5.04783700 & -1.24324900 & $\mathrm{C}$ & 2.31959700 & 0.41846300 & -3.20008600 \\
\hline $\mathrm{H}$ & -3.97798000 & -4.74642800 & -0.60359900 & $\mathrm{H}$ & 3.39173400 & 0.63196500 & -3.15340200 \\
\hline $\mathrm{C}$ & -5.57925900 & -3.67010900 & 0.42216100 & $\mathrm{H}$ & 1.97243400 & 0.67148600 & -4.21035200 \\
\hline $\mathrm{H}$ & -6.32060400 & -4.38613200 & 0.80045900 & $\mathrm{C}$ & 2.80161200 & -1.90636700 & -3.98148600 \\
\hline \multirow[t]{2}{*}{$\mathrm{H}$} & -6.15086900 & -2.77165800 & 0.15372800 & $\mathrm{H}$ & 2.50369700 & -1.57284900 & -4.98429700 \\
\hline & & & & $\mathrm{H}$ & 3.88258100 & -1.75768200 & -3.90225700 \\
\hline \multirow[t]{6}{*}{ 6-ts } & & & \multirow{6}{*}{ C. } & $\mathrm{H}$ & 2.58555200 & -2.97636600 & -3.92170000 \\
\hline & & & & $\mathrm{F}$ & 4.68076600 & 4.16986300 & 1.31289600 \\
\hline & & & & $\mathrm{Rh}$ & 1.40619900 & -0.17753900 & 0.65738800 \\
\hline & & & & $\mathrm{Cl}$ & -0.71066200 & -1.68837200 & 0.53185600 \\
\hline & & & & $\mathrm{H}$ & 1.79982100 & 1.07141100 & -2.49634800 \\
\hline & & & & $\mathrm{C}$ & 0.61193500 & 1.90478100 & 3.02583200 \\
\hline $\mathrm{C}$ & 5.33840800 & 1.13887900 & -0.54163300 & $\mathrm{O}$ & 1.54784000 & 2.47787600 & 3.55631200 \\
\hline $\mathrm{C}$ & 4.11475400 & 0.48854100 & -0.27477400 & $\mathrm{O}$ & -0.57938800 & 2.50703100 & 2.79841200 \\
\hline $\mathrm{C}$ & 3.06246900 & 1.01015000 & 0.49368000 & $\mathrm{C}$ & -0.65161900 & 3.88501400 & 3.19151700 \\
\hline $\mathrm{C}$ & 3.29825900 & 2.28093600 & 1.01292100 & $\mathrm{H}$ & 0.07434400 & 4.48256900 & 2.63374700 \\
\hline $\mathrm{C}$ & 4.52255000 & 2.93719400 & 0.76763400 & $\mathrm{H}$ & -1.66773500 & 4.20239600 & 2.95570700 \\
\hline $\mathrm{C}$ & 5.55749600 & 2.41146200 & 0.01891300 & $\mathrm{H}$ & -0.45284900 & 3.99260500 & 4.26155300 \\
\hline $\mathrm{C}$ & 6.07096000 & 0.26907300 & -1.42458800 & $\mathrm{C}$ & 1.64616200 & -0.38951700 & 2.95031700 \\
\hline $\mathrm{C}$ & 5.28962400 & -0.82716400 & -1.67429500 & $\mathrm{H}$ & 2.55329500 & 0.11786100 & 3.28117100 \\
\hline $\mathrm{H}$ & 2.58253400 & 2.79953000 & 1.63455200 & $\mathrm{C}$ & 1.08570600 & -1.52021300 & 5.11650200 \\
\hline $\mathrm{H}$ & 6.47201900 & 2.97531400 & -0.13127200 & $\mathrm{H}$ & 0.18356300 & -0.91542500 & 5.25832900 \\
\hline $\mathrm{H}$ & 7.06378100 & 0.43482600 & -1.82309100 & $\mathrm{H}$ & 0.93022000 & -2.48574000 & 5.61042000 \\
\hline $\mathrm{H}$ & 5.50435700 & -1.68705700 & -2.28856900 & $\mathrm{H}$ & 1.91447500 & -1.01157100 & 5.62239300 \\
\hline $\mathrm{N}$ & 4.08873600 & -0.73371400 & -0.95926600 & $\mathrm{C}$ & 0.57285700 & 0.48208100 & 2.61525100 \\
\hline $\mathrm{P}$ & 2.49592300 & -1.41490200 & -1.11579900 & $\mathrm{H}$ & 2.32863500 & -1.01017300 & 1.66241300 \\
\hline $\mathrm{C}$ & 2.73037700 & -3.29734000 & -0.81003000 & $\mathrm{H}$ & -0.43023200 & 0.06891500 & 2.57227000 \\
\hline $\mathrm{C}$ & 2.01736100 & -1.07615600 & -2.94594300 & $\mathrm{C}$ & 1.38329800 & -1.73081000 & 3.62170600 \\
\hline $\mathrm{C}$ & 4.01944600 & -3.86509500 & -1.44270500 & $\mathrm{H}$ & 2.25605000 & -2.38541000 & 3.51579500 \\
\hline $\mathrm{H}$ & 4.91337300 & -3.43307200 & -0.98491900 & $\mathrm{H}$ & 0.53646800 & -2.22681800 & 3.13678800 \\
\hline $\mathrm{H}$ & 4.04598200 & -4.94646200 & -1.25615600 & $\mathrm{Cl}$ & -0.15447900 & 1.29546500 & -0.63108000 \\
\hline $\mathrm{H}$ & 4.07457300 & -3.72208500 & -2.52381400 & $\mathrm{Rh}$ & -2.29787800 & 0.05691200 & -0.31543600 \\
\hline $\mathrm{C}$ & 2.83260000 & -3.52050900 & 0.71264000 & $\mathrm{C}$ & -3.85287200 & 4.34589900 & -0.51407300 \\
\hline $\mathrm{H}$ & 3.05243400 & -4.58025300 & 0.89773200 & $\mathrm{C}$ & -3.04816400 & 3.11423000 & -0.03513300 \\
\hline $\mathrm{H}$ & 3.64093000 & -2.93082300 & 1.15906500 & $\mathrm{C}$ & -3.32126100 & 2.87179600 & -3.77497100 \\
\hline $\mathrm{H}$ & 1.89577800 & -3.27696900 & 1.21773700 & $\mathrm{C}$ & -3.57800200 & 1.80087100 & -0.57471400 \\
\hline $\mathrm{C}$ & 1.50530100 & -4.07850200 & -1.33122200 & $\mathrm{C}$ & -2.43001000 & 1.99135700 & -2.86548800 \\
\hline $\mathrm{H}$ & 0.56496500 & -3.66680100 & -0.95333900 & $\mathrm{C}$ & -3.27502800 & 1.26764800 & -1.84248900 \\
\hline
\end{tabular}




\begin{tabular}{|c|c|c|c|c|c|c|c|}
\hline $\mathrm{H}$ & -4.92361200 & 4.18558500 & -0.31687600 & & & & \\
\hline $\mathrm{H}$ & -1.99378300 & 3.24895200 & -0.28875300 & & & & \\
\hline $\mathrm{H}$ & -3.90807900 & 2.22610500 & -4.44400200 & & & & \\
\hline $\mathrm{H}$ & -1.88484200 & 1.27504900 & -3.49212300 & & & & \\
\hline $\mathrm{H}$ & -3.55442500 & 5.19564300 & 0.11549700 & & & & \\
\hline $\mathrm{H}$ & -3.09170100 & 3.07403100 & 1.06077300 & & & & \\
\hline $\mathrm{H}$ & -2.65595700 & 3.45871300 & -4.42360700 & $\mathrm{C}$ & 998300 & 5424100 & 6213800 \\
\hline $\mathrm{H}$ & -4.55198600 & 1.53792000 & -0.16070600 & $\mathrm{C}$ & -3.86793900 & -0.97712000 & -0.71010200 \\
\hline $\mathrm{H}$ & -1.67584700 & 2.61351100 & -2.38105700 & $\mathrm{C}$ & -2.61928000 & -1.46348900 & -0.29991300 \\
\hline $\mathrm{H}$ & -4.02126600 & 0.62049700 & -2.30340500 & $\mathrm{C}$ & -2.53604100 & -2.85305600 & -0.20108700 \\
\hline $\mathrm{C}$ & -3.67387400 & 4.76508700 & -1.98819400 & $\mathrm{C}$ & -3.65649900 & -3.64856500 & -0.51470300 \\
\hline $\mathrm{H}$ & -4.13500600 & 5.75566000 & -2.09358700 & $\mathrm{C}$ & -4.87804200 & -3.15390900 & -0.94226900 \\
\hline $\mathrm{H}$ & -2.60457000 & 4.91562400 & -2.19592800 & $\mathrm{C}$ & -5.99433600 & -0.82292000 & -1.50648900 \\
\hline $\mathrm{C}$ & -4.29345500 & 3.82386600 & -3.05160700 & $\mathrm{C}$ & -5.46829900 & 0.43944400 & -1.42149100 \\
\hline $\mathrm{H}$ & -4.77929600 & 4.43199400 & -3.82572200 & $\mathrm{H}$ & -1.63093400 & -3.34960900 & 0.13153000 \\
\hline $\mathrm{H}$ & -5.09999900 & 3.24127200 & -2.58712500 & $\mathrm{H}$ & -5.69046400 & -3.83334700 & -1.17722500 \\
\hline $\mathrm{C}$ & -4.87713200 & -3.69993400 & -0.68749500 & $\mathrm{H}$ & -6.99702100 & -1.05853800 & -1.84006700 \\
\hline $\mathrm{C}$ & -3.68210100 & -2.73769300 & -0.89118000 & $\mathrm{H}$ & -5.92862900 & 1.38416600 & -1.66307800 \\
\hline $\mathrm{C}$ & -4.94706600 & -2.23308700 & 2.61797300 & $\mathrm{~N}$ & -4.16760400 & 0.38036200 & -0.89899800 \\
\hline $\mathrm{C}$ & -3.98761900 & -1.30365000 & -0.50503000 & $\mathrm{P}$ & -2.74181400 & 1.37423300 & -0.87862600 \\
\hline $\mathrm{C}$ & -3.64454500 & -1.65002000 & 2.02034900 & $\mathrm{C}$ & -3.22045600 & 3.09237500 & -0.17504200 \\
\hline $\mathrm{C}$ & -3.95104700 & -0.79979600 & 0.80942000 & $\mathrm{C}$ & -2.27410200 & 1.51495700 & -2.75229300 \\
\hline $\mathrm{H}$ & -5.77003300 & -3.28423000 & -1.17811400 & $\mathrm{C}$ & -4.62691900 & 3.56217200 & -0.60281600 \\
\hline $\mathrm{H}$ & -2.81611700 & -3.11696500 & -0.34255000 & $\mathrm{H}$ & -5.40696200 & 2.92561100 & -0.17612200 \\
\hline $\mathrm{H}$ & -5.52019700 & -1.42706800 & 3.09790600 & $\mathrm{H}$ & -4.78335300 & 4.57525000 & -0.21050600 \\
\hline $\mathrm{H}$ & -3.14153800 & -1.05184200 & 2.79064200 & $\mathrm{H}$ & -4.76243600 & 3.60812600 & -1.68570900 \\
\hline $\mathrm{H}$ & -4.64232800 & -4.62858100 & -1.22587100 & $\mathrm{C}$ & -3.20042900 & 3.01821600 & 1.36472800 \\
\hline $\mathrm{H}$ & -3.40394200 & -2.75674700 & -1.95346500 & $\mathrm{H}$ & -3.50428000 & 3.99565300 & 1.76082400 \\
\hline $\mathrm{H}$ & -4.66231300 & -2.92559000 & 3.42260500 & $\mathrm{H}$ & -3.89694900 & 2.27324700 & 1.75517800 \\
\hline $\mathrm{H}$ & -4.69339700 & -0.82704000 & -1.18546400 & $\mathrm{H}$ & -2.20041500 & 2.80166000 & 1.74639600 \\
\hline $\mathrm{H}$ & -2.96174400 & -2.46199200 & 1.76612400 & $\mathrm{C}$ & -2.16297400 & 4.13406500 & -0.60102500 \\
\hline $\mathrm{H}$ & -4.61269800 & 0.03200500 & 1.04564400 & $\mathrm{H}$ & -1.14490700 & 3.80538400 & -0.36869400 \\
\hline $\mathrm{C}$ & -5.23582100 & -4.07559700 & 0.76577600 & $\mathrm{H}$ & -2.21971700 & 4.38355700 & -1.66325200 \\
\hline $\mathrm{H}$ & -5.94008500 & -4.91567900 & 0.70835000 & $\mathrm{H}$ & -2.34877200 & 5.05872500 & -0.04013300 \\
\hline $\mathrm{H}$ & -4.34342800 & -4.47475500 & 1.26958600 & $\mathrm{C}$ & -0.85417400 & 2.09148900 & -2.93867400 \\
\hline $\mathrm{C}$ & -5.87874700 & -2.96203000 & 1.62964400 & $\mathrm{H}$ & -0.76753300 & 3.13393100 & -2.62754400 \\
\hline $\mathrm{H}$ & -6.69304600 & -3.39811500 & 2.22284600 & $\mathrm{H}$ & -0.10105100 & 1.51933400 & -2.39121600 \\
\hline \multirow[t]{2}{*}{$\mathrm{H}$} & -6.35979000 & -2.22948500 & 0.96777300 & $\mathrm{H}$ & -0.60455900 & 2.04493200 & -4.00680500 \\
\hline & & & & $\mathrm{C}$ & -2.27719700 & 0.07904900 & -3.32846500 \\
\hline & & & & $\mathrm{H}$ & -3.26066700 & -0.39492000 & -3.27344200 \\
\hline & & & & $\mathrm{H}$ & -1.99025400 & 0.13740000 & -4.38622800 \\
\hline & & & & $\mathrm{C}$ & -3.28626100 & 2.35141200 & -3.55956500 \\
\hline & & & & $\mathrm{H}$ & -3.01492800 & 2.29317600 & -4.62167600 \\
\hline & & & & $\mathrm{H}$ & -4.30668200 & 1.96698800 & -3.46491100 \\
\hline
\end{tabular}




\begin{tabular}{|c|c|c|c|c|c|c|c|}
\hline $\mathrm{H}$ & -3.27943700 & 3.40909800 & -3.28396600 & $\mathrm{H}$ & 3.67670900 & -5.08558600 & -0.87358000 \\
\hline $\mathrm{F}$ & -3.51909200 & -4.99235500 & -0.39049100 & $\mathrm{C}$ & 5.11215200 & -3.94064400 & -2.05915900 \\
\hline $\mathrm{Rh}$ & -1.19353200 & -0.08096900 & 0.05080300 & $\mathrm{H}$ & 5.66525600 & -4.61836500 & -2.72244700 \\
\hline $\mathrm{Cl}$ & 0.71415100 & 1.57926300 & 0.34443500 & $\mathrm{H}$ & 5.82449100 & -3.14941700 & -1.78957200 \\
\hline $\mathrm{H}$ & -1.55798100 & -0.57580400 & -2.82638600 & $\mathrm{C}$ & 4.55869000 & 3.88543700 & -1.36088300 \\
\hline $\mathrm{C}$ & -0.83195400 & -0.98710100 & 2.80662600 & $\mathrm{C}$ & 3.53406300 & 2.72574800 & -1.32706900 \\
\hline $\mathrm{O}$ & -1.07089500 & -2.15737600 & 3.03149400 & $\mathrm{C}$ & 4.74846800 & 3.26312800 & 2.19635600 \\
\hline $\mathrm{O}$ & 0.28655000 & -0.36777800 & 3.25561500 & $\mathrm{C}$ & 4.04376400 & 1.48796100 & -0.61484400 \\
\hline $\mathrm{C}$ & 1.22374000 & -1.21767400 & 3.92998000 & $\mathrm{C}$ & 3.57136900 & 2.36058900 & 1.75707600 \\
\hline $\mathrm{H}$ & 1.60643100 & -1.97496000 & 3.24054800 & $\mathrm{C}$ & 4.04831700 & 1.30891300 & 0.78221500 \\
\hline $\mathrm{H}$ & 2.02862300 & -0.56078500 & 4.26281900 & $\mathrm{H}$ & 5.52073200 & 3.51269500 & -1.74327600 \\
\hline $\mathrm{H}$ & 0.75281900 & -1.71304100 & 4.78364500 & $\mathrm{H}$ & 2.60109100 & 3.08016000 & -0.88111000 \\
\hline $\mathrm{C}$ & -3.20450300 & -0.32563200 & 2.46761500 & $\mathrm{H}$ & 5.42230300 & 2.69196200 & 2.85076400 \\
\hline $\mathrm{H}$ & -3.39115000 & -1.39546100 & 2.34378600 & $\mathrm{H}$ & 3.13041900 & 1.89179100 & 2.64511800 \\
\hline $\mathrm{C}$ & -4.97290800 & -0.21957300 & 4.30603600 & $\mathrm{H}$ & 4.20155400 & 4.61361800 & -2.10232500 \\
\hline $\mathrm{H}$ & -5.19872700 & -1.28709000 & 4.19868700 & $\mathrm{H}$ & 3.29332300 & 2.45195300 & -2.36319800 \\
\hline $\mathrm{H}$ & -5.17807500 & 0.06422500 & 5.34486200 & $\mathrm{H}$ & 4.33405800 & 4.07073500 & 2.81593500 \\
\hline $\mathrm{H}$ & -5.67165000 & 0.33242000 & 3.66445500 & $\mathrm{H}$ & 4.83381200 & 0.98049700 & -1.16970200 \\
\hline $\mathrm{C}$ & -1.75499200 & -0.02484900 & 2.08585100 & $\mathrm{H}$ & 2.78551900 & 2.97424100 & 1.31445700 \\
\hline $\mathrm{H}$ & -3.89991600 & 0.19381800 & 1.80266700 & $\mathrm{H}$ & 4.82136200 & 0.66814400 & 1.20426700 \\
\hline $\mathrm{H}$ & -1.46430400 & 0.99359400 & 2.34793200 & $\mathrm{C}$ & 4.80721900 & 4.64199700 & -0.03895800 \\
\hline $\mathrm{C}$ & -3.51763000 & 0.07450800 & 3.92495600 & $\mathrm{H}$ & 5.37296400 & 5.54681700 & -0.29654000 \\
\hline $\mathrm{H}$ & -3.30731800 & 1.14407900 & 4.07068900 & $\mathrm{H}$ & 3.84776300 & 5.00020500 & 0.36182300 \\
\hline $\mathrm{H}$ & -2.84867900 & -0.46944400 & 4.60397800 & $\mathrm{C}$ & 5.58820100 & 3.87801700 & 1.05949700 \\
\hline $\mathrm{Cl}$ & 0.69512100 & -1.65847100 & 0.11820600 & $\mathrm{H}$ & 6.30564500 & 4.56433300 & 1.52784500 \\
\hline $\mathrm{Rh}$ & 2.57862200 & -0.03564200 & -0.07392000 & $\mathrm{H}$ & 6.19749100 & 3.09651600 & 0.58620100 \\
\hline $\mathrm{C}$ & 4.85590100 & -3.95641300 & 0.55767600 & & & & \\
\hline $\mathrm{C}$ & 3.86085900 & -2.80151800 & 0.82455500 & 8-ts & & & \\
\hline $\mathrm{C}$ & 3.96430200 & -3.33910900 & -2.89365800 & & & & \\
\hline $\mathrm{C}$ & 4.13471300 & -1.56374200 & -0.00738700 & & & & \\
\hline $\mathrm{C}$ & 2.97155600 & -2.43072300 & -2.12909000 & & & & \\
\hline $\mathrm{C}$ & 3.71902700 & -1.37985700 & -1.34082600 & & & & \\
\hline $\mathrm{H}$ & 5.88623200 & -3.57894300 & 0.64085300 & & & & \\
\hline $\mathrm{H}$ & 2.84097300 & -3.16503900 & 0.67324700 & & & & \\
\hline $\mathrm{H}$ & 4.40701700 & -2.77388100 & -3.72630500 & $\mathrm{C}$ & 5.14651300 & 1.85170700 & 0.08183400 \\
\hline $\mathrm{H}$ & 2.29417100 & -1.95987200 & -2.85248000 & $\mathrm{C}$ & 3.97743800 & 1.06223100 & 0.02051900 \\
\hline $\mathrm{H}$ & 4.73443400 & -4.68458700 & 1.37127600 & $\mathrm{C}$ & 2.85402500 & 1.19660400 & 0.85151600 \\
\hline $\mathrm{H}$ & 3.93286100 & -2.52577500 & 1.88514300 & $\mathrm{C}$ & 2.95299800 & 2.22365600 & 1.78976600 \\
\hline $\mathrm{H}$ & 3.38477300 & -4.15307600 & -3.35069400 & $\mathrm{C}$ & 4.12239900 & 3.00782500 & 1.87928600 \\
\hline $\mathrm{H}$ & 5.05537600 & -1.05702100 & 0.28544600 & $\mathrm{C}$ & 5.23042800 & 2.85219100 & 1.06999100 \\
\hline $\mathrm{H}$ & 2.34606100 & -3.04071600 & -1.47562000 & $\mathrm{C}$ & 5.98164500 & 1.41611200 & -1.00614900 \\
\hline $\mathrm{H}$ & 4.33400100 & -0.74249600 & -1.97546600 & $\mathrm{C}$ & 5.30589400 & 0.43389200 & -1.68154800 \\
\hline $\mathrm{C}$ & 4.70685000 & -4.71280500 & -0.77878200 & $\mathrm{H}$ & 2.14223300 & 2.47481100 & 2.45998900 \\
\hline $\mathrm{H}$ & 5.33617600 & -5.60908800 & -0.70443200 & $\mathrm{H}$ & 6.09583300 & 3.49535000 & 1.18925500 \\
\hline
\end{tabular}




\begin{tabular}{|c|c|c|c|c|c|c|c|}
\hline $\mathrm{H}$ & 6.96711700 & 1.78663700 & -1.25803600 & $\mathrm{H}$ & -0.83377300 & 1.57650700 & 5.21230300 \\
\hline $\mathrm{H}$ & 5.61322600 & -0.12312400 & -2.55246800 & $\mathrm{H}$ & -1.68056400 & 0.36892900 & 4.22739100 \\
\hline $\mathrm{N}$ & 4.08126700 & 0.17563000 & -1.05807900 & $\mathrm{C}$ & 1.60971300 & -1.04486500 & 2.57248900 \\
\hline $\mathrm{P}$ & 2.55701900 & -0.52815400 & -1.54188100 & $\mathrm{H}$ & 2.27532600 & -1.17053300 & 1.27634900 \\
\hline $\mathrm{C}$ & 2.96295600 & -2.36751800 & -1.91357300 & $\mathrm{H}$ & 2.48200800 & -0.64297800 & 3.08878500 \\
\hline $\mathrm{C}$ & 2.09896300 & 0.41621000 & -3.14797800 & $\mathrm{C}$ & 0.25344500 & 0.99147100 & 3.41411800 \\
\hline $\mathrm{C}$ & 4.30684800 & -2.57428900 & -2.64518600 & $\mathrm{H}$ & -0.16534000 & 1.81552200 & 2.82462700 \\
\hline $\mathrm{H}$ & 5.15312800 & -2.26780700 & -2.02427800 & $\mathrm{H}$ & 1.21629600 & 1.32718100 & 3.81076800 \\
\hline $\mathrm{H}$ & 4.42560900 & -3.64591100 & -2.85255700 & $\mathrm{Cl}$ & -0.33790900 & 1.63735100 & -0.16300500 \\
\hline $\mathrm{H}$ & 4.36574000 & -2.05142500 & -3.60212900 & $\mathrm{Rh}$ & -2.38940000 & 0.20865100 & -0.24779500 \\
\hline $\mathrm{C}$ & 3.05603300 & -3.10303800 & -0.56071500 & $\mathrm{C}$ & -4.10714900 & 4.05835600 & 1.50976600 \\
\hline $\mathrm{H}$ & 3.36887300 & -4.13926200 & -0.74600200 & $\mathrm{C}$ & -3.22119300 & 2.79772500 & 1.36499500 \\
\hline $\mathrm{H}$ & 3.79566400 & -2.64667300 & 0.10558000 & $\mathrm{C}$ & -3.85885400 & 4.12269700 & -2.10019200 \\
\hline $\mathrm{H}$ & 2.08976500 & -3.12938900 & -0.05109500 & $\mathrm{C}$ & -3.75732900 & 1.79373700 & 0.36376400 \\
\hline $\mathrm{C}$ & 1.82105900 & -3.00502400 & -2.73405100 & $\mathrm{C}$ & -2.85727100 & 3.00742000 & -1.71693400 \\
\hline $\mathrm{H}$ & 0.84222400 & -2.83732900 & -2.27525200 & $\mathrm{C}$ & -3.56934200 & 1.86472600 & -1.02975200 \\
\hline $\mathrm{H}$ & 1.79336100 & -2.64021800 & -3.76482300 & $\mathrm{H}$ & -5.14837400 & 3.75606200 & 1.69819300 \\
\hline $\mathrm{H}$ & 1.98794000 & -4.08923900 & -2.77846400 & $\mathrm{H}$ & -2.20180200 & 3.09973700 & 1.11188400 \\
\hline $\mathrm{C}$ & 0.61643400 & 0.14337400 & -3.48178700 & $\mathrm{H}$ & -4.49236500 & 3.77520700 & -2.92887300 \\
\hline $\mathrm{H}$ & 0.43437300 & -0.89466500 & -3.77079600 & $\mathrm{H}$ & -2.35505100 & 2.65674100 & -2.62698600 \\
\hline $\mathrm{H}$ & -0.03761600 & 0.38356300 & -2.64145100 & $\mathrm{H}$ & -3.77740600 & 4.58530400 & 2.41570700 \\
\hline $\mathrm{H}$ & 0.32912500 & 0.77834800 & -4.33063300 & $\mathrm{H}$ & -3.15963400 & 2.30553800 & 2.34496900 \\
\hline $\mathrm{C}$ & 2.26725800 & 1.91835700 & -2.82300300 & $\mathrm{H}$ & -3.27829400 & 4.96869300 & -2.49397600 \\
\hline $\mathrm{H}$ & 3.31163000 & 2.18250700 & -2.63115900 & $\mathrm{H}$ & -4.67103900 & 1.30683800 & 0.70505000 \\
\hline $\mathrm{H}$ & 1.92888200 & 2.49829300 & -3.69160600 & $\mathrm{H}$ & -2.07797000 & 3.42430300 & -1.07740000 \\
\hline $\mathrm{C}$ & 2.97048200 & 0.08151600 & -4.37344500 & $\mathrm{H}$ & -4.33596700 & 1.41406000 & -1.65902400 \\
\hline $\mathrm{H}$ & 2.66874400 & 0.73534100 & -5.20235500 & $\mathrm{C}$ & -4.08195700 & 5.06634700 & 0.34214200 \\
\hline $\mathrm{H}$ & 4.03384400 & 0.26364900 & -4.19063300 & $\mathrm{H}$ & -4.57540200 & 5.97814700 & 0.70296600 \\
\hline $\mathrm{H}$ & 2.84336500 & -0.94963100 & -4.71458400 & $\mathrm{H}$ & -3.04164800 & 5.35789700 & 0.13706000 \\
\hline $\mathrm{F}$ & 4.14186600 & 3.97767400 & 2.83022400 & $\mathrm{C}$ & -4.77879100 & 4.62398500 & -0.96919500 \\
\hline $\mathrm{Rh}$ & 1.31877500 & -0.09863600 & 0.50530700 & $\mathrm{H}$ & -5.34731100 & 5.47170300 & -1.37329500 \\
\hline $\mathrm{Cl}$ & -0.65367600 & -1.58239000 & -0.26913600 & $\mathrm{H}$ & -5.52708800 & 3.85498600 & -0.73550200 \\
\hline $\mathrm{H}$ & 1.66443900 & 2.22288200 & -1.96423100 & $\mathrm{C}$ & -4.75373100 & -3.25062000 & -2.06515400 \\
\hline $\mathrm{C}$ & 1.46346500 & -2.54508600 & 2.75604800 & $\mathrm{C}$ & -3.63732400 & -2.19463600 & -1.88169300 \\
\hline $\mathrm{O}$ & 0.42457600 & -3.16340000 & 2.75478900 & $\mathrm{C}$ & -4.67171100 & -3.32546000 & 1.54982700 \\
\hline $\mathrm{O}$ & 2.67553900 & -3.10480000 & 2.96684800 & $\mathrm{C}$ & -4.00612600 & -1.09046900 & -0.90999 \\
\hline $\mathrm{C}$ & 2.66707500 & -4.52412100 & 3.19932100 & $\mathrm{C}$ & -3.45276800 & -2.43498100 & 1.20967800 \\
\hline $\mathrm{H}$ & 2.27104100 & -5.05088800 & 2.32721200 & $\mathrm{C}$ & -3.89890300 & -1.18282500 & 0.49095500 \\
\hline $\mathrm{H}$ & 3.70789100 & -4.79659400 & 3.37398700 & $\mathrm{H}$ & -5.70267800 & -2.74499100 & -2.29911500 \\
\hline $\mathrm{H}$ & 2.05350700 & -4.76398800 & 4.07151000 & $\mathrm{H}$ & -2.71496000 & -2.69637600 & -1.57820700 \\
\hline $\mathrm{C}$ & 0.43851500 & -0.22815600 & 2.53617800 & $\mathrm{H}$ & -5.25690300 & -2.85189000 & 2.35117500 \\
\hline $\mathrm{H}$ & -0.48407900 & -0.77114600 & 2.33923100 & $\mathrm{H}$ & -2.92879600 & -2.18555000 & 2.14036100 \\
\hline $\mathrm{C}$ & -0.69504000 & 0.68568400 & 4.58883000 & $\mathrm{H}$ & -4.49936500 & -3.84165300 & -2.95589400 \\
\hline $\mathrm{H}$ & -0.30061100 & -0.11551900 & 5.22509100 & $\mathrm{H}$ & -3.43074500 & -1.73809600 & -2.85906600 \\
\hline
\end{tabular}




\begin{tabular}{|c|c|c|c|c|c|c|c|}
\hline $\mathrm{H}$ & -4.28714800 & -4.26602700 & 1.96803000 & $\mathrm{H}$ & 2.83656300 & -3.83991900 & -2.40215800 \\
\hline $\mathrm{H}$ & -4.79026700 & -0.43833500 & -1.29504700 & $\mathrm{H}$ & 2.72438400 & -4.83426800 & -0.95001900 \\
\hline $\mathrm{H}$ & -2.74094500 & -2.99896900 & 0.60631300 & $\mathrm{C}$ & 1.66746700 & -1.30882600 & -3.43739600 \\
\hline $\mathrm{H}$ & -4.59182200 & -0.58604600 & 1.08295000 & $\mathrm{H}$ & 1.55891100 & -2.39257900 & -3.36285600 \\
\hline $\mathrm{C}$ & -4.98559100 & -4.23256700 & -0.89720100 & $\mathrm{H}$ & 0.80442300 & -0.85519300 & -2.94354400 \\
\hline $\mathrm{H}$ & -5.64229200 & -5.02538000 & -1.27851400 & $\mathrm{H}$ & 1.62509800 & -1.04633900 & -4.50263800 \\
\hline $\mathrm{H}$ & -4.03764700 & -4.73083400 & -0.64726000 & $\mathrm{C}$ & 3.06528700 & 0.73544900 & -3.11206600 \\
\hline $\mathrm{C}$ & -5.62810200 & -3.64307900 & 0.38328100 & $\mathrm{H}$ & 4.00344700 & 1.17871000 & -2.76743800 \\
\hline $\mathrm{H}$ & -6.37288100 & -4.35307600 & 0.76618300 & $\mathrm{H}$ & 2.98771100 & 0.90874900 & -4.19299300 \\
\hline \multirow[t]{2}{*}{$\mathrm{H}$} & -6.19461800 & -2.74129000 & 0.11527700 & $\mathrm{C}$ & 4.17910300 & -1.43881300 & -3.62623600 \\
\hline & & & & $\mathrm{H}$ & 4.11174000 & -1.14565200 & -4.68203600 \\
\hline \multirow[t]{7}{*}{9} & & & & $\mathrm{H}$ & 5.15087900 & -1.09493800 & -3.25745800 \\
\hline & & & & $\mathrm{H}$ & 4.15353200 & -2.53128300 & -3.59484600 \\
\hline & & & & $\mathrm{F}$ & 3.43428700 & 5.04369700 & 1.07053100 \\
\hline & & & & $\mathrm{Rh}$ & 1.31906200 & 0.15583300 & -0.10016000 \\
\hline & & & & $\mathrm{Cl}$ & -0.56474900 & -1.49803100 & -0.56502000 \\
\hline & & & & $\mathrm{H}$ & 2.24005800 & 1.26965200 & -2.63151700 \\
\hline & & & & $\mathrm{C}$ & -0.35832900 & -0.60193600 & 3.77168600 \\
\hline $\mathrm{C}$ & 5.17459000 & 2.04281200 & 0.03798000 & $\mathrm{O}$ & -0.51890300 & -1.80577800 & 3.77711100 \\
\hline $\mathrm{C}$ & 4.04617800 & 1.20600700 & -0.04438400 & $\mathrm{O}$ & -0.67574700 & 0.18793100 & 4.82484000 \\
\hline $\mathrm{C}$ & 2.71531600 & 1.58176800 & 0.18113100 & $\mathrm{C}$ & -1.23368400 & -0.49415900 & 5.95964400 \\
\hline $\mathrm{C}$ & 2.54445800 & 2.91643600 & 0.55608300 & $\mathrm{H}$ & -0.53054900 & -1.23729500 & 6.34533800 \\
\hline $\mathrm{C}$ & 3.66514600 & 3.76232300 & 0.68599400 & $\mathrm{H}$ & -1.42002900 & 0.28094700 & 6.70378200 \\
\hline $\mathrm{C}$ & 4.97320500 & 3.37843000 & 0.44130500 & $\mathrm{H}$ & -2.16569600 & -0.99711600 & 5.68718000 \\
\hline $\mathrm{C}$ & 6.29646600 & 1.23821800 & -0.37676500 & $\mathrm{C}$ & 1.39054300 & -0.40849600 & 1.93139700 \\
\hline $\mathrm{C}$ & 5.82509800 & -0.00923800 & -0.69403400 & $\mathrm{H}$ & 1.22977900 & -1.48503100 & 1.85854400 \\
\hline $\mathrm{H}$ & 1.56218000 & 3.33471600 & 0.75147400 & $\mathrm{C}$ & 2.75873200 & -0.76451000 & 4.08548100 \\
\hline $\mathrm{H}$ & 5.78177300 & 4.09394400 & 0.54683300 & $\mathrm{H}$ & 2.59382900 & -1.84651300 & 4.02651100 \\
\hline $\mathrm{H}$ & 7.33404900 & 1.54320700 & -0.42709800 & $\mathrm{H}$ & 3.74970400 & -0.59464200 & 4.52338800 \\
\hline $\mathrm{H}$ & 6.37089300 & -0.87075700 & -1.04387100 & $\mathrm{H}$ & 2.02276800 & -0.35066600 & 4.78272200 \\
\hline $\mathrm{N}$ & 4.44359200 & -0.07056300 & -0.46492400 & $\mathrm{C}$ & 0.15069800 & 0.21477300 & 2.59382500 \\
\hline $\mathrm{P}$ & 3.09305200 & -1.05723700 & -0.95051000 & $\mathrm{H}$ & 0.33325300 & 1.24731000 & 2.90290700 \\
\hline $\mathrm{C}$ & 3.53638500 & -2.87899500 & -0.54115000 & $\mathrm{H}$ & -0.69210100 & 0.22893800 & 1.89668100 \\
\hline $\mathrm{C}$ & 3.00528300 & -0.79026700 & -2.86697400 & $\mathrm{C}$ & 2.67870000 & -0.09438300 & 2.69809600 \\
\hline $\mathrm{C}$ & 5.00988600 & -3.23901900 & -0.82678500 & $\mathrm{H}$ & 2.77693100 & 0.98962500 & 2.82028300 \\
\hline $\mathrm{H}$ & 5.68604700 & -2.72098800 & -0.14132100 & $\mathrm{H}$ & 3.54866300 & -0.40613200 & 2.11462900 \\
\hline $\mathrm{H}$ & 5.13655000 & -4.31550800 & -0.65425700 & $\mathrm{Cl}$ & -0.58642100 & 1.75330100 & -0.31582400 \\
\hline $\mathrm{H}$ & 5.32308200 & -3.03481200 & -1.85227100 & $\mathrm{Rh}$ & -2.47938600 & 0.10949700 & -0.51276900 \\
\hline $\mathrm{C}$ & 3.28513800 & -3.12797800 & 0.95902600 & $\mathrm{C}$ & -4.69223800 & 3.87082800 & 0.87485000 \\
\hline $\mathrm{H}$ & 3.60116000 & -4.15213000 & 1.19517100 & $\mathrm{C}$ & -3.67379300 & 2.70473200 & 0.89194200 \\
\hline $\mathrm{H}$ & 3.85902800 & -2.45071000 & 1.59619500 & $\mathrm{C}$ & -4.20120200 & 3.70268900 & -2.70431200 \\
\hline $\mathrm{H}$ & 2.22766300 & -3.04228300 & 1.21377900 & $\mathrm{C}$ & -4.03296900 & 1.57508800 & -0.05303500 \\
\hline $\mathrm{C}$ & 2.59954200 & -3.81448400 & -1.33557800 & $\mathrm{C}$ & -3.12853500 & 2.72609500 & -2.16642900 \\
\hline $\mathrm{H}$ & 1.54596200 & -3.54237300 & -1.21461600 & $\mathrm{C}$ & -3.77898800 & 1.57315700 & -1.43808100 \\
\hline
\end{tabular}




\begin{tabular}{|c|c|c|c|c|c|c|c|}
\hline $\mathrm{H}$ & -5.70859800 & 3.47341400 & 1.01614500 & & & & \\
\hline $\mathrm{H}$ & -2.67589500 & 3.09713700 & 0.68054400 & & & & \\
\hline $\mathrm{H}$ & -4.73727300 & 3.23093600 & -3.54017100 & & & & \\
\hline $\mathrm{H}$ & -2.53018300 & 2.36062600 & -3.01057400 & & & & \\
\hline $\mathrm{H}$ & -4.48592100 & 4.49276000 & 1.75659900 & & & & \\
\hline $\mathrm{H}$ & -3.63169500 & 2.30058200 & 1.91217100 & & & & \\
\hline $\mathrm{H}$ & -3.67643200 & 4.57005900 & -3.12833000 & $\mathrm{C}$ & -5.35482500 & 1.50557900 & 0.34721200 \\
\hline $\mathrm{H}$ & -4.90534300 & 1.01047900 & 0.27829600 & $\mathrm{C}$ & -4.16996400 & 0.79658000 & 0.04947100 \\
\hline $\mathrm{H}$ & -2.44090800 & 3.26380200 & -1.51170700 & $\mathrm{C}$ & -2.95207500 & 1.36824200 & -0.35558700 \\
\hline $\mathrm{H}$ & -4.45790900 & 1.00555200 & -2.07364200 & $\mathrm{C}$ & -2.98007400 & 2.75979100 & -0.45211000 \\
\hline $\mathrm{C}$ & -4.68332500 & 4.79233200 & -0.36330900 & $\mathrm{C}$ & -4.16062600 & 3.48209500 & -0.17893500 \\
\hline $\mathrm{H}$ & -5.28616000 & 5.67234300 & -0.10400200 & $\mathrm{C}$ & -5.35489500 & 2.90711800 & 0.20647600 \\
\hline $\mathrm{H}$ & -3.66465600 & 5.17273600 & -0.52628600 & $\mathrm{C}$ & -6.30927600 & 0.52512600 & 0.79166800 \\
\hline $\mathrm{C}$ & -5.24213300 & 4.19290700 & -1.67808600 & $\mathrm{C}$ & -5.68953800 & -0.69664100 & 0.77126700 \\
\hline $\mathrm{H}$ & -5.85483200 & 4.95100400 & -2.18306200 & $\mathrm{H}$ & -2.10673200 & 3.34135100 & -0.71143000 \\
\hline $\mathrm{H}$ & -5.93252400 & 3.37468500 & -1.43281300 & $\mathrm{H}$ & -6.22548200 & 3.52227400 & 0.40751400 \\
\hline $\mathrm{C}$ & -4.58732200 & -3.66464500 & -2.01496700 & $\mathrm{H}$ & -7.33439300 & 0.70425600 & 1.08982500 \\
\hline $\mathrm{C}$ & -3.57397800 & -2.49507500 & -1.96803400 & $\mathrm{H}$ & -6.08686400 & -1.66209000 & 1.04144900 \\
\hline $\mathrm{C}$ & -4.30371300 & -3.49981000 & 1.58581500 & $\mathrm{~N}$ & -4.38213000 & -0.56520200 & 0.29458300 \\
\hline $\mathrm{C}$ & -3.99216600 & -1.36986200 & -1.04150700 & $\mathrm{P}$ & -2.91783400 & -1.51103500 & 0.38798000 \\
\hline $\mathrm{C}$ & -3.19858400 & -2.52606600 & 1.11244700 & $\mathrm{C}$ & -3.31426500 & -3.11782300 & -0.58623200 \\
\hline $\mathrm{C}$ & -3.81140500 & -1.37070900 & 0.35542700 & $\mathrm{C}$ & -2.71425700 & -1.87775500 & 2.26119300 \\
\hline $\mathrm{H}$ & -5.59352800 & -3.26860500 & -2.21877300 & $\mathrm{C}$ & -4.73408400 & -3.66574100 & -0.33128900 \\
\hline $\mathrm{H}$ & -2.58802300 & -2.88239800 & -1.69796000 & $\mathrm{H}$ & -5.50127700 & -2.98288200 & -0.70645100 \\
\hline $\mathrm{H}$ & -4.88541300 & -3.02895900 & 2.39123800 & $\mathrm{H}$ & -4.84398700 & -4.61170600 & -0.87762600 \\
\hline $\mathrm{H}$ & -2.63684700 & -2.17607900 & 1.98622600 & $\mathrm{H}$ & -4.93473600 & -3.87363900 & 0.72159100 \\
\hline $\mathrm{H}$ & -4.32636500 & -4.28556200 & -2.88323500 & $\mathrm{C}$ & -3.20837200 & -2.78807900 & -2.08982800 \\
\hline $\mathrm{H}$ & -3.47452600 & -2.08723500 & -2.98293800 & $\mathrm{H}$ & -3.51338500 & -3.67115900 & -2.66676700 \\
\hline $\mathrm{H}$ & -3.80596400 & -4.36903800 & 2.03725900 & $\mathrm{H}$ & -3.86790300 & -1.96054200 & -2.37405300 \\
\hline $\mathrm{H}$ & -4.85202100 & -0.81370100 & -1.41779600 & $\mathrm{H}$ & -2.18422000 & -2.53542000 & -2.37334000 \\
\hline $\mathrm{H}$ & -2.48283400 & -3.06316100 & 0.48845800 & $\mathrm{C}$ & -2.26813700 & -4.20354300 & -0.25444200 \\
\hline $\mathrm{H}$ & -4.52495300 & -0.80587800 & 0.95477900 & $\mathrm{H}$ & -1.24386800 & -3.83465500 & -0.36372300 \\
\hline $\mathrm{C}$ & -4.65490700 & -4.58657700 & -0.77916000 & $\mathrm{H}$ & -2.39022400 & -4.60499000 & 0.75556200 \\
\hline $\mathrm{H}$ & -5.24551000 & -5.46382400 & -1.07398300 & $\mathrm{H}$ & -2.39922500 & -5.03983000 & -0.95350400 \\
\hline $\mathrm{H}$ & -3.64913800 & -4.97133900 & -0.55644600 & $\mathrm{C}$ & -1.27843200 & -2.38854500 & 2.51249600 \\
\hline $\mathrm{C}$ & -5.28618200 & -3.98428300 & 0.50107000 & $\mathrm{H}$ & -1.10013500 & -3.37617500 & 2.07911300 \\
\hline $\mathrm{H}$ & -5.93192200 & -4.73942800 & 0.96807900 & $\mathrm{H}$ & -0.52944200 & -1.70003200 & 2.11636200 \\
\hline \multirow[t]{2}{*}{$\mathrm{H}$} & -5.95674100 & -3.16250400 & 0.21582900 & $\mathrm{H}$ & -1.12455800 & -2.47250700 & 3.59679300 \\
\hline & & & & $\mathrm{C}$ & -2.89487500 & -0.52262000 & 2.98208100 \\
\hline \multirow[t]{5}{*}{ 10-ts } & & & & $\mathrm{H}$ & -3.91474200 & -0.13842200 & 2.88282900 \\
\hline & & & & $\mathrm{H}$ & -2.69572000 & -0.67105200 & 4.05146100 \\
\hline & & & & $\mathrm{C}$ & -3.72319600 & -2.88055500 & 2.85333000 \\
\hline & & & & $\mathrm{H}$ & -3.55031000 & -2.94571100 & 3.93570600 \\
\hline & & & & $\mathrm{H}$ & -4.76071000 & -2.56166800 & 2.71326500 \\
\hline
\end{tabular}




\begin{tabular}{|c|c|c|c|c|c|c|c|}
\hline $\mathrm{H}$ & -3.60672000 & -3.88971300 & 2.44855400 & $\mathrm{H}$ & 2.56973700 & 3.42757600 & 4.06728900 \\
\hline $\mathrm{F}$ & -4.09867000 & 4.83314900 & -0.30763500 & $\mathrm{C}$ & 4.14480800 & 1.98505900 & 4.53210000 \\
\hline $\mathrm{Rh}$ & -1.42092300 & 0.06709500 & -0.70624300 & $\mathrm{H}$ & 4.60358800 & 2.23582700 & 5.49742100 \\
\hline $\mathrm{Cl}$ & 0.49289800 & -1.67448300 & -0.86635000 & $\mathrm{H}$ & 4.95463900 & 1.56021900 & 3.92433900 \\
\hline $\mathrm{H}$ & -2.19695300 & 0.23311800 & 2.61533300 & $\mathrm{C}$ & 4.57153300 & -4.13242300 & -0.47668200 \\
\hline $\mathrm{C}$ & 1.30229500 & 3.16097500 & -2.59961800 & $\mathrm{C}$ & 3.40696500 & -3.26496400 & 0.05826600 \\
\hline $\mathrm{O}$ & 2.25537100 & 2.45888200 & -2.87042800 & $\mathrm{C}$ & 4.77084200 & -1.54829000 & -3.00104800 \\
\hline $\mathrm{O}$ & 1.27571400 & 4.49165100 & -2.82517800 & $\mathrm{C}$ & 3.77471800 & -1.80525200 & 0.23814200 \\
\hline $\mathrm{C}$ & 2.47281800 & 5.05077300 & -3.39037000 & $\mathrm{C}$ & 3.47952100 & -1.16869100 & -2.23769900 \\
\hline $\mathrm{H}$ & 2.69868600 & 4.58251200 & -4.35201400 & $\mathrm{C}$ & 3.79694700 & -0.85036100 & -0.79610800 \\
\hline $\mathrm{H}$ & 2.26546000 & 6.11340300 & -3.51942800 & $\mathrm{H}$ & 5.46675800 & -3.96707900 & 0.14153000 \\
\hline $\mathrm{H}$ & 3.32129900 & 4.90471200 & -2.71614500 & $\mathrm{H}$ & 2.54382900 & -3.37559100 & -0.60317500 \\
\hline $\mathrm{C}$ & -0.23140000 & 1.22689000 & -2.13956900 & $\mathrm{H}$ & 5.38341100 & -0.64735600 & -3.14620300 \\
\hline $\mathrm{H}$ & 0.69677000 & 0.66387600 & -2.18700600 & $\mathrm{H}$ & 3.02635100 & -0.29765400 & -2.72550800 \\
\hline $\mathrm{C}$ & -1.03367300 & -0.32980400 & -4.00886400 & $\mathrm{H}$ & 4.29067600 & -5.18390400 & -0.32481000 \\
\hline $\mathrm{H}$ & -0.31570800 & -1.07842900 & -3.66411000 & $\mathrm{H}$ & 3.09823700 & -3.66529600 & 1.03327700 \\
\hline $\mathrm{H}$ & -1.94681900 & -0.83563800 & -4.33873500 & $\mathrm{H}$ & 4.47659800 & -1.88215800 & -4.00602300 \\
\hline $\mathrm{H}$ & -0.60023700 & 0.18736300 & -4.87430600 & $\mathrm{H}$ & 4.47493300 & -1.64667300 & 1.05950100 \\
\hline $\mathrm{C}$ & 0.01130800 & 2.70599800 & -1.93094500 & $\mathrm{H}$ & 2.75737100 & -1.98387000 & -2.30575200 \\
\hline $\mathrm{H}$ & -0.80590500 & 3.32793300 & -2.30350800 & $\mathrm{H}$ & 4.49016200 & -0.01704700 & -0.69829800 \\
\hline $\mathrm{H}$ & 0.13365700 & 2.93743900 & -0.86348400 & $\mathrm{C}$ & 4.95123400 & -3.95629600 & -1.96234700 \\
\hline $\mathrm{C}$ & -1.30606100 & 0.70472200 & -2.92382800 & $\mathrm{H}$ & 5.62327700 & -4.78685800 & -2.21508000 \\
\hline $\mathrm{H}$ & -2.10071600 & 1.41363700 & -3.16569800 & $\mathrm{H}$ & 4.05717800 & -4.10228000 & -2.58597700 \\
\hline $\mathrm{H}$ & -2.19295400 & -0.17998100 & -2.10459800 & $\mathrm{C}$ & 5.65364200 & -2.62990700 & -2.34690000 \\
\hline $\mathrm{Cl}$ & 0.04832900 & 0.82521600 & 1.17171800 & $\mathrm{H}$ & 6.46509600 & -2.84953000 & -3.05309900 \\
\hline $\mathrm{Rh}$ & 2.14034400 & -0.39101300 & 0.53726900 & $\mathrm{H}$ & 6.14523200 & -2.21475100 & -1.45682700 \\
\hline $\mathrm{C}$ & 3.89142500 & 3.42476200 & 2.34501100 & & & & \\
\hline $\mathrm{C}$ & 3.05664900 & 2.51447900 & 1.41286700 & 11 & & & \\
\hline $\mathrm{C}$ & 3.08458400 & 0.90050300 & 4.80910200 & & & & \\
\hline $\mathrm{C}$ & 3.48622000 & 1.06128600 & 1.44717800 & & & & \\
\hline $\mathrm{C}$ & 2.21425700 & 0.47917100 & 3.60125800 & & & & \\
\hline $\mathrm{C}$ & 3.08741100 & 0.12336400 & 2.42006000 & & & & \\
\hline $\mathrm{H}$ & 4.96254600 & 3.27093600 & 2.14518300 & & & & \\
\hline $\mathrm{H}$ & 1.99746200 & 2.62011100 & 1.66181500 & $\mathrm{C}$ & -5.49401500 & 1.25215800 & 0.50000300 \\
\hline $\mathrm{H}$ & 3.59675700 & 0.01626300 & 5.21466000 & $\mathrm{C}$ & -4.27872100 & 0.63174500 & 0.14336900 \\
\hline $\mathrm{H}$ & 1.59175800 & -0.37510700 & 3.89483600 & $\mathrm{C}$ & -3.15983800 & 1.26082400 & -0.42245700 \\
\hline $\mathrm{H}$ & 3.67800100 & 4.46490300 & 2.06219600 & $\mathrm{C}$ & -3.33721300 & 2.62643800 & -0.65052500 \\
\hline $\mathrm{H}$ & 3.16766900 & 2.87724500 & 0.38240800 & $\mathrm{C}$ & -4.54950800 & 3.26536100 & -0.31620100 \\
\hline $\mathrm{H}$ & 2.40765100 & 1.25129800 & 5.60070800 & $\mathrm{C}$ & -5.63912800 & 2.63043700 & 0.24698100 \\
\hline $\mathrm{H}$ & 4.46839800 & 0.90506800 & 0.99985400 & $\mathrm{C}$ & -6.30993000 & 0.22406300 & 1.09061900 \\
\hline $\mathrm{H}$ & 1.52770900 & 1.28844600 & 3.34782500 & $\mathrm{C}$ & -5.58596400 & -0.93925900 & 1.08348200 \\
\hline $\mathrm{H}$ & 3.77428600 & -0.69388800 & 2.63830200 & $\mathrm{H}$ & -2.56419300 & 3.25092900 & -1.07693000 \\
\hline $\mathrm{C}$ & 3.64116300 & 3.28821700 & 3.86201200 & $\mathrm{H}$ & -6.54098600 & 3.18523100 & 0.48241900 \\
\hline $\mathrm{H}$ & 4.14384700 & 4.13804900 & 4.34178600 & $\mathrm{H}$ & -7.31877300 & 0.33054500 & 1.46837300 \\
\hline
\end{tabular}




\begin{tabular}{|c|c|c|c|c|c|c|c|}
\hline $\mathrm{H}$ & -5.87169700 & -1.91703400 & 1.43696300 & $\mathrm{H}$ & -0.74054100 & 0.47241100 & -4.92337600 \\
\hline $\mathrm{N}$ & -4.34007800 & -0.72333800 & 0.48442200 & $\mathrm{C}$ & -0.03031900 & 2.96246800 & -1.64072100 \\
\hline $\mathrm{P}$ & -2.80379600 & -1.54148200 & 0.44439100 & $\mathrm{H}$ & -0.84543500 & 3.61391000 & -1.96154100 \\
\hline $\mathrm{C}$ & -3.14741200 & -3.20538000 & -0.46433300 & $\mathrm{H}$ & 0.00598200 & 2.98832300 & -0.54357700 \\
\hline $\mathrm{C}$ & -2.39271500 & -1.84268200 & 2.29823500 & $\mathrm{C}$ & -1.20637600 & 1.10552500 & -2.94442200 \\
\hline $\mathrm{C}$ & -4.50509700 & -3.82716500 & -0.06916200 & $\mathrm{H}$ & -2.07288000 & 1.75277500 & -3.07547200 \\
\hline $\mathrm{H}$ & -5.34207800 & -3.20087100 & -0.38902800 & $\mathrm{H}$ & -2.50219700 & -0.48384500 & -1.86471400 \\
\hline $\mathrm{H}$ & -4.60147900 & -4.79098800 & -0.58534000 & $\mathrm{Cl}$ & 0.11822300 & 0.91389300 & 1.04158000 \\
\hline $\mathrm{H}$ & -4.60188200 & -4.02312400 & 1.00042400 & $\mathrm{Rh}$ & 2.14995400 & -0.37234900 & 0.38487100 \\
\hline $\mathrm{C}$ & -3.19453800 & -2.94388600 & -1.98429800 & $\mathrm{C}$ & 4.07998800 & 3.37624000 & 2.14975800 \\
\hline $\mathrm{H}$ & -3.47422300 & -3.87981200 & -2.48572800 & $\mathrm{C}$ & 3.19320500 & 2.49466100 & 1.23835300 \\
\hline $\mathrm{H}$ & -3.94324700 & -2.18922300 & -2.24757200 & $\mathrm{C}$ & 3.20328800 & 0.90842700 & 4.64533800 \\
\hline $\mathrm{H}$ & -2.22402100 & -2.62715600 & -2.37238800 & $\mathrm{C}$ & 3.56421700 & 1.02617900 & 1.27891800 \\
\hline $\mathrm{C}$ & -2.01136000 & -4.21196800 & -0.18258600 & $\mathrm{C}$ & 2.29864600 & 0.51328700 & 3.45404300 \\
\hline $\mathrm{H}$ & -1.02546000 & -3.78757600 & -0.39251700 & $\mathrm{C}$ & 3.13851700 & 0.11328000 & 2.26315000 \\
\hline $\mathrm{H}$ & -2.02517000 & -4.58073700 & 0.84655800 & $\mathrm{H}$ & 5.14063200 & 3.17636700 & 1.93503600 \\
\hline $\mathrm{H}$ & -2.14936200 & -5.08054300 & -0.83925200 & $\mathrm{H}$ & 2.14397200 & 2.64220200 & 1.50747800 \\
\hline $\mathrm{C}$ & -0.92126500 & -2.28272400 & 2.44745600 & $\mathrm{H}$ & 3.68430300 & 0.00717200 & 5.05184000 \\
\hline $\mathrm{H}$ & -0.73087200 & -3.26993000 & 2.01971300 & $\mathrm{H}$ & 1.64891200 & -0.31432300 & 3.76397300 \\
\hline $\mathrm{H}$ & -0.23486700 & -1.57041700 & 1.98912800 & $\mathrm{H}$ & 3.90631400 & 4.42231500 & 1.86110200 \\
\hline $\mathrm{H}$ & -0.68808800 & -2.33773800 & 3.51926800 & $\mathrm{H}$ & 3.29831500 & 2.84205800 & 0.20324000 \\
\hline $\mathrm{C}$ & -2.59275700 & -0.48454400 & 3.00808200 & $\mathrm{H}$ & 2.55286600 & 1.29343300 & 5.44336700 \\
\hline $\mathrm{H}$ & -3.63840100 & -0.16282500 & 2.98856700 & $\mathrm{H}$ & 4.53159400 & 0.82638000 & 0.81759300 \\
\hline $\mathrm{H}$ & -2.29823000 & -0.60101300 & 4.05910300 & $\mathrm{H}$ & 1.63888600 & 1.34509900 & 3.20346000 \\
\hline $\mathrm{C}$ & -3.29803100 & -2.88469200 & 2.98619400 & $\mathrm{H}$ & 3.79297400 & -0.73026400 & 2.47934100 \\
\hline $\mathrm{H}$ & -3.02818000 & -2.91781900 & 4.04969300 & $\mathrm{C}$ & 3.84807900 & 3.26371300 & 3.67153100 \\
\hline $\mathrm{H}$ & -4.35811200 & -2.62097800 & 2.93298400 & $\mathrm{H}$ & 4.39516600 & 4.09436100 & 4.13625100 \\
\hline $\mathrm{H}$ & -3.16260700 & -3.89400600 & 2.58883800 & $\mathrm{H}$ & 2.78711800 & 3.45171200 & 3.89210600 \\
\hline $\mathrm{F}$ & -4.63408800 & 4.59639800 & -0.57472700 & $\mathrm{C}$ & 4.30417300 & 1.94540800 & 4.34562200 \\
\hline $\mathrm{Rh}$ & -1.56094100 & 0.05698500 & -0.78385200 & $\mathrm{H}$ & 4.78559200 & 2.18388000 & 5.30304800 \\
\hline $\mathrm{Cl}$ & 0.45383000 & -1.54540000 & -1.05848300 & $\mathrm{H}$ & 5.08784100 & 1.48245100 & 3.73133300 \\
\hline $\mathrm{H}$ & -1.97166600 & 0.30086400 & 2.57354200 & $\mathrm{C}$ & 4.32089800 & -4.29236000 & -0.50593600 \\
\hline $\mathrm{C}$ & 1.28137500 & 3.56623300 & -2.12108900 & $\mathrm{C}$ & 3.22116800 & -3.32406000 & -0.00989100 \\
\hline $\mathrm{O}$ & 2.29539200 & 2.95291100 & -2.38527900 & $\mathrm{C}$ & 4.74945000 & -1.79577300 & -3.09293400 \\
\hline $\mathrm{O}$ & 1.18844400 & 4.91081700 & -2.18518900 & $\mathrm{C}$ & 3.69307600 & -1.88978500 & 0.12779400 \\
\hline $\mathrm{C}$ & 2.39004200 & 5.59956700 & -2.56882000 & $\mathrm{C}$ & 3.47459600 & -1.30057000 & -2.36734100 \\
\hline $\mathrm{H}$ & 2.72740000 & 5.26399400 & -3.55301400 & $\mathrm{C}$ & 3.78546300 & -0.96297200 & -0.92798900 \\
\hline $\mathrm{H}$ & 2.12452600 & 6.65679200 & -2.59297700 & $\mathrm{H}$ & 5.21984200 & -4.17865700 & 0.11843800 \\
\hline $\mathrm{H}$ & 3.18542300 & 5.42058100 & -1.84002800 & $\mathrm{H}$ & 2.35611800 & -3.39354800 & -0.67454100 \\
\hline $\mathrm{C}$ & -0.18074100 & 1.54883300 & -2.14787100 & $\mathrm{H}$ & 5.43144100 & -0.94765900 & -3.24681800 \\
\hline $\mathrm{H}$ & 0.73801800 & 0.97036300 & -2.12651000 & $\mathrm{H}$ & 3.09298400 & -0.41655300 & -2.89253900 \\
\hline $\mathrm{C}$ & -1.04926200 & 0.01057900 & -3.97418000 & $\mathrm{H}$ & 3.95905000 & -5.31503500 & -0.33046400 \\
\hline $\mathrm{H}$ & -0.28257600 & -0.71035600 & -3.68081700 & $\mathrm{H}$ & 2.87755500 & -3.66710900 & 0.97540000 \\
\hline $\mathrm{H}$ & -1.98724100 & -0.52147900 & -4.15839200 & $\mathrm{H}$ & 4.45112600 & -2.13314800 & -4.09552800 \\
\hline
\end{tabular}




\begin{tabular}{|c|c|c|c|c|c|c|c|}
\hline $\mathrm{H}$ & 4.39847300 & -1.75775100 & 0.94856400 & $\mathrm{C}$ & 0.13126200 & -0.70065900 & 3.31160000 \\
\hline $\mathrm{H}$ & 2.69599600 & -2.06213600 & -2.43132300 & $\mathrm{H}$ & -0.01079700 & 0.30196600 & 3.72280900 \\
\hline $\mathrm{H}$ & 4.53229400 & -0.17586000 & -0.83985100 & $\mathrm{H}$ & -0.45241600 & -0.78166100 & 2.39232800 \\
\hline $\mathrm{C}$ & 4.72636100 & -4.18175600 & -1.99041700 & $\mathrm{H}$ & -0.28012400 & -1.41464800 & 4.03792700 \\
\hline $\mathrm{H}$ & 5.33290800 & -5.06780300 & -2.21889800 & $\mathrm{C}$ & 1.71997100 & -2.50843500 & 2.58994500 \\
\hline $\mathrm{H}$ & 3.82890300 & -4.26977100 & -2.61989500 & $\mathrm{H}$ & 2.75947700 & -2.83107900 & 2.47744400 \\
\hline $\mathrm{C}$ & 5.53434100 & -2.92241500 & -2.39259300 & $\mathrm{H}$ & 1.24813200 & -3.15602200 & 3.34069700 \\
\hline $\mathrm{H}$ & 6.34077000 & -3.22092500 & -3.07519400 & $\mathrm{C}$ & 2.38380000 & -0.92702400 & 4.41111700 \\
\hline \multirow[t]{2}{*}{$\mathrm{H}$} & 6.03811600 & -2.52159100 & -1.50290700 & $\mathrm{H}$ & 1.96186100 & -1.65316700 & 5.11861900 \\
\hline & & & & $\mathrm{H}$ & 3.44630700 & -1.16411300 & 4.30241400 \\
\hline \multirow[t]{6}{*}{ 12-ts } & & & & $\mathrm{H}$ & 2.28808600 & 0.06129400 & 4.86953300 \\
\hline & & & & $\mathrm{F}$ & 3.92234200 & -4.27257300 & -2.88055600 \\
\hline & & & & $\mathrm{Rh}$ & 1.26567300 & -0.08362900 & -0.56178100 \\
\hline & & & & $\mathrm{Cl}$ & -0.63858600 & 1.57472600 & 0.07738900 \\
\hline & & & & $\mathrm{H}$ & 1.20090100 & -2.66432800 & 1.64141200 \\
\hline & & & & $\mathrm{C}$ & 3.03277800 & 3.23180200 & -2.36305500 \\
\hline $\mathrm{C}$ & 4.84496300 & -2.40570700 & 0.07627900 & $\mathrm{O}$ & 4.10835700 & 2.68754400 & -2.22567600 \\
\hline $\mathrm{C}$ & 3.74952800 & -1.51501800 & 0.08513400 & $\mathrm{O}$ & 2.86718100 & 4.57035400 & -2.36753400 \\
\hline $\mathrm{C}$ & 2.70420100 & -1.49544400 & -0.85206800 & $\mathrm{C}$ & 4.06884100 & 5.34438600 & -2.21001300 \\
\hline $\mathrm{C}$ & 2.80026500 & -2.47575200 & -1.83901500 & $\mathrm{H}$ & 4.77913900 & 5.11674600 & -3.00921100 \\
\hline $\mathrm{C}$ & 3.89984300 & -3.35843000 & -1.87547100 & $\mathrm{H}$ & 3.75302200 & 6.38655500 & -2.26129000 \\
\hline $\mathrm{C}$ & 4.93696900 & -3.35052200 & -0.96433500 & $\mathrm{H}$ & 4.53775300 & 5.13195300 & -1.24562400 \\
\hline $\mathrm{C}$ & 5.60222200 & -2.12245300 & 1.26654700 & $\mathrm{C}$ & 1.79987300 & 1.00846500 & -2.51427900 \\
\hline $\mathrm{C}$ & 4.94825000 & -1.13101100 & 1.94890200 & $\mathrm{H}$ & 2.69677100 & 0.60021000 & -2.98065200 \\
\hline $\mathrm{H}$ & 2.03608700 & -2.61526800 & -2.59034800 & $\mathrm{C}$ & 0.48339500 & -0.91300100 & -3.62690800 \\
\hline $\mathrm{H}$ & 5.75145400 & -4.06220400 & -1.04748900 & $\mathrm{H}$ & 1.45359100 & -1.27374200 & -3.97616700 \\
\hline $\mathrm{H}$ & 6.52374200 & -2.59691800 & 1.57904900 & $\mathrm{H}$ & -0.08082700 & -1.75241500 & -3.20705300 \\
\hline $\mathrm{H}$ & 5.21469200 & -0.66449700 & 2.88398300 & $\mathrm{H}$ & -0.06993600 & -0.55991200 & -4.50957800 \\
\hline $\mathrm{N}$ & 3.81951000 & -0.71622100 & 1.23391000 & $\mathrm{C}$ & 1.70793000 & 2.52669500 & -2.58158800 \\
\hline $\mathrm{P}$ & 2.30008000 & 0.04179700 & 1.64760400 & $\mathrm{H}$ & 1.35701700 & 2.80450900 & -3.58590600 \\
\hline $\mathrm{C}$ & 2.77516100 & 1.78711500 & 2.28877600 & $\mathrm{H}$ & 0.95632300 & 2.91243100 & -1.88586500 \\
\hline $\mathrm{C}$ & 1.61900600 & -1.04487700 & 3.07883600 & $\mathrm{C}$ & 0.61187500 & 0.22696800 & -2.64177600 \\
\hline $\mathrm{C}$ & 4.04884000 & 1.81101700 & 3.16028800 & $\mathrm{H}$ & -0.32131200 & 0.76787600 & -2.48936200 \\
\hline $\mathrm{H}$ & 4.93271800 & 1.52285200 & 2.58486900 & $\mathrm{H}$ & 2.39017500 & 0.93407600 & -1.12601700 \\
\hline $\mathrm{H}$ & 4.20823600 & 2.83979600 & 3.50914700 & $\mathrm{Cl}$ & -0.64258100 & -1.66314400 & -0.19534200 \\
\hline $\mathrm{H}$ & 3.98194100 & 1.17583800 & 4.04612700 & $\mathrm{Rh}$ & -2.53726800 & -0.06093300 & -0.01886500 \\
\hline $\mathrm{C}$ & 3.04583700 & 2.67107100 & 1.05525400 & $\mathrm{C}$ & -4.48097000 & 3.41941000 & 2.20601800 \\
\hline $\mathrm{H}$ & 3.39110900 & 3.65591700 & 1.39846900 & $\mathrm{C}$ & -3.48225800 & 2.28335800 & 1.87609700 \\
\hline $\mathrm{H}$ & 3.82070900 & 2.25099300 & 0.40767700 & $\mathrm{C}$ & -4.56420500 & 3.81161400 & -1.38872200 \\
\hline $\mathrm{H}$ & 2.13771700 & 2.82072400 & 0.46570800 & $\mathrm{C}$ & -3.99968600 & 1.31181500 & 0.83387800 \\
\hline $\mathrm{C}$ & 1.59574100 & 2.39685200 & 3.07524800 & $\mathrm{C}$ & -3.42285200 & 2.78636200 & -1.19080900 \\
\hline $\mathrm{H}$ & 0.66024000 & 2.35646800 & 2.50912400 & $\mathrm{C}$ & -3.94654600 & 1.51749000 & -0.55782300 \\
\hline $\mathrm{H}$ & 1.44313800 & 1.90949800 & 4.04216200 & $\mathrm{H}$ & -5.46411300 & 2.98700500 & 2.44494800 \\
\hline $\mathrm{H}$ & 1.81923700 & 3.45298400 & 3.27543800 & $\mathrm{H}$ & -2.52865800 & 2.72119300 & 1.57066100 \\
\hline
\end{tabular}




\begin{tabular}{|c|c|c|c|c|c|c|c|}
\hline $\mathrm{H}$ & -5.23055200 & 3.46498200 & -2.19157700 & $\mathrm{C}$ & 4.14264600 & 1.27904600 & -0.09190100 \\
\hline $\mathrm{H}$ & -2.97258600 & 2.57170400 & -2.16843800 & $\mathrm{C}$ & 2.78331300 & 1.61563800 & -0.14469700 \\
\hline $\mathrm{H}$ & -4.13105500 & 3.90117600 & 3.12943900 & $\mathrm{C}$ & 2.53039400 & 2.98197500 & -0.28798000 \\
\hline $\mathrm{H}$ & -3.28050400 & 1.72351000 & 2.79900800 & $\mathrm{C}$ & 3.60163200 & 3.89554700 & -0.36469000 \\
\hline $\mathrm{H}$ & -4.11221500 & 4.74605700 & -1.75024200 & $\mathrm{C}$ & 4.94033400 & 3.54243600 & -0.33358400 \\
\hline $\mathrm{H}$ & -4.82272600 & 0.70067900 & 1.20404400 & $\mathrm{C}$ & 6.41402300 & 1.34920800 & -0.20484000 \\
\hline $\mathrm{H}$ & -2.63359100 & 3.22816200 & -0.58083600 & $\mathrm{C}$ & 6.02150500 & 0.04037600 & -0.09558100 \\
\hline $\mathrm{H}$ & -4.72127900 & 1.04301900 & -1.15837700 & $\mathrm{H}$ & 1.51977300 & 3.37242500 & -0.35081500 \\
\hline $\mathrm{C}$ & -4.66413100 & 4.51956200 & 1.13968200 & $\mathrm{H}$ & 5.70897300 & 4.30338400 & -0.41748500 \\
\hline $\mathrm{H}$ & -5.21494100 & 5.33719300 & 1.62265300 & $\mathrm{H}$ & 7.44025800 & 1.68871300 & -0.26622300 \\
\hline $\mathrm{H}$ & -3.68217000 & 4.94112900 & 0.87988000 & $\mathrm{H}$ & 6.63030200 & -0.84895700 & -0.06160200 \\
\hline $\mathrm{C}$ & -5.42605800 & 4.11530700 & -0.14719300 & $\mathrm{~N}$ & 4.62595600 & -0.03240600 & 0.01392400 \\
\hline $\mathrm{H}$ & -6.11040900 & 4.92729400 & -0.42546600 & $\mathrm{P}$ & 3.37326900 & -1.22095700 & -0.21118300 \\
\hline $\mathrm{H}$ & -6.06969900 & 3.25351500 & 0.07430800 & $\mathrm{C}$ & 3.83215300 & -2.73374800 & 0.87775000 \\
\hline $\mathrm{C}$ & -4.56653600 & -3.55950800 & -2.13752300 & $\mathrm{C}$ & 3.50609800 & -1.67216200 & -2.08977600 \\
\hline $\mathrm{C}$ & -3.55966900 & -2.41754500 & -1.85965400 & $\mathrm{C}$ & 5.33609700 & -3.08329100 & 0.86277100 \\
\hline $\mathrm{C}$ & -4.40763200 & -3.97827100 & 1.45266200 & $\mathrm{H}$ & 5.92846100 & -2.31284900 & 1.36385900 \\
\hline $\mathrm{C}$ & -4.02399400 & -1.46019100 & -0.77995000 & $\mathrm{H}$ & 5.47497900 & -4.01499700 & 1.42635400 \\
\hline $\mathrm{C}$ & -3.29258500 & -2.93763900 & 1.19147700 & $\mathrm{H}$ & 5.74224900 & -3.24367700 & -0.13703900 \\
\hline $\mathrm{C}$ & -3.87418900 & -1.67239000 & 0.60413600 & $\mathrm{C}$ & 3.45314700 & -2.42792700 & 2.34050400 \\
\hline $\mathrm{H}$ & -5.56618100 & -3.13410500 & -2.31282800 & $\mathrm{H}$ & 3.78659900 & -3.26637500 & 2.96551300 \\
\hline $\mathrm{H}$ & -2.58583500 & -2.84898900 & -1.61621800 & $\mathrm{H}$ & 3.93890300 & -1.52318200 & 2.71463100 \\
\hline $\mathrm{H}$ & -5.02205300 & -3.64848200 & 2.30274200 & $\mathrm{H}$ & 2.37396300 & -2.32874800 & 2.46929700 \\
\hline $\mathrm{H}$ & -2.78051700 & -2.72422800 & 2.13794300 & $\mathrm{C}$ & 3.00091900 & -3.95043400 & 0.41683100 \\
\hline $\mathrm{H}$ & -4.26928600 & -4.03174500 & -3.08386200 & $\mathrm{H}$ & 1.93376300 & -3.71659300 & 0.34148600 \\
\hline $\mathrm{H}$ & -3.41926200 & -1.84806400 & -2.78810300 & $\mathrm{H}$ & 3.34010100 & -4.35116400 & -0.54172900 \\
\hline $\mathrm{H}$ & -3.92227100 & -4.91136000 & 1.77141200 & $\mathrm{H}$ & 3.11440400 & -4.74896600 & 1.16105900 \\
\hline $\mathrm{H}$ & -4.88174900 & -0.864499000 & -1.09083400 & $\mathrm{C}$ & 2.25400800 & -2.44357900 & -2.55944500 \\
\hline $\mathrm{H}$ & -2.54122700 & -3.36741500 & 0.52748600 & $\mathrm{H}$ & 2.15688500 & -3.42856200 & -2.09808300 \\
\hline $\mathrm{H}$ & -4.61262600 & -1.21149200 & 1.25884300 & $\mathrm{H}$ & 1.33147100 & -1.89270300 & -2.36051100 \\
\hline $\mathrm{C}$ & -4.67492600 & -4.66863200 & -1.06999900 & $\mathrm{H}$ & 2.33109100 & -2.59449300 & -3.64426600 \\
\hline $\mathrm{H}$ & -5.25576900 & -5.48332400 & -1.52155700 & $\mathrm{C}$ & 3.56142700 & -0.33686800 & -2.86891100 \\
\hline $\mathrm{H}$ & -3.67708000 & -5.09025700 & -0.88076200 & $\mathrm{H}$ & 4.44803600 & 0.25424900 & -2.62380900 \\
\hline $\mathrm{C}$ & -5.34898500 & -4.27679900 & 0.26890900 & $\mathrm{H}$ & 3.59826700 & -0.57003300 & -3.94076400 \\
\hline $\mathrm{H}$ & -6.00951700 & -5.09412000 & 0.58645200 & $\mathrm{C}$ & 4.77114300 & -2.47811100 & -2.44180100 \\
\hline \multirow[t]{2}{*}{$\mathrm{H}$} & -6.00981300 & -3.41619800 & 0.09881200 & $\mathrm{H}$ & 4.82284000 & -2.58059800 & -3.53348200 \\
\hline & & & & $\mathrm{H}$ & 5.68746300 & -1.97229500 & -2.12098500 \\
\hline \multirow[t]{6}{*}{13} & & & & $\mathrm{H}$ & 4.75875500 & -3.48896700 & -2.02566200 \\
\hline & & & & $\mathrm{F}$ & 3.28804100 & 5.21096400 & -0.48742700 \\
\hline & & & & $\mathrm{Rh}$ & 1.47012000 & 0.08791300 & -0.04109500 \\
\hline & & & & $\mathrm{Cl}$ & -0.30875200 & -1.73825100 & -0.06481400 \\
\hline & & & & $\mathrm{H}$ & 2.67845700 & 0.28636500 & -2.69741100 \\
\hline & & & & $\mathrm{C}$ & 1.30716700 & 0.28184400 & 2.04866400 \\
\hline $\mathrm{C}$ & & 2.16788600 & -0.21090600 & $\mathrm{H}$ & 1.21140500 & -0.76647000 & 2.34516100 \\
\hline
\end{tabular}




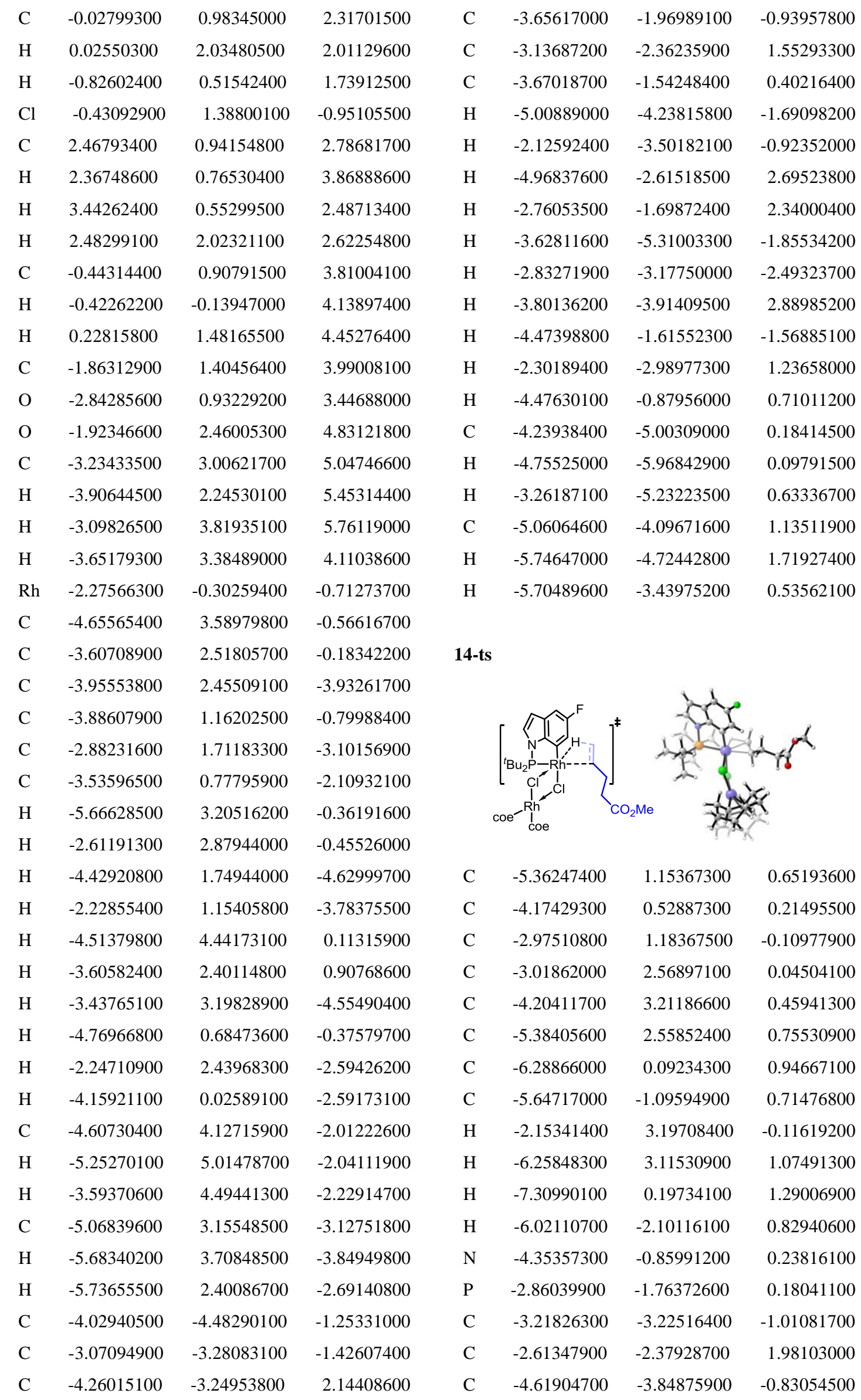




\begin{tabular}{|c|c|c|c|c|c|c|c|}
\hline $\mathrm{H}$ & -5.41025600 & -3.13554300 & -1.07760700 & $\mathrm{H}$ & 0.14026400 & 2.95691100 & -0.65359500 \\
\hline $\mathrm{H}$ & -4.71277400 & -4.69416800 & -1.52464900 & $\mathrm{Cl}$ & 0.10223900 & 0.51448700 & 1.19886800 \\
\hline $\mathrm{H}$ & -4.79650400 & -4.23575400 & 0.17533400 & $\mathrm{Rh}$ & 2.18112700 & -0.59147000 & 0.35468400 \\
\hline $\mathrm{C}$ & -3.13877400 & -2.68215000 & -2.45356500 & $\mathrm{C}$ & 3.82995100 & 3.40369500 & 1.83215300 \\
\hline $\mathrm{H}$ & -3.41513600 & -3.48636200 & -3.14809100 & $\mathrm{C}$ & 2.95421900 & 2.39787500 & 1.04688500 \\
\hline $\mathrm{H}$ & -3.83326700 & -1.85012100 & -2.61506300 & $\mathrm{C}$ & 3.53266800 & 0.93339900 & 4.45837300 \\
\hline $\mathrm{H}$ & -2.12718300 & -2.35281900 & -2.70365700 & $\mathrm{C}$ & 3.50944300 & 0.98812800 & 1.06903000 \\
\hline $\mathrm{C}$ & -2.13877100 & -4.31572200 & -0.84287600 & $\mathrm{C}$ & 2.56680800 & 0.38171100 & 3.38179300 \\
\hline $\mathrm{H}$ & -1.12662200 & -3.90534300 & -0.90734200 & $\mathrm{C}$ & 3.31110400 & 0.05663200 & 2.10668300 \\
\hline $\mathrm{H}$ & -2.23947400 & -4.85772100 & 0.10170400 & $\mathrm{H}$ & 4.87761200 & 3.31424100 & 1.50734200 \\
\hline $\mathrm{H}$ & -2.25447900 & -5.04948600 & -1.65127800 & $\mathrm{H}$ & 1.93600900 & 2.43247600 & 1.43975900 \\
\hline $\mathrm{C}$ & -1.16391600 & -2.88588500 & 2.14612800 & $\mathrm{H}$ & 4.16389400 & 0.11445300 & 4.83243100 \\
\hline $\mathrm{H}$ & -0.96715200 & -3.79391900 & 1.57076000 & $\mathrm{H}$ & 2.07376900 & -0.51525300 & 3.77746500 \\
\hline $\mathrm{H}$ & -0.43347600 & -2.12827200 & 1.85513800 & $\mathrm{H}$ & 3.50423600 & 4.40728700 & 1.53030500 \\
\hline $\mathrm{H}$ & -1.00015100 & -3.12497500 & 3.20547100 & $\mathrm{H}$ & 2.89773700 & 2.74575300 & 0.00863000 \\
\hline $\mathrm{C}$ & -2.81052600 & -1.13896200 & 2.88288300 & $\mathrm{H}$ & 2.92467300 & 1.26370800 & 5.31235000 \\
\hline $\mathrm{H}$ & -3.83947100 & -0.76762300 & 2.85167400 & $\mathrm{H}$ & 4.43261800 & 0.89811300 & 0.49811500 \\
\hline $\mathrm{H}$ & -2.58950700 & -1.42727300 & 3.91887700 & $\mathrm{H}$ & 1.77445100 & 1.10869800 & 3.19447900 \\
\hline $\mathrm{C}$ & -3.59323700 & -3.47734400 & 2.43735000 & $\mathrm{H}$ & 4.09853200 & -0.68150500 & 2.25847300 \\
\hline $\mathrm{H}$ & -3.40700200 & -3.68954700 & 3.49843000 & $\mathrm{C}$ & 3.77335500 & 3.31694800 & 3.37107100 \\
\hline $\mathrm{H}$ & -4.63836000 & -3.16514000 & 2.35051700 & $\mathrm{H}$ & 4.25988500 & 4.22231600 & 3.75682800 \\
\hline $\mathrm{H}$ & -3.45920700 & -4.41614200 & 1.89258900 & $\mathrm{H}$ & 2.72629600 & 3.38337600 & 3.70075600 \\
\hline $\mathrm{F}$ & -4.16354300 & 4.56527200 & 0.57098300 & $\mathrm{C}$ & 4.45636800 & 2.08630500 & 4.01844300 \\
\hline $\mathrm{Rh}$ & -1.43371000 & -0.01245400 & -0.69910700 & $\mathrm{H}$ & 5.00353200 & 2.41232800 & 4.91275100 \\
\hline $\mathrm{Cl}$ & 0.50574900 & -1.65211500 & -1.20359700 & $\mathrm{H}$ & 5.22257300 & 1.70113700 & 3.33204900 \\
\hline $\mathrm{H}$ & -2.13434700 & -0.32418700 & 2.61285700 & $\mathrm{C}$ & 4.11777000 & -4.63798400 & -0.44267900 \\
\hline $\mathrm{C}$ & 1.25805600 & 4.87974500 & -2.12404200 & $\mathrm{C}$ & 3.04942900 & -3.59641700 & -0.03002200 \\
\hline $\mathrm{O}$ & 2.09912000 & 5.15643700 & -1.29322000 & $\mathrm{C}$ & 4.91458900 & -2.17128500 & -2.96448400 \\
\hline $\mathrm{O}$ & 0.45588000 & 5.79921600 & -2.70487500 & $\mathrm{C}$ & 3.62305100 & -2.20775000 & 0.17159500 \\
\hline $\mathrm{C}$ & 0.64452400 & 7.15249100 & -2.25941800 & $\mathrm{C}$ & 3.63701000 & -1.58331900 & -2.32015100 \\
\hline $\mathrm{H}$ & 1.66391100 & 7.48705900 & -2.47009400 & $\mathrm{C}$ & 3.86116000 & -1.27534000 & -0.85730300 \\
\hline $\mathrm{H}$ & -0.07822000 & 7.74792400 & -2.81790300 & $\mathrm{H}$ & 4.96894100 & -4.58804600 & 0.25276200 \\
\hline $\mathrm{H}$ & 0.45996000 & 7.23188600 & -1.18477600 & $\mathrm{H}$ & 2.24802500 & -3.59275900 & -0.77217200 \\
\hline $\mathrm{C}$ & -0.16207600 & 2.84015600 & -1.70033300 & $\mathrm{H}$ & 5.66797000 & -1.37719900 & -3.06701000 \\
\hline $\mathrm{H}$ & -1.09888200 & 3.38478700 & -1.84645300 & $\mathrm{H}$ & 3.35617800 & -0.67333500 & -2.86596300 \\
\hline $\mathrm{C}$ & -1.38916900 & 0.84151500 & -2.80687800 & $\mathrm{H}$ & 3.67240500 & -5.63271600 & -0.30349200 \\
\hline $\mathrm{H}$ & -2.20873000 & 1.50699100 & -3.07814100 & $\mathrm{H}$ & 2.59450600 & -3.92316100 & 0.91444800 \\
\hline $\mathrm{H}$ & -1.15372100 & 0.08435700 & -3.55485200 & $\mathrm{H}$ & 4.65951600 & -2.48297200 & -3.98723900 \\
\hline $\mathrm{H}$ & -2.25521300 & -0.12951200 & -2.08439000 & $\mathrm{H}$ & 4.26684800 & -2.14994700 & 1.04731700 \\
\hline $\mathrm{C}$ & 0.93216300 & 3.47953000 & -2.59737400 & $\mathrm{H}$ & 2.80733700 & -2.28116000 & -2.44519500 \\
\hline $\mathrm{H}$ & 1.85238700 & 2.89103500 & -2.53328900 & $\mathrm{H}$ & 4.67375700 & -0.56749000 & -0.69650700 \\
\hline $\mathrm{H}$ & 0.59871300 & 3.50106700 & -3.63987400 & $\mathrm{C}$ & 4.64772000 & -4.55606200 & -1.88960400 \\
\hline $\mathrm{C}$ & -0.32304700 & 1.37338100 & -2.02591900 & $\mathrm{H}$ & 5.21091500 & -5.48087500 & -2.07051900 \\
\hline $\mathrm{H}$ & 0.62510200 & 0.84563200 & -2.10510200 & $\mathrm{H}$ & 3.79978000 & -4.58745700 & -2.58953100 \\
\hline
\end{tabular}




\begin{tabular}{|c|c|c|c|c|c|c|c|}
\hline $\mathrm{C}$ & 5.56726200 & -3.35535900 & -2.22387800 & $\mathrm{H}$ & 1.96121700 & -4.37065200 & 1.44406100 \\
\hline $\mathrm{H}$ & 6.39424500 & -3.70707900 & -2.85439600 & $\mathrm{C}$ & 2.82879700 & -2.86611000 & 3.53119300 \\
\hline \multirow[t]{2}{*}{$\mathrm{H}$} & 6.03770500 & -2.99657200 & -1.29865100 & $\mathrm{H}$ & 2.51859400 & -3.89402400 & 3.75998100 \\
\hline & & & & $\mathrm{H}$ & 3.90776000 & -2.88997200 & 3.35222600 \\
\hline \multirow[t]{6}{*}{15} & & & & $\mathrm{H}$ & 2.63045500 & -2.26473600 & 4.42242300 \\
\hline & & & & $\mathrm{F}$ & 4.80015100 & -1.07239800 & -4.41743300 \\
\hline & & & & $\mathrm{Rh}$ & 1.46158000 & 0.23621500 & -0.27934400 \\
\hline & & & & $\mathrm{Cl}$ & -0.67115900 & 0.94207300 & 0.98793400 \\
\hline & & & & $\mathrm{H}$ & 1.76720000 & -3.10079900 & 0.22942900 \\
\hline & & & & $\mathrm{C}$ & 0.51738400 & 5.79666000 & -1.61978100 \\
\hline $\mathrm{C}$ & 02600 & -1.38667000 & 0.86339300 & $\mathrm{O}$ & 0.72438200 & 6.09977800 & -0.46445900 \\
\hline $\mathrm{C}$ & 4.16594800 & -0.87134200 & -0.42505900 & $\mathrm{O}$ & 0.03960100 & 6.66071800 & -2.54308600 \\
\hline $\mathrm{C}$ & 3.11686900 & -0.43404600 & -1.24428800 & $\mathrm{C}$ & -0.19765800 & 7.99677100 & -2.07100800 \\
\hline $\mathrm{C}$ & 3.38205900 & -0.52144500 & -2.61225900 & $\mathrm{H}$ & 0.72894800 & 8.44558100 & -1.70269300 \\
\hline $\mathrm{C}$ & 4.61884500 & -1.02030500 & -3.07238600 & $\mathrm{H}$ & -0.57481100 & 8.54885000 & -2.93243700 \\
\hline $\mathrm{C}$ & 5.64270800 & -1.45173800 & -2.25052700 & $\mathrm{H}$ & -0.93463600 & 7.99376000 & -1.26338000 \\
\hline $\mathrm{C}$ & 6.12694500 & -1.75111600 & 0.32645000 & $\mathrm{C}$ & 0.97413000 & 3.34360400 & -1.16915300 \\
\hline $\mathrm{C}$ & 5.32896300 & -1.46713500 & 1.40409300 & $\mathrm{H}$ & 0.05945700 & 3.22156400 & -0.58230700 \\
\hline $\mathrm{H}$ & 2.66270300 & -0.22741600 & -3.36967300 & $\mathrm{C}$ & 0.45404200 & 1.06382700 & -2.18151200 \\
\hline $\mathrm{H}$ & 6.56752900 & -1.82735200 & -2.67507300 & $\mathrm{H}$ & -0.59455600 & 1.15937300 & -1.91616200 \\
\hline $\mathrm{H}$ & 7.12536000 & -2.16600300 & 0.37976100 & $\mathrm{H}$ & 0.69121400 & 0.30165000 & -2.91361300 \\
\hline $\mathrm{H}$ & 5.53635100 & -1.59911600 & 2.45410200 & $\mathrm{H}$ & 2.39452200 & 1.39216400 & 0.11157200 \\
\hline $\mathrm{N}$ & 4.12402100 & -0.90216400 & 0.97291700 & $\mathrm{C}$ & 0.76931200 & 4.43245300 & -2.23643500 \\
\hline $\mathrm{P}$ & 2.53790900 & -0.65305900 & 1.65218500 & $\mathrm{H}$ & 1.65739400 & 4.52290200 & -2.87846900 \\
\hline $\mathrm{C}$ & 2.79684300 & 0.58051700 & 3.10356900 & $\mathrm{H}$ & -0.06126700 & 4.18768400 & -2.90782500 \\
\hline $\mathrm{C}$ & 2.03487200 & -2.39111300 & 2.29824400 & $\mathrm{C}$ & 1.35748000 & 2.03516500 & -1.82175500 \\
\hline $\mathrm{C}$ & 4.08322700 & 0.29800800 & 3.91034300 & $\mathrm{H}$ & 2.35292800 & 2.00158400 & -2.25977800 \\
\hline $\mathrm{H}$ & 4.97909500 & 0.45267400 & 3.30290000 & $\mathrm{H}$ & 1.75462400 & 3.67709100 & -0.47889700 \\
\hline $\mathrm{H}$ & 4.12723000 & 1.00959300 & 4.74480400 & $\mathrm{Cl}$ & -0.21890100 & -1.67955600 & -0.87432400 \\
\hline $\mathrm{H}$ & 4.11971300 & -0.70520100 & 4.33953100 & $\mathrm{Rh}$ & -2.31621100 & -0.66521800 & -0.00911800 \\
\hline $\mathrm{C}$ & 2.92579400 & 2.00613000 & 2.52616700 & $\mathrm{C}$ & -4.69087100 & 0.87381600 & 3.57522600 \\
\hline $\mathrm{H}$ & 3.15049800 & 2.69385500 & 3.35185200 & $\mathrm{C}$ & -3.54348000 & 0.22553000 & 2.76325000 \\
\hline $\mathrm{H}$ & 3.74075000 & 2.08242700 & 1.79860200 & $\mathrm{C}$ & -4.91280500 & 3.04678400 & 0.69199400 \\
\hline $\mathrm{H}$ & 1.99902200 & 2.33779800 & 2.05233200 & $\mathrm{C}$ & -3.93982400 & -0.13181100 & 1.34426200 \\
\hline $\mathrm{C}$ & 1.57215300 & 0.55324600 & 4.04313700 & $\mathrm{C}$ & -3.62838900 & 2.23633100 & 0.39618700 \\
\hline $\mathrm{H}$ & 0.63399300 & 0.68942100 & 3.49717200 & $\mathrm{C}$ & -3.94625900 & 0.76499300 & 0.25948600 \\
\hline $\mathrm{H}$ & 1.51068500 & -0.36944000 & 4.62660100 & $\mathrm{H}$ & -5.59084300 & 0.24461600 & 3.50453100 \\
\hline $\mathrm{H}$ & 1.66558700 & 1.38159900 & 4.75712800 & $\mathrm{H}$ & -2.67461600 & 0.88816500 & 2.77692500 \\
\hline $\mathrm{C}$ & 0.52763100 & -2.40726300 & 2.63157400 & $\mathrm{H}$ & -5.53823600 & 3.08039100 & -0.21166900 \\
\hline $\mathrm{H}$ & 0.28156100 & -1.78639300 & 3.49625000 & $\mathrm{H}$ & -3.17695200 & 2.61960100 & -0.52781900 \\
\hline $\mathrm{H}$ & -0.08050600 & -2.08041300 & 1.78704500 & $\mathrm{H}$ & -4.39230000 & 0.85584900 & 4.63249700 \\
\hline $\mathrm{H}$ & 0.24198000 & -3.43924600 & 2.87557800 & $\mathrm{H}$ & -3.23588400 & -0.69486500 & 3.27743200 \\
\hline $\mathrm{C}$ & 2.30791400 & -3.37523700 & 1.13730800 & $\mathrm{H}$ & -4.61238300 & 4.08458900 & 0.89361400 \\
\hline $\mathrm{H}$ & 3.37492000 & -3.45011800 & 0.90605900 & $\mathrm{H}$ & -4.65827600 & -0.95021200 & 1.30485500 \\
\hline
\end{tabular}




\begin{tabular}{|c|c|c|c|c|c|c|c|}
\hline $\mathrm{H}$ & -2.89590600 & 2.40613200 & 1.18644700 & $\mathrm{C}$ & 5.17452600 & -1.72967500 & 1.43894800 \\
\hline $\mathrm{H}$ & -4.65736900 & 0.56736600 & -0.54165100 & $\mathrm{H}$ & 2.39150800 & -0.84514600 & -3.34630700 \\
\hline $\mathrm{C}$ & -5.06699500 & 2.32626700 & 3.21476300 & $\mathrm{H}$ & 6.21215900 & -2.62337000 & -2.60881600 \\
\hline $\mathrm{H}$ & -5.72845500 & 2.68744500 & 4.01294800 & $\mathrm{H}$ & 6.86884500 & -2.67328100 & 0.43462100 \\
\hline $\mathrm{H}$ & -4.16921200 & 2.95904100 & 3.26881800 & $\mathrm{H}$ & 5.41068900 & -1.77486600 & 2.49058400 \\
\hline $\mathrm{C}$ & -5.78338200 & 2.53689200 & 1.85739600 & $\mathrm{~N}$ & 3.99954200 & -1.11845800 & 0.99250300 \\
\hline $\mathrm{H}$ & -6.59352800 & 3.26542400 & 1.99194700 & $\mathrm{P}$ & 2.46364900 & -0.68291300 & 1.70397600 \\
\hline $\mathrm{H}$ & -6.27712900 & 1.60078400 & 1.56467500 & $\mathrm{C}$ & 2.89143000 & 0.65082800 & 3.01282600 \\
\hline $\mathrm{C}$ & -3.88434300 & -2.80696500 & -3.72510100 & $\mathrm{C}$ & 1.86499700 & -2.30400000 & 2.53969100 \\
\hline $\mathrm{C}$ & -3.04275500 & -1.84754900 & -2.84938500 & $\mathrm{C}$ & 4.15808300 & 0.34242400 & 3.83849600 \\
\hline $\mathrm{C}$ & -3.58927200 & -4.98710200 & -0.85297300 & $\mathrm{H}$ & 5.05340700 & 0.33973700 & 3.21065800 \\
\hline $\mathrm{C}$ & -3.61088600 & -1.65257900 & -1.45730400 & $\mathrm{H}$ & 4.28684600 & 1.13543300 & 4.58672800 \\
\hline $\mathrm{C}$ & -2.63701000 & -3.82408200 & -0.48724900 & $\mathrm{H}$ & 4.10563600 & -0.60571800 & 4.37675600 \\
\hline $\mathrm{C}$ & -3.40276000 & -2.52641400 & -0.37298500 & $\mathrm{C}$ & 3.14384800 & 1.97576900 & 2.26244200 \\
\hline $\mathrm{H}$ & -4.93740600 & -2.48782100 & -3.71234800 & $\mathrm{H}$ & 3.46904300 & 2.73458900 & 2.98604600 \\
\hline $\mathrm{H}$ & -2.01103100 & -2.20488600 & -2.80853600 & $\mathrm{H}$ & 3.93262300 & 1.87500200 & 1.50855000 \\
\hline $\mathrm{H}$ & -4.22404100 & -5.22502200 & 0.01274400 & $\mathrm{H}$ & 2.23677300 & 2.34284500 & 1.77681900 \\
\hline $\mathrm{H}$ & -2.13778100 & -4.06134200 & 0.46041400 & $\mathrm{C}$ & 1.68434100 & 0.85038100 & 3.95372500 \\
\hline $\mathrm{H}$ & -3.54399900 & -2.68234800 & -4.76224400 & $\mathrm{H}$ & 0.75568600 & 1.01562700 & 3.39824000 \\
\hline $\mathrm{H}$ & -3.00884200 & -0.86920700 & -3.34768100 & $\mathrm{H}$ & 1.54115800 & 0.00432400 & 4.63211100 \\
\hline $\mathrm{H}$ & -2.97204400 & -5.87857100 & -1.03249400 & $\mathrm{H}$ & 1.86532100 & 1.73810600 & 4.57345600 \\
\hline $\mathrm{H}$ & -4.55084000 & -1.10062100 & -1.46383300 & $\mathrm{C}$ & 0.36282900 & -2.16441400 & 2.87059600 \\
\hline $\mathrm{H}$ & -1.84873200 & -3.74651700 & -1.23743700 & $\mathrm{H}$ & 0.16817600 & -1.41535400 & 3.64287300 \\
\hline $\mathrm{H}$ & -4.18409200 & -2.56929700 & 0.38470300 & $\mathrm{H}$ & -0.22277600 & -1.90578600 & 1.98617500 \\
\hline $\mathrm{C}$ & -3.81304800 & -4.30998600 & -3.38249100 & $\mathrm{H}$ & -0.00108300 & -3.12929600 & 3.24879900 \\
\hline $\mathrm{H}$ & -4.28263600 & -4.84538400 & -4.21792300 & $\mathrm{C}$ & 2.04010600 & -3.41740300 & 1.48127500 \\
\hline $\mathrm{H}$ & -2.76191900 & -4.63293700 & -3.38105300 & $\mathrm{H}$ & 3.09437300 & -3.60695300 & 1.25750700 \\
\hline $\mathrm{C}$ & -4.50650900 & -4.75387500 & -2.06999000 & $\mathrm{H}$ & 1.61012300 & -4.34499200 & 1.88122800 \\
\hline $\mathrm{H}$ & -5.04149000 & -5.69524000 & -2.25110200 & $\mathrm{C}$ & 2.62954700 & -2.71957100 & 3.81116100 \\
\hline \multirow[t]{2}{*}{$\mathrm{H}$} & -5.28233700 & -4.02152100 & -1.81006200 & $\mathrm{H}$ & 2.25026200 & -3.69650000 & 4.13926800 \\
\hline & & & & $\mathrm{H}$ & 3.70325400 & -2.83578500 & 3.63425000 \\
\hline \multirow[t]{6}{*}{ 16-ts } & & \multirow{6}{*}{ $\mathrm{Me}$ के } & & $\mathrm{H}$ & 2.48264500 & -2.02348200 & 4.64176800 \\
\hline & & & & $\mathrm{F}$ & 4.42601900 & -1.95161100 & -4.36739300 \\
\hline & & & & $\mathrm{Rl}$ & 1.37215300 & 0.10879900 & -0.33158700 \\
\hline & & & & $\mathrm{Cl}$ & -0.68318000 & 1.09202800 & 0.87320200 \\
\hline & & & & $\mathrm{H}$ & 1.52013600 & -3.18399000 & 0.54947100 \\
\hline & & & & $\mathrm{C}$ & 1.15188800 & 5.79776100 & -1.74680800 \\
\hline $\mathrm{C}$ & 4600 & -1.88997200 & -0.82397500 & $\mathrm{O}$ & 1.32904600 & 6.19193200 & -0.61431200 \\
\hline $\mathrm{C}$ & 6900 & -1.23322700 & -0.40169400 & $\mathrm{O}$ & 0.77975100 & 6.60035300 & -2.76808800 \\
\hline $\mathrm{C}$ & 2.93829700 & -0.81423800 & -1.23682700 & $\mathrm{C}$ & 0.61445400 & 7.98792500 & -2.43349800 \\
\hline $\mathrm{C}$ & 3.13185600 & -1.08659800 & -2.59146400 & $\mathrm{H}$ & 1.55320100 & 8.40918500 & -2.06341700 \\
\hline $\mathrm{C}$ & 4.30972600 & -1.72552000 & -3.03301500 & $\mathrm{H}$ & 0.31345300 & 8.47817100 & -3.35981200 \\
\hline $\mathrm{C}$ & 5.33558700 & -2.13098500 & -2.20141400 & $\mathrm{H}$ & -0.15501400 & 8.10935000 & -1.66637700 \\
\hline $\mathrm{C}$ & 5.90467000 & -2.18513500 & 0.37196900 & $\mathrm{C}$ & 1.50605500 & 3.38448200 & -1.05964700 \\
\hline
\end{tabular}




\begin{tabular}{|c|c|c|c|c|c|c|c|}
\hline $\mathrm{H}$ & 0.59935100 & 3.36278900 & -0.44774000 & $\mathrm{H}$ & -2.34960900 & -3.87790800 & 0.64436500 \\
\hline $\mathrm{C}$ & 0.75751300 & 1.15914300 & -2.10536800 & $\mathrm{H}$ & -3.59385700 & -2.75605000 & -4.67667100 \\
\hline $\mathrm{H}$ & -0.27382600 & 1.47588300 & -1.97035200 & $\mathrm{H}$ & -3.01210000 & -0.88521100 & -3.35879100 \\
\hline $\mathrm{H}$ & 0.94190700 & 0.53947400 & -2.97550200 & $\mathrm{H}$ & -3.22714000 & -5.74869300 & -0.75480200 \\
\hline $\mathrm{H}$ & 2.32824300 & 1.41870500 & -0.31678600 & $\mathrm{H}$ & -4.60394300 & -0.94481300 & -1.50357400 \\
\hline $\mathrm{C}$ & 1.32781700 & 4.36769100 & -2.22443400 & $\mathrm{H}$ & -2.02009600 & -3.67857300 & -1.06429900 \\
\hline $\mathrm{H}$ & 2.20056900 & 4.34883600 & -2.89278900 & $\mathrm{H}$ & -4.34072100 & -2.32147500 & 0.43291600 \\
\hline $\mathrm{H}$ & 0.46922900 & 4.09675200 & -2.84932400 & $\mathrm{C}$ & -3.95575400 & -4.28871600 & -3.21221300 \\
\hline $\mathrm{C}$ & 1.80177500 & 1.98663400 & -1.59182500 & $\mathrm{H}$ & -4.43022300 & -4.85190300 & -4.02632600 \\
\hline $\mathrm{H}$ & 2.76758500 & 1.88943800 & -2.09178400 & $\mathrm{H}$ & -2.91918300 & -4.65314400 & -3.16943300 \\
\hline $\mathrm{H}$ & 2.31870300 & 3.73878700 & -0.41767200 & $\mathrm{C}$ & -4.69327400 & -4.63026000 & -1.89290300 \\
\hline $\mathrm{Cl}$ & -0.30818400 & -1.67430000 & -0.78729500 & $\mathrm{H}$ & -5.25798000 & -5.56127600 & -2.03256200 \\
\hline $\mathrm{Rh}$ & -2.38425100 & -0.51863000 & -0.02288800 & $\mathrm{H}$ & -5.44823900 & -3.85776900 & 59430700 \\
\hline $\mathrm{C}$ & -4.65079200 & 1.27822300 & 3.50158400 & & & & \\
\hline $\mathrm{C}$ & -3.53500800 & 0.55681500 & 2.70814000 & 17 & & & \\
\hline $\mathrm{C}$ & -4.88414400 & 3.28280400 & 0.49703900 & & & & \\
\hline $\mathrm{C}$ & -3.96878800 & 0.12779300 & 1.31988700 & & & & \\
\hline $\mathrm{C}$ & -3.62475000 & 2.42650700 & 0.21915800 & & & & \\
\hline $\mathrm{C}$ & -3.97747500 & 0.95733000 & 0.18162600 & & & & \\
\hline $\mathrm{H}$ & -5.56589400 & 0.66727200 & 3.48717500 & & & & \\
\hline $\mathrm{H}$ & -2.65100900 & 1.19737500 & 2.66745300 & & & & \\
\hline $\mathrm{H}$ & -5.52879400 & 3.28031000 & -0.39363100 & $\mathrm{C}$ & 4.41580900 & -0.79622600 & -2.78292600 \\
\hline $\mathrm{H}$ & -3.19029200 & 2.74138300 & -0.73819300 & $\mathrm{C}$ & 3.33696600 & -0.92876000 & -1.88649300 \\
\hline $\mathrm{H}$ & -4.33024200 & 1.31677500 & 4.55181700 & $\mathrm{C}$ & 2.25942200 & -0.04561000 & -1.73538500 \\
\hline $\mathrm{H}$ & -3.23872900 & -0.34000300 & 3.26850700 & $\mathrm{C}$ & 2.29964600 & 1.05784100 & -2.59152900 \\
\hline $\mathrm{H}$ & -4.55668600 & 4.32309300 & 0.63337000 & $\mathrm{C}$ & 3.37073500 & 1.21663300 & -3.49422400 \\
\hline $\mathrm{H}$ & -4.70882600 & -0.67232700 & 1.34656100 & $\mathrm{C}$ & 4.43304300 & 0.33684400 & -3.62064200 \\
\hline $\mathrm{H}$ & -2.86740200 & 2.62662200 & 0.97843100 & $\mathrm{C}$ & 5.25310100 & -1.95025800 & -2.56933800 \\
\hline $\mathrm{H}$ & -4.71225000 & 0.72685800 & -0.58892700 & $\mathrm{C}$ & 4.66712800 & -2.71973000 & -1.59630300 \\
\hline $\mathrm{C}$ & -5.00072100 & 2.71453200 & 3.06051300 & $\mathrm{H}$ & 1.52493800 & 1.81749400 & -2.58325700 \\
\hline $\mathrm{H}$ & -5.63682700 & 3.13995500 & 3.84757700 & $\mathrm{H}$ & 5.22163200 & 0.53103000 & -4.33988900 \\
\hline $\mathrm{H}$ & -4.08735300 & 3.32709800 & 3.05876900 & $\mathrm{H}$ & 6.18305600 & -2.18261000 & -3.07242500 \\
\hline $\mathrm{C}$ & -5.73965300 & 2.85907700 & 1.70679300 & $\mathrm{H}$ & 4.99816200 & -3.65835400 & -1.17740300 \\
\hline $\mathrm{H}$ & -6.53367000 & 3.60969300 & 1.81352100 & $\mathrm{~N}$ & 3.48980600 & -2.10894200 & -1.15562900 \\
\hline $\mathrm{H}$ & -6.25627800 & 1.91718100 & 1.47939200 & $\mathrm{P}$ & 2.14311800 & -2.46863900 & -0.12265000 \\
\hline $\mathrm{C}$ & -3.95889400 & -2.80633000 & -3.64174600 & $\mathrm{C}$ & 2.90670200 & -3.04701200 & 1.52757900 \\
\hline $\mathrm{C}$ & -3.09638200 & -1.83007300 & -2.80529900 & $\mathrm{C}$ & 1.21101000 & -3.87890600 & -1.02958700 \\
\hline $\mathrm{C}$ & -3.81217100 & -4.82511500 & -0.64279300 & $\mathrm{C}$ & 3.50953800 & -4.46661100 & 1.47960600 \\
\hline $\mathrm{C}$ & -3.68799200 & -1.53232300 & -1.44138600 & $\mathrm{H}$ & 4.29127700 & -4.56592100 & 0.72064200 \\
\hline $\mathrm{C}$ & -2.82265000 & -3.67958400 & -0.32534600 & $\mathrm{H}$ & 3.97361400 & -4.67823600 & 2.45155100 \\
\hline $\mathrm{C}$ & -3.53849200 & -2.34884700 & -0.30380900 & $\mathrm{H}$ & 2.75644100 & -5.23923600 & 1.30527700 \\
\hline $\mathrm{H}$ & -4.99831400 & -2.44607400 & -3.67001900 & $\mathrm{C}$ & 4.03637200 & -2.05681100 & 1.89166200 \\
\hline $\mathrm{H}$ & -2.08128600 & -2.22562400 & -2.72110000 & $\mathrm{H}$ & 4.43008100 & -2.32856000 & 2.87926000 \\
\hline $\mathrm{H}$ & -4.47364000 & -4.98592300 & 0.22066400 & $\mathrm{H}$ & 4.86197500 & -2.09707200 & 1.17554000 \\
\hline
\end{tabular}




\begin{tabular}{|c|c|c|c|c|c|c|c|}
\hline $\mathrm{H}$ & 3.68779100 & -1.02358000 & 1.94741900 & $\mathrm{C}$ & -3.92665000 & 2.22062600 & -0.35428100 \\
\hline $\mathrm{C}$ & 1.81339400 & -3.00349100 & 2.61880400 & $\mathrm{C}$ & -3.42060000 & 1.65620300 & -2.81129100 \\
\hline $\mathrm{H}$ & 1.34639700 & -2.01970200 & 2.70493700 & $\mathrm{C}$ & -4.03210800 & 1.35702500 & -1.46197200 \\
\hline $\mathrm{H}$ & 1.01739000 & -3.73099400 & 2.44022600 & $\mathrm{H}$ & -4.91692300 & 4.77834200 & -0.46518800 \\
\hline $\mathrm{H}$ & 2.27360900 & -3.24700000 & 3.58504400 & $\mathrm{H}$ & -2.18858800 & 3.41810800 & -0.85320500 \\
\hline $\mathrm{C}$ & 0.18001000 & -4.54434200 & -0.09574700 & $\mathrm{H}$ & -5.23103400 & 1.75130400 & -4.01389500 \\
\hline $\mathrm{H}$ & 0.65202400 & -5.14646400 & 0.68677600 & $\mathrm{H}$ & -3.13685500 & 0.72724100 & -3.32130400 \\
\hline $\mathrm{H}$ & -0.48297000 & -3.81234900 & 0.37513400 & $\mathrm{H}$ & -3.39140500 & 5.62789000 & -0.64705500 \\
\hline $\mathrm{H}$ & -0.44379200 & -5.22331000 & -0.69185100 & $\mathrm{H}$ & -2.90018500 & 3.75733700 & 0.71051900 \\
\hline $\mathrm{C}$ & 0.45506700 & -3.18150200 & -2.18585100 & $\mathrm{H}$ & -3.89664700 & 2.72066700 & -4.61959300 \\
\hline $\mathrm{H}$ & 1.13198200 & -2.66056300 & -2.87009400 & $\mathrm{H}$ & -4.77038800 & 2.23024300 & 0.33450300 \\
\hline $\mathrm{H}$ & -0.08974600 & -3.93911100 & -2.76462600 & $\mathrm{H}$ & -2.50452700 & 2.23893000 & -2.70503100 \\
\hline $\mathrm{C}$ & 2.14194000 & -4.94628900 & -1.63768000 & $\mathrm{H}$ & -4.94043700 & 0.76091500 & -1.54078900 \\
\hline $\mathrm{H}$ & 1.52685200 & -5.66914600 & -2.18938100 & $\mathrm{C}$ & -4.09620600 & 4.72855900 & -2.46954300 \\
\hline $\mathrm{H}$ & 2.85447700 & -4.51144000 & -2.34418400 & $\mathrm{H}$ & -4.46489200 & 5.72815600 & -2.73381100 \\
\hline $\mathrm{H}$ & 2.69987000 & -5.50616300 & -0.88286700 & $\mathrm{H}$ & -3.10907400 & 4.64457500 & -2.94677700 \\
\hline $\mathrm{F}$ & 3.35178600 & 2.31667700 & -4.29092200 & $\mathrm{C}$ & -5.06659600 & 3.67947100 & -3.06799800 \\
\hline $\mathrm{Rh}$ & 0.84723200 & -0.56382700 & -0.38319800 & $\mathrm{H}$ & -5.66789100 & 4.15866800 & -3.85156600 \\
\hline $\mathrm{Cl}$ & -1.15418100 & -1.19757700 & 1.06712300 & $\mathrm{H}$ & -5.78441100 & 3.37418000 & -2.29483000 \\
\hline $\mathrm{H}$ & -0.28960200 & -2.46468600 & -1.81489500 & $\mathrm{C}$ & -5.53842300 & -3.07503200 & 1.15286500 \\
\hline $\mathrm{C}$ & 4.68707300 & 4.08867000 & 3.13204000 & $\mathrm{C}$ & -4.37283800 & -2.29384500 & 0.50058600 \\
\hline $\mathrm{O}$ & 4.40239600 & 3.78948800 & 4.27215000 & $\mathrm{C}$ & -4.93236300 & -0.81748900 & 3.91709200 \\
\hline $\mathrm{O}$ & 5.47354000 & 5.14524100 & 2.81725600 & $\mathrm{C}$ & -4.55545000 & -0.78959600 & 0.54845700 \\
\hline $\mathrm{C}$ & 5.97109800 & 5.89533500 & 3.93519200 & $\mathrm{C}$ & -3.74476200 & -0.50984000 & 2.97338900 \\
\hline $\mathrm{H}$ & 6.58282400 & 5.26234500 & 4.58435100 & $\mathrm{C}$ & -4.24375700 & 0.02820700 & 1.65212700 \\
\hline $\mathrm{H}$ & 6.57283600 & 6.69745200 & 3.50613900 & $\mathrm{H}$ & -6.49399200 & -2.72946800 & 0.73073400 \\
\hline $\mathrm{H}$ & 5.14518100 & 6.30691700 & 4.52192500 & $\mathrm{H}$ & -3.43198200 & -2.59154100 & 0.96984400 \\
\hline $\mathrm{C}$ & 3.11931500 & 2.34773600 & 2.14392700 & $\mathrm{H}$ & -5.37736100 & 0.12691700 & 4.26133000 \\
\hline $\mathrm{H}$ & 2.23546700 & 2.88708700 & 2.50825700 & $\mathrm{H}$ & -3.08482100 & 0.21810700 & 3.46128500 \\
\hline $\mathrm{C}$ & 1.59553300 & 0.59264400 & 1.16186300 & $\mathrm{H}$ & -5.43705800 & -4.12574700 & 0.84759100 \\
\hline $\mathrm{H}$ & 1.82957800 & -0.09788200 & 1.97586200 & $\mathrm{H}$ & -4.30047000 & -2.59800600 & -0.55229200 \\
\hline $\mathrm{H}$ & 0.69764600 & 1.15326100 & 1.43980600 & $\mathrm{H}$ & -4.52648800 & -1.30763600 & 4.81320700 \\
\hline $\mathrm{H}$ & 3.63936100 & 0.98483000 & 0.54142300 & $\mathrm{H}$ & -5.36359900 & -0.44649000 & -0.09723500 \\
\hline $\mathrm{C}$ & 4.24920600 & 3.34817100 & 1.88316800 & $\mathrm{H}$ & -3.15050600 & -1.41330600 & 2.82761200 \\
\hline $\mathrm{H}$ & 5.13475100 & 2.83500200 & 1.48053400 & $\mathrm{H}$ & -4.82339000 & 0.94376300 & 1.76824700 \\
\hline $\mathrm{H}$ & 3.96572400 & 4.08466000 & 1.12175900 & $\mathrm{C}$ & -5.62841300 & -3.03721200 & 2.69289600 \\
\hline $\mathrm{C}$ & 2.75547900 & 1.53799700 & 0.88354700 & $\mathrm{H}$ & -6.35871300 & -3.80335400 & 2.98410600 \\
\hline $\mathrm{H}$ & 2.48943500 & 2.22411300 & 0.07185500 & $\mathrm{H}$ & -4.67061800 & -3.36738600 & 3.12107600 \\
\hline $\mathrm{H}$ & 3.41853500 & 1.67487200 & 2.95694500 & $\mathrm{C}$ & -6.05753400 & -1.69089800 & 3.32810400 \\
\hline $\mathrm{Cl}$ & -0.70798500 & 1.21579100 & -1.02859700 & $\mathrm{H}$ & -6.76410500 & -1.89255500 & 4.14378300 \\
\hline $\mathrm{Rh}$ & -2.80212200 & 0.40474500 & 0.06402700 & $\mathrm{H}$ & -6.62437500 & -1.11115700 & 2.58752000 \\
\hline $\mathrm{C}$ & -3.92466500 & 4.71199500 & -0.93627900 & & & & \\
\hline $\mathrm{C}$ & -3.14447200 & 3.51924600 & -0.33321900 & 18-ts & & & \\
\hline $\mathrm{C}$ & -4.42411100 & 2.42924300 & -3.70072500 & & & & \\
\hline
\end{tabular}



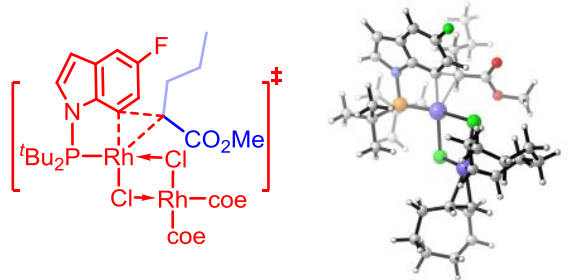

C $\quad-4.51140800$

C $\quad-3.59984100$

C $\quad-2.53037200$

C $\quad-2.35467200$

C $\quad-3.23632000$

C $\quad-4.33548700$

$-0.98777600$

$-2.00424100$

$-0.44076000$

$-1.08311200$

$-1.13745900$

$-0.45997300$

$-2.45451200$

$-0.94299100$

$-2.99354500$

$-1.88605600$

C $\quad-5.42598300$

$-2.32397200$

$-2.40850700$

0.07522400

$-2.34180200$

C $\quad-5.02014100$

1.19655000

$-1.67200700$

$-1.57693800$

$-3.09411300$

$-0.54879900$

$-2.82508400$

$-3.10331400$

$\mathrm{H} \quad-5.00102000$

H $\quad-6.28119000$

0.01105600

$-3.00224800$

$-5.45175100$

2.18487900

$-1.67705300$

0.91007600

$-0.88287100$

$\mathrm{N} \quad-3.89322700$

P $\quad-2.45495700$

1.83017700

$-0.44608100$

3.14169800

0.80589600

C $\quad-3.05842100$

C $\quad-1.92610700$

2.63424400

$-2.12160200$

C $\quad-4.43058600$

3.75788700

0.46698700

0.46958700

$\mathrm{H} \quad-5.22102300$

3.00136600

4.49463400

1.24069700

4.27842100

$-0.49338100$

2.16897700

2.42878000

3.15386300

2.91930800

2.13671200

1.61743700

2.49927600

2.02274400

4.25088000

0.93731300

1.12670500

3.84068900

4.88886200

0.05048600

4.89241500

1.78771800

3.22162800

$-2.01657600$

4.03782800

$-1.29415500$

2.46317300

$-1.74276500$

$-3.00045600$

3.62541700

1.49253700

$-3.16332500$

1.07867100

$-3.36398300$

1.89862300

$-4.10605700$

3.73854400

$-2.62482600$

4.06068500

$-3.61695400$

3.38944500
H $\quad-2.87290800$

F $\quad-3.01771900$

4.62370100

$-1.98205300$

Rh $\quad-1.03958700$

$-4.26812500$

0.16988600

1.58870900

0.67710200

H $\quad-1.23378100$

C $\quad-1.84882000$

$-2.23299400$

$-3.41165200$

$-1.77168800$

$-2.76017600$

$-3.20819900$

$-2.22117600$

$-3.54152400$

$-1.35942600$

$-2.26956300$

$-1.69994600$

$-2.60357800$

$-1.80331400$

$-0.85134000$

$-1.09693100$

$-0.53156600$

$-0.19504700$

$-1.49787100$

$-0.60363700$

$-2.34704500$

$-1.57128400$

$-0.08480700$

$-4.15544300$

$-2.94647800$

$-3.37436700$

$-1.70338700$

$-2.42850200$

$-1.45152900$

$-3.84917800$

$-3.23562300$

$-2.81251400$

$-1.89547600$

$-4.89825600$

$-2.70938200$

$-4.13103800$

$-1.26574300$

$-3.01524400$

$-0.83235600$

$-4.86114000$

$-5.79999300$
$-2.29032000$

0.19162000

0.72851600

$-2.84742000$

2.08566800

1.81801200

3.09076300

3.75055300

3.03750400

4.52814500

4.18681900

1.62180800

1.07632000

3.24162300

2.71482700

4.29423500

2.82482200

1.45966000

1.17579400

2.02213600

3.09723900

3.65689400

3.55494200

0.35831800

0.10852400

0.50586300

0.85470400

$-2.87687300$

0.04568100

$-2.03874400$

$-1.25925800$

0.54563100

0.74256900

$-3.71223300$

$-2.71462200$

1.30523100

1.91793700

$-3.33063700$

0.30548800

$-1.37142300$

$-1.90381900$

$-0.84058200$

$-0.82090400$ 


\begin{tabular}{|c|c|c|c|c|c|c|c|}
\hline $\mathrm{H}$ & 3.39149600 & -5.15926500 & -0.89257200 & $\mathrm{H}$ & -6.25884700 & 1.10600700 & -0.00144400 \\
\hline $\mathrm{C}$ & 4.84389200 & -4.08161400 & -2.11950000 & $\mathrm{~N}$ & -4.30134500 & 0.18549700 & 0.01237800 \\
\hline $\mathrm{H}$ & 5.31133100 & -4.77753600 & -2.82842200 & $\mathrm{P}$ & -3.02800600 & 1.34601000 & -0.36192800 \\
\hline $\mathrm{H}$ & 5.62626200 & -3.35346400 & -1.86694900 & $\mathrm{C}$ & -3.22294500 & 2.75466900 & 0.92111600 \\
\hline $\mathrm{C}$ & 4.77419600 & 3.76916200 & -1.16907300 & $\mathrm{C}$ & -3.49519300 & 1.93966000 & -2.14007500 \\
\hline $\mathrm{C}$ & 3.69154700 & 2.66520200 & -1.10126500 & $\mathrm{C}$ & -4.68955600 & 3.08648900 & 1.26186400 \\
\hline $\mathrm{C}$ & 5.15674000 & 3.00314300 & 2.34515100 & $\mathrm{H}$ & -5.20133200 & 2.22974700 & 1.71030200 \\
\hline $\mathrm{C}$ & 4.18103700 & 1.37472800 & -0.47306000 & $\mathrm{H}$ & -4.70331200 & 3.89879400 & 2.00029500 \\
\hline $\mathrm{C}$ & 3.90967700 & 2.17369500 & 1.95595700 & $\mathrm{H}$ & -5.26466500 & 3.42374400 & 0.39585800 \\
\hline $\mathrm{C}$ & 4.26641500 & 1.13925300 & 0.91344300 & $\mathrm{C}$ & -2.52020300 & 2.28665500 & 2.21357800 \\
\hline $\mathrm{H}$ & 5.68577500 & 3.36072800 & -1.63058100 & $\mathrm{H}$ & -2.60503400 & 3.08016300 & 2.96775900 \\
\hline $\mathrm{H}$ & 2.81628700 & 3.05281300 & -0.57298300 & $\mathrm{H}$ & -2.98366900 & 1.38766800 & 2.62941800 \\
\hline $\mathrm{H}$ & 5.83899200 & 2.37689700 & 2.93748300 & $\mathrm{H}$ & -1.45775700 & 2.08561000 & 2.04844600 \\
\hline $\mathrm{H}$ & 3.50881600 & 1.69037600 & 2.85553800 & $\mathrm{C}$ & -2.49466400 & 4.01962500 & 0.41973800 \\
\hline $\mathrm{H}$ & 4.40840000 & 4.54352600 & -1.85759700 & $\mathrm{H}$ & -1.46026900 & 3.80764900 & 0.13093300 \\
\hline $\mathrm{H}$ & 3.35996700 & 2.44615200 & -2.12511800 & $\mathrm{H}$ & -3.00355700 & 4.49327400 & -0.42416400 \\
\hline $\mathrm{H}$ & 4.82571000 & 3.81030400 & 3.01364300 & $\mathrm{H}$ & -2.46867100 & 4.75277500 & 1.23626900 \\
\hline $\mathrm{H}$ & 4.90636900 & 0.85299500 & -1.09898200 & $\mathrm{C}$ & -2.32129900 & 2.71687900 & -2.77330000 \\
\hline $\mathrm{H}$ & 3.12646900 & 2.84059900 & 1.59099200 & $\mathrm{H}$ & -2.07209500 & 3.63477100 & -2.23568000 \\
\hline $\mathrm{H}$ & 5.03354500 & 0.44635000 & 1.25870600 & $\mathrm{H}$ & -1.41725500 & 2.10825500 & -2.83490100 \\
\hline $\mathrm{C}$ & 5.15304900 & 4.45914300 & 0.15837800 & $\mathrm{H}$ & -2.61158300 & 3.00060600 & -3.79365800 \\
\hline $\mathrm{H}$ & 5.75370700 & 5.33966800 & -0.10450200 & $\mathrm{C}$ & -3.72850100 & 0.66616200 & -2.98420500 \\
\hline $\mathrm{H}$ & 4.24426900 & 4.85562000 & 0.63435500 & $\mathrm{H}$ & -4.59696000 & 0.09372300 & -2.64614400 \\
\hline $\mathrm{C}$ & 5.95571500 & 3.60925400 & 1.17483000 & $\mathrm{H}$ & -3.91311400 & 0.97130000 & -4.02250300 \\
\hline $\mathrm{H}$ & 6.74426600 & 4.23296100 & 1.61573300 & $\mathrm{C}$ & -4.76413800 & 2.81281100 & -2.19810300 \\
\hline $\mathrm{H}$ & 6.48100300 & 2.80976000 & 0.63580200 & $\mathrm{H}$ & -4.98358800 & 3.03195500 & -3.25109400 \\
\hline & & & & $\mathrm{H}$ & -5.64198500 & 2.30753500 & -1.78649200 \\
\hline 19-ts & & & & $\mathrm{H}$ & -4.63964500 & 3.77322600 & -1.69024900 \\
\hline & & & & $\mathrm{F}$ & -3.23842900 & -5.01302400 & -1.17510800 \\
\hline & & & & $\mathrm{Rh}$ & -1.20209400 & 0.01696800 & -0.39665700 \\
\hline & & & & $\mathrm{Cl}$ & 0.44601500 & 1.79546400 & -0.88000200 \\
\hline & & & & $\mathrm{H}$ & -2.85386500 & 0.00906400 & -2.97426400 \\
\hline & & & & $\mathrm{C}$ & 0.38698400 & -1.97078900 & 2.85936400 \\
\hline $\mathrm{C}$ & 0 & -1.93606 & 15100 & $\mathrm{O}$ & 0.72686900 & -0.84626800 & 3.16837700 \\
\hline $\mathrm{C}$ & -3.88 & -1.13178700 & 62700 & $\mathrm{O}$ & 0.90670100 & -3.08080600 & 3.43064400 \\
\hline $\mathrm{C}$ & 1900 & -1.56938700 & -0.13727800 & $\mathrm{C}$ & 1.91161200 & -2.85138500 & 4.43169000 \\
\hline $\mathrm{C}$ & -2.37352800 & -2.91971400 & -0.52957300 & $\mathrm{H}$ & 1.51585000 & -2.22758700 & 5.23741000 \\
\hline $\mathrm{C}$ & -3.478 & -3.72203600 & -0.83559200 & $\mathrm{H}$ & 2.18047300 & -3.83938600 & 4.80676700 \\
\hline $\mathrm{C}$ & 63900 & -3.29185700 & -0.78933700 & $\mathrm{H}$ & 2.78426000 & -2.35767500 & 3.99515900 \\
\hline $\mathrm{C}$ & -6.15315300 & -1.06730300 & -0.40050500 & $\mathrm{C}$ & -1.67433100 & -1.25484300 & 1.57723000 \\
\hline $\mathrm{C}$ & -5.69794600 & 0.19325700 & -0.12633800 & $\mathrm{H}$ & -1.21701600 & -0.29146700 & 1.82581900 \\
\hline $\mathrm{H}$ & -1.39278800 & -3.37848400 & -0.57472600 & $\mathrm{C}$ & -2.52481100 & -1.42455700 & 3.99572000 \\
\hline $\mathrm{H}$ & -5.60672200 & -3.98135200 & -1.00519100 & $\mathrm{H}$ & -2.02375200 & -0.49075100 & 4.27302600 \\
\hline $\mathrm{H}$ & -7.18997400 & -1.34727400 & -0.53542700 & $\mathrm{H}$ & -3.43737000 & -1.51337400 & 4.59700200 \\
\hline
\end{tabular}




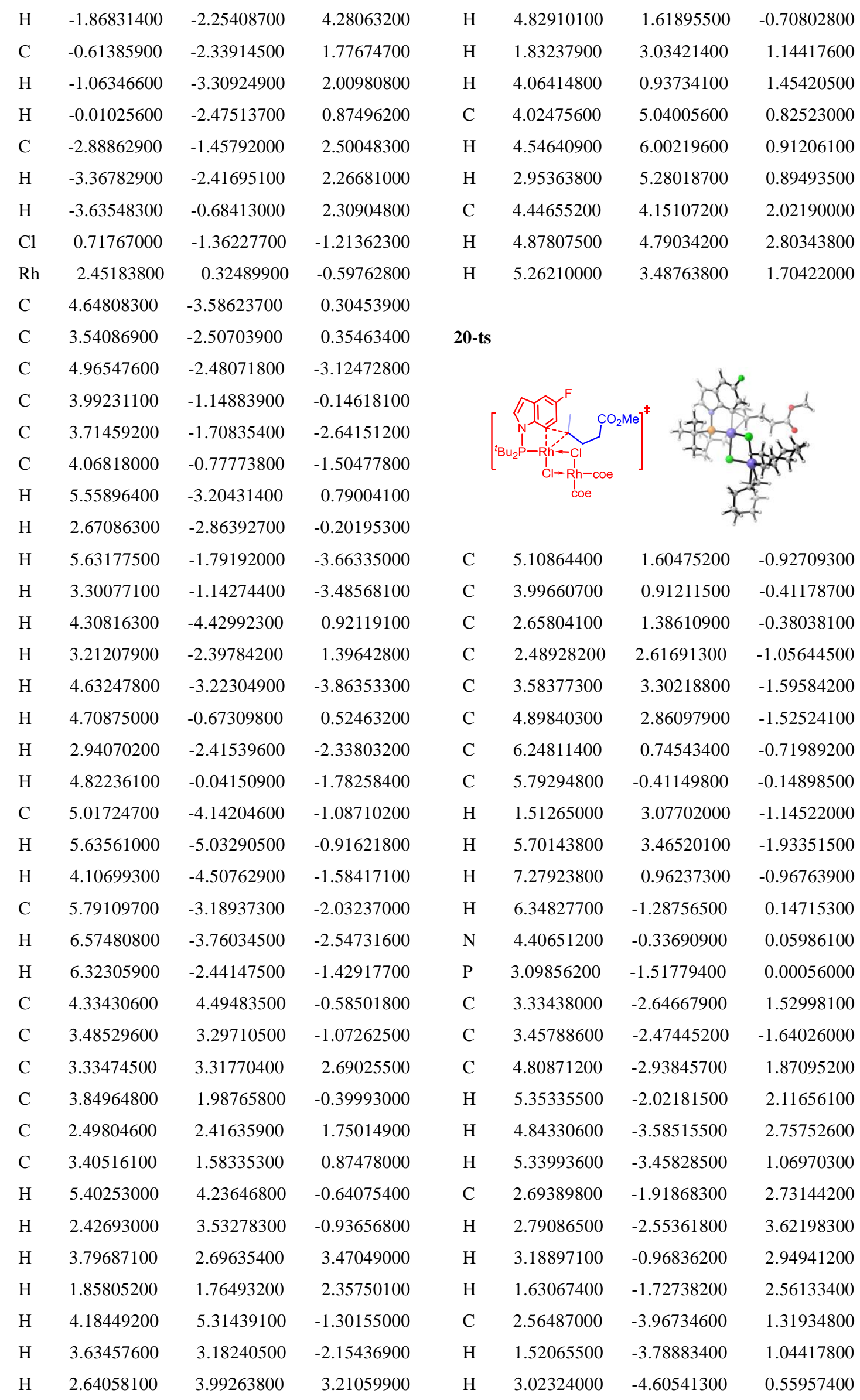




\begin{tabular}{|c|c|c|c|c|c|c|c|}
\hline $\mathrm{H}$ & 2.56996900 & -4.52846900 & 2.26272500 & $\mathrm{H}$ & -2.75666900 & 2.62775100 & -0.36692300 \\
\hline $\mathrm{C}$ & 2.23495400 & -3.32393800 & -2.04619400 & $\mathrm{H}$ & -5.30242000 & 1.23155900 & -4.01899000 \\
\hline $\mathrm{H}$ & 1.99594300 & -4.10671500 & -1.32291400 & $\mathrm{H}$ & -2.99450100 & 0.65434600 & -3.54293500 \\
\hline $\mathrm{H}$ & 1.34323900 & -2.70998400 & -2.18399300 & $\mathrm{H}$ & -4.51532100 & 4.23816700 & 0.46407300 \\
\hline $\mathrm{H}$ & 2.46297200 & -3.81518700 & -3.00148000 & $\mathrm{H}$ & -3.44522300 & 2.28492300 & 1.20140300 \\
\hline $\mathrm{C}$ & 3.67541100 & -1.40578900 & -2.73523800 & $\mathrm{H}$ & -4.31428300 & 2.67108500 & -4.21554000 \\
\hline $\mathrm{H}$ & 4.57781900 & -0.81181100 & -2.56651300 & $\mathrm{H}$ & -4.83900300 & 0.46148400 & 0.31062600 \\
\hline $\mathrm{H}$ & 3.78841600 & -1.91933900 & -3.69885100 & $\mathrm{H}$ & -2.78240100 & 2.01758400 & -2.46415000 \\
\hline $\mathrm{C}$ & 4.70250300 & -3.38296800 & -1.57839800 & $\mathrm{H}$ & -4.67256900 & -0.35421200 & -1.93531100 \\
\hline $\mathrm{H}$ & 4.86261500 & -3.81674200 & -2.57406400 & $\mathrm{C}$ & -5.01553600 & 3.78319800 & -1.58041900 \\
\hline $\mathrm{H}$ & 5.61132400 & -2.83357300 & -1.31837300 & $\mathrm{H}$ & -5.66802500 & 4.66568000 & -1.54757800 \\
\hline $\mathrm{H}$ & 4.58369200 & -4.21700000 & -0.88093000 & $\mathrm{H}$ & -4.06638800 & 4.13564200 & -2.01044200 \\
\hline $\mathrm{F}$ & 3.34043400 & 4.49006500 & -2.20341200 & $\mathrm{C}$ & -5.66341500 & 2.73567200 & -2.52022600 \\
\hline $\mathrm{Rh}$ & 1.28371100 & -0.17486300 & -0.18783600 & $\mathrm{H}$ & -6.40574800 & 3.23732900 & -3.15487600 \\
\hline $\mathrm{Cl}$ & -0.39812600 & -1.98616100 & -0.18756300 & $\mathrm{H}$ & -6.23222100 & 2.01722600 & -1.91517600 \\
\hline $\mathrm{H}$ & 2.82107700 & -0.72674500 & -2.81050900 & $\mathrm{C}$ & -4.23825400 & -4.77884900 & -0.41946300 \\
\hline $\mathrm{C}$ & 1.91396800 & 1.48980000 & 1.41285200 & $\mathrm{C}$ & -3.35175700 & -3.58400700 & -0.84357500 \\
\hline $\mathrm{H}$ & 1.41783900 & 0.62437800 & 1.87094100 & $\mathrm{C}$ & -3.78755000 & -3.37648100 & 2.88586700 \\
\hline $\mathrm{C}$ & 0.95276300 & 2.68431600 & 1.45716700 & $\mathrm{C}$ & -3.84225200 & -2.24850800 & -0.31832600 \\
\hline $\mathrm{H}$ & 1.51676100 & 3.61766700 & 1.35432000 & $\mathrm{C}$ & -2.82982800 & -2.51141000 & 2.03063900 \\
\hline $\mathrm{H}$ & 0.22936900 & 2.64111900 & 0.64295000 & $\mathrm{C}$ & -3.59698900 & -1.74773900 & 0.97606200 \\
\hline $\mathrm{Cl}$ & -0.68742400 & 1.05408200 & -1.08923500 & $\mathrm{H}$ & -5.28971100 & -4.55978800 & -0.65856200 \\
\hline $\mathrm{C}$ & 3.18996500 & 1.77211600 & 2.20789300 & $\mathrm{H}$ & -2.32075900 & -3.77711800 & -0.53645300 \\
\hline $\mathrm{H}$ & 2.91664600 & 1.98188800 & 3.25157500 & $\mathrm{H}$ & -4.37720200 & -2.72159700 & 3.54302400 \\
\hline $\mathrm{H}$ & 3.88321300 & 0.93015000 & 2.21501300 & $\mathrm{H}$ & -2.29605900 & -1.81796300 & 2.69308400 \\
\hline $\mathrm{H}$ & 3.71949700 & 2.64580300 & 1.81424800 & $\mathrm{H}$ & -3.95742600 & -5.63370100 & -1.05020800 \\
\hline $\mathrm{C}$ & 0.15800100 & 2.70319900 & 2.78649900 & $\mathrm{H}$ & -3.33720600 & -3.53712300 & -1.94055200 \\
\hline $\mathrm{H}$ & -0.44895200 & 1.79790300 & 2.87580800 & $\mathrm{H}$ & -3.17173900 & -3.99978100 & 3.54965300 \\
\hline $\mathrm{H}$ & 0.84212100 & 2.75305100 & 3.64167100 & $\mathrm{H}$ & -4.77165700 & -1.92858900 & -0.79042000 \\
\hline $\mathrm{C}$ & -0.78736900 & 3.88431500 & 2.86527300 & $\mathrm{H}$ & -2.06891000 & -3.14533500 & 1.57260400 \\
\hline $\mathrm{O}$ & -1.99902300 & 3.82731400 & 2.82649900 & $\mathrm{H}$ & -4.34123100 & -1.07665700 & 1.40230900 \\
\hline $\mathrm{O}$ & -0.10641300 & 5.04852800 & 2.97666600 & $\mathrm{C}$ & -4.14383200 & -5.22831600 & 1.05372100 \\
\hline $\mathrm{C}$ & -0.91492700 & 6.23408500 & 3.02933100 & $\mathrm{H}$ & -4.65406400 & -6.19802800 & 1.12103400 \\
\hline $\mathrm{H}$ & -1.58250600 & 6.20609300 & 3.89492800 & $\mathrm{H}$ & -3.09241400 & -5.43203800 & 1.30472600 \\
\hline $\mathrm{H}$ & -0.21285400 & 7.06427200 & 3.11311700 & $\mathrm{C}$ & -4.76562200 & -4.27839600 & 2.10795200 \\
\hline $\mathrm{H}$ & -1.51668800 & 6.32992500 & 2.12149300 & $\mathrm{H}$ & -5.30512000 & -4.87857900 & 2.85229600 \\
\hline $\mathrm{Rh}$ & -2.45519300 & -0.57591900 & -0.44194400 & $\mathrm{H}$ & -5.52991000 & -3.65764000 & 1.62229200 \\
\hline $\mathrm{C}$ & -4.78250100 & 3.34660200 & -0.11877500 & & & & \\
\hline $\mathrm{C}$ & -3.67246500 & 2.29772000 & 0.12945200 & 21-ts & & & \\
\hline $\mathrm{C}$ & -4.71154000 & 1.97438700 & -3.46403800 & & & & \\
\hline $\mathrm{C}$ & -4.05847800 & 0.90034000 & -0.31165000 & & & & \\
\hline $\mathrm{C}$ & -3.50643900 & 1.27089200 & -2.79368600 & & & & \\
\hline $\mathrm{C}$ & -3.96866200 & 0.42074300 & -1.63321700 & & & & \\
\hline $\mathrm{H}$ & -5.73356600 & 2.98480800 & 0.30066300 & & & & \\
\hline
\end{tabular}




\begin{tabular}{|c|c|c|c|c|c|c|c|}
\hline $\mathrm{C}$ & 5.08823500 & 1.50797700 & -1.28705900 & $\mathrm{O}$ & -2.12198900 & 4.97837100 & 2.60355900 \\
\hline $\mathrm{C}$ & 3.95486300 & 0.95837500 & -0.66286600 & $\mathrm{O}$ & -1.21370000 & 6.99857700 & 2.16949900 \\
\hline $\mathrm{C}$ & 2.60436500 & 1.25313900 & -0.97198900 & $\mathrm{C}$ & -2.12381700 & 7.57345500 & 3.12114500 \\
\hline C & 2.45866200 & 2.04483700 & -2.13209000 & $\mathrm{H}$ & -1.97585900 & 7.13129200 & 4.11001000 \\
\hline C & 3.58199700 & 2.55381200 & -2.79805300 & $\mathrm{H}$ & -1.89436300 & 8.63935300 & 3.13853000 \\
\hline C & 4.89497800 & 2.35472800 & -2.39585400 & $\mathrm{H}$ & -3.15941800 & 7.40794900 & 2.81152300 \\
\hline C & 6.22765300 & 0.97955600 & -0.57476900 & $\mathrm{C}$ & 0.25419400 & 3.80017800 & 1.30568400 \\
\hline C & 5.75674100 & 0.12078200 & 0.38067100 & $\mathrm{H}$ & -0.54424200 & 3.13417500 & 1.65142200 \\
\hline $\mathrm{H}$ & 1.47958600 & 2.31917400 & -2.50645300 & $\mathrm{C}$ & 1.66185500 & 1.84752000 & 0.51941100 \\
\hline $\mathrm{H}$ & 5.71073600 & 2.82486700 & -2.93418000 & $\mathrm{H}$ & 2.53531900 & 2.01780300 & 1.14671500 \\
\hline $\mathrm{H}$ & 7.26944600 & 1.21365900 & -0.75211200 & $\mathrm{H}$ & 0.94385900 & 1.27727600 & 1.13936800 \\
\hline $\mathrm{H}$ & 6.30722300 & -0.47318900 & 1.09464600 & $\mathrm{H}$ & 1.74285700 & 3.85365000 & -0.27963300 \\
\hline $\mathrm{N}$ & 4.35437200 & 0.07907900 & 0.34549900 & $\mathrm{C}$ & -0.36679900 & 5.15582200 & 0.93491000 \\
\hline $\mathrm{P}$ & 3.15522500 & -1.17279800 & 0.64754000 & $\mathrm{H}$ & 0.39765600 & 5.91356200 & 0.73841200 \\
\hline C & 3.18196000 & -1.43417900 & 2.54222700 & $\mathrm{H}$ & -0.95100900 & 5.04690100 & 0.00944400 \\
\hline $\mathrm{C}$ & 3.81968300 & -2.72012400 & -0.29384900 & $\mathrm{C}$ & 0.99047200 & 3.16109100 & 0.11761900 \\
\hline $\mathrm{C}$ & 4.42785700 & -2.16607300 & 3.07804900 & $\mathrm{H}$ & 0.26545900 & 2.98262300 & -0.68161500 \\
\hline $\mathrm{H}$ & 5.35725000 & -1.64026300 & 2.83535700 & $\mathrm{H}$ & 0.94174100 & 3.93597900 & 2.15237000 \\
\hline $\mathrm{H}$ & 4.36243300 & -2.21656200 & 4.17283400 & $\mathrm{Cl}$ & -0.68721500 & 0.56733900 & -1.54430100 \\
\hline $\mathrm{H}$ & 4.50343700 & -3.19252400 & 2.71068000 & $\mathrm{Rh}$ & -2.25071200 & -1.08377200 & -0.53103500 \\
\hline $\mathrm{C}$ & 3.11195700 & -0.04191300 & 3.20568100 & $\mathrm{C}$ & -4.42339100 & 2.75813100 & 0.57007200 \\
\hline $\mathrm{H}$ & 3.08772200 & -0.17527300 & 4.29485200 & $\mathrm{C}$ & -3.30639800 & 1.69135100 & 0.47692300 \\
\hline $\mathrm{H}$ & 3.97903700 & 0.57866800 & 2.96078600 & $\mathrm{C}$ & -5.10912100 & 1.66787800 & -2.81369100 \\
\hline $\mathrm{H}$ & 2.20624600 & 0.49952300 & 2.91972600 & $\mathrm{C}$ & -3.80052600 & 0.33786700 & 0.00610000 \\
\hline $\mathrm{C}$ & 1.91042500 & -2.21847400 & 2.93508300 & $\mathrm{C}$ & -3.78892800 & 0.92585100 & -2.49502100 \\
\hline $\mathrm{H}$ & 1.00568700 & -1.72345800 & 2.57055600 & $\mathrm{C}$ & -3.97970400 & -0.03233000 & -1.34167900 \\
\hline H & 1.90970300 & -3.24108000 & 2.54998900 & $\mathrm{H}$ & -5.26373200 & 2.35932000 & 1.15812000 \\
\hline $\mathrm{H}$ & 1.85555100 & -2.27576600 & 4.03001200 & $\mathrm{H}$ & -2.50756800 & 2.06458000 & -0.16860000 \\
\hline $\mathrm{C}$ & 3.05706700 & -3.98399800 & 0.15876700 & $\mathrm{H}$ & -5.81565400 & 0.96692600 & -3.28084000 \\
\hline $\mathrm{H}$ & 3.33263500 & -4.29450800 & 1.17156500 & $\mathrm{H}$ & -3.45893500 & 0.38802200 & -3.39240500 \\
\hline $\mathrm{H}$ & 1.97289100 & -3.86077700 & 0.11020700 & $\mathrm{H}$ & -4.01454900 & 3.59499500 & 1.15045600 \\
\hline $\mathrm{H}$ & 3.32665300 & -4.80700000 & -0.51582900 & $\mathrm{H}$ & -2.86567500 & 1.57216800 & 1.47534300 \\
\hline $\mathrm{C}$ & 3.53267100 & -2.46531600 & -1.79146400 & $\mathrm{H}$ & -4.88779600 & 2.42936100 & -3.57472500 \\
\hline $\mathrm{H}$ & 4.04073200 & -1.56682700 & -2.15620000 & $\mathrm{H}$ & -4.44523600 & -0.14782900 & 0.73806900 \\
\hline $\mathrm{H}$ & 3.90792300 & -3.32116300 & -2.36869400 & $\mathrm{H}$ & -3.00700900 & 1.65299900 & -2.26987300 \\
\hline $\mathrm{C}$ & 5.33463900 & -2.97573200 & -0.14431900 & $\mathrm{H}$ & -4.73419000 & -0.79085900 & -1.54787500 \\
\hline $\mathrm{H}$ & 5.57588100 & -3.89679400 & -0.69097300 & $\mathrm{C}$ & -4.96637900 & 3.30969600 & -0.76447100 \\
\hline $\mathrm{H}$ & 5.93036100 & -2.17174200 & -0.58185200 & $\mathrm{H}$ & -5.58393200 & 4.18313100 & -0.51721800 \\
\hline $\mathrm{H}$ & 5.65360500 & -3.12394600 & 0.89014900 & $\mathrm{H}$ & -4.13140500 & 3.70169200 & -1.36416700 \\
\hline F & 3.35768800 & 3.32751900 & -3.89000800 & $\mathrm{C}$ & -5.81793700 & 2.33724700 & -1.61929600 \\
\hline $\mathrm{Rh}$ & 1.29719700 & -0.25467200 & -0.32403000 & $\mathrm{H}$ & -6.68006500 & 2.88181000 & -2.02619900 \\
\hline $\mathrm{Cl}$ & -0.06268700 & -2.29157600 & -0.35391800 & $\mathrm{H}$ & -6.24232600 & 1.56438900 & -0.96457000 \\
\hline $\mathrm{H}$ & 2.46440700 & -2.35307500 & -1.99124200 & $\mathrm{C}$ & -3.66798900 & -5.39251500 & -0.83234700 \\
\hline $\mathrm{C}$ & -1.32615300 & 5.66372200 & 1.99334700 & $\mathrm{C}$ & -2.89370700 & -4.09118900 & -1.15178600 \\
\hline
\end{tabular}




\begin{tabular}{|c|c|c|c|c|c|c|c|}
\hline $\mathrm{C}$ & -3.24992200 & -4.28116900 & 2.58646000 & $\mathrm{C}$ & -5.22894400 & 1.15527600 & 3.33872600 \\
\hline C & -3.48463400 & -2.86547500 & -0.48292700 & $\mathrm{H}$ & -5.95686900 & 0.44391000 & 2.93784500 \\
\hline $\mathrm{C}$ & -2.37861400 & -3.27176200 & 1.80172700 & $\mathrm{H}$ & -5.13377200 & 0.95234800 & 4.41321600 \\
\hline C & -3.22518000 & -2.46541500 & 0.84296700 & $\mathrm{H}$ & -5.63005400 & 2.16629200 & 3.23470800 \\
\hline $\mathrm{H}$ & -4.73943000 & -5.24056500 & -1.03135700 & $\mathrm{C}$ & -3.40233900 & -0.48713400 & 2.87989100 \\
\hline $\mathrm{H}$ & -1.84294800 & -4.22513400 & -0.88232400 & $\mathrm{H}$ & -3.40657000 & -0.72036900 & 3.95286100 \\
\hline $\mathrm{H}$ & -3.87548100 & -3.73689300 & 3.30834600 & $\mathrm{H}$ & -4.08480400 & -1.18505800 & 2.38173300 \\
\hline $\mathrm{H}$ & -1.87428400 & -2.61046700 & 2.51777400 & $\mathrm{H}$ & -2.39505000 & -0.65758500 & 2.48994100 \\
\hline $\mathrm{H}$ & -3.33290300 & -6.15486600 & -1.54893200 & $\mathrm{C}$ & -2.81592600 & 1.90594200 & 3.36652800 \\
\hline $\mathrm{H}$ & -2.91222000 & -3.93420400 & -2.23814300 & $\mathrm{H}$ & -1.82716200 & 1.83252400 & 2.90283400 \\
\hline $\mathrm{H}$ & -2.57569500 & -4.91256600 & 3.18235100 & $\mathrm{H}$ & -3.13043300 & 2.95425300 & 3.35514200 \\
\hline $\mathrm{H}$ & -4.45490700 & -2.59185000 & -0.89689500 & $\mathrm{H}$ & -2.71427500 & 1.60770900 & 4.41781500 \\
\hline $\mathrm{H}$ & -1.58996300 & -3.80668000 & 1.26992800 & $\mathrm{C}$ & -2.79314800 & 3.90368700 & 0.44644900 \\
\hline $\mathrm{H}$ & -4.01111200 & -1.90823500 & 1.35117000 & $\mathrm{H}$ & -2.40143200 & 3.93053900 & 1.46680200 \\
\hline $\mathrm{C}$ & -3.50059700 & -5.97216600 & 0.58810000 & $\mathrm{H}$ & -2.01745900 & 3.49012500 & -0.20099600 \\
\hline $\mathrm{H}$ & -3.92895300 & -6.98270500 & 0.56802000 & $\mathrm{H}$ & -2.97795400 & 4.94057600 & 0.13605400 \\
\hline $\mathrm{H}$ & -2.43039400 & -6.11158000 & 0.80003400 & $\mathrm{C}$ & -4.54274700 & 3.11338400 & -1.15750600 \\
\hline $\mathrm{C}$ & -4.17110600 & -5.18351600 & 1.74057900 & $\mathrm{H}$ & -5.52166400 & 2.64858600 & -1.30833600 \\
\hline $\mathrm{H}$ & -4.64109300 & -5.89508500 & 2.43203000 & $\mathrm{H}$ & -4.60690500 & 4.15178500 & -1.50702300 \\
\hline \multirow[t]{2}{*}{$\mathrm{H}$} & 800 & -4.58518000 & 1.33180400 & $\mathrm{C}$ & -5.19515600 & 3.84027500 & 1.16249300 \\
\hline & & & & $\mathrm{H}$ & -5.33185800 & 4.85001700 & 0.75400600 \\
\hline \multirow[t]{7}{*}{22} & & & & $\mathrm{H}$ & -6.16836500 & 3.34389400 & 1.11950700 \\
\hline & & & & $\mathrm{H}$ & -4.90458600 & 3.95433300 & 2.21093900 \\
\hline & & & & $\mathrm{F}$ & -5.10217900 & -2.48759400 & -4.34456400 \\
\hline & & & & $\mathrm{Rh}$ & -2.18294100 & 0.25491800 & -0.40590400 \\
\hline & & & & $\mathrm{Cl}$ & -0.30178200 & 1.50276300 & 0.47417300 \\
\hline & & & & $\mathrm{H}$ & -3.81049400 & 2.59160900 & -1.78202500 \\
\hline & & & & $\mathrm{H}$ & -3.10052500 & -1.20270200 & -0.51345100 \\
\hline $\mathrm{C}$ & -6.32634300 & -0.53952900 & -1.54408700 & $\mathrm{Cl}$ & -0.49818900 & -0.87545900 & -1.79988800 \\
\hline $\mathrm{C}$ & -5.09150100 & -0.33019000 & -0.89623700 & $\mathrm{C}$ & 4.14436600 & -1.07381700 & 3.13871900 \\
\hline $\mathrm{C}$ & -3.86034500 & -0.83137300 & -1.34919800 & $\mathrm{C}$ & 3.26333500 & -1.07559700 & 2.03896000 \\
\hline $\mathrm{C}$ & -3.89208700 & -1.57275300 & -2.53904800 & $\mathrm{C}$ & 2.32317300 & -0.08757300 & 1.71628700 \\
\hline $\mathrm{C}$ & -5.11378500 & -1.76794200 & -3.19751900 & $\mathrm{C}$ & 2.28849300 & 0.98305000 & 2.61408200 \\
\hline $\mathrm{C}$ & -6.33288500 & -1.29288200 & -2.73217900 & $\mathrm{C}$ & 3.16161600 & 1.01031400 & 3.72061900 \\
\hline $\mathrm{C}$ & -7.30909500 & 0.15048700 & -0.74857000 & $\mathrm{C}$ & 4.09206700 & 0.02799300 & 4.01593700 \\
\hline $\mathrm{C}$ & -6.64771100 & 0.74821500 & 0.29140600 & $\mathrm{C}$ & 4.90117400 & -2.29691400 & 3.04321700 \\
\hline $\mathrm{H}$ & -2.98909800 & -2.01239300 & -2.94692600 & $\mathrm{C}$ & 4.45951900 & -2.97775900 & 1.93695800 \\
\hline $\mathrm{H}$ & -7.24148200 & -1.50817400 & -3.28490600 & $\mathrm{H}$ & 1.60765000 & 1.81770300 & 2.48478500 \\
\hline $\mathrm{H}$ & -8.37620500 & 0.19266000 & -0.92268200 & $\mathrm{H}$ & 4.73173000 & 0.12396800 & 4.88688400 \\
\hline $\mathrm{H}$ & -7.04960500 & 1.34629700 & 1.09412800 & $\mathrm{H}$ & 5.68431900 & -2.63265400 & 3.71092000 \\
\hline $\mathrm{N}$ & -5.27587300 & 0.46616100 & 0.22656300 & $\mathrm{H}$ & 4.78299900 & -3.93227100 & 1.54971700 \\
\hline $\mathrm{P}$ & -3.81333800 & 1.28197900 & 0.80079200 & $\mathrm{~N}$ & 3.45655100 & -2.24394200 & 1.29870300 \\
\hline $\mathrm{C}$ & -3.84420800 & 0.98057000 & 2.68457600 & $\mathrm{P}$ & 2.32185300 & -2.44769400 & 0.00059400 \\
\hline $\mathrm{C}$ & -4.11810800 & 3.12009400 & 0.32856600 & $\mathrm{C}$ & 3.35102900 & -3.04703900 & -1.49312400 \\
\hline
\end{tabular}




\begin{tabular}{|c|c|c|c|c|c|c|c|}
\hline C & 1.11354600 & -3.79932300 & 0.63608100 & $\mathrm{H}$ & 3.21065900 & 2.15917300 & 0.09201400 \\
\hline $\mathrm{C}$ & 3.80625200 & -4.51835400 & -1.39996100 & $\mathrm{H}$ & 4.60572000 & 1.55152000 & -2.58583300 \\
\hline $\mathrm{H}$ & 4.41935400 & -4.71550800 & -0.51520300 & & & & \\
\hline $\mathrm{H}$ & 4.42599800 & -4.74228700 & -2.27777800 & 23-ts & & & \\
\hline $\mathrm{H}$ & 2.96957800 & -5.22108900 & -1.40691500 & & & & \\
\hline $\mathrm{C}$ & 4.61350300 & -2.16000100 & -1.57691100 & & & & \\
\hline $\mathrm{H}$ & 5.17685000 & -2.44263800 & -2.47524200 & & & & \\
\hline $\mathrm{H}$ & 5.26735000 & -2.29669200 & -0.71100600 & & & & \\
\hline $\mathrm{H}$ & 4.37597700 & -1.09787900 & -1.65704200 & & & & \\
\hline $\mathrm{C}$ & 2.50647000 & -2.86485700 & -2.77449900 & & & & \\
\hline $\mathrm{H}$ & 2.14520900 & -1.84137200 & -2.89635600 & $\mathrm{C}$ & -6.29879900 & -0.59471100 & -1.58777500 \\
\hline $\mathrm{H}$ & 1.63479900 & -3.52379100 & -2.79605600 & $\mathrm{C}$ & -5.07730600 & -0.27605900 & -0.96515300 \\
\hline $\mathrm{H}$ & 3.13072800 & -3.11168100 & -3.64276600 & $\mathrm{C}$ & -3.80091600 & -0.63740400 & -1.42373800 \\
\hline C & 0.23835700 & -4.33590800 & -0.51454700 & $\mathrm{C}$ & -3.78656800 & -1.41633700 & -2.58753500 \\
\hline $\mathrm{H}$ & 0.80979700 & -4.94322200 & -1.22271300 & $\mathrm{C}$ & -4.99903100 & -1.74648500 & -3.21914000 \\
\hline $\mathrm{H}$ & -0.25893300 & -3.53115600 & -1.06415900 & $\mathrm{C}$ & 25700 & -1.36990600 & -2.76296700 \\
\hline $\mathrm{H}$ & -0.54024400 & -4.98206200 & -0.08831800 & $\mathrm{C}$ & -7.32139500 & 0.02242700 & -0.78121800 \\
\hline $\mathrm{C}$ & 0.20528800 & -3.08192200 & 1.66250200 & $\mathrm{C}$ & -6.69951100 & 0.67295000 & 0.25341400 \\
\hline $\mathrm{H}$ & 0.77773800 & -2.65807300 & 2.49362300 & $\mathrm{H}$ & -2.85843700 & -1.77208000 & -3.01980800 \\
\hline $\mathrm{H}$ & -0.50345300 & -3.80962300 & 2.08027000 & $\mathrm{H}$ & -7.14232300 & -1.67374900 & -3.30528000 \\
\hline $\mathrm{C}$ & 1.81622400 & -4.96712300 & 1.35647000 & $\mathrm{H}$ & -8.39101100 & -0.01896600 & -0.94114700 \\
\hline $\mathrm{H}$ & 1.04616100 & -5.65596500 & 1.72735700 & $\mathrm{H}$ & -7.13843700 & 1.23754600 & 1.06135700 \\
\hline $\mathrm{H}$ & 2.39618200 & -4.62566500 & 2.21849900 & $\mathrm{~N}$ & -5.31326600 & 0.49075300 & 0.17197300 \\
\hline $\mathrm{H}$ & 2.47427100 & -5.54149100 & 0.70041500 & $\mathrm{P}$ & -3.87916600 & 1.26964100 & 0.80978400 \\
\hline $\mathrm{F}$ & 3.07852800 & 2.08463700 & 4.54855200 & $\mathrm{C}$ & -3.92822400 & 0.91705300 & 2.68282700 \\
\hline $\mathrm{Rh}$ & 1.18649500 & -0.43150400 & 0.07774500 & $\mathrm{C}$ & -4.14006700 & 3.12042200 & 0.37663800 \\
\hline $\mathrm{H}$ & -0.38840100 & -2.28296300 & 1.19961300 & $\mathrm{C}$ & -5.32513100 & 1.07278400 & 3.31626100 \\
\hline $\mathrm{C}$ & 6.18413700 & 3.77692000 & -2.40037600 & $\mathrm{H}$ & -6.04032500 & 0.36103400 & 2.89438500 \\
\hline $\mathrm{O}$ & 6.06527000 & 3.57155600 & -3.58935200 & $\mathrm{H}$ & -5.24475200 & 0.85735900 & 4.38940300 \\
\hline $\mathrm{O}$ & 7.05402800 & 4.67929400 & -1.88701600 & $\mathrm{H}$ & -5.73251200 & 2.08176300 & 3.21757500 \\
\hline $\mathrm{C}$ & 7.85089600 & 5.38177700 & -2.85218700 & $\mathrm{C}$ & -3.48578300 & -0.55394300 & 2.85099000 \\
\hline $\mathrm{H}$ & 8.45766600 & 4.68304900 & -3.43503800 & $\mathrm{H}$ & -3.51342500 & -0.81191200 & 3.91777300 \\
\hline $\mathrm{H}$ & 8.48831400 & 6.05234500 & -2.27456000 & $\mathrm{H}$ & -4.15416600 & -1.24189000 & 2.32143300 \\
\hline $\mathrm{H}$ & 7.21538100 & 5.95121300 & -3.53610300 & $\mathrm{H}$ & -2.46919700 & -0.71310200 & 2.48128000 \\
\hline $\mathrm{C}$ & 4.24420300 & 2.24112200 & -1.81305700 & $\mathrm{C}$ & -2.91485900 & 1.82862600 & 3.40495900 \\
\hline $\mathrm{H}$ & 3.52558300 & 2.90321100 & -2.31280800 & $\mathrm{H}$ & -1.91954300 & 1.77704800 & 2.95240000 \\
\hline C & 2.35041000 & 0.68069400 & -1.22430400 & $\mathrm{H}$ & -3.23771000 & 2.87424200 & 3.42350600 \\
\hline $\mathrm{H}$ & 2.64895500 & -0.00151100 & -2.02443300 & $\mathrm{H}$ & -2.82442800 & 1.49754800 & 4.44727800 \\
\hline $\mathrm{H}$ & 1.59057200 & 1.35869700 & -1.62529000 & $\mathrm{C}$ & -2.81955400 & 3.89674200 & 0.56283200 \\
\hline $\mathrm{H}$ & 4.26509400 & 0.78994200 & -0.20434100 & $\mathrm{H}$ & -2.47042200 & 3.90386200 & 1.59858600 \\
\hline $\mathrm{C}$ & 5.42173800 & 3.07172100 & -1.29524900 & $\mathrm{H}$ & -2.01716700 & 3.49319900 & -0.05848900 \\
\hline $\mathrm{H}$ & 6.13905600 & 2.43457500 & -0.75776000 & $\mathrm{H}$ & -2.98748000 & 4.93961100 & 0.26372400 \\
\hline $\mathrm{H}$ & 5.09364000 & 3.82218700 & -0.56562000 & $\mathrm{C}$ & -4.51773900 & 3.15622200 & -1.12191900 \\
\hline $\mathrm{C}$ & 3.54118000 & 1.46030900 & -0.68461400 & $\mathrm{H}$ & -5.48611300 & 2.68614600 & -1.31736200 \\
\hline
\end{tabular}




\begin{tabular}{|c|c|c|c|c|c|c|c|}
\hline $\mathrm{H}$ & -4.58204400 & 4.20464300 & -1.43959100 & $\mathrm{H}$ & 0.85034100 & -2.69753200 & 2.47299300 \\
\hline $\mathrm{C}$ & -5.25004000 & 3.81135700 & 1.19227200 & $\mathrm{H}$ & -0.41978100 & -3.86368400 & 2.06594300 \\
\hline $\mathrm{H}$ & -5.37943900 & 4.83129400 & 0.80766400 & $\mathrm{C}$ & 1.90001900 & -4.98367800 & 1.29183900 \\
\hline $\mathrm{H}$ & -6.21672000 & 3.30802300 & 1.09902800 & $\mathrm{H}$ & 1.14292300 & -5.68649600 & 1.66318700 \\
\hline $\mathrm{H}$ & -4.99802100 & 3.89717500 & 2.25329000 & $\mathrm{H}$ & 2.48794800 & -4.64789700 & 2.15068000 \\
\hline $\mathrm{F}$ & -4.92652100 & -2.49333800 & -4.34870800 & $\mathrm{H}$ & 2.55554800 & -5.54096600 & 0.61873000 \\
\hline $\mathrm{Rh}$ & -2.22957200 & 0.23341700 & -0.40103500 & $\mathrm{~F}$ & 3.12815300 & 2.01072400 & 4.59017800 \\
\hline $\mathrm{Cl}$ & -0.31575400 & 1.45799100 & 0.57184900 & $\mathrm{Rh}$ & 1.20076300 & -0.44351200 & 0.10125100 \\
\hline $\mathrm{H}$ & -3.76347500 & 2.66216500 & -1.74261400 & $\mathrm{H}$ & -0.34144700 & -2.32731600 & 1.20027700 \\
\hline $\mathrm{H}$ & -2.99983300 & -1.14117900 & -0.26915600 & $\mathrm{C}$ & 6.09808700 & 3.88347700 & -2.36958900 \\
\hline $\mathrm{Cl}$ & -0.50625900 & -0.87072600 & -1.75959200 & $\mathrm{O}$ & 5.95921900 & 3.70440600 & -3.56064700 \\
\hline $\mathrm{C}$ & 4.21578700 & -1.10398700 & 3.10189700 & $\mathrm{O}$ & 6.96597200 & 4.78470300 & -1.85117600 \\
\hline $\mathrm{C}$ & 3.31786600 & -1.09655300 & 2.01600400 & $\mathrm{C}$ & 7.73565900 & 5.52007900 & -2.81401800 \\
\hline $\mathrm{C}$ & 2.35935200 & -0.11585400 & 1.72765400 & $\mathrm{H}$ & 8.34024500 & 4.84314300 & -3.42417500 \\
\hline $\mathrm{C}$ & 2.32311400 & 0.93626800 & 2.64679100 & $\mathrm{H}$ & 8.37512000 & 6.18531100 & -2.23251400 \\
\hline $\mathrm{C}$ & 3.21310300 & 0.95423800 & 3.74002200 & $\mathrm{H}$ & 7.08018000 & 6.09683600 & -3.47245900 \\
\hline $\mathrm{C}$ & 4.16178500 & -0.02035400 & 4.00132600 & $\mathrm{C}$ & 4.19019100 & 2.30782500 & -1.78390500 \\
\hline $\mathrm{C}$ & 4.98756700 & -2.31440500 & 2.97032600 & $\mathrm{H}$ & 3.45423200 & 2.97125100 & -2.25592600 \\
\hline $\mathrm{C}$ & 4.53793800 & -2.97952200 & 1.85776800 & $\mathrm{C}$ & 2.32584100 & 0.71155500 & -1.19762100 \\
\hline $\mathrm{H}$ & 1.62829100 & 1.76304300 & 2.54447200 & $\mathrm{H}$ & 2.61558200 & 0.05358900 & -2.02132400 \\
\hline $\mathrm{H}$ & 4.81357700 & 0.06773300 & 4.86407500 & $\mathrm{H}$ & 1.55049300 & 1.39071200 & -1.56591400 \\
\hline $\mathrm{H}$ & 5.78571400 & -2.65223100 & 3.61893000 & $\mathrm{H}$ & 4.25828200 & 0.81899300 & -0.21136300 \\
\hline $\mathrm{H}$ & 4.86851900 & -3.92138100 & 1.44618100 & $\mathrm{C}$ & 5.36530900 & 3.14276400 & -1.26756800 \\
\hline $\mathrm{N}$ & 3.51516800 & -2.24698100 & 1.24974900 & $\mathrm{H}$ & 6.10062200 & 2.50386600 & -0.75721200 \\
\hline $\mathrm{P}$ & 2.36128600 & -2.44092200 & -0.03266100 & $\mathrm{H}$ & 5.03989200 & 3.87202800 & -0.51557000 \\
\hline $\mathrm{C}$ & 3.37106300 & -3.00375100 & -1.55237100 & $\mathrm{C}$ & 3.51777600 & 1.49199800 & -0.66170800 \\
\hline $\mathrm{C}$ & 1.17455300 & -3.81392000 & 0.59765100 & $\mathrm{H}$ & 3.19348200 & 2.16769700 & 0.13767600 \\
\hline $\mathrm{C}$ & 3.84175200 & -4.47164300 & -1.48943200 & $\mathrm{H}$ & 4.54735400 & 1.64109000 & -2.57844000 \\
\hline $\mathrm{H}$ & 4.46961300 & -4.67640700 & -0.61687400 & 24 & & & \\
\hline $\mathrm{H}$ & 4.45024500 & -4.67589300 & -2.37978500 & & & & \\
\hline $\mathrm{H}$ & 3.01148400 & -5.18191400 & -1.49483100 & & & & \\
\hline $\mathrm{C}$ & 4.62228700 & -2.10160500 & -1.64175600 & & & & \\
\hline $\mathrm{H}$ & 5.17233800 & -2.36074500 & -2.55525200 & & & & \\
\hline $\mathrm{H}$ & 5.29328200 & -2.24690800 & -0.79055700 & & & & \\
\hline $\mathrm{H}$ & 4.37162500 & -1.04088100 & -1.69701800 & & & & \\
\hline $\mathrm{C}$ & 2.50388300 & -2.80872000 & -2.81635300 & $\mathrm{C}$ & -6.32457200 & -0.88169900 & -1.53195400 \\
\hline $\mathrm{H}$ & 2.13478400 & -1.78568400 & -2.91604300 & $\mathrm{C}$ & -5.13715300 & -0.51587400 & -0.86991900 \\
\hline $\mathrm{H}$ & 1.63617000 & -3.47284700 & -2.83368800 & $\mathrm{C}$ & -3.83551800 & -0.89398100 & -1.22443400 \\
\hline $\mathrm{H}$ & 3.11504200 & -3.03764300 & -3.69865900 & $\mathrm{C}$ & -3.76169100 & -1.76190800 & -2.31656300 \\
\hline $\mathrm{C}$ & 0.28964100 & -4.34333400 & -0.54890300 & $\mathrm{C}$ & -4.94013200 & -2.16553000 & -2.97681800 \\
\hline $\mathrm{H}$ & 0.85753500 & -4.93438800 & -1.27342700 & $\mathrm{C}$ & -6.21756800 & -1.75899300 & -2.62956600 \\
\hline $\mathrm{H}$ & -0.22482100 & -3.53693600 & -1.07990100 & $\mathrm{C}$ & -7.38652500 & -0.17785500 & -0.85756500 \\
\hline $\mathrm{H}$ & -0.47621000 & -5.00374400 & -0.12163200 & $\mathrm{C}$ & -6.82557900 & 0.56657500 & 0.14815600 \\
\hline $\mathrm{C}$ & 0.27195100 & -3.12116900 & 1.64594600 & $\mathrm{H}$ & -2.81424100 & -2.14032500 & -2.68396300 \\
\hline
\end{tabular}




\begin{tabular}{|c|c|c|c|c|c|c|c|}
\hline $\mathrm{H}$ & -7.07475200 & -2.10558700 & -3.19703900 & $\mathrm{H}$ & 1.76820500 & 1.40092500 & 2.85121300 \\
\hline $\mathrm{H}$ & -8.44431600 & -0.22311400 & -1.08337800 & $\mathrm{H}$ & 5.59988400 & 0.24163600 & 4.39663000 \\
\hline $\mathrm{H}$ & -7.30730200 & 1.21426300 & 0.86407100 & $\mathrm{H}$ & 6.83313500 & -2.10079700 & 2.69809800 \\
\hline $\mathrm{N}$ & -5.44132700 & 0.35602100 & 0.17970600 & $\mathrm{H}$ & 5.79431500 & -3.35453100 & 0.57225900 \\
\hline $\mathrm{P}$ & -4.03772400 & 1.11320000 & 0.87048800 & $\mathrm{~N}$ & 4.12738900 & -1.99585100 & 0.80041200 \\
\hline $\mathrm{C}$ & -4.26716500 & 1.00149400 & 2.75986800 & $\mathrm{P}$ & 2.80961300 & -2.33110200 & -0.27928800 \\
\hline $\mathrm{C}$ & -4.08224300 & 2.91007900 & 0.20865100 & $\mathrm{C}$ & 3.60350400 & -2.56601300 & -1.99837000 \\
\hline $\mathrm{C}$ & -5.71007200 & 1.30760900 & 3.21350500 & $\mathrm{C}$ & 2.04175400 & -3.95490500 & 0.39377900 \\
\hline $\mathrm{H}$ & -6.41668800 & 0.56233800 & 2.83862900 & $\mathrm{C}$ & 4.34518700 & -3.90803300 & -2.17108000 \\
\hline $\mathrm{H}$ & -5.74217900 & 1.26667600 & 4.30960400 & $\mathrm{H}$ & 5.14268300 & -4.04834900 & -1.43523900 \\
\hline $\mathrm{H}$ & -6.05527200 & 2.30080400 & 2.91565100 & $\mathrm{H}$ & 4.81536100 & -3.91640500 & -3.16287800 \\
\hline $\mathrm{C}$ & -3.93730600 & -0.45117900 & 3.17000400 & $\mathrm{H}$ & 3.67443000 & -4.76931800 & -2.12391000 \\
\hline $\mathrm{H}$ & -4.15423700 & -0.57012100 & 4.23929800 & $\mathrm{C}$ & 4.62178600 & -1.42221800 & -2.19951400 \\
\hline $\mathrm{H}$ & -4.54412900 & -1.17994900 & 2.62169900 & $\mathrm{H}$ & 5.02007800 & -1.48647200 & -3.21996300 \\
\hline $\mathrm{H}$ & -2.88173000 & -0.68439900 & 3.00906400 & $\mathrm{H}$ & 5.46136000 & -1.49513500 & -1.50265000 \\
\hline $\mathrm{C}$ & -3.28153100 & 1.95323500 & 3.46900500 & $\mathrm{H}$ & 4.16937600 & -0.43623500 & -2.08026700 \\
\hline $\mathrm{H}$ & -2.25189800 & 1.81420600 & 3.12559000 & $\mathrm{C}$ & 2.49668300 & -2.46385200 & -3.07181600 \\
\hline $\mathrm{H}$ & -3.55702000 & 3.00485400 & 3.34384100 & $\mathrm{H}$ & 1.95240500 & -1.51822600 & -3.02091000 \\
\hline $\mathrm{H}$ & -3.30385900 & 1.73794300 & 4.54478200 & $\mathrm{H}$ & 1.76310200 & -3.27065200 & -2.99583300 \\
\hline $\mathrm{C}$ & -2.73202900 & 3.60693300 & 0.48153100 & $\mathrm{H}$ & 2.96392500 & -2.53289900 & -4.06249100 \\
\hline $\mathrm{H}$ & -2.54867200 & 3.77362300 & 1.54559500 & $\mathrm{C}$ & 1.07425000 & -4.57559100 & -0.63438700 \\
\hline $\mathrm{H}$ & -1.88631100 & 3.03849800 & 0.08564900 & $\mathrm{H}$ & 1.59692600 & -4.99230200 & -1.50051600 \\
\hline $\mathrm{H}$ & -2.74209900 & 4.58811700 & -0.01029000 & $\mathrm{H}$ & 0.32742100 & -3.85746400 & -0.98552100 \\
\hline $\mathrm{C}$ & -4.27156100 & 2.78858100 & -1.32085100 & $\mathrm{H}$ & 0.53767900 & -5.40286500 & -0.15211500 \\
\hline $\mathrm{H}$ & -5.23022900 & 2.33373000 & -1.58622600 & $\mathrm{C}$ & 1.23186500 & -3.53315400 & 1.64340400 \\
\hline $\mathrm{H}$ & -4.24165000 & 3.79476300 & -1.75750800 & $\mathrm{H}$ & 1.85733700 & -3.05367600 & 2.40261500 \\
\hline $\mathrm{C}$ & -5.22763900 & 3.76375500 & 0.78549100 & $\mathrm{H}$ & 0.78197500 & -4.42772800 & 2.09392500 \\
\hline $\mathrm{H}$ & -5.22472900 & 4.73897100 & 0.28151000 & $\mathrm{C}$ & 3.09217700 & -4.99514900 & 0.83076800 \\
\hline $\mathrm{H}$ & -6.21032600 & 3.31381900 & 0.61289800 & $\mathrm{H}$ & 2.56629900 & -5.86048900 & 1.25484900 \\
\hline $\mathrm{H}$ & -5.10895400 & 3.95388400 & 1.85615900 & $\mathrm{H}$ & 3.75874000 & -4.60186400 & 1.60339400 \\
\hline $\mathrm{F}$ & -4.80061000 & -3.00958600 & -4.03137200 & $\mathrm{H}$ & 3.70149000 & -5.36191000 & 0.00118100 \\
\hline $\mathrm{Rh}$ & -2.34946900 & -0.09770000 & -0.11251200 & $\mathrm{~F}$ & 3.54925100 & 1.79967400 & 4.63434600 \\
\hline $\mathrm{Cl}$ & -0.40345600 & 0.86214000 & 1.21797700 & $\mathrm{Rh}$ & 1.33121900 & -0.63420700 & 0.27799000 \\
\hline $\mathrm{H}$ & -3.47596900 & 2.19970900 & -1.78991900 & $\mathrm{H}$ & 0.40675800 & -2.85515700 & 1.38777700 \\
\hline $\mathrm{H}$ & -2.69418000 & -1.22433800 & 0.83866800 & $\mathrm{C}$ & 4.72226900 & 4.87227500 & -2.36891900 \\
\hline $\mathrm{Cl}$ & -0.57942000 & -1.27943700 & -1.31856800 & $\mathrm{O}$ & 4.16657200 & 4.97651100 & -3.44130100 \\
\hline $\mathrm{C}$ & 4.92373300 & -0.88468100 & 2.63351000 & $\mathrm{O}$ & 5.70210600 & 5.71106500 & -1.95547800 \\
\hline $\mathrm{C}$ & 3.85590900 & -0.98012000 & 1.71911500 & $\mathrm{C}$ & 6.05066500 & 6.75286100 & -2.87904900 \\
\hline $\mathrm{C}$ & 2.68908900 & -0.20498500 & 1.71160800 & $\mathrm{H}$ & 6.40576700 & 6.32985000 & -3.82298900 \\
\hline $\mathrm{C}$ & 2.61965600 & 0.73801200 & 2.74021900 & $\mathrm{H}$ & 6.84348600 & 7.32284000 & -2.39308600 \\
\hline $\mathrm{C}$ & 3.67710700 & 0.85677900 & 3.66469600 & $\mathrm{H}$ & 5.18783700 & 7.39323000 & -3.08274100 \\
\hline $\mathrm{C}$ & 4.82867700 & 0.08724500 & 3.64941900 & $\mathrm{C}$ & 3.33076300 & 2.84132900 & -1.72119700 \\
\hline $\mathrm{C}$ & 5.87792100 & -1.88910100 & 2.23483800 & $\mathrm{H}$ & 2.41013400 & 3.40331200 & -1.92111900 \\
\hline $\mathrm{C}$ & 5.36785400 & -2.54200300 & 1.14141600 & $\mathrm{C}$ & 1.94929500 & 0.83654900 & -1.03997000 \\
\hline
\end{tabular}




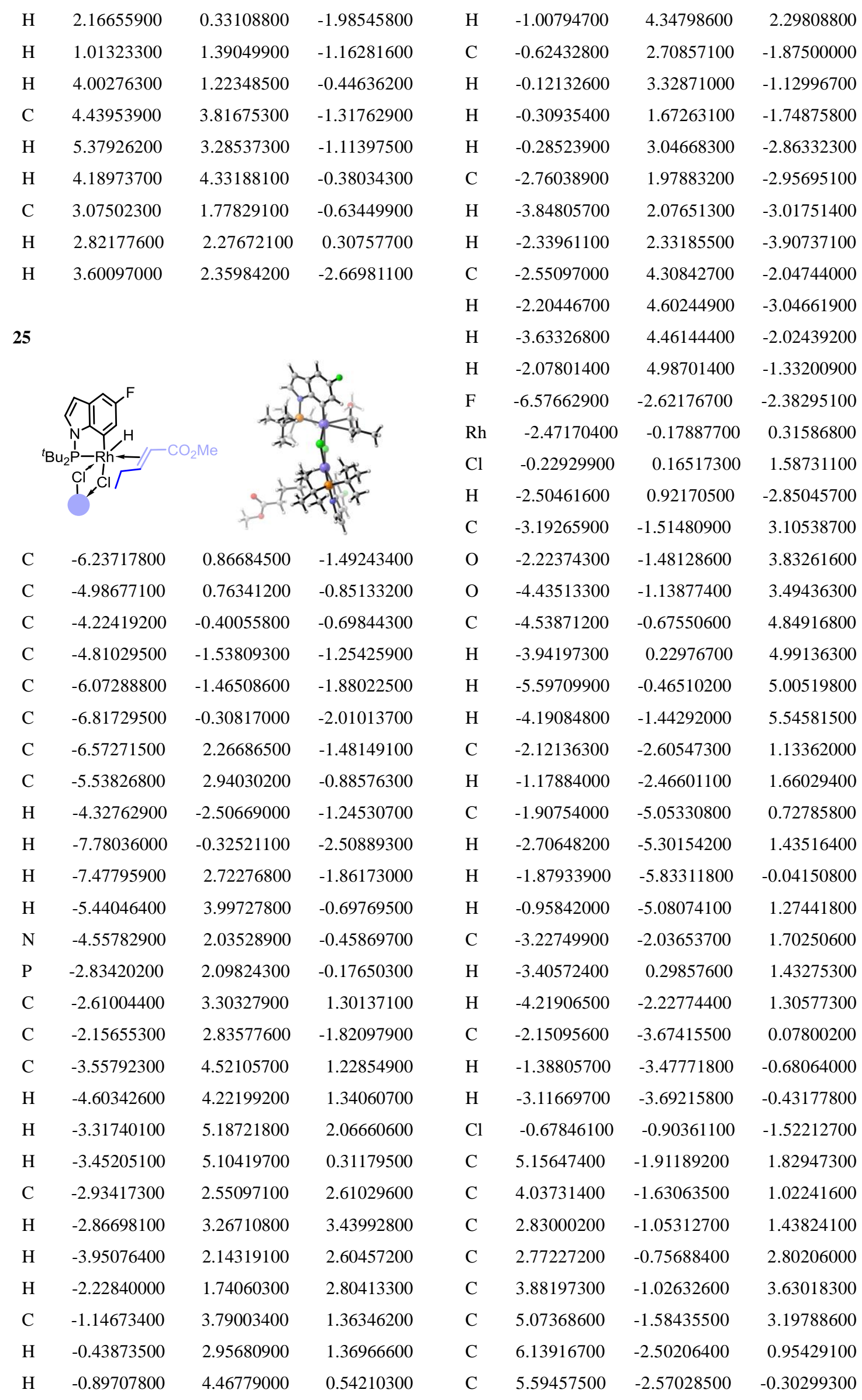




\begin{tabular}{|c|c|c|c|c|c|c|c|}
\hline $\mathrm{H}$ & 1.89282300 & -0.31092500 & 3.25405800 & $\mathrm{H}$ & 2.13190100 & 1.14270800 & -1.52210100 \\
\hline $\mathrm{H}$ & 5.88326300 & -1.75657700 & 3.89920100 & $\mathrm{H}$ & 1.30413000 & 1.70462300 & -0.06066800 \\
\hline $\mathrm{H}$ & 7.13601700 & -2.82923500 & 1.22115500 & $\mathrm{H}$ & 4.28404900 & 0.96125900 & -0.10020300 \\
\hline $\mathrm{H}$ & 6.02863400 & -2.95188600 & -1.21552400 & $\mathrm{C}$ & 5.02776300 & 3.61175700 & 0.26884300 \\
\hline $\mathrm{N}$ & 4.30572000 & -2.02891700 & -0.29054700 & $\mathrm{H}$ & 5.89328700 & 2.97507600 & 0.04122200 \\
\hline $\mathrm{P}$ & 2.90612500 & -1.99200300 & -1.31678500 & $\mathrm{H}$ & 4.96346400 & 3.63198700 & 1.36515100 \\
\hline $\mathrm{C}$ & 3.51508600 & -1.40650700 & -3.03454000 & $\mathrm{C}$ & 3.44392300 & 1.61753200 & 0.15508000 \\
\hline $\mathrm{C}$ & 2.32411000 & -3.82068900 & -1.36940600 & $\mathrm{H}$ & 3.38399300 & 1.62201700 & 1.24866500 \\
\hline $\mathrm{C}$ & 4.25728400 & -2.48773500 & -3.84757300 & $\mathrm{H}$ & 3.83450600 & 3.05468000 & -1.43908000 \\
\hline $\mathrm{H}$ & 5.13811900 & -2.87577500 & -3.32690000 & & & & \\
\hline $\mathrm{H}$ & 4.60799700 & -2.03287100 & -4.78282100 & 26-ts & & & \\
\hline $\mathrm{H}$ & 3.61607400 & -3.32900800 & -4.11926000 & & & & \\
\hline $\mathrm{C}$ & 4.48282800 & -0.22021600 & -2.83529900 & & & & \\
\hline $\mathrm{H}$ & 4.80230900 & 0.13353900 & -3.82383300 & & & & \\
\hline $\mathrm{H}$ & 5.37754600 & -0.50543200 & -2.27466200 & & & & \\
\hline $\mathrm{H}$ & 4.01234800 & 0.61883000 & -2.32226100 & & & & \\
\hline $\mathrm{C}$ & 2.28539100 & -0.92685900 & -3.83769600 & & & & \\
\hline $\mathrm{H}$ & 1.73454000 & -0.13882700 & -3.31903500 & $\mathrm{C}$ & -6.11432700 & 0.98589500 & -1.32610100 \\
\hline $\mathrm{H}$ & 1.57952300 & -1.73461100 & -4.04680200 & $\mathrm{C}$ & -4.89177900 & 0.92535200 & -0.62682800 \\
\hline $\mathrm{H}$ & 2.62950900 & -0.52747600 & -4.80051200 & $\mathrm{C}$ & -4.07812700 & -0.20822000 & -0.50239800 \\
\hline $\mathrm{C}$ & 1.24814300 & -4.01740800 & -2.45680600 & $\mathrm{C}$ & -4.53865500 & -1.33356000 & -1.17983700 \\
\hline $\mathrm{H}$ & 1.66507500 & -3.97155200 & -3.46730000 & $\mathrm{C}$ & -5.77123200 & -1.29831600 & -1.86402700 \\
\hline $\mathrm{H}$ & 0.44232200 & -3.28114500 & -2.38079300 & $\mathrm{C}$ & $-6.5894 \mathrm{C}$ & -0.18741000 & -1.94424400 \\
\hline $\mathrm{H}$ & 0.80603900 & -5.01513600 & -2.33675800 & $\mathrm{C}$ & -6.52839800 & 2.36494000 & -1.28311100 \\
\hline $\mathrm{C}$ & 1.69347200 & -4.08120900 & 0.01748800 & $\mathrm{C}$ & -5.55615800 & 3.07291300 & -0.62802200 \\
\hline $\mathrm{H}$ & 2.41103600 & -3.94337100 & 0.83125800 & $\mathrm{H}$ & -3.98055900 & -2.25901600 & -1.21583800 \\
\hline $\mathrm{H}$ & 1.33198300 & -5.11679800 & 0.05497700 & $\mathrm{H}$ & -7.52406100 & -0.23080200 & -2.49324200 \\
\hline $\mathrm{C}$ & 3.46469300 & -4.84100400 & -1.55973600 & $\mathrm{H}$ & -7.43999200 & 2.78295800 & -1.69094400 \\
\hline $\mathrm{H}$ & 3.03575500 & -5.84994400 & -1.49999400 & $\mathrm{H}$ & -5.51792200 & 4.12868600 & -0.41209000 \\
\hline $\mathrm{H}$ & 4.22100800 & -4.75904600 & -0.77408600 & $\mathrm{~N}$ & -4.54496700 & 2.20967000 & -0.18219600 \\
\hline $\mathrm{H}$ & 3.96013400 & -4.75864600 & -2.52904700 & $\mathrm{P}$ & -2.82266600 & 2.37802600 & 0.08599600 \\
\hline $\mathrm{F}$ & 3.76596400 & -0.70489400 & 4.94402500 & $\mathrm{C}$ & -2.66493200 & 3.59038100 & 1.56272600 \\
\hline $\mathrm{Rh}$ & 1.41889300 & -0.79571900 & 0.01455700 & $\mathrm{C}$ & -2.18359500 & 3.17768100 & -1.54299800 \\
\hline $\mathrm{H}$ & 0.83487400 & -3.42670800 & 0.20564100 & $\mathrm{C}$ & -3.65133600 & 4.77634100 & 1.50292500 \\
\hline $\mathrm{C}$ & 5.34315400 & 5.01724800 & -0.20505500 & $\mathrm{H}$ & -4.68651500 & 4.43957200 & 1.60566600 \\
\hline $\mathrm{O}$ & 4.67207500 & 5.67736300 & -0.96959900 & $\mathrm{H}$ & -3.43961000 & 5.44116500 & 2.35020600 \\
\hline $\mathrm{O}$ & 6.49714200 & 5.46375600 & 0.34462400 & $\mathrm{H}$ & -3.56291600 & 5.37253700 & 0.59325200 \\
\hline $\mathrm{C}$ & 6.88497400 & 6.79303900 & -0.03305300 & $\mathrm{C}$ & -2.96865900 & 2.80680600 & 2.85558600 \\
\hline $\mathrm{H}$ & 7.03565600 & 6.85922200 & -1.11424300 & $\mathrm{H}$ & -2.94767300 & 3.50623200 & 3.70161800 \\
\hline $\mathrm{H}$ & 7.81869100 & 6.98786500 & 0.49582600 & $\mathrm{H}$ & -3.96329300 & 2.34790000 & 2.82890900 \\
\hline $\mathrm{H}$ & 6.11965300 & 7.51680300 & 0.26089000 & $\mathrm{H}$ & -2.22313200 & 2.03100200 & 3.03977000 \\
\hline $\mathrm{C}$ & 3.74561000 & 3.04404000 & -0.34480000 & $\mathrm{C}$ & -1.21576500 & 4.11571000 & 1.64501000 \\
\hline $\mathrm{H}$ & 2.90810100 & 3.71133600 & -0.10642300 & $\mathrm{H}$ & -0.48468200 & 3.30134800 & 1.64115400 \\
\hline $\mathrm{C}$ & 2.13403600 & 1.09811400 & -0.42725900 & $\mathrm{H}$ & -0.97711100 & 4.81134900 & 0.83567200 \\
\hline
\end{tabular}




\begin{tabular}{|c|c|c|c|c|c|c|c|}
\hline $\mathrm{H}$ & -1.09649500 & 4.66260000 & 2.58902400 & $\mathrm{H}$ & 2.17118800 & -0.12856500 & 3.29241300 \\
\hline $\mathrm{C}$ & -0.64778600 & 3.06675700 & -1.59759000 & $\mathrm{H}$ & 6.04925900 & -1.92405900 & 3.69363100 \\
\hline $\mathrm{H}$ & -0.14984400 & 3.65535000 & -0.82310700 & $\mathrm{H}$ & 6.95823800 & -3.24740400 & 0.98711900 \\
\hline $\mathrm{H}$ & -0.32557900 & 2.02818200 & -1.51388500 & $\mathrm{H}$ & 5.63466000 & -3.38329700 & -1.33764600 \\
\hline $\mathrm{H}$ & -0.30561600 & 3.44935800 & -2.56840400 & $\mathrm{~N}$ & 4.09351000 & -2.24988100 & -0.32991000 \\
\hline $\mathrm{C}$ & -2.77371900 & 2.33669400 & -2.69801400 & $\mathrm{P}$ & 2.62301400 & -2.11235200 & -1.24308600 \\
\hline $\mathrm{H}$ & -3.86207500 & 2.42615400 & -2.75942400 & $\mathrm{C}$ & 3.14951700 & -1.66022100 & -3.02656200 \\
\hline $\mathrm{H}$ & -2.35334600 & 2.71039900 & -3.64088300 & $\mathrm{C}$ & 1.84946400 & -3.86693800 & -1.15506800 \\
\hline $\mathrm{C}$ & -2.59781600 & 4.64799900 & -1.74541500 & $\mathrm{C}$ & 3.72315200 & -2.84580000 & -3.83051800 \\
\hline $\mathrm{H}$ & -2.25966100 & 4.96524300 & -2.74057800 & $\mathrm{H}$ & 4.60023700 & -3.29461500 & -3.35406700 \\
\hline $\mathrm{H}$ & -3.68269600 & 4.78524900 & -1.71591900 & $\mathrm{H}$ & 4.04329300 & -2.47202200 & -4.81154100 \\
\hline $\mathrm{H}$ & -2.13390600 & 5.32201100 & -1.01978500 & $\mathrm{H}$ & 2.98587200 & -3.63169600 & -4.00946100 \\
\hline $\mathrm{F}$ & -6.16454700 & -2.44059700 & -2.48297700 & $\mathrm{C}$ & 4.23658800 & -0.56647900 & -2.95215500 \\
\hline $\mathrm{Rh}$ & -2.37138600 & 0.03792600 & 0.57062200 & $\mathrm{H}$ & 4.51481800 & -0.28696300 & -3.97628500 \\
\hline $\mathrm{Cl}$ & -0.08029100 & 0.42321400 & 1.79522700 & $\mathrm{H}$ & 5.13891300 & -0.91336200 & -2.44044200 \\
\hline $\mathrm{H}$ & -2.51078900 & 1.27960000 & -2.61127700 & $\mathrm{H}$ & 3.88489100 & 0.33521100 & -2.44928100 \\
\hline $\mathrm{C}$ & -3.03356500 & -3.14346300 & 0.90751400 & $\mathrm{C}$ & 1.91721100 & -1.09776500 & -3.77008800 \\
\hline $\mathrm{O}$ & -4.19718400 & -3.46504800 & 1.06056500 & $\mathrm{H}$ & 1.48442100 & -0.23336100 & -3.26113200 \\
\hline $\mathrm{O}$ & -2.17110700 & -3.87152000 & 0.15533200 & $\mathrm{H}$ & 1.12246900 & -1.83852300 & -3.88880000 \\
\hline $\mathrm{C}$ & -2.73601100 & -5.05642700 & -0.42857600 & $\mathrm{H}$ & 2.22994300 & -0.78170600 & -4.77366200 \\
\hline $\mathrm{H}$ & -3.55493500 & -4.80110000 & -1.10602300 & $\mathrm{C}$ & 0.68603600 & -3.99568500 & -2.15767000 \\
\hline $\mathrm{H}$ & -1.92111000 & -5.52894000 & -0.97697200 & $\mathrm{H}$ & 1.02677200 & -3.98819800 & -3.19714600 \\
\hline $\mathrm{H}$ & -3.11565900 & -5.72493300 & 0.34905200 & $\mathrm{H}$ & -0.06189500 & -3.20875300 & -2.02400900 \\
\hline $\mathrm{C}$ & -3.17248000 & -1.16497400 & 2.39095900 & $\mathrm{H}$ & 0.18989300 & -4.96031500 & -1.99032900 \\
\hline $\mathrm{H}$ & -4.24266000 & -1.34212600 & 2.28191000 & $\mathrm{C}$ & 1.28872400 & -3.99397700 & 0.28066000 \\
\hline $\mathrm{C}$ & -2.96531900 & -1.92146800 & 4.76182500 & $\mathrm{H}$ & 2.06660900 & -3.87640800 & 1.04073300 \\
\hline $\mathrm{H}$ & -2.34834600 & -2.78523600 & 4.49190500 & $\mathrm{H}$ & 0.84468400 & -4.99051900 & 0.40086400 \\
\hline $\mathrm{H}$ & -2.69704100 & -1.61679200 & 5.77929300 & $\mathrm{C}$ & 2.86725800 & -5.00551700 & -1.36613800 \\
\hline $\mathrm{H}$ & -4.01267900 & -2.24460500 & 4.76969400 & $\mathrm{H}$ & 2.34767200 & -5.96105500 & -1.21766600 \\
\hline $\mathrm{C}$ & -2.37182000 & -1.97918300 & 1.55036600 & $\mathrm{H}$ & 3.68536100 & -4.95978100 & -0.64194800 \\
\hline $\mathrm{H}$ & -3.33865600 & 0.36523100 & 1.78272800 & $\mathrm{H}$ & 3.29287100 & -5.02279500 & -2.37140700 \\
\hline $\mathrm{H}$ & -1.32272000 & -2.11849100 & 1.79548200 & $\mathrm{~F}$ & 4.13606800 & -0.61993500 & 4.84541300 \\
\hline $\mathrm{C}$ & -2.74054100 & -0.75102200 & 3.78634000 & $\mathrm{Rh}$ & 1.37322000 & -0.72835600 & 0.13580800 \\
\hline $\mathrm{H}$ & -3.32529000 & 0.11281500 & 4.12149900 & $\mathrm{H}$ & 0.48985000 & -3.26955300 & 0.47397500 \\
\hline $\mathrm{H}$ & -1.68702500 & -0.46039600 & 3.78664800 & $\mathrm{C}$ & 5.65511200 & 4.76308200 & -0.66663600 \\
\hline $\mathrm{Cl}$ & -0.78140300 & -0.60479400 & -1.29437300 & $\mathrm{O}$ & 4.99069700 & 5.41961300 & -1.43991400 \\
\hline $\mathrm{C}$ & 5.13437300 & -2.11077100 & 1.70229800 & $\mathrm{O}$ & 6.86073800 & 5.16369100 & -0.19850600 \\
\hline $\mathrm{C}$ & 3.97997600 & -1.76230200 & 0.97472200 & $\mathrm{C}$ & 7.31476300 & 6.43788000 & -0.67870700 \\
\hline $\mathrm{C}$ & 2.87146100 & -1.05099400 & 1.45278200 & $\mathrm{H}$ & 7.41721000 & 6.42628400 & -1.76749400 \\
\hline $\mathrm{C}$ & 2.96134500 & -0.68285300 & 2.79738200 & $\mathrm{H}$ & 8.28345100 & 6.60189900 & -0.20517900 \\
\hline $\mathrm{C}$ & 4.10870200 & -1.01663000 & 3.54662800 & $\mathrm{H}$ & 6.61263500 & 7.22840400 & -0.39941000 \\
\hline $\mathrm{C}$ & 5.20140200 & -1.70911800 & 3.05187000 & $\mathrm{C}$ & 3.91717600 & 2.90926900 & -0.58938100 \\
\hline $\mathrm{C}$ & 5.97703000 & -2.83721700 & 0.78491100 & $\mathrm{H}$ & 3.14550800 & 3.64382900 & -0.32755500 \\
\hline $\mathrm{C}$ & 5.32004000 & -2.91420700 & -0.41713200 & $\mathrm{C}$ & 2.17440000 & 1.08363600 & -0.46499100 \\
\hline
\end{tabular}




\begin{tabular}{|c|c|c|c|c|c|c|c|}
\hline $\mathrm{H}$ & 2.09682700 & 1.07828600 & -1.55778200 & $\mathrm{H}$ & 2.08524200 & 2.05186100 & -3.60096100 \\
\hline $\mathrm{H}$ & 1.41451900 & 1.76325300 & -0.07351300 & $\mathrm{H}$ & 3.36669700 & 1.38344800 & -4.61949200 \\
\hline $\mathrm{H}$ & 4.32620100 & 0.80441000 & -0.28186700 & $\mathrm{C}$ & 1.24710300 & 3.53019700 & -1.39386000 \\
\hline $\mathrm{C}$ & 5.27567200 & 3.41101300 & -0.09415500 & $\mathrm{H}$ & 1.66625800 & 3.90435400 & -2.33266000 \\
\hline $\mathrm{H}$ & 6.07583100 & 2.70146800 & -0.34504200 & $\mathrm{H}$ & 0.62040500 & 2.66138200 & -1.61547300 \\
\hline $\mathrm{H}$ & 5.29159900 & 3.49045100 & 1.00106400 & $\mathrm{H}$ & 0.59828400 & 4.32024800 & -0.99407500 \\
\hline C & 3.55515900 & 1.53118700 & -0.00044300 & $\mathrm{C}$ & 1.66232600 & 2.87973400 & 1.00158000 \\
\hline $\mathrm{H}$ & 3.57581000 & 1.58734100 & 1.09323300 & $\mathrm{H}$ & 2.39287400 & 2.67593700 & 1.78907400 \\
\hline \multirow[t]{2}{*}{$\mathrm{H}$} & 3.92811600 & 2.86498600 & -1.68622000 & $\mathrm{H}$ & 1.05255900 & 3.73671900 & 1.31565400 \\
\hline & & & & $\mathrm{C}$ & 3.22941600 & 4.45251600 & -0.12334000 \\
\hline \multirow[t]{8}{*}{27} & & & & $\mathrm{H}$ & 2.59374200 & 5.26853900 & 0.24428700 \\
\hline & & & & $\mathrm{H}$ & 3.99825200 & 4.26939300 & 0.63257900 \\
\hline & & & & $\mathrm{H}$ & 3.71465000 & 4.80729200 & -1.03466400 \\
\hline & & & & $\mathrm{F}$ & 4.33222200 & -1.33984500 & 4.79561500 \\
\hline & & & & $\mathrm{Rh}$ & 2.06392300 & -0.14617000 & -0.02076200 \\
\hline & & & & $\mathrm{Cl}$ & 0.13882700 & -0.03075600 & -1.75387900 \\
\hline & & & & $\mathrm{H}$ & 0.98878000 & 2.01629700 & 0.93488200 \\
\hline & & & & $\mathrm{Cl}$ & 0.34442000 & -1.39305600 & 1.25294500 \\
\hline $\mathrm{C}$ & 5.48345300 & 1.02098100 & 2.30647100 & $\mathrm{C}$ & -5.60369400 & -0.86619800 & -1.98536000 \\
\hline $\mathrm{C}$ & 4.44992300 & 0.80625500 & 1.37505000 & $\mathrm{C}$ & -4.42660800 & -0.95333600 & -1.21697400 \\
\hline $\mathrm{C}$ & 3.37633200 & -0.08355700 & 1.51291300 & $\mathrm{C}$ & -3.14565700 & -0.53131700 & -1.59701400 \\
\hline $\mathrm{C}$ & 3.36655600 & -0.80317200 & 2.70840500 & $\mathrm{C}$ & -3.07655500 & 0.01409600 & -2.88107400 \\
\hline $\mathrm{C}$ & 4.39239400 & -0.60605000 & 3.65558400 & $\mathrm{C}$ & -4.24243900 & 0.11873100 & -3.66763500 \\
\hline $\mathrm{C}$ & 5.45180600 & 0.27327800 & 3.50047500 & $\mathrm{C}$ & -5.50361300 & -0.29270900 & -3.26904800 \\
\hline $\mathrm{C}$ & 6.34739800 & 2.01580500 & 1.71917100 & $\mathrm{C}$ & -6.65031100 & -1.43655800 & -1.17380100 \\
\hline $\mathrm{C}$ & 5.82123600 & 2.36410400 & 0.50169300 & $\mathrm{C}$ & -6.08809000 & -1.84797300 & 0.00808100 \\
\hline $\mathrm{H}$ & 2.59721700 & -1.53463100 & 2.92952800 & $\mathrm{H}$ & -2.14274400 & 0.37385100 & -3.30016600 \\
\hline $\mathrm{H}$ & 6.20547200 & 0.36207900 & 4.27569400 & $\mathrm{H}$ & -6.35256000 & -0.17523100 & -3.93419400 \\
\hline $\mathrm{H}$ & 7.25879500 & 2.41796000 & 2.14279300 & $\mathrm{H}$ & -7.69851700 & -1.52430300 & -1.42967600 \\
\hline $\mathrm{H}$ & 6.18869000 & 3.07506400 & -0.22358000 & $\mathrm{H}$ & -6.55708900 & -2.32081600 & 0.85839200 \\
\hline $\mathrm{N}$ & 4.65929100 & 1.62346600 & 0.25934000 & $\mathrm{~N}$ & -4.72203200 & -1.55101700 & 0.01130800 \\
\hline $\mathrm{P}$ & 3.31892000 & 1.63537200 & -0.84002000 & $\mathrm{P}$ & -3.31007200 & -1.93482100 & 0.94569900 \\
\hline $\mathrm{C}$ & 4.03982200 & 1.77648300 & -2.60519600 & $\mathrm{C}$ & -3.76916800 & -1.58268900 & 2.76619400 \\
\hline $\mathrm{C}$ & 2.33678500 & 3.21526200 & -0.34960000 & $\mathrm{C}$ & -3.02627000 & -3.80532800 & 0.62805300 \\
\hline $\mathrm{C}$ & 4.55245000 & 3.18786300 & -2.96119900 & $\mathrm{C}$ & -4.68079300 & -2.65413600 & 3.39914900 \\
\hline $\mathrm{H}$ & 5.33028000 & 3.54115500 & -2.27717900 & $\mathrm{H}$ & -5.61985300 & -2.78735400 & 2.85335300 \\
\hline $\mathrm{H}$ & 4.99618400 & 3.14754900 & -3.96413000 & $\mathrm{H}$ & -4.93907600 & -2.32917300 & 4.41510700 \\
\hline $\mathrm{H}$ & 3.75366900 & 3.93226100 & -2.99238200 & $\mathrm{H}$ & -4.18887900 & -3.62560400 & 3.48582800 \\
\hline $\mathrm{C}$ & 5.21869200 & 0.78992500 & -2.73710100 & $\mathrm{C}$ & -4.50822300 & -0.22835400 & 2.82211800 \\
\hline $\mathrm{H}$ & 5.63429500 & 0.88396900 & -3.74832100 & $\mathrm{H}$ & -4.72758500 & 0.00269400 & 3.87223000 \\
\hline $\mathrm{H}$ & 6.02029700 & 0.99648700 & -2.02231400 & $\mathrm{H}$ & -5.45555700 & -0.24958800 & 2.27603200 \\
\hline $\mathrm{H}$ & 4.90276100 & -0.24574500 & -2.60810000 & $\mathrm{H}$ & -3.90496500 & 0.58810700 & 2.42325700 \\
\hline $\mathrm{C}$ & 2.93811100 & 1.36899300 & -3.60919800 & $\mathrm{C}$ & -2.46582800 & -1.48259700 & 3.58985200 \\
\hline $\mathrm{H}$ & 2.55553000 & 0.36330600 & -3.41815100 & $\mathrm{H}$ & -1.78552900 & -0.72017000 & 3.20407300 \\
\hline
\end{tabular}




\begin{tabular}{|c|c|c|c|c|c|c|c|}
\hline $\mathrm{H}$ & -1.91524700 & -2.42607200 & 3.61944300 & $\mathrm{C}$ & 6.80622400 & -3.09633000 & -1.06318000 \\
\hline $\mathrm{H}$ & -2.72612400 & -1.21775400 & 4.62260200 & $\mathrm{H}$ & 6.89231500 & -3.46799100 & -0.03529200 \\
\hline $\mathrm{C}$ & -1.96607900 & -4.36825800 & 1.59687100 & $\mathrm{H}$ & 7.31250200 & -3.81143700 & -1.72207000 \\
\hline $\mathrm{H}$ & -2.33452500 & -4.43607200 & 2.62500100 & $\mathrm{H}$ & 7.35166300 & -2.14591100 & -1.11848800 \\
\hline $\mathrm{H}$ & -1.04325300 & -3.77997200 & 1.59504400 & $\mathrm{C}$ & 3.14098000 & -1.70373300 & -0.94772200 \\
\hline $\mathrm{H}$ & -1.71036200 & -5.38791500 & 1.28056500 & $\mathrm{H}$ & 5.14648200 & -0.97688800 & -0.53368700 \\
\hline $\mathrm{C}$ & -2.47846700 & -3.88687900 & -0.81614900 & $\mathrm{H}$ & 3.05872400 & -1.42414700 & -2.00207200 \\
\hline $\mathrm{H}$ & -3.18737600 & -3.49507500 & -1.55104700 & $\mathrm{C}$ & 5.33630300 & -2.91715900 & -1.46005400 \\
\hline $\mathrm{H}$ & -2.28334400 & -4.93898700 & -1.06175000 & $\mathrm{H}$ & 5.27723400 & -2.57425700 & -2.50380300 \\
\hline $\mathrm{C}$ & -4.31145200 & -4.65488300 & 0.69113700 & $\mathrm{H}$ & 4.83180700 & -3.89078600 & -1.42491900 \\
\hline $\mathrm{H}$ & -4.05724200 & -5.68373300 & 0.40452800 & & & & \\
\hline $\mathrm{H}$ & -5.07213600 & -4.29730100 & -0.00886500 & 28-ts & & & \\
\hline $\mathrm{H}$ & -4.75067200 & -4.69792000 & 1.68992100 & & & & \\
\hline $\mathrm{F}$ & -4.10900800 & 0.66945000 & -4.90208900 & & & & \\
\hline $\mathrm{Rh}$ & -1.66960800 & -0.81551900 & -0.24456300 & & & & \\
\hline $\mathrm{H}$ & -1.53014500 & -3.34681900 & -0.93224600 & & & & \\
\hline $\mathrm{C}$ & -3.94439300 & 5.66458100 & 1.07600600 & & & & \\
\hline $\mathrm{O}$ & -2.91167800 & 6.13436900 & 1.50518700 & & & & \\
\hline $\mathrm{O}$ & -5.11333300 & 6.34569600 & 1.04774400 & $\mathrm{C}$ & -6.07663500 & 0.49050900 & -1.75784500 \\
\hline $\mathrm{C}$ & -5.05256000 & 7.69230000 & 1.54258300 & $\mathrm{C}$ & -4.87036200 & 0.50940600 & -1.02565200 \\
\hline $\mathrm{H}$ & -4.73024800 & 7.70463800 & 2.58735900 & $\mathrm{C}$ & -3.98059500 & -0.56591300 & -0.88815700 \\
\hline $\mathrm{H}$ & -6.06567100 & 8.08506300 & 1.44935800 & $\mathrm{C}$ & -4.35093600 & -1.71565400 & -1.58415100 \\
\hline $\mathrm{H}$ & -4.35349400 & 8.28996100 & 0.95087600 & $\mathrm{C}$ & -5.56543900 & -1.75996800 & -2.30107000 \\
\hline $\mathrm{C}$ & -2.99007900 & 3.31746600 & 0.90741200 & $\mathrm{C}$ & -6.45469600 & -0.70716900 & -2.39662200 \\
\hline $\mathrm{H}$ & -2.02252500 & 3.79661500 & 0.71769900 & $\mathrm{C}$ & -6.59301500 & 1.83324500 & -1.70948600 \\
\hline $\mathrm{C}$ & -1.91709300 & 1.05448800 & 0.59963000 & $\mathrm{C}$ & -5.69447100 & 2.59939300 & -1.01431700 \\
\hline $\mathrm{H}$ & -1.91235900 & 0.89310900 & 1.68287800 & $\mathrm{H}$ & -3.72058400 & -2.59403900 & -1.62444600 \\
\hline $\mathrm{H}$ & -0.96366500 & 1.51157900 & 0.32370600 & $\mathrm{H}$ & -7.36950400 & -0.80885300 & -2.97072100 \\
\hline $\mathrm{H}$ & -4.03464600 & 1.49413700 & 0.36063300 & $\mathrm{H}$ & -7.51961700 & 2.18984700 & -2.14099800 \\
\hline $\mathrm{C}$ & -4.10556400 & 4.27799800 & 0.48251700 & $\mathrm{H}$ & -5.73848700 & 3.65228100 & -0.78597500 \\
\hline $\mathrm{H}$ & -5.09745200 & 3.89272800 & 0.74472400 & $\mathrm{~N}$ & -4.63662800 & 1.80785400 & -0.55293300 \\
\hline $\mathrm{H}$ & -4.10986300 & 4.39347600 & -0.61146200 & $\mathrm{P}$ & -2.94834300 & 2.09923100 & -0.17909700 \\
\hline $\mathrm{C}$ & -3.06242400 & 1.96521900 & 0.17242900 & $\mathrm{C}$ & -2.96965600 & 3.27259200 & 1.33866600 \\
\hline $\mathrm{H}$ & -3.01460000 & 2.14066300 & -0.90766800 & $\mathrm{C}$ & -2.29078400 & 2.99568500 & -1.74645500 \\
\hline $\mathrm{H}$ & -3.04146400 & 3.16199000 & 1.99406400 & $\mathrm{C}$ & -4.04464500 & 4.37677600 & 1.26085200 \\
\hline $\mathrm{C}$ & 2.39118200 & -3.00651800 & -0.75025900 & $\mathrm{H}$ & -5.05351000 & 3.95671700 & 1.30202800 \\
\hline $\mathrm{O}$ & 2.53840900 & -3.77365500 & 0.17985700 & $\mathrm{H}$ & -3.93021800 & 5.03284500 & 2.13362900 \\
\hline $\mathrm{O}$ & 1.60027100 & -3.29811900 & -1.81555200 & $\mathrm{H}$ & -3.96024600 & 5.00355200 & 0.37122400 \\
\hline $\mathrm{C}$ & 1.01408100 & -4.60611800 & -1.79629900 & $\mathrm{C}$ & -3.27386500 & 2.41991200 & 2.58845400 \\
\hline $\mathrm{H}$ & 0.45617300 & -4.76937500 & -0.87208300 & $\mathrm{H}$ & -3.36135900 & 3.09085100 & 3.45355300 \\
\hline $\mathrm{H}$ & 0.35254000 & -4.64523500 & -2.66259200 & $\mathrm{H}$ & -4.21972800 & 1.87630000 & 2.49431400 \\
\hline $\mathrm{H}$ & 1.79231300 & -5.37192800 & -1.87618700 & $\mathrm{H}$ & -2.47286800 & 1.70656900 & 2.79784000 \\
\hline $\mathrm{C}$ & 4.60198900 & -1.92362100 & -0.53495300 & $\mathrm{C}$ & -1.57484900 & 3.90991900 & 1.51296200 \\
\hline $\mathrm{H}$ & 4.62969400 & -2.30593500 & 0.48857900 & $\mathrm{H}$ & -0.78057700 & 3.15716100 & 1.52842000 \\
\hline
\end{tabular}




\begin{tabular}{|c|c|c|c|c|c|c|c|}
\hline $\mathrm{H}$ & -1.35295900 & 4.64571500 & 0.73493600 & $\mathrm{C}$ & 5.47269200 & -2.77751000 & -0.26257000 \\
\hline $\mathrm{H}$ & -1.54985600 & 4.43687000 & 2.47549700 & $\mathrm{H}$ & 1.99537200 & -0.10941800 & 3.23689800 \\
\hline $\mathrm{C}$ & -0.74986500 & 2.98618000 & -1.71415900 & $\mathrm{H}$ & 5.90227700 & -1.76662300 & 3.88443300 \\
\hline $\mathrm{H}$ & -0.33706400 & 3.58006200 & -0.89490600 & $\mathrm{H}$ & 7.02623300 & -3.05763400 & 1.24533100 \\
\hline $\mathrm{H}$ & -0.36627900 & 1.96763300 & -1.63748100 & $\mathrm{H}$ & 5.85997700 & -3.23748900 & -1.15976800 \\
\hline $\mathrm{H}$ & -0.37714700 & 3.41926900 & -2.65191500 & $\mathrm{~N}$ & 4.22179400 & -2.15367700 & -0.25580500 \\
\hline $\mathrm{C}$ & -2.75447000 & 2.14916600 & -2.95440800 & $\mathrm{P}$ & 2.81383500 & -2.05887400 & -1.26654200 \\
\hline $\mathrm{H}$ & -3.84081100 & 2.17033700 & -3.08044000 & $\mathrm{C}$ & 3.44307400 & -1.57644800 & -3.00792200 \\
\hline $\mathrm{H}$ & -2.30460700 & 2.57306600 & -3.86179500 & $\mathrm{C}$ & 2.09887400 & -3.84175800 & -1.24611100 \\
\hline $\mathrm{C}$ & -2.79331600 & 4.43952000 & -1.93777400 & $\mathrm{C}$ & 4.10903600 & -2.73367400 & -3.78062300 \\
\hline $\mathrm{H}$ & -2.42326000 & 4.80841300 & -2.90349800 & $\mathrm{H}$ & 4.96742300 & -3.15587700 & -3.24923000 \\
\hline $\mathrm{H}$ & -3.88535400 & 4.50282200 & -1.96732000 & $\mathrm{H}$ & 4.48057900 & -2.34061000 & -4.73569000 \\
\hline $\mathrm{H}$ & -2.41977400 & 5.12260200 & -1.16973800 & $\mathrm{H}$ & 3.41256600 & -3.54224000 & -4.01300100 \\
\hline $\mathrm{F}$ & -5.86459100 & -2.92395400 & -2.93413400 & $\mathrm{C}$ & 4.48522900 & -0.44819100 & -2.85232700 \\
\hline $\mathrm{Rh}$ & -2.34264900 & -0.22525400 & 0.27384800 & $\mathrm{H}$ & 4.80590600 & -0.13382000 & -3.85359000 \\
\hline $\mathrm{Cl}$ & -0.17153400 & 0.34719000 & 1.59643800 & $\mathrm{H}$ & 5.37122900 & -0.77902200 & -2.30288200 \\
\hline $\mathrm{H}$ & -2.42910000 & 1.10858700 & -2.87620700 & $\mathrm{H}$ & 4.07887200 & 0.42790800 & -2.34581400 \\
\hline $\mathrm{C}$ & -3.08205500 & -1.15755600 & 3.37512000 & $\mathrm{C}$ & 2.24260000 & -1.04987700 & -3.82622800 \\
\hline $\mathrm{O}$ & -2.07950000 & -1.29724600 & 4.03467200 & $\mathrm{H}$ & 1.75449400 & -0.19825100 & -3.34636200 \\
\hline $\mathrm{O}$ & -4.24944200 & -0.68073800 & 3.86271700 & $\mathrm{H}$ & 1.47901100 & -1.81418100 & -3.99187000 \\
\hline $\mathrm{C}$ & -4.23606800 & -0.33434000 & 5.25921300 & $\mathrm{H}$ & 2.60584300 & -0.72431100 & -4.80956500 \\
\hline $\mathrm{H}$ & -3.51612300 & 0.46656600 & 5.44606000 & $\mathrm{C}$ & 1.01748400 & -4.01207400 & -2.33240900 \\
\hline $\mathrm{H}$ & -5.24933400 & -0.00302600 & 5.48641200 & $\mathrm{H}$ & 1.43820600 & -4.02556600 & -3.34218600 \\
\hline $\mathrm{H}$ & -3.96896600 & -1.20396500 & 5.86502400 & $\mathrm{H}$ & 0.25512500 & -3.22888700 & -2.28226800 \\
\hline $\mathrm{C}$ & -2.19757600 & -2.23033100 & 1.24478600 & $\mathrm{H}$ & 0.51724800 & -4.97735400 & -2.17967300 \\
\hline $\mathrm{H}$ & -1.23400800 & -2.18396700 & 1.74995900 & $\mathrm{C}$ & 1.43739100 & -3.99687700 & 0.14283800 \\
\hline $\mathrm{C}$ & -2.16486900 & -4.74394500 & 1.37962600 & $\mathrm{H}$ & 2.15122100 & -3.86350800 & 0.96084800 \\
\hline $\mathrm{H}$ & -2.83575900 & -4.76722100 & 2.24642800 & $\mathrm{H}$ & 1.01473800 & -5.00676000 & 0.22398600 \\
\hline $\mathrm{H}$ & -2.31245300 & -5.67132700 & 0.81366500 & $\mathrm{C}$ & 3.16859700 & -4.94384400 & -1.38213000 \\
\hline $\mathrm{H}$ & -1.13684100 & -4.73733800 & 1.75911400 & $\mathrm{H}$ & 2.67190000 & -5.91841100 & -1.28878400 \\
\hline $\mathrm{C}$ & -3.24560500 & -1.53538400 & 1.91394300 & $\mathrm{H}$ & 3.92189600 & -4.88100300 & -0.59181300 \\
\hline $\mathrm{H}$ & -3.41204300 & -0.10619800 & 1.47746500 & $\mathrm{H}$ & 3.67750600 & -4.93254300 & -2.34822300 \\
\hline $\mathrm{H}$ & -4.26886300 & -1.81820800 & 1.66697600 & $\mathrm{~F}$ & 3.87718700 & -0.53107000 & 4.91084500 \\
\hline $\mathrm{C}$ & -2.44829100 & -3.51741200 & 0.49024600 & $\mathrm{Rh}$ & 1.42535200 & -0.72990000 & 0.03654500 \\
\hline $\mathrm{H}$ & -1.81186300 & -3.55906600 & -0.40189700 & $\mathrm{H}$ & 0.61244900 & -3.28856200 & 0.28506500 \\
\hline $\mathrm{H}$ & -3.48552900 & -3.56241000 & 0.14908200 & $\mathrm{C}$ & 5.48308000 & 4.96061500 & -0.44400300 \\
\hline $\mathrm{Cl}$ & -0.65187600 & -0.73555100 & -1.52003600 & $\mathrm{O}$ & 4.85521400 & 5.58976700 & -1.26883800 \\
\hline $\mathrm{C}$ & 5.12428400 & -1.98025000 & 1.83880800 & $\mathrm{O}$ & 6.62192400 & 5.41776200 & 0.12861400 \\
\hline $\mathrm{C}$ & 4.00843600 & -1.66901000 & 1.03788200 & $\mathrm{C}$ & 7.04869400 & 6.71815400 & -0.30338500 \\
\hline $\mathrm{C}$ & 2.84874500 & -0.99544300 & 1.44458200 & $\mathrm{H}$ & 7.24160300 & 6.72404000 & -1.37986500 \\
\hline $\mathrm{C}$ & 2.83772400 & -0.62830300 & 2.79227800 & $\mathrm{H}$ & 7.96505500 & 6.92547600 & 0.25055100 \\
\hline $\mathrm{C}$ & 3.94558900 & -0.92464500 & 3.61320200 & $\mathrm{H}$ & 6.28639000 & 7.46926600 & -0.07813700 \\
\hline $\mathrm{C}$ & 5.09082000 & -1.57856500 & 3.18938000 & $\mathrm{C}$ & 3.83527000 & 3.02482400 & -0.51405600 \\
\hline $\mathrm{C}$ & 6.04765200 & -2.67891300 & 0.97904900 & $\mathrm{H}$ & 3.01172700 & 3.72031900 & -0.30995600 \\
\hline
\end{tabular}




\begin{tabular}{|c|c|c|c|c|c|c|c|}
\hline $\mathrm{C}$ & 2.17556900 & 1.11648800 & -0.52246400 & $\mathrm{H}$ & 3.06481700 & 2.82939300 & -3.65106800 \\
\hline $\mathrm{H}$ & 2.17960900 & 1.10433200 & -1.61806000 & $\mathrm{H}$ & 3.55689100 & 1.48121300 & -4.67633300 \\
\hline $\mathrm{H}$ & 1.35797000 & 1.76089100 & -0.19263100 & $\mathrm{C}$ & 1.43459300 & 3.30421200 & -1.25999700 \\
\hline $\mathrm{H}$ & 4.31940800 & 0.93976300 & -0.18437300 & $\mathrm{H}$ & 1.54227900 & 3.39279100 & -2.34284300 \\
\hline $\mathrm{C}$ & 5.12607400 & 3.58602700 & 0.08734800 & $\mathrm{H}$ & 0.73745500 & 2.48781200 & -1.06197100 \\
\hline $\mathrm{H}$ & 5.97675300 & 2.91832400 & -0.10589300 & $\mathrm{H}$ & 0.98053600 & 4.23624600 & -0.89901600 \\
\hline $\mathrm{H}$ & 5.05406000 & 3.65406600 & 1.18104000 & $\mathrm{C}$ & 2.52234000 & 3.17274300 & 0.98865800 \\
\hline $\mathrm{C}$ & 3.49590500 & 1.62764500 & 0.04197400 & $\mathrm{H}$ & 3.44052900 & 3.08110400 & 1.57500700 \\
\hline $\mathrm{H}$ & 3.43377600 & 1.67729200 & 1.13437600 & $\mathrm{H}$ & 2.06528500 & 4.14197300 & 1.22457700 \\
\hline \multirow[t]{2}{*}{$\mathrm{H}$} & 3.93257100 & 2.98828700 & -1.60693800 & $\mathrm{C}$ & 3.71816100 & 4.28406300 & -0.90634800 \\
\hline & & & & $\mathrm{H}$ & 3.26142500 & 5.21655300 & -0.55010400 \\
\hline \multirow[t]{7}{*}{29} & & & & $\mathrm{H}$ & 4.70098000 & 4.20296500 & -0.43215300 \\
\hline & & & & $\mathrm{H}$ & 3.84961400 & 4.38582500 & -1.98715900 \\
\hline & & & & $\mathrm{F}$ & 4.20889900 & -1.05287900 & 5.06941500 \\
\hline & & & & $\mathrm{Rh}$ & 2.08626800 & -0.16433300 & 0.12897200 \\
\hline & & & & $\mathrm{Cl}$ & 0.15703700 & -0.08065900 & -1.60950900 \\
\hline & & & & $\mathrm{H}$ & 1.82896800 & 2.39349400 & 1.32443600 \\
\hline & & & & $\mathrm{C}$ & 1.80857100 & -3.94153500 & -1.85290900 \\
\hline $\mathrm{C}$ & 5.63 & 0.85222400 & 2.34103700 & $\mathrm{O}$ & 2.24558400 & -3.65934300 & -2.94895600 \\
\hline $\mathrm{C}$ & 4.59942800 & 0.62300500 & 1.40830100 & $\mathrm{O}$ & 1.21765200 & -5.13058500 & -1.57985300 \\
\hline $\mathrm{C}$ & 3.42295200 & -0.10206200 & 1.63739700 & $\mathrm{C}$ & 1.12477900 & -6.03776000 & -2.69049900 \\
\hline $\mathrm{C}$ & 3.32485800 & -0.65879700 & 2.91501500 & $\mathrm{H}$ & 2.11963400 & -6.27888300 & -3.07437200 \\
\hline $\mathrm{C}$ & 4.36166500 & -0.47268700 & 3.85098900 & $\mathrm{H}$ & 0.63878800 & -6.93200100 & -2.29833900 \\
\hline $\mathrm{C}$ & 5.51170500 & 0.26097200 & 3.61435200 & $\mathrm{H}$ & 0.53071600 & -5.59964400 & -3.49725500 \\
\hline $\mathrm{C}$ & 6.57510600 & 1.71856000 & 1.68397800 & $\mathrm{C}$ & 2.86266700 & -1.95238200 & -0.67919400 \\
\hline $\mathrm{C}$ & 6.09870100 & 1.98260000 & 0.42674200 & $\mathrm{H}$ & 2.98940700 & -1.66569600 & -1.71891400 \\
\hline $\mathrm{H}$ & 2.46246300 & -1.24155800 & 3.21917500 & $\mathrm{C}$ & 4.78929700 & -3.65421100 & -0.82209700 \\
\hline $\mathrm{H}$ & 6.26048200 & 0.37273000 & 4.39132700 & $\mathrm{H}$ & 4.90909400 & -3.47048200 & -1.89506000 \\
\hline $\mathrm{H}$ & 7.50450200 & 2.09669300 & 2.09046800 & $\mathrm{H}$ & 5.77421400 & -3.88992800 & -0.40179900 \\
\hline $\mathrm{H}$ & 6.53578200 & 2.59242400 & -0.34692300 & $\mathrm{H}$ & 4.16518700 & -4.54759200 & -0.70605900 \\
\hline $\mathrm{N}$ & 4.89630800 & 1.29372500 & 0.21521400 & $\mathrm{C}$ & 1.77304400 & -3.02905500 & -0.63535800 \\
\hline $\mathrm{P}$ & 3.54300200 & 1.36944000 & -0.88037100 & $\mathrm{H}$ & 1.81113200 & -3.62756100 & 0.28001900 \\
\hline $\mathrm{C}$ & 4.27925000 & 1.28443100 & -2.65339700 & $\mathrm{H}$ & 0.77934100 & -2.57564800 & -0.66805700 \\
\hline $\mathrm{C}$ & 2.78393700 & 3.11623200 & -0.53494800 & $\mathrm{C}$ & 4.19237800 & -2.43342100 & -0.09127700 \\
\hline $\mathrm{C}$ & 5.56311400 & 2.12660900 & -2.82723900 & $\mathrm{H}$ & 4.06142000 & -2.68390800 & 0.96699300 \\
\hline $\mathrm{H}$ & 6.40273200 & 1.68841200 & -2.28132700 & $\mathrm{H}$ & 4.92461800 & -1.62113600 & -0.12035500 \\
\hline $\mathrm{H}$ & 5.83013100 & 2.11751200 & -3.89178300 & $\mathrm{Cl}$ & 0.28005200 & -1.05738700 & 1.59227500 \\
\hline $\mathrm{H}$ & 5.45501900 & 3.17033600 & -2.52875400 & $\mathrm{C}$ & -5.56519500 & -0.84013000 & -1.87655300 \\
\hline $\mathrm{C}$ & 4.65772500 & -0.17601800 & -2.98053200 & $\mathrm{C}$ & -4.41122600 & -0.86046900 & -1.06838800 \\
\hline $\mathrm{H}$ & 5.25412400 & -0.17661700 & -3.90183500 & $\mathrm{C}$ & -3.12074300 & -0.46251400 & -1.44227000 \\
\hline $\mathrm{H}$ & 5.26481200 & -0.63689900 & -2.19592300 & $\mathrm{C}$ & -3.01645300 & -0.01903800 & -2.76326100 \\
\hline $\mathrm{H}$ & 3.78273100 & -0.80126900 & -3.16559500 & $\mathrm{C}$ & -4.15815400 & 0.01838000 & -3.58930600 \\
\hline $\mathrm{C}$ & 3.21230100 & 1.74624900 & -3.66886500 & $\mathrm{C}$ & -5.42916400 & -0.36854300 & -3.19733700 \\
\hline $\mathrm{H}$ & 2.24486800 & 1.25725900 & -3.51234000 & $\mathrm{C}$ & -6.63377700 & -1.35269800 & -1.05561900 \\
\hline
\end{tabular}




\begin{tabular}{|c|c|c|c|c|c|c|c|}
\hline $\mathrm{C}$ & -6.10667300 & -1.66748600 & 0.17108600 & $\mathrm{C}$ & -1.81875000 & 1.28953200 & 0.65778400 \\
\hline $\mathrm{H}$ & -2.07153200 & 0.30728600 & -3.18441400 & $\mathrm{H}$ & -1.99747500 & 1.22146900 & 1.73479800 \\
\hline $\mathrm{H}$ & -6.25836900 & -0.30885200 & -3.89432500 & $\mathrm{H}$ & -0.78523600 & 1.62005400 & 0.51506500 \\
\hline $\mathrm{H}$ & -7.67267300 & -1.46921100 & -1.33665600 & $\mathrm{H}$ & -3.81976900 & 1.92646600 & 0.10381700 \\
\hline $\mathrm{H}$ & -6.59950000 & -2.07859400 & 1.03962200 & $\mathrm{C}$ & -3.57717300 & 4.68749900 & -0.07341900 \\
\hline $\mathrm{N}$ & -4.74212500 & -1.36472200 & 0.19152700 & $\mathrm{H}$ & -4.62933600 & 4.42157400 & 0.08398400 \\
\hline $\mathrm{P}$ & -3.36805800 & -1.62210200 & 1.21991500 & $\mathrm{H}$ & -3.43411300 & 4.68951500 & -1.16387000 \\
\hline $\mathrm{C}$ & -3.91237200 & -1.03945000 & 2.95517000 & $\mathrm{C}$ & -2.78161800 & 2.26367700 & -0.00799600 \\
\hline $\mathrm{C}$ & -3.06277600 & -3.51427200 & 1.15252500 & $\mathrm{H}$ & -2.58810400 & 8100 & -1.08542200 \\
\hline $\mathrm{C}$ & -4.86146900 & -2.01818600 & 3.67786800 & $\mathrm{H}$ & -2.82350300 & 3.6 & 1.66808700 \\
\hline $\mathrm{H}$ & -5.77164500 & -2.22491000 & 3.10695000 & & & & \\
\hline $\mathrm{H}$ & -5.17095000 & -1.56060400 & 4.62633900 & 30-ts & & & \\
\hline $\mathrm{H}$ & -4.37996600 & -2.96881800 & 3.91991400 & & & & \\
\hline $\mathrm{C}$ & -4.64837200 & 0.30801200 & 2.78548700 & & & & \\
\hline $\mathrm{H}$ & -4.88764100 & 0.70018400 & 3.78201100 & & & & \\
\hline $\mathrm{H}$ & -5.58410100 & 0.19668500 & 2.23073200 & & & & \\
\hline $\mathrm{H}$ & -4.04010000 & 1.05459300 & 2.27210900 & & & & \\
\hline $\mathrm{C}$ & -2.65195600 & -0.83325600 & 3.82445200 & & & & \\
\hline $\mathrm{H}$ & -1.94116500 & -0.13714100 & 3.37397400 & & & & \\
\hline $\mathrm{H}$ & -2.11825200 & -1.76819100 & 4.01356600 & $\mathrm{C}$ & -6.13921400 & 1.24907500 & -1.26810700 \\
\hline $\mathrm{H}$ & -2.96008100 & -0.42501600 & 4.79563400 & $\mathrm{C}$ & -4.90880300 & 1.12973800 & -0.58724600 \\
\hline $\mathrm{C}$ & -2.06688600 & -3.94273800 & 2.24949700 & $\mathrm{C}$ & -4.07602300 & 0.00023300 & -0.59258900 \\
\hline $\mathrm{H}$ & -2.50263900 & -3.88755000 & 3.25158600 & $\mathrm{C}$ & -4.53197000 & -1.04555300 & -1.39499600 \\
\hline $\mathrm{H}$ & -1.15040700 & -3.34511200 & 2.23325000 & $\mathrm{C}$ & -5.77164300 & -0.95314900 & -2.06269800 \\
\hline $\mathrm{H}$ & -1.78484100 & -4.98927800 & 2.07604000 & $\mathrm{C}$ & -6.60651400 & 0.14569000 & -2.00960600 \\
\hline $\mathrm{C}$ & -2.42151600 & -3.77320000 & -0.23118600 & $\mathrm{C}$ & -6.57543300 & 2.60623700 & -1.06946200 \\
\hline $\mathrm{H}$ & -3.06743700 & -3.45658600 & -1.05553600 & $\mathrm{C}$ & -5.60822800 & 3.25164500 & -0.34476100 \\
\hline $\mathrm{H}$ & -2.23225000 & -4.84876400 & -0.34137100 & $\mathrm{H}$ & -3.94543200 & -1.93829500 & -1.56678800 \\
\hline $\mathrm{C}$ & -4.35157600 & -4.35469700 & 1.24449500 & $\mathrm{H}$ & -7.54544500 & 0.15206600 & -2.55284900 \\
\hline $\mathrm{H}$ & -4.08268600 & -5.41267700 & 1.12749100 & $\mathrm{H}$ & -7.49524100 & 3.05242900 & -1.42556900 \\
\hline $\mathrm{H}$ & -5.05873600 & -4.10461700 & 0.44839000 & $\mathrm{H}$ & -5.58197600 & 4.27865400 & -0.01646000 \\
\hline $\mathrm{H}$ & -4.86082200 & -4.25531500 & 2.20576900 & $\mathrm{~N}$ & -4.58178100 & 2.36258300 & -0.00553300 \\
\hline $\mathrm{F}$ & -3.98952700 & 0.47089300 & -4.85864700 & $\mathrm{P}$ & -2.86401000 & 2.52802600 & 0.30741500 \\
\hline $\mathrm{Rh}$ & -1.67825100 & -0.65078000 & -0.03857400 & $\mathrm{C}$ & -2.75595300 & 3.55972400 & 1.91832100 \\
\hline $\mathrm{H}$ & -1.45167100 & -3.27002500 & -0.33560600 & $\mathrm{C}$ & -2.22118500 & 3.52709000 & -1.20265000 \\
\hline $\mathrm{C}$ & -3.35894500 & 6.10817200 & 0.41062200 & $\mathrm{C}$ & -3.75598400 & 4.73409000 & 1.98914400 \\
\hline $\mathrm{O}$ & -2.39286400 & 6.50789800 & 1.02517200 & $\mathrm{H}$ & -4.78882400 & 4.37681800 & 2.02737300 \\
\hline $\mathrm{O}$ & -4.38954200 & 6.90698800 & 0.04715900 & $\mathrm{H}$ & -3.56979100 & 5.28760400 & 2.91862000 \\
\hline $\mathrm{C}$ & -4.25956800 & 8.28706500 & 0.42076500 & $\mathrm{H}$ & -3.65938500 & 5.44416700 & 1.16528000 \\
\hline $\mathrm{H}$ & -4.18051900 & 8.38695300 & 1.50683300 & $\mathrm{C}$ & -3.07342200 & 2.61935400 & 3.09992500 \\
\hline $\mathrm{H}$ & -5.16348900 & 8.77621100 & 0.05596200 & $\mathrm{H}$ & -3.07362700 & 3.20828200 & 4.02663100 \\
\hline $\mathrm{H}$ & -3.37170300 & 8.72981800 & -0.03939900 & $\mathrm{H}$ & -4.06280000 & 2.15949300 & 2.99895600 \\
\hline $\mathrm{C}$ & -2.63298600 & 3.67826500 & 0.58644700 & $\mathrm{H}$ & -2.32511000 & 1.83098000 & 3.20194400 \\
\hline $\mathrm{H}$ & -1.60071200 & 4.02803000 & 0.46964100 & $\mathrm{C}$ & -1.31656900 & 4.08915200 & 2.09302000 \\
\hline
\end{tabular}




\begin{tabular}{|c|c|c|c|c|c|c|c|}
\hline $\mathrm{H}$ & -0.57344400 & 3.29030100 & 2.01012400 & $\mathrm{C}$ & 5.81868000 & -2.98356300 & 0.66820100 \\
\hline $\mathrm{H}$ & -1.07234600 & 4.87647300 & 1.37439000 & $\mathrm{C}$ & 5.16598000 & -2.98056700 & -0.53851200 \\
\hline $\mathrm{H}$ & -1.22460700 & 4.52477600 & 3.09623200 & $\mathrm{H}$ & 2.15480200 & -0.16083500 & 3.25954300 \\
\hline $\mathrm{C}$ & -0.68261200 & 3.43271500 & -1.24422900 & $\mathrm{H}$ & 5.92666400 & -2.17971800 & 3.60697400 \\
\hline $\mathrm{H}$ & -0.20133300 & 3.92639700 & -0.39618100 & $\mathrm{H}$ & 6.77408700 & -3.45527700 & 0.85955000 \\
\hline $\mathrm{H}$ & -0.35324700 & 2.39280300 & -1.27834200 & $\mathrm{H}$ & 5.45963600 & -3.43276600 & -1.47425800 \\
\hline $\mathrm{H}$ & -0.32692900 & 3.92985100 & -2.15654300 & $\mathrm{~N}$ & 3.97713800 & -2.25334700 & -0.43163800 \\
\hline $\mathrm{C}$ & -2.78189200 & 2.82114200 & -2.45878300 & $\mathrm{P}$ & 2.53213100 & -1.98127700 & -1.35485600 \\
\hline $\mathrm{H}$ & -3.86982900 & 2.91037500 & -2.53099900 & $\mathrm{C}$ & 3.11041400 & -1.47775300 & -3.10616900 \\
\hline $\mathrm{H}$ & -2.34723700 & 3.30268200 & -3.34455200 & $\mathrm{C}$ & 1.64617300 & -3.68518000 & -1.36140100 \\
\hline $\mathrm{C}$ & -2.64833400 & 5.00665700 & -1.23967400 & $\mathrm{C}$ & 3.63904000 & -2.65383300 & -3.95341100 \\
\hline $\mathrm{H}$ & -2.29738300 & 5.44426500 & -2.18355200 & $\mathrm{H}$ & 4.48739100 & -3.16429100 & -3.48692300 \\
\hline $\mathrm{H}$ & -3.73549600 & 5.12762900 & -1.21356900 & $\mathrm{H}$ & 3.98973300 & -2.25537800 & -4.91412500 \\
\hline $\mathrm{H}$ & -2.20721100 & 5.59422100 & -0.42960700 & $\mathrm{H}$ & 2.86729600 & -3.39510900 & -4.17382600 \\
\hline $\mathrm{F}$ & -6.15267500 & -2.02551200 & -2.80447400 & $\mathrm{C}$ & 4.24512000 & -0.44140000 & -2.96100300 \\
\hline $\mathrm{Rh}$ & -2.37657800 & 0.14274900 & 0.51616800 & $\mathrm{H}$ & 4.55671400 & -0.12770900 & -3.96534300 \\
\hline $\mathrm{Cl}$ & -0.10671600 & 0.47480900 & 1.79619800 & $\mathrm{H}$ & 5.11955800 & -0.85290900 & -2.44911700 \\
\hline $\mathrm{H}$ & -2.51239200 & 1.76222100 & -2.48942900 & $\mathrm{H}$ & 3.92279600 & 0.45106200 & -2.42257500 \\
\hline $\mathrm{C}$ & -2.63651700 & -4.40263700 & 1.22044700 & $\mathrm{C}$ & 1.91965700 & -0.82225700 & -3.83962800 \\
\hline $\mathrm{O}$ & -2.88648000 & -4.50212700 & 2.40334000 & $\mathrm{H}$ & 1.51606800 & 0.03130200 & -3.29028600 \\
\hline $\mathrm{O}$ & -2.28126100 & -5.45417900 & 0.44641700 & $\mathrm{H}$ & 1.09709200 & -1.52083700 & -4.01059900 \\
\hline $\mathrm{C}$ & -2.26512000 & -6.72865800 & 1.11385300 & $\mathrm{H}$ & 2.26489700 & -0.46725600 & -4.81916200 \\
\hline $\mathrm{H}$ & -3.25344900 & -6.96164900 & 1.51857900 & $\mathrm{C}$ & 0.49588700 & -3.70236500 & -2.38936100 \\
\hline $\mathrm{H}$ & -1.98250500 & -7.45466400 & 0.35092200 & $\mathrm{H}$ & 0.86023000 & -3.72656400 & -3.42084500 \\
\hline $\mathrm{H}$ & -1.53897200 & -6.72450700 & 1.93101000 & $\mathrm{H}$ & -0.17349900 & -2.84392300 & -2.27753900 \\
\hline $\mathrm{C}$ & -2.28970100 & -1.93408600 & 1.25639700 & $\mathrm{H}$ & -0.09744800 & -4.61242600 & -2.23322500 \\
\hline $\mathrm{H}$ & -1.27252700 & -2.01585300 & 1.63733400 & $\mathrm{C}$ & 1.04997200 & -3.83245500 & 0.05768800 \\
\hline $\mathrm{C}$ & -2.87356700 & -1.06633200 & 3.59622000 & $\mathrm{H}$ & 1.82197100 & -3.84161400 & 0.83256900 \\
\hline $\mathrm{H}$ & -1.83876300 & -0.73864700 & 3.72124200 & $\mathrm{H}$ & 0.49005200 & -4.77423900 & 0.11534400 \\
\hline $\mathrm{H}$ & -3.54221600 & -0.33989600 & 4.06817900 & $\mathrm{C}$ & 2.58973200 & -4.87989000 & -1.60122100 \\
\hline $\mathrm{H}$ & -2.99365800 & -2.03069200 & 4.10530600 & $\mathrm{H}$ & 2.00446600 & -5.80419300 & -1.50896500 \\
\hline $\mathrm{C}$ & -2.71643100 & -3.11355900 & 0.41218500 & $\mathrm{H}$ & 3.39179100 & -4.92246000 & -0.85898200 \\
\hline $\mathrm{H}$ & -3.76451300 & -3.01474800 & 0.11001400 & $\mathrm{H}$ & 3.03744200 & -4.87929200 & -2.59712900 \\
\hline $\mathrm{H}$ & -2.12181500 & -3.21289800 & -0.49931500 & $\mathrm{~F}$ & 4.08445200 & -0.81140900 & 4.79864400 \\
\hline $\mathrm{C}$ & -3.20873300 & -1.30038900 & 2.13596300 & $\mathrm{Rh}$ & 1.33674800 & -0.60822800 & 0.08200500 \\
\hline $\mathrm{H}$ & -4.26229200 & -1.50468600 & 1.93981900 & $\mathrm{H}$ & 0.34235200 & -3.02670700 & 0.28884900 \\
\hline $\mathrm{H}$ & -3.37106900 & 0.23280900 & 1.75952000 & $\mathrm{C}$ & 5.80814800 & 4.75327600 & -0.48616000 \\
\hline $\mathrm{Cl}$ & -0.76895900 & -0.30571400 & -1.38952800 & $\mathrm{O}$ & 5.17997300 & 5.45214900 & -1.25249800 \\
\hline $\mathrm{C}$ & 5.01216200 & -2.24486900 & 1.60797700 & $\mathrm{O}$ & 7.01985200 & 5.09873700 & 0.00984800 \\
\hline $\mathrm{C}$ & 3.88225400 & -1.80759000 & 0.88962700 & $\mathrm{C}$ & 7.52642300 & 6.36693600 & -0.43196900 \\
\hline $\mathrm{C}$ & 2.81160000 & -1.05438400 & 1.39012300 & $\mathrm{H}$ & 7.64342000 & 6.37874300 & -1.51928500 \\
\hline $\mathrm{C}$ & 2.91657600 & -0.73838200 & 2.74692000 & $\mathrm{H}$ & 8.49376900 & 6.48355800 & 0.05809100 \\
\hline $\mathrm{C}$ & 4.04039800 & -1.16058400 & 3.48692900 & $\mathrm{H}$ & 6.84970700 & 7.17553000 & -0.14184900 \\
\hline $\mathrm{C}$ & 5.09498800 & -1.89549000 & 2.97100800 & $\mathrm{C}$ & 4.00005800 & 2.96652200 & -0.47528200 \\
\hline
\end{tabular}




\begin{tabular}{|c|c|c|c|c|c|c|c|}
\hline $\mathrm{H}$ & 3.25333200 & 3.71936000 & -0.19391000 & $\mathrm{H}$ & -0.31995000 & 3.22558100 & 1.55233800 \\
\hline $\mathrm{C}$ & 2.19243500 & 1.20436000 & -0.43233000 & $\mathrm{H}$ & -0.77712100 & 4.75775000 & 0.76480200 \\
\hline $\mathrm{H}$ & 2.12510400 & 1.25069400 & -1.52504600 & $\mathrm{H}$ & -0.78115500 & 4.62807800 & 2.52367000 \\
\hline $\mathrm{H}$ & 1.44848000 & 8300 & -0.01608000 & $\mathrm{C}$ & -0.71839800 & 3.00066900 & -1.66854200 \\
\hline $\mathrm{H}$ & 4.32861600 & 0.83789000 & -0.23592300 & $\mathrm{H}$ & -0.14911600 & 3.59317500 & -0.94891000 \\
\hline $\mathrm{C}$ & 5.37234100 & 3.40143700 & 0.04512300 & $\mathrm{H}$ & -0.43233900 & 1.95327900 & -1.57194200 \\
\hline $\mathrm{H}$ & 6.14689800 & 2.66909300 & -0.22050000 & $\mathrm{H}$ & -0.42719300 & 3.33911900 & -2.67188600 \\
\hline $\mathrm{H}$ & 5.38222100 & 3.44884500 & 1.14219300 & $\mathrm{C}$ & -2.94026700 & 2.34332900 & -2.61481100 \\
\hline $\mathrm{C}$ & 3.58274600 & 1.58376600 & 0.06309900 & $\mathrm{H}$ & -4.02586700 & 2.47704900 & -2.60722000 \\
\hline $\mathrm{H}$ & 3.59269400 & 1.60070800 & 1.15819000 & $\mathrm{H}$ & -2.56872400 & 2.68243900 & -3.59051900 \\
\hline \multirow[t]{2}{*}{$\mathrm{H}$} & 4.01779000 & 2.9 & -1.57281200 & $\mathrm{C}$ & -2.59779800 & 4.66370800 & -1.72238800 \\
\hline & & & & $\mathrm{H}$ & -2.30834600 & 4.94675100 & -2.74276600 \\
\hline \multirow[t]{7}{*}{31} & & & & $\mathrm{H}$ & -3.67021600 & 4.85488800 & -1.62793200 \\
\hline & & & & $\mathrm{H}$ & -2.05536700 & 5.32498700 & -1.04078600 \\
\hline & & & & $\mathrm{F}$ & -6.88582500 & -2.13530500 & -1.79931000 \\
\hline & & & & $\mathrm{Rh}$ & -2.51320800 & 0.13239400 & 0.63502200 \\
\hline & & & & $\mathrm{Cl}$ & -0.16406600 & 0.43802400 & 1.78037600 \\
\hline & & & & $\mathrm{H}$ & -2.71391900 & 1.27790700 & -2.52343800 \\
\hline & & & & $\mathrm{C}$ & -2.05072800 & -4.588586000 & 0.80923200 \\
\hline $\mathrm{C}$ & 17900 & 1.32826700 & -0.91112200 & $\mathrm{O}$ & -1.58172400 & -4.84361400 & 1.89746900 \\
\hline $\mathrm{C}$ & -5.06672300 & 1.17620000 & -0.35888400 & $\mathrm{O}$ & -2.24602800 & -5.51912300 & -0.15390100 \\
\hline $\mathrm{C}$ & -4.34032100 & -0.01994400 & -0.26018700 & $\mathrm{C}$ & -1.91487200 & -6.86792900 & 0.21464200 \\
\hline $\mathrm{C}$ & -5.00964400 & -1.12556600 & -0.78670100 & $\mathrm{H}$ & -2.49219500 & -7.18045500 & 1.08877500 \\
\hline $\mathrm{C}$ & -6.30879300 & -1.00175800 & -1.32284900 & $\mathrm{H}$ & -2.17001500 & -7.47830300 & -0.65215600 \\
\hline $\mathrm{C}$ & -7.01724100 & 0.18137700 & -1.39163000 & $\mathrm{H}$ & -0.84957400 & -6.95590200 & 0.44573800 \\
\hline $\mathrm{C}$ & -6.64053000 & 2.73849600 & -0.87722000 & $\mathrm{C}$ & -2.21069200 & -2.15592000 & 1.34622600 \\
\hline $\mathrm{C}$ & -5.54329700 & 3.37318800 & -0.35751900 & $\mathrm{H}$ & -1.17115700 & -2.10208900 & 1.65587000 \\
\hline $\mathrm{H}$ & -4.56467000 & -2.10867700 & -0.83413400 & $\mathrm{C}$ & -2.83611700 & -1.15707200 & 3.60061800 \\
\hline $\mathrm{H}$ & -8.01286400 & 0.20622100 & -1.82131100 & $\mathrm{H}$ & -1.80298800 & -0.81741100 & 3.70152800 \\
\hline $\mathrm{H}$ & -7.55357800 & 3.22572800 & -1.19467500 & $\mathrm{H}$ & -3.50962700 & -0.36432900 & 3.93966900 \\
\hline $\mathrm{H}$ & -5.39279000 & 4.42585300 & -0.18064100 & $\mathrm{H}$ & -2.97733600 & -2.02074900 & 4.26614600 \\
\hline $\mathrm{N}$ & -4.56815600 & 2.43383800 & -0.00054500 & $\mathrm{C}$ & -2.52065000 & -3.22253500 & 0.32736400 \\
\hline $\mathrm{P}$ & -2.82879400 & 2.45068000 & 0.16268300 & $\mathrm{H}$ & -3.59629500 & -3.31764600 & 0.15781900 \\
\hline $\mathrm{C}$ & -2.47881100 & 3.64875600 & 1.62155700 & $\mathrm{H}$ & -2.06152500 & -3.01664100 & -0.64523700 \\
\hline $\mathrm{C}$ & -2.23970000 & 3.17869900 & -1.51902900 & $\mathrm{C}$ & -3.14311100 & -1.60536000 & 2.19257300 \\
\hline $\mathrm{C}$ & -3.38277500 & 4.90139300 & 1.61076800 & $\mathrm{H}$ & -4.19557600 & -1.78230700 & 1.97303100 \\
\hline $\mathrm{H}$ & -4.42936200 & 4.64209500 & 1.79126900 & $\mathrm{H}$ & -3.37438400 & 0.62064700 & 1.80420700 \\
\hline $\mathrm{H}$ & -3.06394100 & 5.55701300 & 2.43099100 & $\mathrm{Cl}$ & -0.84383300 & -0.61480300 & -1.30402600 \\
\hline $\mathrm{H}$ & -3.31547900 & 5.48120800 & 0.68844500 & $\mathrm{C}$ & 5.13887200 & -1.90364500 & 1.67604700 \\
\hline $\mathrm{C}$ & -2.75278100 & 2.90311900 & 2.94543800 & $\mathrm{C}$ & 3.97413600 & -1.59076000 & 0.94892800 \\
\hline $\mathrm{H}$ & -2.61078700 & 3.60980500 & 3.77364100 & $\mathrm{C}$ & 2.83923400 & -0.92508300 & 1.43116100 \\
\hline $\mathrm{H}$ & -3.78250300 & 2.53321600 & 2.99799900 & $\mathrm{C}$ & 2.91357800 & -0.56512200 & 2.77878700 \\
\hline $\mathrm{H}$ & -2.06831700 & 2.06559000 & 3.09287300 & $\mathrm{C}$ & 4.07155000 & -0.86233400 & 3.52717600 \\
\hline $\mathrm{C}$ & -0.99780800 & 4.08253600 & 1.59644600 & $\mathrm{C}$ & 5.18952200 & -1.51032800 & 3.02873800 \\
\hline
\end{tabular}




\begin{tabular}{|c|c|c|c|c|c|c|c|}
\hline $\mathrm{C}$ & 6.00778700 & -2.59448200 & 0.75548600 & $\mathrm{H}$ & 2.96249400 & 3.77630200 & -0.30452000 \\
\hline $\mathrm{C}$ & 5.35580100 & -2.68689200 & -0.44811300 & $\mathrm{C}$ & 2.08240900 & 1.18746100 & -0.47135500 \\
\hline $\mathrm{H}$ & 2.10207800 & -0.04651900 & 3.27781500 & $\mathrm{H}$ & 2.00524600 & 1.18763400 & -1.56421500 \\
\hline $\mathrm{H}$ & 6.04340600 & -1.70011000 & 3.67041700 & $\mathrm{H}$ & 1.30242200 & 1.84027300 & -0.07519400 \\
\hline $\mathrm{H}$ & 7.00232600 & -2.97156600 & 0.95706800 & $\mathrm{H}$ & 4.24391600 & 0.98029900 & -0.29436800 \\
\hline $\mathrm{H}$ & 5.68785500 & -3.13824900 & -1.37141500 & $\mathrm{C}$ & 5.09760200 & 3.61926300 & -0.06192500 \\
\hline $\mathrm{N}$ & 4.10708200 & -2.06516500 & -0.35916500 & $\mathrm{H}$ & 5.92833000 & 2.95310800 & -0.33106100 \\
\hline $\mathrm{P}$ & 2.63209500 & -1.98161400 & -1.27051500 & $\mathrm{H}$ & 5.10863900 & 3.66734100 & 1.03535100 \\
\hline $\mathrm{C}$ & 3.13685900 & -1.50677900 & -3.05373300 & $\mathrm{C}$ & 3.44816900 & 1.67609100 & -0.00312300 \\
\hline $\mathrm{C}$ & 1.92528700 & -3.76375700 & -1.18490900 & $\mathrm{H}$ & 3.46839800 & 1.71927100 & 1.09106200 \\
\hline $\mathrm{C}$ & 3.74858100 & -2.67054200 & -3.86045000 & $\mathrm{H}$ & 9700 & 3.04547000 & -1.66980300 \\
\hline $\mathrm{H}$ & 4.63564100 & -3.09663300 & -3.38190900 & & & & \\
\hline $\mathrm{H}$ & 4.06157600 & -2.28413600 & -4.83889400 & 32-ts & & & \\
\hline $\mathrm{H}$ & 3.03292900 & -3.47507900 & -4.04459300 & & & & \\
\hline $\mathrm{C}$ & 4.18430800 & -0.37495500 & -2.97970900 & & & & \\
\hline $\mathrm{H}$ & 4.44382800 & -0.07808600 & -4.00388900 & & & & \\
\hline $\mathrm{H}$ & 5.10277200 & -0.69279800 & -2.47785500 & & & & \\
\hline $\mathrm{H}$ & 3.80516100 & 0.50982900 & -2.46679000 & & & & \\
\hline $\mathrm{C}$ & 1.88279700 & -0.99006600 & -3.79398200 & & & & \\
\hline $\mathrm{H}$ & 1.41264900 & -0.14824000 & -3.28051100 & $\mathrm{C}$ & -5.96480500 & 0.03702600 & -2.06301300 \\
\hline $\mathrm{H}$ & 1.12017700 & -1.76333000 & -3.91712600 & $\mathrm{C}$ & -4.78050700 & 0.15639500 & -1.30460200 \\
\hline $\mathrm{H}$ & 2.18266800 & -0.65611200 & -4.79576400 & $\mathrm{C}$ & -3.86018400 & -0.87417100 & -1.05925300 \\
\hline $\mathrm{C}$ & 0.74555900 & -3.91854500 & -2.16596400 & $\mathrm{C}$ & -4.17503900 & -2.08600000 & -1.67206900 \\
\hline $\mathrm{H}$ & 1.07676900 & -3.98293800 & -3.20712700 & $\mathrm{C}$ & -5.36629900 & -2.22836200 & -2.41428300 \\
\hline $\mathrm{H}$ & 0.02881400 & -3.09627100 & -2.08082100 & $\mathrm{C}$ & -6.28614400 & -1.21843000 & -2.61552300 \\
\hline $\mathrm{H}$ & 0.21077200 & -4.84904600 & -1.93617300 & $\mathrm{C}$ & -6.53056400 & 1.35845800 & -2.13112900 \\
\hline $\mathrm{C}$ & 1.40351800 & -3.91694000 & 0.26187700 & $\mathrm{C}$ & -5.68045100 & 2.20879500 & -1.47469300 \\
\hline $\mathrm{H}$ & 2.19646800 & -3.79706300 & 1.00552200 & $\mathrm{H}$ & -3.52006100 & -2.94462800 & -1.62510200 \\
\hline $\mathrm{H}$ & 0.97510700 & -4.91870500 & 0.38696900 & $\mathrm{H}$ & -7.18184900 & -1.39590700 & -3.20117200 \\
\hline $\mathrm{C}$ & 2.96934700 & -4.87297300 & -1.42150400 & $\mathrm{H}$ & -7.45831400 & 1.64465200 & -2.60996900 \\
\hline $\mathrm{H}$ & 2.47992200 & -5.84286200 & -1.26302500 & $\mathrm{H}$ & -5.77024100 & 3.27301900 & -1.32573200 \\
\hline $\mathrm{H}$ & 3.80160400 & -4.80568300 & -0.71508600 & $\mathrm{~N}$ & -4.60736800 & 1.49517600 & -0.92915800 \\
\hline $\mathrm{H}$ & 3.37240100 & -4.87727900 & -2.43601200 & $\mathrm{P}$ & -2.94698300 & 1.88011000 & -0.52932600 \\
\hline $\mathrm{F}$ & 4.08281700 & -0.47518900 & 4.82878700 & $\mathrm{C}$ & -3.05820000 & 3.17839100 & 0.87514100 \\
\hline $\mathrm{Rh}$ & 1.33005400 & -0.64925100 & 0.11462100 & $\mathrm{C}$ & -2.27349300 & 2.67630300 & -2.14710100 \\
\hline $\mathrm{H}$ & 0.61003200 & -3.19755700 & 0.49542600 & $\mathrm{C}$ & -4.16710300 & 4.23547800 & 0.68283400 \\
\hline $\mathrm{C}$ & 5.41751600 & 5.00383800 & -0.59133600 & $\mathrm{H}$ & -5.16136500 & 3.78214200 & 0.72345100 \\
\hline $\mathrm{O}$ & 4.71561400 & 5.66336000 & -1.32813600 & $\mathrm{H}$ & -4.10359800 & 4.95313200 & 1.51130100 \\
\hline $\mathrm{O}$ & 6.61534600 & 5.43197900 & -0.12758900 & $\mathrm{H}$ & -4.07579700 & 4.80404200 & -0.24473500 \\
\hline $\mathrm{C}$ & 7.01162500 & 6.74118000 & -0.56247900 & $\mathrm{C}$ & -3.37307700 & 2.42002900 & 2.17985700 \\
\hline $\mathrm{H}$ & 7.09464600 & 6.77774400 & -1.65235300 & $\mathrm{H}$ & -3.49754300 & 3.15278200 & 2.98863100 \\
\hline $\mathrm{H}$ & 7.98175700 & 6.92345900 & -0.09868100 & $\mathrm{H}$ & -4.29848000 & 1.84175700 & 2.10570300 \\
\hline $\mathrm{H}$ & 6.28501600 & 7.49181900 & -0.23913200 & $\mathrm{H}$ & -2.55570700 & 1.75023500 & 2.45713600 \\
\hline $\mathrm{C}$ & 3.76161700 & 3.07406100 & -0.57244100 & $\mathrm{C}$ & -1.69242200 & 3.87836800 & 1.03687200 \\
\hline
\end{tabular}




\begin{tabular}{|c|c|c|c|c|c|c|c|}
\hline $\mathrm{H}$ & -0.87332200 & 3.15909100 & 1.13306700 & $\mathrm{C}$ & 6.14321800 & -2.51698800 & 1.44283700 \\
\hline $\mathrm{H}$ & -1.47201200 & 4.55895800 & 0.21002100 & $\mathrm{C}$ & 5.62861500 & -2.74167500 & 0.19114000 \\
\hline $\mathrm{H}$ & -1.71493600 & 4.47874900 & 1.95548900 & $\mathrm{H}$ & 1.95587200 & 0.17499800 & 3.26409100 \\
\hline $\mathrm{C}$ & -0.73498900 & 2.74391900 & -2.07526100 & $\mathrm{H}$ & 5.85022800 & -1.34049200 & 4.23913000 \\
\hline $\mathrm{H}$ & -0.37277200 & 3.41462200 & -1.29215800 & $\mathrm{H}$ & 7.11514300 & -2.84743400 & 1.78642800 \\
\hline $\mathrm{H}$ & -0.30558500 & 1.75340400 & -1.91613000 & $\mathrm{H}$ & 6.06642900 & -3.27153500 & -0.64185000 \\
\hline $\mathrm{H}$ & -0.35654400 & 3.12553300 & -3.03303100 & $\mathrm{~N}$ & 4.36626500 & -2.15109700 & 0.08576400 \\
\hline $\mathrm{C}$ & -2.65939100 & 1.71696000 & -3.29610400 & $\mathrm{P}$ & 3.01857100 & -2.15536100 & -1.00834700 \\
\hline $\mathrm{H}$ & -3.74132500 & 1.67022200 & -3.44974000 & $\mathrm{C}$ & 3.74434000 & -1.78071700 & -2.73630200 \\
\hline $\mathrm{H}$ & -2.20606200 & 2.09242300 & -4.22299800 & $\mathrm{C}$ & 2.33500100 & -3.94633900 & -0.90015600 \\
\hline $\mathrm{C}$ & -2.83516900 & 4.07456700 & -2.46635000 & $\mathrm{C}$ & 4.48747400 & -2.97057400 & -3.37926500 \\
\hline $\mathrm{H}$ & -2.45534100 & 4.38449100 & -3.44896900 & $\mathrm{H}$ & 5.32433900 & -3.32691800 & -2.77063000 \\
\hline $\mathrm{H}$ & -3.92754200 & 4.08088800 & -2.52726400 & $\mathrm{H}$ & 4.90460500 & -2.63685400 & -4.33804000 \\
\hline $\mathrm{H}$ & -2.51642900 & 4.83295300 & -1.74559300 & $\mathrm{H}$ & 3.82769300 & -3.81503700 & -3.59030800 \\
\hline $\mathrm{F}$ & -5.60839600 & -3.44872000 & -2.96092400 & $\mathrm{C}$ & 4.74689300 & -0.61485300 & -2.59493500 \\
\hline $\mathrm{Rh}$ & -2.27176700 & -0.38852100 & 0.11692600 & $\mathrm{H}$ & 5.13616400 & -0.37122400 & -3.59153200 \\
\hline $\mathrm{Cl}$ & -0.17111500 & 0.36936500 & 1.52394000 & $\mathrm{H}$ & 5.59498600 & -0.87597000 & -1.95566700 \\
\hline $\mathrm{H}$ & -2.28324200 & 0.70463800 & -3.12800100 & $\mathrm{H}$ & 4.28394400 & 0.28707800 & -2.19301600 \\
\hline $\mathrm{C}$ & -4.07517000 & -0.51364500 & 3.87341400 & $\mathrm{C}$ & 2.58774900 & -1.35073900 & -3.66595300 \\
\hline $\mathrm{O}$ & -5.14176900 & -0.34903400 & 3.31805300 & $\mathrm{H}$ & 2.03146800 & -0.49857100 & -3.26938900 \\
\hline $\mathrm{O}$ & -3.81256500 & -0.09676300 & 5.12767800 & $\mathrm{H}$ & 1.87095000 & -2.15681800 & -3.84192100 \\
\hline $\mathrm{C}$ & -4.89614400 & 0.56576800 & 5.80231100 & $\mathrm{H}$ & 3.00890600 & -1.06201800 & -4.63750300 \\
\hline $\mathrm{H}$ & -5.76244100 & -0.09670200 & 5.87855700 & $\mathrm{C}$ & 1.31630000 & -4.20986900 & -2.02750700 \\
\hline $\mathrm{H}$ & -4.51371800 & 0.81467200 & 6.79229900 & $\mathrm{H}$ & 1.79347900 & -4.29659100 & -3.00827100 \\
\hline $\mathrm{H}$ & -5.18603500 & 1.47140700 & 5.26320300 & $\mathrm{H}$ & 0.54848600 & -3.43217100 & -2.08077300 \\
\hline $\mathrm{C}$ & -3.07880500 & -1.68061000 & 1.84517100 & $\mathrm{H}$ & 0.81392600 & -5.16560400 & -1.82905500 \\
\hline $\mathrm{H}$ & -4.08677600 & -2.03778100 & 1.63500300 & $\mathrm{C}$ & 1.60526900 & -4.01784800 & 0.46123500 \\
\hline $\mathrm{C}$ & -2.21647700 & -3.64928900 & 0.43512200 & $\mathrm{H}$ & 2.27661600 & -3.82151000 & 1.30250100 \\
\hline $\mathrm{H}$ & -3.26745800 & -3.84833400 & 0.21504900 & $\mathrm{H}$ & 1.18770200 & -5.02524300 & 0.58871400 \\
\hline $\mathrm{H}$ & -1.64505500 & -3.70320100 & -0.49711900 & $\mathrm{C}$ & 3.42707600 & -5.03481100 & -0.90380000 \\
\hline $\mathrm{H}$ & -1.85821000 & -4.46151200 & 1.08485600 & $\mathrm{H}$ & 2.94078000 & -6.01014600 & -0.77141300 \\
\hline $\mathrm{C}$ & -2.87464500 & -1.22783600 & 3.28311200 & $\mathrm{H}$ & 4.13490600 & -4.90537800 & -0.08024900 \\
\hline $\mathrm{H}$ & -2.69428400 & -2.12054500 & 3.89925000 & $\mathrm{H}$ & 3.98838300 & -5.07910700 & -1.83945000 \\
\hline $\mathrm{H}$ & -1.97828000 & -0.60898500 & 3.38777500 & $\mathrm{~F}$ & 3.76036700 & -0.05212900 & 5.05239300 \\
\hline $\mathrm{C}$ & -2.02082500 & -2.32848600 & 1.14532600 & $\mathrm{Rh}$ & 1.52910200 & -0.77056300 & 0.11577300 \\
\hline $\mathrm{H}$ & -1.03671300 & -2.21770000 & 1.59877800 & $\mathrm{H}$ & 0.76858700 & -3.31153200 & 0.51371400 \\
\hline $\mathrm{H}$ & -3.38502100 & -0.28055700 & 1.27217700 & $\mathrm{C}$ & 5.34870100 & 5.03729800 & -0.71531100 \\
\hline $\mathrm{Cl}$ & -0.44150700 & -0.92187200 & -1.55640200 & $\mathrm{O}$ & 4.84736300 & 5.46372900 & -1.73372200 \\
\hline $\mathrm{C}$ & 5.16811500 & -1.76399800 & 2.19181700 & $\mathrm{O}$ & 6.33963800 & 5.67799100 & -0.05133300 \\
\hline $\mathrm{C}$ & 4.08387800 & -1.55458100 & 1.31694200 & $\mathrm{C}$ & 6.77379900 & 6.91401500 & -0.63797200 \\
\hline $\mathrm{C}$ & 2.89488900 & -0.87076500 & 1.60516100 & $\mathrm{H}$ & 7.15997500 & 6.74816200 & -1.64765400 \\
\hline $\mathrm{C}$ & 2.82112200 & -0.37450300 & 2.90938800 & $\mathrm{H}$ & 7.56196700 & 7.28861000 & 0.01620600 \\
\hline $\mathrm{C}$ & 3.89461000 & -0.56810300 & 3.80299400 & $\mathrm{H}$ & 5.94701900 & 7.62790200 & -0.69005100 \\
\hline $\mathrm{C}$ & 5.06750300 & -1.23700600 & 3.49512100 & $\mathrm{C}$ & 3.78498200 & 3.03297000 & -0.65834500 \\
\hline
\end{tabular}




\begin{tabular}{|c|c|c|c|c|c|c|c|}
\hline $\mathrm{H}$ & 2.91152900 & 3.69642100 & -0.62411800 & $\mathrm{H}$ & -1.96068000 & 1.99452400 & 3.35479100 \\
\hline $\mathrm{C}$ & 2.22375500 & 1.04997000 & -0.57813500 & $\mathrm{H}$ & -2.82569200 & 3.54781300 & 3.30348200 \\
\hline $\mathrm{H}$ & 2.32863900 & 0.92718000 & -1.66112800 & $\mathrm{H}$ & -3.15513900 & 2.39576700 & 4.59591900 \\
\hline $\mathrm{H}$ & 1.34425300 & 1.66958600 & -0.39009400 & $\mathrm{C}$ & -1.40036000 & 3.56002600 & 0.75522200 \\
\hline $\mathrm{H}$ & 4.32901000 & 1.03246900 & -0.02993700 & $\mathrm{H}$ & -1.38257900 & 3.82002000 & 1.81486000 \\
\hline $\mathrm{C}$ & 4.98027200 & 3.74240900 & -0.01709300 & $\mathrm{H}$ & -0.74116800 & 2.70123600 & 0.61386600 \\
\hline $\mathrm{H}$ & 5.86960800 & 3.09661300 & -0.02156400 & $\mathrm{H}$ & -0.97713400 & 4.40858600 & 0.20242100 \\
\hline $\mathrm{H}$ & 4.78957800 & 3.96604600 & 48300 & $\mathrm{C}$ & -2.74027200 & 3.14950400 & -1.31578400 \\
\hline $\mathrm{C}$ & 3.45854200 & 1.69625300 & 0.03632400 & $\mathrm{H}$ & -3.71939500 & 2.99083100 & -1.77538600 \\
\hline $\mathrm{H}$ & 3.28894800 & 1.87132100 & 1.10423500 & $\mathrm{H}$ & -2.32044800 & 4.07702500 & -1.72503000 \\
\hline \multirow[t]{2}{*}{$\mathrm{H}$} & 3.99410300 & 2.86864100 & -1.72310000 & $\mathrm{C}$ & -3.71966100 & 4.51019700 & 0.53919400 \\
\hline & & & & $\mathrm{H}$ & -3.30962900 & 5.38659900 & 0.02081800 \\
\hline \multirow[t]{7}{*}{33} & & & & $\mathrm{H}$ & -4.74600600 & 4.37457900 & 0.18432100 \\
\hline & & & & $\mathrm{H}$ & -3.74028100 & 4.75016900 & 1.60581400 \\
\hline & & & & $\mathrm{F}$ & -4.67533900 & -1.58319800 & -4.57824400 \\
\hline & & & & $\mathrm{Rh}$ & -2.17211100 & -0.03426100 & 0.01127300 \\
\hline & & & & $\mathrm{Cl}$ & -0.20027400 & 0.15873300 & 1.66244500 \\
\hline & & & & $\mathrm{H}$ & -2.08588500 & 2.32725300 & -1.62392900 \\
\hline & & & & $\mathrm{C}$ & -2.94020800 & -1.72013700 & 1.01459900 \\
\hline $\mathrm{C}$ & 4900 & 0.67619300 & -2.02391900 & $\mathrm{H}$ & -2.71758000 & -1.45239500 & 2.05127900 \\
\hline $\mathrm{C}$ & 2100 & 0.57987400 & -1.16316300 & $\mathrm{C}$ & -2.06763600 & -2.91572900 & 0.61575700 \\
\hline $\mathrm{C}$ & -3.61319300 & -0.16488600 & -1.39160700 & $\mathrm{H}$ & -2.22056500 & -3.16808500 & -0.43857700 \\
\hline $\mathrm{C}$ & -3.61434400 & -0.89335100 & -2.58338300 & $\mathrm{H}$ & -1.01204600 & -2.66463300 & 0.72940600 \\
\hline $\mathrm{C}$ & -4.73111500 & -0.84119200 & -3.44231300 & $\mathrm{Cl}$ & -0.38679700 & -0.93913000 & -1.49003900 \\
\hline $\mathrm{C}$ & -5.86753000 & -0.08465900 & -3.21013000 & $\mathrm{C}$ & -4.42904900 & -2.01428700 & 0.84422500 \\
\hline $\mathrm{C}$ & -6.78710300 & 1.61861900 & -1.40786400 & $\mathrm{H}$ & -4.76083100 & -2.72481800 & 1.61667200 \\
\hline $\mathrm{C}$ & -6.20983500 & 2.05097700 & -0.24245000 & $\mathrm{H}$ & -5.05597600 & -1.12668200 & 0.93721300 \\
\hline $\mathrm{H}$ & -2.76848400 & -1.50426100 & -2.88075400 & $\mathrm{H}$ & -4.63834300 & -2.45692300 & -0.13457200 \\
\hline $\mathrm{H}$ & -6.68086900 & -0.08443300 & -3.92803600 & $\mathrm{C}$ & -2.36236900 & -4.15562800 & 1.48133400 \\
\hline $\mathrm{H}$ & -7.75394800 & 1.93461400 & -1.77861400 & $\mathrm{H}$ & -2.24786600 & -3.92672900 & 2.55005500 \\
\hline $\mathrm{H}$ & -6.58927300 & 2.75622600 & 0.47917300 & $\mathrm{H}$ & -3.39521600 & -4.50256200 & 1.36209500 \\
\hline $\mathrm{N}$ & -4.98260000 & 1.40207900 & -0.04686900 & $\mathrm{C}$ & -1.45658100 & -5.33045100 & 1.16608500 \\
\hline $\mathrm{P}$ & -3.53291300 & 1.62567900 & 0.89439000 & $\mathrm{O}$ & -0.56318200 & -5.35168800 & 0.34448700 \\
\hline $\mathrm{C}$ & -4.07090900 & 1.81942700 & 2.73051500 & $\mathrm{O}$ & -1.77486400 & -6.39585300 & 1.93700900 \\
\hline $\mathrm{C}$ & -2.82426300 & 3.29674400 & 0.22224000 & $\mathrm{C}$ & -0.98072000 & -7.57299400 & 1.72739400 \\
\hline $\mathrm{C}$ & -5.37169900 & 2.63212000 & 2.91010500 & $\mathrm{H}$ & 0.06940100 & -7.37680900 & 1.96229800 \\
\hline $\mathrm{H}$ & -6.23753600 & 2.08945800 & 2.52131500 & $\mathrm{H}$ & -1.38761300 & -8.32534700 & 2.40389800 \\
\hline $\mathrm{H}$ & -5.53673000 & 2.77749700 & 3.98559100 & $\mathrm{H}$ & -1.05488000 & -7.90939700 & 0.68963600 \\
\hline $\mathrm{H}$ & -5.34048500 & 3.62127800 & 2.44945500 & $\mathrm{C}$ & 5.51857700 & -0.71983500 & 1.89225600 \\
\hline $\mathrm{C}$ & -4.31888100 & 0.42492700 & 3.33974000 & $\mathrm{C}$ & 4.35323100 & -0.75359000 & 1.10107000 \\
\hline $\mathrm{H}$ & -4.68221900 & 0.56014800 & 4.36653900 & $\mathrm{C}$ & 3.07837900 & -0.30318300 & 1.46851200 \\
\hline $\mathrm{H}$ & -5.07737600 & -0.14111400 & 2.79415400 & $\mathrm{C}$ & 3.00418100 & 0.21707300 & 2.76298000 \\
\hline $\mathrm{H}$ & -3.40343700 & -0.16734400 & 3.39054200 & $\mathrm{C}$ & 4.15825300 & 0.27087000 & 3.57108100 \\
\hline $\mathrm{C}$ & -2.92582800 & 2.48274100 & 3.52640400 & $\mathrm{C}$ & 5.41319500 & -0.17099300 & 3.18608400 \\
\hline
\end{tabular}




\begin{tabular}{|c|c|c|c|c|c|c|c|}
\hline $\mathrm{C}$ & 6.56066600 & -1.30735500 & 1.08796400 & $\mathrm{H}$ & 1.70814100 & 4.11290700 & -0.67537200 \\
\hline $\mathrm{C}$ & 6.00697200 & -1.67527600 & -0.11244200 & $\mathrm{C}$ & 1.81699500 & 1.36153900 & -0.70985400 \\
\hline $\mathrm{H}$ & 2.07429200 & 0.59306400 & 3.17630100 & $\mathrm{H}$ & 1.96223400 & 1.22523400 & -1.78531100 \\
\hline $\mathrm{H}$ & 6.25313100 & -0.09455800 & 3.86849000 & $\mathrm{H}$ & 0.80223900 & 1.74206400 & -0.56159000 \\
\hline $\mathrm{H}$ & 7.59996800 & -1.43741200 & 1.36156900 & $\mathrm{H}$ & 3.85580000 & 1.95001800 & -0.24308200 \\
\hline $\mathrm{H}$ & 6.47568000 & -2.14904100 & -0.96233100 & $\mathrm{C}$ & 3.71992100 & 4.72757900 & -0.22319200 \\
\hline $\mathrm{N}$ & 4.65194900 & -1.33493700 & -0.13261200 & $\mathrm{H}$ & 4.75751200 & 4.42854300 & -0.41041500 \\
\hline $\mathrm{P}$ & 3.25295400 & -1.61745700 & -1.12297900 & $\mathrm{H}$ & 3.61772800 & 4.77957000 & 0.87098500 \\
\hline $\mathrm{C}$ & 3.78176500 & -1.16506600 & -2.90041500 & $\mathrm{C}$ & 2.83437000 & 2.33372800 & -0.12662000 \\
\hline $\mathrm{C}$ & 2.88629400 & -3.48523400 & -0.91809700 & $\mathrm{H}$ & 2.67094200 & 2.44375200 & 0.95098900 \\
\hline $\mathrm{C}$ & 4.68221700 & -2.22453100 & -3.56854200 & $\mathrm{H}$ & 8300 & 3.62513900 & -1.88135500 \\
\hline $\mathrm{H}$ & 5.59477400 & -2.42482900 & -2.99907100 & & & & \\
\hline $\mathrm{H}$ & 4.98952300 & -1.84558200 & -4.55177800 & 34-ts & & & \\
\hline $\mathrm{H}$ & 4.16146700 & -3.17084400 & -3.73350300 & & & & \\
\hline $\mathrm{C}$ & 4.56186700 & 0.16640500 & -2.83945400 & & & & \\
\hline $\mathrm{H}$ & 4.79685200 & 0.47790000 & -3.86517900 & & & & \\
\hline $\mathrm{H}$ & 5.50321200 & 0.06696700 & -2.29186400 & & & & \\
\hline $\mathrm{H}$ & 3.98388800 & 0.96640600 & -2.37326900 & & & & \\
\hline $\mathrm{C}$ & 2.51152500 & -0.97667100 & -3.75958800 & $\mathrm{C}$ & 5.91183300 & -2.04891200 & -1.04978600 \\
\hline $\mathrm{H}$ & 1.84350900 & -0.21255800 & -3.35607900 & $\mathrm{C}$ & 4.67180500 & -1.82254700 & -0.41551200 \\
\hline $\mathrm{H}$ & 1.93250400 & -1.89851800 & -3.85751500 & $\mathrm{C}$ & 3.99578800 & -0.595002 & -0.35409700 \\
\hline $\mathrm{H}$ & 2.81492300 & -0.66622300 & -4.76779300 & $\mathrm{C}$ & 4.63157600 & 0.44840700 & -1.02537600 \\
\hline $\mathrm{C}$ & 1.84112000 & -3.94942400 & -1.95306600 & $\mathrm{C}$ & 5.88304400 & 0.24582700 & -1.64467100 \\
\hline $\mathrm{H}$ & 2.25871800 & -4.00378100 & -2.96371700 & $\mathrm{C}$ & 6.55932900 & -0.95854500 & -1.66411400 \\
\hline $\mathrm{H}$ & 0.95906500 & -3.30323500 & -1.97017700 & $\mathrm{C}$ & 6.15829900 & -3.46372000 & -0.94972000 \\
\hline $\mathrm{H}$ & 1.49846200 & -4.95347600 & -1.67790900 & $\mathrm{C}$ & 5.08052200 & -4.03021800 & -0.32113800 \\
\hline $\mathrm{C}$ & 2.28527300 & -3.61463700 & 0.50179300 & $\mathrm{H}$ & 4.18495700 & 1.42898900 & -1.12260700 \\
\hline $\mathrm{H}$ & 2.99006700 & -3.30007600 & 1.27718600 & $\mathrm{H}$ & 7.51792200 & -1.04437700 & -2.16461900 \\
\hline $\mathrm{H}$ & 2.00917700 & -4.66013600 & 0.67886600 & $\mathrm{H}$ & 7.03031700 & -3.99869400 & -1.30375600 \\
\hline $\mathrm{C}$ & 4.13843200 & -4.38142800 & -0.98523300 & $\mathrm{H}$ & 4.90628400 & -5.06690700 & -0.08008700 \\
\hline $\mathrm{H}$ & 3.83132300 & -5.41374900 & -0.77346200 & $\mathrm{~N}$ & 4.15996400 & -3.04243700 & 0.04713200 \\
\hline $\mathrm{H}$ & 4.88410000 & -4.09878400 & -0.23632200 & $P$ & 2.42551000 & -3.00359400 & 0.29819800 \\
\hline $\mathrm{H}$ & 4.61473200 & -4.38036700 & -1.96829000 & $\mathrm{C}$ & 2.12585500 & -4.10074500 & 1.84220900 \\
\hline $\mathrm{F}$ & 4.01884800 & 0.79937500 & 4.81524100 & $\mathrm{C}$ & 1.71895500 & -3.82044800 & -1.29111600 \\
\hline $\mathrm{Rh}$ & 1.61231400 & -0.52937100 & 0.09353100 & $\mathrm{C}$ & 2.96202300 & -5.39819300 & 1.86477500 \\
\hline $\mathrm{H}$ & 1.35945300 & -3.03445700 & 0.61314700 & $\mathrm{H}$ & 4.03110300 & -5.18388500 & 1.94911800 \\
\hline $\mathrm{C}$ & 3.51020000 & 6.13217200 & -0.75540500 & $\mathrm{H}$ & 2.67595800 & -5.97812500 & 2.75190700 \\
\hline $\mathrm{O}$ & 2.48861700 & 6.55585400 & -1.25348100 & $\mathrm{H}$ & 2.79842400 & -6.03578900 & 0.99414800 \\
\hline $\mathrm{O}$ & 4.61882900 & 6.88921500 & -0.58117700 & $\mathrm{C}$ & 2.51892400 & -3.27967600 & 3.08945700 \\
\hline $\mathrm{C}$ & 4.50263600 & 8.25598100 & -1.00470400 & $\mathrm{H}$ & 2.41363200 & -3.91846800 & 3.97607400 \\
\hline $\mathrm{H}$ & 4.27971100 & 8.31079500 & -2.07384100 & $\mathrm{H}$ & 3.56158700 & -2.94517400 & 3.04514000 \\
\hline $\mathrm{H}$ & 5.46978200 & 8.71260600 & -0.79120800 & $\mathrm{H}$ & 1.87002900 & -2.41068800 & 3.22189600 \\
\hline $\mathrm{H}$ & 3.70779100 & 8.76537700 & -0.45256600 & $\mathrm{C}$ & 0.62404000 & -4.44511200 & 1.94236700 \\
\hline $\mathrm{C}$ & 2.72253300 & 3.71654500 & -0.79812900 & $\mathrm{H}$ & -0.00588200 & -3.55221600 & 1.88341700 \\
\hline
\end{tabular}




\begin{tabular}{|c|c|c|c|c|c|c|c|}
\hline $\mathrm{H}$ & 0.30651400 & -5.15456600 & 1.17280700 & $\mathrm{C}$ & -4.75016100 & 3.59474400 & -0.45490600 \\
\hline $\mathrm{H}$ & 0.43822000 & -4.91763500 & 2.91544500 & $\mathrm{H}$ & -2.27179700 & 0.22981400 & 3.28281100 \\
\hline $\mathrm{C}$ & 0.21002500 & -3.51793400 & -1.37520900 & $\mathrm{H}$ & -5.74530000 & 2.72800000 & 3.62654900 \\
\hline $\mathrm{H}$ & -0.36298900 & -3.98008000 & -0.56737400 & $\mathrm{H}$ & -6.32287600 & 4.22566900 & 0.92185400 \\
\hline $\mathrm{H}$ & 0.02556200 & -2.44239500 & -1.36601700 & $\mathrm{H}$ & -4.94723600 & 4.12431400 & -1.37521400 \\
\hline $\mathrm{H}$ & -0.17590500 & -3.91892500 & -2.32181400 & $\mathrm{~N}$ & -3.67627900 & 2.70662400 & -0.35197000 \\
\hline $\mathrm{C}$ & 2.41920300 & -3.12190500 & -2.47899600 & $\mathrm{P}$ & -2.25639900 & 2.27993700 & -1.25620000 \\
\hline $\mathrm{H}$ & 3.49019600 & -3.34160500 & -2.51461500 & $\mathrm{C}$ & -2.85838200 & 1.91232300 & -3.03083100 \\
\hline $\mathrm{H}$ & 1.96971100 & -3.49398000 & -3.40886100 & $\mathrm{C}$ & -1.15355100 & 3.84724400 & -1.18450100 \\
\hline $\mathrm{C}$ & 1.94883700 & -5.33879300 & -1.40676300 & $\mathrm{C}$ & -3.19629200 & 3.17460800 & -3.85172900 \\
\hline $\mathrm{H}$ & 1.58225900 & -5.67052700 & -2.38710800 & $\mathrm{H}$ & -3.96953900 & 3.79018700 & -3.38194700 \\
\hline $\mathrm{H}$ & 3.00858200 & -5.60597100 & -1.35252200 & $\mathrm{H}$ & -3.58457000 & 2.85641700 & -4.82784200 \\
\hline $\mathrm{H}$ & 1.40130700 & -5.90795500 & -0.65016100 & $\mathrm{H}$ & -2.32106700 & 3.80028900 & -4.03937900 \\
\hline $\mathrm{F}$ & 6.44678500 & 1.31853200 & -2.25884200 & $\mathrm{C}$ & -4.13297700 & 1.04546300 & -2.94025400 \\
\hline $\mathrm{Rh}$ & 2.23262500 & -0.60082100 & 0.66027600 & $\mathrm{H}$ & -4.44904800 & 0.78763900 & -3.95914200 \\
\hline $\mathrm{Cl}$ & -0.09654700 & -0.68528500 & 1.85276000 & $\mathrm{H}$ & -4.95716000 & 1.57543200 & -2.45447700 \\
\hline $\mathrm{H}$ & 2.28217000 & -2.03781900 & -2.45361600 & $\mathrm{H}$ & -3.96436300 & 0.11272400 & -2.40064200 \\
\hline $\mathrm{C}$ & 3.27779600 & 5.10454600 & 0.94581400 & $\mathrm{C}$ & -1.75179600 & 1.12048200 & -3.76218800 \\
\hline $\mathrm{O}$ & 2.53696800 & 5.67739300 & 0.16968400 & $\mathrm{H}$ & -1.47789900 & 0.20500300 & -3.23308600 \\
\hline $\mathrm{O}$ & 4.56346400 & 5.46125400 & 1.14167700 & $\mathrm{H}$ & -0.83917600 & 1.70651800 & -3.89602100 \\
\hline $\mathrm{C}$ & 5.02729700 & 6.57069300 & 0.35336200 & $\mathrm{H}$ & -2.11931300 & 0.84317500 & -4.75860500 \\
\hline $\mathrm{H}$ & 4.44672900 & 7.46936000 & 0.57830800 & $\mathrm{C}$ & 0.02485900 & 3.73674300 & -2.17401300 \\
\hline $\mathrm{H}$ & 6.07250100 & 6.70898600 & 0.63062500 & $\mathrm{H}$ & -0.29399000 & 3.85306100 & -3.21458700 \\
\hline $\mathrm{H}$ & 4.93813900 & 6.34667400 & -0.71288500 & $\mathrm{H}$ & 0.55723400 & 2.78591100 & -2.07673400 \\
\hline $\mathrm{C}$ & 3.01747200 & 2.59874900 & 0.89704100 & $\mathrm{H}$ & 0.73778200 & 4.54111900 & -1.95438900 \\
\hline $\mathrm{H}$ & 4.07460900 & 2.40557500 & 0.69853200 & $\mathrm{C}$ & -0.59255100 & 3.87078000 & 0.25595600 \\
\hline $\mathrm{C}$ & 3.09759000 & 0.52916900 & 2.42699900 & $\mathrm{H}$ & -1.38360100 & 3.96316000 & 1.00633300 \\
\hline $\mathrm{H}$ & 4.18397600 & 0.61176600 & 2.45446000 & $\mathrm{H}$ & 0.09331400 & 4.72028200 & 0.35574300 \\
\hline $\mathrm{H}$ & 2.64151100 & 0.19714200 & 3.35862000 & $\mathrm{C}$ & -1.91796700 & 5.16635500 & -1.41343200 \\
\hline $\mathrm{H}$ & 3.13636400 & -0.92722500 & 1.93747900 & $\mathrm{H}$ & -1.21738300 & 5.99659200 & -1.25751900 \\
\hline $\mathrm{C}$ & 2.90072600 & 3.88570100 & 1.76048800 & $\mathrm{H}$ & -2.74058700 & 5.29131600 & -0.70331500 \\
\hline $\mathrm{H}$ & 1.86614000 & 4.01902700 & 2.09107900 & $\mathrm{H}$ & -2.31502200 & 5.26366900 & -2.42663500 \\
\hline $\mathrm{H}$ & 3.54159000 & 3.80564500 & 2.64340700 & $\mathrm{~F}$ & -4.14209700 & 1.07365100 & 4.80180400 \\
\hline $\mathrm{C}$ & 2.37419300 & 1.42354800 & 1.59434600 & $\mathrm{Rh}$ & -1.30046000 & 0.69669200 & 0.15394800 \\
\hline $\mathrm{H}$ & 1.34057500 & 1.59676100 & 1.88694400 & $\mathrm{H}$ & -0.01395000 & 2.96424400 & 0.47601000 \\
\hline $\mathrm{H}$ & 2.52354900 & 2.78004000 & -0.06412800 & $\mathrm{C}$ & -6.28771800 & -4.15152900 & -0.65953900 \\
\hline $\mathrm{Cl}$ & 0.80720300 & 0.18293300 & -1.26278000 & $\mathrm{O}$ & -5.71461700 & -4.88706800 & -1.43474200 \\
\hline $\mathrm{C}$ & -4.76877300 & 2.75095300 & 1.65635000 & $\mathrm{O}$ & -7.54075900 & -4.38624200 & -0.20286300 \\
\hline $\mathrm{C}$ & -3.68678200 & 2.19364700 & 0.94693000 & $\mathrm{C}$ & -8.16090400 & -5.58303300 & -0.69587400 \\
\hline $\mathrm{C}$ & -2.74472300 & 1.28071200 & 1.43966400 & $\mathrm{H}$ & -8.24815600 & -5.55230600 & -1.78559000 \\
\hline $\mathrm{C}$ & -2.93271400 & 0.92625900 & 2.77795700 & $\mathrm{H}$ & -9.14834100 & -5.61387500 & -0.23377600 \\
\hline $\mathrm{C}$ & -4.01135400 & 1.46695700 & 3.50799500 & $\mathrm{H}$ & -7.57813800 & -6.46453300 & -0.41408500 \\
\hline $\mathrm{C}$ & -4.94059800 & 2.35907000 & 2.99940200 & $\mathrm{C}$ & -4.32905600 & -2.53264800 & -0.58912700 \\
\hline $\mathrm{C}$ & -5.43591400 & 3.63452900 & 0.73280800 & $\mathrm{H}$ & -3.65393400 & -3.36946100 & -0.37177900 \\
\hline
\end{tabular}




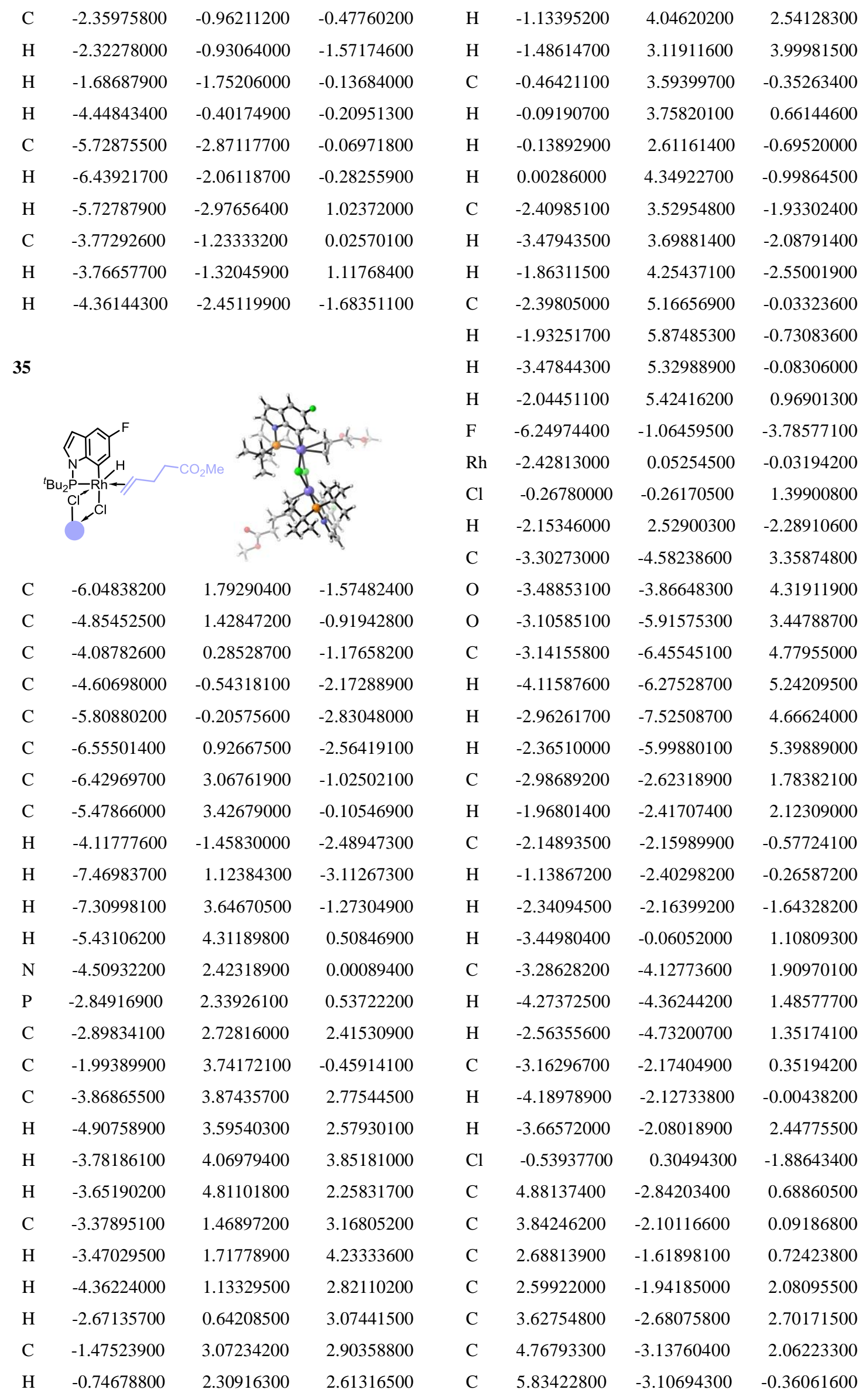




\begin{tabular}{|c|c|c|c|c|c|c|c|}
\hline C & 5.34981800 & -2.55097300 & -1.51740100 & $\mathrm{C}$ & 2.27642100 & 1.21647200 & 0.01279100 \\
\hline $\mathrm{H}$ & 1.75782700 & -1.62716100 & 2.68920100 & $\mathrm{H}$ & 2.36706600 & 1.72944700 & -0.95085200 \\
\hline $\mathrm{H}$ & 5.51707800 & -3.69699800 & 2.61251300 & $\mathrm{H}$ & 1.46627400 & 1.68625900 & 0.57444800 \\
\hline $\mathrm{H}$ & 6.77263300 & -3.63889500 & -0.26911200 & $\mathrm{H}$ & 4.37478900 & 0.72614500 & 0.32998700 \\
\hline $\mathrm{H}$ & 5.78459200 & -2.54639600 & -2.50606600 & $\mathrm{C}$ & 5.28262400 & 2.87647400 & 1.84796600 \\
\hline $\mathrm{N}$ & 4.13060400 & -1.91625200 & -1.26369500 & $\mathrm{H}$ & 6.11717900 & 2.31867400 & 1.40282000 \\
\hline$P$ & 2.81008600 & -1.23847800 & -2.16634600 & $\mathrm{H}$ & 5.13129600 & 2.42936100 & 2.84011500 \\
\hline $\mathrm{C}$ & 3.59432600 & -0.01513000 & -3.41227400 & $\mathrm{C}$ & 3.57052000 & 1.2927 & 0.81402400 \\
\hline $\mathrm{C}$ & 2.03252100 & -2.74946600 & -3.06570800 & $\mathrm{H}$ & 101600 & 0.83394600 & 1.79773100 \\
\hline $\mathrm{C}$ & 4.26767500 & -0.69039200 & -4.62524400 & $\mathrm{H}$ & 04700 & 3.22549200 & 0.01933600 \\
\hline $\mathrm{H}$ & 5.06111800 & -1.38647700 & -4.33605800 & & & & \\
\hline $\mathrm{H}$ & 4.73179600 & 0.09237100 & -5.23861800 & 36-ts & & & \\
\hline $\mathrm{H}$ & 3.55651300 & -1.22041300 & -5.26267300 & & & & \\
\hline $\mathrm{C}$ & 4.67015400 & 0.80718700 & -2.67039500 & & & & \\
\hline $\mathrm{H}$ & 5.09680500 & 1.53273500 & -3.37471100 & & & & \\
\hline $\mathrm{H}$ & 5.48505600 & 0.18127400 & -2.29539900 & & & & \\
\hline $\mathrm{H}$ & 4.25805900 & 1.36696600 & -1.83040600 & & & & \\
\hline $\mathrm{C}$ & 2.48714900 & 0.93658300 & -3.91825900 & & & & \\
\hline $\mathrm{H}$ & 1.97484200 & 1.45006300 & -3.10153900 & $\mathrm{C}$ & -5.92581700 & 1.642 & -1.77739700 \\
\hline $\mathrm{H}$ & 1.72513200 & 0.41793000 & -4.50533400 & $\mathrm{C}$ & 7800 & 1.32 & -1.06231200 \\
\hline $\mathrm{H}$ & 2.94748200 & 1.69465200 & -4.56520300 & $\mathrm{C}$ & -3.89073100 & 0.25364300 & -1.34064400 \\
\hline $\mathrm{C}$ & 1.01687800 & -2.28406800 & -4.12877700 & $\mathrm{C}$ & -4.27500400 & -0.53758800 & -2.42317200 \\
\hline $\mathrm{H}$ & 1.49984200 & -1.82872900 & -4.99853100 & $\mathrm{C}$ & -5.45607500 & -0.24530000 & -3.13787200 \\
\hline $\mathrm{H}$ & 0.29086400 & -1.57436000 & -3.72075000 & $\mathrm{C}$ & -6.30191700 & 0.80860100 & -2.84977400 \\
\hline $\mathrm{H}$ & 0.46303400 & -3.16020200 & -4.49089700 & $\mathrm{C}$ & -6.43555700 & 2.85255700 & -1.18858800 \\
\hline $\mathrm{C}$ & 1.27823600 & -3.53147400 & -1.96815900 & $\mathrm{C}$ & -5.57236400 & 3.22486500 & -0.19072700 \\
\hline $\mathrm{H}$ & 1.93911900 & -3.85478300 & -1.15880100 & $\mathrm{H}$ & -3.68620500 & -1.38085600 & -2.76801400 \\
\hline $\mathrm{H}$ & 0.82322700 & -4.42545900 & -2.41450300 & $\mathrm{H}$ & -7.19282100 & 0.97683100 & -3.44543100 \\
\hline $\mathrm{C}$ & 3.06866400 & -3.70246800 & -3.69333600 & $\mathrm{H}$ & -7.33709700 & 3.38266300 & -1.46792500 \\
\hline $\mathrm{H}$ & 2.53144300 & -4.55620700 & -4.12662600 & $\mathrm{H}$ & -5.62521000 & 4.07921700 & 0.46570200 \\
\hline $\mathrm{H}$ & 3.76410500 & -4.09525000 & -2.94647400 & $\mathrm{~N}$ & -4.54036400 & 2.28951100 & -0.07042600 \\
\hline $\mathrm{H}$ & 3.64573600 & -3.24178800 & -4.49693800 & $\mathrm{P}$ & -2.91432800 & 2.30202300 & 0.57646100 \\
\hline $\mathrm{F}$ & 3.48240200 & -2.95319000 & 4.02438600 & $\mathrm{C}$ & -3.12819900 & 2.58522800 & 2.45934300 \\
\hline $\mathrm{Rh}$ & 1.38618800 & -0.59860300 & -0.43717500 & $\mathrm{C}$ & -2.10905700 & 3.82588100 & -0.27162800 \\
\hline $\mathrm{H}$ & 0.46860400 & -2.93465700 & -1.53688300 & $\mathrm{C}$ & -4.18175600 & 3.65086200 & 2.82874100 \\
\hline $\mathrm{C}$ & 5.73029400 & 4.31114700 & 2.05034800 & $\mathrm{H}$ & -5.18756700 & 3.33821700 & 2.53410900 \\
\hline $\mathrm{O}$ & 5.14648100 & 5.29999100 & 1.66078600 & $\mathrm{H}$ & -4.18526800 & 3.76970200 & 3.92005800 \\
\hline $\mathrm{O}$ & 6.89265300 & 4.36028600 & 2.74313800 & $\mathrm{H}$ & -3.97886000 & 4.63261800 & 2.39654500 \\
\hline C & 7.39832100 & 5.67811100 & 3.00358800 & $\mathrm{C}$ & -3.58349200 & 1.24993600 & 3.08478900 \\
\hline $\mathrm{H}$ & 7.59513500 & 6.20840500 & 2.06757900 & $\mathrm{H}$ & -3.78097300 & 1.41019800 & 4.15262500 \\
\hline $\mathrm{H}$ & 8.32343600 & 5.53243800 & 3.56254900 & $\mathrm{H}$ & -4.50848600 & 0.87976000 & 2.62850500 \\
\hline $\mathrm{H}$ & 6.68173600 & 6.25739100 & 3.59260200 & $\mathrm{H}$ & -2.81272800 & 0.48065600 & 2.99876900 \\
\hline $\mathrm{C}$ & 4.01626000 & 2.75715300 & 0.99634100 & $\mathrm{C}$ & -1.76014700 & 2.96203700 & 3.06755600 \\
\hline $\mathrm{H}$ & 3.21302500 & 3.33885900 & 1.46488100 & $\mathrm{H}$ & -0.97579100 & 2.25471800 & 2.77864500 \\
\hline
\end{tabular}




\begin{tabular}{|c|c|c|c|c|c|c|c|}
\hline $\mathrm{H}$ & -1.44434000 & 3.97219000 & 2.79073400 & $\mathrm{C}$ & 5.75111500 & -3.21916000 & -0.51303000 \\
\hline $\mathrm{H}$ & -1.84270900 & 2.93558200 & 4.16169700 & $\mathrm{C}$ & 5.25593700 & -2.62863100 & -1.64790300 \\
\hline $\mathrm{C}$ & -0.57890400 & 3.74013900 & -0.09852700 & $\mathrm{H}$ & 1.82378000 & -1.62784500 & 2.67235600 \\
\hline $\mathrm{H}$ & -0.26256200 & 3.83931600 & 0.94321700 & $\mathrm{H}$ & 5.49722000 & -3.83705100 & 2.46025900 \\
\hline $\mathrm{H}$ & -0.19023100 & 2.80087800 & -0.49498100 & $\mathrm{H}$ & 6.66947100 & -3.78951500 & -0.45618300 \\
\hline $\mathrm{H}$ & -0.11576700 & 4.56181600 & -0.66099300 & $\mathrm{H}$ & 5.66092300 & -2.62935000 & -2.64905000 \\
\hline $\mathrm{C}$ & -2.43952400 & 3.69763800 & -1.77646100 & $\mathrm{~N}$ & 4.06972300 & -1.95164600 & -1.35058300 \\
\hline $\mathrm{H}$ & -3.50928700 & 3.81570500 & -1.97382300 & $\mathrm{P}$ & 2.76233700 & -1.18983600 & -2.20302200 \\
\hline $\mathrm{H}$ & -1.90860600 & 4.49370200 & -2.31467200 & $\mathrm{C}$ & 3.57754200 & 0.03259500 & -3.42591900 \\
\hline $\mathrm{C}$ & -2.60375900 & 5.19709500 & 0.22545400 & $\mathrm{C}$ & 1.89673400 & -2.63644200 & -3.12557400 \\
\hline $\mathrm{H}$ & -2.13719200 & 5.97695600 & -0.39073800 & $\mathrm{C}$ & 4.21548400 & -0.64107600 & -4.65870500 \\
\hline $\mathrm{H}$ & -3.68714500 & 5.31354800 & 0.12469300 & $\mathrm{H}$ & 4.98561500 & -1.37042200 & -4.38955800 \\
\hline $\mathrm{H}$ & -2.32189900 & 5.39644700 & 1.26307100 & $\mathrm{H}$ & 4.70215100 & 0.13619500 & -5.26149300 \\
\hline $\mathrm{F}$ & -5.76766900 & -1.06489500 & -4.17534800 & $\mathrm{H}$ & 3.47979300 & -1.13310600 & -5.29873400 \\
\hline $\mathrm{Rh}$ & -2.30302100 & 0.03090100 & -0.09676800 & $\mathrm{C}$ & 4.68832100 & 0.79857400 & -2.67426300 \\
\hline $\mathrm{Cl}$ & -0.21385400 & -0.23615900 & 1.43948400 & $\mathrm{H}$ & 5.13153300 & 1.52827300 & -3.36378500 \\
\hline $\mathrm{H}$ & -2.10978400 & 2.74001500 & -2.18703800 & $\mathrm{H}$ & 5.48546600 & 0.13542000 & -2.32620500 \\
\hline $\mathrm{C}$ & -3.29772000 & -4.48692300 & 3.51933300 & $\mathrm{H}$ & 4.30526100 & 1.34905600 & -1.81418900 \\
\hline $\mathrm{O}$ & -3.47960400 & -3.81247600 & 4.51001400 & $\mathrm{C}$ & 2.50084800 & 1.03370100 & -3.90142600 \\
\hline $\mathrm{O}$ & -3.09431800 & -5.82134700 & 3.54972600 & $\mathrm{H}$ & 2.00556300 & 1.53906100 & -3.06926800 \\
\hline $\mathrm{C}$ & -3.11509300 & -6.41802200 & 4.85739200 & $\mathrm{H}$ & 1.72349600 & 0.55681900 & -4.50376500 \\
\hline $\mathrm{H}$ & -4.08572700 & -6.26220900 & 5.33617800 & $\mathrm{H}$ & 2.98354400 & 1.79626300 & -4.52619900 \\
\hline $\mathrm{H}$ & -2.93288100 & -7.48090300 & 4.69632800 & $\mathrm{C}$ & 0.89861700 & -2.10258100 & -4.17349400 \\
\hline $\mathrm{H}$ & -2.33449100 & -5.98495600 & 5.48829500 & $\mathrm{H}$ & 1.39917500 & -1.65578300 & -5.03765100 \\
\hline $\mathrm{C}$ & -3.07092100 & -2.45300000 & 2.03150000 & $\mathrm{H}$ & 0.20654800 & -1.36846700 & -3.75030000 \\
\hline $\mathrm{H}$ & -2.06465800 & -2.20972100 & 2.38493900 & $\mathrm{H}$ & 0.30337400 & -2.94559600 & -4.54790500 \\
\hline $\mathrm{C}$ & -2.22485200 & -2.11043900 & -0.37142900 & $\mathrm{C}$ & 1.10962600 & -3.39428300 & -2.03288700 \\
\hline $\mathrm{H}$ & -1.25916900 & -2.47818600 & -0.03408100 & $\mathrm{H}$ & 1.76029100 & -3.77256100 & -1.23849100 \\
\hline $\mathrm{H}$ & -2.48994900 & -2.35442100 & -1.39406300 & $\mathrm{H}$ & 0.59815300 & -4.25195300 & -2.48949500 \\
\hline $\mathrm{H}$ & -3.39581900 & -0.48288700 & 0.97049700 & $\mathrm{C}$ & 2.87479900 & -3.63093200 & -3.78198800 \\
\hline $\mathrm{C}$ & -3.29276000 & -3.97153200 & 2.09068400 & $\mathrm{H}$ & 2.28872900 & -4.44451400 & -4.22896700 \\
\hline $\mathrm{H}$ & -4.26137400 & -4.24083500 & 1.64451700 & $\mathrm{H}$ & 3.55473900 & -4.07814500 & -3.05155700 \\
\hline $\mathrm{H}$ & -2.53348100 & -4.51319800 & 1.51708300 & $\mathrm{H}$ & 3.46937300 & -3.18343400 & -4.58096800 \\
\hline $\mathrm{C}$ & -3.25043000 & -1.94849200 & 0.60521400 & $\mathrm{~F}$ & 3.53428900 & -3.03484100 & 3.94045300 \\
\hline $\mathrm{H}$ & -4.27397700 & -2.00417800 & 0.23049900 & $\mathrm{H}$ & 0.33955100 & -2.75957500 & -1.58101100 \\
\hline $\mathrm{H}$ & -3.77914100 & -1.96851600 & 2.71031700 & $\mathrm{C}$ & 5.81773800 & 4.24834100 & 2.10761600 \\
\hline $\mathrm{Cl}$ & -0.51940700 & 0.46416600 & -1.82575200 & $\mathrm{O}$ & 5.39915900 & 5.23162900 & 1.53460800 \\
\hline $\mathrm{Rh}$ & 1.39660300 & -0.55315900 & -0.42976800 & $\mathrm{O}$ & 6.83778000 & 4.28592800 & 2.99724300 \\
\hline $\mathrm{C}$ & 4.84009000 & -2.93199400 & 0.56691000 & $\mathrm{C}$ & 7.40942800 & 5.58298500 & 3.22327200 \\
\hline $\mathrm{C}$ & 3.81394600 & -2.14360500 & 0.00996200 & $\mathrm{H}$ & 7.82057600 & 5.99123100 & 2.29567600 \\
\hline $\mathrm{C}$ & 2.69729400 & -1.62738800 & 0.68157800 & $\mathrm{H}$ & 8.20091000 & 5.43101100 & 3.95809300 \\
\hline $\mathrm{C}$ & 2.63547000 & -1.96455700 & 2.03632100 & $\mathrm{H}$ & 6.65565600 & 6.27507700 & 3.60889400 \\
\hline $\mathrm{C}$ & 3.65194700 & -2.75013500 & 2.61760700 & $\mathrm{C}$ & 4.06097100 & 2.74905400 & 1.04411800 \\
\hline $\mathrm{C}$ & 4.75479900 & -3.24181200 & 1.93925200 & $\mathrm{H}$ & 3.25916400 & 3.35489900 & 1.48489800 \\
\hline
\end{tabular}




\begin{tabular}{|c|c|c|c|c|c|c|c|}
\hline $\mathrm{C}$ & 2.30182500 & 1.25321200 & 0.02129400 & $\mathrm{H}$ & 1.64426400 & -2.56045000 & 3.76726000 \\
\hline $\mathrm{H}$ & 2.42657100 & 1.76014800 & -0.94111600 & $\mathrm{H}$ & 2.99599400 & -1.77208700 & 4.58757300 \\
\hline $\mathrm{H}$ & 1.48791800 & 1.74270100 & 0.56131300 & $\mathrm{C}$ & 0.61114800 & -4.29374900 & 1.83739400 \\
\hline $\mathrm{H}$ & 4.37771800 & 0.70931800 & 0.38528400 & $\mathrm{H}$ & 1.05791800 & -4.54502100 & 2.80404800 \\
\hline $\mathrm{C}$ & 5.30197100 & 2.83260500 & 1.93667700 & $\mathrm{H}$ & 0.04663800 & -3.36244400 & 1.94234200 \\
\hline $\mathrm{H}$ & 6.12211800 & 2.22633700 & 1.52689300 & $\mathrm{H}$ & -0.10410500 & -5.09057300 & 1.59563100 \\
\hline $\mathrm{H}$ & 5.10374800 & 2.41718400 & 2.93309900 & $\mathrm{C}$ & 0.92185400 & -4.01613900 & -0.63868700 \\
\hline $\mathrm{C}$ & 3.57834800 & 1.29770900 & 0.85184800 & $\mathrm{H}$ & 1.61240800 & -3.93189600 & -1.48327000 \\
\hline $\mathrm{H}$ & 3.39718400 & 0.84220000 & 1.83142300 & $\mathrm{H}$ & 0.27048300 & -4.88080300 & -0.82228700 \\
\hline \multirow[t]{2}{*}{$\mathrm{H}$} & 4.28521500 & 3.20924800 & 0.07345300 & $\mathrm{C}$ & 2.44929600 & -5.53813900 & 0.62812200 \\
\hline & & & & $\mathrm{H}$ & 1.73931200 & -6.35698400 & 0.45333400 \\
\hline \multirow[t]{7}{*}{37} & & & & $\mathrm{H}$ & 3.16215300 & -5.53767300 & -0.20122100 \\
\hline & & & & $\mathrm{H}$ & 2.98890800 & -5.76945500 & 1.54966800 \\
\hline & & & & $\mathrm{F}$ & 4.19922800 & -0.38309000 & -4.96430000 \\
\hline & & & & $\mathrm{Rh}$ & 1.65766500 & -0.93472600 & -0.16229000 \\
\hline & & & & $\mathrm{Cl}$ & -0.28567400 & -0.65669100 & 1.50722100 \\
\hline & & & & $\mathrm{H}$ & 0.27676000 & -3.12745300 & -0.62431400 \\
\hline & & & $\phi$ & $\mathrm{C}$ & 6.24818700 & 4.22029100 & 0.91264400 \\
\hline $\mathrm{C}$ & 5.04 & -2.59210600 & -2.22678200 & $\mathrm{O}$ & 5.67716500 & 4.82954800 & 1.79174100 \\
\hline $\mathrm{C}$ & 4.01216900 & -2.19590000 & -1.35927300 & $\mathrm{O}$ & 7.45021100 & 4.59236500 & 0.41201600 \\
\hline $\mathrm{C}$ & 3.02954900 & -1.23061000 & -1.61577100 & $\mathrm{C}$ & 8.01532800 & 5.77921700 & 0.98844300 \\
\hline $\mathrm{C}$ & 3.12568900 & -0.63464600 & -2.87584100 & $\mathrm{H}$ & 8.17960000 & 5.64679800 & 2.06152000 \\
\hline $\mathrm{C}$ & 4.15833600 & -1.00879800 & -3.75922900 & $\mathrm{H}$ & 8.96376400 & 5.93331100 & 0.47244800 \\
\hline $\mathrm{C}$ & 5.12 & -1.95978600 & -3.48368300 & $\mathrm{H}$ & 7.35269800 & 6.63592800 & 0.83691900 \\
\hline $\mathrm{C}$ & 5.78648700 & -3.61255600 & -1.52397600 & $\mathrm{C}$ & 4.44500200 & 2.42902700 & 0.83497700 \\
\hline $\mathrm{C}$ & 5.18479800 & -3.80087100 & -0.30560600 & $\mathrm{H}$ & 3.68459300 & 3.21931000 & 0.82608500 \\
\hline $\mathrm{H}$ & 2.42421300 & 0.12492200 & -3.20405500 & $\mathrm{C}$ & 2.62067600 & 0.68394900 & 0.69504700 \\
\hline $\mathrm{H}$ & 5.89078200 & -2.19176300 & -4.21834100 & $\mathrm{H}$ & 2.73566300 & 0.44551300 & 1.75654300 \\
\hline $\mathrm{H}$ & 6.66261700 & -4.14233300 & -1.87556600 & $\mathrm{H}$ & 1.83876700 & 1.44498300 & 0.60548900 \\
\hline $\mathrm{H}$ & 5.44990000 & -4.48445700 & 0.48713100 & $\mathrm{H}$ & 4.68940200 & 0.39997300 & 0.10635700 \\
\hline $\mathrm{N}$ & 4.09568600 & -2.93442600 & -0.17672200 & $\mathrm{C}$ & 5.73807700 & 2.97105300 & 0.22058200 \\
\hline $\mathrm{P}$ & 2.76884800 & -2.66611400 & 0.90943500 & $\mathrm{H}$ & 6.53665100 & 2.21810100 & 0.24006700 \\
\hline $\mathrm{C}$ & 3.54740100 & -2.55312500 & 2.64916800 & $\mathrm{H}$ & 5.59363500 & 3.21365200 & -0.84149100 \\
\hline $\mathrm{C}$ & 1.65815400 & -4.21799400 & 0.70738600 & $\mathrm{C}$ & 3.92349500 & 1.18540000 & 0.08770100 \\
\hline $\mathrm{C}$ & 4.00290600 & -3.91043000 & 3.22325500 & $\mathrm{H}$ & 3.76993100 & 1.43538200 & -0.96768600 \\
\hline $\mathrm{H}$ & 4.73856700 & -4.41245200 & 2.58754600 & $\mathrm{H}$ & 4.61879400 & 2.19252400 & 1.89301700 \\
\hline $\mathrm{H}$ & 4.48289000 & -3.73080100 & 4.19387100 & $\mathrm{Cl}$ & 0.24766700 & 0.53189300 & -1.56574700 \\
\hline $\mathrm{H}$ & 3.16858600 & -4.59425600 & 3.39539400 & $\mathrm{C}$ & -4.95655000 & 2.64080000 & 2.26226400 \\
\hline $\mathrm{C}$ & 4.78332900 & -1.63132300 & 2.54898200 & $\mathrm{C}$ & -3.94754700 & 2.21313600 & 1.37657600 \\
\hline $\mathrm{H}$ & 5.19965100 & -1.50056400 & 3.55590600 & $\mathrm{C}$ & -2.99983800 & 1.20785600 & 1.61005900 \\
\hline $\mathrm{H}$ & 5.56395800 & -2.05771100 & 1.91261100 & $\mathrm{C}$ & -3.10184800 & 0.60386900 & 2.86593400 \\
\hline $\mathrm{H}$ & 4.53436300 & -0.64119200 & 2.16271400 & $\mathrm{C}$ & -4.10690300 & 1.00907600 & 3.76722200 \\
\hline $\mathrm{C}$ & 2.51675700 & -1.92129900 & 3.61153900 & $\mathrm{C}$ & -5.04147400 & 1.99960200 & 3.51416300 \\
\hline $\mathrm{H}$ & 2.15506300 & -0.95259300 & 3.25971000 & $\mathrm{C}$ & -5.66240200 & 3.69639500 & 1.57966500 \\
\hline
\end{tabular}




\begin{tabular}{|c|c|c|c|c|c|c|c|}
\hline $\mathrm{C}$ & -5.07042600 & 3.87340900 & 0.35485700 & $\mathrm{C}$ & -2.69117700 & -0.70314700 & -0.71966500 \\
\hline $\mathrm{H}$ & -2.42587700 & -0.18538300 & 3.17731600 & $\mathrm{H}$ & -2.82394200 & -0.44731700 & -1.77499400 \\
\hline $\mathrm{H}$ & -5.78550800 & 2.25376000 & 4.26163900 & $\mathrm{H}$ & -1.93096200 & -1.48840300 & -0.65752200 \\
\hline $\mathrm{H}$ & -6.51209600 & 4.25657600 & 1.94857400 & $\mathrm{H}$ & -4.73697500 & -0.36303100 & -0.08096100 \\
\hline $\mathrm{H}$ & -5.31941100 & 4.57478900 & -0.42755300 & $\mathrm{C}$ & -5.86247300 & -2.90422300 & -0.20329100 \\
\hline $\mathrm{N}$ & -4.01803800 & 2.96632200 & 0.20245000 & $\mathrm{H}$ & -6.63863600 & -2.12780200 & -0.19313300 \\
\hline $\mathrm{P}$ & -2.71997000 & 2.65476400 & -0.90603900 & $\mathrm{H}$ & -5.70272200 & -3.16870400 & 0.85121800 \\
\hline $\mathrm{C}$ & -3.52987600 & 2.58306100 & -2.63337800 & $\mathrm{C}$ & -3.99496100 & -1.17135700 & -0.08776500 \\
\hline $\mathrm{C}$ & -1.54752100 & 4.16240700 & -0.71473600 & $\mathrm{H}$ & -3.82572800 & -1.43775700 & 0.96128900 \\
\hline $\mathrm{C}$ & -3.94040300 & 3.96107300 & -3.19177600 & $\mathrm{H}$ & -4.75747600 & -2.13500100 & -1.88915900 \\
\hline $\mathrm{H}$ & -4.64770000 & 4.48643900 & -2.54279100 & & & & \\
\hline $\mathrm{H}$ & -4.43973200 & 3.80753600 & -4.15710600 & 38-ts & & & \\
\hline $\mathrm{H}$ & -3.08252300 & 4.61357500 & -3.37012000 & & & & \\
\hline $\mathrm{C}$ & -4.79893600 & 1.70957400 & -2.51738700 & & & & \\
\hline $\mathrm{H}$ & -5.23948700 & 1.60466100 & -3.51694200 & & & & \\
\hline $\mathrm{H}$ & -5.54932100 & 2.16019400 & -1.86155200 & & & & \\
\hline $\mathrm{H}$ & -4.58113400 & 0.70696000 & -2.14460300 & & & & \\
\hline $\mathrm{C}$ & -2.53959100 & 1.91817300 & -3.61545900 & $\mathrm{C}$ & -5.09506100 & 1.28364500 & -2.15694400 \\
\hline $\mathrm{H}$ & -2.20841700 & 0.93517200 & -3.27356600 & $\mathrm{C}$ & -4.27940700 & 0.80289700 & -1.11634800 \\
\hline $\mathrm{H}$ & -1.64658500 & 2.52531000 & -3.78212800 & $\mathrm{C}$ & -3.33659100 & -0.25396800 & -1.21731600 \\
\hline $\mathrm{H}$ & -3.03967200 & 1.79162400 & -4.58418400 & $\mathrm{C}$ & -3.14469700 & -0.71386700 & -2.53959200 \\
\hline $\mathrm{C}$ & -0.51879800 & 4.20317300 & -1.86281000 & $\mathrm{C}$ & -3.91177600 & -0.19984800 & -3.59171600 \\
\hline $\mathrm{H}$ & -0.97155700 & 4.48232800 & -2.81903800 & $\mathrm{C}$ & -4.91224400 & 0.75263500 & -3.44725100 \\
\hline $\mathrm{H}$ & 0.00198900 & 3.24893900 & -1.98601700 & $\mathrm{C}$ & -5.95200100 & 2.28221700 & -1.56461700 \\
\hline $\mathrm{H}$ & 0.23525100 & 4.96576800 & -1.62827200 & $\mathrm{C}$ & -5.60144000 & 2.39820600 & -0.24717900 \\
\hline $\mathrm{C}$ & -0.79771000 & 3.92496900 & 0.61793800 & $\mathrm{H}$ & -2.45661100 & -1.51848900 & -2.75846200 \\
\hline $\mathrm{H}$ & -1.47776000 & 3.86245400 & 1.47300100 & $\mathrm{H}$ & -5.50339300 & 1.05886600 & -4.30363900 \\
\hline $\mathrm{H}$ & -0.11107100 & 4.76315900 & 0.79563400 & $\mathrm{H}$ & -6.73388300 & 2.84307900 & -2.06012400 \\
\hline $\mathrm{C}$ & -2.28606700 & 5.51137500 & -0.61373500 & $\mathrm{H}$ & -6.00521200 & 3.04955300 & 0.51305600 \\
\hline $\mathrm{H}$ & -1.54241500 & 6.30115700 & -0.44529200 & $\mathrm{~N}$ & -4.56174900 & 1.50654100 & 0.05520600 \\
\hline $\mathrm{H}$ & -2.98423300 & 5.53154100 & 0.22784400 & $\mathrm{P}$ & -3.20678300 & 1.52683100 & 1.17243200 \\
\hline $\mathrm{H}$ & -2.83219800 & 5.77031900 & -1.52404900 & $\mathrm{C}$ & -3.98389400 & 1.38319500 & 2.91814700 \\
\hline $\mathrm{F}$ & -4.15548200 & 0.37375400 & 4.96698500 & $\mathrm{C}$ & -2.40969100 & 3.26745800 & 0.90550100 \\
\hline $\mathrm{Rh}$ & -1.65957800 & 0.87361500 & 0.13592700 & $\mathrm{C}$ & -4.66858600 & 2.66295000 & 3.43763200 \\
\hline $\mathrm{H}$ & -0.18718000 & 3.01255500 & 0.58921000 & $\mathrm{H}$ & -5.47664400 & 3.00299400 & 2.78148700 \\
\hline $\mathrm{C}$ & -6.42536300 & -4.12503900 & -0.90506700 & $\mathrm{H}$ & -5.11713900 & 2.44581000 & 4.41579500 \\
\hline $\mathrm{O}$ & -5.90167100 & -4.72382400 & -1.82005100 & $\mathrm{H}$ & -3.96707600 & 3.48822400 & 3.58093300 \\
\hline $\mathrm{O}$ & -7.61611000 & -4.48362600 & -0.36865300 & $\mathrm{C}$ & -5.03858800 & 0.25835600 & 2.87652100 \\
\hline $\mathrm{C}$ & -8.22956000 & -5.64200600 & -0.95340300 & $\mathrm{H}$ & -5.45272700 & 0.12976800 & 3.88473300 \\
\hline $\mathrm{H}$ & -8.42841200 & -5.47869900 & -2.01632300 & $\mathrm{H}$ & -5.86597000 & 0.48955800 & 2.19944200 \\
\hline $\mathrm{H}$ & -9.16257700 & -5.78723500 & -0.40758000 & $\mathrm{H}$ & -4.60532200 & -0.69988500 & 2.57866300 \\
\hline $\mathrm{H}$ & -7.58274600 & -6.51739300 & -0.84683000 & $\mathrm{C}$ & -2.86481000 & 0.96441500 & 3.89777100 \\
\hline $\mathrm{C}$ & -4.56788000 & -2.38993800 & -0.83820500 & $\mathrm{H}$ & -2.37834900 & 0.03691700 & 3.58259300 \\
\hline $\mathrm{H}$ & -3.82965700 & -3.20090600 & -0.85566500 & $\mathrm{H}$ & -2.08916100 & 1.72709800 & 4.00372600 \\
\hline
\end{tabular}




\begin{tabular}{|c|c|c|c|c|c|c|c|}
\hline $\mathrm{H}$ & -3.30759600 & 0.80052400 & 4.88883200 & $\mathrm{H}$ & 2.93706400 & -1.87095100 & -4.35907700 \\
\hline $\mathrm{C}$ & -1.39458000 & 3.56344300 & 2.02933000 & $\mathrm{C}$ & 2.05234100 & -4.58015400 & -0.97194300 \\
\hline $\mathrm{H}$ & -1.88126100 & 3.75729600 & 2.99036500 & $\mathrm{H}$ & 2.48293200 & -4.82361600 & -1.94783200 \\
\hline $\mathrm{H}$ & -0.66944900 & 2.75725500 & 2.15894900 & $\mathrm{H}$ & 1.16027900 & -3.96560800 & -1.12653500 \\
\hline $\mathrm{H}$ & -0.83917800 & 4.47142200 & 1.76056900 & $\mathrm{H}$ & 1.73264600 & -5.52663500 & -0.51650300 \\
\hline $\mathrm{C}$ & -1.66610400 & 3.19798500 & -0.44661900 & $\mathrm{C}$ & 2.43412900 & -3.75683600 & 1.37167000 \\
\hline $\mathrm{H}$ & -2.34331300 & 2.96684600 & -1.27506700 & $\mathrm{H}$ & 3.10369000 & -3.25833000 & 2.07930200 \\
\hline $\mathrm{H}$ & -1.21010600 & 4.17665600 & -0.64738900 & $\mathrm{H}$ & 2.21273200 & -4.75697500 & 1.76758600 \\
\hline $\mathrm{C}$ & -3.41621200 & 4.43519700 & 0.81898500 & $\mathrm{C}$ & 4.33218300 & -4.77654900 & 0.10140100 \\
\hline $\mathrm{H}$ & -2.84153700 & 5.36414500 & 0.70978500 & $\mathrm{H}$ & 4.03439700 & -5.76069800 & 0.48640100 \\
\hline $\mathrm{H}$ & -4.06680900 & 4.35079800 & -0.05426000 & $\mathrm{H}$ & 5.05065800 & -4.34993800 & 0.80705300 \\
\hline $\mathrm{H}$ & -4.03827900 & 4.54439700 & 1.70951800 & $\mathrm{H}$ & 4.83916900 & -4.94223300 & -0.85226200 \\
\hline $\mathrm{F}$ & -3.68574500 & -0.70090600 & -4.83045900 & $\mathrm{~F}$ & 4.32624100 & 1.61023100 & 4.59455400 \\
\hline $\mathrm{Rh}$ & -1.91101300 & -0.15037300 & 0.30956000 & $\mathrm{Rh}$ & 1.80798800 & -0.77555500 & 0.37423400 \\
\hline $\mathrm{Cl}$ & 0.05477400 & 0.28996700 & 1.74468200 & $\mathrm{H}$ & 1.48508500 & -3.20525300 & 1.33984600 \\
\hline $\mathrm{H}$ & -0.87594500 & 2.44385100 & -0.43949800 & $\mathrm{C}$ & 3.57594100 & 5.56363000 & -1.94499200 \\
\hline $\mathrm{Cl}$ & -0.24813700 & -1.57779100 & -0.91898600 & $\mathrm{O}$ & 2.66259900 & 5.77421500 & -2.71413600 \\
\hline $\mathrm{C}$ & 5.75376900 & -0.57823000 & 2.09045900 & $\mathrm{O}$ & 4.60669600 & 6.42349700 & -1.76429100 \\
\hline $\mathrm{C}$ & 4.57273600 & -0.78143100 & 1.34941500 & $\mathrm{C}$ & 4.54253000 & 7.63081500 & -2.53791500 \\
\hline $\mathrm{C}$ & 3.30987900 & -0.23740900 & 1.61786500 & $\mathrm{H}$ & 4.53850000 & 7.40391400 & -3.60766600 \\
\hline $\mathrm{C}$ & 3.26824800 & 0.57571200 & 2.75355100 & $\mathrm{H}$ & 5.43448600 & 8.19867400 & -2.27046100 \\
\hline $\mathrm{C}$ & 4.43696200 & 0.80077200 & 3.50887100 & $\mathrm{H}$ & 3.63959300 & 8.19818400 & -2.29513800 \\
\hline $\mathrm{C}$ & 5.67929500 & 0.26061000 & 3.22043800 & $\mathrm{C}$ & 2.78754000 & 3.19807500 & -1.43871000 \\
\hline $\mathrm{C}$ & 6.77505900 & -1.35120500 & 1.42889400 & $\mathrm{H}$ & 1.75659000 & 3.56925100 & -1.46991100 \\
\hline $\mathrm{C}$ & 6.19546900 & -1.98251100 & 0.35747800 & $\mathrm{C}$ & 1.95678000 & 0.88049300 & -0.85786700 \\
\hline $\mathrm{H}$ & 2.35254700 & 1.05769500 & 3.07972800 & $\mathrm{H}$ & 2.17851700 & 0.50652400 & -1.86039800 \\
\hline $\mathrm{H}$ & 6.53297800 & 0.48268700 & 3.85196900 & $\mathrm{H}$ & 0.91635600 & 1.21775800 & -0.85264100 \\
\hline $\mathrm{H}$ & 7.81807300 & -1.42776000 & 1.70849900 & $\mathrm{H}$ & 3.93603700 & 1.65960200 & -0.42994900 \\
\hline $\mathrm{H}$ & 6.64310500 & -2.64857600 & -0.36505200 & $\mathrm{C}$ & 3.72262300 & 4.34755100 & -1.05111000 \\
\hline $\mathrm{N}$ & 4.84253200 & -1.64083300 & 0.28241000 & $\mathrm{H}$ & 4.77334700 & 4.03324500 & -1.05935500 \\
\hline $\mathrm{P}$ & 3.42860500 & -2.11062600 & -0.60828800 & $\mathrm{H}$ & 3.52213500 & 4.67429100 & -0.02026100 \\
\hline $\mathrm{C}$ & 3.93418700 & -2.02901300 & -2.44725200 & $\mathrm{C}$ & 2.89668000 & 2.01041700 & -0.46210800 \\
\hline $\mathrm{C}$ & 3.06987100 & -3.90272400 & -0.03196600 & $\mathrm{H}$ & 2.65959500 & 2.35385100 & 0.55105300 \\
\hline $\mathrm{C}$ & 4.84375000 & -3.18917300 & -2.90339800 & $\mathrm{H}$ & 3.02064900 & 2.87063900 & -2.46090300 \\
\hline $\mathrm{H}$ & 5.77315300 & -3.24757600 & -2.32895200 & $\mathrm{C}$ & -3.04800900 & -2.99247200 & -0.44912400 \\
\hline $\mathrm{H}$ & 5.12139400 & -3.01764200 & -3.95135500 & $\mathrm{O}$ & -3.13849500 & -3.50889500 & -1.54285400 \\
\hline $\mathrm{H}$ & 4.34453200 & -4.15992300 & -2.85602100 & $\mathrm{O}$ & -2.44779900 & -3.59744200 & 0.60411400 \\
\hline $\mathrm{C}$ & 4.70545900 & -0.70636400 & -2.66110700 & $\mathrm{C}$ & -1.86050000 & -4.87456000 & 0.32113900 \\
\hline $\mathrm{H}$ & 4.92728700 & -0.60473300 & -3.73104700 & $\mathrm{H}$ & -1.07714500 & -4.77054400 & -0.43285900 \\
\hline $\mathrm{H}$ & 5.65366500 & -0.69351800 & -2.11632400 & $\mathrm{H}$ & -1.44142100 & -5.21861800 & 1.26741800 \\
\hline $\mathrm{H}$ & 4.13089800 & 0.17074500 & -2.35767800 & $\mathrm{H}$ & -2.61809900 & -5.57524600 & -0.04160400 \\
\hline $\mathrm{C}$ & 2.65239300 & -2.02917800 & -3.31104800 & $\mathrm{C}$ & -5.22693900 & -1.80027100 & -0.22909900 \\
\hline $\mathrm{H}$ & 1.95097600 & -1.24178000 & -3.02653100 & $\mathrm{H}$ & -5.43017300 & -1.91252000 & -1.29931600 \\
\hline $\mathrm{H}$ & 2.11591500 & -2.97968900 & -3.25502500 & $\mathrm{C}$ & -7.38032500 & -3.01768700 & 0.36618800 \\
\hline
\end{tabular}




\begin{tabular}{|c|c|c|c|c|c|c|c|}
\hline $\mathrm{H}$ & -7.65360800 & -3.12333300 & -0.69049000 & $\mathrm{H}$ & -2.73565200 & 2.23178100 & 4.77945600 \\
\hline $\mathrm{H}$ & -7.81781200 & -3.86214800 & 0.91072500 & $\mathrm{C}$ & -1.33452800 & 3.96147400 & 0.87870600 \\
\hline $\mathrm{H}$ & -7.84964300 & -2.09926000 & 0.74010400 & $\mathrm{H}$ & -1.13890200 & 3.92495100 & 1.95247500 \\
\hline $\mathrm{C}$ & -3.69957600 & -1.69398400 & -0.01375100 & $\mathrm{H}$ & -0.65769400 & 3.25756400 & 0.39210200 \\
\hline $\mathrm{H}$ & -5.70264100 & -0.87286300 & 0.09934900 & $\mathrm{H}$ & -1.08544200 & 4.97270000 & 0.53093300 \\
\hline $\mathrm{H}$ & -3.51621700 & -1.59315700 & 1.05934400 & $\mathrm{C}$ & -2.93629800 & 3.75213500 & -1.02212700 \\
\hline $\mathrm{C}$ & -5.85752800 & -2.97539900 & 0.54091500 & $\mathrm{H}$ & -3.97099800 & 3.65278000 & -1.36111900 \\
\hline $\mathrm{H}$ & -5.61146900 & -2.89649400 & 1.60909600 & $\mathrm{H}$ & -2.57043400 & 4.73295800 & -1.35266100 \\
\hline \multirow[t]{2}{*}{$\mathrm{H}$} & -5.42932700 & -3.92334000 & 192200 & $\mathrm{C}$ & -3.69459500 & 4.77895600 & 1.13834200 \\
\hline & & & & $\mathrm{H}$ & -3.37537800 & 5.74868100 & 0.73488000 \\
\hline \multirow[t]{7}{*}{ 39-ts } & & & & $\mathrm{H}$ & -4.75084200 & 4.65825500 & 0.88424500 \\
\hline & & & & $\mathrm{H}$ & -3.59763100 & 4.83686700 & 2.22587900 \\
\hline & & & & $\mathrm{F}$ & -4.51440500 & -0.52224900 & -4.76552800 \\
\hline & & & & $\mathrm{Rh}$ & -2.01455700 & 0.31467400 & 0.03842800 \\
\hline & & & & $\mathrm{Cl}$ & -0.00145400 & 0.81688400 & 1.40823500 \\
\hline & & & & $\mathrm{H}$ & -2.33383200 & 2.98268900 & -1.51369300 \\
\hline & & & & $\mathrm{C}$ & -2.72894600 & -3.85511300 & 0.04877800 \\
\hline $\mathrm{C}$ & 400 & 1.32157400 & -1.89278300 & $\mathrm{O}$ & -2.11704900 & -3.77776300 & 1.09507300 \\
\hline $\mathrm{C}$ & -4.68524600 & 0.95403900 & -0.99408400 & $\mathrm{O}$ & -3.09803500 & -5.03437300 & -0.50339200 \\
\hline $\mathrm{C}$ & -3.63711700 & 0.02914200 & -1.24938700 & $\mathrm{C}$ & -2.71194900 & -6.20883800 & 0.22743000 \\
\hline $\mathrm{C}$ & -3.60958100 & -0.41122900 & -2.59444700 & $\mathrm{H}$ & -3.12755600 & -6.18726400 & 1.23830900 \\
\hline $\mathrm{C}$ & -4.60133400 & -0.03001600 & -3.50462400 & $\mathrm{H}$ & -3.11569200 & -7.05014000 & -0.33675800 \\
\hline $\mathrm{C}$ & -5.67704700 & 0.79147800 & -3.19539100 & $\mathrm{H}$ & -1.62271100 & -6.28110200 & 0.29449500 \\
\hline $\mathrm{C}$ & -6.54469500 & 2.25796200 & -1.18698200 & $\mathrm{C}$ & -3.60753200 & -1.47954500 & -0.02241000 \\
\hline $\mathrm{C}$ & -5.99383300 & 2.45295000 & 0.04979900 & $\mathrm{H}$ & -3.03839600 & -1.45708600 & 0.91517500 \\
\hline $\mathrm{H}$ & -2.83545500 & -1.07966700 & -2.95087900 & $\mathrm{C}$ & -5.44752700 & -2.79113600 & 1.20022100 \\
\hline $\mathrm{H}$ & -6.43060400 & 1.01353500 & -3.94331700 & $\mathrm{H}$ & -4.85380000 & -2.81160300 & 2.12103800 \\
\hline $\mathrm{H}$ & -7.44564400 & 2.72997700 & -1.55717700 & $\mathrm{H}$ & -6.50692000 & -2.75402800 & 1.48024800 \\
\hline $\mathrm{H}$ & -6.33447400 & 3.08528100 & 0.85435500 & $\mathrm{H}$ & -5.28548100 & -3.73762200 & 0.67364200 \\
\hline $\mathrm{N}$ & -4.84589700 & 1.65898600 & 0.20001000 & $\mathrm{C}$ & -3.13232800 & -2.68797600 & -0.83508400 \\
\hline $\mathrm{P}$ & -3.30857300 & 1.88546800 & 1.03277400 & $\mathrm{H}$ & -3.89300500 & -3.01174500 & -1.55094200 \\
\hline $\mathrm{C}$ & -3.70246200 & 1.77885100 & 2.90406200 & $\mathrm{H}$ & -2.22925000 & -2.44626700 & -1.40198400 \\
\hline $\mathrm{C}$ & -2.80784400 & 3.68144600 & 0.51624500 & $\mathrm{C}$ & -5.10230000 & -1.57286100 & 0.32507200 \\
\hline $\mathrm{C}$ & -5.06435400 & 2.39207600 & 3.28640600 & $\mathrm{H}$ & -5.68892800 & -1.61478100 & -0.60109200 \\
\hline $\mathrm{H}$ & -5.89326600 & 1.86829800 & 2.80078300 & $\mathrm{H}$ & -5.41899500 & -0.66918400 & 0.84972700 \\
\hline $\mathrm{H}$ & -5.20302500 & 2.28721600 & 4.37043000 & $\mathrm{Cl}$ & -0.31907800 & -0.88730100 & -1.38942700 \\
\hline $\mathrm{H}$ & -5.13698800 & 3.45731200 & 3.05198300 & $\mathrm{C}$ & 5.28549300 & -1.27086400 & 2.42504200 \\
\hline $\mathrm{C}$ & -3.72277600 & 0.28052100 & 3.27606300 & $\mathrm{C}$ & 4.19129000 & -1.20827700 & 1.53968800 \\
\hline $\mathrm{H}$ & -3.92315300 & 0.18757600 & 4.35151800 & $\mathrm{C}$ & 3.05667200 & -0.39548000 & 1.66516400 \\
\hline $\mathrm{H}$ & -4.51033900 & -0.26257400 & 2.74677300 & $\mathrm{C}$ & 3.05509200 & 0.41250200 & 2.80503200 \\
\hline $\mathrm{H}$ & -2.76419300 & -0.20134100 & 3.06393200 & $\mathrm{C}$ & 4.14052500 & 0.37326500 & 3.70360700 \\
\hline $\mathrm{C}$ & -2.57377400 & 2.44929600 & 3.71596100 & $\mathrm{C}$ & 5.25777600 & -0.43210800 & 3.55732600 \\
\hline $\mathrm{H}$ & -1.58768000 & 2.05908600 & 3.44601300 & $\mathrm{C}$ & 6.18559700 & -2.25311400 & 1.87440100 \\
\hline $\mathrm{H}$ & -2.56360400 & 3.53675900 & 3.60523900 & $\mathrm{C}$ & 5.62029700 & -2.74178300 & 0.72359700 \\
\hline
\end{tabular}




\begin{tabular}{|c|c|c|c|c|c|c|c|}
\hline $\mathrm{H}$ & 2.23622700 & 1.08906100 & 3.02641600 & $\mathrm{H}$ & 2.48189800 & 0.62787500 & -1.91159500 \\
\hline $\mathrm{H}$ & 6.05340700 & -0.40331700 & 4.29428500 & $\mathrm{H}$ & 1.38389600 & 1.61684700 & -0.94025600 \\
\hline $\mathrm{H}$ & 7.14174600 & -2.56003700 & 2.27880700 & $\mathrm{H}$ & 4.37339800 & 1.24291300 & -0.29798400 \\
\hline $\mathrm{H}$ & 5.99613900 & -3.49369500 & 0.04576000 & $\mathrm{C}$ & 4.92518100 & 3.89691600 & -0.89681000 \\
\hline $\mathrm{N}$ & 4.39664000 & -2.10852100 & 0.49179800 & $\mathrm{H}$ & 5.83600800 & 3.30154600 & -0.74451300 \\
\hline $\mathrm{P}$ & 3.04437500 & -2.24165400 & -0.58841500 & $\mathrm{H}$ & 4.69984600 & 4.33845900 & 0.08290400 \\
\hline $\mathrm{C}$ & 3.79385700 & -2.26795700 & -2.34391300 & $\mathrm{C}$ & 3.47217100 & 1.85804700 & -0.41461600 \\
\hline $\mathrm{C}$ & 2.21778800 & -3.90687300 & -0.12091700 & $\mathrm{H}$ & 3.24959000 & 2.26298600 & 0.57882400 \\
\hline $\mathrm{C}$ & 4.47982500 & -3.59924800 & -2.71389700 & $\mathrm{H}$ & 4.01346800 & 2.62715900 & -2.38194800 \\
\hline $\mathrm{H}$ & 5.29181200 & -3.86001900 & -2.02840400 & & & & \\
\hline $\mathrm{H}$ & 4.92109200 & -3.49435000 & -3.71344700 & 40-ts & & & \\
\hline $\mathrm{H}$ & 3.77848900 & -4.43613100 & -2.75528600 & & & & \\
\hline $\mathrm{C}$ & 4.85030200 & -1.14307700 & -2.41863300 & & & & \\
\hline $\mathrm{H}$ & 5.24824700 & -1.10872600 & -3.44082200 & & & & \\
\hline $\mathrm{H}$ & 5.68616600 & -1.31851800 & -1.73545300 & & & & \\
\hline $\mathrm{H}$ & 4.42915800 & -0.16120800 & -2.19464200 & & & & \\
\hline $\mathrm{C}$ & 2.66950200 & -1.98255800 & -3.36488300 & $\mathrm{C}$ & -5.79482800 & 1.25072000 & -1.67701100 \\
\hline $\mathrm{H}$ & 2.13548700 & -1.05378600 & -3.15188000 & $\mathrm{C}$ & -4.72083300 & 1.01099600 & -0.79875500 \\
\hline $\mathrm{H}$ & 1.92927300 & -2.78553300 & -3.40238200 & $\mathrm{C}$ & -3.72161300 & 0.01277400 & -0.95383200 \\
\hline $\mathrm{H}$ & 3.11751300 & -1.89731100 & -4.36323800 & $\mathrm{C}$ & -3.80978200 & -0.67468800 & -2.18760000 \\
\hline $\mathrm{C}$ & 1.20931600 & -4.33409300 & -1.20585800 & $\mathrm{C}$ & -4.85775000 & -0.42605700 & -3.08019100 \\
\hline $\mathrm{H}$ & 1.70096200 & -4.66989700 & -2.12421900 & $\mathrm{C}$ & -5.88003200 & 0.48733100 & -2.85564200 \\
\hline $\mathrm{H}$ & 0.50906500 & -3.53123100 & -1.45458200 & $\mathrm{C}$ & -6.55126000 & 2.33411200 & -1.09927900 \\
\hline $\mathrm{H}$ & 0.62452700 & -5.18107100 & -0.82393400 & $\mathrm{C}$ & -5.90544400 & 2.72434300 & 0.04172100 \\
\hline $\mathrm{C}$ & 1.45011300 & -3.61880200 & 1.19158700 & $\mathrm{H}$ & -3.08313600 & -1.43161500 & -2.45753000 \\
\hline $\mathrm{H}$ & 2.11651500 & -3.28905200 & 1.99466300 & $\mathrm{H}$ & -6.68162400 & 0.59965000 & -3.57784200 \\
\hline $\mathrm{H}$ & 0.94877800 & -4.53668500 & 1.52374400 & $\mathrm{H}$ & -7.46278700 & 2.76989500 & -1.48758600 \\
\hline $\mathrm{C}$ & 3.22325400 & -5.04310800 & 0.14700400 & $\mathrm{H}$ & -6.16676000 & 3.50509800 & 0.73835300 \\
\hline $\mathrm{H}$ & 2.66265600 & -5.93555000 & 0.45478600 & $\mathrm{~N}$ & -4.77295500 & 1.92179400 & 0.25733300 \\
\hline $\mathrm{H}$ & 3.91332400 & -4.79255500 & 0.95753700 & $\mathrm{P}$ & -3.17323500 & 2.22413100 & 0.93644000 \\
\hline $\mathrm{H}$ & 3.80793300 & -5.31582100 & -0.73515200 & $\mathrm{C}$ & -3.44037800 & 2.47639700 & 2.81643300 \\
\hline $\mathrm{F}$ & 4.07782900 & 1.19181100 & 4.78653800 & $\mathrm{C}$ & -2.64641900 & 3.86458500 & 0.06592100 \\
\hline $\mathrm{Rh}$ & 1.64826400 & -0.58587600 & 0.22692700 & $\mathrm{C}$ & -4.75250700 & 3.20660900 & 3.16395700 \\
\hline $\mathrm{H}$ & 0.65885000 & -2.87036100 & 1.05580100 & $\mathrm{H}$ & -5.62852800 & 2.64219800 & 2.83048100 \\
\hline $\mathrm{C}$ & 5.26347900 & 5.02339900 & -1.85389500 & $\mathrm{H}$ & -4.82152800 & 3.30261700 & 4.25529200 \\
\hline $\mathrm{O}$ & 4.74414600 & 5.22187500 & -2.93130700 & $\mathrm{H}$ & -4.80507300 & 4.21524700 & 2.74592700 \\
\hline $\mathrm{O}$ & 6.24871100 & 5.80526400 & -1.35054000 & $\mathrm{C}$ & -3.48197400 & 1.07146500 & 3.45631300 \\
\hline $\mathrm{C}$ & 6.64956600 & 6.90124700 & -2.18579500 & $\mathrm{H}$ & -3.60946400 & 1.18122600 & 4.54121300 \\
\hline $\mathrm{H}$ & 7.02087800 & 6.53826100 & -3.14830100 & $\mathrm{H}$ & -4.32033100 & 0.47548400 & 3.08493500 \\
\hline $\mathrm{H}$ & 7.44195800 & 7.41325700 & -1.63843700 & $\mathrm{H}$ & -2.55424000 & 0.52113500 & 3.27534000 \\
\hline $\mathrm{H}$ & 5.80908500 & 7.57755600 & -2.36553000 & $\mathrm{C}$ & -2.23803300 & 3.23227900 & 3.42123200 \\
\hline $\mathrm{C}$ & 3.77082800 & 3.01805800 & -1.38553200 & $\mathrm{H}$ & -1.28601100 & 2.75382100 & 3.17179000 \\
\hline $\mathrm{H}$ & 2.87501000 & 3.63774700 & -1.51676600 & $\mathrm{H}$ & -2.19729800 & 4.27897500 & 3.10869400 \\
\hline $\mathrm{C}$ & 2.30043400 & 1.01971300 & -0.90668000 & $\mathrm{H}$ & -2.33696600 & 3.22284100 & 4.51427900 \\
\hline
\end{tabular}




\begin{tabular}{|c|c|c|c|c|c|c|c|}
\hline C & -1.13995700 & 4.12549600 & 0.27901200 & $\mathrm{H}$ & 6.12728300 & -0.36905000 & 6800 \\
\hline $\mathrm{H}$ & -0.87242800 & 4.28185800 & 1.32631700 & $\mathrm{H}$ & 7.06015800 & -2.69955700 & 2.30213300 \\
\hline $\mathrm{H}$ & -0.52990400 & 3.30460000 & -0.10219700 & $\mathrm{H}$ & 5.82737500 & -3.70044700 & 0.14642400 \\
\hline $\mathrm{H}$ & -0.87014200 & 5.03660200 & -0.27108100 & $\mathrm{~N}$ & 4.31290600 & -2.20559000 & 0.52963600 \\
\hline C & -2.88216300 & 3.65743800 & -1.44742800 & $\mathrm{P}$ & 2.95101100 & -2.31783100 & -0.54254700 \\
\hline $\mathrm{H}$ & -3.94267600 & 3.55730600 & -1.69435000 & $\mathrm{C}$ & 3.70422300 & -2.41312300 & -2.29337600 \\
\hline $\mathrm{H}$ & -2.49651500 & 4.53704000 & -1.97899500 & $\mathrm{C}$ & 2.06005300 & -3.93433500 & -0.03842300 \\
\hline $\mathrm{C}$ & -3.44332400 & 5.10109100 & 0.52708000 & $\mathrm{C}$ & 4.34677500 & -3.77579200 & -2.62656500 \\
\hline $\mathrm{H}$ & -3.11924000 & 5.96275300 & -0.07081400 & $\mathrm{H}$ & 5.14166000 & -4.04968300 & -1.92645200 \\
\hline $\mathrm{H}$ & -4.51922800 & 4.98769800 & 0.36904200 & $\mathrm{H}$ & 4.80279600 & -3.70789400 & -3.62283000 \\
\hline $\mathrm{H}$ & -3.26521200 & 5.35321000 & 1.57604200 & $\mathrm{H}$ & 3.61708700 & -4.58838000 & -2.65852900 \\
\hline F & -4.88711200 & -1.14704700 & -4.22860500 & $\mathrm{C}$ & 4.80235800 & -1.32970900 & -2.38448900 \\
\hline $\mathrm{Rh}$ & -2.00354700 & 0.45717000 & 0.14292200 & $\mathrm{H}$ & 5.18453900 & -1.30683700 & -3.41298200 \\
\hline $\mathrm{Cl}$ & 0.09884800 & 1.00719700 & 1.36376200 & $\mathrm{H}$ & 5.64122300 & -1.54259100 & -1.71576900 \\
\hline $\mathrm{H}$ & -2.35432600 & 2.77505900 & -1.82120400 & $\mathrm{H}$ & 4.42791100 & -0.33245100 & -2.14639700 \\
\hline $\mathrm{C}$ & -3.65680800 & -1.24432600 & 0.52968600 & $\mathrm{C}$ & 2.59609100 & -2.11871200 & -3.32939600 \\
\hline $\mathrm{H}$ & -3.03768900 & -1.03909100 & 1.41324800 & $\mathrm{H}$ & 2.09024500 & -1.16826000 & -3.14627100 \\
\hline $\mathrm{C}$ & -3.26085500 & -2.61111500 & -0.03930400 & $\mathrm{H}$ & 1.83207500 & -2.89951800 & -3.34953900 \\
\hline $\mathrm{H}$ & -4.02842000 & -2.96069700 & -0.73769600 & $\mathrm{H}$ & 3.05238400 & -2.07433300 & -4.32670300 \\
\hline $\mathrm{H}$ & -2.31905500 & -2.54352800 & -0.58554500 & $\mathrm{C}$ & 1.04471800 & -4.35225000 & 32900 \\
\hline $\mathrm{Cl}$ & -0.44108600 & -0.94354900 & -1.24514900 & $\mathrm{H}$ & 1.53566300 & -4.75599000 & -2.01322400 \\
\hline $\mathrm{C}$ & -5.12328400 & -1.21486200 & 0.96220700 & $\mathrm{H}$ & 0.39949400 & -3.52103700 & -1.42102300 \\
\hline $\mathrm{H}$ & -5.27914400 & -1.96410600 & 1.75064100 & $\mathrm{H}$ & 0.39929500 & -5.13674300 & -0.71088800 \\
\hline $\mathrm{H}$ & -5.42681500 & -0.24926900 & 1.36799700 & $\mathrm{C}$ & 1.29723800 & -3.58077400 & 1.26165100 \\
\hline $\mathrm{H}$ & -5.78871900 & -1.45643500 & 0.12666100 & $\mathrm{H}$ & 1.97672400 & -3.29024600 & 2.06948700 \\
\hline $\mathrm{C}$ & -3.08386200 & -3.65651900 & 1.09123200 & $\mathrm{H}$ & 0.71824700 & -4.45363500 & 1.58550300 \\
\hline $\mathrm{H}$ & -2.28937000 & -3.34976100 & 1.77723200 & $\mathrm{C}$ & 3.01512900 & -5.10335800 & 0.26983200 \\
\hline $\mathrm{H}$ & -4.01327500 & -3.76249000 & 1.66229100 & $\mathrm{H}$ & 2.41317000 & -5.96175100 & 0.59453600 \\
\hline $\mathrm{C}$ & -2.70926900 & -5.02130600 & 0.55177300 & $\mathrm{H}$ & 3.70847700 & -4.86041000 & 1.08001900 \\
\hline $\mathrm{O}$ & -1.64693900 & -5.58579900 & 0.72427100 & $\mathrm{H}$ & 3.59471500 & -5.42272300 & -0.60028400 \\
\hline $\mathrm{O}$ & -3.71804400 & -5.55617800 & -0.17034900 & $\mathrm{~F}$ & 4.25015200 & 1.36022700 & 4.61884700 \\
\hline $\mathrm{C}$ & -3.45605900 & -6.84622700 & -0.74508100 & $\mathrm{Rh}$ & 1.63192200 & -0.56700400 & 0.22826000 \\
\hline $\mathrm{H}$ & -3.22235600 & -7.57450300 & 0.03610500 & $\mathrm{H}$ & 0.57548300 & -2.76833300 & 1.10226000 \\
\hline $\mathrm{H}$ & -4.37102000 & -7.12440400 & -1.26872600 & $\mathrm{C}$ & 5.04897200 & 5.06439200 & -2.07712700 \\
\hline $\mathrm{H}$ & -2.61638700 & -6.79259700 & -1.44344600 & $\mathrm{O}$ & 4.43247600 & 5.29297100 & -3.09572600 \\
\hline $\mathrm{C}$ & 5.28043900 & -1.30244300 & 2.39402100 & $\mathrm{O}$ & 6.08674800 & 5.82468900 & -1.65210300 \\
\hline C & 4.17567000 & -1.23336000 & 1.52157100 & $\mathrm{C}$ & 6.41503200 & 6.94080900 & -2.49221700 \\
\hline $\mathrm{C}$ & 3.09041000 & -0.35154500 & 1.61300400 & $\mathrm{H}$ & 6.69126200 & 6.60218700 & -3.49476700 \\
\hline $\mathrm{C}$ & 3.15326800 & 0.52200900 & 2.70204900 & $\mathrm{H}$ & 7.25938000 & 7.43395200 & -2.00894900 \\
\hline $\mathrm{C}$ & 4.24981700 & 0.47629900 & 3.58625500 & $\mathrm{H}$ & 5.56652700 & 7.62604200 & -2.57388000 \\
\hline $\mathrm{C}$ & 5.31844100 & -0.39735100 & 3.47349700 & $\mathrm{C}$ & 3.65882400 & 2.99444300 & -1.56900200 \\
\hline $\mathrm{C}$ & 6.11613500 & -2.36397400 & 1.89210100 & $\mathrm{H}$ & 2.74405900 & 3.58083800 & -1.71840100 \\
\hline C & 5.50505100 & -2.88872300 & 0.78127400 & $\mathrm{C}$ & 2.26762300 & 0.95468600 & -1.02097400 \\
\hline $\mathrm{H}$ & 2.37619800 & 1.25422600 & 2.89478100 & $\mathrm{H}$ & 2.49075700 & 0.49749100 & -1.98839600 \\
\hline
\end{tabular}




\begin{tabular}{|c|c|c|c|c|c|c|c|}
\hline $\mathrm{H}$ & 1.33632500 & 1.52020200 & -1.12538500 & $\mathrm{C}$ & 1.32176900 & -4.59878400 & 1.96217000 \\
\hline $\mathrm{H}$ & 4.32310600 & 1.28396300 & -0.41374700 & $\mathrm{H}$ & 1.69451800 & -4.68397500 & 2.98759600 \\
\hline $\mathrm{C}$ & 4.78469100 & 3.92530500 & -1.11187100 & $\mathrm{H}$ & 0.52877900 & -3.84828200 & 1.93648700 \\
\hline $\mathrm{H}$ & 5.72426400 & 3.37430300 & -0.97201200 & $\mathrm{H}$ & 0.87024000 & -5.56494000 & 1.70204300 \\
\hline $\mathrm{H}$ & 4.55739400 & 4.36352200 & -0.13031500 & $\mathrm{C}$ & 1.87980100 & -4.40755300 & -0.48372700 \\
\hline $\mathrm{C}$ & 3.40001300 & 1.85952400 & -0.55786500 & $\mathrm{H}$ & 2.63732500 & -4.18297900 & -1.24149400 \\
\hline $\mathrm{H}$ & 3.15470200 & 2.29323400 & 0.41801500 & $\mathrm{H}$ & 1.53466600 & -5.43724800 & -0.64883900 \\
\hline \multirow[t]{2}{*}{$\mathrm{H}$} & 000 & 2.57729300 & 237300 & $\mathrm{C}$ & 3.54267900 & -5.38663000 & 1.09636800 \\
\hline & & & & $\mathrm{H}$ & 3.05887300 & -6.36861700 & 1.01409600 \\
\hline \multirow[t]{7}{*}{ 41-ts } & & & & $\mathrm{H}$ & 4.29039100 & -5.32263700 & 0.30268400 \\
\hline & & & & $\mathrm{H}$ & 4.05483900 & -5.36010200 & 2.06097700 \\
\hline & & & & $\mathrm{F}$ & 4.32925100 & -0.83946800 & -4.89756000 \\
\hline & & & & $\mathrm{Rh}$ & 1.84471900 & -0.97462200 & -0.03917400 \\
\hline & & & & $\mathrm{Cl}$ & -0.30450800 & -1.55518800 & 1.01326200 \\
\hline & & & & $\mathrm{H}$ & 1.03529400 & -3.73154300 & -0.63821100 \\
\hline & & & & $\mathrm{C}$ & 3.76643800 & 5.59001200 & -0.32351300 \\
\hline C & 5.46243900 & -2.40880500 & -1.84205100 & $\mathrm{O}$ & 2.82682400 & 5.82408800 & 0.41247200 \\
\hline $\mathrm{C}$ & 4.46837500 & -1.89391700 & -0.99133000 & $\mathrm{O}$ & 4.78738700 & 6.45174800 & -0.51081000 \\
\hline $\mathrm{C}$ & 3.48907100 & -0.93143200 & -1.33792900 & $\mathrm{C}$ & 4.69383500 & 7.68923000 & 0.21455600 \\
\hline $\mathrm{C}$ & 3.45887000 & -0.63804900 & -2.71922700 & $\mathrm{H}$ & 4.66632200 & 7.50159500 & 1.29123100 \\
\hline $\mathrm{C}$ & 4.40851500 & -1.18953800 & -3.58904200 & $\mathrm{H}$ & 5.58687800 & 8.25389800 & -0.05450300 \\
\hline $\mathrm{C}$ & 5.44099000 & -2.03103200 & -3.19835100 & $\mathrm{H}$ & 3.79149800 & 8.23560300 & -0.07288600 \\
\hline $\mathrm{C}$ & 6.27701100 & -3.27278600 & -1.02076300 & $\mathrm{C}$ & 3.78384300 & 3.06137100 & -0.26586600 \\
\hline $\mathrm{C}$ & 5.73100200 & -3.28644900 & 0.23368400 & $\mathrm{H}$ & 2.89981900 & 3.18240900 & 0.37044400 \\
\hline $\mathrm{H}$ & 2.73347300 & 0.05420100 & -3.12925600 & $\mathrm{C}$ & 3.54944600 & 0.54775300 & -0.21280700 \\
\hline $\mathrm{H}$ & 6.17063000 & -2.37605200 & -3.92287000 & $\mathrm{H}$ & 4.50752700 & 0.30847300 & 0.24558800 \\
\hline $\mathrm{H}$ & 7.16218800 & -3.81482500 & -1.32768800 & $\mathrm{H}$ & 2.86378800 & 0.79030900 & 0.62183600 \\
\hline $\mathrm{H}$ & 6.05071700 & -3.82965900 & 1.10992600 & $\mathrm{H}$ & 4.49055500 & 1.68231400 & -1.79516500 \\
\hline $\mathrm{N}$ & 4.60521400 & -2.44922500 & 0.28155500 & $\mathrm{C}$ & 3.93340800 & 4.32213900 & -1.13636300 \\
\hline $\mathrm{P}$ & 3.07786400 & -2.49173300 & 1.15689600 & $\mathrm{H}$ & 4.89354300 & 4.33844700 & -1.65961600 \\
\hline $\mathrm{C}$ & 3.54489400 & -2.11241300 & 2.97307300 & $\mathrm{H}$ & 3.14211800 & 4.32840000 & -1.89843500 \\
\hline $\mathrm{C}$ & 2.44984400 & -4.30661400 & 0.94968800 & $\mathrm{C}$ & 3.64158200 & 1.78329400 & -1.10782800 \\
\hline $\mathrm{C}$ & 4.28902000 & -3.24926400 & 3.70064500 & $\mathrm{H}$ & 2.73948300 & 1.87031600 & -1.72043300 \\
\hline $\mathrm{H}$ & 5.22472000 & -3.52306100 & 3.20239100 & $\mathrm{H}$ & 4.64989600 & 2.98065900 & 0.40602900 \\
\hline $\mathrm{H}$ & 4.54974100 & -2.90724900 & 4.71071000 & $\mathrm{Cl}$ & 0.39184200 & 0.45569300 & -1.47896400 \\
\hline $\mathrm{H}$ & 3.67866700 & -4.14869400 & 3.81211600 & $\mathrm{C}$ & -4.73089700 & 1.70253800 & 2.79749000 \\
\hline $\mathrm{C}$ & 4.45061200 & -0.86243600 & 2.96722400 & $\mathrm{C}$ & -3.74424500 & 1.48899300 & 1.81420500 \\
\hline $\mathrm{H}$ & 4.69794100 & -0.60625000 & 4.00525100 & $\mathrm{C}$ & -2.87879900 & 0.39073500 & 1.72009300 \\
\hline $\mathrm{H}$ & 5.38735200 & -1.03100900 & 2.42792400 & $\mathrm{C}$ & -3.04400100 & -0.55624200 & 2.73467100 \\
\hline $\mathrm{H}$ & 3.94558400 & 0.00102000 & 2.52641200 & $\mathrm{C}$ & -4.02633400 & -0.36780600 & 3.72790600 \\
\hline $\mathrm{C}$ & 2.25099000 & -1.76525500 & 3.74308300 & $\mathrm{C}$ & -4.88001000 & 0.72051700 & 3.79718800 \\
\hline $\mathrm{H}$ & 1.69547700 & -0.95878300 & 3.25641400 & $\mathrm{C}$ & -5.34841600 & 2.96367700 & 2.47244100 \\
\hline $\mathrm{H}$ & 1.57719500 & -2.61919300 & 3.84374600 & $\mathrm{C}$ & -4.72865600 & 3.45731600 & 1.35224000 \\
\hline $\mathrm{H}$ & 2.52124400 & -1.43529400 & 4.75463700 & $\mathrm{H}$ & -2.43795700 & -1.45488200 & 2.78504500 \\
\hline
\end{tabular}




\begin{tabular}{|c|c|c|c|c|c|c|c|}
\hline $\mathrm{H}$ & -5.61303300 & 0.79116100 & 4.59384700 & $\mathrm{H}$ & -1.92435700 & -1.54566100 & -1.30263100 \\
\hline $\mathrm{H}$ & -6.15871500 & 3.44834100 & 3.00198100 & $\mathrm{H}$ & -4.67276300 & -0.58493800 & -0.29625500 \\
\hline $\mathrm{H}$ & -4.91321200 & 4.37948100 & 0.82203600 & $\mathrm{C}$ & -5.89761600 & -2.91621300 & -1.20587600 \\
\hline $\mathrm{N}$ & -3.74255400 & 2.56509700 & 0.92480700 & $\mathrm{H}$ & -6.60699800 & -2.16190500 & -0.83426600 \\
\hline $\mathrm{P}$ & -2.47224600 & 2.49854300 & -0.25734100 & $\mathrm{H}$ & -5.71130700 & -3.58776800 & -0.35963500 \\
\hline $\mathrm{C}$ & -3.29802300 & 2.96593100 & -1.91495000 & $\mathrm{C}$ & -3.96422900 & -1.36828000 & -0.59369800 \\
\hline $\mathrm{C}$ & -1.20703000 & 3.81642300 & 0.32147400 & $\mathrm{H}$ & -3.78476400 & -1.97330400 & 0.30195400 \\
\hline $\mathrm{C}$ & -3.62198200 & 4.46772500 & -2.05829000 & $\mathrm{H}$ & 100 & -1.64435100 & 49300 \\
\hline $\mathrm{H}$ & -4.28705500 & 4.83376000 & -1.27034600 & & & & \\
\hline $\mathrm{H}$ & -4.14037100 & 4.61795200 & -3.01412500 & 42 & & & \\
\hline $\mathrm{H}$ & -2.72527200 & 5.09147700 & -2.07337500 & & & & \\
\hline $\mathrm{C}$ & -4.62258700 & 2.17768800 & -2.01145300 & & & & \\
\hline $\mathrm{H}$ & -5.06837900 & 2.36477700 & -2.99661200 & & & & \\
\hline $\mathrm{H}$ & -5.34081200 & 2.49270200 & -1.24913300 & & & & \\
\hline $\mathrm{H}$ & -4.47726400 & 1.10079700 & -1.91225800 & & & & \\
\hline $\mathrm{C}$ & -2.35923300 & 2.54629700 & -3.06835100 & & & & \\
\hline $\mathrm{H}$ & -2.09127600 & 1.48830500 & -3.02679200 & $\mathrm{C}$ & -5.86389700 & -2.50514000 & 1.53842800 \\
\hline $\mathrm{H}$ & -1.43006800 & 3.12155500 & -3.07633800 & $\mathrm{C}$ & -4.93511300 & -1.64057100 & 0.91101000 \\
\hline $\mathrm{H}$ & -2.87184500 & 2.72977400 & -4.02135000 & $\mathrm{C}$ & -4.39308300 & -0.52483400 & 1.58178100 \\
\hline $\mathrm{C}$ & -0.19000400 & 4.12348700 & -0.79643600 & $\mathrm{C}$ & -4.60596000 & -0.47535200 & 2.96239800 \\
\hline $\mathrm{H}$ & -0.63763700 & 4.69426800 & -1.61608400 & $\mathrm{C}$ & -5.40893300 & -1.42963000 & 3.60201100 \\
\hline $\mathrm{H}$ & 0.25049000 & 3.20957100 & -1.20671500 & $\mathrm{C}$ & -6.09862400 & -2.41260800 & 2.92125800 \\
\hline $\mathrm{H}$ & 0.62109200 & 4.73135100 & -0.37654200 & $\mathrm{C}$ & -6.44356200 & -3.31285400 & 0.49867800 \\
\hline $\mathrm{C}$ & -0.45915900 & 3.17035600 & 1.51252700 & $\mathrm{C}$ & -5.82820600 & -2.97040700 & -0.66664100 \\
\hline $\mathrm{H}$ & -1.13254000 & 2.92191000 & 2.33931000 & $\mathrm{H}$ & -4.21312300 & 0.33621700 & 3.56231600 \\
\hline $\mathrm{H}$ & 0.28990800 & 3.88043900 & 1.88617300 & $\mathrm{H}$ & -6.79420000 & -3.06295200 & 3.44063800 \\
\hline $\mathrm{C}$ & -1.85552400 & 5.12346400 & 0.81891100 & $\mathrm{H}$ & -7.23874300 & -4.03808800 & 0.61010000 \\
\hline $\mathrm{H}$ & -1.05699200 & 5.79145000 & 1.16628000 & $\mathrm{H}$ & -5.99331900 & -3.36721200 & -1.65428000 \\
\hline $\mathrm{H}$ & -2.53240500 & 4.94849600 & 1.65996700 & $\mathrm{~N}$ & -4.85322300 & -1.97710800 & -0.46316800 \\
\hline $\mathrm{H}$ & -2.40377600 & 5.65310300 & 0.03589100 & $\mathrm{P}$ & -3.28068200 & -1.94171900 & -1.33068800 \\
\hline $\mathrm{F}$ & -4.13759100 & -1.33163000 & 4.67959300 & $\mathrm{C}$ & -3.75449800 & -1.20920600 & -3.04108000 \\
\hline $\mathrm{Rh}$ & -1.54435200 & 0.42200700 & 0.20024300 & $\mathrm{C}$ & -2.75470300 & -3.80772800 & -1.48818200 \\
\hline $\mathrm{H}$ & 0.07906600 & 2.26086200 & 1.21409900 & $\mathrm{C}$ & -4.60080500 & -2.12450300 & -3.94593400 \\
\hline $\mathrm{C}$ & -6.60420300 & -3.69509600 & -2.29844100 & $\mathrm{H}$ & -5.57089000 & -2.37139200 & -3.50292200 \\
\hline $\mathrm{O}$ & -6.41502400 & -3.57739100 & -3.49033800 & $\mathrm{H}$ & -4.80488000 & -1.59776200 & -4.88742900 \\
\hline $\mathrm{O}$ & -7.51523800 & -4.54881400 & -1.77343400 & $\mathrm{H}$ & -4.08480600 & -3.05340500 & -4.20169800 \\
\hline $\mathrm{C}$ & -8.26948700 & -5.30648900 & -2.73110600 & $\mathrm{C}$ & -4.54266600 & 0.09208000 & -2.78041400 \\
\hline $\mathrm{H}$ & -8.83964500 & -4.64252500 & -3.38706000 & $\mathrm{H}$ & -4.80288700 & 0.54617600 & -3.74528500 \\
\hline $\mathrm{H}$ & -8.94155900 & -5.93455200 & -2.14502900 & $\mathrm{H}$ & -5.47090200 & -0.08870700 & -2.23014900 \\
\hline $\mathrm{H}$ & -7.60586300 & -5.92221500 & -3.34446500 & $\mathrm{H}$ & -3.94038100 & 0.81901100 & -2.22699700 \\
\hline $\mathrm{C}$ & -4.60732600 & -2.24483400 & -1.68669200 & $\mathrm{C}$ & -2.44606500 & -0.83436400 & -3.77358200 \\
\hline $\mathrm{H}$ & -3.89712700 & -3.01664700 & -2.01097800 & $\mathrm{H}$ & -1.79906000 & -0.21711900 & -3.14271500 \\
\hline $\mathrm{C}$ & -2.65625000 & -0.76227000 & -1.08298100 & $\mathrm{H}$ & -1.87376400 & -1.70967800 & -4.08912100 \\
\hline $\mathrm{H}$ & -2.80374300 & -0.17591100 & -1.99407700 & $\mathrm{H}$ & -2.69854200 & -0.26224300 & -4.67603700 \\
\hline
\end{tabular}




\begin{tabular}{|c|c|c|c|c|c|c|c|}
\hline $\mathrm{C}$ & -1.63563900 & -3.92729100 & -2.54898700 & $\mathrm{H}$ & 5.88392900 & -0.26738600 & -4.42902000 \\
\hline $\mathrm{H}$ & -2.03959100 & -3.88062800 & -3.56560700 & $\mathrm{H}$ & 6.68067400 & 2.46417700 & -3.08961400 \\
\hline $\mathrm{H}$ & -0.85389100 & -3.17532000 & -2.44815500 & $\mathrm{H}$ & 5.47555900 & 3.75688100 & -1.07689300 \\
\hline $\mathrm{H}$ & -1.16076800 & -4.91038300 & -2.43515700 & $\mathrm{~N}$ & 4.10458500 & 2.08521200 & -1.05204800 \\
\hline $\mathrm{C}$ & -2.21245800 & -4.19397300 & -0.09265200 & $\mathrm{P}$ & 2.78432500 & 2.29460100 & 0.05688200 \\
\hline $\mathrm{H}$ & -2.98449000 & -4.09514700 & 0.67879400 & $\mathrm{C}$ & 3.58785600 & 2.85629400 & 1.69417000 \\
\hline $\mathrm{H}$ & -1.89875600 & -5.24629600 & -0.11620000 & $\mathrm{C}$ & 1.69933100 & 3.66038900 & -0.73020400 \\
\hline $\mathrm{C}$ & -3.83146400 & -4.85165600 & -1.86180700 & $\mathrm{C}$ & 4.07322500 & 4.32073500 & 1.68783400 \\
\hline $\mathrm{H}$ & -3.31232000 & -5.80272900 & -2.03894300 & $\mathrm{H}$ & 4.79577900 & 4.52153200 & 0.89121400 \\
\hline $\mathrm{H}$ & -4.54695100 & -5.01967400 & -1.05607900 & $\mathrm{H}$ & 4.57848800 & 4.52161400 & 2.64143200 \\
\hline $\mathrm{H}$ & -4.37860700 & -4.61324600 & -2.77748300 & $\mathrm{H}$ & 3.25142800 & 5.03550400 & 1.59929700 \\
\hline $\mathrm{F}$ & -5.57556900 & -1.31347700 & 4.94038300 & $\mathrm{C}$ & 4.81001100 & 1.94499300 & 1.94614800 \\
\hline $\mathrm{Rh}$ & -1.84535900 & -0.66036300 & -0.11518600 & $\mathrm{H}$ & 5.22656500 & 2.18601800 & 2.93231700 \\
\hline $\mathrm{Cl}$ & 0.21998600 & -1.60189200 & -0.87803500 & $\mathrm{H}$ & 5.59450200 & 2.09833200 & 1.19974700 \\
\hline $\mathrm{H}$ & -1.35518600 & -3.58675700 & 0.20328900 & $\mathrm{H}$ & 4.54864800 & 0.88540500 & 1.94571700 \\
\hline $\mathrm{C}$ & -2.97386400 & 5.69380800 & 0.71798700 & $\mathrm{C}$ & 2.56191600 & 2.67457800 & 2.83535000 \\
\hline $\mathrm{O}$ & -2.46766000 & 5.68446300 & -0.38613200 & $\mathrm{H}$ & 2.17568800 & 1.65441400 & 2.89324500 \\
\hline $\mathrm{O}$ & -3.32879000 & 6.82986700 & 1.35757800 & $\mathrm{H}$ & 1.70612800 & 3.34650200 & 2.73202300 \\
\hline $\mathrm{C}$ & -3.04774900 & 8.05024300 & 0.65364200 & $\mathrm{H}$ & 3.05338800 & 2.90664900 & 3.78887700 \\
\hline $\mathrm{H}$ & -3.57699200 & 8.07307700 & -0.30266700 & $\mathrm{C}$ & 0.67527700 & 4.18891400 & 0.29371400 \\
\hline $\mathrm{H}$ & -3.39796000 & 8.85128700 & 1.30528000 & $\mathrm{H}$ & 1.14306100 & 4.81754700 & 1.05858100 \\
\hline $\mathrm{H}$ & -1.97479100 & 8.14881300 & 0.46651400 & $\mathrm{H}$ & 0.13845300 & 3.37372100 & 0.78875800 \\
\hline $\mathrm{C}$ & -3.40472000 & 3.18788300 & 0.74665200 & $\mathrm{H}$ & -0.06768700 & 4.80358000 & -0.22786800 \\
\hline $\mathrm{H}$ & -2.49012900 & 3.03224600 & 0.16500400 & $\mathrm{C}$ & 0.93901600 & 2.96034800 & -1.88261900 \\
\hline $\mathrm{C}$ & -3.92726500 & 0.69538700 & 0.81199300 & $\mathrm{H}$ & 1.61803500 & 2.52742100 & -2.62413500 \\
\hline $\mathrm{H}$ & -4.71482700 & 0.90428600 & 0.07498600 & $\mathrm{H}$ & 0.30747400 & 3.69991000 & -2.39166700 \\
\hline $\mathrm{H}$ & -3.03708200 & 0.63470800 & 0.08874100 & $\mathrm{C}$ & 2.50584900 & 4.82847000 & -1.32962400 \\
\hline $\mathrm{H}$ & -4.57368600 & 2.15571900 & 2.24295200 & $\mathrm{H}$ & 1.80188700 & 5.53024500 & -1.79547200 \\
\hline $\mathrm{C}$ & -3.26764200 & 4.47361300 & 1.56804400 & $\mathrm{H}$ & 3.19754100 & 4.48994900 & -2.10635000 \\
\hline $\mathrm{H}$ & -4.16593700 & 4.67068500 & 2.16414600 & $\mathrm{H}$ & 3.07214300 & 5.38679300 & -0.58015300 \\
\hline $\mathrm{H}$ & -2.44269900 & 4.37560700 & 2.28898400 & $\mathrm{~F}$ & 4.17000500 & -2.20467900 & -4.37199000 \\
\hline $\mathrm{C}$ & -3.68060200 & 1.96670000 & 1.63188000 & $\mathrm{Rh}$ & 1.63667500 & 0.29741700 & -0.22734300 \\
\hline $\mathrm{H}$ & -2.84131200 & 1.81295000 & 2.31946100 & $\mathrm{H}$ & 0.27121100 & 2.16951900 & -1.51605600 \\
\hline $\mathrm{H}$ & -4.21989500 & 3.31203600 & 0.02028100 & $\mathrm{C}$ & 6.07501800 & -4.11717000 & 2.86615400 \\
\hline $\mathrm{Cl}$ & -0.36977100 & 0.70651200 & 1.32667300 & $\mathrm{O}$ & 5.83414400 & -3.89834300 & 4.03409000 \\
\hline $\mathrm{C}$ & 5.05135600 & 0.92260200 & -2.77788600 & $\mathrm{O}$ & 6.94697800 & -5.06833400 & 2.45428900 \\
\hline $\mathrm{C}$ & 4.01182200 & 0.92765300 & -1.82628500 & $\mathrm{C}$ & 7.59208100 & -5.80616400 & 3.50269700 \\
\hline $\mathrm{C}$ & 3.01824500 & -0.04706300 & -1.66492900 & $\mathrm{H}$ & 8.16944400 & -5.13766600 & 4.14768000 \\
\hline $\mathrm{C}$ & 3.10673400 & -1.10776300 & -2.57061100 & $\mathrm{H}$ & 8.25068700 & -6.51556600 & 3.00007600 \\
\hline $\mathrm{C}$ & 4.14026600 & -1.13959200 & -3.52875100 & $\mathrm{H}$ & 6.85439600 & -6.33410000 & 4.11345400 \\
\hline $\mathrm{C}$ & 5.11930500 & -0.16984000 & -3.66562500 & $\mathrm{C}$ & 4.28336500 & -2.50240700 & 2.05473600 \\
\hline $\mathrm{C}$ & 5.80019700 & 2.13362700 & -2.55352700 & $\mathrm{H}$ & 3.47713900 & -3.13808300 & 2.44284700 \\
\hline $\mathrm{C}$ & 5.20223300 & 2.81119800 & -1.52102100 & $\mathrm{C}$ & 2.54691400 & -0.86053300 & 1.23043700 \\
\hline $\mathrm{H}$ & 2.40020600 & -1.93069500 & -2.56063400 & $\mathrm{H}$ & 2.75208600 & -0.19480400 & 2.07300900 \\
\hline
\end{tabular}




\begin{tabular}{|c|c|c|c|c|c|c|c|}
\hline $\mathrm{H}$ & 3200 & -1.51035400 & 1.51062800 & $\mathrm{H}$ & 2.33719500 & -1.17077300 & 4.49462900 \\
\hline $\mathrm{H}$ & 4.58062800 & -1.03141500 & 0.49179700 & $\mathrm{C}$ & 1.57313500 & -4.40769200 & 1.66279600 \\
\hline $\mathrm{C}$ & 5.48338800 & -3.37780000 & 1.68178600 & $\mathrm{H}$ & 1.85763500 & -4.51994200 & 2.71415600 \\
\hline $\mathrm{H}$ & 6.28619300 & -2.76627200 & 1.24452000 & $\mathrm{H}$ & 0.79035700 & -3.65307700 & 1.58911700 \\
\hline $\mathrm{H}$ & 5.22062200 & -4.11078600 & 0.90946000 & $\mathrm{H}$ & 1.14024000 & -5.36367700 & 1.34082000 \\
\hline $\mathrm{C}$ & 3.77303600 & -1.68000300 & 0.85469200 & $\mathrm{C}$ & 2.42357200 & -4.26352800 & -0.72207200 \\
\hline $\mathrm{H}$ & 3.52920300 & -2.35502400 & 0.02665900 & $\mathrm{H}$ & 3.27156600 & -4.03086300 & -1.37603500 \\
\hline \multirow[t]{2}{*}{$\mathrm{H}$} & 0 & -1.83776200 & 57600 & $\mathrm{H}$ & 2.13879800 & -5.30814500 & -0.90667200 \\
\hline & & & & $\mathrm{C}$ & 3.85861300 & -5.19171000 & 1.08663400 \\
\hline \multirow[t]{8}{*}{ 43-ts } & & & & $\mathrm{H}$ & 3.35085100 & -6.16439800 & 1.04881800 \\
\hline & & & & $\mathrm{H}$ & 4.66084400 & -5.21619300 & 0.34815600 \\
\hline & & & & $\mathrm{H}$ & 4.29831200 & -5.10018000 & 2.08300400 \\
\hline & & & & $\mathrm{F}$ & 6.16774900 & -0.46110500 & -4.80453900 \\
\hline & & & & $\mathrm{Rh}$ & 1.95589700 & -0.78489900 & -0.13311500 \\
\hline & & & & $\mathrm{Cl}$ & -0.15714800 & -1.85189800 & 0.19006300 \\
\hline & & & & $\mathrm{H}$ & 1.58674400 & -3.62367400 & -1.00767100 \\
\hline & & & & $\mathrm{C}$ & 2.90610600 & 5.57825900 & 0.39190700 \\
\hline $\mathrm{C}$ & 6.15494600 & -2.25535700 & -1.66443800 & $\mathrm{O}$ & 1.78762400 & 6.03146300 & 0.53824600 \\
\hline $\mathrm{C}$ & 3600 & -1.52939900 & -0.98783500 & $\mathrm{O}$ & 3.93842200 & 5.89241300 & 1.20495200 \\
\hline $\mathrm{C}$ & 4.63803700 & -0.31619100 & -1.49733700 & $\mathrm{C}$ & 3.63026800 & 6.79624500 & 2.27871900 \\
\hline $\mathrm{C}$ & 4.98444100 & -0.01112200 & -2.81649900 & $\mathrm{H}$ & 2.86714300 & 6.36837600 & 2.93454800 \\
\hline $\mathrm{C}$ & 5.87372600 & -0.82343700 & -3.53363200 & $\mathrm{H}$ & 4.56615000 & 6.93726800 & 2.82009800 \\
\hline $\mathrm{C}$ & 6.52064100 & -1.90817100 & -2.97679000 & $\mathrm{H}$ & 3.26600700 & 7.74907500 & 1.88549300 \\
\hline $\mathrm{C}$ & 6.65162300 & -3.23533000 & -0.73551000 & $\mathrm{C}$ & 3.43769500 & 3.15417000 & -0.09553100 \\
\hline $\mathrm{C}$ & 5.91607900 & -3.11737800 & 0.40458200 & $\mathrm{H}$ & 2.47285700 & 2.86086100 & 0.33571500 \\
\hline $\mathrm{H}$ & 4.62998500 & 0.89316300 & -3.29562300 & $\mathrm{C}$ & 4.06430400 & 0.73577900 & -0.56983600 \\
\hline $\mathrm{H}$ & 7.28097100 & -2.44565400 & -3.53314300 & $\mathrm{H}$ & 4.77703100 & 0.81968600 & 0.26205900 \\
\hline $\mathrm{H}$ & 7.47337200 & -3.92071000 & -0.89575400 & $\mathrm{H}$ & 3.11835200 & 0.53165800 & 0.05150400 \\
\hline $\mathrm{H}$ & 5.99596600 & -3.68375000 & 1.31732400 & $\mathrm{H}$ & 4.79229600 & 2.45670800 & -1.62762000 \\
\hline $\mathrm{N}$ & 4.93967300 & -2.11279000 & 0.28667600 & $\mathrm{C}$ & 3.32669900 & 4.58259600 & -0.66805200 \\
\hline $\mathrm{P}$ & 3.29034900 & -2.24283100 & 0.98530400 & $\mathrm{H}$ & 4.29553500 & 4.88867800 & -1.07957100 \\
\hline $\mathrm{C}$ & 3.57353200 & -1.81226500 & 2.83754500 & $\mathrm{H}$ & 2.58216900 & 4.61349100 & -1.46816200 \\
\hline $\mathrm{C}$ & 2.80028900 & -4.11034000 & 0.76973300 & $\mathrm{C}$ & 3.85106000 & 2.13318600 & -1.16298100 \\
\hline $\mathrm{C}$ & 4.32125000 & -2.87616200 & 3.66357400 & $\mathrm{H}$ & 3.09135900 & 2.09773200 & -1.95145300 \\
\hline $\mathrm{H}$ & 5.32949400 & -3.07149700 & 3.28399500 & $\mathrm{H}$ & 4.17144200 & 3.15676300 & 0.72124700 \\
\hline $\mathrm{H}$ & 4.43314800 & -2.50827600 & 4.69204400 & $\mathrm{Cl}$ & 0.56810000 & 0.76661500 & -1.47497200 \\
\hline $\mathrm{H}$ & 3.77798500 & -3.82289700 & 3.71824400 & $\mathrm{C}$ & -4.36872200 & 1.38565300 & 2.68257400 \\
\hline $\mathrm{C}$ & 4.37963900 & -0.49759000 & 2.88788500 & $\mathrm{C}$ & -3.65555400 & 1.07281600 & 1.51024800 \\
\hline $\mathrm{H}$ & 4.51887800 & -0.20924000 & 3.93775400 & $\mathrm{C}$ & -2.82865500 & -0.06036500 & 1.31714100 \\
\hline $\mathrm{H}$ & 5.36844900 & -0.60009900 & 2.43097500 & $\mathrm{C}$ & -2.63031300 & -0.81806000 & 2.49316900 \\
\hline $\mathrm{H}$ & 3.84479700 & 0.31743400 & 2.39130100 & $\mathrm{C}$ & -3.29625700 & -0.48728900 & 3.68024700 \\
\hline $\mathrm{C}$ & 2.19005100 & -1.54728000 & 3.47369100 & $\mathrm{C}$ & -4.19285000 & 0.56414300 & 3.81322100 \\
\hline $\mathrm{H}$ & 1.62657700 & -0.79936000 & 2.90701700 & $\mathrm{C}$ & -5.12832900 & 2.57409900 & 2.37893000 \\
\hline $\mathrm{H}$ & 1.57749400 & -2.44944200 & 3.54187600 & $\mathrm{C}$ & -4.81624400 & 2.94882600 & 1.09949000 \\
\hline
\end{tabular}




\begin{tabular}{|c|c|c|c|c|c|c|c|}
\hline $\mathrm{H}$ & -2.00821000 & -1.70634400 & 2.48175200 & $\mathrm{H}$ & -3.08571100 & -1.11542600 & -1.14072300 \\
\hline $\mathrm{H}$ & -4.71033100 & 0.72793800 & 4.75228200 & $\mathrm{H}$ & -2.86949500 & -2.13966600 & 0.28109400 \\
\hline $\mathrm{H}$ & -5.82159900 & 3.08671600 & 3.03327200 & $\mathrm{H}$ & -5.28548900 & -0.28813000 & -0.16504500 \\
\hline $\mathrm{H}$ & -5.17296500 & 3.79485800 & 0.53141200 & $\mathrm{C}$ & -6.96786100 & -2.48035900 & -0.57581800 \\
\hline $\mathrm{N}$ & -3.90161100 & 2.04644900 & 0.54113600 & $\mathrm{H}$ & -7.46795400 & -1.51764400 & -0.75613300 \\
\hline $\mathrm{P}$ & -2.59814500 & 2.16735100 & -0.63462500 & $\mathrm{H}$ & -7.20787500 & -2.74608200 & 0.46086200 \\
\hline $\mathrm{C}$ & -3.46392700 & 2.43974300 & -2.31935800 & $\mathrm{C}$ & -4.84111400 & -1.25452200 & 0.09484200 \\
\hline $\mathrm{C}$ & -1.60110700 & 3.72574200 & -0.09948900 & $\mathrm{H}$ & -5.08955700 & -1.44144100 & 1.14695100 \\
\hline $\mathrm{C}$ & -4.06306000 & 3.84529300 & -2.52170000 & $\mathrm{H}$ & -5.25910000 & 5200 & -1.84695300 \\
\hline $\mathrm{H}$ & -4.80585400 & 4.09551400 & -1.75681300 & & & & \\
\hline $\mathrm{H}$ & -4.57716400 & 3.87134700 & -3.49145000 & 44 & & & \\
\hline $\mathrm{H}$ & -3.30380700 & 4.63056100 & -2.53676800 & & & & \\
\hline $\mathrm{C}$ & -4.60666400 & 1.40969800 & -2.43387100 & & & & \\
\hline $\mathrm{H}$ & -5.07046900 & 1.50938600 & -3.42355000 & & & & \\
\hline $\mathrm{H}$ & -5.38275600 & 1.57036900 & -1.67998100 & & & & \\
\hline $\mathrm{H}$ & -4.24207500 & 0.38296600 & -2.34440200 & & & & \\
\hline $\mathrm{C}$ & -2.43538300 & 2.14436400 & -3.43434000 & $\mathrm{C}$ & -2.28257100 & 3.08430700 & -0.02630900 \\
\hline $\mathrm{H}$ & -1.99765300 & 1.14766600 & -3.32413500 & $\mathrm{C}$ & -1.58232300 & 1.88017400 & -0.27149000 \\
\hline $\mathrm{H}$ & -1.61725200 & 2.86808300 & -3.45527800 & $\mathrm{C}$ & -0.19413600 & 1.81490700 & -0.46825900 \\
\hline $\mathrm{H}$ & -2.94525800 & 2.19042400 & -4.40544200 & $\mathrm{C}$ & 0.50578800 & 3.02637800 & -0.39545000 \\
\hline $\mathrm{C}$ & -0.65382500 & 4.16256500 & -1.23780900 & $\mathrm{C}$ & -0.18228100 & 4.21558400 & -0.12092900 \\
\hline $\mathrm{H}$ & -1.19404700 & 4.61824900 & -2.07382400 & $\mathrm{C}$ & -1.55650700 & 4.28585300 & 0.04911800 \\
\hline $\mathrm{H}$ & -0.05494700 & 3.33115300 & -1.61729100 & $\mathrm{C}$ & -3.66948100 & 2.72223300 & 0.08851400 \\
\hline $\mathrm{H}$ & 0.03264000 & 4.91777600 & -0.83730500 & $\mathrm{C}$ & -3.74881400 & 1.36466700 & -0.06416000 \\
\hline $\mathrm{C}$ & -0.76074100 & 3.30214100 & 1.12708300 & $\mathrm{H}$ & 1.57371300 & 3.07736200 & -0.57959100 \\
\hline $\mathrm{H}$ & -1.39410900 & 2.98203100 & 1.96126700 & $\mathrm{H}$ & -2.03139900 & 5.24524400 & 0.22603700 \\
\hline $\mathrm{H}$ & -0.16835800 & 4.16565900 & 1.45459200 & $\mathrm{H}$ & -4.50560200 & 3.38906200 & 0.25189300 \\
\hline $\mathrm{C}$ & -2.46440000 & 4.93363600 & 0.32287700 & $\mathrm{H}$ & -4.62356200 & 0.73480500 & -0.05257000 \\
\hline $\mathrm{H}$ & -1.78497200 & 5.76303100 & 0.55604700 & $\mathrm{~N}$ & -2.47861300 & 0.81613500 & -0.28364600 \\
\hline $\mathrm{H}$ & -3.04902500 & 4.72302600 & 1.22159900 & $\mathrm{P}$ & -1.79850000 & -0.80076600 & -0.03201100 \\
\hline $\mathrm{H}$ & -3.14235700 & 5.28024600 & -0.46077700 & $\mathrm{C}$ & -2.58420700 & -1.84202400 & -1.42365700 \\
\hline $\mathrm{F}$ & -3.07431300 & -1.27978500 & 4.75996400 & $\mathrm{C}$ & -2.43351000 & -1.27004700 & 1.72107900 \\
\hline $\mathrm{Rh}$ & -1.50333200 & 0.19409500 & -0.27355500 & $\mathrm{C}$ & -4.07011900 & -1.53307600 & -1.69069600 \\
\hline $\mathrm{H}$ & -0.07967600 & 2.48128000 & 0.88937200 & $\mathrm{H}$ & -4.21976600 & -0.49703300 & -2.00822900 \\
\hline $\mathrm{C}$ & -7.61018700 & -3.50227900 & -1.49464900 & $\mathrm{H}$ & -4.41733600 & -2.17792400 & -2.50833700 \\
\hline $\mathrm{O}$ & -7.08817500 & -4.01313700 & -2.46202800 & $\mathrm{H}$ & -4.71096000 & -1.73291700 & -0.82753300 \\
\hline $\mathrm{O}$ & -8.87828000 & -3.77207800 & -1.10484600 & $\mathrm{C}$ & -1.77712300 & -1.49698900 & -2.69617300 \\
\hline $\mathrm{C}$ & -9.58790000 & -4.70906800 & -1.92957300 & $\mathrm{H}$ & -2.18673400 & -2.06354700 & -3.54275900 \\
\hline $\mathrm{H}$ & -9.67891200 & -4.33192400 & -2.95210300 & $\mathrm{H}$ & -1.84559700 & -0.43148000 & -2.94426500 \\
\hline $\mathrm{H}$ & -10.57219200 & -4.81589000 & -1.47218100 & $\mathrm{H}$ & -0.72070000 & -1.75710700 & -2.58236100 \\
\hline $\mathrm{H}$ & -9.06892800 & -5.67115600 & -1.95375300 & $\mathrm{C}$ & -2.40665400 & -3.34188000 & -1.10973700 \\
\hline $\mathrm{C}$ & -5.45654800 & -2.35301700 & -0.78774200 & $\mathrm{H}$ & -1.36980700 & -3.58740600 & -0.86182600 \\
\hline $\mathrm{H}$ & -4.97997300 & -3.31805500 & -0.57237100 & $\mathrm{H}$ & -3.05236000 & -3.66893400 & -0.28864800 \\
\hline $\mathrm{C}$ & -3.31745300 & -1.21012800 & -0.06754300 & $\mathrm{H}$ & -2.69111000 & -3.92067500 & -1.99798500 \\
\hline
\end{tabular}




\begin{tabular}{|c|c|c|c|c|c|c|c|}
\hline $\mathrm{C}$ & -1.68468200 & -2.52100500 & 2.22840100 & $\mathrm{C}$ & -2.22503500 & 3.10868700 & -0.10077100 \\
\hline $\mathrm{H}$ & -1.90124600 & -3.41283900 & 1.63541200 & $\mathrm{C}$ & -1.54932100 & 1.87216600 & -0.17021200 \\
\hline $\mathrm{H}$ & -0.60274700 & -2.38159800 & 2.22753500 & $\mathrm{C}$ & -0.15502900 & 1.69398800 & -0.14296600 \\
\hline $\mathrm{H}$ & -2.01321300 & -2.71743900 & 3.25759800 & $\mathrm{C}$ & 0.57740400 & 2.88826000 & -0.09206900 \\
\hline $\mathrm{C}$ & -2.06143900 & -0.08124400 & 2.63597200 & $\mathrm{C}$ & -0.08058100 & 4.12910900 & -0.01792400 \\
\hline $\mathrm{H}$ & -2.62140300 & 0.82663000 & 2.39191000 & $\mathrm{C}$ & -1.45563500 & 4.28509200 & -0.02079100 \\
\hline $\mathrm{H}$ & -2.29697000 & -0.35156600 & 3.67313000 & $\mathrm{C}$ & -3.62919000 & 2.79775200 & -0.14697500 \\
\hline $\mathrm{C}$ & -3.94870100 & -1.53604400 & 1.81564700 & $\mathrm{C}$ & -3.74857000 & 1.43577800 & -0.23886000 \\
\hline $\mathrm{H}$ & -4.19759300 & -1.74320100 & 2.86456600 & $\mathrm{H}$ & 1.66177400 & 2.90722900 & -0.11190700 \\
\hline $\mathrm{H}$ & -4.55297800 & -0.67930700 & 1.50761000 & $\mathrm{H}$ & -1.89410800 & 5.27609500 & 0.02672200 \\
\hline $\mathrm{H}$ & -4.25516000 & -2.41051700 & 1.23402400 & $\mathrm{H}$ & -4.45252800 & 3.49989800 & -0.13098700 \\
\hline F & 0.53873000 & 5.35878100 & -0.05169200 & $\mathrm{H}$ & -4.64327800 & 0.83829700 & -0.31136000 \\
\hline $\mathrm{Rh}$ & 0.51984500 & -0.36568200 & -0.18922600 & $\mathrm{~N}$ & -2.48250900 & 0.84121400 & -0.26822900 \\
\hline $\mathrm{Cl}$ & 1.02868300 & -2.65703700 & 0.12565300 & $\mathrm{P}$ & -1.83421700 & -0.76954000 & -0.03665700 \\
\hline $\mathrm{H}$ & -0.99113200 & 0.14458800 & 2.58110300 & $\mathrm{C}$ & -2.53656600 & -1.80372100 & -1.47726800 \\
\hline $\mathrm{H}$ & 0.22444700 & 1.02112100 & -1.21145400 & $\mathrm{C}$ & -2.53341000 & -1.27429700 & 1.68042900 \\
\hline $\mathrm{C}$ & 4.65601000 & -0.08100600 & 1.91422200 & $\mathrm{C}$ & -4.00197500 & -1.47425800 & -1.82463300 \\
\hline $\mathrm{C}$ & 3.17968000 & -0.45881000 & 1.64243700 & $\mathrm{H}$ & -4.11594100 & -0.44086500 & -2.16339200 \\
\hline $\mathrm{C}$ & 4.95684700 & -0.46325200 & -1.66593400 & $\mathrm{H}$ & -4.31568900 & -2.12598500 & -2.65010000 \\
\hline $\mathrm{C}$ & 2.54053300 & 0.37794100 & 0.55547400 & $\mathrm{H}$ & -4.68985400 & -1.65102900 & -0.99351000 \\
\hline $\mathrm{C}$ & 3.51447800 & -0.95366800 & -1.39563700 & $\mathrm{C}$ & -1.65999100 & -1.46828500 & -2.70493300 \\
\hline $\mathrm{C}$ & 2.66363700 & 0.14291800 & -0.80673700 & $\mathrm{H}$ & -2.04044600 & -2.02305700 & -3.57250400 \\
\hline $\mathrm{H}$ & 4.73851800 & 1.00534900 & 2.06789500 & $\mathrm{H}$ & -1.69437000 & -0.40074700 & -2.94994400 \\
\hline $\mathrm{H}$ & 3.11376600 & -1.52318600 & 1.40615600 & $\mathrm{H}$ & -0.61681400 & -1.75140600 & -2.54128900 \\
\hline $\mathrm{H}$ & 4.95401300 & 0.22400400 & -2.52426800 & $\mathrm{C}$ & -2.39765200 & -3.30665300 & -1.15850900 \\
\hline $\mathrm{H}$ & 3.07870800 & -1.30676900 & -2.33820800 & $\mathrm{H}$ & -1.38204700 & -3.56636700 & -0.84617700 \\
\hline $\mathrm{H}$ & 4.93621500 & -0.54350100 & 2.87045500 & $\mathrm{H}$ & -3.10003700 & -3.62917700 & -0.38363900 \\
\hline $\mathrm{H}$ & 2.60931200 & -0.31288800 & 2.56869400 & $\mathrm{H}$ & -2.63091500 & -3.87728100 & -2.06659900 \\
\hline $\mathrm{H}$ & 5.55072600 & -1.33435300 & -1.97621100 & $\mathrm{C}$ & -1.85462400 & -2.55812800 & 2.20134200 \\
\hline $\mathrm{H}$ & 2.25472700 & 1.37850100 & 0.87606900 & $\mathrm{H}$ & -2.07742300 & -3.43400500 & 1.58771900 \\
\hline $\mathrm{H}$ & 3.53071900 & -1.81637900 & -0.72739400 & $\mathrm{H}$ & -0.76905500 & -2.46014900 & 2.25148400 \\
\hline $\mathrm{H}$ & 2.48440900 & 0.98163000 & -1.48276900 & $\mathrm{H}$ & -2.23529200 & -2.75476100 & 3.21225900 \\
\hline $\mathrm{C}$ & 5.69164200 & -0.51823900 & 0.85713300 & $\mathrm{C}$ & -2.17717400 & -0.11401800 & 2.63796400 \\
\hline $\mathrm{H}$ & 6.68163000 & -0.37937600 & 1.31105100 & $\mathrm{H}$ & -2.67543200 & 0.82107700 & 2.36561000 \\
\hline $\mathrm{H}$ & 5.59754200 & -1.59964600 & 0.68192900 & $\mathrm{H}$ & -2.50292000 & -0.38658700 & 3.64976400 \\
\hline $\mathrm{C}$ & 5.66649500 & 0.24103700 & -0.49270200 & $\mathrm{C}$ & -4.06132100 & -1.48181700 & 1.68654700 \\
\hline $\mathrm{H}$ & 6.69941200 & 0.43034100 & -0.81308000 & $\mathrm{H}$ & -4.37641000 & -1.69650300 & 2.71586900 \\
\hline \multirow[t]{2}{*}{$\mathrm{H}$} & 5.22270900 & 1.23349900 & -0.33637500 & $\mathrm{H}$ & -4.61042100 & -0.59364900 & 1.36221300 \\
\hline & & & & $\mathrm{H}$ & -4.36932800 & -2.33259900 & 1.07172900 \\
\hline \multirow[t]{5}{*}{ 45-ts } & & & & $\mathrm{F}$ & 0.69690100 & 5.23831600 & 0.04919800 \\
\hline & & & & $\mathrm{Rh}$ & 0.47170600 & -0.30196900 & -0.09565600 \\
\hline & & & & $\mathrm{Cl}$ & 0.97918400 & -2.63540400 & 0.22829200 \\
\hline & & & & $\mathrm{H}$ & -1.09777700 & 0.06702000 & 2.67100400 \\
\hline & & & & $\mathrm{H}$ & 0.23590000 & 0.70201600 & -1.27411300 \\
\hline
\end{tabular}




\begin{tabular}{|c|c|c|c|c|c|c|c|}
\hline $\mathrm{C}$ & 4.78731400 & -0.27742000 & 1.81411300 & $\mathrm{C}$ & -2.55051200 & -1.20480400 & 1.68297500 \\
\hline $\mathrm{C}$ & 3.28443800 & -0.58769000 & 1.60468500 & $\mathrm{C}$ & -3.97781400 & -1.49903800 & -1.83270100 \\
\hline $\mathrm{C}$ & 4.86632900 & -0.40499500 & -1.79475300 & $\mathrm{H}$ & -4.05883300 & -0.49133100 & -2.24830400 \\
\hline $\mathrm{C}$ & 2.62340000 & 0.35531700 & 0.62449800 & $\mathrm{H}$ & -4.30333600 & -2.20217300 & -2.60961600 \\
\hline $\mathrm{C}$ & 3.41815300 & -0.84941500 & -1.47442100 & $\mathrm{H}$ & -4.67617100 & -1.59378800 & -0.99687800 \\
\hline $\mathrm{C}$ & 2.66642000 & 0.23554700 & -0.74795200 & $\mathrm{C}$ & -1.63246000 & -1.59598300 & -2.68906800 \\
\hline $\mathrm{H}$ & 4.92014800 & 0.79130500 & 2.03787800 & $\mathrm{H}$ & -2.04893800 & -2.15647000 & -3.53597300 \\
\hline $\mathrm{H}$ & 3.16805900 & -1.62709500 & 1.29195600 & $\mathrm{H}$ & -1.60928200 & -0.53705100 & -2.97010000 \\
\hline $\mathrm{H}$ & 4.84797800 & 0.34174400 & -2.60121200 & $\mathrm{H}$ & -0.60893000 & -1.93967800 & -2.52096300 \\
\hline $\mathrm{H}$ & 2.91065300 & -1.09781400 & -2.41430100 & $\mathrm{C}$ & -2.42687900 & -3.33417900 & -1.06109600 \\
\hline $\mathrm{H}$ & 5.10191300 & -0.81687800 & 2.71758300 & $\mathrm{H}$ & -1.42152800 & -3.60326700 & -0.72545800 \\
\hline $\mathrm{H}$ & 2.77364600 & -0.49687400 & 2.57149700 & $\mathrm{H}$ & -3.14860100 & -3.60308900 & -0.28387600 \\
\hline $\mathrm{H}$ & 5.39645300 & -1.27784500 & -2.20001300 & $\mathrm{H}$ & -2.66330900 & -3.93826800 & -1.94632600 \\
\hline $\mathrm{H}$ & 2.34920800 & 1.31909000 & 1.04622800 & $\mathrm{C}$ & -1.89173900 & -2.48650600 & 2.23540300 \\
\hline $\mathrm{H}$ & 3.42654100 & -1.76447200 & -0.87917300 & $\mathrm{H}$ & -2.15927300 & -3.37925300 & 1.66620100 \\
\hline $\mathrm{H}$ & 2.45244100 & 1.12444000 & -1.34042800 & $\mathrm{H}$ & -0.80150800 & -2.42204900 & 2.24657400 \\
\hline $\mathrm{C}$ & 5.74266000 & -0.67498500 & 0.66972800 & $\mathrm{H}$ & -2.24307700 & -2.63227400 & 3.26529300 \\
\hline $\mathrm{H}$ & 6.76096700 & -0.60485500 & 1.07372200 & $\mathrm{C}$ & -2.17597400 & -0.02577700 & 2.61116800 \\
\hline $\mathrm{H}$ & 5.59564900 & -1.73687100 & 0.42556500 & $\mathrm{H}$ & -2.63893300 & 0.91549800 & 2.30137700 \\
\hline $\mathrm{C}$ & 5.67486000 & 0.17992400 & -0.61989800 & $\mathrm{H}$ & -2.52492000 & -0.25760400 & 3.62523200 \\
\hline $\mathrm{H}$ & 6.69507300 & 0.34580400 & -0.98963500 & $\mathrm{C}$ & -4.08245300 & -1.37595500 & 1.68946000 \\
\hline \multirow[t]{2}{*}{$\mathrm{H}$} & 5.29007400 & 1.17745400 & -0.36850600 & $\mathrm{H}$ & -4.40766300 & -1.55390300 & 2.72265200 \\
\hline & & & & $\mathrm{H}$ & -4.60464100 & -0.48189400 & 1.33643700 \\
\hline \multirow[t]{6}{*}{46} & & & & $\mathrm{H}$ & -4.40932600 & -2.23500700 & 1.09632100 \\
\hline & & & & $\mathrm{F}$ & 0.72453000 & 5.21273800 & 0.15551000 \\
\hline & & & & $\mathrm{Rh}$ & 0.43768100 & -0.29381100 & 0.01520300 \\
\hline & & & & $\mathrm{Cl}$ & 0.97747000 & -2.68710300 & 0.15798800 \\
\hline & & & & $\mathrm{H}$ & -1.09234400 & 0.12829200 & 2.66182700 \\
\hline & & & & $\mathrm{H}$ & 0.33744300 & -0.11150700 & -1.48343700 \\
\hline $\mathrm{C}$ & -2.19860100 & 800 & -0.22608600 & $\mathrm{C}$ & 4.87599100 & -0.44015700 & 1.72874300 \\
\hline $\mathrm{C}$ & -1.53045200 & 1.87389200 & -0.20210400 & $\mathrm{C}$ & 3.36153400 & -0.71553400 & 1.55340000 \\
\hline $\mathrm{C}$ & -0.15200500 & 1.66914100 & -0.02682300 & $\mathrm{C}$ & 4.80092900 & -0.23885900 & -1.87453900 \\
\hline $\mathrm{C}$ & 0.57941700 & 2.85802700 & 0.07979500 & $\mathrm{C}$ & 2.66912600 & 0.31306800 & 0.68750500 \\
\hline $\mathrm{C}$ & -0.06213200 & 4.11213900 & 0.03843200 & $\mathrm{C}$ & 3.36332500 & -0.70250300 & -1.53372800 \\
\hline $\mathrm{C}$ & -1.42542900 & 4.28776100 & -0.10897700 & $\mathrm{C}$ & 2.65471300 & 0.31916700 & -0.68534000 \\
\hline $\mathrm{C}$ & -3.59986900 & 2.81700100 & -0.36204400 & $\mathrm{H}$ & 5.03104300 & 0.60117300 & 2.04692800 \\
\hline $\mathrm{C}$ & -3.73244500 & 1.45400900 & -0.41096300 & $\mathrm{H}$ & 3.21570800 & -1.72198400 & 1.15484100 \\
\hline $\mathrm{H}$ & 1.65848500 & 2.87527100 & 0.19852900 & $\mathrm{H}$ & 4.75536500 & 0.57661500 & -2.60978900 \\
\hline $\mathrm{H}$ & -1.85332700 & 5.28442300 & -0.12477800 & $\mathrm{H}$ & 2.81503800 & -0.86205600 & -2.46974300 \\
\hline $\mathrm{H}$ & -4.41421900 & 3.52704200 & -0.42803900 & $\mathrm{H}$ & 5.21871400 & -1.06614500 & 2.56342000 \\
\hline $\mathrm{H}$ & -4.62876700 & 0.86436100 & -0.51927000 & $\mathrm{H}$ & 2.89395400 & -0.70689900 & 2.54629200 \\
\hline $\mathrm{N}$ & -2.47332800 & 0.8480 & -0.33748200 & $\mathrm{H}$ & 5.30430200 & -1.07569500 & -2.37758000 \\
\hline $\mathrm{P}$ & -1.84560300 & -0.74962800 & -0.04188000 & $\mathrm{H}$ & 2.37971800 & 1.22757300 & 1.20172200 \\
\hline $\mathrm{C}$ & -2.52305300 & -1.84525000 & -1.45182800 & $\mathrm{H}$ & 3.38600100 & -1.66686300 & -1.02185600 \\
\hline
\end{tabular}




\begin{tabular}{|c|c|c|c|c|c|c|c|}
\hline $\mathrm{H}$ & 2.37177000 & 1.24105300 & -1.18866200 & $\mathrm{H}$ & 1.20557600 & -1.90742500 & -2.47390000 \\
\hline $\mathrm{C}$ & 5.78007000 & -0.73486300 & 0.51367600 & $\mathrm{H}$ & 2.43937600 & -1.37682500 & -3.63708400 \\
\hline $\mathrm{H}$ & 6.81446300 & -0.70415900 & 0.87975100 & $\mathrm{C}$ & 1.43990500 & 0.87363200 & -2.56666300 \\
\hline $\mathrm{H}$ & 5.61654100 & -1.76991800 & 0.18071700 & $\mathrm{H}$ & 1.51725500 & 1.87672300 & -2.13792600 \\
\hline $\mathrm{C}$ & 5.66309900 & 0.23425200 & -0.68796800 & $\mathrm{H}$ & 1.71726800 & 0.93616800 & -3.62646100 \\
\hline $\mathrm{H}$ & 6.66736300 & 0.42959600 & -1.08565300 & $\mathrm{C}$ & 3.83648300 & 0.34334200 & -2.03098600 \\
\hline \multirow[t]{2}{*}{$\mathrm{H}$} & 5.29665700 & 1.20587400 & \multirow[t]{2}{*}{-0.33050900} & $\mathrm{H}$ & 4.06022700 & 0.43227600 & -3.10182600 \\
\hline & \multirow{7}{*}{$\mathrm{Cl} \quad \mathrm{CO}_{2} \mathrm{M}$} & \multirow{7}{*}{$\frac{1}{5}$} & & $\mathrm{H}$ & 3.99828400 & 1.32836900 & -1.58355600 \\
\hline \multirow[t]{6}{*}{47} & & & & $\mathrm{H}$ & 4.55831900 & -0.36139600 & -1.60800100 \\
\hline & & & & $\mathrm{F}$ & -2.84737000 & 4.21216200 & 0.83600300 \\
\hline & & & & $\mathrm{Rh}$ & -0.44894600 & -0.71646800 & 0.13876800 \\
\hline & & & & $\mathrm{Cl}$ & 0.00684800 & -3.10505000 & -0.29015100 \\
\hline & & & & $\mathrm{H}$ & 0.39153500 & 0.56010900 & -2.51624900 \\
\hline & & & & $\mathrm{C}$ & -3.26779800 & -0.42414000 & -1.17549400 \\
\hline $\mathrm{C}$ & 0.67495100 & 3.41224700 & 0.72514700 & $\mathrm{O}$ & -4.09926200 & 0.42178500 & -0.91711500 \\
\hline $\mathrm{C}$ & 0.53689900 & 2.01869300 & 0.55611900 & $\mathrm{O}$ & -2.78943600 & -0.64442100 & -2.42201500 \\
\hline $\mathrm{C}$ & -0.66946000 & 1.30785500 & 0.43449100 & $\mathrm{C}$ & -3.33511200 & 0.20315400 & -3.44846200 \\
\hline $\mathrm{C}$ & -1.80924800 & 2.11297500 & 0.54654200 & $\mathrm{H}$ & -3.11072400 & 1.25151000 & -3.23558500 \\
\hline $\mathrm{C}$ & -1.69389800 & 3.50419700 & 0.74151300 & $\mathrm{H}$ & -2.85560800 & -0.11591900 & -4.37377500 \\
\hline $\mathrm{C}$ & -0.49523100 & 4.18664300 & 0.83616300 & $\mathrm{H}$ & -4.41886500 & 0.07485300 & -3.51131100 \\
\hline $\mathrm{C}$ & 2.08894000 & 3.68266700 & 0.71905100 & $\mathrm{C}$ & -2.63653700 & -1.14282900 & 1.14614800 \\
\hline $\mathrm{C}$ & 2.74145100 & 2.49125500 & 0.54747300 & $\mathrm{H}$ & -2.97102500 & -0.16821000 & 1.49376500 \\
\hline $\mathrm{H}$ & -2.81550100 & 1.72161500 & 0.45521300 & $\mathrm{C}$ & -3.88353000 & -2.60129200 & 2.74764700 \\
\hline $\mathrm{H}$ & -0.48283200 & 5.26204300 & 0.97813300 & $\mathrm{H}$ & -4.51166600 & -3.02788600 & 1.95813100 \\
\hline $\mathrm{H}$ & 2.56788800 & 4.64591400 & 0.83930600 & $\mathrm{H}$ & -3.77673100 & -3.34976800 & 3.54023600 \\
\hline $\mathrm{H}$ & 3.80149700 & 2.29968200 & 0.50040200 & $\mathrm{H}$ & -4.41026800 & -1.73694800 & 3.16846400 \\
\hline $\mathrm{N}$ & 1.81234300 & 1.44552500 & 0.46923100 & $\mathrm{C}$ & -2.66368000 & -1.38357900 & -0.20424900 \\
\hline $\mathrm{P}$ & 1.81364900 & -0.21284200 & -0.04536800 & $\mathrm{H}$ & -0.21517900 & -0.65496200 & 1.63248500 \\
\hline $\mathrm{C}$ & 3.04427500 & -1.11771400 & 1.10254400 & $\mathrm{H}$ & -2.46106600 & -2.37856200 & -0.59031900 \\
\hline $\mathrm{C}$ & 2.38117800 & -0.14039800 & -1.87621100 & $\mathrm{C}$ & -2.49509300 & -2.20893600 & 2.20103400 \\
\hline $\mathrm{C}$ & 4.27757700 & -0.25386100 & 1.44243800 & $\mathrm{H}$ & -1.88197800 & -1.82929800 & 3.02745300 \\
\hline $\mathrm{H}$ & 4.00747600 & 0.63049500 & 2.02491700 & $\mathrm{H}$ & -1.98395600 & -3.08238900 & 1.78698400 \\
\hline $\mathrm{H}$ & 4.95580600 & -0.85856300 & 2.05716400 & & & & \\
\hline $\mathrm{H}$ & 4.83778100 & 0.06260600 & 0.55827300 & 48-ts & & & \\
\hline $\mathrm{C}$ & 2.29737000 & -1.42714800 & 2.41852900 & & & & \\
\hline $\mathrm{H}$ & 3.00969600 & -1.87163700 & 3.12537100 & & & & \\
\hline $\mathrm{H}$ & 1.89100000 & -0.52117200 & 2.88202100 & & & & \\
\hline $\mathrm{H}$ & 1.48425200 & -2.13893100 & 2.25723800 & & $\mathrm{Cl}$ & & \\
\hline $\mathrm{C}$ & 3.50689300 & -2.44619500 & 0.46896300 & & & & \\
\hline $\mathrm{H}$ & 2.66323800 & -3.06638800 & 0.15600100 & $\mathrm{C}$ & -0.80627600 & 3.41028200 & -0.59587900 \\
\hline $\mathrm{H}$ & 4.17220300 & -2.28635600 & -0.38508500 & $\mathrm{C}$ & -0.63059100 & 2.01478900 & -0.45598800 \\
\hline $\mathrm{H}$ & 4.07690900 & -3.00355100 & 1.22318800 & $\mathrm{C}$ & 0.60089000 & 1.33135000 & -0.45497100 \\
\hline $\mathrm{C}$ & 2.21797200 & -1.50895200 & -2.56993900 & $\mathrm{C}$ & 1.70626800 & 2.16711600 & -0.65688400 \\
\hline $\mathrm{H}$ & 2.90490300 & -2.26340000 & -2.18151400 & $\mathrm{C}$ & 1.54862400 & 3.55748700 & -0.81521800 \\
\hline
\end{tabular}




\begin{tabular}{|c|c|c|c|c|c|c|c|}
\hline $\mathrm{C}$ & 0.33240300 & 4.21335300 & -0.79083400 & $\mathrm{H}$ & 4.46987600 & 0.37680300 & 3.42566600 \\
\hline $\mathrm{C}$ & -2.21991600 & 3.65662600 & -0.48973700 & $\mathrm{C}$ & 2.57922500 & -1.06276900 & -1.15658700 \\
\hline $\mathrm{C}$ & -2.83878400 & 2.45045800 & -0.29849200 & $\mathrm{H}$ & 3.07105200 & -0.14726600 & -1.49104500 \\
\hline $\mathrm{H}$ & 2.72513100 & 1.79832500 & -0.65489400 & $\mathrm{C}$ & 4.33887100 & -2.62941700 & -2.04776700 \\
\hline $\mathrm{H}$ & 0.28301800 & 5.29011200 & -0.91325000 & $\mathrm{H}$ & 4.68374600 & -2.89712100 & -1.04271800 \\
\hline $\mathrm{H}$ & -2.71956100 & 4.61442900 & -0.55683200 & $\mathrm{H}$ & 4.52620500 & -3.48284100 & -2.70824000 \\
\hline $\mathrm{H}$ & -3.88954700 & 2.23472200 & -0.18664900 & $\mathrm{H}$ & 4.94940800 & -1.78984500 & -2.40033500 \\
\hline $\mathrm{N}$ & -1.88908400 & 1.42420800 & -0.29097600 & $\mathrm{C}$ & 2.53414000 & -1.21089100 & 0.27262300 \\
\hline$P$ & -1.87599100 & -0.27201300 & 0.06637200 & $\mathrm{H}$ & 1.34477300 & -0.71806100 & -1.61379300 \\
\hline $\mathrm{C}$ & -3.03795800 & -1.08305200 & -1.21314600 & $\mathrm{H}$ & 2.46639700 & -2.21537200 & 0.68450900 \\
\hline $\mathrm{C}$ & -2.49229800 & -0.38846400 & 1.87273800 & $\mathrm{C}$ & 2.84318800 & -2.28093600 & -2.04566100 \\
\hline $\mathrm{C}$ & -4.30620900 & -0.25225200 & -1.49545300 & $\mathrm{H}$ & 2.51454700 & -2.06299800 & -3.06939300 \\
\hline $\mathrm{H}$ & -4.06819100 & 0.71735300 & -1.94071800 & $\mathrm{H}$ & 2.24697500 & -3.12291000 & -1.68349400 \\
\hline $\mathrm{H}$ & -4.92254000 & -0.80341200 & -2.21713400 & & & & \\
\hline $\mathrm{H}$ & -4.91856000 & -0.09025600 & -0.60364700 & 49 & & & \\
\hline $\mathrm{C}$ & -2.21670300 & -1.18371400 & -2.51826700 & & & & \\
\hline $\mathrm{H}$ & -2.85742500 & -1.59753800 & -3.30772400 & & & & \\
\hline $\mathrm{H}$ & -1.86362000 & -0.20228700 & -2.85423100 & & & & \\
\hline $\mathrm{H}$ & -1.35336900 & -1.84304500 & -2.39397500 & & & & \\
\hline $\mathrm{C}$ & -3.43526000 & -2.50033300 & -0.75422000 & & & & \\
\hline $\mathrm{H}$ & -2.56381800 & -3.10092000 & -0.47982900 & $\mathrm{C}$ & -0.67276100 & 3.42050600 & 0.11375700 \\
\hline $\mathrm{H}$ & -4.13584200 & -2.47901100 & 0.08673500 & $\mathrm{C}$ & -0.53707800 & 2.02961600 & -0.05635900 \\
\hline $\mathrm{H}$ & -3.94418800 & -3.00193300 & -1.58737300 & $\mathrm{C}$ & 0.50036200 & 1.36360600 & -0.72009100 \\
\hline $\mathrm{C}$ & -2.28069700 & -1.81384000 & 2.42481300 & $\mathrm{C}$ & 1.47074000 & 2.20781700 & -1.26587000 \\
\hline $\mathrm{H}$ & -2.92677600 & -2.55132400 & 1.94369000 & $\mathrm{C}$ & 1.36158000 & 3.60562700 & -1.10752300 \\
\hline $\mathrm{H}$ & -1.24939400 & -2.15487300 & 2.30903500 & $\mathrm{C}$ & 0.33374600 & 4.24354300 & -0.43256200 \\
\hline $\mathrm{H}$ & -2.52700000 & -1.80468700 & 3.49462800 & $\mathrm{C}$ & -1.90102100 & 3.61222900 & 0.84395300 \\
\hline $\mathrm{C}$ & -1.60126000 & 0.58586700 & 2.67678900 & $\mathrm{C}$ & -2.45381000 & 2.37630200 & 1.06701900 \\
\hline $\mathrm{H}$ & -1.71248200 & 1.62271300 & 2.34628200 & $\mathrm{H}$ & 2.32420200 & 1.82152500 & -1.81554600 \\
\hline $\mathrm{H}$ & -1.89407000 & 0.53360600 & 3.73301800 & $\mathrm{H}$ & 0.32355600 & 5.32531200 & -0.35144000 \\
\hline $\mathrm{C}$ & -3.96984700 & 0.01462800 & 2.04640700 & $\mathrm{H}$ & -2.32666900 & 4.55280600 & 1.16964100 \\
\hline $\mathrm{H}$ & -4.21947900 & -0.02576400 & 3.11461700 & $\mathrm{H}$ & -3.37541200 & 2.12531400 & 1.57011100 \\
\hline $\mathrm{H}$ & -4.16844800 & 1.03557800 & 1.70687900 & $\mathrm{~N}$ & -1.62913300 & 1.38271000 & 0.53077300 \\
\hline $\mathrm{H}$ & -4.65067200 & -0.66855400 & 1.52974000 & $\mathrm{P}$ & -1.68854800 & -0.32702800 & 0.20454700 \\
\hline $\mathrm{F}$ & 2.67528400 & 4.29161300 & -0.99611300 & $\mathrm{C}$ & -3.01461300 & -0.52204700 & -1.16286000 \\
\hline $\mathrm{Rh}$ & 0.52057200 & -0.71207500 & -0.20890300 & $\mathrm{C}$ & -2.18384200 & -1.15261500 & 1.84825300 \\
\hline $\mathrm{Cl}$ & 0.09709600 & -3.13141500 & 0.10423200 & $\mathrm{C}$ & -4.23599700 & 0.39419100 & -0.95804200 \\
\hline $\mathrm{H}$ & -0.54131000 & 0.31517900 & 2.61243900 & $\mathrm{H}$ & -3.95454300 & 1.45039000 & -0.92634100 \\
\hline $\mathrm{C}$ & 3.19963600 & -0.22602900 & 1.17567100 & $\mathrm{H}$ & -4.91783400 & 0.25689600 & -1.80725800 \\
\hline $\mathrm{O}$ & 4.03025500 & 0.59337200 & 0.83570400 & $\mathrm{H}$ & -4.79887300 & 0.15893100 & -0.05043500 \\
\hline $\mathrm{O}$ & 2.79121300 & -0.39346300 & 2.45054100 & $\mathrm{C}$ & -2.29912800 & -0.10311400 & -2.47028700 \\
\hline $\mathrm{C}$ & 3.38382600 & 0.50040100 & 3.40896600 & $\mathrm{H}$ & -3.00842500 & -0.17829800 & -3.30498600 \\
\hline $\mathrm{H}$ & 3.14491000 & 1.53747700 & 3.15952000 & $\mathrm{H}$ & -1.93354400 & 0.92780400 & -2.43378700 \\
\hline $\mathrm{H}$ & 2.95038800 & 0.22412900 & 4.37007900 & $\mathrm{H}$ & -1.45987400 & -0.77248800 & -2.70633900 \\
\hline
\end{tabular}




\begin{tabular}{|c|c|c|c|c|c|c|c|}
\hline $\mathrm{C}$ & -3.46026000 & -1.99223600 & -1.29144800 & $\mathrm{C}$ & 1.60917300 & 2.69018000 & 1.69185600 \\
\hline $\mathrm{H}$ & -2.60750700 & -2.67069100 & -1.39174600 & $\mathrm{C}$ & 1.10132800 & 1.59649500 & 0.96026500 \\
\hline $\mathrm{H}$ & -4.07463000 & -2.31461900 & -0.44509300 & $\mathrm{C}$ & -0.07182600 & 1.60771500 & 0.19556200 \\
\hline $\mathrm{H}$ & -4.07713400 & -2.08930000 & -2.19425600 & $\mathrm{C}$ & -0.74796000 & 2.82195700 & 0.15222800 \\
\hline $\mathrm{C}$ & -1.94069100 & -2.67447400 & 1.75429900 & $\mathrm{C}$ & -0.25023900 & 3.92634000 & 0.87970000 \\
\hline $\mathrm{H}$ & -2.61952600 & -3.16470900 & 1.05339100 & $\mathrm{C}$ & 0.89224200 & 3.90347300 & 1.65700200 \\
\hline $\mathrm{H}$ & -0.92283500 & -2.91404300 & 1.43835300 & $\mathrm{C}$ & 2.82960000 & 2.22242400 & 2.29858500 \\
\hline $\mathrm{H}$ & -2.11317200 & -3.11119300 & 2.74655900 & $\mathrm{C}$ & 3.02190600 & 0.92283700 & 1.90265900 \\
\hline $\mathrm{C}$ & -1.26834600 & -0.57492800 & 2.94766400 & $\mathrm{H}$ & -1.64494700 & 2.97084000 & -0.43478400 \\
\hline $\mathrm{H}$ & -1.36825300 & 0.50952500 & 3.05021700 & $\mathrm{H}$ & 1.20775100 & 4.79270200 & 2.19191300 \\
\hline $\mathrm{H}$ & -1.54248200 & -1.03419400 & 3.90560400 & $\mathrm{H}$ & 3.48291600 & 2.78090800 & 2.95630500 \\
\hline $\mathrm{C}$ & -3.65425900 & -0.89549100 & 2.23976900 & $\mathrm{H}$ & 3.81908800 & 0.24055100 & 2.15938400 \\
\hline $\mathrm{H}$ & -3.83864500 & -1.36787600 & 3.21306900 & $\mathrm{~N}$ & 1.96638900 & 0.51246300 & 1.08695600 \\
\hline $\mathrm{H}$ & -3.88590800 & 0.16820600 & 2.34974900 & $\mathrm{P}$ & 1.64429300 & -0.75665400 & -0.06278700 \\
\hline $\mathrm{H}$ & -4.35903300 & -1.33373800 & 1.52888100 & $\mathrm{C}$ & 1.69318700 & -2.40029700 & 0.91561400 \\
\hline $\mathrm{F}$ & 2.34213300 & 4.37042000 & -1.65356100 & $\mathrm{C}$ & 3.06310500 & -0.60169900 & -1.33890900 \\
\hline $\mathrm{Rh}$ & 0.34610100 & -0.65865600 & -0.79315000 & $\mathrm{C}$ & 3.09768400 & -2.97431200 & 1.17949800 \\
\hline $\mathrm{Cl}$ & 0.10268700 & -3.07786800 & -1.17816900 & $\mathrm{H}$ & 3.74951800 & -2.26825400 & 1.70411800 \\
\hline $\mathrm{H}$ & -0.21891600 & -0.80965400 & 2.75729300 & $\mathrm{H}$ & 2.99854900 & -3.86338000 & 1.81563900 \\
\hline $\mathrm{H}$ & 1.28194200 & 0.62692500 & 2.10035800 & $\mathrm{H}$ & 3.59504100 & -3.28903700 & 0.25844100 \\
\hline $\mathrm{C}$ & 2.74782600 & -1.24617000 & -0.37438900 & $\mathrm{C}$ & 1.00369100 & -2.15659100 & 2.27378700 \\
\hline $\mathrm{O}$ & 2.43786500 & -0.90430400 & -1.52860800 & $\mathrm{H}$ & 0.89539900 & -3.11889800 & 2.78980000 \\
\hline $\mathrm{O}$ & 3.87904300 & -1.85712500 & -0.07852800 & $\mathrm{H}$ & 1.58419800 & -1.48845200 & 2.91669500 \\
\hline $\mathrm{C}$ & 4.75082600 & -2.18432900 & -1.19034600 & $\mathrm{H}$ & 0.00423000 & -1.72907800 & 2.15483400 \\
\hline $\mathrm{H}$ & 5.08986000 & -1.26998400 & -1.68228400 & $\mathrm{C}$ & 0.86764000 & -3.42812600 & 0.10728400 \\
\hline $\mathrm{H}$ & 5.58884400 & -2.71869400 & -0.74512400 & $\mathrm{H}$ & -0.17338500 & -3.11352300 & -0.01423600 \\
\hline $\mathrm{H}$ & 4.21625100 & -2.81469600 & -1.90324500 & $\mathrm{H}$ & 1.28979500 & -3.61482700 & -0.88521500 \\
\hline $\mathrm{C}$ & 1.74719200 & -0.94640600 & 0.69962900 & $\mathrm{H}$ & 0.86346900 & -4.38368600 & 0.64777800 \\
\hline $\mathrm{H}$ & 1.47871600 & -1.85799000 & 1.24250500 & $\mathrm{C}$ & 2.86390900 & -1.68222700 & -2.42222000 \\
\hline $\mathrm{C}$ & 2.17465800 & 0.18031600 & 1.65069300 & $\mathrm{H}$ & 3.03334500 & -2.69304600 & -2.03438500 \\
\hline $\mathrm{H}$ & 2.65477200 & 0.98617100 & 1.08490400 & $\mathrm{H}$ & 1.86646300 & -1.62990000 & -2.86837600 \\
\hline $\mathrm{C}$ & 3.11379500 & -0.30855800 & 2.77092900 & $\mathrm{H}$ & 3.59565300 & -1.51583000 & -3.22318300 \\
\hline $\mathrm{H}$ & 2.60871700 & -1.09588400 & 3.34876800 & $\mathrm{C}$ & 2.90649000 & 0.80324700 & -1.96685600 \\
\hline $\mathrm{H}$ & 4.00305700 & -0.77536200 & 2.33011500 & $\mathrm{H}$ & 3.08106300 & 1.59444600 & -1.23016300 \\
\hline $\mathrm{C}$ & 3.53667300 & 0.82741300 & 3.70909800 & $\mathrm{H}$ & 3.66286200 & 0.91188800 & -2.75506500 \\
\hline $\mathrm{H}$ & 2.66614700 & 1.29417200 & 4.18620100 & $\mathrm{C}$ & 4.49101000 & -0.69100800 & -0.76148800 \\
\hline $\mathrm{H}$ & 4.19648000 & 0.46011200 & 4.50326100 & $\mathrm{H}$ & 5.19531200 & -0.48063800 & -1.57675300 \\
\hline \multirow[t]{2}{*}{$\mathrm{H}$} & 4.07461500 & 1.61191400 & 3.16330600 & $\mathrm{H}$ & 4.66764200 & 0.05970000 & 0.01417900 \\
\hline & & & & $\mathrm{H}$ & 4.74332600 & -1.67491600 & -0.36459000 \\
\hline \multirow[t]{5}{*}{ 50-ts } & & & & $\mathrm{F}$ & -0.96122600 & 5.07854300 & 0.80257800 \\
\hline & & & & $\mathrm{Rh}$ & -0.61715000 & -0.12664300 & -0.66640700 \\
\hline & & & & $\mathrm{Cl}$ & -0.38919100 & -0.00411600 & -3.04941300 \\
\hline & & & & $\mathrm{H}$ & 1.92396500 & 0.94757200 & -2.41874100 \\
\hline & & & & $\mathrm{C}$ & -2.97553200 & -1.59760200 & 1.01359000 \\
\hline
\end{tabular}




\begin{tabular}{|c|c|c|c|c|c|c|c|}
\hline $\mathrm{O}$ & -2.65249600 & -2.66118000 & 0.52590800 & $\mathrm{H}$ & -4.62843100 & 0.11181500 & -1.10664300 \\
\hline $\mathrm{O}$ & -3.76802600 & -1.46565400 & 2.09097600 & $\mathrm{C}$ & -1.54595000 & -0.05160700 & -2.72505400 \\
\hline $\mathrm{C}$ & -4.26473900 & -2.69670000 & 2.65112300 & $\mathrm{H}$ & -1.99675600 & -0.10610300 & -3.72484100 \\
\hline $\mathrm{H}$ & -3.43458500 & -3.32825700 & 2.97706400 & $\mathrm{H}$ & -1.22362100 & 0.98093600 & -2.55965800 \\
\hline $\mathrm{H}$ & -4.88078100 & -2.40037500 & 3.49972800 & $\mathrm{H}$ & -0.66273600 & -0.70386800 & -2.73683900 \\
\hline $\mathrm{H}$ & -4.85971400 & -3.23759100 & 1.91103700 & $\mathrm{C}$ & -2.95221500 & -1.98875500 & -1.97439200 \\
\hline $\mathrm{C}$ & -2.76488200 & -0.02308700 & -0.93035900 & $\mathrm{H}$ & -2.09317300 & -2.65426900 & -1.84740400 \\
\hline $\mathrm{H}$ & -2.93165100 & -0.92943000 & -1.51736000 & $\mathrm{H}$ & -3.77614300 & -2.34743700 & -1.35003500 \\
\hline $\mathrm{C}$ & -4.90565800 & 0.94403000 & -1.86319100 & $\mathrm{H}$ & -3.28436400 & -2.06053200 & -3.01833500 \\
\hline $\mathrm{H}$ & -5.48800700 & 0.66418800 & -0.97698400 & $\mathrm{C}$ & -2.36774200 & -2.73133200 & 1.39371200 \\
\hline $\mathrm{H}$ & -5.36094600 & 1.84124200 & -2.29745800 & $\mathrm{H}$ & -2.76438600 & -3.22473000 & 0.50373900 \\
\hline $\mathrm{H}$ & -4.99588000 & 0.13425300 & -2.59643500 & $\mathrm{H}$ & -1.29492500 & -2.93365700 & 1.41900800 \\
\hline $\mathrm{C}$ & -2.57609900 & -0.24254000 & 0.47961500 & $\mathrm{H}$ & -2.83341500 & -3.19827000 & 2.27126800 \\
\hline $\mathrm{H}$ & -1.16731100 & -0.38188800 & 0.86113700 & $\mathrm{C}$ & -2.21110100 & -0.62571900 & 2.76369700 \\
\hline $\mathrm{H}$ & -2.80637900 & 0.58961300 & 1.14335800 & $\mathrm{H}$ & -2.41352000 & 0.44633000 & 2.83890400 \\
\hline $\mathrm{C}$ & -3.43040300 & 1.20666200 & -1.50829700 & $\mathrm{H}$ & -2.75205300 & -1.13121600 & 3.57363200 \\
\hline $\mathrm{H}$ & -2.88843300 & 1.50920300 & -2.41148700 & $\mathrm{C}$ & -4.21339400 & -1.02659900 & 1.32140600 \\
\hline \multirow[t]{2}{*}{$\mathrm{H}$} & -3.37520500 & 2.03610500 & -0.79530800 & $\mathrm{H}$ & -4.67739100 & -1.52033300 & 2.18484500 \\
\hline & & & & $\mathrm{H}$ & -4.51176300 & 0.02565900 & 1.35471500 \\
\hline \multirow[t]{6}{*}{51} & & & & $\mathrm{H}$ & -4.63921300 & -1.47918200 & 0.42313000 \\
\hline & & & & $\mathrm{F}$ & 2.47062800 & 4.47112400 & -0.86582600 \\
\hline & & & & $\mathrm{Rh}$ & 0.54374500 & -0.62985900 & -0.36544600 \\
\hline & & & & $\mathrm{Cl}$ & 0.41739100 & -3.04471200 & -0.94629600 \\
\hline & & & & $\mathrm{H}$ & -1.14450600 & -0.78262800 & 2.93354000 \\
\hline & & & & $\mathrm{C}$ & 3.26596200 & -1.32120700 & 0.07893700 \\
\hline $\mathrm{C}$ & -0.83428900 & 3.41508200 & 0.17139700 & $\mathrm{O}$ & 2.67115800 & -0.71030700 & -0.81997600 \\
\hline $\mathrm{C}$ & -0.60018000 & 2.02790400 & 0.09959900 & $\mathrm{O}$ & 4.56316500 & -1.57144600 & 0.01851500 \\
\hline $\mathrm{C}$ & 0.59874400 & 1.39593500 & -0.25693500 & $\mathrm{C}$ & 5.25485800 & -1.14460800 & -1.17730200 \\
\hline $\mathrm{C}$ & 1.62623000 & 2.27920300 & -0.60294700 & $\mathrm{H}$ & 5.20246200 & -0.05789500 & -1.27464200 \\
\hline $\mathrm{C}$ & 1.42223400 & 3.67306000 & -0.53322400 & $\mathrm{H}$ & 6.28508300 & -1.47118100 & -1.04146100 \\
\hline $\mathrm{C}$ & 0.23702900 & 4.27534800 & -0.14618100 & $\mathrm{H}$ & 4.80809600 & -1.61549400 & -2.05552800 \\
\hline $\mathrm{C}$ & -2.21795600 & 3.56523700 & 0.54671700 & $\mathrm{C}$ & 1.25476000 & -1.04143400 & 1.54672200 \\
\hline $\mathrm{C}$ & -2.76018200 & 2.30980800 & 0.65661500 & $\mathrm{C}$ & 2.72500700 & 0.93884700 & 2.45407300 \\
\hline $\mathrm{H}$ & 2.60020900 & 1.92383500 & -0.92573800 & $\mathrm{H}$ & 2.77309900 & 1.46704300 & 1.49940000 \\
\hline $\mathrm{H}$ & 0.15815000 & 5.35692200 & -0.11567500 & $\mathrm{H}$ & 2.74305000 & 1.69163900 & 3.25025700 \\
\hline $\mathrm{H}$ & -2.75056300 & 4.49206100 & 0.71781800 & $\mathrm{H}$ & 3.63333200 & 0.33396900 & 2.56452800 \\
\hline $\mathrm{H}$ & -3.77119600 & 2.02608000 & 0.90788000 & $\mathrm{C}$ & 2.54260100 & -1.85455000 & 1.27633100 \\
\hline $\mathrm{N}$ & -1.78357000 & 1.34410400 & 0.39700000 & $\mathrm{H}$ & 3.21501500 & -1.89875800 & 2.14209600 \\
\hline $\mathrm{P}$ & -1.71462300 & -0.35667400 & 0.01913400 & $\mathrm{H}$ & 2.26720200 & -2.88247000 & 1.01015800 \\
\hline $\mathrm{C}$ & -2.59004400 & -0.51824700 & -1.68175000 & $\mathrm{C}$ & 1.45118200 & 0.09224100 & 2.56532900 \\
\hline $\mathrm{C}$ & -2.68487700 & -1.22022300 & 1.41915700 & $\mathrm{H}$ & 1.44934000 & -0.38210500 & 3.56295700 \\
\hline $\mathrm{C}$ & -3.83768600 & 0.37633600 & -1.81307600 & $\mathrm{H}$ & 0.57889800 & 0.75304400 & 2.54520100 \\
\hline $\mathrm{H}$ & -3.59894900 & 1.43567700 & -1.68386200 & $\mathrm{H}$ & 0.50369300 & -1.73291600 & 1.93383000 \\
\hline $\mathrm{H}$ & -4.24853200 & 0.24877000 & -2.82301800 & & & & \\
\hline
\end{tabular}


52-ts
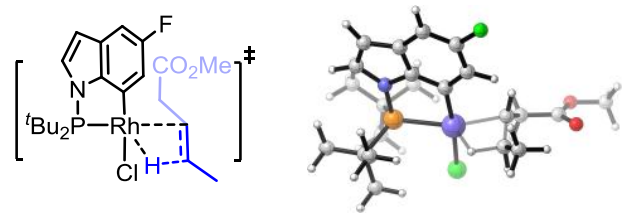

C $\quad-2.05724300$

C $\quad-1.45472000$

C $\quad-0.13743800$

C $\quad 0.57922800$

C $\quad 0.00462600$

C $\quad-1.29196100$

C $\quad-3.41155400$

C $\quad-3.58735500$

$\mathrm{H} \quad 1.60761400$

H $\quad-1.67965800$

H $\quad-4.16462200$

H $\quad-4.46512600$

$\mathrm{N} \quad-2.40030900$

P $\quad-1.90905400$

C $\quad-2.14003900$

C $\quad-3.06094900$

C $\quad-3.42620900$

$\mathrm{H} \quad-3.43540300$

H $\quad-3.46957200$

H $\quad-4.33586000$

C $\quad-0.92137400$

H -1.01019500

H $\quad-0.86580500$

3.07037500

1.83433600

1.64680000

2.84674200

4.08649200

4.24562600

2.77655600

1.42235800

2.88530700

5.23085300

3.48526200

0.83212300

0.81888500

$-0.76694100$

$-1.86940300$

$-1.20914400$

$-1.56874800$

$-0.54794100$

$-2.25110500$

$-1.72792400$

$-2.11597400$

$-0.48905800$

$-1.85354700$

$-3.35976400$

$-3.60239000$

$-3.66250200$

$-3.96004900$

$-2.45072900$

$-3.35851500$

$-2.32798600$

$-2.60896700$

0.00110800

0.90837400

$-0.23297200$

$-1.45877500$

$-1.65058500$

$-0.59419900$

$\mathrm{H} \quad-5.11809800$
$-1.55386800$
$-0.74394100$

$-0.41309000$

0.05580900

0.15969200

$-0.17871000$

$-0.62657700$

$-1.12931000$

$-1.02781500$

0.50011700

$-0.86249000$

$-1.44889000$

$-1.24031900$

$-0.60600400$

$-0.10698600$

$-1.64544600$

1.35265200

$-2.44011000$

$-2.83155700$

$-3.29871000$

$-1.85398000$

$-2.54328000$

$-3.48207000$

$-2.79712100$

$-2.06009000$

$-0.64498000$

$-0.70951400$

$-2.16893700$

2.08867900

1.48414700

2.38746700

2.99029900

2.31129000

1.85797200

3.20194700

0.92972200

1.83096600

0.42717500
$-1.25094700$

H $\quad-4.62334100$

$-2.33382200$

0.28123300

F $\quad 0.78796100$

5.18778200

$-0.04628000$

$\mathrm{Rh} \quad 0.42471900$

$-0.29415700$

0.45311900

$\mathrm{Cl} \quad 0.85573900$

$-2.70031600$

0.84394500

H -1.98412900

0.21321600

2.63683000

C $\quad 4.75477800$

$-0.52334600$

$-0.50535800$

O $\quad 5.30934600$

0.06231900

0.40200600

O $\quad 5.40244900$

$-1.05312300$

$-1.56127600$

C $\quad 6.83383200$

$-0.91411600$

$-1.54259200$

H 7.25469600

$-1.40195200$

$-0.65944300$

H 7.18525200

$-1.39911000$

$-2.45355500$

H 7.11580100

0.14204100

$-1.53486200$

C 2.49592800

0.21473600

0.23976500

H 2.57920500

1.23206300

$-0.13494700$

C 2.68476200

1.20693400

2.62191500

$\mathrm{H} \quad 2.25533900$

2.16121100

2.31313400

H 2.34249700

0.98574600

3.63774700

H 3.77606000

1.29864600

2.63124300

C $\quad 3.25691200$

$-0.78904000$

$-0.59126600$

H 3.07063300

$-1.81075100$

$-0.24189800$

H 2.94796900

$-0.75065200$

$-1.64164400$

C $\quad 2.33305600$

0.06862100

1.66314500

H 2.61132500

$-0.91721700$

2.03867200

H 1.01341200

0.03058900

1.94077200

53
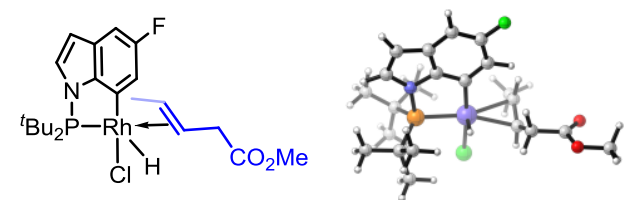

3.42344500

0.01913800

C $\quad-0.98307500$

2.03881000

$-0.06388300$

C $\quad 0.25779800$

1.43754300

$-0.34107000$

C $\quad 1.28508400$

2.36918600

$-0.53779400$

C $\quad 1.04189200$

3.75600600

$-0.47851000$

C $\quad-0.18868300$

4.32132500

$-0.20705900$

C $\quad-2.64014200$

3.54992600

0.36407500

C $\quad-3.15696500$

2.28633900

0.47726800

H 2.31083500

2.08331000

$-0.74123200$

H $\quad-0.30474400$

5.39899100

$-0.16526300$

H $\quad-3.19354400$

4.46861500

0.50962800

H $\quad-4.16522100$

1.98310400

0.71232000

N

$-2.16407700$

1.34075200

0.20768900

$\mathrm{P}$

$-2.01319800$

$-0.38777100$

0.25583200 


\begin{tabular}{|c|c|c|c|c|c|c|c|}
\hline C & -3.21570200 & -1.01232000 & -1.09590000 & $\mathrm{C}$ & 2.25331700 & -0.60450000 & -1.57962700 \\
\hline $\mathrm{C}$ & -2.52792900 & -0.87939400 & 2.02831200 & $\mathrm{H}$ & 2.49068900 & 0.44160600 & -1.74304300 \\
\hline C & -4.52816600 & -0.20500000 & -1.15241200 & $\mathrm{Rh}$ & 0.23939600 & -0.62582400 & -0.33995700 \\
\hline $\mathrm{H}$ & -4.35303400 & 0.84676600 & -1.39247000 & $\mathrm{H}$ & 0.58109100 & -0.3773 & 1.11321500 \\
\hline $\mathrm{H}$ & -5.15400500 & -0.62650000 & -1.94934200 & & & & \\
\hline $\mathrm{H}$ & -5.10455600 & -0.26280200 & -0.22480600 & 54-ts & & & \\
\hline $\mathrm{C}$ & -2.46259100 & -0.81589600 & -2.43220600 & & & & \\
\hline $\mathrm{H}$ & -3.13170900 & -1.08368100 & -3.26024100 & & & & \\
\hline $\mathrm{H}$ & -2.15147400 & 0.22450000 & -2.58089500 & & & & \\
\hline $\mathrm{H}$ & -1.58376900 & -1.46589600 & -2.49221600 & & & & \\
\hline $\mathrm{C}$ & -3.53472400 & -2.50967600 & -0.91297900 & & & & \\
\hline $\mathrm{H}$ & -2.62776400 & -3.11344900 & -0.82821000 & $\mathrm{C}$ & 0700 & 00 & -0.15259900 \\
\hline $\mathrm{H}$ & -4.16965100 & -2.69258100 & -0.04051100 & $\mathrm{C}$ & -0.90586400 & 8900 & -0.15234900 \\
\hline $\mathrm{H}$ & -4.09037100 & -2.85307500 & -1.79519300 & $\mathrm{C}$ & 0.32476100 & 1.34785900 & -0.25839200 \\
\hline $\mathrm{C}$ & -2.12208800 & -2.34272900 & 2.29981700 & $\mathrm{C}$ & 1.41378100 & 2.22086100 & -0.39389400 \\
\hline $\mathrm{H}$ & -2.67764900 & -3.05352000 & 1.68383300 & $\mathrm{C}$ & 1.25117500 & 3.61949900 & -0.41040100 \\
\hline $\mathrm{H}$ & -1.05896900 & -2.51306900 & 2.11754200 & $\mathrm{C}$ & 0.03508000 & 4.26168100 & -0.29149700 \\
\hline $\mathrm{H}$ & -2.33843400 & -2.57024800 & 3.35178400 & $\mathrm{C}$ & -2.50304200 & 3.65731300 & 0.01655300 \\
\hline $\mathrm{C}$ & -1.74801700 & 0.04155000 & 2.99285900 & $\mathrm{C}$ & -3.11096700 & 2.43342700 & 0.10821400 \\
\hline $\mathrm{H}$ & -1.99310600 & 1.09826200 & 2.85141800 & $\mathrm{H}$ & 2.43457900 & 1.87300600 & -0.50785100 \\
\hline $\mathrm{H}$ & -2.01189700 & -0.23261200 & 4.02188200 & $\mathrm{H}$ & -0.02401400 & 5.34471500 & -0.30347600 \\
\hline $\mathrm{C}$ & -4.03778000 & -0.70572800 & 2.29207100 & $\mathrm{H}$ & -3.00772400 & 4.61389800 & 0.05870400 \\
\hline $\mathrm{H}$ & -4.23839100 & -0.96641600 & 3.33896600 & $\mathrm{H}$ & -4.15746900 & 2.20155500 & 0.23017100 \\
\hline $\mathrm{H}$ & -4.37258600 & 0.32579600 & 2.14786500 & $\mathrm{~N}$ & -2.15811600 & 1.41920800 & -0.00711200 \\
\hline $\mathrm{H}$ & -4.65119800 & -1.36440100 & 1.67146400 & $\mathrm{P}$ & -2.13500200 & -0.30715300 & 0.16340400 \\
\hline F & 2.10140200 & 4.57651500 & -0.69567200 & $\mathrm{C}$ & -3.28738400 & -0.96059500 & -1.20833000 \\
\hline $\mathrm{Cl}$ & -0.01130100 & -3.07530100 & -0.47613300 & $\mathrm{C}$ & -2.76714500 & -0.62987300 & 1.93884200 \\
\hline $\mathrm{H}$ & -0.66578400 & -0.07869500 & 2.88544100 & $\mathrm{C}$ & -4.56689800 & -0.12324700 & -1.40198700 \\
\hline $\mathrm{C}$ & 4.82118000 & -0.59669400 & 0.53179300 & $\mathrm{H}$ & -4.34171100 & 0.90188800 & -1.70797700 \\
\hline $\mathrm{O}$ & 5.37684600 & -0.80887000 & -0.52346000 & $\mathrm{H}$ & -5.16137100 & -0.58264800 & -2.20210900 \\
\hline $\mathrm{O}$ & 5.47091000 & -0.42377100 & 1.70164200 & $\mathrm{H}$ & -5.19634400 & -0.09202700 & -0.50810300 \\
\hline $\mathrm{C}$ & 6.90560900 & -0.47936400 & 1.62311600 & $\mathrm{C}$ & -2.44792600 & -0.88036200 & -2.50451000 \\
\hline $\mathrm{H}$ & 7.28312100 & 0.29234300 & 0.94696200 & $\mathrm{H}$ & -3.06878200 & -1.19448800 & -3.35366700 \\
\hline $\mathrm{H}$ & 7.26028500 & -0.30685300 & 2.63955300 & $\mathrm{H}$ & -2.10126200 & 0.13990800 & -2.70443300 \\
\hline $\mathrm{H}$ & 7.23153000 & -1.45856000 & 1.26248200 & $\mathrm{H}$ & -1.58016900 & -1.54660100 & -2.45843900 \\
\hline $\mathrm{C}$ & 2.54939700 & -1.15838700 & -0.36080800 & $\mathrm{C}$ & -3.65976300 & -2.43274200 & -0.94032800 \\
\hline $\mathrm{H}$ & 2.47087100 & -2.23694400 & -0.25298500 & $\mathrm{H}$ & -2.77772100 & -3.04869200 & -0.74307200 \\
\hline $\mathrm{C}$ & 1.92557400 & -1.37940900 & -2.82990300 & $\mathrm{H}$ & -4.35954500 & -2.53397100 & -0.10464700 \\
\hline $\mathrm{H}$ & 1.68410500 & -2.42228900 & -2.61785200 & $\mathrm{H}$ & -4.16007100 & -2.83115400 & -1.83249300 \\
\hline $\mathrm{H}$ & 1.09151100 & -0.92588100 & -3.38145100 & $\mathrm{C}$ & -2.52097700 & -2.10160600 & 2.33134000 \\
\hline $\mathrm{H}$ & 2.79934000 & -1.34192700 & -3.49618800 & $\mathrm{H}$ & -3.13339100 & -2.79816800 & 1.75400900 \\
\hline $\mathrm{C}$ & 3.31466200 & -0.44683200 & 0.72822300 & $\mathrm{H}$ & -1.47717000 & -2.39533000 & 2.20007800 \\
\hline $\mathrm{H}$ & 3.09774100 & 0.62766600 & 0.73757300 & $\mathrm{H}$ & -2.78844800 & -2.22270200 & 3.38928600 \\
\hline $\mathrm{H}$ & 3.05099900 & -0.82739800 & 1.71946000 & $\mathrm{C}$ & -1.91657400 & 0.27616300 & 2.85653500 \\
\hline
\end{tabular}




\begin{tabular}{|c|c|c|c|c|c|c|c|}
\hline $\mathrm{H}$ & -2.07994700 & 1.33944400 & 2.65732200 & $\mathrm{H}$ & 2.26129200 & 2.51141500 & -0.36606500 \\
\hline $\mathrm{H}$ & -2.19724200 & 0.07891100 & 3.89874000 & $\mathrm{H}$ & -0.93995200 & 5.36262700 & -0.15763600 \\
\hline $\mathrm{C}$ & -4.25807800 & -0.29069300 & 2.13348500 & $\mathrm{H}$ & -3.71244000 & 3.91507800 & 0.19098700 \\
\hline $\mathrm{H}$ & -4.51739600 & -0.45347000 & 3.18775100 & $\mathrm{H}$ & -4.22551100 & 1.29306700 & 0.32689100 \\
\hline $\mathrm{H}$ & -4.48351100 & 0.75485000 & 1.90395200 & $\mathrm{~N}$ & -2.08492100 & 1.03473000 & 0.16106900 \\
\hline $\mathrm{H}$ & -4.91389300 & -0.93209800 & 1.53754400 & $\mathrm{P}$ & -1.59814200 & -0.62318400 & -0.07398600 \\
\hline $\mathrm{F}$ & 2.37495300 & 4.37004100 & -0.55004400 & $\mathrm{C}$ & -2.04412700 & -1.00085000 & -1.90923200 \\
\hline $\mathrm{Cl}$ & -0.12766300 & -3.15361700 & -0.12912500 & $\mathrm{C}$ & -2.65622300 & -1.67331200 & 1.12760400 \\
\hline $\mathrm{H}$ & -0.84748600 & 0.06663800 & 2.74870800 & $\mathrm{C}$ & -3.38594200 & -0.37817300 & -2.34219600 \\
\hline C & 4.84305200 & -0.56261000 & 0.43574800 & $\mathrm{H}$ & -3.39384400 & 0.70811200 & -2.21640100 \\
\hline o & 5.11927600 & -0.87042900 & -0.70382100 & $\mathrm{H}$ & -3.53576000 & -0.59074600 & -3.40882400 \\
\hline $\mathrm{O}$ & 5.75709300 & -0.29145300 & 1.38537900 & $\mathrm{H}$ & -4.24310100 & -0.79365000 & -1.80670000 \\
\hline $\mathrm{C}$ & 7.13097100 & -0.35911200 & 0.96073200 & $\mathrm{C}$ & -0.92078300 & -0.35587900 & -2.75672800 \\
\hline $\mathrm{H}$ & 7.31866600 & 0.35873500 & 0.15810500 & $\mathrm{H}$ & -1.13837400 & -0.52951200 & -3.81918000 \\
\hline $\mathrm{H}$ & 7.72027400 & -0.11196400 & 1.84374300 & $\mathrm{H}$ & -0.84760500 & 0.72412100 & -2.60161600 \\
\hline $\mathrm{H}$ & 7.37063000 & -1.36439500 & 0.60506900 & $\mathrm{H}$ & 0.05751100 & -0.81225200 & -2.55420200 \\
\hline $\mathrm{C}$ & 2.42352000 & -1.19450800 & 0.16079700 & $\mathrm{C}$ & -2.04354400 & -2.51850400 & -2.18217400 \\
\hline $\mathrm{H}$ & 2.47060300 & -2.27011300 & 0.33751600 & $\mathrm{H}$ & -1.12020200 & -2.99438800 & -1.83817000 \\
\hline $\mathrm{C}$ & 2.13164400 & -1.83079600 & -2.33604300 & $\mathrm{H}$ & -2.89713700 & -3.02633800 & -1.72404700 \\
\hline $\mathrm{H}$ & 1.84174300 & -2.82994600 & -2.00886200 & $\mathrm{H}$ & -2.12093400 & -2.67355900 & -3.26628100 \\
\hline $\mathrm{H}$ & 1.44442400 & -1.50365600 & -3.12601000 & $\mathrm{C}$ & -2.05274400 & -3.09254200 & 1.22063000 \\
\hline $\mathrm{H}$ & 3.14038400 & -1.86637800 & -2.77443000 & $\mathrm{H}$ & -2.14913200 & -3.65374600 & 0.28918000 \\
\hline $\mathrm{C}$ & 3.43332400 & -0.39465200 & 0.98712200 & $\mathrm{H}$ & -0.99143000 & -3.07448100 & 1.47835800 \\
\hline $\mathrm{H}$ & 3.19675300 & 0.67459600 & 0.97672300 & $\mathrm{H}$ & -2.59400000 & -3.64417900 & 2.00047000 \\
\hline $\mathrm{H}$ & 3.42101100 & -0.70438700 & 2.03678700 & $\mathrm{C}$ & -2.59593900 & -1.03243100 & 2.52960200 \\
\hline $\mathrm{C}$ & 2.15197700 & -0.83914600 & -1.20766100 & $\mathrm{H}$ & -2.95000500 & 0.00251000 & 2.53661300 \\
\hline $\mathrm{H}$ & 2.44102600 & 0.16153100 & -1.51551900 & $\mathrm{H}$ & -3.24098800 & -1.61395600 & 3.20039900 \\
\hline $\mathrm{Rh}$ & 0.23375500 & -0.71423600 & -0.20799200 & $\mathrm{C}$ & -4.13807600 & -1.77788900 & 0.70734800 \\
\hline \multirow[t]{2}{*}{$\mathrm{H}$} & 1.36219100 & -0.90316500 & 0.95000400 & $\mathrm{H}$ & -4.67016500 & -2.35974600 & 1.47076800 \\
\hline & & & & $\mathrm{H}$ & -4.63137000 & -0.80339900 & 0.64271300 \\
\hline \multirow[t]{6}{*}{55} & & & & $\mathrm{H}$ & -4.27032200 & -2.29649000 & -0.24441400 \\
\hline & & & & $\mathrm{F}$ & 1.60539500 & 4.97891500 & -0.41592300 \\
\hline & & & & $\mathrm{Rh}$ & 0.69543900 & -0.40824100 & -0.04880200 \\
\hline & & & & $\mathrm{Cl}$ & 1.15624900 & -2.84442100 & -0.33547600 \\
\hline & & & & $\mathrm{H}$ & -1.58592300 & -1.05810100 & 2.94249100 \\
\hline & & & & $\mathrm{C}$ & 3.66865300 & -0.71551400 & 0.03279200 \\
\hline $\mathrm{C}$ & -1.55339100 & 3.25621200 & 0.00073300 & $\mathrm{O}$ & 2.77359900 & -0.13551200 & -0.59195900 \\
\hline $\mathrm{C}$ & -1.03331400 & 1.94797500 & 0.01572900 & $\mathrm{O}$ & 4.84514700 & -0.96956700 & -0.51895500 \\
\hline $\mathrm{C}$ & 0.31421600 & 1.58077200 & -0.09909900 & $\mathrm{C}$ & 4.99699000 & -0.63688600 & -1.91722300 \\
\hline $\mathrm{C}$ & 1.19064600 & 2.65699800 & -0.25649700 & $\mathrm{H}$ & 4.88135800 & 0.43953600 & -2.06295800 \\
\hline $\mathrm{C}$ & 0.69701100 & 3.97840800 & -0.26993000 & $\mathrm{H}$ & 6.00557500 & -0.95616000 & -2.17691600 \\
\hline $\mathrm{C}$ & -0.63870100 & 4.32056700 & -0.14066700 & $\mathrm{H}$ & 4.25291800 & -1.17256800 & -2.51025300 \\
\hline $\mathrm{C}$ & -2.98137500 & 3.11800900 & 0.14318100 & $\mathrm{C}$ & 2.60535900 & -0.22279900 & 2.24688900 \\
\hline $\mathrm{C}$ & -3.26681700 & 1.77836200 & 0.22215000 & $\mathrm{H}$ & 2.76193400 & -0.43566400 & 3.31596700 \\
\hline
\end{tabular}




\begin{tabular}{|c|c|c|c|c|c|c|c|}
\hline $\mathrm{C}$ & 0.40484200 & 0.76472300 & 2.82553600 & $\mathrm{H}$ & -5.06027300 & -2.18892200 & -1.04001500 \\
\hline $\mathrm{H}$ & 0.85075300 & 1.74500600 & 2.63429400 & $\mathrm{C}$ & -1.81639400 & -2.61035100 & 2.04762700 \\
\hline $\mathrm{H}$ & -0.66270400 & 0.85365000 & 2.62853400 & $\mathrm{H}$ & -2.72479900 & -3.10329600 & 1.69371700 \\
\hline $\mathrm{H}$ & 0.52811900 & 0.54408000 & 3.89835700 & $\mathrm{H}$ & -1.00485400 & -2.89475000 & 1.37303700 \\
\hline $\mathrm{C}$ & 3.53978300 & -1.15362700 & 1.46192200 & $\mathrm{H}$ & -1.59373900 & -3.00559400 & 3.04724200 \\
\hline $\mathrm{H}$ & 4.53869000 & -1.18498400 & 1.90829000 & $\mathrm{C}$ & -0.69418200 & -0.48931800 & 2.78370200 \\
\hline $\mathrm{H}$ & 3.14856400 & -2.17870900 & 1.44276400 & $\mathrm{H}$ & -0.75090000 & 0.59620600 & 2.90585900 \\
\hline $\mathrm{C}$ & 1.09063200 & -0.32402700 & 1.99942400 & $\mathrm{H}$ & -0.54913500 & -0.93585500 & 3.77528700 \\
\hline $\mathrm{H}$ & 0.74100500 & -1.31931000 & 2.30078800 & $\mathrm{C}$ & -3.17979100 & -0.75289900 & 3.06622900 \\
\hline \multirow[t]{2}{*}{$\mathrm{H}$} & 2.92730200 & 0.81624500 & 2.09914100 & $\mathrm{H}$ & -2.99732800 & -1.18733000 & 4.05763500 \\
\hline & & & & $\mathrm{H}$ & -3.31835900 & 0.32336900 & 3.20600800 \\
\hline \multirow[t]{6}{*}{ 56-ts } & & & & $\mathrm{H}$ & -4.11294800 & -1.18291100 & 2.69097100 \\
\hline & & & & $\mathrm{F}$ & 2.11788500 & 4.34276300 & -1.44173100 \\
\hline & & & & $\mathrm{Rh}$ & -0.13850900 & -0.65730900 & -1.02182300 \\
\hline & & & & $\mathrm{Cl}$ & -0.73946400 & -3.01431600 & -1.41717700 \\
\hline & & & & $\mathrm{H}$ & 0.19300200 & -0.71906300 & 2.18354200 \\
\hline & & & & $\mathrm{C}$ & 5.16494200 & -0.76009100 & 0.88369400 \\
\hline $\mathrm{C}$ & -0.83709100 & 3.38100800 & 0.40942100 & $\mathrm{O}$ & 4.79048800 & -0.32450400 & 1.95104800 \\
\hline $\mathrm{C}$ & -0.82863400 & 2.00803700 & 0.07281800 & $\mathrm{O}$ & 6.46441200 & -0.88689600 & 0.54112100 \\
\hline $\mathrm{C}$ & 0.13558300 & 1.35330000 & -0.71855200 & $\mathrm{C}$ & 7.40885300 & -0.48571200 & 1.54846000 \\
\hline $\mathrm{C}$ & 1.11559800 & 2.21680900 & -1.22498800 & $\mathrm{H}$ & 7.28226300 & -1.08509800 & 2.45404400 \\
\hline $\mathrm{C}$ & 1.12030700 & 3.58907300 & -0.91206800 & $\mathrm{H}$ & 8.39273800 & -0.65495000 & 1.11018700 \\
\hline $\mathrm{C}$ & 0.18242600 & 4.20433700 & -0.10472500 & $\mathrm{H}$ & 7.27526500 & 0.56971500 & 1.79984900 \\
\hline $\mathrm{C}$ & -1.98538000 & 3.58849100 & 1.25105500 & $\mathrm{C}$ & 2.85237900 & -0.63721400 & -0.14446900 \\
\hline $\mathrm{C}$ & -2.62385900 & 2.38445500 & 1.38673300 & $\mathrm{H}$ & 2.90455300 & 0.43333100 & -0.35474900 \\
\hline $\mathrm{H}$ & 1.91478100 & 1.87132400 & -1.87336100 & $\mathrm{C}$ & 1.57628400 & -0.92698300 & -2.39898300 \\
\hline $\mathrm{H}$ & 0.24607800 & 5.26685500 & 0.10425900 & $\mathrm{H}$ & 2.04086800 & -0.02627200 & -2.79542100 \\
\hline $\mathrm{H}$ & -2.30475900 & 4.52157300 & 1.69693700 & $\mathrm{H}$ & 1.36091300 & -1.70328600 & -3.13022300 \\
\hline $\mathrm{H}$ & -3.52513100 & 2.14995500 & 1.93152300 & $\mathrm{H}$ & 0.12978500 & -0.31223100 & -2.52781100 \\
\hline $\mathrm{N}$ & -1.94269400 & 1.39974400 & 0.66690400 & $\mathrm{C}$ & 4.25822300 & -1.26393600 & -0.22618600 \\
\hline $\mathrm{P}$ & -2.10022500 & -0.30929100 & 0.41417000 & $\mathrm{H}$ & 4.19644700 & -2.35670200 & -0.11969000 \\
\hline $\mathrm{C}$ & -3.78724900 & -0.55702600 & -0.43805400 & $\mathrm{H}$ & 4.73318300 & -1.07715200 & -1.19482400 \\
\hline $\mathrm{C}$ & -1.97845100 & -1.07932700 & 2.15896500 & $\mathrm{C}$ & 1.89878600 & -1.34029900 & -1.07460600 \\
\hline $\mathrm{C}$ & -4.91153700 & 0.31593600 & 0.15350500 & $\mathrm{H}$ & 1.79172000 & -2.40696100 & -0.88082000 \\
\hline $\mathrm{H}$ & -4.70697100 & 1.38295500 & 0.03090000 & $\mathrm{H}$ & 2.50033800 & -0.73707900 & 0.88891000 \\
\hline $\mathrm{H}$ & -5.83972400 & 0.09463100 & -0.38882800 & & & & \\
\hline $\mathrm{H}$ & -5.09854400 & 0.11379100 & 1.21199000 & Iנ & & & \\
\hline $\mathrm{C}$ & -3.56521700 & -0.14345000 & -1.91098800 & & & & \\
\hline $\mathrm{H}$ & -4.51837800 & -0.22588900 & -2.44916000 & & & & \\
\hline $\mathrm{H}$ & -3.22098000 & 0.89318500 & -1.99605700 & & & & \\
\hline $\mathrm{H}$ & -2.83662700 & -0.79537300 & -2.40213300 & & & & \\
\hline $\mathrm{C}$ & -4.19122900 & -2.04415300 & -0.38545100 & $\mathrm{C}$ & 2.71141600 & 2.69547100 & 1.01306900 \\
\hline $\mathrm{H}$ & -3.38987800 & -2.70027800 & -0.73807500 & $\mathrm{C}$ & 1.90413400 & 1.62357800 & 0.58067800 \\
\hline $\mathrm{H}$ & -4.48896400 & -2.35227200 & 0.62182300 & $\mathrm{C}$ & 0.71473800 & 1.72168100 & -0.15741500 \\
\hline
\end{tabular}




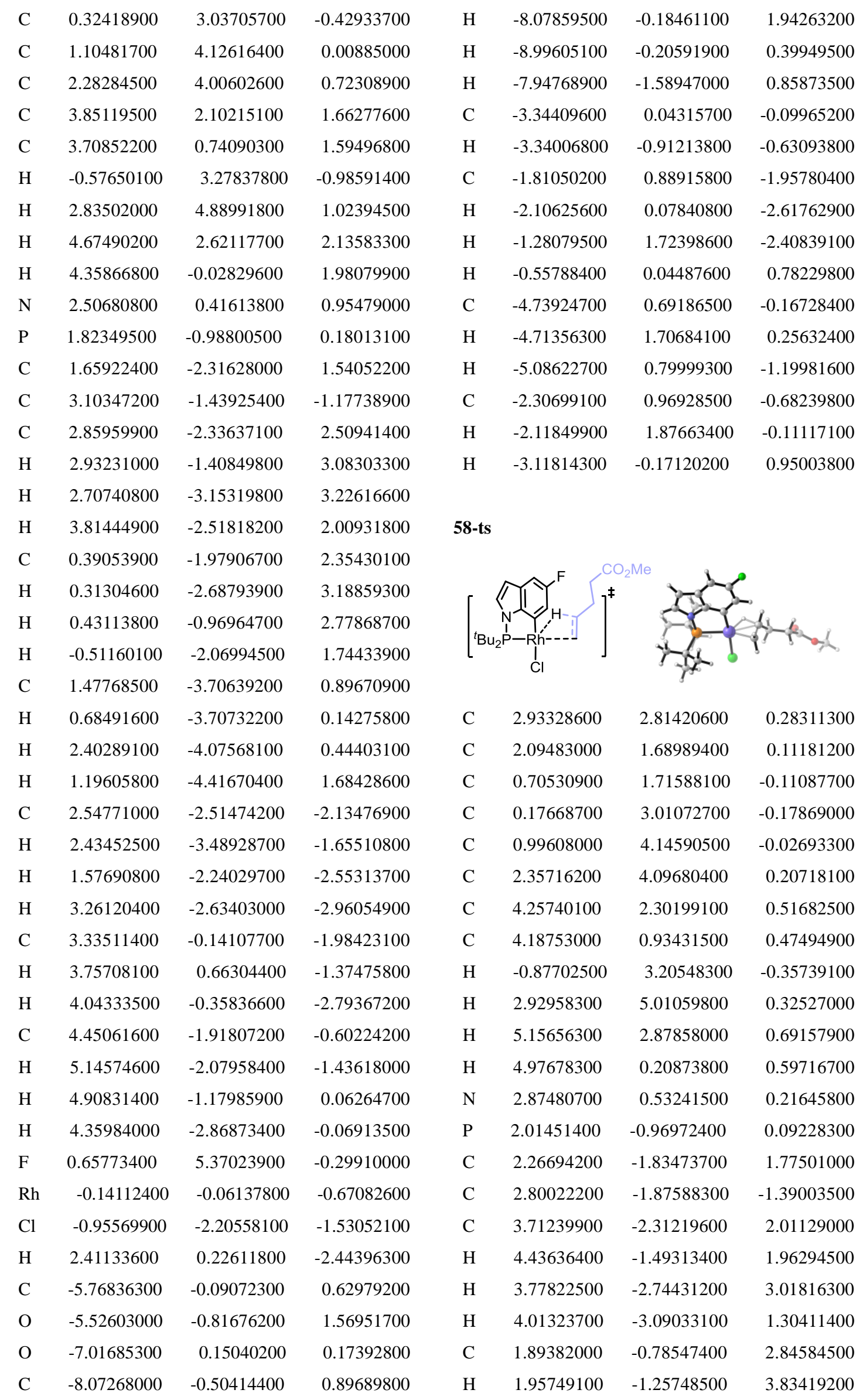




\begin{tabular}{|c|c|c|c|c|c|c|c|}
\hline $\mathrm{H}$ & 2.56900100 & 0.07538900 & 2.83663500 & & & & \\
\hline $\mathrm{H}$ & 0.87026800 & -0.41960700 & 2.71292000 & & & & \\
\hline $\mathrm{C}$ & 1.29868100 & -3.03074800 & 1.89265900 & & & & \\
\hline $\mathrm{H}$ & 0.26076400 & -2.74126100 & 1.71075400 & & & & \\
\hline $\mathrm{H}$ & 1.54206000 & -3.83765800 & 1.19747000 & & & & \\
\hline $\mathrm{H}$ & 1.37465900 & -3.43522800 & 2.91031700 & $\mathrm{C}$ & 1.54913100 & 3.31670100 & -0.10593000 \\
\hline $\mathrm{C}$ & 2.38720100 & -3.36151000 & -1.39168100 & $\mathrm{C}$ & 1.07108100 & 1.99223200 & -0.07908500 \\
\hline $\mathrm{H}$ & 2.87974700 & -3.92522200 & -0.59293800 & $\mathrm{C}$ & -0.26617500 & 1.58104900 & -0.02416900 \\
\hline $\mathrm{H}$ & 1.30420500 & -3.48713700 & -1.30247300 & $\mathrm{C}$ & -1.18469100 & 2.63333600 & 0.00826200 \\
\hline $\mathrm{H}$ & 2.70198500 & -3.80619600 & -2.34459800 & $\mathrm{C}$ & -0.73579500 & 3.97056100 & -0.01922200 \\
\hline $\mathrm{C}$ & 2.20713700 & -1.18295800 & -2.63893200 & $\mathrm{C}$ & 0.59351200 & 4.35368500 & -0.07886500 \\
\hline $\mathrm{H}$ & 2.41225300 & -0.10657700 & -2.64557500 & $\mathrm{C}$ & 2.98629400 & 3.21750500 & -0.15292200 \\
\hline $\mathrm{H}$ & 2.66342900 & -1.61589000 & -3.53860300 & $\mathrm{C}$ & 3.31526700 & 1.88482500 & -0.14176200 \\
\hline $\mathrm{C}$ & 4.33630300 & -1.75753900 & -1.44406700 & $\mathrm{H}$ & -2.25541500 & 2.45893500 & 0.05633900 \\
\hline $\mathrm{H}$ & 4.69251000 & -2.30878700 & -2.32373900 & $\mathrm{H}$ & 0.85857500 & 5.40546800 & -0.09978300 \\
\hline $\mathrm{H}$ & 4.66271000 & -0.71973300 & -1.55238600 & $\mathrm{H}$ & 3.69588300 & 4.03401600 & -0.19490400 \\
\hline $\mathrm{H}$ & 4.82954100 & -2.19041800 & -0.56886600 & $\mathrm{H}$ & 4.29217900 & 1.42524900 & -0.16203800 \\
\hline $\mathrm{F}$ & 0.39428800 & 5.36005300 & -0.11363900 & $\mathrm{~N}$ & 2.15374400 & 1.11000400 & -0.10231100 \\
\hline $\mathrm{Rh}$ & -0.23588200 & -0.09170900 & -0.32059100 & $\mathrm{P}$ & 1.70461600 & -0.56411900 & 0.06157400 \\
\hline $\mathrm{Cl}$ & -1.12515100 & -2.34015700 & -0.76333000 & $\mathrm{C}$ & 2.18841500 & -1.02218100 & 1.86148100 \\
\hline $\mathrm{H}$ & 1.12472800 & -1.33624600 & -2.70250700 & $\mathrm{C}$ & 2.75584400 & -1.49815600 & -1.23199600 \\
\hline $\mathrm{C}$ & -6.02775800 & -0.10345800 & 0.44775100 & $\mathrm{C}$ & 3.53919400 & -0.42139200 & 2.29771000 \\
\hline $\mathrm{O}$ & -6.03823500 & -0.62320400 & 1.54243800 & $\mathrm{H}$ & 3.54264400 & 0.66999000 & 2.22936300 \\
\hline $\mathrm{O}$ & -7.11639600 & -0.00480800 & -0.34482700 & $\mathrm{H}$ & 3.71104200 & -0.68856500 & 3.34849300 \\
\hline $\mathrm{C}$ & -8.32759800 & -0.55173700 & 0.20363300 & $\mathrm{H}$ & 4.38491300 & -0.80829500 & 1.72415900 \\
\hline $\mathrm{H}$ & -8.59447400 & -0.03988200 & 1.13223600 & $\mathrm{C}$ & 1.08020600 & -0.41171400 & 2.75339400 \\
\hline $\mathrm{H}$ & -9.09175900 & -0.39035500 & -0.55718100 & $\mathrm{H}$ & 1.32379200 & -0.61218400 & 3.80531300 \\
\hline $\mathrm{H}$ & -8.20726000 & -1.61868900 & 0.40873600 & $\mathrm{H}$ & 0.99651200 & 0.67263900 & 2.62956400 \\
\hline $\mathrm{C}$ & -3.50171100 & 0.00004900 & 0.35610000 & $\mathrm{H}$ & 0.10276700 & -0.86939500 & 2.55553900 \\
\hline $\mathrm{H}$ & -3.36118300 & -1.04154800 & 0.05629500 & $\mathrm{C}$ & 2.19140100 & -2.55130100 & 2.05873300 \\
\hline $\mathrm{C}$ & -1.81702500 & 0.68624600 & -1.48031600 & $\mathrm{H}$ & 1.26032200 & -3.00852900 & 1.71077300 \\
\hline $\mathrm{H}$ & -2.20844900 & -0.12096000 & -2.09470200 & $\mathrm{H}$ & 3.03408900 & -3.03515900 & 1.55596400 \\
\hline $\mathrm{H}$ & -1.46017100 & 1.56151700 & -2.01726200 & $\mathrm{H}$ & 2.29230300 & -2.76193600 & 3.13148300 \\
\hline $\mathrm{H}$ & -1.36580300 & 0.46515500 & 0.72698300 & $\mathrm{C}$ & 2.14539600 & -2.90013900 & -1.44599200 \\
\hline $\mathrm{C}$ & -4.82088200 & 0.54975900 & -0.20395700 & $\mathrm{H}$ & 2.25333900 & -3.53905200 & -0.56641600 \\
\hline $\mathrm{H}$ & -4.90362100 & 1.63076600 & -0.01583900 & $\mathrm{H}$ & 1.08020600 & -2.85754300 & -1.68120400 \\
\hline $\mathrm{H}$ & -4.88496100 & 0.42232200 & -1.28957500 & $\mathrm{H}$ & 2.67070700 & -3.38512900 & -2.27902700 \\
\hline $\mathrm{C}$ & -2.31849100 & 0.83341100 & -0.13666800 & $\mathrm{C}$ & 2.69560800 & -0.70724100 & -2.55852900 \\
\hline $\mathrm{H}$ & -2.32881200 & 1.86386700 & 0.22974100 & $\mathrm{H}$ & 3.12166400 & 0.29559000 & -2.46262100 \\
\hline \multirow[t]{2}{*}{$\mathrm{H}$} & -3.54838100 & 0.01871900 & 1.44966200 & $\mathrm{H}$ & 3.28127200 & -1.25317100 & -3.30901100 \\
\hline & & & & $\mathrm{C}$ & 4.23904100 & -1.64844600 & -0.83354100 \\
\hline \multirow[t]{3}{*}{59} & & & & $\mathrm{H}$ & 4.76420800 & -2.15564900 & -1.65300900 \\
\hline & & & & $\mathrm{H}$ & 4.73651700 & -0.68595400 & -0.67984600 \\
\hline & & & & $\mathrm{H}$ & 4.37408700 & -2.25711500 & 0.06323300 \\
\hline
\end{tabular}




\begin{tabular}{|c|c|c|c|c|c|c|c|}
\hline $\mathrm{F}$ & -1.68469700 & 4.94361000 & 0.01323100 & $\mathrm{C}$ & -3.14273400 & 0.55441200 & -0.97749100 \\
\hline $\mathrm{Rh}$ & -0.57934000 & -0.42337600 & 0.00026600 & $\mathrm{C}$ & -2.50006800 & -0.60184300 & 1.92378800 \\
\hline $\mathrm{Cl}$ & -1.03387300 & -2.83971300 & 0.32409400 & $\mathrm{C}$ & -3.90812500 & 1.84118700 & -0.60396800 \\
\hline $\mathrm{H}$ & 1.67807700 & -0.61108800 & -2.94013500 & $\mathrm{H}$ & -3.26119100 & 2.72126900 & -0.61274100 \\
\hline $\mathrm{C}$ & -3.78947000 & -0.56601700 & 0.23762200 & $\mathrm{H}$ & -4.68723900 & 1.99880800 & -1.36090100 \\
\hline $\mathrm{O}$ & -2.67482300 & -0.13884500 & 0.53564600 & $\mathrm{H}$ & -4.40896200 & 1.78509200 & 0.36577800 \\
\hline $\mathrm{O}$ & -4.75984400 & -0.63315600 & 1.14607900 & $\mathrm{C}$ & -2.42498200 & 0.79955400 & -2.32445600 \\
\hline $\mathrm{C}$ & -4.42474800 & -0.23938700 & 2.49367800 & $\mathrm{H}$ & -3.17546900 & 1.10564400 & -3.06541500 \\
\hline $\mathrm{H}$ & -4.12826200 & 0.81198000 & 2.51944700 & $\mathrm{H}$ & -1.68201900 & 1.60093600 & -2.25300700 \\
\hline $\mathrm{H}$ & -5.33545400 & -0.39756700 & 3.07049900 & $\mathrm{H}$ & -1.92757100 & -0.10253200 & -2.68824000 \\
\hline $\mathrm{H}$ & -3.61110100 & -0.86070300 & 2.87380500 & $\mathrm{C}$ & -4.15152200 & -0.60186700 & -1.14845800 \\
\hline $\mathrm{C}$ & -3.17979200 & -1.29831800 & -2.15906600 & $\mathrm{H}$ & -3.65631400 & -1.55006500 & -1.37165500 \\
\hline $\mathrm{H}$ & -3.70053200 & -1.61440000 & -3.07043900 & $\mathrm{H}$ & -4.78817100 & -0.72596000 & -0.26674800 \\
\hline $\mathrm{C}$ & -0.77527500 & -0.33057200 & -2.04466300 & $\mathrm{H}$ & -4.80980100 & -0.35824300 & -1.99229100 \\
\hline $\mathrm{H}$ & -0.14848100 & 0.47716000 & -2.42977700 & $\mathrm{C}$ & -2.93491200 & -2.07392700 & 1.74793300 \\
\hline $\mathrm{H}$ & -2.60555600 & 0.82297900 & -2.08925900 & $\mathrm{H}$ & -3.78511000 & -2.18357600 & 1.07085400 \\
\hline $\mathrm{C}$ & -4.26952900 & -0.98357400 & -1.12963400 & $\mathrm{H}$ & -2.12177900 & -2.69280500 & 1.35934900 \\
\hline $\mathrm{H}$ & -4.90897700 & -0.16088700 & -1.48548400 & $\mathrm{H}$ & -3.23677700 & -2.46769100 & 2.72733600 \\
\hline $\mathrm{H}$ & -4.93829100 & -1.83991200 & -0.99143500 & $\mathrm{C}$ & -1.36421700 & -0.55423000 & 2.96778800 \\
\hline $\mathrm{C}$ & -2.22234200 & -0.12537900 & -2.48625400 & $\mathrm{H}$ & -0.98973000 & 0.46045400 & 3.13161300 \\
\hline $\mathrm{H}$ & -2.19226600 & 0.00916700 & -3.57964700 & $\mathrm{H}$ & -1.74857700 & -0.93378800 & 3.92283500 \\
\hline $\mathrm{H}$ & -2.60369500 & -2.16078100 & -1.80843500 & $\mathrm{C}$ & -3.68839100 & 0.22687700 & 2.45015400 \\
\hline \multirow[t]{2}{*}{$\mathrm{H}$} & -0.40219200 & -1.29427600 & -2.40888100 & $\mathrm{H}$ & -3.99379000 & -0.17802200 & 3.42373600 \\
\hline & & & & $\mathrm{H}$ & -3.43260400 & 1.27998100 & 2.60644000 \\
\hline \multirow[t]{6}{*}{ 60-ts } & & & & $\mathrm{H}$ & -4.55891300 & 0.17439400 & 1.79149500 \\
\hline & & & & $\mathrm{F}$ & 3.25166900 & 2.80938900 & -2.53338600 \\
\hline & & & & $\mathrm{Rh}$ & -0.08112100 & -1.15716100 & -0.59629500 \\
\hline & & & & $\mathrm{Cl}$ & -1.38718400 & -2.83173600 & -1.59120200 \\
\hline & & & & $\mathrm{H}$ & -0.52529600 & -1.19291400 & 2.67493200 \\
\hline & & & & $\mathrm{H}$ & 1.84521100 & 1.12743500 & 2.00727200 \\
\hline $\mathrm{C}$ & 0.48670500 & 3.073 & -0.21821700 & $\mathrm{C}$ & 2.56303900 & -1.46917800 & -0.22165100 \\
\hline $\mathrm{C}$ & 0.25431600 & 1.69668500 & -0.03177800 & $\mathrm{O}$ & 1.93947000 & -2.15486100 & -1.04126600 \\
\hline $\mathrm{C}$ & 1.06135800 & 0.64711100 & -0.54645700 & $\mathrm{O}$ & 3.88324700 & -1.49956200 & -0.09735700 \\
\hline $\mathrm{C}$ & 4700 & 1.0995 & 44500 & $\mathrm{C}$ & 4.58813600 & -2.40913100 & -0.97371600 \\
\hline $\mathrm{C}$ & 2.25700300 & 2.46570800 & -1.67914600 & $\mathrm{H}$ & 4.39709300 & -2.14923700 & -2.01713300 \\
\hline $\mathrm{C}$ & 1.54421100 & 3.47873300 & -1.05505200 & $\mathrm{H}$ & 5.64167200 & -2.28282900 & -0.72754400 \\
\hline $\mathrm{C}$ & -0.55272400 & 3.75530600 & 0.51268500 & $\mathrm{H}$ & 4.26345700 & -3.43538200 & -0.78866400 \\
\hline $\mathrm{C}$ & -1.38531200 & 2.80179500 & 1.03017000 & $\mathrm{C}$ & 1.78882600 & -0.52245300 & 0.64018700 \\
\hline $\mathrm{H}$ & 2.73213500 & 0.40868200 & -1.94094200 & $\mathrm{H}$ & 1.11029300 & -1.16816500 & 1.23019200 \\
\hline $\mathrm{H}$ & 1.79081600 & 4.51855700 & -1.23877600 & $\mathrm{C}$ & 2.54591800 & 0.37671800 & 1.62636900 \\
\hline $\mathrm{H}$ & -0.66844300 & 4.82512500 & 0.62830400 & $\mathrm{H}$ & 3.32780700 & 0.92391200 & 1.08661100 \\
\hline $\mathrm{H}$ & -2.28531800 & 2.92885300 & 1.61216200 & $\mathrm{C}$ & 3.15076000 & -0.38803100 & 2.81596100 \\
\hline $\mathrm{N}$ & -0.92671100 & 1.51896700 & 0.69851700 & $\mathrm{H}$ & 2.35075700 & -0.92057100 & 3.35008100 \\
\hline $\mathrm{P}$ & -1.77498300 & 0.02354100 & 0.26792900 & $\mathrm{H}$ & 3.84635000 & -1.15244300 & 2.45116900 \\
\hline
\end{tabular}




\begin{tabular}{|c|c|c|c|c|c|c|c|}
\hline $\mathrm{C}$ & 3.87947900 & 0.54554300 & 3.78933900 & $\mathrm{H}$ & -1.94688300 & 0.76622400 & 2.95612100 \\
\hline $\mathrm{H}$ & 3.20031100 & 1.30470600 & 4.19642900 & $\mathrm{H}$ & -2.54854700 & -0.69616500 & 3.75679700 \\
\hline $\mathrm{H}$ & 4.29992200 & -0.01346000 & 4.63267600 & $\mathrm{C}$ & -4.19834400 & -0.19968600 & 1.66807200 \\
\hline \multirow[t]{2}{*}{$\mathrm{H}$} & 4.70391200 & 1.07066600 & 3.29216200 & $\mathrm{H}$ & -4.67756200 & -0.55036500 & 2.59141100 \\
\hline & & & & $\mathrm{H}$ & -4.21318000 & 0.89503700 & 1.69367400 \\
\hline \multirow[t]{6}{*}{ 61-ts } & & & & $\mathrm{H}$ & -4.81829100 & -0.53597600 & 0.83369100 \\
\hline & & & & $\mathrm{F}$ & 3.20474700 & 3.26330300 & -1.94314800 \\
\hline & & & & $\mathrm{Rh}$ & 0.33958200 & -1.05540100 & -0.15935700 \\
\hline & & & & $\mathrm{Cl}$ & -0.40284800 & -3.09621600 & -1.11247600 \\
\hline & & & & $\mathrm{H}$ & -0.99867500 & -0.73509200 & 2.90798800 \\
\hline & & & & $\mathrm{C}$ & 3.23286500 & -1.41638800 & 0.33217600 \\
\hline $\mathrm{C}$ & -0.07460700 & 3.19599800 & -0.41923100 & $\mathrm{O}$ & 2.36535800 & -1.83046500 & -0.43729600 \\
\hline $\mathrm{C}$ & -0.08446300 & 1.83777600 & -0.05187000 & $\mathrm{O}$ & 4.46577600 & -1.90639900 & 0.33565000 \\
\hline $\mathrm{C}$ & 1.00986200 & 0.93778700 & -0.15282400 & $\mathrm{C}$ & 4.74177900 & -2.96344300 & -0.61426000 \\
\hline $\mathrm{C}$ & 2.10209200 & 1.48380200 & -0.86800000 & $\mathrm{H}$ & 4.60595200 & -2.59580200 & -1.63371100 \\
\hline $\mathrm{C}$ & 2.10100900 & 2.82192200 & -1.28854500 & $\mathrm{H}$ & 5.77985200 & -3.24060500 & -0.43514200 \\
\hline $\mathrm{C}$ & 1.07519200 & 3.72113700 & -1.04491300 & $\mathrm{H}$ & 4.07256000 & -3.80850600 & -0.44056600 \\
\hline $\mathrm{C}$ & -1.37756100 & 3.71135100 & -0.07661700 & $\mathrm{C}$ & 1.53565100 & 0.10135000 & 1.44948600 \\
\hline $\mathrm{C}$ & -2.12155400 & 2.66630400 & 0.39943200 & $\mathrm{C}$ & 2.20102500 & 2.45952700 & 2.43575000 \\
\hline $\mathrm{H}$ & 2.98849500 & 0.90229300 & -1.09523100 & $\mathrm{H}$ & 2.03502600 & 3.02173600 & 1.51460200 \\
\hline $\mathrm{H}$ & 1.16284200 & 4.75618900 & -1.35617600 & $\mathrm{H}$ & 1.95988900 & 3.11823200 & 3.27802000 \\
\hline $\mathrm{H}$ & -1.71898100 & 4.73316500 & -0.18079000 & $\mathrm{H}$ & 3.27036200 & 2.22905900 & 2.50625500 \\
\hline $\mathrm{H}$ & -3.14980700 & 2.65708000 & 0.72802900 & $\mathrm{C}$ & 3.00597600 & -0.31041800 & 1.32655100 \\
\hline $\mathrm{N}$ & -1.35797500 & 1.49141500 & 0.41514100 & $\mathrm{H}$ & 3.64262800 & 0.53111000 & 1.03153100 \\
\hline $\mathrm{P}$ & -1.74517400 & -0.20545100 & 0.09548700 & $\mathrm{H}$ & 3.38219400 & -0.64184400 & 2.30442000 \\
\hline $\mathrm{C}$ & -2.81380300 & -0.17370800 & -1.51262300 & $\mathrm{C}$ & 1.33177400 & 1.19886900 & 2.51868300 \\
\hline $\mathrm{C}$ & -2.76779000 & -0.77102000 & 1.60900600 & $\mathrm{H}$ & 1.52059600 & 0.71434600 & 3.49050300 \\
\hline $\mathrm{C}$ & -3.86561400 & 0.95458300 & -1.56553800 & $\mathrm{H}$ & 0.27748500 & 1.48336000 & 2.52850200 \\
\hline $\mathrm{H}$ & -3.40613600 & 1.94484100 & -1.59806900 & $\mathrm{H}$ & 0.99304800 & -0.77230400 & 1.85490700 \\
\hline $\mathrm{H}$ & -4.44200100 & 0.82956500 & -2.49138100 & & & & \\
\hline $\mathrm{H}$ & -4.57882000 & 0.92740100 & -0.73776400 & 62-ts & & & \\
\hline $\mathrm{C}$ & -1.82584500 & 0.04183700 & -2.68107300 & & & & \\
\hline $\mathrm{H}$ & -2.39990700 & 0.08900700 & -3.61619300 & & & & \\
\hline $\mathrm{H}$ & -1.27361100 & 0.98202200 & -2.58042200 & & & & \\
\hline $\mathrm{H}$ & -1.10528200 & -0.77546400 & -2.75594200 & & & & \\
\hline $\mathrm{C}$ & -3.52660300 & -1.53078900 & -1.69926500 & & & & \\
\hline $\mathrm{H}$ & -2.83547900 & -2.37288900 & -1.62095900 & $\mathrm{C}$ & -0.81560600 & 3.27374600 & -0.31004400 \\
\hline $\mathrm{H}$ & -4.34772100 & -1.66954300 & -0.98849900 & $\mathrm{C}$ & -0.55017700 & 1.93747800 & 0.04281800 \\
\hline $\mathrm{H}$ & -3.96583700 & -1.54791200 & -2.70535900 & $\mathrm{C}$ & 0.73700600 & 1.33321500 & 0.11274900 \\
\hline $\mathrm{C}$ & -2.82864500 & -2.31503100 & 1.60315800 & $\mathrm{C}$ & 1.75598400 & 2.15503000 & -0.42705000 \\
\hline $\mathrm{H}$ & -3.40687300 & -2.70925300 & 0.76497700 & $\mathrm{C}$ & 1.49099400 & 3.46744700 & -0.83782700 \\
\hline $\mathrm{H}$ & -1.83146200 & -2.75994500 & 1.55078700 & $\mathrm{C}$ & 0.25053900 & 4.08139500 & -0.75292400 \\
\hline $\mathrm{H}$ & -3.31374500 & -2.64820100 & 2.53014800 & $\mathrm{C}$ & -2.23509000 & 3.46001200 & -0.13483800 \\
\hline $\mathrm{C}$ & -2.01127000 & -0.32259800 & 2.87581200 & $\mathrm{C}$ & -2.76414800 & 2.25672400 & 0.24455700 \\
\hline
\end{tabular}




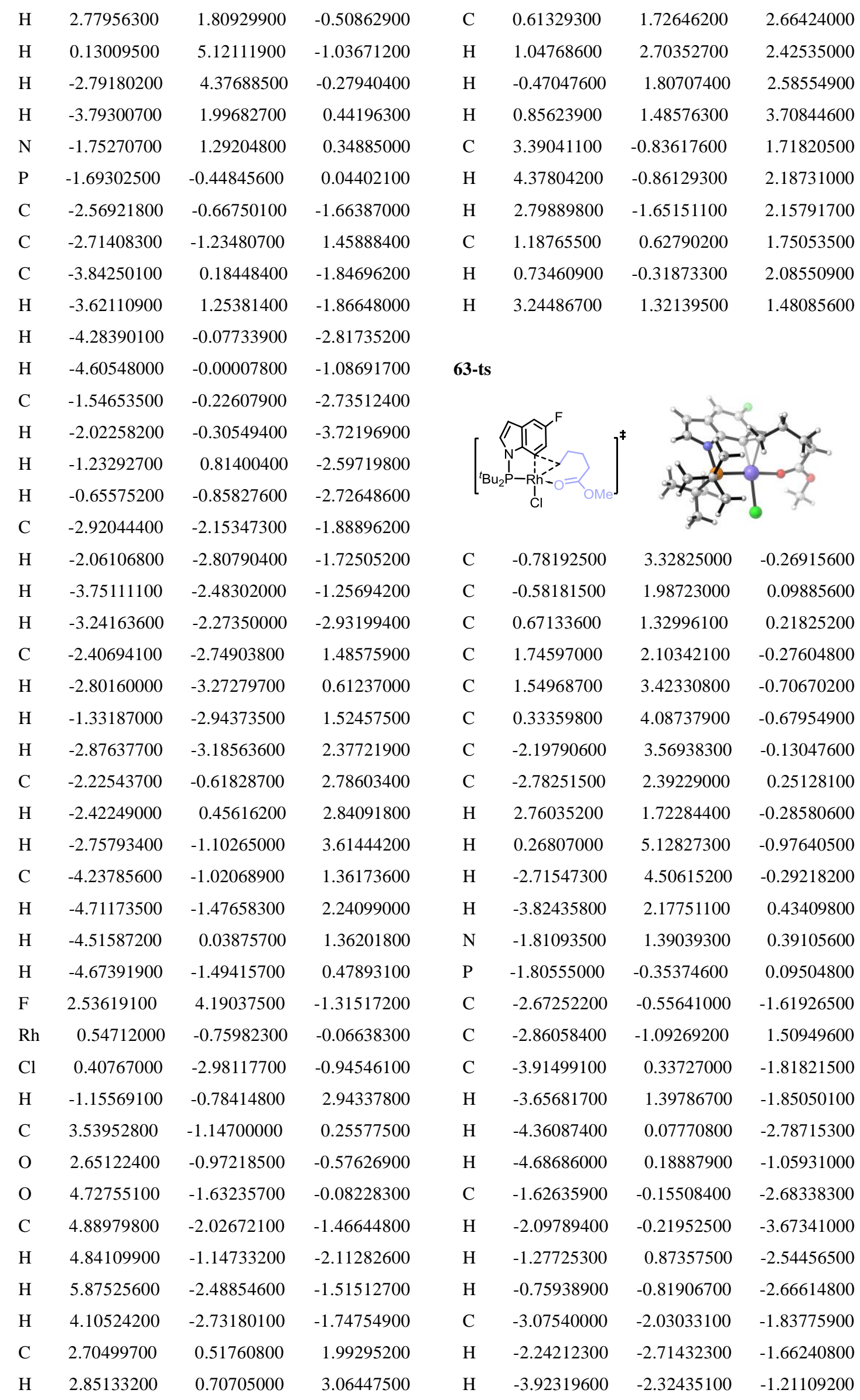




\begin{tabular}{|c|c|c|c|c|c|c|c|}
\hline $\mathrm{H}$ & -3.39182500 & -2.14498800 & -2.88287100 & $\mathrm{C}$ & 1.04293000 & 1.66091700 & 0.54908900 \\
\hline $\mathrm{C}$ & -2.60923200 & -2.61677400 & 1.55064000 & $\mathrm{C}$ & 2.15238600 & 2.38435900 & 0.10081800 \\
\hline $\mathrm{H}$ & -3.01563100 & -3.13265400 & 0.67777700 & $\mathrm{C}$ & 1.99574600 & 3.54534300 & -0.67078100 \\
\hline $\mathrm{H}$ & -1.54213500 & -2.84983400 & 1.60133600 & $\mathrm{C}$ & 0.76619400 & 4.09813900 & -0.96349900 \\
\hline $\mathrm{H}$ & -3.10107100 & -3.02920600 & 2.44148600 & $\mathrm{C}$ & -1.77657100 & 3.64385100 & -0.58931500 \\
\hline $\mathrm{C}$ & -2.36168000 & -0.48218600 & 2.83680800 & $\mathrm{C}$ & -2.41011900 & 2.56533100 & -0.04868200 \\
\hline $\mathrm{H}$ & -2.48330500 & 0.60459900 & 2.86678600 & $\mathrm{H}$ & 3.15889800 & 2.12392100 & 0.40373300 \\
\hline $\mathrm{H}$ & -2.94645300 & -0.91218300 & 3.65983300 & $\mathrm{H}$ & 0.69389400 & 5.05491300 & -1.46911800 \\
\hline $\mathrm{C}$ & -4.37432900 & -0.82280300 & 1.39460000 & $\mathrm{H}$ & -2.25068300 & 4.54704000 & -0.95007100 \\
\hline $\mathrm{H}$ & -4.87256300 & -1.25148400 & 2.27394300 & $\mathrm{H}$ & -3.46694900 & 2.40773100 & 0.09047900 \\
\hline $\mathrm{H}$ & -4.61137000 & 0.24634200 & 1.38157000 & $\mathrm{~N}$ & -1.49613100 & 1.57142700 & 0.33894700 \\
\hline $\mathrm{H}$ & -4.81997200 & -1.28880600 & 0.51252600 & $\mathrm{P}$ & -1.78803000 & -0.19826700 & 0.12873700 \\
\hline $\mathrm{F}$ & 2.64468900 & 4.09956700 & -1.14083900 & $\mathrm{C}$ & -2.74952900 & -0.35457100 & -1.55181400 \\
\hline $\mathrm{Rh}$ & 0.42390700 & -0.76019800 & 0.05056700 & $\mathrm{C}$ & -2.92091900 & -0.63379100 & 1.61788400 \\
\hline $\mathrm{Cl}$ & 0.21485000 & -2.93276100 & -0.92755200 & $\mathrm{C}$ & -3.88886500 & 0.65069300 & -1.83526100 \\
\hline $\mathrm{H}$ & -1.31098800 & -0.71895200 & 3.02366000 & $\mathrm{H}$ & -3.51661900 & 1.65610300 & -2.03632400 \\
\hline $\mathrm{C}$ & 3.71996800 & -1.04368800 & -0.04863300 & $\mathrm{H}$ & -4.40443000 & 0.30796500 & -2.74177500 \\
\hline $\mathrm{O}$ & 2.53997100 & -1.04823700 & -0.37019800 & $\mathrm{H}$ & -4.63947000 & 0.70277300 & -1.04310200 \\
\hline $\mathrm{O}$ & 4.65690100 & -1.54390000 & -0.85330100 & $\mathrm{C}$ & -1.68592700 & -0.16745900 & -2.65767900 \\
\hline $\mathrm{C}$ & 4.19429800 & -2.09153500 & -2.11200500 & $\mathrm{H}$ & -2.18491600 & -0.23043300 & -3.63431000 \\
\hline $\mathrm{H}$ & 3.84017000 & -1.28478100 & -2.75874200 & $\mathrm{H}$ & -1.20603900 & 0.81567700 & -2.59234200 \\
\hline $\mathrm{H}$ & 5.06703200 & -2.57854700 & -2.54603400 & $\mathrm{H}$ & -0.91422900 & -0.93776800 & -2.61244200 \\
\hline $\mathrm{H}$ & 3.38138500 & -2.79982500 & -1.94063000 & $\mathrm{C}$ & -3.35616200 & -1.77383100 & -1.64324500 \\
\hline $\mathrm{C}$ & 3.32518600 & -0.37029300 & 2.41050400 & $\mathrm{H}$ & -2.63891800 & -2.55657900 & -1.39262100 \\
\hline $\mathrm{H}$ & 3.95417700 & -0.27268300 & 3.30348700 & $\mathrm{H}$ & -4.24645700 & -1.87652000 & -1.01340500 \\
\hline $\mathrm{C}$ & 0.92816000 & 0.58402600 & 1.83868000 & $\mathrm{H}$ & -3.67610600 & -1.93785900 & -2.68075400 \\
\hline $\mathrm{H}$ & 0.21244800 & 1.23298500 & 2.34064500 & $\mathrm{C}$ & -2.94056000 & -2.17373600 & 1.74900800 \\
\hline $\mathrm{H}$ & 2.80145200 & 1.70291500 & 1.97423500 & $\mathrm{H}$ & -3.46129100 & -2.65955400 & 0.92096500 \\
\hline $\mathrm{C}$ & 4.29966200 & -0.48466500 & 1.22739700 & $\mathrm{H}$ & -1.92683100 & -2.58310900 & 1.79004900 \\
\hline $\mathrm{H}$ & 4.72601000 & 0.50001700 & 0.98527000 & $\mathrm{H}$ & -3.46525900 & -2.44166800 & 2.67576500 \\
\hline $\mathrm{H}$ & 5.15396400 & -1.11547100 & 1.49577300 & $\mathrm{C}$ & -2.26867200 & -0.05499300 & 2.89081600 \\
\hline $\mathrm{C}$ & 2.33319000 & 0.81452200 & 2.41467300 & $\mathrm{H}$ & -2.19912500 & 1.03653500 & 2.86046600 \\
\hline $\mathrm{H}$ & 2.15871100 & 1.06822100 & 3.46954900 & $\mathrm{H}$ & -2.88296900 & -0.33177600 & 3.75728800 \\
\hline $\mathrm{H}$ & 2.77954700 & -1.31513500 & 2.52814600 & $\mathrm{C}$ & -4.36552200 & -0.10695100 & 1.52881400 \\
\hline \multirow[t]{2}{*}{$\mathrm{H}$} & .61982500 & -0.43927000 & 2.13245700 & $\mathrm{H}$ & -4.90357400 & -0.39907200 & 2.44024600 \\
\hline & & & & $\mathrm{H}$ & -4.41114700 & 0.98536100 & 1.46761600 \\
\hline \multirow[t]{6}{*}{64} & & & & $\mathrm{H}$ & -4.91360900 & -0.52876600 & 0.68272900 \\
\hline & & & & $\mathrm{F}$ & 3.12138900 & 4.19055800 & -1.06074000 \\
\hline & & & & $\mathrm{Rh}$ & 0.25161000 & -1.11960300 & 0.11907800 \\
\hline & & & & $\mathrm{Cl}$ & -0.24304400 & -3.09041400 & -1.04634500 \\
\hline & & & & $\mathrm{H}$ & -1.26853200 & -0.46834600 & 3.05234700 \\
\hline & & & & $\mathrm{C}$ & 3.52709900 & -1.40268100 & -0.11810200 \\
\hline $\mathrm{C}$ & 506300 & 3.367 & -0.55503900 & $\mathrm{O}$ & 2.34200100 & -1.68574700 & -0.22761600 \\
\hline $\mathrm{C}$ & 334900 & 2.11521200 & 0.08424900 & $\mathrm{O}$ & 4.41362800 & -1.73779500 & -1.05611300 \\
\hline
\end{tabular}




\begin{tabular}{|c|c|c|c|c|c|c|c|}
\hline $\mathrm{C}$ & 3.88665900 & -2.40694400 & -2.22610200 & $\mathrm{H}$ & -3.96799200 & -2.51222600 & -1.21342300 \\
\hline $\mathrm{H}$ & 3.22331600 & -1.73414900 & -2.77428200 & $\mathrm{H}$ & -3.01416400 & -2.08224500 & 0.21584900 \\
\hline $\mathrm{H}$ & 4.76126900 & -2.66279500 & -2.82349900 & $\mathrm{H}$ & -2.99981500 & -1.03658300 & -1.23286900 \\
\hline $\mathrm{H}$ & 3.32888300 & -3.29874400 & -1.93408200 & $\mathrm{C}$ & -1.89077400 & -2.76385900 & -3.01250600 \\
\hline $\mathrm{C}$ & 3.33581000 & -0.62076300 & 2.32837800 & $\mathrm{H}$ & -1.73041700 & -1.73313900 & -3.34113900 \\
\hline $\mathrm{H}$ & 4.03969900 & -0.62949600 & 3.16918400 & $\mathrm{H}$ & -1.15856200 & -3.41153700 & -3.50373400 \\
\hline $\mathrm{C}$ & 1.10286900 & 0.71564100 & 1.73772900 & $\mathrm{H}$ & -2.88379700 & -3.07973400 & -3.35840100 \\
\hline $\mathrm{H}$ & 0.33935800 & 1.10115100 & 2.42181700 & $\mathrm{C}$ & 1.55556100 & -1.92589100 & -2.90487000 \\
\hline $\mathrm{H}$ & 3.00218200 & 1.52544000 & 2.43267500 & $\mathrm{H}$ & 0.77734700 & -2.10890200 & -3.64944900 \\
\hline $\mathrm{C}$ & 4.17803500 & -0.68530800 & 1.04235700 & $\mathrm{H}$ & 1.57944700 & -0.84915900 & -2.72979900 \\
\hline $\mathrm{H}$ & 4.46325500 & 0.31665300 & 0.69458800 & $\mathrm{H}$ & 2.51583400 & -2.23265000 & -3.33921600 \\
\hline $\mathrm{H}$ & 5.12561200 & -1.20214800 & 1.23216800 & $\mathrm{C}$ & 2.50977700 & -2.52568900 & -0.64077700 \\
\hline $\mathrm{C}$ & 2.42140400 & 0.60166000 & 2.54267800 & $\mathrm{H}$ & 2.41948400 & -3.14445200 & 0.25616900 \\
\hline $\mathrm{H}$ & 2.12759300 & 0.56799200 & 3.59887300 & $\mathrm{H}$ & 3.43447800 & -2.81102200 & -1.15793000 \\
\hline $\mathrm{H}$ & 2.74282000 & -1.53919400 & 2.41889900 & $\mathrm{C}$ & 1.27333800 & -4.23684500 & -1.97278900 \\
\hline \multirow[t]{2}{*}{$\mathrm{H}$} & 0.72824300 & -0.37137700 & 1.65660100 & $\mathrm{H}$ & 2.23641600 & -4.51201800 & -2.42167400 \\
\hline & & & & $\mathrm{H}$ & 1.13056800 & -4.88111700 & -1.10033100 \\
\hline \multirow[t]{6}{*}{65} & & & & $\mathrm{H}$ & 0.49764800 & -4.46646600 & -2.70654000 \\
\hline & \multirow{5}{*}{$\begin{array}{r}\mathrm{Bu}_{2} \mathrm{P}-\mathrm{Rh}- \\
\mathrm{h}- \\
\mathrm{Cl}\end{array}$} & \multirow{5}{*}{$\mathrm{CO}_{2} \mathrm{Me}$} & \multirow{5}{*}{8} & $\mathrm{~F}$ & -1.41738500 & 0.38784400 & 5.14409100 \\
\hline & & & & $\mathrm{Rh}$ & -0.77958600 & 0.13851100 & -0.33019700 \\
\hline & & & & $\mathrm{Cl}$ & -0.89403300 & 0.70465400 & -2.74051900 \\
\hline & & & & $\mathrm{H}$ & 2.61613500 & -1.48737500 & -0.32279700 \\
\hline & & & & $\mathrm{C}$ & 5.58784800 & 1.92222100 & 0.19469900 \\
\hline $\mathrm{C}$ & -0.48002400 & -2.45813400 & 3.11871000 & $\mathrm{O}$ & 5.78570300 & 2.98106900 & 0.75019400 \\
\hline $\mathrm{C}$ & -0.50742800 & -1.79932100 & 1.87084200 & $\mathrm{O}$ & 6.53238800 & 1.27321500 & -0.52699900 \\
\hline $\mathrm{C}$ & -0.81747300 & -0.45044600 & 1.63525400 & $\mathrm{C}$ & 7.80914900 & 1.92643800 & -0.59555400 \\
\hline $\mathrm{C}$ & -1.13020100 & 0.25387600 & 2.80455700 & $\mathrm{H}$ & 8.23573600 & 2.04590800 & 0.40424600 \\
\hline $\mathrm{C}$ & -1.10736300 & -0.37619400 & 4.06482500 & $\mathrm{H}$ & 8.43876400 & 1.27724400 & -1.20517900 \\
\hline $\mathrm{C}$ & -0.79108700 & -1.70719700 & 4.26876000 & $\mathrm{H}$ & 7.71369000 & 2.91260700 & -1.05827000 \\
\hline $\mathrm{C}$ & -0.10833500 & -3.82230400 & 2.84660400 & $\mathrm{C}$ & 3.17694900 & 1.84699800 & 0.98856900 \\
\hline $\mathrm{C}$ & 0.06393900 & -3.94499500 & 1.49204700 & $\mathrm{H}$ & 2.98072200 & 2.82599200 & 0.53331500 \\
\hline $\mathrm{H}$ & -1.38616200 & 1.30950000 & 2.80223600 & $\mathrm{C}$ & 1.16938900 & 0.87230300 & -0.25356000 \\
\hline $\mathrm{H}$ & -0.79195700 & -2.12621800 & 5.26927900 & $\mathrm{H}$ & 1.03645700 & 1.83392600 & -0.75588000 \\
\hline $\mathrm{H}$ & 0.02200900 & -4.61855400 & 3.56817200 & $\mathrm{H}$ & 1.74622700 & 0.23888000 & -0.92766800 \\
\hline $\mathrm{H}$ & 0.33495000 & -4.81765800 & 0.91747700 & $\mathrm{H}$ & 1.20299900 & 1.57453900 & 1.79094100 \\
\hline $\mathrm{N}$ & -0.17093500 & -2.71645300 & 0.87033300 & $\mathrm{C}$ & 4.28599700 & 1.14328100 & 0.20199100 \\
\hline $\mathrm{P}$ & -0.25786900 & -2.09655200 & -0.74891700 & $\mathrm{H}$ & 4.49641100 & 0.14950400 & 0.62160300 \\
\hline $\mathrm{C}$ & -1.84919100 & -2.89183200 & -1.47672700 & $\mathrm{H}$ & 3.99927900 & 0.96895100 & -0.84256600 \\
\hline $\mathrm{C}$ & 1.32561100 & -2.73700800 & -1.61080200 & $\mathrm{C}$ & 1.85905000 & 1.04409300 & 1.09359500 \\
\hline $\mathrm{C}$ & -2.03235700 & -4.36489800 & -1.06051400 & $\mathrm{H}$ & 2.05876400 & 0.06920400 & 1.55650400 \\
\hline $\mathrm{H}$ & -2.07169700 & -4.48326200 & 0.02571100 & $\mathrm{H}$ & 3.54396600 & 2.05555000 & 2.00040400 \\
\hline $\mathrm{H}$ & -2.98869300 & -4.71828200 & -1.46746300 & $\mathrm{C}$ & -1.61029700 & 5.02132000 & -0.52704700 \\
\hline $\mathrm{H}$ & -1.25256400 & -5.02130400 & -1.45426200 & $\mathrm{C}$ & -0.93099000 & 3.64338700 & -0.72758000 \\
\hline $\mathrm{C}$ & -3.01813000 & -2.07442500 & -0.87896600 & $\mathrm{C}$ & -4.61878400 & 3.04314500 & -0.48402200 \\
\hline
\end{tabular}




\begin{tabular}{|c|c|c|c|c|c|c|c|}
\hline $\mathrm{C}$ & -1.36116000 & 2.60291700 & 0.27811700 & $\mathrm{H}$ & 2.44311700 & 5.27881300 & 0.29140100 \\
\hline $\mathrm{C}$ & -3.53200300 & 2.01531900 & -0.88690300 & $\mathrm{H}$ & 0.75385100 & 5.26368000 & -0.20884200 \\
\hline $\mathrm{C}$ & -2.50133800 & 1.85952700 & 0.19966000 & $\mathrm{C}$ & 2.87056000 & 2.58440600 & 0.46860300 \\
\hline $\mathrm{H}$ & -1.48601200 & 5.34244500 & 0.51735900 & $\mathrm{H}$ & 3.73881300 & 3.23984100 & 0.61967800 \\
\hline $\mathrm{H}$ & -1.10676900 & 3.28553600 & -1.74670100 & $\mathrm{H}$ & 2.47036600 & 2.32721900 & 1.45498700 \\
\hline $\mathrm{H}$ & -5.25612400 & 2.61154800 & 0.30030400 & $\mathrm{H}$ & 3.20796700 & 1.66650500 & -0.01779800 \\
\hline $\mathrm{H}$ & -4.02535200 & 1.05653200 & -1.08610300 & $\mathrm{C}$ & 2.45831900 & 3.62232000 & -1.78139900 \\
\hline $\mathrm{H}$ & -1.04765500 & 5.74312100 & -1.13397300 & $\mathrm{H}$ & 2.65722100 & 2.70580300 & -2.34058100 \\
\hline $\mathrm{H}$ & 0.15109600 & 3.79428900 & -0.63462900 & $\mathrm{H}$ & 1.83499200 & 4.28620900 & -2.38844400 \\
\hline $\mathrm{H}$ & -5.26654600 & 3.19007300 & -1.35895300 & $\mathrm{H}$ & 3.41518500 & 4.13671100 & -1.62246600 \\
\hline $\mathrm{H}$ & -0.80405700 & 2.58465300 & 1.21185900 & $\mathrm{C}$ & -0.40001400 & 2.38849200 & -3.29068100 \\
\hline $\mathrm{H}$ & -3.05384300 & 2.31426300 & -1.82185000 & $\mathrm{H}$ & 0.51916300 & 2.90880000 & -3.56790000 \\
\hline $\mathrm{H}$ & -2.81678300 & 1.30681100 & 1.08268700 & $\mathrm{H}$ & -0.20032700 & 1.31606400 & -3.35443700 \\
\hline $\mathrm{C}$ & -3.09864300 & 5.13857100 & -0.91289000 & $\mathrm{H}$ & -1.16668500 & 2.64696900 & -4.03303900 \\
\hline $\mathrm{H}$ & -3.33792600 & 6.20982500 & -0.90729400 & $\mathrm{C}$ & -2.26917000 & 2.08755600 & -1.65984500 \\
\hline $\mathrm{H}$ & -3.23371200 & 4.81312800 & -1.95444100 & $\mathrm{H}$ & -2.68959900 & 2.30509200 & -0.67328300 \\
\hline $\mathrm{C}$ & -4.10910000 & 4.41122300 & 0.00672300 & $\mathrm{H}$ & -2.98362800 & 2.43902400 & -2.41491100 \\
\hline $\mathrm{H}$ & -4.99241200 & 5.04889900 & 0.14064400 & $\mathrm{C}$ & -1.16076700 & 4.31994700 & -1.86627900 \\
\hline $\mathrm{H}$ & -3.67079900 & 4.30394400 & 1.00784600 & $\mathrm{H}$ & -1.89275200 & 4.57024500 & -2.64527600 \\
\hline \multirow{7}{*}{ 66-ts } & & & & $\mathrm{H}$ & -1.57664900 & 4.66406600 & -0.91402700 \\
\hline & & & & $\mathrm{H}$ & -0.25513200 & 4.89251800 & -2.07780700 \\
\hline & & & & $\mathrm{F}$ & 0.27304600 & -1.25663700 & 4.95836400 \\
\hline & & & & $\mathrm{Rh}$ & 0.90229100 & -0.15121500 & -0.41124300 \\
\hline & & & & $\mathrm{Cl}$ & 2.24723500 & 0.14825700 & -2.38956500 \\
\hline & & & & $\mathrm{H}$ & -2.18030700 & 1.00361200 & -1.77406700 \\
\hline & & & & $\mathrm{C}$ & -5.51421400 & -2.08294600 & -0.53149500 \\
\hline $\mathrm{C}$ & -0.59431300 & 1.7671 & 47800 & $\mathrm{O}$ & -5.68059700 & -3.26361100 & -0.74917400 \\
\hline $\mathrm{C}$ & -0.32821200 & 1.30410100 & 1.86777000 & $\mathrm{O}$ & -6.44331500 & -1.13736600 & -0.80202100 \\
\hline $\mathrm{C}$ & 0.02384800 & -0.02395500 & 1.51652300 & $\mathrm{C}$ & -7.67394900 & -1.62607700 & -1.35910500 \\
\hline $\mathrm{C}$ & 0.24124600 & -0.85472100 & 2.63914400 & $\mathrm{H}$ & -8.15486200 & -2.32956800 & -0.67391500 \\
\hline $\mathrm{C}$ & 0.04916700 & -0.38515700 & 3.94343300 & $\mathrm{H}$ & -8.29986700 & -0.74507800 & -1.50514100 \\
\hline $\mathrm{C}$ & -0.39805800 & 0.88997300 & 4.25553000 & $\mathrm{H}$ & -7.49165600 & -2.13006000 & -2.31216500 \\
\hline $\mathrm{C}$ & -1.03637300 & 3.13152400 & 3.03395800 & $\mathrm{C}$ & -3.10448500 & -2.44508200 & 0.17681700 \\
\hline $\mathrm{C}$ & -0.98725800 & 3.44484200 & 1.70379500 & $\mathrm{H}$ & -2.79535800 & -2.74075700 & -0.83436600 \\
\hline $\mathrm{H}$ & 0.49792500 & -1.90277500 & 2.52871300 & $\mathrm{C}$ & -1.22419900 & -0.71747100 & 0.27533300 \\
\hline $\mathrm{H}$ & -0.58169900 & 1.17081800 & 5.28690300 & $\mathrm{H}$ & -1.06093700 & -0.98197600 & -0.78634100 \\
\hline $\mathrm{H}$ & -1.35726800 & 3.79403400 & 3.82720800 & $\mathrm{H}$ & -1.87682200 & 0.14956500 & 0.29268100 \\
\hline $\mathrm{H}$ & -1.22624000 & 4.37716700 & 1.21646500 & $\mathrm{H}$ & -1.18679300 & -2.73638500 & 1.08810100 \\
\hline $\mathrm{N}$ & -0.54462300 & 2.34250100 & 0.95959600 & $\mathrm{C}$ & -4.27440500 & -1.46068300 & 0.08409900 \\
\hline $\mathrm{P}$ & 0.31231600 & 2.15679600 & -0.56928000 & $\mathrm{H}$ & -4.55218700 & -1.09736500 & 1.08440900 \\
\hline $\mathrm{C}$ & 1.83586000 & 3.33592800 & -0.39820100 & $\mathrm{H}$ & -4.02228000 & -0.56541300 & -0.49574700 \\
\hline $\mathrm{C}$ & -0.91846700 & 2.79753500 & -1.89363800 & $\mathrm{C}$ & -1.89291100 & -1.90973700 & 0.96525000 \\
\hline $\mathrm{C}$ & 1.52463700 & 4.67768600 & 0.29775600 & $\mathrm{H}$ & -2.20852200 & -1.62425200 & 1.97582200 \\
\hline $\mathrm{H}$ & 1.23006400 & 4.54229700 & 1.34074900 & $\mathrm{H}$ & -3.46145000 & -3.36093300 & 0.66103800 \\
\hline
\end{tabular}




\begin{tabular}{|c|c|c|c|c|c|c|c|}
\hline $\mathrm{C}$ & 2.21409800 & -4.53925600 & -1.45950800 & $\mathrm{C}$ & -2.88021100 & -0.38841200 & 1.46495300 \\
\hline $\mathrm{C}$ & 1.58953700 & -3.13001700 & -1.58693400 & $\mathrm{C}$ & -3.68509700 & 0.11013400 & -2.19342600 \\
\hline $\mathrm{C}$ & 4.72714900 & -2.76795800 & 0.43599900 & $\mathrm{H}$ & -3.31728100 & 1.12059100 & -2.39228200 \\
\hline $\mathrm{C}$ & 1.44908500 & -2.40739100 & -0.26336200 & $\mathrm{H}$ & -4.05787500 & -0.29974600 & -3.14112500 \\
\hline $\mathrm{C}$ & 3.86916400 & -1.61548400 & -0.14261300 & $\mathrm{H}$ & -4.53859100 & 0.17880200 & -1.51413700 \\
\hline $\mathrm{C}$ & 2.45533600 & -1.70106700 & 0.37083100 & $\mathrm{C}$ & -1.43329400 & -0.84233600 & -2.72129300 \\
\hline $\mathrm{H}$ & 1.66859700 & -5.11723600 & -0.69837500 & $\mathrm{H}$ & -1.84835100 & -1.15760600 & -3.68779900 \\
\hline $\mathrm{H}$ & 2.17097300 & -2.53183200 & -2.29280800 & $\mathrm{H}$ & -0.97346500 & 0.14126000 & -2.85893800 \\
\hline $\mathrm{H}$ & 4.90743100 & -2.58956100 & 1.50558500 & $\mathrm{H}$ & -0.65284600 & -1.56768000 & -2.45599900 \\
\hline $\mathrm{H}$ & 4.32546100 & -0.66149100 & 0.14624900 & $\mathrm{C}$ & -3.11062200 & -2.25199200 & -1.49513800 \\
\hline $\mathrm{H}$ & 2.04126900 & -5.05732000 & -2.41283400 & $\mathrm{H}$ & -2.35433800 & -2.92690100 & -1.08360500 \\
\hline $\mathrm{H}$ & 0.59104000 & -3.23907100 & -2.03077500 & $\mathrm{H}$ & -4.00030900 & -2.28573400 & -0.85904100 \\
\hline $\mathrm{H}$ & 5.71049000 & -2.71750100 & -0.05188900 & $\mathrm{H}$ & -3.40537300 & -2.63426700 & -2.48124800 \\
\hline $\mathrm{H}$ & 0.63135500 & -2.76723600 & 0.35622900 & $\mathrm{C}$ & -2.77176100 & -1.83893200 & 1.98431900 \\
\hline $\mathrm{H}$ & 3.88657700 & -1.64849100 & -1.23216600 & $\mathrm{H}$ & -3.17791500 & -2.56500700 & 1.27646800 \\
\hline $\mathrm{H}$ & 2.35275800 & -1.50681400 & 1.43495700 & $\mathrm{H}$ & -1.74154500 & -2.13465200 & 2.19183000 \\
\hline $\mathrm{C}$ & 3.72419400 & -4.60915300 & -1.15119900 & $\mathrm{H}$ & -3.35101100 & -1.91716300 & 2.91345100 \\
\hline $\mathrm{H}$ & 4.02971600 & -5.65134400 & -1.31146200 & $\mathrm{C}$ & -2.37977300 & 0.58526100 & 2.55469900 \\
\hline $\mathrm{H}$ & 4.27636800 & -4.02283700 & -1.89979300 & $\mathrm{H}$ & -2.47922100 & 1.63116100 & 2.25087300 \\
\hline $\mathrm{C}$ & 4.15701200 & -4.19124100 & 0.27645300 & $\mathrm{H}$ & -2.98615500 & 0.43483200 & 3.45683100 \\
\hline $\mathrm{H}$ & 4.93181000 & -4.88415100 & 0.62987800 & $\mathrm{C}$ & -4.36267200 & -0.07016300 & 1.17766700 \\
\hline \multirow[t]{2}{*}{$\mathrm{H}$} & 3.30943300 & -4.32752000 & 0.96140800 & $\mathrm{H}$ & -4.92183900 & -0.16836800 & 2.11693100 \\
\hline & & & & $\mathrm{H}$ & -4.51551400 & 0.95158500 & 0.81746200 \\
\hline \multirow[t]{6}{*}{67} & & & & $\mathrm{H}$ & -4.80966300 & -0.76274800 & 0.46078900 \\
\hline & & & & $\mathrm{F}$ & 3.21723500 & 3.61096200 & -1.68312400 \\
\hline & & & & $\mathrm{Rh}$ & 0.44107800 & -0.85166000 & -0.14269900 \\
\hline & & & & $\mathrm{Cl}$ & 0.00810300 & -3.29233600 & 0.12121900 \\
\hline & & & & $\mathrm{H}$ & -1.33705000 & 0.40738500 & 2.82273900 \\
\hline & & & & $\mathrm{H}$ & 0.65686200 & 1.44837600 & 2.27392100 \\
\hline $\mathrm{C}$ & -0.31441100 & 3.313 & -0.93746400 & $\mathrm{O}$ & 2.57152600 & -1.31604800 & -0.60378300 \\
\hline $\mathrm{C}$ & -0.30256800 & 1.94216000 & -0.61672300 & $\mathrm{O}$ & 4.26960000 & -2.70722600 & -1.07236500 \\
\hline $\mathrm{C}$ & 0.80530100 & 1.08586100 & -0.62154400 & $\mathrm{C}$ & 5.14712400 & -1.57170600 & -1.26028600 \\
\hline $\mathrm{C}$ & 1.99936300 & 1.70252100 & -1.00458000 & $\mathrm{H}$ & 5.21263800 & -0.99127800 & -0.33734700 \\
\hline C & 2.01892100 & 3.0748 & -1.32768500 & $\mathrm{H}$ & 6.11483700 & -1.99938600 & -1.51816600 \\
\hline $\mathrm{C}$ & 0.91161300 & 3.90642200 & -1.30326800 & $\mathrm{H}$ & 4.77416700 & -0.93853800 & -2.06829500 \\
\hline $\mathrm{C}$ & -1.68322700 & 3.74172400 & -0.79291300 & $\mathrm{C}$ & 1.44166800 & 0.68997100 & 2.38572500 \\
\hline $\mathrm{C}$ & -2.42656500 & 2.65082500 & -0.41719000 & $\mathrm{H}$ & 2.29942100 & 1.05590500 & 1.81022000 \\
\hline $\mathrm{H}$ & 2.93520500 & 1.15480400 & -1.04974400 & $\mathrm{C}$ & 1.83649300 & 0.58855800 & 3.87372000 \\
\hline $\mathrm{H}$ & 1.00934500 & 4.95513100 & -1.56325400 & $\mathrm{H}$ & 0.97469900 & 0.23635300 & 4.45883800 \\
\hline $\mathrm{H}$ & -2.07414500 & 4.74000000 & -0.94331300 & $\mathrm{H}$ & 2.61679900 & -0.17678500 & 3.99169500 \\
\hline $\mathrm{H}$ & -3.48683000 & 2.58422800 & -0.22412000 & $\mathrm{C}$ & 2.33458300 & 1.92034400 & 4.44725000 \\
\hline $\mathrm{N}$ & -1.59823900 & 1.53240400 & -0.29388300 & $\mathrm{H}$ & 1.56326800 & 2.69666600 & 4.37229000 \\
\hline$P$ & -1.74910600 & -0.18543700 & -0.05703800 & $\mathrm{H}$ & 2.60887600 & 1.82225300 & 5.50416300 \\
\hline $\mathrm{C}$ & -2.57048700 & -0.81848500 & -1.67133300 & $\mathrm{H}$ & 3.21729300 & 2.27875600 & 3.90384700 \\
\hline
\end{tabular}




\begin{tabular}{|c|c|c|c|c|c|c|c|}
\hline $\mathrm{C}$ & 3.01722700 & -2.45274000 & -0.74876400 & $\mathrm{C}$ & -2.12114100 & 0.84281200 & 2.58536600 \\
\hline $\mathrm{H}$ & 2.40986900 & -3.35336000 & -0.61939700 & $\mathrm{H}$ & -2.08033500 & 1.86244000 & 2.19151500 \\
\hline $\mathrm{C}$ & 0.98152800 & -0.65530100 & 1.84127000 & $\mathrm{H}$ & -2.69906800 & 0.86249500 & 3.51815400 \\
\hline $\mathrm{H}$ & 0.15234300 & -1.06722500 & 2.42348200 & $\mathrm{C}$ & -4.24388400 & 0.35489900 & 1.35837800 \\
\hline \multirow[t]{2}{*}{$\mathrm{H}$} & 1.79878300 & -1.38624700 & 1.87479700 & $\mathrm{H}$ & -4.76193500 & 0.41624500 & 2.32430300 \\
\hline & & & & $\mathrm{H}$ & -4.27791500 & 1.35398800 & 0.91126400 \\
\hline \multirow[t]{6}{*}{ 68-ts } & & & & $\mathrm{H}$ & -4.81655700 & -0.32833300 & 0.72699700 \\
\hline & & & & $\mathrm{F}$ & 3.39851300 & 2.51515400 & -2.73273000 \\
\hline & & & & $\mathrm{Rh}$ & 0.39629600 & -1.02194800 & 0.21330500 \\
\hline & & & & $\mathrm{Cl}$ & -0.27988000 & -3.32633600 & 0.24787400 \\
\hline & & & & $\mathrm{H}$ & -1.10464600 & 0.52509000 & 2.83341800 \\
\hline & & & & $\mathrm{H}$ & 0.20960900 & 2.47058100 & 1.74471800 \\
\hline $\mathrm{C}$ & -0.05927300 & 2.78754500 & -1.69773000 & $\mathrm{O}$ & 2.50981700 & -1.75109400 & 0.17467400 \\
\hline $\mathrm{C}$ & -0.09335400 & 1.65824500 & -0.85864000 & $\mathrm{O}$ & 4.11792300 & -3.26246000 & -0.25170900 \\
\hline $\mathrm{C}$ & 1.01339300 & 0.84548100 & -0.51149100 & $\mathrm{C}$ & 5.12634100 & -2.30511200 & 0.14019700 \\
\hline $\mathrm{C}$ & 2.18818300 & 1.16685500 & -1.22768000 & $\mathrm{H}$ & 4.96624800 & -1.99166100 & 1.17441500 \\
\hline $\mathrm{C}$ & 2.22162700 & 2.25794100 & -2.10570400 & $\mathrm{H}$ & 6.07490200 & -2.83045800 & 0.03786700 \\
\hline $\mathrm{C}$ & 1.15237700 & 3.11066300 & -2.34050000 & $\mathrm{H}$ & 5.09498000 & -1.43417800 & -0.51865800 \\
\hline $\mathrm{C}$ & -1.39515600 & 3.33142500 & -1.68403800 & $\mathrm{C}$ & 1.26530900 & 2.17910300 & 1.75319100 \\
\hline $\mathrm{C}$ & -2.16789200 & 2.50945600 & -0.90826400 & $\mathrm{H}$ & 1.78127700 & 2.84884000 & 1.05424000 \\
\hline $\mathrm{H}$ & 3.11032300 & 0.62171900 & -1.05587900 & $\mathrm{C}$ & 1.83451500 & 2.38017200 & 3.16799300 \\
\hline $\mathrm{H}$ & 1.26724800 & 3.96752800 & -2.99530500 & $\mathrm{H}$ & 1.30541400 & 1.72480500 & 3.87432100 \\
\hline $\mathrm{H}$ & -1.73790600 & 4.22546500 & -2.18876300 & $\mathrm{H}$ & 2.88694600 & 2.06307600 & 3.18608600 \\
\hline $\mathrm{H}$ & -3.21967000 & 2.58323700 & -0.67744000 & $\mathrm{C}$ & 1.73161300 & 3.83427100 & 3.64313800 \\
\hline $\mathrm{N}$ & -1.39490900 & 1.46109900 & -0.39316800 & $\mathrm{H}$ & 0.68757500 & 4.17000200 & 3.66704600 \\
\hline $\mathrm{P}$ & -1.71300500 & -0.21972500 & 0.03466600 & $\mathrm{H}$ & 2.14383900 & 3.95366900 & 4.65163300 \\
\hline $\mathrm{C}$ & -2.69619500 & -0.91898100 & -1.47207000 & $\mathrm{H}$ & 2.28086400 & 4.50878100 & 2.97518900 \\
\hline $\mathrm{C}$ & -2.80266300 & -0.13269000 & 1.60370400 & $\mathrm{C}$ & 2.85795800 & -2.87045700 & -0.18881900 \\
\hline $\mathrm{C}$ & -3.76224000 & 0.04208300 & -2.04178200 & $\mathrm{H}$ & 2.15544400 & -3.65367800 & -0.48266800 \\
\hline $\mathrm{H}$ & -3.31752200 & 0.94245700 & -2.47162700 & $\mathrm{C}$ & 1.42287900 & 0.72620100 & 1.28947400 \\
\hline $\mathrm{H}$ & -4.28728600 & -0.48299100 & -2.85030000 & $\mathrm{H}$ & 0.95504900 & 0.07978900 & 2.05180400 \\
\hline $\mathrm{H}$ & -4.51748500 & 0.34030600 & -1.31035900 & $\mathrm{H}$ & 2.47578300 & 0.45107900 & 1.23126700 \\
\hline $\mathrm{C}$ & -1.65620200 & -1.18028300 & -2.58474700 & & & & \\
\hline $\mathrm{H}$ & -2.18519500 & -1.54214500 & -3.47676900 & 69 & & & \\
\hline $\mathrm{H}$ & -1.11755200 & -0.26825400 & -2.86215300 & & & & \\
\hline $\mathrm{H}$ & -0.92528400 & -1.93402200 & -2.28397800 & & & & \\
\hline $\mathrm{C}$ & -3.37782200 & -2.25215700 & -1.09655400 & & & & \\
\hline $\mathrm{H}$ & -2.68002800 & -2.95854100 & -0.64213200 & & & & \\
\hline $\mathrm{H}$ & -4.23097100 & -2.10696100 & -0.42607700 & & & & \\
\hline $\mathrm{H}$ & -3.76657800 & -2.70735700 & -2.01699800 & $\mathrm{C}$ & -0.47549500 & 3.44782000 & -0.22306500 \\
\hline $\mathrm{C}$ & -2.83133900 & -1.53475600 & 2.25174000 & $\mathrm{C}$ & -0.38718900 & 2.04760200 & -0.10141100 \\
\hline $\mathrm{H}$ & -3.36472700 & -2.26859600 & 1.64332400 & $\mathrm{C}$ & 0.77829200 & 1.27016900 & -0.14351400 \\
\hline $\mathrm{H}$ & -1.82336800 & -1.92187400 & 2.42312700 & $\mathrm{C}$ & 1.94487700 & 2.01095500 & -0.35737100 \\
\hline $\mathrm{H}$ & -3.34817000 & -1.46321400 & 3.21787700 & $\mathrm{C}$ & 1.88737400 & 3.41497300 & -0.47754000 \\
\hline
\end{tabular}




\begin{tabular}{|c|c|c|c|c|c|c|c|}
\hline $\mathrm{C}$ & 0.72520900 & 4.16417100 & -0.40822700 & $\mathrm{H}$ & 4.88675100 & -0.10760900 & -1.73033500 \\
\hline $\mathrm{C}$ & -1.87893000 & 3.76609700 & -0.13682600 & $\mathrm{C}$ & 0.69571800 & 0.42341500 & 2.91640500 \\
\hline $\mathrm{C}$ & -2.56737300 & 2.58784500 & 0.00561800 & $\mathrm{H}$ & -0.05799400 & 1.11371800 & 2.52763100 \\
\hline $\mathrm{H}$ & 2.91788900 & 1.53505700 & -0.42098200 & $\mathrm{H}$ & 0.33712900 & 0.12368000 & 3.91776800 \\
\hline $\mathrm{H}$ & 0.76289600 & 5.24391900 & -0.50648200 & $\mathrm{C}$ & 0.72686900 & -0.86264100 & 2.07428200 \\
\hline $\mathrm{H}$ & -2.32762200 & 4.75079000 & -0.16921000 & $\mathrm{H}$ & -0.17069200 & -1.44791200 & 2.29260800 \\
\hline $\mathrm{H}$ & -3.63166900 & 2.42843300 & 0.09400600 & $\mathrm{H}$ & 2.62769800 & -2.93015300 & -1.05788100 \\
\hline $\mathrm{N}$ & -1.67218100 & 1.51518300 & 0.04200000 & $\mathrm{C}$ & 1.94355100 & -1.73486200 & 2.37080800 \\
\hline $\mathrm{P}$ & -1.71914300 & -0.21985900 & -0.11855500 & $\mathrm{H}$ & 1.87725800 & -2.69943300 & 1.86288100 \\
\hline $\mathrm{C}$ & -2.25262800 & -0.52035900 & -1.94360400 & $\mathrm{H}$ & 2.88391900 & -1.24877300 & 2.09712800 \\
\hline $\mathrm{C}$ & -3.05619000 & -0.81486700 & 1.11286800 & $\mathrm{C}$ & 2.01841600 & 1.17569600 & 3.10836900 \\
\hline $\mathrm{C}$ & -3.34373600 & 0.45703300 & -2.42446000 & $\mathrm{H}$ & 2.44587200 & 1.49193400 & 2.15357700 \\
\hline $\mathrm{H}$ & -3.02019800 & 1.49936600 & -2.35649900 & $\mathrm{H}$ & 1.85031200 & 2.07566900 & 3.71131600 \\
\hline $\mathrm{H}$ & -3.55449700 & 0.24289200 & -3.48029500 & $\mathrm{H}$ & 2.76091100 & 0.56452500 & 3.63342500 \\
\hline $\mathrm{H}$ & -4.28456100 & 0.35130100 & -1.87934500 & $\mathrm{H}$ & 1800 & -1.926 & 6200 \\
\hline $\mathrm{C}$ & -0.98398800 & -0.27635000 & -2.79531700 & & & & \\
\hline $\mathrm{H}$ & -1.23861500 & -0.41176900 & -3.85489200 & 70-ts & & & \\
\hline $\mathrm{H}$ & -0.58729700 & 0.73543600 & -2.67160300 & & & & \\
\hline $\mathrm{H}$ & -0.19335100 & -0.99970800 & -2.55858500 & & & & \\
\hline $\mathrm{C}$ & -2.70863500 & -1.97833400 & -2.15792100 & & & & \\
\hline $\mathrm{H}$ & -1.97047700 & -2.69611800 & -1.78836100 & & & & \\
\hline $\mathrm{H}$ & -3.67450200 & -2.18953900 & -1.68955500 & & & & \\
\hline $\mathrm{H}$ & -2.83198700 & -2.14518500 & -3.23615600 & $\mathrm{C}$ & -0.09248400 & 3.07814600 & -1.02506700 \\
\hline $\mathrm{C}$ & -2.89183000 & -2.33595300 & 1.32665400 & $\mathrm{C}$ & -0.13838700 & 1.83628500 & -0.36415900 \\
\hline $\mathrm{H}$ & -3.10838700 & -2.91034600 & 0.42332000 & $\mathrm{C}$ & 0.97921100 & 1.03665100 & -0.01110300 \\
\hline $\mathrm{H}$ & -1.88218700 & -2.60690200 & 1.64285800 & $\mathrm{C}$ & 2.19393800 & 1.51715200 & -0.54726900 \\
\hline $\mathrm{H}$ & -3.59808600 & -2.65406300 & 2.10452000 & $\mathrm{C}$ & 2.24563900 & 2.72427600 & -1.25827900 \\
\hline $\mathrm{C}$ & -2.84108300 & -0.08741500 & 2.45775100 & $\mathrm{C}$ & 1.15471100 & 3.55143800 & -1.48118200 \\
\hline $\mathrm{H}$ & -2.93758800 & 0.99782400 & 2.36308600 & $\mathrm{C}$ & -1.45584600 & 3.54491400 & -1.09285700 \\
\hline $\mathrm{H}$ & -3.60692500 & -0.43533700 & 3.16251200 & $\mathrm{C}$ & -2.25131500 & 2.57450200 & -0.54635600 \\
\hline $\mathrm{C}$ & -4.49896000 & -0.52714800 & 0.64439700 & $\mathrm{H}$ & 3.12443400 & 0.98547600 & -0.38715800 \\
\hline $\mathrm{H}$ & -5.18636500 & -0.86936100 & 1.42853700 & $\mathrm{H}$ & 1.27861300 & 4.49831100 & -1.99544000 \\
\hline $\mathrm{H}$ & -4.69154100 & 0.53917700 & 0.49145300 & $\mathrm{H}$ & -1.80067400 & 4.48650100 & -1.50043300 \\
\hline $\mathrm{H}$ & -4.76173200 & -1.06148000 & -0.27091100 & $\mathrm{H}$ & -3.32518700 & 2.55551700 & -0.43917500 \\
\hline $\mathrm{F}$ & 3.06442700 & 4.06887000 & -0.66809000 & $\mathrm{~N}$ & -1.47034100 & 1.50028100 & -0.09955000 \\
\hline $\mathrm{Rh}$ & 0.51994000 & -0.73600100 & -0.02036300 & $\mathrm{P}$ & -1.71345200 & -0.24603500 & -0.01244900 \\
\hline $\mathrm{Cl}$ & 0.21510000 & -3.20611600 & -0.32378300 & $\mathrm{C}$ & -2.40988900 & -0.71002300 & -1.75895800 \\
\hline $\mathrm{H}$ & -1.86706700 & -0.30555700 & 2.89640200 & $\mathrm{C}$ & -3.03806800 & -0.51383000 & 1.34498900 \\
\hline $\mathrm{C}$ & 3.19318700 & -2.00437500 & -0.91644500 & $\mathrm{C}$ & -3.44278500 & 0.29248300 & -2.31841200 \\
\hline $\mathrm{O}$ & 2.69910100 & -0.97815500 & -0.45533100 & $\mathrm{H}$ & -3.00111500 & 1.27122100 & -2.51818200 \\
\hline $\mathrm{O}$ & 4.45598400 & -2.10479300 & -1.28409700 & $\mathrm{H}$ & -3.80997500 & -0.10428600 & -3.27384500 \\
\hline $\mathrm{C}$ & 5.28468600 & -0.92857700 & -1.12981300 & $\mathrm{H}$ & -4.31315200 & 0.42857400 & -1.67214800 \\
\hline $\mathrm{H}$ & 5.32434600 & -0.63189800 & -0.07943800 & $\mathrm{C}$ & -1.20331000 & -0.72539600 & -2.72349500 \\
\hline $\mathrm{H}$ & 6.27018400 & -1.22594000 & -1.48518600 & $\mathrm{H}$ & -1.57054900 & -0.94192600 & -3.73590300 \\
\hline
\end{tabular}




\begin{tabular}{|c|c|c|c|c|c|c|c|}
\hline $\mathrm{H}$ & -0.68980200 & 0.24081400 & -2.75153300 & & & & \\
\hline $\mathrm{H}$ & -0.47701700 & -1.49216800 & -2.44498100 & & & & \\
\hline $\mathrm{C}$ & -3.04748400 & -2.11573900 & -1.73304500 & & & & \\
\hline $\mathrm{H}$ & -2.38096100 & -2.86417800 & -1.29932100 & & & & \\
\hline $\mathrm{H}$ & -4.00123300 & -2.12889300 & -1.19607600 & & & & \\
\hline $\mathrm{H}$ & -3.25871400 & -2.41237800 & -2.76893800 & $\mathrm{C}$ & -0.88447700 & 3.45863000 & 0.26968600 \\
\hline $\mathrm{C}$ & -3.05527700 & -2.01354000 & 1.71668500 & $\mathrm{C}$ & -0.63777600 & 2.07710500 & 0.15526400 \\
\hline $\mathrm{H}$ & -3.40801400 & -2.64796000 & 0.90123900 & $\mathrm{C}$ & 0.61018200 & 1.45224800 & 0.03534800 \\
\hline $\mathrm{H}$ & -2.06265800 & -2.37026800 & 2.00316000 & $\mathrm{C}$ & 1.69083200 & 2.33755600 & 0.01823400 \\
\hline $\mathrm{H}$ & -3.73563900 & -2.15229700 & 2.56725900 & $\mathrm{C}$ & 1.47412900 & 3.72635700 & 0.13342100 \\
\hline $\mathrm{C}$ & -2.60088300 & 0.28143100 & 2.59103700 & $\mathrm{C}$ & 0.23083700 & 4.32200700 & 0.26404800 \\
\hline $\mathrm{H}$ & -2.60614600 & 1.36063300 & 2.41507400 & $\mathrm{C}$ & -2.31584200 & 3.59897000 & 0.36842400 \\
\hline $\mathrm{H}$ & -3.30524900 & 0.06912700 & 3.40545900 & $\mathrm{C}$ & -2.86579800 & 2.34337000 & 0.30124100 \\
\hline $\mathrm{C}$ & -4.45930400 & -0.05994700 & 0.95750100 & $\mathrm{H}$ & 2.71417100 & 1.98628200 & -0.06516400 \\
\hline $\mathrm{H}$ & -5.12031900 & -0.20698100 & 1.82165200 & $\mathrm{H}$ & 0.14713700 & 5.40007500 & 0.35169000 \\
\hline $\mathrm{H}$ & -4.50364100 & 1.00233600 & 0.69568500 & $\mathrm{H}$ & -2.87493500 & 4.51871400 & 0.48463900 \\
\hline $\mathrm{H}$ & -4.87545500 & -0.63954000 & 0.13050300 & $\mathrm{H}$ & -3.90534100 & 2.05346500 & 0.33519600 \\
\hline $\mathrm{F}$ & 3.45931300 & 3.12086500 & -1.72172700 & $\mathrm{~N}$ & -1.85356700 & 1.38769100 & 0.18075000 \\
\hline $\mathrm{Rh}$ & 0.41570600 & -0.95697700 & 0.28234300 & $\mathrm{P}$ & -1.69764100 & -0.31257000 & -0.16249500 \\
\hline $\mathrm{Cl}$ & -0.14929800 & -3.27258300 & -0.07441600 & $\mathrm{C}$ & -2.21435300 & -0.47244900 & -2.00937300 \\
\hline $\mathrm{H}$ & -1.60410000 & -0.01183500 & 2.93163400 & $\mathrm{C}$ & -2.93343400 & -1.19436700 & 0.99918200 \\
\hline $\mathrm{C}$ & 2.88460000 & -2.56106200 & -0.66720500 & $\mathrm{C}$ & -3.41681200 & 0.41954400 & -2.37678700 \\
\hline $\mathrm{O}$ & 2.54884800 & -1.63731500 & 0.06698400 & $\mathrm{H}$ & -3.21696700 & 1.47722400 & -2.18444100 \\
\hline $\mathrm{O}$ & 4.14089000 & -2.91207300 & -0.88046100 & $\mathrm{H}$ & -3.61132800 & 0.30823200 & -3.45139200 \\
\hline $\mathrm{C}$ & 5.16231000 & -2.16059000 & -0.18904300 & $\mathrm{H}$ & -4.33343100 & 0.13919100 & -1.85244500 \\
\hline $\mathrm{H}$ & 5.02659000 & -2.24783600 & 0.89152600 & $\mathrm{C}$ & -0.99128000 & 0.01259700 & -2.82461800 \\
\hline $\mathrm{H}$ & 6.10581500 & -2.60974500 & -0.49625600 & $\mathrm{H}$ & -1.24093100 & -0.02463700 & -3.89347000 \\
\hline $\mathrm{H}$ & 5.12187300 & -1.10914900 & -0.48272500 & $\mathrm{H}$ & -0.70895700 & 1.04115400 & -2.58113700 \\
\hline $\mathrm{C}$ & 0.53149800 & 1.90673400 & 2.52448200 & $\mathrm{H}$ & -0.12199300 & -0.64088400 & -2.67491700 \\
\hline $\mathrm{H}$ & -0.48821000 & 2.05407100 & 2.16760100 & $\mathrm{C}$ & -2.50246100 & -1.93930200 & -2.38699100 \\
\hline $\mathrm{H}$ & 0.44239900 & 1.67644400 & 3.59904600 & $\mathrm{H}$ & -1.67713100 & -2.60177900 & -2.11043200 \\
\hline $\mathrm{C}$ & 1.14148900 & 0.64011100 & 1.88401000 & $\mathrm{H}$ & -3.42496400 & -2.31326700 & -1.93356100 \\
\hline $\mathrm{H}$ & 0.60055000 & -0.20995600 & 2.32822600 & $\mathrm{H}$ & -2.63237900 & -1.99586800 & -3.47573200 \\
\hline $\mathrm{H}$ & 2.17693400 & -3.19340800 & -1.20674700 & $\mathrm{C}$ & -2.58928600 & -2.69968900 & 1.04063900 \\
\hline $\mathrm{C}$ & 2.61492300 & 0.47524100 & 2.24438700 & $\mathrm{H}$ & -2.74770200 & -3.19360500 & 0.07962400 \\
\hline $\mathrm{H}$ & 3.01999500 & -0.47998200 & 1.91343700 & $\mathrm{H}$ & -1.55119000 & -2.88154600 & 1.32674600 \\
\hline $\mathrm{H}$ & 3.24023300 & 1.27671600 & 1.84215500 & $\mathrm{H}$ & -3.24335000 & -3.18294800 & 1.77806000 \\
\hline $\mathrm{C}$ & 1.31348400 & 3.21835200 & 2.38374700 & $\mathrm{C}$ & -2.77258400 & -0.60339300 & 2.41680800 \\
\hline $\mathrm{H}$ & 1.44097600 & 3.51603900 & 1.34055900 & $\mathrm{H}$ & -3.00406100 & 0.46504800 & 2.45115000 \\
\hline $\mathrm{H}$ & 0.77019900 & 4.02130600 & 2.89582300 & $\mathrm{H}$ & -3.47073700 & -1.12224000 & 3.08588200 \\
\hline $\mathrm{H}$ & 2.30598500 & 3.15475700 & 2.84241100 & $\mathrm{C}$ & -4.40864300 & -1.02628300 & 0.57672900 \\
\hline \multirow[t]{2}{*}{$\mathrm{H}$} & 2.69633800 & 0.51316000 & 3.34055100 & $\mathrm{H}$ & -5.03677800 & -1.52701500 & 1.32458500 \\
\hline & & & & $\mathrm{H}$ & -4.72381000 & 0.02095700 & 0.54205100 \\
\hline & & & & $\mathrm{H}$ & -4.62732200 & -1.48688500 & -0.38905700 \\
\hline
\end{tabular}




\begin{tabular}{|c|c|c|c|c|c|c|c|}
\hline$F$ & 2.57264900 & 4.52817400 & 0.11865000 & $\mathrm{~N}$ & -1.60135400 & 1.49518200 & 0.11355200 \\
\hline $\mathrm{Rh}$ & 0.58124000 & -0.56788300 & -0.13201600 & $\mathrm{P}$ & -1.74632400 & -0.25858600 & 0.01663000 \\
\hline $\mathrm{Cl}$ & 0.56767100 & -3.00398300 & -0.71505500 & $\mathrm{C}$ & -2.53059000 & -0.56744000 & -1.72644100 \\
\hline $\mathrm{H}$ & -1.76710900 & -0.74859400 & 2.81397900 & $\mathrm{C}$ & -2.96233400 & -0.75520000 & 1.41091200 \\
\hline $\mathrm{C}$ & 3.34217000 & -1.36170200 & -1.29130700 & $\mathrm{C}$ & -3.64861800 & 0.42843500 & -2.10268000 \\
\hline $\mathrm{O}$ & 2.75856000 & -0.47617300 & -0.67143000 & $\mathrm{H}$ & -3.27381800 & 1.44955100 & -2.20157000 \\
\hline $\mathrm{O}$ & 4.57549800 & -1.24452700 & -1.74475500 & $\mathrm{H}$ & -4.04761700 & 0.12829800 & -3.08031500 \\
\hline $\mathrm{C}$ & 5.25596900 & 0.00668600 & -1.48802200 & $\mathrm{H}$ & -4.48728500 & 0.43115700 & -1.40225800 \\
\hline $\mathrm{H}$ & 5.35718500 & 0.16447600 & -0.41196200 & $\mathrm{C}$ & -1.39204200 & -0.40114800 & -2.75816900 \\
\hline $\mathrm{H}$ & 6.23326700 & -0.09917200 & -1.95666500 & $\mathrm{H}$ & -1.81329900 & -0.53386000 & -3.76403700 \\
\hline $\mathrm{H}$ & 4.69938200 & 0.83528100 & -1.93118300 & $\mathrm{H}$ & -0.93978200 & 0.59464100 & -2.71202800 \\
\hline $\mathrm{C}$ & 0.72262700 & 0.32344500 & 2.83491700 & $\mathrm{H}$ & -0.60457500 & -1.14354900 & -2.61069100 \\
\hline $\mathrm{H}$ & 1.54433400 & 1.03178700 & 2.69210300 & $\mathrm{C}$ & -3.08585200 & -2.00393900 & -1.82535400 \\
\hline $\mathrm{H}$ & -0.20963000 & 0.86686000 & 2.67114000 & $\mathrm{H}$ & -2.34629200 & -2.75192000 & -1.53170300 \\
\hline $\mathrm{H}$ & 0.73487500 & 0.00274000 & 3.88799500 & $\mathrm{H}$ & -3.99198000 & -2.14128700 & -1.22723100 \\
\hline $\mathrm{C}$ & 0.87038700 & -0.89217600 & 1.92598400 & $\mathrm{H}$ & -3.35956100 & -2.19161700 & -2.87198200 \\
\hline $\mathrm{H}$ & 0.08224900 & -1.62459300 & 2.13200600 & $\mathrm{C}$ & -2.86273500 & -2.28326400 & 1.61594600 \\
\hline $\mathrm{H}$ & 2.88890300 & -2.33100600 & -1.51982500 & $\mathrm{H}$ & -3.23801600 & -2.84977800 & 0.76127000 \\
\hline $\mathrm{C}$ & 2.22871500 & -1.58463200 & 2.09079800 & $\mathrm{H}$ & -1.83178000 & -2.60054700 & 1.79199900 \\
\hline $\mathrm{H}$ & 2.32664400 & -2.37748900 & 1.34391900 & $\mathrm{H}$ & -3.46818700 & -2.55640200 & 2.49020500 \\
\hline $\mathrm{H}$ & 3.03777800 & -0.86310000 & 1.91949900 & $\mathrm{C}$ & -2.49734300 & -0.06868400 & 2.71066300 \\
\hline $\mathrm{C}$ & 2.40671400 & -2.22218300 & 3.48460100 & $\mathrm{H}$ & -2.57164200 & 1.02098700 & 2.65390000 \\
\hline $\mathrm{H}$ & 3.35692900 & -2.76701100 & 3.53200400 & $\mathrm{H}$ & -3.14109100 & -0.40640700 & 3.53289000 \\
\hline $\mathrm{H}$ & 2.41194100 & -1.47564100 & 4.28576300 & $\mathrm{C}$ & -4.43163300 & -0.36253100 & 1.15843200 \\
\hline \multirow[t]{2}{*}{$\mathrm{H}$} & 1.60341100 & -2.93907500 & 3.69447700 & $\mathrm{H}$ & -5.02580900 & -0.65316900 & 2.03466400 \\
\hline & & & & $\mathrm{H}$ & -4.56138400 & 0.71681000 & 1.02663400 \\
\hline \multirow[t]{6}{*}{ 72-ts } & & & & $\mathrm{H}$ & -4.86144200 & -0.87232500 & 0.29329800 \\
\hline & & & & $\mathrm{F}$ & 3.13723400 & 3.66328200 & -1.43322400 \\
\hline & & & & $\mathrm{Rh}$ & 0.43094800 & -0.86996400 & 0.10742100 \\
\hline & & & & $\mathrm{Cl}$ & -0.03396900 & -3.16637400 & -0.45957500 \\
\hline & & & & $\mathrm{H}$ & -1.46880700 & -0.33728700 & 2.96672500 \\
\hline & & & & $\mathrm{C}$ & 2.93044200 & -2.25307000 & -1.12409000 \\
\hline $\mathrm{C}$ & -0.37169600 & 3.27152200 & -0.63915100 & $\mathrm{O}$ & 2.58317000 & -1.38625700 & -0.32855100 \\
\hline $\mathrm{C}$ & $-0.30 s$ & 1.9457 & 4200 & $\mathrm{O}$ & 4.18507300 & -2.46122800 & -1.48466100 \\
\hline $\mathrm{C}$ & 0.87017400 & 1.18125900 & 0.02392200 & $\mathrm{C}$ & 5.19169900 & -1.61014700 & -0.89459500 \\
\hline $\mathrm{C}$ & 2.02691300 & 1.81761700 & -0.47911100 & $\mathrm{H}$ & 5.19115100 & -1.72321000 & 0.19210800 \\
\hline $\mathrm{C}$ & 1.97123300 & 3.11791900 & -0.99892300 & $\mathrm{H}$ & 6.13695500 & -1.94934600 & -1.31624600 \\
\hline $\mathrm{C}$ & 0.82185400 & 3.89142400 & -1.05967700 & $\mathrm{H}$ & 5.00437200 & -0.56658000 & -1.15823500 \\
\hline $\mathrm{C}$ & -1.76043800 & 3.65485500 & -0.57267800 & $\mathrm{C}$ & 0.55774300 & 1.80321100 & 2.56995400 \\
\hline $\mathrm{C}$ & -2.46714100 & 2.56859100 & -0.13216400 & $\mathrm{H}$ & 1.01960200 & 2.73948900 & 2.23958200 \\
\hline $\mathrm{H}$ & 2.99822200 & 1.34152500 & -0.42103200 & $\mathrm{H}$ & -0.52005100 & 1.88992500 & 2.43958700 \\
\hline $\mathrm{H}$ & 0.86460600 & 4.91192600 & -1.42446600 & $\mathrm{H}$ & 0.75216700 & 1.68845700 & 3.64499600 \\
\hline $\mathrm{H}$ & -2.18065100 & 4.62044000 & -0.82288200 & $\mathrm{C}$ & 1.15847600 & 0.60311900 & 1.83121600 \\
\hline $\mathrm{H}$ & -3.53033800 & 2.46511800 & 0.02288600 & $\mathrm{H}$ & 0.67968800 & -0.30561000 & 2.22731100 \\
\hline
\end{tabular}




\begin{tabular}{|c|c|c|c|c|c|c|c|}
\hline $\mathrm{H}$ & 2.23453100 & -2.94499600 & -1.60305000 & $\mathrm{H}$ & -2.77623900 & -3.21916000 & 0.63514900 \\
\hline $\mathrm{C}$ & 2.66835700 & 0.50328500 & 2.09193000 & $\mathrm{H}$ & -1.34143600 & -2.81446100 & 1.56432700 \\
\hline $\mathrm{H}$ & 3.15710900 & -0.10032000 & 1.32781000 & $\mathrm{H}$ & -2.88805000 & -3.09379300 & 2.39608700 \\
\hline $\mathrm{H}$ & 3.10761700 & 1.50748900 & 2.03496100 & $\mathrm{C}$ & -2.39385400 & -0.47135500 & 2.75532900 \\
\hline $\mathrm{C}$ & 2.97801700 & -0.11955600 & 3.46415800 & $\mathrm{H}$ & -2.65058000 & 0.59185500 & 2.76034500 \\
\hline $\mathrm{H}$ & 4.06103500 & -0.14945400 & 3.63386400 & $\mathrm{H}$ & -2.93917200 & -0.95491300 & 3.57573600 \\
\hline $\mathrm{H}$ & 2.53338500 & 0.44781700 & 4.28934700 & $\mathrm{C}$ & -4.32883300 & -1.03807600 & 1.27758200 \\
\hline \multirow[t]{2}{*}{$\mathrm{H}$} & 2.60269700 & -1.14853500 & 36900 & $\mathrm{H}$ & -4.80092600 & -1.49689800 & 2.15577400 \\
\hline & & & & $\mathrm{H}$ & -4.66806400 & 0.00160900 & 1.23754900 \\
\hline \multirow[t]{6}{*}{73} & & & & $\mathrm{H}$ & -4.70661700 & -1.56195400 & 0.39667300 \\
\hline & & & & $\mathrm{F}$ & 2.42414100 & 4.53001300 & -0.28246300 \\
\hline & & & & $\mathrm{Rh}$ & 0.43597500 & -0.57341100 & -0.30875500 \\
\hline & & & & $\mathrm{Cl}$ & 0.43669500 & -3.02714400 & -0.72275100 \\
\hline & & & & $\mathrm{H}$ & -1.32768700 & -0.56707500 & 2.96572400 \\
\hline & & & & $\mathrm{C}$ & 3.10135200 & -1.42505100 & -1.66684600 \\
\hline $\mathrm{C}$ & -0.98545600 & 3.44451300 & 0.27944400 & $\mathrm{O}$ & 2.52047700 & -0.48610800 & -1.12833900 \\
\hline $\mathrm{C}$ & -0.74856500 & 2.06467500 & 0.12756000 & $\mathrm{O}$ & 4.28009100 & -1.31502600 & -2.24899000 \\
\hline $\mathrm{C}$ & 0.47617200 & 1.44687800 & -0.15416500 & $\mathrm{C}$ & 4.89885100 & -0.00678400 & -2.24189300 \\
\hline $\mathrm{C}$ & 1.54568000 & 2.33584900 & -0.28773500 & $\mathrm{H}$ & 5.08161000 & 0.31593700 & -1.21462900 \\
\hline $\mathrm{C}$ & 1.33887400 & 3.72310300 & -0.13888000 & $\mathrm{H}$ & 5.83709600 & -0.13250900 & -2.78026300 \\
\hline $\mathrm{C}$ & 0.11756200 & 4.31248000 & 0.14155100 & $\mathrm{H}$ & 4.25431900 & 0.71652700 & -2.74610300 \\
\hline $\mathrm{C}$ & -2.39521100 & 3.57658600 & 0.54936600 & $\mathrm{C}$ & 0.96460300 & -0.69404200 & 1.67989500 \\
\hline $\mathrm{C}$ & -2.94339000 & 2.31822700 & 0.54230400 & $\mathrm{H}$ & 0.57648100 & 0.18280400 & 2.20587000 \\
\hline $\mathrm{H}$ & 2.55107500 & 1.99114700 & -0.50672700 & $\mathrm{H}$ & 0.46242500 & -1.59772800 & 2.03946000 \\
\hline $\mathrm{H}$ & 0.04100700 & 5.38990200 & 0.24277900 & $\mathrm{C}$ & 2.47055500 & -0.81044400 & 1.87467400 \\
\hline $\mathrm{H}$ & -2.94175100 & 4.49302200 & 0.73209300 & $\mathrm{H}$ & 2.84996300 & -1.69636700 & 1.35001500 \\
\hline $\mathrm{H}$ & -3.96971200 & 2.02236200 & 0.70038000 & $\mathrm{H}$ & 2.97990400 & 0.06401400 & 1.45145000 \\
\hline $\mathrm{N}$ & -1.94924900 & 1.36974900 & 0.28957500 & $\mathrm{C}$ & 2.83662800 & -0.92423900 & 3.36941900 \\
\hline $\mathrm{P}$ & -1.81653500 & -0.34338300 & 0.01501200 & $\mathrm{H}$ & 2.32816000 & -1.79686800 & 3.80226600 \\
\hline $\mathrm{C}$ & -2.63671500 & -0.62524300 & -1.69648300 & $\mathrm{H}$ & 2.45244700 & -0.04437800 & 3.90391300 \\
\hline $\mathrm{C}$ & -2.79847600 & -1.16338200 & 1.43396900 & $\mathrm{H}$ & 2.69141300 & -2.43833200 & -1.71045600 \\
\hline $\mathrm{C}$ & -3.91309000 & 0.21405700 & -1.90111000 & $\mathrm{C}$ & 4.34718700 & -1.04456300 & 3.60160300 \\
\hline $\mathrm{H}$ & -3.71648500 & 1.28590700 & -1.80968300 & $\mathrm{H}$ & 4.58173600 & -1.12394000 & 4.66949300 \\
\hline $\mathrm{H}$ & -4.28758000 & 0.03030600 & -2.91650600 & $\mathrm{H}$ & 4.75466200 & -1.93323500 & 3.10374400 \\
\hline $\mathrm{H}$ & -4.71500500 & -0.05187900 & -1.20809400 & $\mathrm{H}$ & 4.87833500 & -0.16883300 & 3.20815800 \\
\hline $\mathrm{C}$ & -1.58529100 & -0.16449700 & -2.73439600 & & & & \\
\hline $\mathrm{H}$ & -2.00787600 & -0.27602600 & -3.74177100 & 74-t & & & \\
\hline $\mathrm{H}$ & -1.30434500 & 0.88501700 & -2.60224600 & & & & \\
\hline $\mathrm{H}$ & -0.67990600 & -0.78427100 & -2.69507100 & & & & \\
\hline $\mathrm{C}$ & -2.93487100 & -2.12067800 & -1.92458700 & & & & \\
\hline $\mathrm{H}$ & -2.05764800 & -2.74642400 & -1.73467700 & & & & \\
\hline $\mathrm{H}$ & -3.76471600 & -2.47754300 & -1.30728200 & & & & \\
\hline $\mathrm{H}$ & -3.22946700 & -2.26013400 & -2.97296800 & $\mathrm{C}$ & 88200 & 3.27632700 & -0.67167700 \\
\hline $\mathrm{C}$ & -2.42023000 & -2.65922600 & 1.50320400 & $\mathrm{C}$ & -0.37404400 & 1.95459200 & 0214700 \\
\hline
\end{tabular}




\begin{tabular}{|c|c|c|c|c|c|c|c|}
\hline $\mathrm{C}$ & 0.78557300 & 1.14000800 & -0.21005800 & $\mathrm{C}$ & 4.96411500 & -2.01732300 & -1.06526200 \\
\hline $\mathrm{C}$ & 1.85875500 & 1.71140400 & -0.92900900 & $\mathrm{H}$ & 4.86784600 & -2.25637500 & -0.00362500 \\
\hline $\mathrm{C}$ & 1.76273500 & 3.00626600 & -1.45791600 & $\mathrm{H}$ & 5.87450600 & -2.45005700 & -1.47778000 \\
\hline $\mathrm{C}$ & 0.65861200 & 3.83372900 & -1.31915700 & $\mathrm{H}$ & 4.95657000 & -0.93350800 & -1.20269300 \\
\hline $\mathrm{C}$ & -1.79905600 & 3.71907100 & -0.35379500 & $\mathrm{C}$ & 1.22567500 & 0.51551700 & 1.46826500 \\
\hline $\mathrm{C}$ & -2.45694300 & 2.66718100 & 0.22441500 & $\mathrm{H}$ & 0.60541400 & 1.22334900 & 2.01697900 \\
\hline $\mathrm{H}$ & 2.80484400 & 1.19271300 & -1.03035400 & $\mathrm{H}$ & 0.99188900 & -0.47925000 & 1.89610600 \\
\hline $\mathrm{H}$ & 0.67857600 & 4.84610600 & -1.70753700 & $\mathrm{C}$ & 2.71250100 & 0.77876500 & 1.70484300 \\
\hline $\mathrm{H}$ & -2.21698600 & 4.70173900 & -0.53070100 & $\mathrm{H}$ & 3.31581200 & 0.13074200 & 1.06048500 \\
\hline $\mathrm{H}$ & -3.47448400 & 2.61391800 & 0.58056500 & $\mathrm{H}$ & 2.95542300 & 1.81373100 & 1.43440700 \\
\hline $\mathrm{N}$ & -1.60548800 & 1.55701500 & 0.32178900 & $\mathrm{C}$ & 3.10131200 & 0.52835600 & 3.17201500 \\
\hline $\mathrm{P}$ & -1.84179900 & -0.18960100 & 0.22552400 & $\mathrm{H}$ & 2.89346200 & -0.51893400 & 3.43274200 \\
\hline $\mathrm{C}$ & -2.97533600 & -0.43699100 & -1.31686500 & $\mathrm{H}$ & 2.46651100 & 1.14068800 & 3.82810400 \\
\hline $\mathrm{C}$ & -2.74740800 & -0.65024200 & 1.84576900 & $\mathrm{H}$ & 1.89906200 & -2.80794400 & -2.06313700 \\
\hline $\mathrm{C}$ & -4.12416200 & 0.58791700 & -1.43977200 & $\mathrm{C}$ & 4.57530600 & 0.84198700 & 3.45427700 \\
\hline $\mathrm{H}$ & -3.75462700 & 1.60197600 & -1.60756200 & $\mathrm{H}$ & 4.83001500 & 0.65014800 & 4.50281400 \\
\hline $\mathrm{H}$ & -4.72770100 & 0.30923000 & -2.31329500 & $\mathrm{H}$ & 5.23731100 & 0.22567900 & 2.83290400 \\
\hline $\mathrm{H}$ & -4.79491300 & 0.59976800 & -0.57716200 & $\mathrm{H}$ & 4.805 & $1.8 \mathrm{~s}$ & 3.24134200 \\
\hline $\mathrm{C}$ & -2.06492200 & -0.27223800 & -2.55453200 & & & & \\
\hline $\mathrm{H}$ & -2.68561500 & -0.36008300 & -3.45651100 & 75-ts & & & \\
\hline $\mathrm{H}$ & -1.57840300 & 0.70841200 & -2.57550400 & & & & \\
\hline $\mathrm{H}$ & -1.29088000 & -1.04205000 & -2.58876500 & & & & \\
\hline $\mathrm{C}$ & -3.57502200 & -1.85962200 & -1.31893700 & & & & \\
\hline $\mathrm{H}$ & -2.80982400 & -2.62983600 & -1.20133400 & & & & \\
\hline $\mathrm{H}$ & -4.33775000 & -1.98867300 & -0.54473600 & & & & \\
\hline $\mathrm{H}$ & -4.06832800 & -2.01919300 & -2.28676700 & $\mathrm{C}$ & -0.77137800 & -2.96743800 & -0.34028300 \\
\hline $\mathrm{C}$ & -2.66800200 & -2.18247500 & 2.02809200 & $\mathrm{C}$ & -0.43815900 & -1.61608500 & -0.12763200 \\
\hline $\mathrm{H}$ & -3.23119400 & -2.72725300 & 1.26704000 & $\mathrm{C}$ & -1.14680500 & -0.50185700 & -0.65236900 \\
\hline $\mathrm{H}$ & -1.63584300 & -2.54135800 & 1.99249000 & $\mathrm{C}$ & -2.13978100 & -0.85740400 & -1.59748900 \\
\hline $\mathrm{H}$ & -3.09341200 & -2.44020200 & 3.00693700 & $\mathrm{C}$ & -2.44282300 & -2.19946400 & -1.84878500 \\
\hline $\mathrm{C}$ & -1.98652800 & 0.01704400 & 3.01110100 & $\mathrm{C}$ & -1.83410600 & -3.27278700 & -1.21139400 \\
\hline $\mathrm{H}$ & -1.99133400 & 1.10871100 & 2.93911500 & $\mathrm{C}$ & 0.19945300 & -3.73818100 & 0.39808600 \\
\hline $\mathrm{H}$ & -2.47548300 & -0.26303600 & 3.95280300 & $\mathrm{C}$ & 1.09431000 & -2.85923300 & 0.94246400 \\
\hline $\mathrm{C}$ & -4.22019700 & -0.19884100 & 1.90931700 & $\mathrm{H}$ & -2.75086200 & -0.10795000 & -2.08621100 \\
\hline $\mathrm{H}$ & -4.62391400 & -0.46605500 & 2.89461900 & $\mathrm{H}$ & -2.15948800 & -4.28669000 & -1.41698600 \\
\hline $\mathrm{H}$ & -4.33229400 & 0.88486900 & 1.79912400 & $\mathrm{H}$ & 0.23240200 & -4.81530500 & 0.49800000 \\
\hline $\mathrm{H}$ & -4.84479500 & -0.69139100 & 1.16025800 & $\mathrm{H}$ & 1.97251200 & -3.06271300 & 1.53601200 \\
\hline $\mathrm{F}$ & 2.85060800 & 3.48948200 & -2.11243800 & $\mathrm{~N}$ & 0.73967700 & -1.54042200 & 0.62133900 \\
\hline $\mathrm{Rh}$ & 0.29559000 & -0.89719900 & -0.08001800 & $\mathrm{P}$ & 1.69604900 & -0.10778100 & 0.23314900 \\
\hline $\mathrm{Cl}$ & -0.32796200 & -3.11941100 & -0.74278600 & $\mathrm{C}$ & 3.03777800 & -0.70171800 & -1.01200100 \\
\hline $\mathrm{H}$ & -0.94947500 & -0.32291900 & 3.06522200 & $\mathrm{C}$ & 2.42421400 & 0.46616900 & 1.90427900 \\
\hline $\mathrm{C}$ & 2.65352800 & -2.28379800 & -1.47365300 & $\mathrm{C}$ & 3.71954500 & -2.03469000 & -0.63781000 \\
\hline $\mathrm{O}$ & 2.38462600 & -1.48048300 & -0.58493600 & $\mathrm{H}$ & 3.02093200 & -2.87391700 & -0.66163500 \\
\hline $\mathrm{O}$ & 3.88479000 & -2.62238100 & -1.81053500 & $\mathrm{H}$ & 4.49787400 & -2.23349100 & -1.38559400 \\
\hline
\end{tabular}




\begin{tabular}{|c|c|c|c|c|c|c|c|}
\hline $\mathrm{H}$ & 4.20936600 & -2.01505000 & 0.33862500 & $76-t$ & & & \\
\hline $\mathrm{C}$ & 2.31896200 & -0.89519400 & -2.36623300 & & & & \\
\hline $\mathrm{H}$ & 3.05579300 & -1.24646700 & -3.10069900 & & & & \\
\hline $\mathrm{H}$ & 1.52385700 & -1.64541500 & -2.30527200 & & & & \\
\hline $\mathrm{H}$ & 1.88404500 & 0.03876600 & -2.73010500 & & & & \\
\hline $\mathrm{C}$ & 4.11343800 & 0.39422700 & -1.16485900 & & & & \\
\hline $\mathrm{H}$ & 3.67851000 & 1.37636500 & -1.36866000 & $\mathrm{C}$ & -0.27429700 & 2.85105300 & -1.31191400 \\
\hline $\mathrm{H}$ & 4.75953300 & 0.46209200 & -0.28398400 & $\mathrm{C}$ & -0.12559100 & 1.59809000 & -0.68818600 \\
\hline $\mathrm{H}$ & 4.75294400 & 0.12778900 & -2.01598800 & $\mathrm{C}$ & -0.71650400 & 1.20398700 & 0.54308100 \\
\hline $\mathrm{C}$ & 3.01860300 & 1.88255700 & 1.74206300 & $\mathrm{C}$ & -1.34785300 & 2.27562100 & 1.21819000 \\
\hline $\mathrm{H}$ & 3.92276400 & 1.89369600 & 1.12942500 & $\mathrm{C}$ & -1.46360200 & 3.54009200 & 0.62338200 \\
\hline $\mathrm{H}$ & 2.30254200 & 2.57535700 & 1.29216900 & $\mathrm{C}$ & -0.99715100 & 3.85858100 & -0.64257400 \\
\hline $\mathrm{H}$ & 3.28837000 & 2.25917000 & 2.73715800 & $\mathrm{C}$ & 0.47182200 & 2.77587600 & -2.54430900 \\
\hline $\mathrm{C}$ & 1.25149400 & 0.54565000 & 2.90490800 & $\mathrm{C}$ & 1.07867200 & 1.55035100 & -2.58500200 \\
\hline $\mathrm{H}$ & 0.71609300 & -0.40182600 & 3.00770900 & $\mathrm{H}$ & -1.81362400 & 2.14348400 & 2.18771800 \\
\hline $\mathrm{H}$ & 1.65072000 & 0.82572700 & 3.88795200 & $\mathrm{H}$ & -1.16679500 & 4.84511800 & -1.05942900 \\
\hline $\mathrm{C}$ & 3.50080900 & -0.48180200 & 2.46972400 & $\mathrm{H}$ & 0.55082000 & 3.54454200 & -3.30207900 \\
\hline $\mathrm{H}$ & 3.81675500 & -0.10239300 & 3.44985700 & $\mathrm{H}$ & 1.73004300 & 1.13649000 & -3.34029700 \\
\hline $\mathrm{H}$ & 3.12926000 & -1.49999300 & 2.62436500 & $\mathrm{~N}$ & 0.74098200 & 0.80401300 & -1.44751400 \\
\hline $\mathrm{H}$ & 4.39204200 & -0.52567300 & 1.83860800 & $\mathrm{P}$ & 1.64387900 & -0.34747300 & -0.46213800 \\
\hline $\mathrm{F}$ & -3.42953900 & -2.45859000 & -2.73775700 & $\mathrm{C}$ & 3.36672100 & 0.45742800 & -0.16468000 \\
\hline $\mathrm{Rh}$ & 0.14722000 & 1.18966100 & -0.66822200 & $\mathrm{C}$ & 1.73772600 & -1.93629500 & -1.52176600 \\
\hline $\mathrm{Cl}$ & 1.49217200 & 2.88480400 & -1.55046500 & $\mathrm{C}$ & 4.0228 & 1.06098600 & -1.42510600 \\
\hline $\mathrm{H}$ & 0.53263200 & 1.31595400 & 2.61161500 & $\mathrm{H}$ & 3.44698800 & 1.89925700 & -1.82418500 \\
\hline $\mathrm{C}$ & -2.72142000 & -0.13506300 & 1.28010100 & $\mathrm{H}$ & 5.00774400 & 1.44885700 & -1.13506200 \\
\hline $\mathrm{O}$ & -3.90302600 & -0.26421500 & 1.04451000 & $\mathrm{H}$ & 4.18672200 & 0.33505500 & -2.22464200 \\
\hline $\mathrm{O}$ & -2.09333300 & -0.73637700 & 2.31260900 & $\mathrm{C}$ & 3.14424300 & 1.60082700 & 0.85093600 \\
\hline $\mathrm{C}$ & -2.90486300 & -1.64225100 & 3.08035600 & $\mathrm{H}$ & 4.10751600 & 2.09699200 & 1.02906300 \\
\hline $\mathrm{H}$ & -3.27819300 & -2.44911100 & 2.44451200 & $\mathrm{H}$ & 2.44474000 & 2.35389700 & 0.47358700 \\
\hline $\mathrm{H}$ & -2.24691000 & -2.03731300 & 3.85448500 & $\mathrm{H}$ & 2.76561300 & 1.22592200 & 1.80474800 \\
\hline $\mathrm{H}$ & -3.75171400 & -1.11406000 & 3.52569700 & $\mathrm{C}$ & 4.31423300 & -0.59047700 & 0.45703100 \\
\hline $\mathrm{C}$ & -2.51261300 & 1.87443600 & -0.24585100 & $\mathrm{H}$ & 3.88005900 & -1.06442800 & 1.34130700 \\
\hline $\mathrm{H}$ & -3.40019300 & 1.46306400 & -0.73534000 & $\mathrm{H}$ & 4.60412800 & -1.36602900 & -0.25861900 \\
\hline $\mathrm{C}$ & -3.64663400 & 4.15287200 & -0.07535800 & $\mathrm{H}$ & 5.23303300 & -0.07718800 & 0.76852000 \\
\hline $\mathrm{H}$ & -4.55650900 & 3.78032200 & -0.56102200 & $\mathrm{C}$ & 2.04910300 & -3.12291100 & -0.58343400 \\
\hline $\mathrm{H}$ & -3.93760100 & 4.96017600 & 0.60553200 & $\mathrm{H}$ & 3.03219400 & -3.04124600 & -0.11277700 \\
\hline $\mathrm{H}$ & -3.00350600 & 4.58560700 & -0.85126000 & $\mathrm{H}$ & 1.30770700 & -3.21128600 & 0.21521800 \\
\hline $\mathrm{C}$ & -1.77218100 & 0.75133000 & 0.49117000 & $\mathrm{H}$ & 2.03583200 & -4.04778400 & -1.17434900 \\
\hline $\mathrm{H}$ & -1.88111700 & 2.29081500 & -1.06033900 & $\mathrm{C}$ & 0.35231100 & -2.15769700 & -2.16393800 \\
\hline $\mathrm{H}$ & -1.08358300 & 1.16958900 & 1.23723200 & $\mathrm{H}$ & 0.09240600 & -1.36106400 & -2.86739700 \\
\hline $\mathrm{C}$ & -2.92352900 & 3.03192200 & 0.67832800 & $\mathrm{H}$ & 0.37810800 & -3.10270700 & -2.72114500 \\
\hline $\mathrm{H}$ & -2.03119500 & 3.43668900 & 1.17399600 & $\mathrm{C}$ & 2.78999000 & -1.87613400 & -2.64684500 \\
\hline \multirow[t]{2}{*}{$\mathrm{H}$} & -3.57754200 & 2.63549700 & 1.46579900 & $\mathrm{H}$ & 2.72114800 & -2.80125700 & -3.23309800 \\
\hline & & & & $\mathrm{H}$ & 2.61779700 & -1.04421000 & -3.33751900 \\
\hline
\end{tabular}




\begin{tabular}{|c|c|c|c|c|c|c|c|}
\hline $\mathrm{H}$ & 3.81240400 & -1.80905600 & -2.26729700 & $\mathrm{P}$ & -1.60503300 & -0.64343800 & 0.46059100 \\
\hline $\mathrm{F}$ & -2.11887100 & 4.49499400 & 1.32724400 & $\mathrm{C}$ & -3.33029400 & 0.00900600 & 1.01030100 \\
\hline $\mathrm{Rh}$ & 0.37845900 & -0.41727100 & 1.35279500 & $\mathrm{C}$ & -1.41241000 & -2.52588200 & 0.72658800 \\
\hline $\mathrm{Cl}$ & 1.79408200 & -1.51199300 & 2.85004700 & $\mathrm{C}$ & -3.56066500 & -0.00840900 & 2.53694700 \\
\hline $\mathrm{H}$ & -0.45139300 & -2.23995200 & -1.42879400 & $\mathrm{H}$ & -2.89023000 & 0.67750600 & 3.05974200 \\
\hline $\mathrm{C}$ & -3.56101500 & -1.15790900 & -1.00832600 & $\mathrm{H}$ & -4.58771400 & 0.33112800 & 2.72244200 \\
\hline $\mathrm{O}$ & -3.09037400 & -2.25861200 & -1.21339600 & $\mathrm{H}$ & -3.46388500 & -1.00065300 & 2.98322200 \\
\hline $\mathrm{O}$ & -4.85671400 & -0.85192100 & -1.21887200 & $\mathrm{C}$ & -3.41686500 & 1.47682600 & 0.53375200 \\
\hline $\mathrm{C}$ & -5.66731000 & -1.91774100 & -1.74621200 & $\mathrm{H}$ & -4.38192000 & 1.88759700 & 0.85890800 \\
\hline $\mathrm{H}$ & -5.67684100 & -2.76739900 & -1.05872500 & $\mathrm{H}$ & -2.62579300 & 2.09597000 & 0.96927000 \\
\hline $\mathrm{H}$ & -6.66740400 & -1.49729800 & -1.85294100 & $\mathrm{H}$ & -3.35562600 & 1.55395300 & -0.55439600 \\
\hline $\mathrm{H}$ & -5.28220000 & -2.24797200 & -2.71456100 & $\mathrm{C}$ & -4.43668000 & -0.81239400 & 0.31563200 \\
\hline $\mathrm{C}$ & -1.76733400 & -0.30397400 & 0.57482200 & $\mathrm{H}$ & -4.28889200 & -0.87283800 & -0.76590900 \\
\hline $\mathrm{H}$ & -1.35203400 & -1.27749500 & 0.27569300 & $\mathrm{H}$ & -4.52129000 & -1.82433900 & 0.72420000 \\
\hline $\mathrm{C}$ & -3.19590400 & -1.74730500 & 2.17594200 & $\mathrm{H}$ & -5.39643100 & -0.31087100 & 0.49423500 \\
\hline $\mathrm{H}$ & -2.59145500 & -2.63146700 & 1.94967000 & $\mathrm{C}$ & -2.07281300 & -3.25829900 & -0.46204300 \\
\hline $\mathrm{H}$ & -3.52924500 & -1.81974200 & 3.21702500 & $\mathrm{H}$ & -3.15686200 & -3.12523100 & -0.48871600 \\
\hline $\mathrm{H}$ & -4.08870000 & -1.77438500 & 1.54258600 & $\mathrm{H}$ & -1.67399700 & -2.91343400 & -1.42006900 \\
\hline $\mathrm{C}$ & -2.77107900 & 0.05365800 & -0.53691100 & $\mathrm{H}$ & -1.87083100 & -4.33285200 & -0.36560100 \\
\hline $\mathrm{H}$ & -3.44731700 & 0.85187500 & -0.21820500 & $\mathrm{C}$ & 0.09673400 & -2.84804500 & 0.72446400 \\
\hline $\mathrm{H}$ & -2.23826700 & 0.41875800 & -1.42176600 & $\mathrm{H}$ & 0.63409700 & -2.35229900 & 1.53755200 \\
\hline $\mathrm{C}$ & -2.39636300 & -0.45169200 & 1.97076000 & $\mathrm{H}$ & 0.22115500 & -3.93130100 & 0.84651600 \\
\hline $\mathrm{H}$ & -3.03725500 & 0.41176400 & 2.18394800 & $\mathrm{C}$ & -2.01806400 & -3.03447900 & 2.05049900 \\
\hline \multirow[t]{2}{*}{$\mathrm{H}$} & -1 & -0.42213800 & 2.74 & $\mathrm{H}$ & -1.80044800 & -4.10614100 & 2.14227700 \\
\hline & & & & $\mathrm{H}$ & -1.58656900 & -2.54185400 & 2.92814800 \\
\hline \multirow[t]{6}{*}{ 77-ts } & & & & $\mathrm{H}$ & -3.10417000 & -2.92087100 & 2.08493400 \\
\hline & & & & $\mathrm{F}$ & 1.53507200 & 4.87282800 & -0.11295200 \\
\hline & & & & $\mathrm{Rh}$ & -0.94015000 & 0.16342100 & -1.49178300 \\
\hline & & & & $\mathrm{Cl}$ & -2.74515200 & -0.38908900 & -2.86325000 \\
\hline & & & & $\mathrm{H}$ & 0.56663100 & -2.56997000 & -0.22286300 \\
\hline & & & & $\mathrm{C}$ & 4.62996200 & -1.32283000 & 0.13866200 \\
\hline $\mathrm{C}$ & 0.51859000 & 2.12737600 & 2.00878000 & $\mathrm{O}$ & 4.38044800 & -1.47986300 & 1.31504400 \\
\hline $\mathrm{C}$ & 0.16660200 & 1.22402900 & 0.99000000 & $\mathrm{O}$ & 5.85763600 & -1.50382500 & -0.39401500 \\
\hline $\mathrm{C}$ & 800 & 1.42012500 & -0.40266900 & $\mathrm{C}$ & 6.87535800 & -1.92203600 & 0.53107400 \\
\hline $\mathrm{C}$ & 0.77890300 & 2.73025200 & -0.72379300 & $\mathrm{H}$ & 6.60764900 & -2.87681400 & 0.99169700 \\
\hline $\mathrm{C}$ & 1.09731000 & 3.65266000 & 0.28319200 & $\mathrm{H}$ & 7.78508100 & -2.02313200 & -0.06139500 \\
\hline $\mathrm{C}$ & 1.03165000 & 3.38701500 & 1.64289700 & $\mathrm{H}$ & 7.00907300 & -1.17443300 & 1.31743000 \\
\hline $\mathrm{C}$ & 0.17385400 & 1.48055800 & 3.25195800 & $\mathrm{C}$ & 2.53651500 & 0.00270400 & -0.36715100 \\
\hline $\mathrm{C}$ & -0.41340000 & 0.28377800 & 2.94676400 & $\mathrm{H}$ & 2.97538000 & 0.98580000 & -0.15890300 \\
\hline $\mathrm{H}$ & 0.92515800 & 3.04699500 & -1.74985900 & $\mathrm{C}$ & 1.71309700 & 0.72810200 & -2.69792800 \\
\hline $\mathrm{H}$ & 1.33974100 & 4.13584100 & 2.36418200 & $\mathrm{H}$ & 2.36201200 & 1.60556000 & -2.61214900 \\
\hline $\mathrm{H}$ & 0.34251300 & 1.86277700 & 4.25028300 & $\mathrm{H}$ & 2.23532400 & -0.03003200 & -3.29226900 \\
\hline $\mathrm{H}$ & -0.81464100 & -0.46807700 & 3.60958900 & $\mathrm{H}$ & 0.83078400 & 1.02521400 & -3.28556700 \\
\hline $\mathrm{N}$ & -0.44396800 & 0.10012400 & 1.55575500 & $\mathrm{C}$ & 3.61923000 & -0.93617600 & -0.92729500 \\
\hline
\end{tabular}




\begin{tabular}{|c|c|c|c|c|c|c|c|}
\hline $\mathrm{H}$ & 3.16195300 & -1.87276100 & -1.28063500 & $\mathrm{C}$ & 0.04749800 & -2.23687300 & 1.96775400 \\
\hline $\mathrm{H}$ & 4.14705800 & -0.50421700 & -1.78189500 & $\mathrm{H}$ & 0.08098500 & -1.43715600 & 2.71326000 \\
\hline $\mathrm{C}$ & 1.34439900 & 0.15347500 & -1.32674100 & $\mathrm{H}$ & 0.31863100 & -3.17693500 & 2.46415900 \\
\hline $\mathrm{H}$ & 0.96592700 & -0.87155300 & -1.48184100 & $\mathrm{C}$ & -2.34784900 & -2.75697300 & 2.47062400 \\
\hline \multirow[t]{2}{*}{$\mathrm{H}$} & 2.19895800 & -0.40347000 & 0.58878000 & $\mathrm{H}$ & -1.98927300 & -3.66321300 & 2.97512400 \\
\hline & & & & $\mathrm{H}$ & -2.42147600 & -1.97199600 & 3.23048700 \\
\hline \multirow[t]{6}{*}{ 78-ts } & & & & $\mathrm{H}$ & -3.34950200 & -2.97193900 & 2.09158700 \\
\hline & & & & $\mathrm{F}$ & 0.28811700 & 5.10314300 & -0.86580700 \\
\hline & & & & $\mathrm{Rh}$ & -0.55693400 & -0.28312000 & -1.35441900 \\
\hline & & & & $\mathrm{Cl}$ & -1.56702300 & -1.56304900 & -3.01364300 \\
\hline & & & & $\mathrm{H}$ & 0.80852500 & -2.03552900 & 1.20942700 \\
\hline & & & & $\mathrm{C}$ & 5.79077500 & -0.64762500 & 0.17794300 \\
\hline $\mathrm{C}$ & -1.02794500 & 2.78331600 & 1.57655500 & $\mathrm{O}$ & 6.36311000 & -1.09222000 & -0.79330800 \\
\hline $\mathrm{C}$ & -0.75948500 & 1.60022800 & 0.86980700 & $\mathrm{O}$ & 6.28530800 & -0.72340700 & 1.43485200 \\
\hline $\mathrm{C}$ & -0.04142600 & 1.49757900 & -0.34889600 & $\mathrm{C}$ & 7.56667400 & -1.36213300 & 1.55375200 \\
\hline $\mathrm{C}$ & 0.25039600 & 2.75149400 & -0.92972100 & $\mathrm{H}$ & 8.32097700 & -0.82354500 & 0.97361800 \\
\hline $\mathrm{C}$ & -0.05556600 & 3.94347500 & -0.25479400 & $\mathrm{H}$ & 7.80983600 & -1.33558200 & 2.61647600 \\
\hline $\mathrm{C}$ & -0.63956000 & 4.01040500 & 1.00110500 & $\mathrm{H}$ & 7.51676300 & -2.39424600 & 1.19636700 \\
\hline $\mathrm{C}$ & -1.72822100 & 2.37915200 & 2.77285900 & $\mathrm{C}$ & 3.75378600 & -0.00393300 & -1.19305400 \\
\hline $\mathrm{C}$ & -1.90385900 & 1.02357900 & 2.71351600 & $\mathrm{H}$ & 3.53092700 & -1.05147300 & -1.43270800 \\
\hline $\mathrm{H}$ & 0.77084400 & 2.83291800 & -1.87737400 & $\mathrm{C}$ & 1.37064500 & 0.35419700 & -0.32579600 \\
\hline $\mathrm{H}$ & -0.79879500 & 4.96945400 & 1.48137600 & $\mathrm{H}$ & 1.31524200 & -0.74880200 & -0.40841400 \\
\hline $\mathrm{H}$ & -2.05937200 & 3.02147600 & 3.57857100 & $\mathrm{H}$ & 1.60426300 & 0.54346700 & 0.72168000 \\
\hline $\mathrm{H}$ & -2.39329800 & 0.36738900 & 3.41757100 & $\mathrm{H}$ & 2.10828000 & 0.80745400 & -2.31168000 \\
\hline $\mathrm{N}$ & -1.32309500 & 0.51161400 & 1.54190300 & $\mathrm{C}$ & 4.45414500 & 0.07135100 & 0.16708800 \\
\hline $\mathrm{P}$ & -1.77879800 & -0.77651600 & 0.43088200 & $\mathrm{H}$ & 4.64094800 & 1.11763000 & 0.44957500 \\
\hline $\mathrm{C}$ & -3.66978900 & -0.56394600 & 0.12938900 & $\mathrm{H}$ & 3.84002700 & -0.34654200 & 0.97359600 \\
\hline $\mathrm{C}$ & -1.35554400 & -2.40000600 & 1.34634500 & $\mathrm{C}$ & 2.46889500 & 0.84111800 & -1.27367300 \\
\hline $\mathrm{C}$ & -4.49199000 & -0.31227600 & 1.41207800 & $\mathrm{H}$ & 2.70614000 & 1.89097300 & -1.06330200 \\
\hline $\mathrm{H}$ & -4.24015400 & 0.64305700 & 1.87786500 & $\mathrm{H}$ & 4.45211400 & 0.33789300 & -1.96483200 \\
\hline $\mathrm{H}$ & -5.55004800 & -0.26849700 & 1.12431000 & & & & \\
\hline $\mathrm{H}$ & -4.39471700 & -1.10372700 & 2.15837600 & 79 & & & \\
\hline $\mathrm{C}$ & -3.83091300 & 0.66992000 & -0.78676000 & & & & \\
\hline $\mathrm{H}$ & -4.90331400 & 0.83968900 & -0.95064600 & & & & \\
\hline $\mathrm{H}$ & -3.41433500 & 1.57336500 & -0.32969500 & & & & \\
\hline $\mathrm{H}$ & -3.35090100 & 0.52217200 & -1.75680800 & & & & \\
\hline $\mathrm{C}$ & -4.22007500 & -1.81143100 & -0.59303500 & & & & \\
\hline $\mathrm{H}$ & -3.64398300 & -2.06053900 & -1.48797600 & & & & \\
\hline $\mathrm{H}$ & -4.26118100 & -2.68666200 & 0.06316800 & $\mathrm{C}$ & -4.31348200 & 2.25898100 & 0.20510000 \\
\hline $\mathrm{H}$ & -5.24881700 & -1.59554500 & -0.90857600 & $\mathrm{C}$ & -4.64845300 & 0.86915000 & 0.23653100 \\
\hline $\mathrm{C}$ & -1.30857500 & -3.54494500 & 0.31074600 & $\mathrm{C}$ & -6.69991600 & 1.37211200 & 1.37331500 \\
\hline $\mathrm{H}$ & -2.28612700 & -3.75310600 & -0.13054300 & $\mathrm{C}$ & -6.35919000 & 2.73201300 & 1.34077600 \\
\hline $\mathrm{H}$ & -0.61827700 & -3.32172300 & -0.50747400 & $\mathrm{C}$ & -5.19405900 & 3.20211100 & 0.76552600 \\
\hline $\mathrm{H}$ & -0.96834300 & -4.45879600 & 0.81461800 & $\mathrm{C}$ & -3.05464500 & 2.39068800 & -0.46710100 \\
\hline
\end{tabular}




\begin{tabular}{|c|c|c|c|c|c|c|c|}
\hline $\mathrm{C}$ & -2.64629200 & 1.12264300 & -0.81380000 & $\mathrm{C}$ & 4.12431000 & -2.50947500 & -0.77354700 \\
\hline $\mathrm{H}$ & -7.63998000 & 1.07823800 & 1.82782300 & $\mathrm{C}$ & 4.21629500 & -1.17948500 & -0.06202400 \\
\hline $\mathrm{H}$ & -4.98060800 & 4.26585600 & 0.75495900 & $\mathrm{H}$ & 4.60920800 & -2.38876700 & 3.36964300 \\
\hline $\mathrm{H}$ & -2.53224600 & 3.30930600 & -0.69628500 & $\mathrm{H}$ & 2.24131500 & -2.55208000 & 1.41798100 \\
\hline $\mathrm{N}$ & -3.63225000 & 0.17697100 & -0.42399700 & $\mathrm{H}$ & 6.24111900 & -2.96341500 & -0.97541800 \\
\hline $\mathrm{P}$ & -2.73037100 & -1.34705500 & -0.36006900 & $\mathrm{H}$ & 4.05820000 & -2.36080600 & -1.85838000 \\
\hline $\mathrm{C}$ & -3.65018700 & -2.46817300 & -1.59291800 & $\mathrm{H}$ & 3.24278500 & -3.47571300 & 3.55108100 \\
\hline $\mathrm{C}$ & -2.74838500 & -2.00951400 & 1.43250700 & $\mathrm{H}$ & 2.31224800 & -1.44942400 & 2.77801600 \\
\hline $\mathrm{C}$ & -5.17174000 & -2.52290400 & -1.35912600 & $\mathrm{H}$ & 5.19495700 & -4.37290200 & -0.90119600 \\
\hline $\mathrm{H}$ & -5.61822300 & -1.52444800 & -1.40071100 & $\mathrm{H}$ & 4.21426300 & -0.22601100 & 1.84439800 \\
\hline $\mathrm{H}$ & -5.63202800 & -3.12129800 & -2.15620400 & $\mathrm{H}$ & 3.22038200 & -3.04714400 & -0.48129000 \\
\hline $\mathrm{H}$ & -5.44189300 & -2.98727700 & -0.40746700 & $\mathrm{H}$ & 5.10241100 & -0.61340500 & -0.34778900 \\
\hline $\mathrm{C}$ & -3.39150200 & -1.85634200 & -2.98807100 & $\mathrm{C}$ & 4.57929100 & -4.03453400 & 1.96210500 \\
\hline $\mathrm{H}$ & -3.87652000 & -2.48516000 & -3.74615500 & $\mathrm{H}$ & 5.00517500 & -4.75429600 & 2.67329700 \\
\hline $\mathrm{H}$ & -3.81228100 & -0.84862600 & -3.07067700 & $\mathrm{H}$ & 3.83700500 & -4.60108600 & 1.38114900 \\
\hline $\mathrm{H}$ & -2.32238700 & -1.79798000 & -3.21298000 & $\mathrm{C}$ & 5.71619800 & -3.54240300 & 1.03198800 \\
\hline $\mathrm{C}$ & -3.03977900 & -3.88318000 & -1.55219600 & $\mathrm{H}$ & 6.55170700 & -4.25246300 & 1.08678700 \\
\hline $\mathrm{H}$ & -1.95026000 & -3.86003200 & -1.66793700 & $\mathrm{H}$ & 6.11165300 & -2.59470300 & 1.42127600 \\
\hline $\mathrm{H}$ & -3.28047500 & -4.41650000 & -0.62734600 & $\mathrm{C}$ & 4.85233700 & 3.96923100 & -1.35020600 \\
\hline $\mathrm{H}$ & -3.44823900 & -4.47180600 & -2.38335600 & $\mathrm{C}$ & 4.02223700 & 2.67551800 & -1.53023800 \\
\hline $\mathrm{C}$ & -1.60020700 & -3.03999300 & 1.54536000 & $\mathrm{C}$ & 2.86198300 & 4.30973900 & 1.65069600 \\
\hline $\mathrm{H}$ & -1.77927700 & -3.93744400 & 0.94633400 & $\mathrm{C}$ & 4.02056900 & 1.78639700 & -0.30277000 \\
\hline $\mathrm{H}$ & -0.63966100 & -2.61526500 & 1.24421100 & $\mathrm{C}$ & 2.20072400 & 3.10085000 & 0.94715700 \\
\hline $\mathrm{H}$ & -1.52295400 & -3.35566600 & 2.59396900 & $\mathrm{C}$ & 3.18353600 & 1.96043200 & 0.81720700 \\
\hline $\mathrm{C}$ & -2.41493500 & -0.80895400 & 2.34507700 & $\mathrm{H}$ & 5.85531300 & 3.71396000 & -0.97607500 \\
\hline $\mathrm{H}$ & -3.21778100 & -0.06635500 & 2.36124200 & $\mathrm{H}$ & 3.00501700 & 2.94113000 & -1.82808500 \\
\hline $\mathrm{H}$ & -2.27633500 & -1.17670200 & 3.36991600 & $\mathrm{H}$ & 3.01129600 & 4.07529800 & 2.71446700 \\
\hline $\mathrm{C}$ & -4.05765000 & -2.66775900 & 1.90373800 & $\mathrm{H}$ & 1.32427600 & 2.78868800 & 1.52863100 \\
\hline $\mathrm{H}$ & -3.93800800 & -2.96577800 & 2.95364300 & $\mathrm{H}$ & 5.00415900 & 4.39929100 & -2.34974800 \\
\hline $\mathrm{H}$ & -4.91126500 & -1.98755300 & 1.85686000 & $\mathrm{H}$ & 4.44582900 & 2.10626800 & -2.36799600 \\
\hline $\mathrm{H}$ & -4.29767300 & -3.57342800 & 1.33886500 & $\mathrm{H}$ & 2.14660100 & 5.14348600 & 1.62005000 \\
\hline $\mathrm{F}$ & -7.22259000 & 3.61508900 & 1.89275400 & $\mathrm{H}$ & 4.99134400 & 1.32623000 & -0.11536800 \\
\hline $\mathrm{Cl}$ & 0.93578700 & -1.67163900 & -0.79160200 & $\mathrm{H}$ & 1.82637400 & 3.40332800 & -0.03157100 \\
\hline $\mathrm{H}$ & -1.49056700 & -0.31254800 & 2.03240700 & $\mathrm{H}$ & 3.56057600 & 1.62102800 & 1.78134900 \\
\hline $\mathrm{C}$ & -5.84456400 & 0.42714000 & 0.81446200 & $\mathrm{C}$ & 4.24795400 & 5.06901600 & -0.45146300 \\
\hline $\mathrm{H}$ & -6.12347000 & -0.62014200 & 0.81103400 & $\mathrm{H}$ & 4.84916200 & 5.97300100 & -0.61423800 \\
\hline $\mathrm{Rh}$ & -0.90803300 & -0.16090500 & -1.00223600 & $\mathrm{H}$ & 3.23927200 & 5.31875800 & -0.81161100 \\
\hline $\mathrm{H}$ & -2.07236000 & 0.89256600 & -1.81590700 & $\mathrm{C}$ & 4.21094600 & 4.78003100 & 1.07003100 \\
\hline $\mathrm{Cl}$ & 0.85043200 & 1.41886900 & -1.59492500 & $\mathrm{H}$ & 4.48791600 & 5.69398600 & 1.61161500 \\
\hline $\mathrm{Rh}$ & 2.54582300 & 0.18283100 & -0.24808100 & $\mathrm{H}$ & 4.99250000 & 4.04806800 & 1.31415700 \\
\hline $\mathrm{C}$ & 3.85828600 & -2.95859900 & 2.80189900 & & & & \\
\hline $\mathrm{C}$ & 2.93191400 & -1.97926500 & 2.04226700 & 80-ts & & & \\
\hline $\mathrm{C}$ & 5.36425500 & -3.38056400 & -0.46019000 & & & & \\
\hline $\mathrm{C}$ & 3.68427800 & -0.94834300 & 1.22258300 & & & & \\
\hline
\end{tabular}



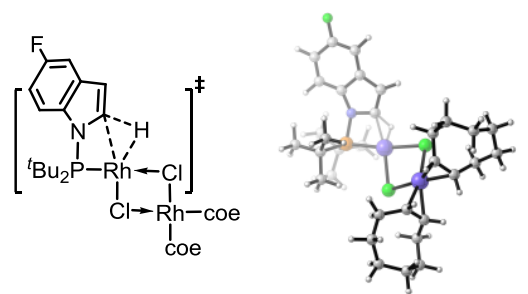$$
\text { C }
$$

C -4.24306900

C $\quad-4.63482300$

C $\quad-6.73695600$

C $\quad-6.34869200$

C $\quad-5.12933300$

C $\quad-2.92357200$

C $\quad-2.52498700$

$\mathrm{H} \quad-7.71060800$

H $\quad-4.88066100$

H $\quad-2.34617700$

$\mathrm{N} \quad-3.57980700$

P $\quad-2.78007500$

C $\quad-3.76127300$

C $\quad-2.73656300$

C $\quad-5.27887700$

H $\quad-5.68565400$

H $\quad-5.77846200$

H $\quad-5.54652000$

C $\quad-3.50857400$

H $\quad-4.05210600$

$\mathrm{H} \quad-3.87018400$

H $\quad-2.44577700$

C $\quad-3.21164500$

H $\quad-2.12535400$

H $\quad-3.45217200$

H $\quad-3.66653400$

C $\quad-1.64225400$

H $\quad-1.89477100$

H $\quad-0.67404200$

$\mathrm{H} \quad-1.53366300$

C $\quad-2.31886200$

H $\quad-3.08431700$

$-2.17196800$

C $\quad-4.07154100$

H $\quad-3.94666000$

H $\quad-4.88002500$

H $\quad-4.38205000$

F $\quad-7.21507500$
2.25856500

0.88444900

1.49909100

2.84188200

3.24899600

2.32174700

1.02804000

1.25506600

4.30457400

3.21375400

0.14500100

$-1.40150900$

$-2.43545900$

$-2.15439500$

$-2.43561000$

$-1.41997300$

$-2.99542700$

$-2.91321500$

$-1.78617500$

$-2.35770300$

$-0.75318400$

$-1.78273400$

$-3.87566900$

$-3.89701100$

$-4.42780800$

$-4.41729700$

$-3.24528900$

0.24454300

0.31741000

1.29191400

1.21421500

0.70127600

$-0.32643100$

$-0.56709800$

1.70287500

0.66359000

$-0.52542400$

$-0.22460700$

$-0.31235200$

$-1.57120000$

1.43816500

$-1.30358300$

$-1.30994400$

$-2.10480500$

$-0.35776300$

$-2.95005500$

$-3.71359100$

$-2.98376500$

$-3.20866500$

$-1.59206100$

$-1.73599100$

$-0.67850400$

$-2.43084200$

1.46787500

$-4.11187400$

0.85025000

1.13661400

$-2.86025800$

$-3.60045700$

2.50089700

2.38546000

2.44903000

$-0.22869300$

$-1.42215500$

3.39086200

1.92258800

$-2.74917600$

$-3.08987400$

2.95869500

$-2.01261700$

1.91808900

1.33344500

$-3.61710100$

3.78086500
$\mathrm{Cl} \quad 0.95475400$

H $\quad-1.38053400$

$-1.68612500$

$-0.77333400$

C $\quad-5.87412400$

$-0.54035500$

0.50299400

2.07000000

H $\quad-6.17700200$

$-0.53760500$

0.83668700

$\mathrm{Rh}$

$\mathrm{H}$

$-0.94611700$

$-0.17831700$

0.87966600

H -1.95226500

0.63122400

$-0.96661200$

Cl $\quad 0.79296800$

1.43218000

0.19308000

$\mathrm{Rh} \quad 2.55660300$

$-2.96082000$

$-1.98314500$

$-3.33924000$

$-0.94009700$

$-2.47882500$

$-1.15380200$

$-2.38961700$

$-2.55580800$

$-2.90732100$

$-2.32252500$

$-3.48927500$

$-1.46374200$

$-4.32822800$

$-0.21655400$

$-3.02850500$

$-0.57312700$

$-4.02339200$

$-4.74656400$

$-4.58982800$

$-3.51458000$

$-4.22030400$

$-2.56897300$

4.01664800

2.71765000

4.29990600

1.81321600

3.09379000

1.96563800

3.76517300

2.97864000

4.05382100

2.76515500

4.46091400

2.16323000

5.12693100

1.36166800
$-1.89523800$

$-1.50621300$

$-0.25537400$

2.73262100

2.00273000

$-0.56532900$

1.17539300

$-0.84376600$

$-0.12355300$

3.28829600

1.38885100

$-1.09400900$

$-1.92569500$

3.49088100

2.75641800

$-1.01404000$

1.79011200

$-0.53604100$

$-0.42675700$

1.86711000

2.56202700

1.29706200

0.94597600

1.30797300

$-1.39099200$

$-1.55748100$

1.68645000

$-0.34232500$

0.81026900

$-1.05827600$

$-1.81427500$

2.74090100

1.60343500

$-2.38967900$

$-2.41783000$

1.69263800

$-0.19690800$
0.91727100

0.99262900 


\begin{tabular}{|c|c|c|c|c|c|c|c|}
\hline $\mathrm{H}$ & 1.80853400 & 3.40605500 & 0.03365000 & $\mathrm{H}$ & -1.09909500 & -2.63681300 & 1.88 \\
\hline $\mathrm{H}$ & 3.62864900 & 1.61861300 & 1.75509400 & $\mathrm{H}$ & -2.15860000 & -3.28214400 & 3.15580600 \\
\hline $\mathrm{C}$ & 4.20086700 & 5.09935200 & -0.45543000 & $\mathrm{C}$ & -3.01919000 & -0.77178200 & 2.66251700 \\
\hline $\mathrm{H}$ & 4.78968800 & 6.00963300 & -0.62795200 & $\mathrm{H}$ & -3.78575300 & -0.01144200 & 2.48826300 \\
\hline $\mathrm{H}$ & 3.17819100 & 5.34720700 & -0.77508200 & $\mathrm{H}$ & -3.08662200 & -1.09190200 & 3.70982100 \\
\hline $\mathrm{C}$ & 4.22229700 & 4.79027100 & 1.06244300 & $\mathrm{C}$ & -4.59349500 & -2.62156400 & 2.02534200 \\
\hline $\mathrm{H}$ & 4.51048800 & 5.69983200 & 1.60547200 & $\mathrm{H}$ & -4.66585500 & -2.87497700 & 3.09096500 \\
\hline \multirow[t]{2}{*}{$\mathrm{H}$} & 5.01944300 & 4.06 & 1.26799700 & $\mathrm{H}$ & -5.40413600 & -1.92129000 & 1.80095500 \\
\hline & & & & $\mathrm{H}$ & -4.75989500 & -3.54427200 & 1.46184100 \\
\hline \multirow[t]{7}{*}{81} & \multirow{7}{*}{ 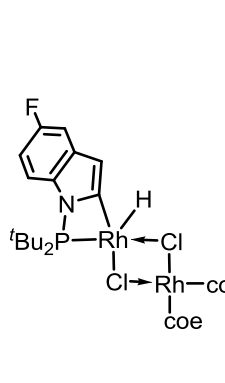 } & \multirow{7}{*}{ (3) } & \multirow{7}{*}{ a } & $\mathrm{F}$ & -7.93309800 & 3.65356800 & 0.19655400 \\
\hline & & & & $\mathrm{Cl}$ & 0.95262300 & -1.57937900 & -0.01088300 \\
\hline & & & & $\mathrm{H}$ & -2.03791900 & -0.30307700 & 2.52453500 \\
\hline & & & & $\mathrm{C}$ & -6.29908200 & 0.40564800 & 0.12675200 \\
\hline & & & & $\mathrm{H}$ & -6.56309600 & -0.64361000 & 0.20746700 \\
\hline & & & & $\mathrm{Rh}$ & -1.04624200 & -0.10818700 & -0.27626000 \\
\hline & & & & $\mathrm{H}$ & -1.20540400 & -0.23341000 & -1.77451500 \\
\hline $\mathrm{C}$ & -4.62701200 & 2.19989500 & -0.17029000 & $\mathrm{Cl}$ & 0.63645800 & 1.62436200 & -0.41710100 \\
\hline $\mathrm{C}$ & -4.97952700 & 0.81703500 & -0.07089400 & $\mathrm{Rh}$ & 2.69186600 & 0.23664100 & -0.05076900 \\
\hline $\mathrm{C}$ & -7.29108100 & 1.38176200 & 0.21776500 & $\mathrm{C}$ & 5.04050000 & -3.16900100 & 1.89180500 \\
\hline $\mathrm{C}$ & -6.94254300 & 2.73274600 & 0.10736100 & $\mathrm{C}$ & 3.92022800 & -2.12294400 & 1.67624500 \\
\hline $\mathrm{C}$ & -5.64215300 & 3.16785700 & -0.08396700 & $\mathrm{C}$ & 5.05474000 & -3.27533700 & -1.72406300 \\
\hline $\mathrm{C}$ & -3.19776800 & 2.29402100 & -0.33608800 & $\mathrm{C}$ & 4.30104300 & -1.02151100 & 0.70595700 \\
\hline $\mathrm{C}$ & -2.69507600 & 1.01713500 & -0.33303700 & $\mathrm{C}$ & 3.82458300 & -2.38798400 & -1.41944300 \\
\hline $\mathrm{H}$ & -8.33115700 & 1.11549100 & 0.37199400 & $\mathrm{C}$ & 4.24519100 & -1.12910000 & -0.69768800 \\
\hline $\mathrm{H}$ & -5.43117000 & 4.22985500 & -0.15740400 & $\mathrm{H}$ & 5.97984200 & -2.65413100 & 2.14348000 \\
\hline $\mathrm{H}$ & -2.62273700 & 3.20514300 & -0.42917200 & $\mathrm{H}$ & 3.00744500 & -2.63321400 & 1.35799600 \\
\hline $\mathrm{N}$ & -3.78146000 & 0.10833300 & -0.21012100 & $\mathrm{H}$ & 5.65691600 & -2.80411800 & -2.51379200 \\
\hline $\mathrm{P}$ & -2.93953000 & -1.38722500 & -0.02392700 & $\mathrm{H}$ & 3.32351900 & -2.14168400 & -2.36386900 \\
\hline $\mathrm{C}$ & -3.56408500 & -2.58008100 & -1.36566000 & $\mathrm{H}$ & 4.77068100 & -3.75525700 & 2.78109600 \\
\hline $\mathrm{C}$ & -3.20659100 & -2.00983600 & 1.75591400 & $\mathrm{H}$ & 3.68930800 & -1.66173400 & 2.64573900 \\
\hline $\mathrm{C}$ & -5.10427600 & -2.63564200 & -1.42072400 & $\mathrm{H}$ & 4.68596100 & -4.22120700 & -2.14502700 \\
\hline $\mathrm{H}$ & -5.53011500 & -1.65743300 & -1.66095200 & $\mathrm{H}$ & 5.06189100 & -0.35255200 & 1.10877000 \\
\hline $\mathrm{H}$ & -5.40239900 & -3.33048700 & -2.21627300 & $\mathrm{H}$ & 3.10317700 & -2.95304500 & -0.82762600 \\
\hline $\mathrm{H}$ & -5.55327500 & -2.99268100 & -0.49079800 & $\mathrm{H}$ & 4.95052400 & -0.53186100 & -1.27433000 \\
\hline $\mathrm{C}$ & -3.05255500 & -2.04689100 & -2.72157800 & $\mathrm{C}$ & 5.30775600 & -4.15936000 & 0.73841500 \\
\hline $\mathrm{H}$ & -3.47115000 & -2.67018700 & -3.52207800 & $\mathrm{H}$ & 5.95804700 & -4.94539200 & 1.14381300 \\
\hline $\mathrm{H}$ & -3.36742800 & -1.01380500 & -2.89992800 & $\mathrm{H}$ & 4.36921700 & -4.66530100 & 0.46865400 \\
\hline $\mathrm{H}$ & -1.96213300 & -2.09241100 & -2.79037000 & $\mathrm{C}$ & 5.98160600 & -3.57951300 & -0.53044200 \\
\hline $\mathrm{C}$ & -2.97982200 & -3.98737400 & -1.13032700 & $\mathrm{H}$ & 6.73797800 & -4.29047700 & -0.88770500 \\
\hline $\mathrm{H}$ & -1.88962700 & -3.96726800 & -1.02221500 & $\mathrm{H}$ & 6.53819200 & -2.67336200 & -0.25661300 \\
\hline $\mathrm{H}$ & -3.40890900 & -4.47625900 & -0.25045400 & $\mathrm{C}$ & 4.50484300 & 4.11372300 & -1.65372600 \\
\hline $\mathrm{H}$ & -3.21409300 & -4.61547700 & -1.99889100 & $\mathrm{C}$ & 3.62377700 & 2.84213100 & -1.60036100 \\
\hline $\mathrm{C}$ & -2.09913800 & -3.03544500 & 2.08799100 & $\mathrm{C}$ & 3.89957100 & 4.16420200 & 1.91302900 \\
\hline $\mathrm{H}$ & -2.20958300 & -3.96963600 & 1.53076100 & $\mathrm{C}$ & 4.07869400 & 1.83959700 & -0.55775100 \\
\hline
\end{tabular}




\begin{tabular}{|c|c|c|c|c|c|c|c|}
\hline $\mathrm{C}$ & 2.96745700 & 3.02551700 & 1.43520300 & $\mathrm{H}$ & -4.48821000 & 0.84756700 & -1.13577700 \\
\hline $\mathrm{C}$ & 3.77140800 & 1.90610300 & 0.81584000 & $\mathrm{C}$ & -1.91280800 & -0.97573800 & -2.61000700 \\
\hline $\mathrm{H}$ & 5.56311100 & 3.82385900 & -1.73736500 & $\mathrm{H}$ & -2.48301400 & -1.17677200 & -3.52672300 \\
\hline $\mathrm{H}$ & 2.58202800 & 3.13398600 & -1.44514600 & $\mathrm{H}$ & -1.23114100 & -0.14353700 & -2.81762300 \\
\hline $\mathrm{H}$ & 4.45397300 & 3.83156200 & 2.80220300 & $\mathrm{H}$ & -1.31002700 & -1.86337600 & -2.38844500 \\
\hline $\mathrm{H}$ & 2.39154900 & 2.65677500 & 2.29318600 & $\mathrm{C}$ & -3.75125400 & -1.88708000 & -1.16508000 \\
\hline $\mathrm{H}$ & 4.26115900 & 4.63846200 & -2.58767800 & $\mathrm{H}$ & -3.15115800 & -2.72797400 & -0.79790100 \\
\hline $\mathrm{H}$ & 3.65911000 & 2.35531400 & -2.58385500 & $\mathrm{H}$ & -4.53818000 & -1.68107900 & -0.43198100 \\
\hline $\mathrm{H}$ & 3.26327700 & 4.99677400 & 2.24394900 & $\mathrm{H}$ & -4.24445400 & -2.21296200 & -2.09010200 \\
\hline $\mathrm{H}$ & 5.02636900 & 1.36595800 & -0.81683500 & $\mathrm{C}$ & -3.00334100 & -1.44154100 & 2.28367300 \\
\hline $\mathrm{H}$ & 2.23920000 & 3.42192100 & 0.72616600 & $\mathrm{H}$ & -3.68925800 & -2.03669900 & 1.67247200 \\
\hline $\mathrm{H}$ & 4.49015400 & 1.47305900 & 1.51094900 & $\mathrm{H}$ & -2.07884700 & -2.01474000 & 2.41043100 \\
\hline $\mathrm{C}$ & 4.35260200 & 5.12055400 & -0.49419400 & $\mathrm{H}$ & -3.46489800 & -1.32233100 & 3.27280800 \\
\hline $\mathrm{H}$ & 4.87166600 & 6.03690100 & -0.80383700 & $\mathrm{C}$ & -1.74673000 & 0.69752100 & 2.61053200 \\
\hline $\mathrm{H}$ & 3.29449800 & 5.40303800 & -0.39398400 & $\mathrm{H}$ & -1.56920900 & 1.72608500 & 2.28278200 \\
\hline $\mathrm{C}$ & 4.91874200 & 4.68486700 & 0.88042300 & $\mathrm{H}$ & -2.17504800 & 0.73419900 & 3.62111000 \\
\hline $\mathrm{H}$ & 5.42869300 & 5.54059200 & 1.34181000 & $\mathrm{C}$ & -4.04947500 & 0.73232100 & 1.59269200 \\
\hline \multirow[t]{2}{*}{$\mathrm{H}$} & 5.70016100 & 3.92944100 & 0.72255200 & $\mathrm{H}$ & -4.46991200 & 0.83650000 & 2.60254700 \\
\hline & & & & $\mathrm{H}$ & -3.91976400 & 1.74369700 & 1.19645600 \\
\hline \multirow[t]{6}{*}{$\mathbf{P \alpha}$} & & & & $\mathrm{H}$ & -4.79888200 & 0.21593500 & 0.98453400 \\
\hline & & & & $\mathrm{F}$ & 4.20356700 & 3.10082300 & -0.71705900 \\
\hline & & & & $\mathrm{H}$ & -0.77697200 & 0.19054100 & 2.67446400 \\
\hline & & & & $\mathrm{C}$ & 2.42232000 & -1.25379700 & 1.18281000 \\
\hline & & & & $\mathrm{O}$ & 3.58309100 & -1.60776600 & 1.19439900 \\
\hline & & & & $\mathrm{O}$ & 1.69847700 & -1.06711100 & 2.31260400 \\
\hline $\mathrm{C}$ & 0.59946600 & 2.81414700 & -0.56988000 & $\mathrm{C}$ & 2.41433900 & -1.26979500 & 3.54178900 \\
\hline $\mathrm{C}$ & 0.44986600 & 1.40691000 & -0.36278300 & $\mathrm{H}$ & 3.25987700 & -0.58019500 & 3.61134800 \\
\hline $\mathrm{C}$ & 1.59442700 & 0.57855700 & -0.26914800 & $\mathrm{H}$ & 1.69375900 & -1.07383900 & 4.33645000 \\
\hline $\mathrm{C}$ & 2.84076400 & 1.20158900 & -0.39441300 & $\mathrm{H}$ & 2.78754100 & -2.29551100 & 3.60501200 \\
\hline $\mathrm{C}$ & 2.95987000 & 2.57670900 & -0.60675900 & $\mathrm{C}$ & 2.11790000 & -1.69818200 & -1.29425900 \\
\hline $\mathrm{C}$ & 1.86456400 & 3.40742700 & -0.69886900 & $\mathrm{H}$ & 3.17629900 & -1.45800500 & -1.44712900 \\
\hline $\mathrm{C}$ & -0.70894700 & 3.39302100 & -0.59720600 & $\mathrm{C}$ & 2.43997400 & -3.94832700 & -2.45462200 \\
\hline $\mathrm{C}$ & -1.59499600 & 2.37486100 & -0.42018100 & $\mathrm{H}$ & 3.50608800 & -3.75722100 & -2.62704800 \\
\hline $\mathrm{H}$ & 3.74876000 & 0.61353800 & -0.31131700 & $\mathrm{H}$ & 2.30630500 & -5.03259600 & -2.36535700 \\
\hline $\mathrm{H}$ & 1.98614500 & 4.47374600 & -0.85790100 & $\mathrm{H}$ & 1.89558300 & -3.61730500 & -3.34799800 \\
\hline $\mathrm{H}$ & -0.95046700 & 4.43875400 & -0.73153000 & $\mathrm{C}$ & 1.59446100 & -0.93794900 & -0.05784000 \\
\hline $\mathrm{H}$ & -2.67040600 & 2.41600500 & -0.37358900 & $\mathrm{H}$ & 1.57805700 & -1.32239900 & -2.17333200 \\
\hline $\mathrm{N}$ & -0.94349700 & 1.14683500 & -0.28545100 & $\mathrm{H}$ & 0.57520800 & -1.26391900 & 0.15073800 \\
\hline $\mathrm{P}$ & -1.74155900 & -0.39604500 & 0.06013700 & $\mathrm{C}$ & 1.94582600 & -3.22056500 & -1.19968000 \\
\hline $\mathrm{C}$ & -2.88769300 & -0.64127400 & -1.45698900 & $\mathrm{H}$ & 0.88522000 & -3.45777400 & -1.03150300 \\
\hline $\mathrm{C}$ & -2.72528300 & -0.04615000 & 1.67495000 & $\mathrm{H}$ & 2.49288000 & -3.59304300 & -0.32489400 \\
\hline $\mathrm{C}$ & -3.79847000 & 0.51303500 & -1.91563700 & & & & \\
\hline $\mathrm{H}$ & -3.21963600 & 1.37351600 & -2.26200100 & $\mathbf{P \beta}$ & & & \\
\hline $\mathrm{H}$ & -4.40770200 & 0.17143100 & -2.76394500 & & & & \\
\hline
\end{tabular}




\begin{tabular}{|c|c|c|c|c|c|c|c|}
\hline & & & & $\mathrm{F}$ & -2.46836000 & 4.87302300 & -0.13240300 \\
\hline & & & & $\mathrm{H}$ & -0.03443800 & -1.45361800 & -2.13456600 \\
\hline & & & & $\mathrm{C}$ & -3.14953200 & -1.68944300 & -0.48979600 \\
\hline & & & & $\mathrm{O}$ & -2.26229700 & -2.46492400 & -0.78712800 \\
\hline & & & & $\mathrm{O}$ & -4.44240200 & -2.06332000 & -0.36679300 \\
\hline & 0.58144400 & 3.01638800 & -0.72492500 & $\mathrm{C}$ & -4.71121400 & -3.45111700 & -0.62580000 \\
\hline & 0.19749800 & 1.72113800 & -0.25505600 & $\mathrm{H}$ & -4.15616200 & -4.08564000 & 0.07027900 \\
\hline & -1.12618200 & 1.48345600 & 0.18999800 & $\mathrm{H}$ & -5.78542800 & -3.57238900 & -0.48306600 \\
\hline & -1.98025800 & 2.59129100 & 0.22390500 & $\mathrm{H}$ & -4.42678600 & -3.71344400 & -1.64839800 \\
\hline & -1.57074800 & 3.85931600 & -0.19562600 & $\mathrm{C}$ & -1.70287400 & 0.12562600 & 0.57947100 \\
\hline & -0.30786100 & 4.10165600 & -0.69000600 & $\mathrm{H}$ & -0.96968900 & -0.64401400 & 0.34094800 \\
\hline & 1.92735000 & 2.92472100 & -1.20255700 & $\mathrm{C}$ & -2.46592400 & -1.30872400 & 2.60177600 \\
\hline & 2.32940800 & 1.63858300 & -1.00462000 & $\mathrm{H}$ & -1.77734700 & -2.11368800 & 2.32006700 \\
\hline & -3.00595100 & 2.47885600 & 0.56048800 & $\mathrm{H}$ & -2.55584400 & -1.30863000 & 3.69428600 \\
\hline & -0.02638000 & 5.08896100 & -1.04082000 & $\mathrm{H}$ & -3.45392000 & -1.55379900 & 2.19529100 \\
\hline & 2.51213500 & 3.71962300 & -1.64547200 & $\mathrm{C}$ & -2.96811200 & -0.20160000 & -0.25845400 \\
\hline & 3.27772900 & 1.18447700 & -1.23963900 & $\mathrm{H}$ & -3.88012500 & 0.21554300 & 0.17933600 \\
\hline & 1.32448200 & 0.87287700 & -0.41174700 & $\mathrm{H}$ & -2.86230600 & 0.24356100 & -1.25595500 \\
\hline & 1.52015300 & -0.78158800 & 0.20171600 & $\mathrm{C}$ & -1.96317100 & 0.05142000 & 2.10274600 \\
\hline & 2.98580800 & -0.62850200 & 1.43636700 & $\mathrm{H}$ & -2.67793900 & 0.83612000 & 2.38705900 \\
\hline & 1.99873900 & -1.78006000 & -1.36903400 & $\mathrm{H}$ & -1.02399100 & 0.29807800 & 2.61389900 \\
\hline & 4.28762800 & 0.07570600 & 1.00984700 & & & & \\
\hline & 4.12854600 & 1.14004100 & 0.81869700 & & & & \\
\hline & 5.01625600 & 0.00072400 & 1.82917600 & & & & \\
\hline & 4.74836400 & -0.37195400 & 0.12502500 & & & & \\
\hline & 2.38609500 & 0.15273100 & 5280340 & & & & \\
\hline & 3.14020100 & 0.25036500 & 3.42059100 & & & & \\
\hline & 2.07777200 & 1.16247100 & 2.33580400 & & & & \\
\hline & 1.51653900 & -0.36002800 & 3.05356500 & & & & \\
\hline & 3.31566400 & -2.06248800 & 1.90555500 & $\mathrm{C}$ & -2.92544900 & 1.20569300 & 0.95533700 \\
\hline & 2.41483800 & -2.62417900 & 2.17946400 & $\mathrm{C}$ & -1.73903200 & 0.88246600 & 0.22704800 \\
\hline & 3.85687600 & -2.63311700 & 1.14341700 & $\mathrm{C}$ & -0.99659800 & 1.90044300 & -0.42728300 \\
\hline & 3.95855400 & -2.01490100 & .79441600 & $\mathrm{C}$ & -1.47889900 & 3.20496500 & -0.28772900 \\
\hline & 1.69562400 & -3.25665900 & -1.01671300 & $\mathrm{C}$ & -2.63526900 & 3.49967400 & 0.43993100 \\
\hline & 2.35200700 & -3.64564800 & -0.23167900 & $\mathrm{C}$ & -3.38274100 & 2.52836900 & 1.06604600 \\
\hline & 0.65721200 & -3.38162900 & -0.69280400 & $\mathrm{C}$ & -3.466999000 & -0.01245300 & 1.47487100 \\
\hline & 1.84768500 & -3.87988700 & -1.90809800 & $\mathrm{C}$ & -2.63880000 & -1.01486000 & 1.07231600 \\
\hline & 1.00363300 & -1.35041700 & -2.46952200 & $\mathrm{H}$ & -0.96141400 & 4.03381200 & -0.75596700 \\
\hline & 1.16605600 & -0.31848000 & -2.79426800 & $\mathrm{H}$ & -4.28290900 & 2.78263100 & 1.61567800 \\
\hline & 1.13464000 & -2.00445400 & -3.34196700 & $\mathrm{H}$ & -4.36337200 & -0.11954700 & 2.07076000 \\
\hline & 3.43636700 & -1.67451000 & -1.90556700 & $\mathrm{H}$ & -2.71485000 & -2.07140100 & 1.26904900 \\
\hline & 3.53290800 & -2.30128400 & -2.80304000 & $\mathrm{~N}$ & -1.57390300 & -0.52618400 & 0.31216800 \\
\hline & 3.70357800 & -0.65471800 & -2.19904700 & $\mathrm{P}$ & -0.21026400 & -1.50652100 & -0.26407700 \\
\hline & 4.17728900 & -2.03195300 & -1.18364900 & $\mathrm{C}$ & -1.05415100 & -2.64545800 & 5981500 \\
\hline
\end{tabular}




\begin{tabular}{|c|c|c|c|c|c|c|c|}
\hline $\mathrm{C}$ & 0.35777700 & -2.50373900 & 1.28379700 & $\mathrm{H}$ & 5.91312000 & 1.63767100 & 1.71065900 \\
\hline $\mathrm{C}$ & -2.36321000 & -3.35859600 & -1.17591800 & & & & \\
\hline $\mathrm{H}$ & -3.15704100 & -2.64451200 & -0.93970300 & $\mathbf{P} \delta$ & & & \\
\hline $\mathrm{H}$ & -2.70861500 & -3.95734700 & -2.03024300 & & & & \\
\hline $\mathrm{H}$ & -2.24643000 & -4.03986900 & -0.32913300 & & & & \\
\hline $\mathrm{C}$ & -1.35453800 & -1.70494000 & -2.75026300 & & & & \\
\hline $\mathrm{H}$ & -1.80965600 & -2.28041200 & -3.56751300 & & & & \\
\hline $\mathrm{H}$ & -2.05582000 & -0.91072300 & -2.47215100 & & & & \\
\hline $\mathrm{H}$ & -0.44323400 & -1.23665600 & -3.13851000 & $\mathrm{C}$ & 3.13127100 & 1.82805000 & -0.55970500 \\
\hline $\mathrm{C}$ & -0.01012800 & -3.68675400 & -2.01483300 & $\mathrm{C}$ & 1.93635000 & 1.23598100 & -0.04686100 \\
\hline $\mathrm{H}$ & 0.94801600 & -3.22073600 & -2.27410500 & $\mathrm{C}$ & 0.90823000 & 2.04445200 & 0.49708500 \\
\hline $\mathrm{H}$ & 0.17718100 & -4.44931600 & -1.25215500 & $\mathrm{C}$ & 1.13182600 & 3.42476500 & 0.48607300 \\
\hline $\mathrm{H}$ & -0.37782300 & -4.20402300 & -2.91079900 & $\mathrm{C}$ & 2.30242800 & 3.98629900 & -0.03102800 \\
\hline $\mathrm{C}$ & 1.80757100 & -2.93867000 & 0.95382100 & $\mathrm{C}$ & 3.31918000 & 3.21939800 & -0.55619400 \\
\hline $\mathrm{H}$ & 1.85887100 & -3.61976200 & 0.09815100 & $\mathrm{C}$ & 3.98377200 & 0.76948400 & -1.00955200 \\
\hline $\mathrm{H}$ & 2.44665100 & -2.07369800 & 0.75168000 & $\mathrm{C}$ & 3.32508600 & -0.39773000 & -0.76872800 \\
\hline $\mathrm{H}$ & 2.22920900 & -3.46843200 & 1.81843800 & $\mathrm{H}$ & 0.38117900 & 4.09376300 & 0.89627000 \\
\hline $\mathrm{C}$ & 0.42798600 & -1.50694900 & 2.46095700 & $\mathrm{H}$ & 4.22310600 & 3.67977400 & -0.94095500 \\
\hline $\mathrm{H}$ & -0.56049000 & -1.15398700 & 2.76885200 & $\mathrm{H}$ & 4.96630400 & 0.87293000 & -1.45003100 \\
\hline $\mathrm{H}$ & 0.89070200 & -2.00796800 & 3.32162000 & $\mathrm{H}$ & 3.64433000 & -1.40782600 & -0.96539600 \\
\hline $\mathrm{C}$ & -0.44374300 & -3.74676900 & 1.71264600 & $\mathrm{~N}$ & 2.07678900 & -0.16874900 & -0.18607800 \\
\hline $\mathrm{H}$ & 0.04176300 & -4.19947700 & 2.58819000 & $\mathrm{P}$ & 0.86400400 & -1.40901700 & 0.16779100 \\
\hline $\mathrm{H}$ & -1.47092900 & -3.51257700 & 2.00677300 & $\mathrm{C}$ & 1.71317500 & -2.42937700 & 1.55411500 \\
\hline $\mathrm{H}$ & -0.47547600 & -4.51306600 & 0.93212200 & $\mathrm{C}$ & 0.73339900 & -2.41886400 & -1.46962100 \\
\hline $\mathrm{F}$ & -3.02553600 & 4.79572100 & 0.51521600 & $\mathrm{C}$ & 3.18004600 & -2.85547500 & 1.36223300 \\
\hline $\mathrm{H}$ & 1.05101200 & -0.63898900 & 2.22007700 & $\mathrm{H}$ & 3.84406000 & -1.99095700 & 1.27518800 \\
\hline $\mathrm{C}$ & 0.28969700 & 1.67094300 & -1.21714400 & $\mathrm{H}$ & 3.50464500 & -3.42677200 & 2.24302100 \\
\hline $\mathrm{H}$ & 0.24867200 & 0.65929900 & -1.62993500 & $\mathrm{H}$ & 3.32857600 & -3.49564500 & 0.48838600 \\
\hline $\mathrm{C}$ & 1.50145000 & 1.70559400 & -0.25684900 & $\mathrm{C}$ & 1.63820000 & -1.50848500 & 2.79424100 \\
\hline $\mathrm{H}$ & 1.61234400 & 2.71950400 & 0.15087100 & $\mathrm{H}$ & 2.07570800 & -2.02244000 & 3.66048500 \\
\hline $\mathrm{H}$ & 1.30031400 & 1.04710800 & 0.59233400 & $\mathrm{H}$ & 2.19665700 & -0.57849200 & 2.64211500 \\
\hline $\mathrm{C}$ & 0.46336400 & 2.63448700 & -2.40517300 & $\mathrm{H}$ & 0.60401200 & -1.24951700 & 3.04632900 \\
\hline $\mathrm{H}$ & 0.68995800 & 3.65811800 & -2.08437200 & $\mathrm{C}$ & 0.84158200 & -3.67547900 & 1.81699800 \\
\hline $\mathrm{H}$ & -0.44097300 & 2.66804500 & -3.02192100 & $\mathrm{H}$ & -0.21833300 & -3.41940700 & 1.93229700 \\
\hline $\mathrm{H}$ & 1.29090900 & 2.30697700 & -3.04400400 & $\mathrm{H}$ & 0.92919800 & -4.41993100 & 1.01879300 \\
\hline $\mathrm{C}$ & 2.82053500 & 1.25188100 & -0.90405000 & $\mathrm{H}$ & 1.16667800 & -4.15678800 & 2.74858400 \\
\hline $\mathrm{H}$ & 2.64468000 & 0.31109600 & -1.44472800 & $\mathrm{C}$ & -0.62982100 & -3.14641600 & -1.37316400 \\
\hline $\mathrm{H}$ & 3.20636500 & 1.97882800 & -1.62291500 & $\mathrm{H}$ & -0.64512700 & -3.90393600 & -0.58331400 \\
\hline $\mathrm{C}$ & 3.88349500 & 0.95798600 & 0.13557300 & $\mathrm{H}$ & -1.44845500 & -2.44486200 & -1.18100900 \\
\hline $\mathrm{O}$ & 3.71003300 & 0.30701400 & 1.14722700 & $\mathrm{H}$ & -0.83887900 & -3.65778500 & -2.32210700 \\
\hline $\mathrm{O}$ & 5.07752100 & 1.49384300 & -0.19848900 & $\mathrm{C}$ & 0.64388700 & -1.38880400 & -2.61613600 \\
\hline $\mathrm{C}$ & 6.14942700 & 1.23363400 & 0.72266100 & $\mathrm{H}$ & 1.59396500 & -0.87384000 & -2.78397900 \\
\hline $\mathrm{H}$ & 6.32581100 & 0.15836600 & 0.81355600 & $\mathrm{H}$ & 0.37013900 & -1.90678600 & -3.54484700 \\
\hline $\mathrm{H}$ & 7.02364900 & 1.73212200 & 0.30278600 & $\mathrm{C}$ & 1.81734400 & -3.45865100 & -1.80815000 \\
\hline
\end{tabular}




\begin{tabular}{|c|c|c|c|c|c|c|c|}
\hline $\mathrm{H}$ & 1.55958800 & -3.95036600 & -2.75651200 & $\mathrm{~N}$ & 4.08989300 & -0.64083500 & -0.93495100 \\
\hline $\mathrm{H}$ & 2.80700500 & -3.01260900 & -1.94168600 & $\mathrm{P}$ & 2.55397700 & -1.44220300 & -1.10981200 \\
\hline $\mathrm{H}$ & 1.89301400 & -4.24340800 & -1.04970000 & $\mathrm{C}$ & 2.91830800 & -3.28156000 & -0.70236700 \\
\hline $\mathrm{F}$ & 2.43223000 & 5.33479500 & 0.00143000 & $\mathrm{C}$ & 2.10996200 & -1.22331700 & -2.96382800 \\
\hline $\mathrm{H}$ & -0.12234100 & -0.62852400 & -2.42486200 & $\mathrm{C}$ & 4.23371900 & -3.81879800 & -1.30498700 \\
\hline $\mathrm{C}$ & -5.29618400 & 0.42391500 & 0.13321600 & $\mathrm{H}$ & 5.10447000 & -3.30592800 & -0.88725500 \\
\hline $\mathrm{O}$ & -5.49629700 & 0.11115100 & 1.28714900 & $\mathrm{H}$ & 4.32505400 & -4.88144100 & -1.04453900 \\
\hline $\mathrm{O}$ & -6.25538400 & 0.39228700 & -0.82229000 & $\mathrm{H}$ & 4.27458600 & -3.74748500 & -2.39375600 \\
\hline $\mathrm{C}$ & -7.55046000 & -0.04033600 & -0.37895800 & $\mathrm{C}$ & 3.03721300 & -3.38819600 & 0.83197100 \\
\hline $\mathrm{H}$ & -7.93408700 & 0.62509200 & 0.39944200 & $\mathrm{H}$ & 3.32459600 & -4.41421300 & 1.09652500 \\
\hline $\mathrm{H}$ & -8.18995200 & -0.00497700 & -1.26173600 & $\mathrm{H}$ & 3.80577800 & -2.71561600 & 1.22936900 \\
\hline $\mathrm{H}$ & -7.50253000 & -1.05762800 & 0.01955900 & $\mathrm{H}$ & 2.08567800 & -3.17012500 & 1.32205200 \\
\hline $\mathrm{C}$ & -2.86045900 & 0.98458300 & 0.60081500 & $\mathrm{C}$ & 1.73356900 & -4.15843600 & -1.15850300 \\
\hline $\mathrm{H}$ & -2.73041900 & 0.00462400 & 1.07624700 & $\mathrm{H}$ & 0.77707400 & -3.77969200 & -0.78551300 \\
\hline $\mathrm{C}$ & -0.41597400 & 1.56683000 & 1.06173700 & $\mathrm{H}$ & 1.67459000 & -4.24605000 & -2.24705800 \\
\hline $\mathrm{H}$ & -0.73888500 & 2.28451500 & 1.82684600 & $\mathrm{H}$ & 1.87145200 & -5.17134400 & -0.75828800 \\
\hline $\mathrm{H}$ & -0.30567200 & 0.60227500 & 1.56271700 & $\mathrm{C}$ & 0.61146400 & -1.54445700 & -3.15536000 \\
\hline $\mathrm{H}$ & -1.65646100 & 2.42478800 & -0.49131400 & $\mathrm{H}$ & 0.38548800 & -2.60271100 & -2.99977100 \\
\hline $\mathrm{C}$ & -3.97460000 & 0.89369100 & -0.44394900 & $\mathrm{H}$ & -0.02044300 & -0.95956600 & -2.48388600 \\
\hline $\mathrm{H}$ & -4.14436000 & 1.86371300 & -0.93018200 & $\mathrm{H}$ & 0.33186700 & -1.29730200 & -4.18839900 \\
\hline $\mathrm{H}$ & -3.70058300 & 0.20617500 & -1.25613200 & $\mathrm{C}$ & 2.34982900 & 0.26948700 & -3.28853600 \\
\hline $\mathrm{C}$ & -1.52881000 & 1.45121700 & 0.00214500 & $\mathrm{H}$ & 3.40937100 & 0.53671700 & -3.22775500 \\
\hline $\mathrm{H}$ & -1.21023000 & 0.74865500 & -0.77922300 & $\mathrm{H}$ & 2.01526600 & 0.45650100 & -4.31740500 \\
\hline \multirow[t]{2}{*}{$\mathrm{H}$} & 286600 & 1.66774100 & 0097600 & $\mathrm{C}$ & 2.94035000 & -2.06990600 & -3.94760800 \\
\hline & & & & $\mathrm{H}$ & 2.64633400 & -1.79943800 & -4.97044300 \\
\hline \multirow[t]{7}{*}{82} & \multirow{7}{*}{$\mathrm{CO}_{2} \mathrm{~N}$} & & & $\mathrm{H}$ & 4.01385100 & -1.87634100 & -3.86084800 \\
\hline & & \multirow{6}{*}{\multicolumn{2}{|c|}{ sen }} & $\mathrm{H}$ & 2.76533700 & -3.14350400 & -3.83609400 \\
\hline & & & & $\mathrm{F}$ & 4.38878700 & 4.31744000 & 1.28928300 \\
\hline & & & & $\mathrm{Rh}$ & 1.33422500 & -0.17124600 & 0.62842800 \\
\hline & & & & $\mathrm{Cl}$ & -0.73956000 & -1.73562700 & 0.48956400 \\
\hline & & & & $\mathrm{H}$ & 1.78497600 & 0.93107300 & -2.62839800 \\
\hline & & & & $\mathrm{C}$ & 0.61026700 & 1.79151100 & 3.08024900 \\
\hline $\mathrm{C}$ & 5.23805800 & 1.28981500 & -0.49407400 & $\mathrm{O}$ & 1.52407300 & 2.31700500 & 3.69308200 \\
\hline C & 5100 & 0.58674200 & -0.26066100 & $\mathrm{O}$ & -0.56160100 & 2.43179600 & 2.83373100 \\
\hline $\mathrm{C}$ & 2.94178700 & 1.07403400 & 0.47469300 & $\mathrm{C}$ & -0.63442400 & 3.77989500 & 3.31714900 \\
\hline $\mathrm{C}$ & 3.10223900 & 2.36388200 & 0.97477800 & $\mathrm{H}$ & 0.12986800 & 4.40277100 & 2.84422900 \\
\hline $\mathrm{C}$ & 4.30319400 & 3.07055700 & 0.76061400 & $\mathrm{H}$ & -1.63105900 & 4.13275300 & 3.04953900 \\
\hline $\mathrm{C}$ & 5.38315700 & 2.57807700 & 0.05412600 & $\mathrm{H}$ & -0.49438800 & 3.81122700 & 4.40147000 \\
\hline $\mathrm{C}$ & 6.03963900 & 0.44676000 & -1.34173000 & $\mathrm{C}$ & 1.67867500 & -0.50473500 & 2.94625700 \\
\hline $\mathrm{C}$ & 5.31796800 & -0.68670300 & -1.60409400 & $\mathrm{H}$ & 2.52154600 & 0.04874700 & 3.37127900 \\
\hline $\mathrm{H}$ & 2.34047500 & 2.86040700 & 1.55902200 & $\mathrm{C}$ & 0.89801200 & -1.39379700 & 5.18934500 \\
\hline $\mathrm{H}$ & 6.27723000 & 3.17848700 & -0.07631300 & $\mathrm{H}$ & 0.00726400 & -0.75588800 & 5.19440400 \\
\hline $\mathrm{H}$ & 7.03522900 & 0.65684700 & -1.71124600 & $\mathrm{H}$ & 0.66957000 & -2.29808300 & 5.76440700 \\
\hline $\mathrm{H}$ & 5.59071000 & -1.54123500 & -2.20298000 & $\mathrm{H}$ & 1.69779000 & -0.85452000 & 5.71001700 \\
\hline
\end{tabular}




\begin{tabular}{|c|c|c|c|c|c|c|c|}
\hline C & 0.58567500 & 0.40788200 & 2.55278900 & $\mathrm{H}$ & -3.00824200 & -2.46843500 & 1.71727500 \\
\hline $\mathrm{H}$ & 2.20476800 & -0.98008800 & 1.96333400 & $\mathrm{H}$ & -4.60653600 & 0.06093800 & 1.00057200 \\
\hline $\mathrm{H}$ & -0.41901100 & -0.00951000 & 2.56226000 & $\mathrm{C}$ & -5.31580100 & -4.03227500 & 0.70623300 \\
\hline . & 1.31117800 & -1.75760800 & 3.75741300 & $\mathrm{H}$ & -6.03889800 & -4.85581 & 0.64356300 \\
\hline $\mathrm{H}$ & 2.17059700 & -2.43927800 & 3.78696100 & $\mathrm{H}$ & -4.43426500 & -4.45397900 & 1.21073400 \\
\hline $\mathrm{H}$ & 0.49464200 & -2.28567300 & 3.25166900 & $\mathrm{C}$ & -5.93592200 & -2.90790000 & 1.57267500 \\
\hline $\mathrm{Cl}$ & -0.10727400 & 1.21938700 & -0.70620200 & $\mathrm{H}$ & -6.75952300 & -3.32870800 & 2.16404400 \\
\hline $\mathrm{Rh}$ & -2.29009900 & 0.03714900 & -0.36131600 & $\mathrm{H}$ & 77000 & -2.164 & 0.91233200 \\
\hline $\mathrm{C}$ & -3.73757000 & 4.36262500 & -0.52260700 & & & & \\
\hline C & -2.96325300 & 3.10719700 & -0.05505900 & 83 & & & \\
\hline $\mathrm{C}$ & -3.24929700 & 2.90207400 & -3.79672600 & & & & \\
\hline $\mathrm{C}$ & -3.52762300 & 1.81272400 & -0.60553800 & & & & \\
\hline $\mathrm{C}$ & -2.37802400 & 1.99327200 & -2.89575200 & & & & \\
\hline $\mathrm{C}$ & -3.23930700 & 1.28177700 & -1.87757800 & & & & \\
\hline $\mathrm{H}$ & -4.81147700 & 4.22725300 & -0.32408900 & & & & \\
\hline $\mathrm{H}$ & -1.90607600 & 3.21898600 & -0.30871800 & & & & \\
\hline $\mathrm{H}$ & -3.85276300 & 2.27549500 & -4.46901100 & $\mathrm{C}$ & 25800 & 1.82367100 & 0.23556300 \\
\hline $\mathrm{H}$ & -1.84959900 & 1.26979500 & -3.52834500 & $\mathrm{C}$ & 3.95769200 & 1.04273000 & 0.10809100 \\
\hline $\mathrm{H}$ & -3.41637700 & 5.19924700 & 0.11309500 & $\mathrm{C}$ & 2.80181400 & 1.17728800 & 0.89806400 \\
\hline $\mathrm{H}$ & -3.00557900 & 3.05926300 & 1.04044900 & $\mathrm{C}$ & 2.86364000 & 2.20435900 & 1.83996900 \\
\hline $\mathrm{H}$ & -2.57118900 & 3.47737400 & -4.44239700 & $\mathrm{C}$ & 4.03363100 & 2.97578800 & 1.99317300 \\
\hline $\mathrm{H}$ & -4.50742300 & 1.57175500 & -0.19249500 & $\mathrm{C}$ & 5.17734900 & 2.81287100 & 1.23693900 \\
\hline $\mathrm{H}$ & -1.61025400 & 2.59698100 & -2.40828000 & $\mathrm{C}$ & 6.01049700 & 1.40487600 & -0.82167100 \\
\hline $\mathrm{H}$ & -4.00155900 & 0.65714000 & -2.34338400 & $\mathrm{C}$ & 5.35977000 & 0.44212500 & -1.54691000 \\
\hline $\mathrm{C}$ & -3.55186600 & 4.78907500 & -1.99379400 & $\mathrm{H}$ & 2.01964500 & 2.45872300 & 2.46628100 \\
\hline $\mathrm{H}$ & -3.98966100 & 5.79104400 & -2.09000600 & $\mathrm{H}$ & 6.04283700 & 3.44611700 & 1.40077300 \\
\hline $\mathrm{H}$ & -2.47988100 & 4.91630800 & -2.20308000 & $\mathrm{H}$ & 7.00697100 & 1.77721000 & -1.02230300 \\
\hline $\mathrm{C}$ & -4.19624000 & 3.87166600 & -3.06323500 & $\mathrm{H}$ & 5.69911600 & -0.09573900 & -2.41814300 \\
\hline $\mathrm{H}$ & -4.66853400 & 4.49754400 & -3.83152800 & $\mathrm{~N}$ & 4.10695500 & 0.17943100 & -0.98625300 \\
\hline $\mathrm{H}$ & -5.01613500 & 3.30523500 & -2.60209500 & $\mathrm{P}$ & 2.61464300 & -0.52868300 & -1.55819900 \\
\hline $\mathrm{C}$ & -4.94481000 & -3.65978900 & -0.74478400 & $\mathrm{C}$ & 3.05932700 & -2.35691000 & -1.92729000 \\
\hline C & -3.72890800 & -2.72252200 & -0.94124200 & $\mathrm{C}$ & 2.22400600 & 0.42909300 & -3.17327000 \\
\hline $\mathrm{C}$ & -4.99091700 & -2.20076200 & 2.56444800 & $\mathrm{C}$ & 4.41699400 & -2.56672500 & -2.63008400 \\
\hline C & -4.00750300 & -1.28390800 & -0.55288900 & $\mathrm{H}$ & 5.24981400 & -2.24716200 & -1.99752500 \\
\hline $\mathrm{C}$ & -3.67476700 & -1.64326800 & 1.97189800 & $\mathrm{H}$ & 4.54725900 & -3.64030500 & -2.82100400 \\
\hline $\mathrm{C}$ & -3.96207200 & -0.78401900 & 0.76305300 & $\mathrm{H}$ & 4.49085300 & -2.05550600 & -3.59226400 \\
\hline $\mathrm{H}$ & -5.82718200 & -3.22396400 & -1.23703300 & $\mathrm{C}$ & 3.12615900 & -3.07440700 & -0.56331800 \\
\hline $\mathrm{H}$ & -2.87387200 & -3.12039800 & -0.38885300 & $\mathrm{H}$ & 3.44883600 & -4.11219400 & -0.72085600 \\
\hline $\mathrm{H}$ & -5.54828000 & -1.38442200 & 3.04553000 & $\mathrm{H}$ & 3.84747800 & -2.60295400 & 0.11309400 \\
\hline $\mathrm{H}$ & -3.16129400 & -1.05736200 & 2.74445600 & $\mathrm{H}$ & 2.15020100 & -3.09952000 & -0.07258200 \\
\hline $\mathrm{H}$ & -4.72756300 & -4.59135400 & -1.28532400 & $\mathrm{C}$ & 1.93394400 & -3.00342700 & -2.76330900 \\
\hline $\mathrm{H}$ & -3.44555300 & -2.74458500 & -2.00198500 & $\mathrm{H}$ & 0.94572200 & -2.83516400 & -2.32361700 \\
\hline $\mathrm{H}$ & -4.72211500 & -2.90101400 & 3.36774900 & $\mathrm{H}$ & 1.92309100 & -2.64291000 & -3.79622500 \\
\hline $\mathrm{H}$ & -4.70224600 & -0.79134300 & -1.23362600 & $\mathrm{H}$ & 2.10287500 & -4.08775600 & -2.80003100 \\
\hline
\end{tabular}




\begin{tabular}{|c|c|c|c|c|c|c|c|}
\hline $\mathrm{C}$ & 0.74956000 & 0.16252800 & -3.54732400 & $\mathrm{H}$ & -4.31779700 & 3.97262700 & -2.86836800 \\
\hline $\mathrm{H}$ & 0.57042800 & -0.87643200 & -3.83591200 & $\mathrm{H}$ & -2.21585500 & 2.78358500 & -2.57683300 \\
\hline $\mathrm{H}$ & 0.07430900 & 0.40781200 & -2.72440800 & $\mathrm{H}$ & -3.66386300 & 4.56060000 & 2.51191100 \\
\hline $\mathrm{H}$ & 0.48602800 & 0.79457100 & -4.40608400 & $\mathrm{H}$ & -3.11982900 & 2.26702900 & 2.36404600 \\
\hline $\mathrm{C}$ & 2.38402200 & 1.92713100 & -2.82758100 & $\mathrm{H}$ & -3.07404800 & 5.11216400 & -2.37612400 \\
\hline $\mathrm{H}$ & 3.42368000 & 2.18844200 & -2.60795000 & $\mathrm{H}$ & -4.63009600 & 1.37867600 & 0.66168700 \\
\hline $\mathrm{H}$ & 2.06493500 & 2.51959900 & -3.69511400 & $\mathrm{H}$ & -1.94666100 & 3.48536900 & -0.99366700 \\
\hline $\mathrm{C}$ & 3.12911300 & 0.10240800 & -4.37506000 & $\mathrm{H}$ & -4.24975500 & 1.56263600 & -1.69081000 \\
\hline $\mathrm{H}$ & 2.86266800 & 0.76848800 & -5.20655700 & $\mathrm{C}$ & -3.91577300 & 5.13054500 & 0.45355400 \\
\hline $\mathrm{H}$ & 4.18772800 & 0.26991700 & -4.15391300 & $\mathrm{H}$ & -4.38353200 & 6.04447300 & 0.84200600 \\
\hline $\mathrm{H}$ & 3.00336800 & -0.92401500 & -4.73151700 & $\mathrm{H}$ & -2.86308400 & 5.39362400 & 0.27510300 \\
\hline $\mathrm{F}$ & 4.01705300 & 3.93995200 & 2.95009400 & $\mathrm{C}$ & -4.60621900 & 4.76287000 & -0.88397600 \\
\hline $\mathrm{Rh}$ & 1.27135200 & -0.10054400 & 0.49883900 & $\mathrm{H}$ & -5.13776100 & 5.64488200 & -1.26419600 \\
\hline $\mathrm{Cl}$ & -0.67582600 & -1.58899800 & -0.33190000 & $\mathrm{H}$ & -5.38519800 & 4.01329300 & -0.69087900 \\
\hline $\mathrm{H}$ & 1.76342300 & 2.21981800 & -1.97698100 & $\mathrm{C}$ & -4.81506400 & -3.07582500 & -2.22671900 \\
\hline $\mathrm{C}$ & 1.21933800 & -2.70325200 & 2.84173600 & $\mathrm{C}$ & -3.67262900 & -2.05412500 & -2.00906900 \\
\hline $\mathrm{O}$ & 0.12211200 & -3.12256500 & 3.12626600 & $\mathrm{C}$ & -4.75693300 & -3.26729800 & 1.38505900 \\
\hline $\mathrm{O}$ & 2.32801400 & -3.47314300 & 2.78340200 & $\mathrm{C}$ & -4.01417000 & -0.97450000 & -1.00075400 \\
\hline $\mathrm{C}$ & 2.12952100 & -4.86598700 & 3.08459100 & $\mathrm{C}$ & -3.50996700 & -2.40659500 & 1.07411600 \\
\hline $\mathrm{H}$ & 1.44280800 & -5.31760900 & 2.36392200 & $\mathrm{C}$ & -3.91625100 & -1.11858700 & 0.39681600 \\
\hline $\mathrm{H}$ & 3.11672700 & -5.32267800 & 3.01417700 & $\mathrm{H}$ & -5.74977100 & -2.53871300 & -2.44742600 \\
\hline $\mathrm{H}$ & 1.72060900 & -4.98398500 & 4.09133500 & $\mathrm{H}$ & -2.76159100 & -2.58609400 & -1.72302100 \\
\hline $\mathrm{C}$ & 0.44588500 & -0.28390500 & 2.45142000 & $\mathrm{H}$ & -5.33197000 & -2.79846500 & 2.19635300 \\
\hline $\mathrm{H}$ & -0.51136300 & -0.77337500 & 2.27084800 & $\mathrm{H}$ & -2.97656800 & -2.20537900 & 2.01081300 \\
\hline $\mathrm{C}$ & -0.54133200 & 0.38090700 & 4.68372500 & $\mathrm{H}$ & -4.57282400 & -3.64491100 & -3.13491300 \\
\hline $\mathrm{H}$ & -0.10365300 & -0.48781200 & 5.18965400 & $\mathrm{H}$ & -3.45607500 & -1.57042600 & -2.97099700 \\
\hline $\mathrm{H}$ & -0.62839700 & 1.19188100 & 5.41653700 & $\mathrm{H}$ & -4.40333600 & -4.23015400 & 1.77909300 \\
\hline $\mathrm{H}$ & -1.55243800 & 0.10003400 & 4.36621400 & $\mathrm{H}$ & -4.77711700 & -0.28612900 & -1.36537000 \\
\hline $\mathrm{C}$ & 1.56696000 & -1.24542300 & 2.54681100 & $\mathrm{H}$ & -2.81907700 & -2.97462400 & 0.45046900 \\
\hline $\mathrm{H}$ & 2.15819100 & -1.34378800 & 1.51320100 & $\mathrm{H}$ & -4.59205500 & -0.52127700 & 1.00746600 \\
\hline $\mathrm{H}$ & 2.38426000 & -0.91007300 & 3.19472000 & $\mathrm{C}$ & -5.07695600 & -4.08761300 & -1.09097100 \\
\hline $\mathrm{C}$ & 0.31450400 & 0.82333600 & 3.48104400 & $\mathrm{H}$ & -5.74920300 & -4.85266100 & -1.50079100 \\
\hline $\mathrm{H}$ & -0.15228100 & 1.70108900 & 3.01698400 & $\mathrm{H}$ & -4.14234400 & -4.61529600 & -0.85163800 \\
\hline $\mathrm{H}$ & 1.30022300 & 1.13529600 & 3.84096700 & $\mathrm{C}$ & -5.71409600 & -3.52374900 & 0.20379000 \\
\hline $\mathrm{Cl}$ & -0.26468100 & 1.62960800 & -0.15856800 & $\mathrm{H}$ & -6.47896100 & -4.22657200 & 0.55908500 \\
\hline $\mathrm{Rh}$ & -2.36423700 & 0.25101200 & -0.28905000 & $\mathrm{H}$ & -6.25619900 & -2.59989100 & -0.03870600 \\
\hline $\mathrm{C}$ & -3.99440300 & 4.08020700 & 1.58089500 & & & & \\
\hline $\mathrm{C}$ & -3.14708100 & 2.79737500 & 1.40274900 & & & & \\
\hline $\mathrm{C}$ & -3.68629200 & 4.27203100 & -2.01971300 & & & & \\
\hline $\mathrm{C}$ & -3.69643600 & 1.85021500 & 0.35455800 & & & & \\
\hline $\mathrm{C}$ & -2.72467700 & 3.11344300 & -1.66266100 & & & & \\
\hline $\mathrm{C}$ & -3.48205800 & 1.96746000 & -1.03203100 & & & & \\
\hline $\mathrm{H}$ & -5.04793400 & 3.80513000 & 1.73991800 & & & & \\
\hline $\mathrm{H}$ & -2.11369700 & 3.07580800 & 1.18041100 & $\mathrm{C}$ & 5.27177200 & 1.5265 & 51700 \\
\hline
\end{tabular}




\begin{tabular}{|c|c|c|c|c|c|c|c|}
\hline C & -4.09932500 & 0.80976800 & 0.11709200 & $\mathrm{O}$ & 1.06511000 & 4.57854100 & -2.96528900 \\
\hline $\mathrm{C}$ & -2.85340200 & 1.38572500 & -0.18910200 & $\mathrm{C}$ & 2.13023900 & 5.14958700 & -3.74234500 \\
\hline $\mathrm{C}$ & -2.83010400 & 2.77872900 & -0.11850700 & $\mathrm{H}$ & 2.17179300 & 4.69013000 & -4.73360800 \\
\hline $\mathrm{C}$ & -3.99719300 & 3.50768600 & 0.18926200 & $\mathrm{H}$ & 1.89705100 & 6.21181600 & -3.82131900 \\
\hline 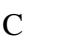 & -5.22319100 & 2.93423800 & 0.46132000 & $\mathrm{H}$ & 3.09227600 & 5.00340600 & -3.24346300 \\
\hline $\mathrm{C}$ & -6.27131300 & 0.54130600 & 0.76460400 & $\mathrm{C}$ & -0.24551900 & 1.28879800 & -2.00272200 \\
\hline $\mathrm{C}$ & -5.68911900 & -0.69298700 & 0.64497700 & $\mathrm{H}$ & 0.69546600 & 0.75403800 & -2.11676500 \\
\hline $\mathrm{H}$ & -1.92323500 & 3.34789300 & -0.27086400 & $\mathrm{C}$ & -0.89934100 & -0.06842200 & -4.09560300 \\
\hline $\mathrm{H}$ & -6.08206000 & 3.55300300 & 0.69867900 & $\mathrm{H}$ & -0.22290900 & -0.86849000 & -3.78173300 \\
\hline $\mathrm{H}$ & -7.29889400 & 0.72691900 & 1.05004200 & $\mathrm{H}$ & -1.76551200 & -0.51237000 & -4.59848500 \\
\hline $\mathrm{H}$ & -6.12267400 & -1.66610500 & 0.81454400 & $\mathrm{H}$ & -0.36709800 & 0.56129400 & -4.81723200 \\
\hline $\mathrm{N}$ & -4.36334600 & -0.56276800 & 0.22356900 & $\mathrm{C}$ & 0.00941700 & 2.77415700 & -1.85343700 \\
\hline$P$ & -2.93872500 & -1.57309600 & 0.27392500 & $\mathrm{H}$ & -0.87095400 & 3.38394000 & -2.06916300 \\
\hline $\mathrm{C}$ & -3.35574900 & -3.05592200 & -0.86696200 & $\mathrm{H}$ & 0.31376500 & 3.00789700 & -0.82288100 \\
\hline $\mathrm{C}$ & -2.81341400 & -2.12559400 & 2.10746800 & $\mathrm{C}$ & -1.31523400 & 0.79040100 & -2.89576900 \\
\hline $\mathrm{C}$ & -4.78704000 & -3.61121900 & -0.71355900 & $\mathrm{H}$ & -2.04335500 & 1.56573000 & -3.15756100 \\
\hline $\mathrm{H}$ & -5.53768500 & -2.86960900 & -1.00109900 & $\mathrm{H}$ & -2.04570900 & 0.03705400 & -2.28028300 \\
\hline $\mathrm{H}$ & -4.90138700 & -4.46917200 & -1.38931400 & $\mathrm{Cl}$ & 0.00710500 & 0.69521800 & 1.27532100 \\
\hline $\mathrm{H}$ & -5.01097100 & -3.96292600 & 0.29599400 & $\mathrm{Rh}$ & 2.14967700 & -0.41654800 & 0.57856100 \\
\hline $\mathrm{C}$ & -3.20609900 & -2.55629800 & -2.31962900 & $\mathrm{C}$ & 3.76822300 & 3.32629700 & 2.64639100 \\
\hline $\mathrm{H}$ & -3.49169400 & -3.36443900 & -3.00592700 & $\mathrm{C}$ & 2.97177300 & 2.45323400 & 1.64719800 \\
\hline $\mathrm{H}$ & -3.85848700 & -1.70016200 & -2.52601000 & $\mathrm{C}$ & 3.01034500 & 0.62783400 & 4.93246000 \\
\hline $\mathrm{H}$ & -2.17334700 & -2.27674900 & -2.54206800 & $\mathrm{C}$ & 3.44602100 & 1.01408700 & 1.58956000 \\
\hline $\mathrm{C}$ & -2.32764500 & -4.18369300 & -0.63547500 & $\mathrm{C}$ & 2.17184700 & 0.25348100 & 3.68660200 \\
\hline $\mathrm{H}$ & -1.29752300 & -3.81613800 & -0.68138100 & $\mathrm{C}$ & 3.06984000 & 0.00417700 & 2.49689600 \\
\hline $\mathrm{H}$ & -2.47560400 & -4.68943800 & 0.32290900 & $\mathrm{H}$ & 4.84573300 & 3.21511200 & 2.45312300 \\
\hline $\mathrm{H}$ & -2.44898100 & -4.93852500 & -1.42346100 & $\mathrm{H}$ & 1.90740800 & 2.51104800 & 1.89049600 \\
\hline C & -1.39667300 & -2.69209300 & 2.34952600 & $\mathrm{H}$ & 3.54538300 & -0.26297000 & 5.29117800 \\
\hline $\mathrm{H}$ & -1.22785800 & -3.63954900 & 1.83085200 & $\mathrm{H}$ & 1.57721300 & -0.63931800 & 3.91640200 \\
\hline $\mathrm{H}$ & -0.61995700 & -1.99017900 & 2.03744700 & $\mathrm{H}$ & 3.52839200 & 4.37542500 & 2.42521700 \\
\hline $\mathrm{H}$ & -1.27506200 & -2.87954400 & 3.42489200 & $\mathrm{H}$ & 3.08151900 & 2.88960800 & 0.64537400 \\
\hline $\mathrm{C}$ & -2.98355600 & -0.84120100 & 2.95138800 & $\mathrm{H}$ & 2.30999800 & 0.90617600 & 5.73227900 \\
\hline $\mathrm{H}$ & -3.98960100 & -0.42017100 & 2.86023700 & $\mathrm{H}$ & 4.43511600 & 0.91640600 & 1.14118500 \\
\hline $\mathrm{H}$ & -2.82224300 & -1.09671700 & 4.00698400 & $\mathrm{H}$ & 1.45939200 & 1.05292900 & 3.47604100 \\
\hline $\mathrm{C}$ & -3.86286000 & -3.15425700 & 2.56817200 & $\mathrm{H}$ & 3.77827500 & -0.80563300 & 2.66855700 \\
\hline $\mathrm{H}$ & -3.73357100 & -3.32808100 & 3.64481300 & $\mathrm{C}$ & 3.50225500 & 3.08810500 & 4.14767900 \\
\hline $\mathrm{H}$ & -4.88675300 & -2.79690500 & 2.42043600 & $\mathrm{H}$ & 3.97236700 & 3.92081400 & 4.68669800 \\
\hline $\mathrm{H}$ & -3.75254200 & -4.12180700 & 2.07047400 & $\mathrm{H}$ & 2.42450600 & 3.18240600 & 4.34516900 \\
\hline $\mathrm{F}$ & -3.88961800 & 4.86163500 & 0.21840100 & $\mathrm{C}$ & 4.03763100 & 1.76131000 & 4.74236000 \\
\hline $\mathrm{Rh}$ & -1.34391100 & 0.09951100 & -0.63904800 & $\mathrm{H}$ & 4.47282500 & 1.96442700 & 5.72952200 \\
\hline $\mathrm{Cl}$ & 0.55225900 & -1.64981000 & -0.91074600 & $\mathrm{H}$ & 4.87072300 & 1.40221500 & 4.12353400 \\
\hline $\mathrm{H}$ & -2.25695200 & -0.07120000 & 2.68050800 & $\mathrm{C}$ & 4.69802000 & -4.01222400 & -0.64800200 \\
\hline $\mathrm{C}$ & 1.15305700 & 3.24912400 & -2.74261800 & $\mathrm{C}$ & 3.50354500 & -3.21394500 & -0.07219300 \\
\hline $\mathrm{O}$ & 2.05309000 & 2.56133300 & -3.18082700 & $\mathrm{C}$ & 4.84306600 & -1.26940400 & -3.00217600 \\
\hline
\end{tabular}




\begin{tabular}{|c|c|c|c|c|c|c|c|}
\hline $\mathrm{C}$ & 3.82770000 & -1.75868800 & 0.20051600 & $\mathrm{H}$ & 4.75125500 & 1.65227300 & 2.75815400 \\
\hline $\mathrm{C}$ & 3.53246800 & -0.97573100 & -2.23321300 & $\mathrm{H}$ & 3.93191600 & 2.92772500 & 3.66321000 \\
\hline $\mathrm{C}$ & 3.82655100 & -0.73938100 & -0.77078100 & $\mathrm{H}$ & 3.73764100 & 1.24870600 & 4.16135200 \\
\hline $\mathrm{H}$ & 5.58212100 & -3.86034200 & -0.01061300 & $\mathrm{C}$ & 2.90113900 & 2.78491600 & 1.16083300 \\
\hline $\mathrm{H}$ & 2.64946700 & -3.30814100 & -0.74749600 & $\mathrm{H}$ & 3.17247800 & 3.77225900 & 1.55804500 \\
\hline $\mathrm{H}$ & 5.43017900 & -0.34388600 & -3.08474800 & $\mathrm{H}$ & 3.73876400 & 2.42519000 & 0.55472800 \\
\hline $\mathrm{H}$ & 3.05760600 & -0.09183400 & -2.67525800 & $\mathrm{H}$ & 2.02845300 & 2.90832900 & 0.51597500 \\
\hline $\mathrm{H}$ & 4.44647700 & -5.07865100 & -0.56486200 & $\mathrm{C}$ & 1.36408100 & 2.39837900 & 3.09520100 \\
\hline $\mathrm{H}$ & 3.19971800 & -3.68293600 & 0.87321600 & $\mathrm{H}$ & 0.45805700 & 2.33386400 & 2.48623000 \\
\hline $\mathrm{H}$ & 4.56824900 & -1.54773200 & -4.02917100 & $\mathrm{H}$ & 1.18812100 & 1.88851700 & 4.04641300 \\
\hline $\mathrm{H}$ & 4.51722400 & -1.63057000 & 1.03592300 & $\mathrm{H}$ & 1.53853900 & 3.45778700 & 3.32363700 \\
\hline $\mathrm{H}$ & 2.83689500 & -1.80657900 & -2.36087100 & $\mathrm{C}$ & 0.00557900 & -0.74089600 & 3.22843600 \\
\hline $\mathrm{H}$ & 4.49693500 & 0.10448900 & -0.61414000 & $\mathrm{H}$ & -0.17725700 & 0.24875400 & 3.65360900 \\
\hline $\mathrm{C}$ & 5.08623000 & -3.73222700 & -2.11532500 & $\mathrm{H}$ & -0.54337100 & -0.82131800 & 2.28956400 \\
\hline $\mathrm{H}$ & 5.78545100 & -4.52502900 & -2.41163900 & $\mathrm{H}$ & -0.40891100 & -1.48049100 & 3.92667400 \\
\hline $\mathrm{H}$ & 4.20302000 & -3.86487200 & -2.75704500 & $\mathrm{C}$ & 1.67776900 & -2.49288600 & 2.53914900 \\
\hline $\mathrm{C}$ & 5.75165300 & -2.36381300 & -2.40726300 & $\mathrm{H}$ & 2.73125200 & -2.77816600 & 2.46213200 \\
\hline $\mathrm{H}$ & 6.57578800 & -2.51391000 & -3.11696500 & $\mathrm{H}$ & 1.20035100 & -3.16658600 & 3.26254600 \\
\hline \multirow[t]{2}{*}{$\mathrm{H}$} & 6.22285900 & -1.99180100 & -1.48755500 & $\mathrm{C}$ & 2.21267000 & -0.92129800 & 4.41203900 \\
\hline & & & & $\mathrm{H}$ & 1.77178100 & -1.66528100 & 5.08826600 \\
\hline \multirow[t]{7}{*}{85} & & & & $\mathrm{H}$ & 3.28301700 & -1.13664200 & 4.35224200 \\
\hline & & & & $\mathrm{H}$ & 2.07306300 & 0.05821900 & 4.87746300 \\
\hline & & & & $\mathrm{F}$ & 4.61860500 & -3.65967500 & -3.04447600 \\
\hline & & & & $\mathrm{Rh}$ & 1.37968100 & -0.02060700 & -0.53084100 \\
\hline & & & & $\mathrm{Cl}$ & -0.63163800 & 1.52482000 & 0.05715300 \\
\hline & & & & $\mathrm{H}$ & 1.19815900 & -2.64958000 & 1.57076000 \\
\hline & & & & $\mathrm{C}$ & 2.76334100 & 2.99510400 & -2.49826000 \\
\hline $\mathrm{C}$ & 5.07385800 & -2.10897100 & 0.19020100 & $\mathrm{O}$ & 3.85800200 & 2.47720500 & -2.55125600 \\
\hline $\mathrm{C}$ & 3.90760900 & -1.31676900 & 0.17536400 & $\mathrm{O}$ & 2.57309400 & 4.30947000 & -2.24299200 \\
\hline $\mathrm{C}$ & 2.94950000 & -1.27280400 & -0.84760800 & $\mathrm{C}$ & 3.77063000 & 5.08395300 & -2.06625000 \\
\hline $\mathrm{C}$ & 3.24204000 & -2.09924700 & -1.93236600 & $\mathrm{H}$ & 4.40309000 & 5.02255300 & -2.95603300 \\
\hline $\mathrm{C}$ & 4.41184300 & -2.88677200 & -1.94643100 & $\mathrm{H}$ & 3.43439500 & 6.10839500 & -1.90411400 \\
\hline $\mathrm{C}$ & 5.34517100 & -2.91998100 & -0.92897100 & $\mathrm{H}$ & 4.33663800 & 4.72480200 & -1.20276800 \\
\hline $\mathrm{C}$ & 5.70380000 & -1.86926100 & 96200 & $\mathrm{C}$ & 1.51493300 & 0.79742900 & -2.78706700 \\
\hline $\mathrm{C}$ & 4.92086700 & -0.98514000 & 2.15617200 & $\mathrm{H}$ & 2.44716300 & 0.39412400 & -3.17652400 \\
\hline $\mathrm{H}$ & 2.59329500 & -2.18102700 & -2.79277400 & $\mathrm{C}$ & 0.26487500 & -1.32400200 & -3.48072000 \\
\hline $\mathrm{H}$ & 6.22293200 & -3.55275400 & -1.00445200 & $\mathrm{H}$ & 1.18151400 & -1.60763200 & -4.00295100 \\
\hline $\mathrm{H}$ & 6.63462100 & -2.29276000 & 1.81709200 & $\mathrm{H}$ & -0.04163000 & -2.13278400 & -2.81228200 \\
\hline $\mathrm{H}$ & 5.07873000 & -0.56909900 & 3.13807200 & $\mathrm{H}$ & -0.52139200 & -1.21740700 & -4.24113500 \\
\hline $\mathrm{N}$ & 3.81949700 & -0.60778700 & 1.37850500 & $\mathrm{C}$ & 1.43536900 & 2.30759200 & -2.75103700 \\
\hline $\mathrm{P}$ & 2.25121300 & 0.08506700 & 1.67706100 & $\mathrm{H}$ & 1.09963200 & 2.65631100 & -3.74153300 \\
\hline $\mathrm{C}$ & 2.60228900 & 1.85201100 & 2.35274600 & $\mathrm{H}$ & 0.68626300 & 2.66517800 & -2.03991900 \\
\hline $\mathrm{C}$ & 1.50810200 & -1.04191400 & 3.04569400 & $\mathrm{C}$ & 0.39758800 & -0.00190500 & -2.76808800 \\
\hline $\mathrm{C}$ & 3.82914300 & 1.90060900 & 3.29007100 & $\mathrm{H}$ & -0.54618000 & 0.45994500 & -2.48531900 \\
\hline
\end{tabular}




\begin{tabular}{|c|c|c|c|c|c|c|c|}
\hline $\mathrm{H}$ & 2.49472100 & 1.01794900 & -0.69313700 & $\mathrm{C}$ & -5.32632200 & -4.32785700 & 0.35504000 \\
\hline $\mathrm{Cl}$ & -0.61858300 & -1.70164200 & -0.20761500 & $\mathrm{H}$ & -5.98603100 & -5.13732100 & 0.69382800 \\
\hline $\mathrm{Rh}$ & -2.52111100 & -0.11755100 & -0.02886400 & $\mathrm{H}$ & -5.98632400 & -3.46837900 & 0.17628000 \\
\hline $\mathrm{C}$ & -4.50953300 & 3.39553400 & 2.10942200 & & & & \\
\hline $\mathrm{C}$ & -3.50001700 & 2.26095500 & 1.81106600 & 86 & & & \\
\hline $\mathrm{C}$ & -4.54230300 & 3.72495200 & -1.49278300 & & & & \\
\hline $\mathrm{C}$ & -3.99917000 & 1.26742200 & 0.78046500 & & & & \\
\hline $\mathrm{C}$ & -3.39957100 & 2.70813400 & -1.26145300 & & & & \\
\hline $\mathrm{C}$ & -3.92769300 & 1.44858000 & -0.61357500 & & & & \\
\hline $\mathrm{H}$ & -5.49300000 & 2.96081600 & 2.34278400 & & & & \\
\hline $\mathrm{H}$ & -2.54636000 & 2.70049600 & 1.50800500 & & & & \\
\hline $\mathrm{H}$ & -5.19525800 & 3.36237900 & -2.29958500 & $\mathrm{C}$ & 4.72048900 & -2.55153500 & -0.07329100 \\
\hline $\mathrm{H}$ & -2.93436500 & 2.47830600 & -2.22878600 & $\mathrm{C}$ & 3.65739100 & -1.62416900 & 0.01155400 \\
\hline $\mathrm{H}$ & -4.17515500 & 3.89563700 & 3.02890900 & $\mathrm{C}$ & 2.59445100 & -1.52562500 & -0.90246600 \\
\hline $\mathrm{H}$ & -3.30408900 & 1.71839500 & 2.74558500 & $\mathrm{C}$ & 2.62338700 & -2.46683700 & -1.93093800 \\
\hline $\mathrm{H}$ & -4.08904300 & 4.65544800 & -1.86264400 & $\mathrm{C}$ & 3.68900000 & -3.38354200 & -2.03887400 \\
\hline $\mathrm{H}$ & -4.82532700 & 0.66026800 & 1.15068900 & $\mathrm{C}$ & 4.75033400 & -3.44949400 & -1.15805900 \\
\hline $\mathrm{H}$ & -2.62117500 & 3.16438900 & -0.64833300 & $\mathrm{C}$ & 5.52014400 & -2.35593400 & 1.10667400 \\
\hline $\mathrm{H}$ & -4.69052200 & 0.95959800 & -1.21771500 & $\mathrm{C}$ & 4.92093700 & -1.37853200 & 1.85614500 \\
\hline $\mathrm{C}$ & -4.68578700 & 4.47632400 & 1.02197500 & $\mathrm{H}$ & 1.82993600 & -2.53993200 & -2.66220100 \\
\hline $\mathrm{H}$ & -5.25115500 & 5.29670100 & 1.48291800 & $\mathrm{H}$ & 5.53710500 & -4.18369100 & -1.29535300 \\
\hline $\mathrm{H}$ & -3.70365000 & 4.90259100 & 0.77038500 & $\mathrm{H}$ & 6.43065300 & -2.87971400 & 1.36845600 \\
\hline $\mathrm{C}$ & -5.42439700 & 4.04434200 & -0.26948700 & $\mathrm{H}$ & 5.22505300 & -0.97163300 & 2.80744900 \\
\hline $\mathrm{H}$ & -6.10948100 & 4.84682900 & -0.57241700 & $\mathrm{~N}$ & 3.78809100 & -0.88962200 & 1.19854800 \\
\hline $\mathrm{H}$ & -6.06585300 & 3.18217800 & -0.04314500 & $\mathrm{P}$ & 2.31991300 & -0.08259900 & 1.70460300 \\
\hline $\mathrm{C}$ & -4.56454500 & -3.65605500 & -2.07100200 & $\mathrm{C}$ & 2.90271700 & 1.60778400 & 2.39524700 \\
\hline $\mathrm{C}$ & -3.55409000 & -2.51190000 & -1.81948500 & $\mathrm{C}$ & 1.64184600 & -1.20023800 & 3.11124400 \\
\hline $\mathrm{C}$ & -4.37292300 & -4.01082100 & 1.52414100 & $\mathrm{C}$ & 4.18883100 & 1.55407900 & 3.24583700 \\
\hline $\mathrm{C}$ & -4.01067000 & -1.53361600 & -0.75546500 & $\mathrm{H}$ & 5.04947500 & 1.23930500 & 2.64927400 \\
\hline $\mathrm{C}$ & -3.26152000 & -2.97296100 & 1.23626000 & $\mathrm{H}$ & 4.40308200 & 2.56545900 & 3.61633700 \\
\hline $\mathrm{C}$ & -3.85047000 & -1.72009300 & 0.63091900 & $\mathrm{H}$ & 4.10672800 & 0.90030500 & 4.11702400 \\
\hline $\mathrm{H}$ & -5.56474300 & -3.23196800 & -2.24645000 & $\mathrm{C}$ & 3.18921400 & 2.50565200 & 1.17483700 \\
\hline $\mathrm{H}$ & -2.57988200 & -2.93978500 & -1.57148200 & $\mathrm{H}$ & 3.58740900 & 3.46703600 & 1.52636700 \\
\hline $\mathrm{H}$ & -4.97943400 & -3.66872400 & 2.37507600 & $\mathrm{H}$ & 3.92988600 & 2.06865500 & 0.49891400 \\
\hline $\mathrm{H}$ & -2.74401200 & -2.73991200 & 2.17487400 & $\mathrm{H}$ & 2.27636000 & 2.70926200 & 0.60793000 \\
\hline $\mathrm{H}$ & -4.27535000 & -4.14575600 & -3.01101000 & $\mathrm{C}$ & 1.76110800 & 2.24815400 & 3.21238500 \\
\hline $\mathrm{H}$ & -3.41677400 & -1.95970900 & -2.75887600 & $\mathrm{H}$ & 0.81760600 & 2.26009300 & 2.65732500 \\
\hline $\mathrm{H}$ & -3.88373800 & -4.93867900 & 1.85226800 & $\mathrm{H}$ & 1.59900700 & 1.74374600 & 4.16930300 \\
\hline $\mathrm{H}$ & -4.87173700 & -0.94472600 & -1.07109500 & $\mathrm{H}$ & 2.02985400 & 3.28921200 & 3.43493200 \\
\hline $\mathrm{H}$ & -2.51280100 & -3.41090000 & 0.57496700 & $\mathrm{C}$ & 0.17149800 & -0.80995100 & 3.38053100 \\
\hline $\mathrm{H}$ & -4.58337700 & -1.24743800 & 1.28349900 & $\mathrm{H}$ & 0.07371200 & 0.18502100 & 3.82286900 \\
\hline $\mathrm{C}$ & -4.66742000 & -4.74610900 & -0.98331800 & $\mathrm{H}$ & -0.42794600 & -0.83959100 & 2.46752200 \\
\hline $\mathrm{H}$ & -5.25625500 & -5.56589700 & -1.41483700 & $\mathrm{H}$ & -0.25692000 & -1.52928800 & 4.09164400 \\
\hline $\mathrm{H}$ & -3.66957500 & -5.16881000 & -0.79623600 & $\mathrm{C}$ & 1.67581300 & -2.64443600 & 2.56100700 \\
\hline
\end{tabular}




\begin{tabular}{|c|c|c|c|c|c|c|c|}
\hline $\mathrm{H}$ & 2.69936500 & -3.00306200 & 2.41543100 & $\mathrm{H}$ & -4.83243100 & 0.64460600 & 1.22305700 \\
\hline $\mathrm{H}$ & 1.18979000 & -3.30537600 & 3.29091200 & $\mathrm{H}$ & -2.63650700 & 3.27004700 & -0.41186600 \\
\hline $\mathrm{C}$ & 2.43357600 & -1.16263700 & 4.43126400 & $\mathrm{H}$ & -4.70816800 & 1.10649800 & -1.11653200 \\
\hline $\mathrm{H}$ & 1.99945000 & -1.89762600 & 5.12241000 & $\mathrm{C}$ & -4.69580500 & 4.46361300 & 1.34913500 \\
\hline $\mathrm{H}$ & 3.48444000 & -1.43374900 & 4.29068200 & $\mathrm{H}$ & -5.25590800 & 5.25487200 & 1.86421600 \\
\hline $\mathrm{H}$ & 2.38534300 & -0.18805400 & 4.92502100 & $\mathrm{H}$ & -3.71284700 & 4.90090100 & 1.12131500 \\
\hline $\mathrm{F}$ & 3.65332700 & -4.25300300 & -3.08255200 & $\mathrm{C}$ & -5.44073200 & 4.11859400 & 0.03517100 \\
\hline $\mathrm{Rh}$ & 1.21521500 & -0.07955000 & -0.53941300 & $\mathrm{H}$ & -6.12760100 & 4.93893300 & -0.21040200 \\
\hline $\mathrm{Cl}$ & -0.64318900 & 1.59593900 & 0.18935300 & $\mathrm{H}$ & -6.08085900 & 3.24289200 & 0.20655300 \\
\hline $\mathrm{H}$ & 1.13629900 & -2.73655200 & 1.61514900 & $\mathrm{C}$ & -4.52759200 & -3.44395400 & -2.30863800 \\
\hline $\mathrm{C}$ & 3.07481200 & 3.50513700 & -2.28660600 & $\mathrm{C}$ & -3.53558400 & -2.30236100 & -1.97608700 \\
\hline $\mathrm{O}$ & 4.14235500 & 3.01027400 & -1.98938700 & $\mathrm{C}$ & -4.36218300 & -4.03983500 & 1.25633000 \\
\hline $\mathrm{O}$ & 2.89526700 & 4.82735400 & -2.48628500 & $\mathrm{C}$ & -4.00773900 & -1.40751300 & -0.84731100 \\
\hline $\mathrm{C}$ & 4.06766000 & 5.64329600 & -2.32268400 & $\mathrm{C}$ & -3.25880600 & -2.97603500 & 1.04509300 \\
\hline $\mathrm{H}$ & 4.84559400 & 5.34297300 & -3.02967900 & $\mathrm{C}$ & -3.85471900 & -1.68856800 & 0.52383700 \\
\hline $\mathrm{H}$ & 3.74225400 & 6.66480900 & -2.52082400 & $\mathrm{H}$ & -5.53303600 & -3.02382100 & -2.46176700 \\
\hline $\mathrm{H}$ & 4.45823600 & 5.55445200 & -1.30545400 & $\mathrm{H}$ & -2.55471800 & -2.73292400 & -1.76005500 \\
\hline $\mathrm{C}$ & 1.93476700 & 1.22891600 & -2.36713600 & $\mathrm{H}$ & -4.97924700 & -3.75725700 & 2.12124400 \\
\hline $\mathrm{H}$ & 2.77711100 & 0.84639600 & -2.95041000 & $\mathrm{H}$ & -2.74545400 & -2.80610700 & 1.99955400 \\
\hline $\mathrm{C}$ & 0.59168700 & -0.54460000 & -3.69504900 & $\mathrm{H}$ & -4.22491600 & -3.86400700 & -3.27747800 \\
\hline $\mathrm{H}$ & 1.55456700 & -0.97855700 & -3.97527400 & $\mathrm{H}$ & -3.40771400 & -1.68469000 & -2.87500300 \\
\hline $\mathrm{H}$ & -0.10827400 & -1.35724600 & -3.47128500 & $\mathrm{H}$ & -3.86661000 & -4.98179900 & 1.52989000 \\
\hline $\mathrm{H}$ & 0.20070900 & -0.01781900 & -4.57953800 & $\mathrm{H}$ & -4.87053800 & -0.80365800 & -1.12619300 \\
\hline $\mathrm{C}$ & 1.77830500 & 2.74637400 & -2.49251600 & $\mathrm{H}$ & -2.50661600 & -3.36619100 & 0.35741500 \\
\hline $\mathrm{H}$ & 1.39847700 & 2.99060200 & -3.49279100 & $\mathrm{H}$ & -4.59704000 & -1.26931000 & 1.20189500 \\
\hline $\mathrm{H}$ & 1.02482000 & 3.11535500 & -1.78708600 & $\mathrm{C}$ & -4.61894500 & -4.60569800 & -1.29726100 \\
\hline $\mathrm{C}$ & 0.70295700 & 0.42097200 & -2.53389700 & $\mathrm{H}$ & -5.18652300 & -5.40627600 & -1.78908200 \\
\hline $\mathrm{H}$ & -0.21582200 & 0.99768800 & -2.41035400 & $\mathrm{H}$ & -3.61473900 & -5.02042300 & -1.12778000 \\
\hline $\mathrm{H}$ & 2.39257900 & 1.09293500 & -1.26006600 & $\mathrm{C}$ & -5.29977900 & -4.29068900 & 0.05847300 \\
\hline $\mathrm{Cl}$ & -0.61673800 & -1.62551200 & -0.25882700 & $\mathrm{H}$ & -5.95008100 & -5.13106300 & 0.33386500 \\
\hline $\mathrm{Rh}$ & -2.53046100 & -0.03870600 & -0.01147300 & $\mathrm{H}$ & -5.97090200 & -3.43094100 & -0.06978100 \\
\hline $\mathrm{C}$ & -4.51984000 & 3.31436400 & 2.36320500 & & & & \\
\hline $\mathrm{C}$ & -3.51146900 & 2.19972300 & 1.99102600 & 87 & & & \\
\hline $\mathrm{C}$ & -4.56316400 & 3.88194200 & -1.21004100 & & & & \\
\hline $\mathrm{C}$ & -4.01003400 & 1.27973700 & 0.89461300 & & & & \\
\hline $\mathrm{C}$ & -3.41774500 & 2.85438900 & -1.04993200 & & & & \\
\hline $\mathrm{C}$ & -3.94261800 & 1.55460500 & -0.48462600 & & & & \\
\hline $\mathrm{H}$ & -5.50348900 & 2.86644200 & 2.56942400 & & & & \\
\hline $\mathrm{H}$ & -2.55527700 & 2.65490200 & 1.72147200 & & & & \\
\hline $\mathrm{H}$ & -5.21826500 & 3.57175300 & -2.03666100 & $\mathrm{C}$ & -3.20788800 & -1.63915300 & -2.27974500 \\
\hline $\mathrm{H}$ & -2.95640900 & 2.68799600 & -2.03169100 & $\mathrm{C}$ & -3.01125700 & -0.85158600 & -1.08459000 \\
\hline $\mathrm{H}$ & -4.18277700 & 3.75208100 & 3.31289200 & $\mathrm{C}$ & -2.64114500 & -1.48253700 & 0.16235800 \\
\hline $\mathrm{H}$ & -3.32087400 & 1.59527300 & 2.88777200 & $\mathrm{C}$ & -2.14928200 & -2.82101100 & 0.01590100 \\
\hline $\mathrm{H}$ & -4.11283200 & 4.83555700 & -1.51968500 & $\mathrm{C}$ & -2.15156600 & -3.46705100 & -1.19989000 \\
\hline
\end{tabular}




\begin{tabular}{|c|c|c|c|c|c|c|c|}
\hline C & -2.73846100 & -2.93872200 & -2.35897300 & $\mathrm{H}$ & -1.14006100 & -0.81999200 & 5.00000900 \\
\hline $\mathrm{C}$ & -4.08782300 & -0.88130500 & -3.13788800 & $\mathrm{C}$ & -4.48796700 & -2.01808200 & 1.81717800 \\
\hline $\mathrm{C}$ & -4.37391300 & 0.29304100 & -2.51613000 & $\mathrm{H}$ & -4.15985400 & -3.06098400 & 1.89434100 \\
\hline$\Pi$ & -1.81420300 & -3.35595100 & 0.89669600 & $\mathrm{C}$ & -6.48492800 & -2.49723100 & 3.33104700 \\
\hline $\mathrm{H}$ & -2.88322100 & -3.56527600 & -3.23267000 & $\mathrm{H}$ & -6.20334200 & -3.55157700 & 3.43921800 \\
\hline $\mathrm{H}$ & -4.48944400 & -1.21393900 & -4.08650400 & $\mathrm{H}$ & -7.01317200 & -2.19750300 & 4.24338000 \\
\hline $\mathrm{H}$ & -4.99030600 & 1.10908400 & -2.86752000 & $\mathrm{H}$ & -7.19343100 & -2.42736900 & 2.49628200 \\
\hline $\mathrm{N}$ & -3.74036100 & 0.39468900 & -1.26586700 & $\mathrm{C}$ & -3.25191600 & -1.12747300 & 1.52697200 \\
\hline $\mathrm{P}$ & -2.57661300 & 1.67301400 & -0.84135100 & $\mathrm{H}$ & -5.16041900 & -1.95727100 & 0.95092500 \\
\hline C & -3.64534700 & 2.93481800 & 0.14780500 & $\mathrm{H}$ & -3.56177000 & -0.08996000 & 1.50831400 \\
\hline $\mathrm{C}$ & -1.91721100 & 2.52782700 & -2.45829300 & $\mathrm{C}$ & -5.25163200 & -1.62026300 & 3.08772000 \\
\hline $\mathrm{C}$ & -5.01115400 & 3.18051800 & -0.52573000 & $\mathrm{H}$ & -5.55821600 & -0.56670500 & 3.01851100 \\
\hline $\mathrm{H}$ & -5.57212900 & 2.24662700 & -0.63246800 & $\mathrm{H}$ & -4.58197600 & -1.69110800 & 3.95531400 \\
\hline $\mathrm{H}$ & -5.60506400 & 3.84851100 & 0.11231400 & $\mathrm{Cl}$ & 0.75232900 & -1.58292500 & -0.17896000 \\
\hline $\mathrm{H}$ & -4.93094100 & 3.65390900 & -1.50620900 & $\mathrm{Rh}$ & 2.65662900 & -0.04739000 & 0.13419800 \\
\hline $\mathrm{C}$ & -3.91404500 & 2.35918700 & 1.55257600 & $\mathrm{C}$ & 4.66502100 & -4.07803400 & 4400 \\
\hline $\mathrm{H}$ & -4.38999800 & 3.13642300 & 2.16491100 & $\mathrm{C}$ & 3.67436700 & -2.90122300 & 1.00080400 \\
\hline $\mathrm{H}$ & -4.61048900 & 1.51767600 & 1.50982200 & $\mathrm{C}$ & 4.46958200 & -3.17006800 & -2.66601900 \\
\hline $\mathrm{H}$ & -3.00066600 & 2.03538100 & 2.05889500 & $\mathrm{C}$ & 4.14166300 & -1.62367000 & 0.33174100 \\
\hline $\mathrm{C}$ & -2.88636600 & 4.26531000 & 0.32703800 & $\mathrm{C}$ & 3.37323400 & -2.28015600 & -2.03227900 \\
\hline $\mathrm{H}$ & -1.89065800 & 4.11492100 & 0.75855500 & $\mathrm{C}$ & 3.98097400 & -1.32097600 & -1.03492400 \\
\hline $\mathrm{H}$ & -2.78144900 & 4.82324700 & -0.60630000 & $\mathrm{H}$ & 5.67336500 & -3.75789500 & 1.13671300 \\
\hline $\mathrm{H}$ & -3.45290400 & 4.90010200 & 1.02006000 & $\mathrm{H}$ & 2.69119200 & -3.20556900 & 0.63380100 \\
\hline C & -0.70448700 & 3.40970100 & -2.07372200 & $\mathrm{H}$ & 5.08199900 & -2.56358200 & -3.34881100 \\
\hline $\mathrm{H}$ & -0.96889800 & 4.27179400 & -1.45919300 & $\mathrm{H}$ & 2.85667400 & -1.73061100 & -2.82895900 \\
\hline $\mathrm{H}$ & 0.06260900 & 2.83672600 & -1.54899900 & $\mathrm{H}$ & 4.36741400 & -4.85707500 & 1.54852000 \\
\hline $\mathrm{H}$ & -0.26235400 & 3.79468600 & -3.00210200 & $\mathrm{H}$ & 3.54922000 & -2.70193300 & 2.07327800 \\
\hline $\mathrm{C}$ & -1.37803200 & 1.41812900 & -3.39023000 & $\mathrm{H}$ & 3.96766800 & -3.92125800 & -3.29177600 \\
\hline $\mathrm{H}$ & -2.17089400 & 0.78659700 & -3.79385900 & $\mathrm{H}$ & 5.00554700 & -1.18150600 & 0.82868900 \\
\hline $\mathrm{H}$ & -0.86601000 & 1.89791600 & -4.23525300 & $\mathrm{H}$ & 2.61928800 & -2.90907900 & -1.55720200 \\
\hline $\mathrm{C}$ & -2.93021400 & 3.38925900 & -3.23614100 & $\mathrm{H}$ & 4.72091200 & -0.66107500 & -1.48609800 \\
\hline $\mathrm{H}$ & -2.44489800 & 3.75223500 & -4.15174100 & $\mathrm{C}$ & 4.74489500 & -4.72553600 & -0.56512700 \\
\hline $\mathrm{H}$ & -3.81678000 & 2.82853300 & -3.54497000 & $\mathrm{H}$ & 5.31286900 & -5.65783000 & -0.44934300 \\
\hline $\mathrm{H}$ & -3.25442200 & 4.27042200 & -2.67591400 & $\mathrm{H}$ & 3.73732600 & -5.03287300 & -0.88034100 \\
\hline $\mathrm{F}$ & -1.67148100 & -4.72858500 & -1.26183400 & $\mathrm{C}$ & 5.41550200 & -3.88290600 & -1.67901700 \\
\hline $\mathrm{Rh}$ & -1.06411400 & 0.09248800 & -0.22612200 & $\mathrm{H}$ & 6.06630400 & -4.53527100 & -2.27592700 \\
\hline $\mathrm{Cl}$ & 0.72042400 & 1.47274000 & 0.63497100 & $\mathrm{H}$ & 6.08487500 & -3.14572900 & -1.21577700 \\
\hline $\mathrm{H}$ & -0.65777500 & 0.77738800 & -2.87144300 & $\mathrm{C}$ & 4.57640700 & 4.00004500 & -0.70962000 \\
\hline $\mathrm{C}$ & -2.22837700 & -1.28143800 & 2.64898300 & $\mathrm{C}$ & 3.59546400 & 2.80771400 & -0.81053400 \\
\hline $\mathrm{O}$ & -1.78525100 & -2.34125400 & 3.04370600 & $\mathrm{C}$ & 4.61045500 & 3.10840000 & 2.79650600 \\
\hline $\mathrm{O}$ & -1.89247100 & -0.09201400 & 3.19036400 & $\mathrm{C}$ & 4.11837100 & 1.53661600 & -0.16977300 \\
\hline $\mathrm{C}$ & -0.86287900 & -0.13556300 & 4.19431000 & $\mathrm{C}$ & 3.49162000 & 2.19945900 & 2.23341800 \\
\hline $\mathrm{H}$ & 0.07909500 & -0.45985800 & 3.74536200 & $\mathrm{C}$ & 4.04649900 & 1.24063600 & 1.20492700 \\
\hline $\mathrm{H}$ & -0.77553900 & 0.88579800 & 4.56525800 & $\mathrm{H}$ & 5.56785500 & 3.69128700 & -1.07335700 \\
\hline
\end{tabular}




\begin{tabular}{|c|c|c|c|c|c|c|c|}
\hline $\mathrm{H}$ & 2.63333300 & 3.09792500 & -0.38043100 & $\mathrm{H}$ & -3.48259500 & 1.17375700 & 2.51592300 \\
\hline $\mathrm{H}$ & 5.27138100 & 2.51294600 & 3.44229800 & $\mathrm{H}$ & -1.98433600 & 2.00463600 & 2.04737800 \\
\hline $\mathrm{H}$ & 3.03257000 & 1.64873400 & 3.06401100 & $\mathrm{C}$ & -3.19535900 & 3.81459500 & 0.29688000 \\
\hline $\mathrm{H}$ & 4.22551400 & 4.77172200 & -1.40885600 & $\mathrm{H}$ & -2.11985200 & 3.80180800 & 0.08977400 \\
\hline $\mathrm{H}$ & 3.40665300 & 2.60466500 & -1.87325800 & $\mathrm{H}$ & -3.71704800 & 4.20011000 & -0.58270900 \\
\hline $\mathrm{H}$ & 4.13727000 & 3.85498300 & 3.44995300 & $\mathrm{H}$ & -3.36621900 & 4.52783300 & 1.11320700 \\
\hline $\mathrm{H}$ & 4.95397400 & 1.10290700 & -0.71913000 & $\mathrm{C}$ & -2.68088500 & 2.62407100 & -2.82859100 \\
\hline $\mathrm{H}$ & 2.70273400 & 2.81909600 & 1.80292200 & $\mathrm{H}$ & -2.67788200 & 3.56281500 & -2.27191700 \\
\hline $\mathrm{H}$ & 4.81735000 & 0.58881700 & 1.61386800 & $\mathrm{H}$ & -1.66901100 & 2.21476300 & -2.81600700 \\
\hline $\mathrm{C}$ & 4.73527000 & 4.65742500 & 0.67750100 & $\mathrm{H}$ & -2.93505100 & 2.86139500 & -3.87016600 \\
\hline $\mathrm{H}$ & 5.28287500 & 5.59586800 & 0.52034800 & $\mathrm{C}$ & -3.60784100 & 0.35671500 & -3.22029200 \\
\hline $\mathrm{H}$ & 3.74557700 & 4.95499200 & 1.05418100 & $\mathrm{H}$ & -4.36089700 & -0.39972600 & -2.99295500 \\
\hline $\mathrm{C}$ & 5.48469200 & 3.83157300 & 1.75281200 & $\mathrm{H}$ & -3.75698500 & 0.67879100 & -4.25973100 \\
\hline $\mathrm{H}$ & 6.15807000 & 4.49796000 & 2.30754400 & $\mathrm{C}$ & -5.12666000 & 2.21151400 & -2.45728400 \\
\hline \multirow[t]{2}{*}{$\mathrm{H}$} & 6.13784300 & 3.10396400 & 1.25334600 & $\mathrm{H}$ & -5.31357000 & 2.39122500 & -3.52421600 \\
\hline & & & & $\mathrm{H}$ & -5.92137900 & 1.55031900 & -2.10078400 \\
\hline \multirow[t]{6}{*}{88} & & & & $\mathrm{H}$ & -5.22188200 & 3.17560000 & -1.95053900 \\
\hline & & & & $\mathrm{F}$ & -1.52841100 & -5.17254000 & -0.64489600 \\
\hline & & & & $\mathrm{Rh}$ & -1.28621800 & -0.14059300 & -0.67337100 \\
\hline & & & & $\mathrm{Cl}$ & 0.28497800 & 1.61659400 & -1.13754200 \\
\hline & & & & $\mathrm{H}$ & -2.61825200 & -0.10548000 & -3.14495300 \\
\hline & & & & $\mathrm{C}$ & 0.18930100 & -0.34812800 & 3.47167400 \\
\hline $\mathrm{C}$ & -3.92342700 & -2.49452800 & -0.84672800 & $\mathrm{O}$ & -0.18820400 & 0.80544300 & 3.53023700 \\
\hline $\mathrm{C}$ & -3.22496500 & -1.47604100 & -0.10383100 & $\mathrm{O}$ & 1.18766200 & -0.83203000 & 4.24776900 \\
\hline $\mathrm{C}$ & -2.08148800 & -1.79716600 & 0.71367900 & $\mathrm{C}$ & 1.79167300 & 0.11613200 & 5.14255800 \\
\hline $\mathrm{C}$ & -1.53058600 & -3.08793000 & 0.43094700 & $\mathrm{H}$ & 1.04214300 & 0.54084800 & 5.81550800 \\
\hline $\mathrm{C}$ & -2.12068400 & -3.97368400 & -0.44671600 & $\mathrm{H}$ & 2.53765900 & -0.44680900 & 5.70445300 \\
\hline $\mathrm{C}$ & -3.35851900 & -3.74433700 & -1.05339000 & $\mathrm{H}$ & 2.26571300 & 0.92701600 & 4.58253200 \\
\hline $\mathrm{C}$ & -5.25031000 & -1.98933400 & -1.10554300 & $\mathrm{C}$ & -1.79832200 & -1.22791100 & 2.11526200 \\
\hline $\mathrm{C}$ & -5.32414000 & -0.73231000 & -0.59422600 & $\mathrm{H}$ & -1.99007100 & -0.15966100 & 2.11267300 \\
\hline $\mathrm{H}$ & -0.64799600 & -3.42497000 & 0.96045900 & $\mathrm{C}$ & -2.62877600 & -1.40569200 & 4.57611900 \\
\hline $\mathrm{H}$ & -3.86201600 & -4.53702900 & -1.59622700 & $\mathrm{H}$ & -2.74408500 & -0.31833800 & 4.64626000 \\
\hline $\mathrm{H}$ & -6.05365400 & -2.53184100 & -1.58697800 & $\mathrm{H}$ & -3.39459400 & -1.86840300 & 5.20945000 \\
\hline $\mathrm{H}$ & -6.15360700 & -0.03968200 & -0.62008700 & $\mathrm{H}$ & -1.65414500 & -1.66535100 & 5.00576600 \\
\hline $\mathrm{N}$ & -4.11929400 & -0.34076900 & 0.01141200 & $\mathrm{C}$ & -0.31819400 & -1.40760400 & 2.51756500 \\
\hline $\mathrm{P}$ & -3.18076800 & 1.07883800 & -0.51578500 & $\mathrm{H}$ & -0.12001100 & -2.39341200 & 2.95054200 \\
\hline $\mathrm{C}$ & -3.71272900 & 2.43092000 & 0.73799300 & $\mathrm{H}$ & 0.31563000 & -1.31935900 & 1.62533400 \\
\hline $\mathrm{C}$ & -3.71973100 & 1.60082400 & -2.30917100 & $\mathrm{C}$ & -2.76536000 & -1.89635100 & 3.12917500 \\
\hline $\mathrm{C}$ & -5.24225300 & 2.46742400 & 0.93932600 & $\mathrm{H}$ & -2.61334200 & -2.98418900 & 3.09457700 \\
\hline $\mathrm{H}$ & -5.62051400 & 1.49876700 & 1.28149600 & $\mathrm{H}$ & -3.79270500 & -1.71923900 & 2.78515300 \\
\hline $\mathrm{H}$ & -5.47487300 & 3.20233400 & 1.72139000 & $\mathrm{Cl}$ & 0.67948900 & -1.51658900 & -1.30968700 \\
\hline $\mathrm{H}$ & -5.79190800 & 2.76285900 & 0.04336100 & $\mathrm{Rh}$ & 2.32123600 & 0.24901600 & -0.71748200 \\
\hline $\mathrm{C}$ & -3.07204700 & 2.09714800 & 2.09832300 & $\mathrm{C}$ & 4.48339600 & -3.39963900 & 0.98057000 \\
\hline $\mathrm{H}$ & -3.30931600 & 2.90458300 & 2.80390000 & $\mathrm{C}$ & 3.37611500 & -2.33831600 & 0.77384100 \\
\hline
\end{tabular}




\begin{tabular}{|c|c|c|c|c|c|c|c|}
\hline $\mathrm{C}$ & 5.00486500 & -2.93868400 & -2.56921500 & & & & \\
\hline $\mathrm{C}$ & 3.85430300 & -1.09696900 & 0.04619300 & & & & \\
\hline $\mathrm{C}$ & 3.71716100 & -2.12209200 & -2.30880200 & & & & \\
\hline $\mathrm{C}$ & 3.99141100 & -0.98621200 & -1.35138700 & & & & \\
\hline $\mathrm{H}$ & 5.36462900 & -2.92442500 & 1.43756800 & & & & \\
\hline $\mathrm{H}$ & 2.52965200 & -2.79401600 & 0.25274200 & & & & \\
\hline $\mathrm{H}$ & 5.69791400 & -2.34271900 & -3.18019900 & $\mathrm{C}$ & -5.25427700 & 1.32787700 & 0.68302800 \\
\hline $\mathrm{H}$ & 3.34172700 & -1.73714000 & -3.26474000 & $\mathrm{C}$ & -4.09671600 & 0.65090300 & 0.23739500 \\
\hline $\mathrm{H}$ & 4.10991100 & -4.11866900 & 1.72287800 & $\mathrm{C}$ & -2.85026800 & 1.25079300 & -0.01393700 \\
\hline $\mathrm{H}$ & 3.00388200 & -2.03742400 & 1.76244700 & $\mathrm{C}$ & -2.80575700 & 2.62062300 & 0.24466300 \\
\hline $\mathrm{H}$ & 4.72814100 & -3.80802700 & -3.18202300 & $\mathrm{C}$ & -3.95919500 & 3.31317400 & 0.66684600 \\
\hline $\mathrm{H}$ & 4.53445200 & -0.49118600 & 0.64608300 & $\mathrm{C}$ & -5.18831700 & 2.72102600 & 0.88002600 \\
\hline $\mathrm{H}$ & 2.94116200 & -2.78205600 & -1.91792600 & $\mathrm{C}$ & -6.25642300 & 0.31692000 & 0.89314700 \\
\hline $\mathrm{H}$ & 4.74527500 & -0.29369300 & -1.72593200 & $\mathrm{C}$ & -5.68794000 & -0.89647400 & 0.60886800 \\
\hline $\mathrm{C}$ & 4.93469600 & -4.19674500 & -0.26139700 & $\mathrm{H}$ & -1.89168900 & 3.19251400 & 0.15757800 \\
\hline $\mathrm{H}$ & 5.54340900 & -5.03295500 & 0.10666700 & $\mathrm{H}$ & -6.03517500 & 3.31151200 & 1.21323600 \\
\hline $\mathrm{H}$ & 4.05702700 & -4.65955300 & -0.73558900 & $\mathrm{H}$ & -7.27668700 & 0.47179500 & 1.22019700 \\
\hline $\mathrm{C}$ & 5.76337900 & -3.42304000 & -1.31746300 & $\mathrm{H}$ & -6.12759700 & -1.88015200 & 0.66248300 \\
\hline $\mathrm{H}$ & 6.58003900 & -4.06715000 & -1.66905300 & $\mathrm{~N}$ & -4.36945700 & -0.72342100 & 0.17715100 \\
\hline $\mathrm{H}$ & 6.25375600 & -2.56909200 & -0.83109400 & $\mathrm{P}$ & -2.94307000 & -1.73337900 & 0.11352400 \\
\hline $\mathrm{C}$ & 4.19014000 & 4.36128000 & -1.38342100 & $\mathrm{C}$ & -3.37277000 & -3.09992200 & -1.15965300 \\
\hline $\mathrm{C}$ & 3.36225600 & 3.08273800 & -1.65756900 & $\mathrm{C}$ & -2.79351900 & -2.45585600 & 1.88410100 \\
\hline $\mathrm{C}$ & 3.11292500 & 3.80834400 & 2.02509000 & $\mathrm{C}$ & -4.80 & -3.65747600 & -1.04745300 \\
\hline $\mathrm{C}$ & 3.70846100 & 1.92789000 & -0.73758900 & $\mathrm{H}$ & -5.55337500 & -2.88658200 & -1.25833900 \\
\hline $\mathrm{C}$ & 2.29400700 & 2.75332200 & 1.24436100 & $\mathrm{H}$ & -4.93342600 & -4.44884400 & -1.79809900 \\
\hline $\mathrm{C}$ & 3.21225400 & 1.76005400 & 0.56959200 & $\mathrm{H}$ & -5.02460600 & -4.09869100 & -0.07218400 \\
\hline $\mathrm{H}$ & 5.26085500 & 4.10794100 & -1.36539800 & $\mathrm{C}$ & -3.22785900 & -2.46732900 & -2.56063600 \\
\hline $\mathrm{H}$ & 2.29805800 & 3.32816600 & -1.60333100 & $\mathrm{H}$ & -3.52631000 & -3.20468700 & -3.31736900 \\
\hline $\mathrm{H}$ & 3.55786600 & 3.33947900 & 2.91515100 & $\mathrm{H}$ & -3.87422400 & -1.59017600 & -2.68059600 \\
\hline $\mathrm{H}$ & 1.61517100 & 2.24732300 & 1.94125100 & $\mathrm{H}$ & -2.19358300 & -2.17694600 & -2.76422300 \\
\hline $\mathrm{H}$ & 4.05299500 & 5.02955000 & -2.24483600 & $\mathrm{C}$ & -2.35251600 & -4.25174300 & -1.03994300 \\
\hline $\mathrm{H}$ & 3.54827800 & 2.76535900 & -2.69210400 & $\mathrm{H}$ & -1.31988700 & -3.88936700 & -1.06304000 \\
\hline $\mathrm{H}$ & 2.40886800 & 4.56422600 & 2.40040600 & $\mathrm{H}$ & -2.49737400 & -4.84108000 & -0.12988300 \\
\hline $\mathrm{H}$ & 4.69732200 & 1.51309100 & -0.93791300 & $\mathrm{H}$ & -2.48706300 & -4.93179100 & -1.89140700 \\
\hline $\mathrm{H}$ & 1.66030900 & 3.25323700 & 0.51059700 & $\mathrm{C}$ & -1.37422000 & -3.04269100 & 2.05210000 \\
\hline $\mathrm{H}$ & 3.84143800 & 1.22126200 & 1.27744100 & $\mathrm{H}$ & -1.21095300 & -3.93107700 & 1.43658600 \\
\hline $\mathrm{C}$ & 3.84345700 & 5.15835900 & -0.10837700 & $\mathrm{H}$ & -0.60157000 & -2.31007200 & 1.80723300 \\
\hline $\mathrm{H}$ & 4.35900800 & 6.12414700 & -0.19091400 & $\mathrm{H}$ & -1.24111500 & -3.33955100 & 3.10109500 \\
\hline $\mathrm{H}$ & 2.77005000 & 5.39811300 & -0.11045800 & $\mathrm{C}$ & -2.94686300 & -1.25516100 & 2.84581100 \\
\hline $\mathrm{C}$ & 4.24005600 & 4.51092900 & 1.24218300 & $\mathrm{H}$ & -3.95386000 & -0.82815100 & 2.81302800 \\
\hline $\mathrm{H}$ & 4.65088500 & 5.28702300 & 1.90126600 & $\mathrm{H}$ & -2.76494900 & -1.60695600 & 3.86981500 \\
\hline \multirow[t]{2}{*}{$\mathrm{H}$} & 5.06396500 & 3.80521900 & 1.07133900 & $\mathrm{C}$ & -3.83991400 & -3.52153600 & 2.26008400 \\
\hline & & & & $\mathrm{H}$ & -3.69458800 & -3.79866700 & 3.31281100 \\
\hline V & & & & $\mathrm{H}$ & -4.86439700 & -3.14849900 & 2.16441800 \\
\hline
\end{tabular}




\begin{tabular}{|c|c|c|c|c|c|c|c|}
\hline $\mathrm{H}$ & -3.74270800 & -4.43756000 & 1.67007000 & $\mathrm{H}$ & 2.64962200 & 3.30071700 & 3.76271300 \\
\hline $\mathrm{F}$ & -3.83524200 & 4.64983700 & 0.87569700 & $\mathrm{C}$ & 4.39472500 & 2.02410600 & 4.08106000 \\
\hline $\mathrm{Rh}$ & -1.36475700 & 0.01805800 & -0.65351300 & $\mathrm{H}$ & 4.93460700 & 2.35175400 & 4.97914200 \\
\hline $\mathrm{Cl}$ & 0.50930000 & -1.68914100 & -1.19193700 & $\mathrm{H}$ & 5.16783400 & 1.65152500 & 3.39552800 \\
\hline $\mathrm{H}$ & -2.22432200 & -0.46365100 & 2.63227800 & $\mathrm{C}$ & 4.11283200 & -4.68504500 & -0.43207000 \\
\hline $\mathrm{C}$ & 1.30691400 & 4.83363900 & -2.24314500 & $\mathrm{C}$ & 3.03981000 & -3.64882600 & -0.01755000 \\
\hline $\mathrm{O}$ & 2.08325500 & 5.07692100 & -1.34113800 & $\mathrm{C}$ & 4.93617300 & -2.18785800 & -2.91571300 \\
\hline $\mathrm{O}$ & 0.66041300 & 5.79015100 & -2.94481300 & $\mathrm{C}$ & 3.61165600 & -2.26293500 & 0.20597400 \\
\hline $\mathrm{C}$ & 0.93644200 & 7.14192600 & -2.54245200 & $\mathrm{C}$ & 3.65123700 & -1.60833300 & -2.27850600 \\
\hline $\mathrm{H}$ & 1.99918800 & 7.36883000 & -2.66330200 & $\mathrm{C}$ & 3.85966100 & -1.31803700 & -0.80982400 \\
\hline $\mathrm{H}$ & 0.33264200 & 7.77130200 & -3.19686100 & $\mathrm{H}$ & 4.95663900 & -4.64418600 & 0.27285100 \\
\hline $\mathrm{H}$ & 0.65791900 & 7.29512000 & -1.49643700 & $\mathrm{H}$ & 2.24556600 & -3.63632700 & -0.76722200 \\
\hline $\mathrm{C}$ & -0.10524700 & 2.82513800 & -1.67946000 & $\mathrm{H}$ & 5.69018800 & -1.39224600 & -3.00021000 \\
\hline $\mathrm{H}$ & -1.04883300 & 3.37482300 & -1.74767700 & $\mathrm{H}$ & 3.37589000 & -0.69158700 & -2.81556500 \\
\hline $\mathrm{C}$ & -1.39426500 & 0.87024300 & -2.82312200 & $\mathrm{H}$ & 3.66578700 & -5.68121000 & -0.31002800 \\
\hline $\mathrm{H}$ & -2.11237500 & 1.63614500 & -3.12871000 & $\mathrm{H}$ & 2.57566400 & -3.98639600 & 0.91852500 \\
\hline $\mathrm{H}$ & -1.08253800 & 0.23193800 & -3.65498800 & $\mathrm{H}$ & 4.69249100 & -2.48719700 & -3.94489300 \\
\hline $\mathrm{H}$ & -2.13560900 & 0.09519200 & -2.26531900 & $\mathrm{H}$ & 4.24571400 & -2.21455600 & 1.08944000 \\
\hline $\mathrm{C}$ & 0.90751300 & 3.44153100 & -2.68222700 & $\mathrm{H}$ & 2.82296000 & -2.30441800 & -2.42097900 \\
\hline $\mathrm{H}$ & 1.81780500 & 2.83257300 & -2.69806100 & $\mathrm{H}$ & 4.67077700 & -0.61265100 & -0.63193700 \\
\hline $\mathrm{H}$ & 0.48474600 & 3.46610000 & -3.69121700 & $\mathrm{C}$ & 4.65769200 & -4.58505600 & -1.87233500 \\
\hline $\mathrm{C}$ & -0.31132000 & 1.34755000 & -1.93846300 & $\mathrm{H}$ & 5.22247400 & -5.50772000 & -2.05907000 \\
\hline $\mathrm{H}$ & 0.63857600 & 0.83441000 & -2.09145100 & $\mathrm{H}$ & 3.81700000 & -4.60727500 & -2.58123200 \\
\hline $\mathrm{H}$ & 0.29283800 & 2.96795500 & -0.66864800 & $\mathrm{C}$ & 5.58091600 & -3.38059100 & -2.18211800 \\
\hline $\mathrm{Cl}$ & 0.06178000 & 0.40792000 & 1.25848300 & $\mathrm{H}$ & 6.41493700 & -3.72474500 & -2.80750200 \\
\hline $\mathrm{Rh}$ & 2.16769700 & -0.65285600 & 0.39194200 & $\mathrm{H}$ & 6.04114700 & -3.03273700 & -1.24758300 \\
\hline $\mathrm{C}$ & 3.76245600 & 3.34681600 & 1.89973800 & & & & \\
\hline $\mathrm{C}$ & 2.90518800 & 2.33391200 & 1.10300000 & 90 & & & \\
\hline $\mathrm{C}$ & 3.48305300 & 0.85802900 & 4.51117100 & & & & \\
\hline $\mathrm{C}$ & 3.47835000 & 0.93119000 & 1.12281400 & & & & \\
\hline $\mathrm{C}$ & 2.52929100 & 0.30086800 & 3.42663700 & & & & \\
\hline $\mathrm{C}$ & 3.28348900 & -0.00924200 & 2.15335200 & & & & \\
\hline $\mathrm{H}$ & 4.81286800 & 3.27361200 & 1.57993600 & & & & \\
\hline $\mathrm{H}$ & 1.88367800 & 2.35413800 & 1.48872700 & & & & \\
\hline $\mathrm{H}$ & 4.12194800 & 0.04437200 & 4.88359300 & $\mathrm{C}$ & -5.50727200 & 1.02490600 & 0.52598800 \\
\hline $\mathrm{H}$ & 2.04341500 & -0.60309100 & 3.81527700 & $\mathrm{C}$ & -4.29276000 & 0.42975100 & 0.12906500 \\
\hline $\mathrm{H}$ & 3.42490100 & 4.34789700 & 1.60256600 & $\mathrm{C}$ & -3.17886000 & 1.09247400 & -0.40447400 \\
\hline $\mathrm{H}$ & 2.85170800 & 2.68582200 & 0.06580800 & $\mathrm{C}$ & -3.35544600 & 2.46822900 & -0.55460500 \\
\hline $\mathrm{H}$ & 2.86713000 & 1.17651800 & 5.36390500 & $\mathrm{C}$ & -4.56876200 & 3.08345400 & -0.18098900 \\
\hline $\mathrm{H}$ & 4.40591000 & 0.85559600 & 0.55705000 & $\mathrm{C}$ & -5.65556600 & 2.41499800 & 0.34916500 \\
\hline $\mathrm{H}$ & 1.73088800 & 1.02206600 & 3.24038500 & $\mathrm{C}$ & -6.31329600 & -0.03781600 & 1.06775700 \\
\hline $\mathrm{H}$ & 4.07798100 & -0.74002500 & 2.30515400 & $\mathrm{C}$ & -5.58290600 & -1.19519500 & 0.99594300 \\
\hline $\mathrm{C}$ & 3.69904700 & 3.24980700 & 3.43778400 & $\mathrm{H}$ & -2.58094700 & 3.11423400 & -0.94510600 \\
\hline $\mathrm{H}$ & 4.17171500 & 4.15906100 & 3.83144500 & $\mathrm{H}$ & -6.55713600 & 2.95458300 & 0.61867800 \\
\hline
\end{tabular}




\begin{tabular}{|c|c|c|c|c|c|c|c|}
\hline $\mathrm{H}$ & -7.32022500 & 0.04235300 & 1.45700900 & $\mathrm{H}$ & -1.05345800 & 0.12817900 & -3.49393300 \\
\hline $\mathrm{H}$ & -5.86145800 & -2.19143000 & 1.30050500 & $\mathrm{H}$ & -2.53036500 & -0.58462700 & -1.93803800 \\
\hline $\mathrm{N}$ & -4.34255900 & -0.94149700 & 0.39895900 & $\mathrm{C}$ & 1.20862700 & 3.61017100 & -2.28509100 \\
\hline $\mathrm{P}$ & -2.79346900 & -1.73385800 & 0.32009900 & $\mathrm{H}$ & 2.08563400 & 2.98607100 & -2.09493800 \\
\hline $\mathrm{C}$ & -3.11150300 & -3.36719200 & -0.64658900 & $\mathrm{H}$ & 1.08188300 & 3.71023000 & -3.36952800 \\
\hline $\mathrm{C}$ & -2.36137300 & -2.08659500 & 2.15917600 & $\mathrm{C}$ & -0.18314700 & 1.54232000 & -2.15617600 \\
\hline $\mathrm{C}$ & -4.45266400 & -4.02959300 & -0.26207800 & $\mathrm{H}$ & 0.72257800 & 0.94861100 & -2.06475400 \\
\hline $\mathrm{H}$ & -5.30437500 & -3.41053200 & -0.55597200 & $\mathrm{H}$ & 0.03277300 & 2.97385200 & -0.57852900 \\
\hline $\mathrm{H}$ & -4.53338300 & -4.97836200 & -0.80772900 & $\mathrm{Cl}$ & 0.14071900 & 0.72515600 & 0.95673300 \\
\hline $\mathrm{H}$ & -4.53586100 & -4.26027500 & 0.80174300 & $\mathrm{Rh}$ & 2.15272900 & -0.55489800 & 0.24613600 \\
\hline $\mathrm{C}$ & -3.17585700 & -3.05187200 & -2.15621100 & $\mathrm{C}$ & 4.09925700 & 3.20538200 & 1.98456700 \\
\hline $\mathrm{H}$ & -3.44164800 & -3.97467000 & -2.68853900 & $\mathrm{C}$ & 3.20523400 & 2.31675400 & 1.08719400 \\
\hline $\mathrm{H}$ & -3.94073400 & -2.30307100 & -2.38736700 & $\mathrm{C}$ & 3.27951500 & 0.73497900 & 4.49548000 \\
\hline $\mathrm{H}$ & -2.21465700 & -2.70272300 & -2.54057900 & $\mathrm{C}$ & 3.58322300 & 0.85026800 & 1.12264200 \\
\hline $\mathrm{C}$ & -1.95302900 & -4.35910800 & -0.40844400 & $\mathrm{C}$ & 2.35628000 & 0.33741400 & 3.31964200 \\
\hline $\mathrm{H}$ & -0.97777600 & -3.90662900 & -0.60977400 & $\mathrm{C}$ & 3.17537600 & -0.06156700 & 2.11401700 \\
\hline $\mathrm{H}$ & -1.95250600 & -4.76374600 & 0.60725800 & $\mathrm{H}$ & 5.15799100 & 3.01287100 & 1.75380400 \\
\hline $\mathrm{H}$ & -2.07764700 & -5.20648300 & -1.09468800 & $\mathrm{H}$ & 2.15937500 & 2.46120500 & 1.36852200 \\
\hline $\mathrm{C}$ & -0.88370400 & -2.51312600 & 2.28295000 & $\mathrm{H}$ & 3.77270500 & -0.16422800 & 4.89206700 \\
\hline $\mathrm{H}$ & -0.68728700 & -3.48880100 & 1.83202400 & $\mathrm{H}$ & 1.71309000 & -0.49144700 & 3.64005300 \\
\hline $\mathrm{H}$ & -0.21002400 & -1.78240400 & 1.83436400 & $\mathrm{H}$ & 3.90809500 & 4.24451600 & 1.68747800 \\
\hline $\mathrm{H}$ & -0.63813900 & -2.58883000 & 3.35067600 & $\mathrm{H}$ & 3.29822300 & 2.68366200 & 0.05827900 \\
\hline $\mathrm{C}$ & -2.56913900 & -0.75024600 & 2.90747700 & $\mathrm{H}$ & 2.64007100 & 1.11419400 & 5.30505500 \\
\hline $\mathrm{H}$ & -3.61916000 & -0.44249000 & 2.91163100 & $\mathrm{H}$ & 4.54163000 & 0.65001200 & 0.64367400 \\
\hline $\mathrm{H}$ & -2.25722700 & -0.88958500 & 3.95066200 & $\mathrm{H}$ & 1.69134800 & 1.16822400 & 3.08043300 \\
\hline $\mathrm{C}$ & -3.24945600 & -3.15730700 & 2.82529900 & $\mathrm{H}$ & 3.83683900 & -0.90194000 & 2.31956500 \\
\hline $\mathrm{H}$ & -2.97597700 & -3.21093500 & 3.88705700 & $\mathrm{C}$ & 3.89214500 & 3.09400900 & 3.50941400 \\
\hline $\mathrm{H}$ & -4.31341700 & -2.90785200 & 2.78107500 & $\mathrm{H}$ & 4.44130100 & 3.92924600 & 3.96336900 \\
\hline $\mathrm{H}$ & -3.10077800 & -4.15565100 & 2.40498500 & $\mathrm{H}$ & 2.83384400 & 3.27591300 & 3.74750800 \\
\hline $\mathrm{F}$ & -4.65819800 & 4.42697700 & -0.36267700 & $\mathrm{C}$ & 4.36790200 & 1.78025300 & 4.17892400 \\
\hline $\mathrm{Rh}$ & -1.58147300 & -0.08034500 & -0.84225500 & $\mathrm{H}$ & 4.86139200 & 2.02262400 & 5.12925200 \\
\hline $\mathrm{Cl}$ & 0.45236400 & -1.61773900 & -1.28152600 & $\mathrm{H}$ & 5.14632900 & 1.32263100 & 3.55379000 \\
\hline $\mathrm{H}$ & -1.96566000 & 0.05505000 & 2.48418100 & $\mathrm{C}$ & 4.14590200 & -4.57585000 & -0.58488000 \\
\hline $\mathrm{C}$ & 1.48966900 & 4.97313800 & -1.68654700 & $\mathrm{C}$ & 3.07988300 & -3.54899300 & -0.13403100 \\
\hline $\mathrm{O}$ & 2.41620300 & 5.24120000 & -0.95025400 & $\mathrm{C}$ & 4.78505000 & -2.11808700 & -3.16171900 \\
\hline $\mathrm{O}$ & 0.55248800 & 5.87239200 & -2.06022200 & $\mathrm{C}$ & 3.62569700 & -2.14305100 & 0.02020500 \\
\hline $\mathrm{C}$ & 0.70409400 & 7.19078200 & -1.50881100 & $\mathrm{C}$ & 3.51542900 & -1.55451500 & -2.47863300 \\
\hline $\mathrm{H}$ & 1.65770400 & 7.62769200 & -1.81724100 & $\mathrm{C}$ & 3.79385200 & -1.22140300 & -1.03074000 \\
\hline $\mathrm{H}$ & -0.13017000 & 7.77080200 & -1.90406600 & $\mathrm{H}$ & 5.02536000 & -4.50440100 & 0.07256000 \\
\hline $\mathrm{H}$ & 0.66545600 & 7.15678300 & -0.41681600 & $\mathrm{H}$ & 2.24024000 & -3.57266900 & -0.83328500 \\
\hline $\mathrm{C}$ & -0.05843300 & 2.96225600 & -1.66997100 & $\mathrm{H}$ & 5.51445000 & -1.30707800 & -3.29884300 \\
\hline $\mathrm{H}$ & -0.93043700 & 3.55840500 & -1.94993700 & $\mathrm{H}$ & 3.19126900 & -0.66016700 & -3.02589200 \\
\hline $\mathrm{C}$ & -1.20043700 & 1.03306600 & -2.91351100 & $\mathrm{H}$ & 3.72553800 & -5.57741700 & -0.41891400 \\
\hline $\mathrm{H}$ & -2.08052900 & 1.62525100 & -3.14992500 & $\mathrm{H}$ & 2.67978900 & -3.87057300 & 0.83699400 \\
\hline
\end{tabular}




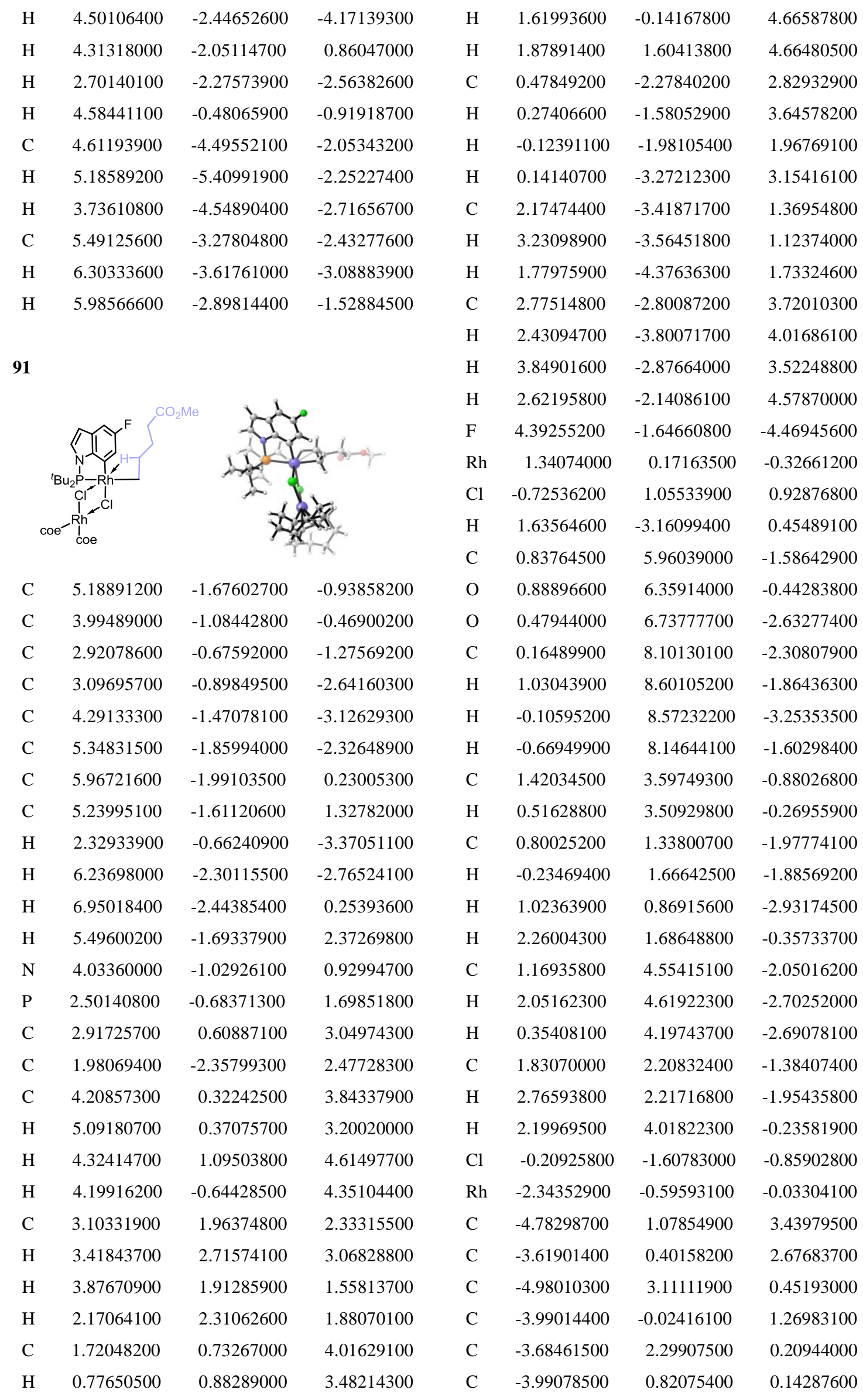




\begin{tabular}{|c|c|c|c|c|c|c|c|}
\hline $\mathrm{H}$ & -5.67770300 & 0.44009600 & 3.38686800 & & & & \\
\hline $\mathrm{H}$ & -2.75480900 & 1.07011500 & 2.67278200 & & & & \\
\hline $\mathrm{H}$ & -5.59393800 & 3.09766800 & -0.46010300 & & & & \\
\hline $\mathrm{H}$ & -3.22704400 & 2.63856300 & -0.72832400 & & & & \\
\hline $\mathrm{H}$ & -4.49957700 & 1.11374700 & 4.50073400 & & coe & & \\
\hline $\mathrm{H}$ & -3.31275500 & -0.49183100 & 3.23725800 & $\mathrm{C}$ & -3.87143600 & -2.59373300 & -0.84040900 \\
\hline $\mathrm{H}$ & -4.69037300 & 4.15952500 & 0.60911600 & $\mathrm{C}$ & -3.21637600 & -1.60362200 & -0.02161100 \\
\hline $\mathrm{H}$ & -4.70207500 & -0.84957300 & 1.25681800 & $\mathrm{C}$ & -2.01472500 & -1.91921400 & 0.71270600 \\
\hline $\mathrm{H}$ & -2.96174100 & 2.51430200 & 0.99748900 & $\mathrm{C}$ & -1.37817500 & -3.12240700 & 0.27107700 \\
\hline $\mathrm{H}$ & -4.68919700 & 0.57829200 & -0.65711800 & $\mathrm{C}$ & -1.93870000 & -3.95079600 & -0.67830800 \\
\hline $\mathrm{C}$ & -5.16238300 & 2.50890700 & 3.00371900 & $\mathrm{C}$ & -3.21898500 & -3.76024600 & -1.20853400 \\
\hline $\mathrm{H}$ & -5.83654200 & 2.90490700 & 3.77424000 & $\mathrm{C}$ & -5.25001200 & -2.18622800 & -0.96781600 \\
\hline $\mathrm{H}$ & -4.26886800 & 3.14907800 & 3.03873000 & $\mathrm{C}$ & -5.40167400 & -1.00987700 & -0.30398000 \\
\hline $\mathrm{C}$ & -5.86133200 & 2.64793900 & 1.62842300 & $\mathrm{H}$ & -0.44641100 & -3.43642700 & 0.72415500 \\
\hline $\mathrm{H}$ & -6.67991200 & 3.37398900 & 1.71754100 & $\mathrm{H}$ & -3.68509800 & -4.53168900 & -1.81197700 \\
\hline $\mathrm{H}$ & -6.34274900 & 1.69433000 & 1.37400100 & $\mathrm{H}$ & -6.03286600 & -2.74258400 & -1.46700200 \\
\hline $\mathrm{C}$ & -3.86758200 & -2.98542800 & -3.61192600 & $\mathrm{H}$ & -6.29033800 & -0.40284900 & -0.20138000 \\
\hline $\mathrm{C}$ & -3.05280400 & -1.95086600 & -2.79850500 & $\mathrm{~N}$ & -4.20063000 & -0.57857100 & 0.28294900 \\
\hline $\mathrm{C}$ & -3.48093000 & -4.98850600 & -0.62393500 & $\mathrm{P}$ & -3.41377200 & 0.97012800 & -0.11731000 \\
\hline $\mathrm{C}$ & -3.61798800 & -1.69711900 & -1.41470600 & $\mathrm{C}$ & -3.97058000 & 2.12123100 & 1.32357200 \\
\hline $\mathrm{C}$ & -2.56467100 & -3.77625100 & -0.33262100 & $\mathrm{C}$ & -4.12618500 & 1.66184300 & -1.78834300 \\
\hline $\mathrm{C}$ & -3.37459100 & -2.50110800 & -0.28442100 & $\mathrm{C}$ & -5.46860500 & 1.95019400 & 1.65028400 \\
\hline $\mathrm{H}$ & -4.93054000 & -2.70077600 & -3.60777400 & $\mathrm{H}$ & -5.69964600 & 0.91342800 & 1.91426200 \\
\hline $\mathrm{H}$ & -2.00947600 & -2.27264400 & -2.74977000 & $\mathrm{H}$ & -5.71509200 & 2.57384300 & 2.52001500 \\
\hline $\mathrm{H}$ & -4.10143100 & -5.19601100 & 0.25965500 & $\mathrm{H}$ & -6.12529900 & 2.25801200 & 0.83407400 \\
\hline $\mathrm{H}$ & -2.04901600 & -3.94366300 & 0.62106500 & $\mathrm{C}$ & -3.17179700 & 1.74389900 & 2.58590700 \\
\hline $\mathrm{H}$ & -3.53861900 & -2.90805100 & -4.65720900 & $\mathrm{H}$ & -3.41971800 & 2.45489400 & 3.38509200 \\
\hline $\mathrm{H}$ & -3.05334100 & -1.00144300 & -3.35035900 & $\mathrm{H}$ & -3.43532300 & 0.74627400 & 2.94584700 \\
\hline $\mathrm{H}$ & -2.83571200 & -5.86813700 & -0.75686200 & $\mathrm{H}$ & -2.09181500 & 1.78985500 & 2.41452200 \\
\hline $\mathrm{H}$ & -4.57519100 & -1.17687600 & -1.44376600 & $\mathrm{C}$ & -3.65472700 & 3.59517500 & 1.00004500 \\
\hline $\mathrm{H}$ & -1.78787600 & -3.71785400 & -1.09696700 & $\mathrm{H}$ & -2.60599000 & 3.73655700 & 0.71684200 \\
\hline $\mathrm{H}$ & -4.14889000 & -2.52971700 & 0.48121100 & $\mathrm{H}$ & -4.28707300 & 4.00032600 & 0.20688300 \\
\hline $\mathrm{C}$ & -3.74423600 & -4.46379200 & -3.18637300 & $\mathrm{H}$ & -3.83777800 & 4.19799500 & 1.89886400 \\
\hline $\mathrm{H}$ & -4.20265500 & -5.06003600 & -3.98591600 & $\mathrm{C}$ & -3.21780900 & 2.83018300 & -2.24415200 \\
\hline $\mathrm{H}$ & -2.68324600 & -4.75253700 & -3.17728700 & $\mathrm{H}$ & -3.26290200 & 3.70120700 & -1.58822900 \\
\hline $\mathrm{C}$ & -4.41291000 & -4.85562300 & -1.84466200 & $\mathrm{H}$ & -2.17404700 & 2.52300500 & -2.33536800 \\
\hline $\mathrm{H}$ & -4.91526300 & -5.82395400 & -1.96757700 & $\mathrm{H}$ & -3.56017500 & 3.15231100 & -3.23650400 \\
\hline \multirow[t]{2}{*}{$\mathrm{H}$} & -5.21330800 & -4.13774600 & -1.62096100 & $\mathrm{C}$ & -3.98581200 & 0.55282200 & -2.85677800 \\
\hline & & & & $\mathrm{H}$ & -4.66077800 & -0.28688800 & -2.68283000 \\
\hline \multirow[t]{5}{*}{92} & & & & $\mathrm{H}$ & -4.23363300 & 0.98720700 & -3.83453900 \\
\hline & & & & $\mathrm{C}$ & -5.58676700 & 2.15246800 & -1.76217100 \\
\hline & & & & $\mathrm{H}$ & -5.86823800 & 2.45239300 & -2.78018400 \\
\hline & & & & $\mathrm{H}$ & -6.29201900 & 1.37671100 & -1.45146500 \\
\hline & & & & $\mathrm{H}$ & -5.72909600 & 3.02742800 & -1.12186600 \\
\hline
\end{tabular}




\begin{tabular}{|c|c|c|c|c|c|c|c|}
\hline $\mathrm{F}$ & -1.26671900 & -5.06853800 & -1.03317000 & $\mathrm{C}$ & 5.58806600 & -2.92421600 & -2.21996800 \\
\hline $\mathrm{Rh}$ & -1.46404100 & -0.08762700 & -0.55684900 & $\mathrm{H}$ & 6.40316300 & -3.48097700 & -2.70077000 \\
\hline $\mathrm{Cl}$ & -0.03343900 & 1.80286300 & -0.95163300 & $\mathrm{H}$ & 6.07443600 & -2.10450600 & -1.67466000 \\
\hline $\mathrm{H}$ & -2.96147600 & 0.16945300 & -2.90317700 & $\mathrm{C}$ & 3.71109600 & 4.75336300 & -1.27675500 \\
\hline $\mathrm{C}$ & -1.72585900 & -1.47629700 & 2.15663900 & $\mathrm{C}$ & 2.90407400 & 3.47544400 & -1.60647300 \\
\hline $\mathrm{H}$ & -2.01790400 & -0.43502500 & 2.25571100 & $\mathrm{C}$ & 3.07680200 & 3.73932300 & 2.13843100 \\
\hline $\mathrm{C}$ & -0.22436700 & -1.55325300 & 2.51223600 & $\mathrm{C}$ & 3.40637700 & 2.23907100 & -0.88573600 \\
\hline $\mathrm{H}$ & 0.07829700 & -2.59787300 & 2.66358200 & $\mathrm{C}$ & 2.20873500 & 2.74081800 & 1.33502600 \\
\hline $\mathrm{H}$ & 0.36527900 & -1.15766500 & 1.67904700 & $\mathrm{C}$ & 3.07738500 & 1.89104700 & 0.43786900 \\
\hline $\mathrm{Cl}$ & 0.46436400 & -1.25729900 & -1.56814000 & $\mathrm{H}$ & 4.78465500 & 4.55554600 & -1.41580300 \\
\hline $\mathrm{C}$ & -2.59281100 & -2.31199100 & 3.12385800 & $\mathrm{H}$ & 1.84741100 & 3.65677500 & -1.39118800 \\
\hline $\mathrm{H}$ & -2.44769200 & -1.99645800 & 4.16376500 & $\mathrm{H}$ & 3.64122800 & 3.18848600 & 2.90342200 \\
\hline $\mathrm{H}$ & -3.65695500 & -2.20281200 & 2.88750200 & $\mathrm{H}$ & 1.66982300 & 2.10173300 & 2.04347900 \\
\hline $\mathrm{H}$ & -2.33928100 & -3.37632000 & 3.05335400 & $\mathrm{H}$ & 3.44426000 & 5.51158900 & -2.02612800 \\
\hline $\mathrm{C}$ & 0.13323200 & -0.75182400 & 3.78333600 & $\mathrm{H}$ & 2.96786900 & 3.29480500 & -2.68768600 \\
\hline $\mathrm{H}$ & -0.11455500 & 0.30416100 & 3.63168000 & $\mathrm{H}$ & 2.39616000 & 4.40962600 & 2.68283200 \\
\hline $\mathrm{H}$ & -0.41723400 & -1.12180500 & 4.65290300 & $\mathrm{H}$ & 4.38004000 & 1.90789100 & -1.24942500 \\
\hline $\mathrm{C}$ & 1.61919700 & -0.83511600 & 4.05896200 & $\mathrm{H}$ & 1.46008500 & 3.28669100 & 0.75799600 \\
\hline $\mathrm{O}$ & 2.47623900 & -0.19888300 & 3.47806600 & $\mathrm{H}$ & 3.79623000 & 1.30133600 & 1.00253600 \\
\hline $\mathrm{O}$ & 1.89850000 & -1.74738000 & 5.01671600 & $\mathrm{C}$ & 3.49405500 & 5.37307700 & 0.12000200 \\
\hline $\mathrm{C}$ & 3.29323500 & -1.93727500 & 5.30358500 & $\mathrm{H}$ & 3.96090700 & 6.36663600 & 0.09999000 \\
\hline $\mathrm{H}$ & 3.74962800 & -0.99940700 & 5.63139500 & $\mathrm{H}$ & 2.42018100 & 5.55628400 & 0.27250000 \\
\hline $\mathrm{H}$ & 3.32964600 & -2.68115800 & 6.10013300 & $\mathrm{C}$ & 4.07318100 & 4.58763300 & 1.32313000 \\
\hline $\mathrm{H}$ & 3.82253500 & -2.29835900 & 4.41735400 & $\mathrm{H}$ & 4.53313300 & 5.29832200 & 2.02259800 \\
\hline $\mathrm{Rh}$ & 2.10009500 & 0.49876100 & -0.92066100 & $\mathrm{H}$ & 4.89551900 & 3.95045000 & 0.97139200 \\
\hline $\mathrm{C}$ & 4.49964200 & -3.22567900 & 0.15394000 & & & & \\
\hline $\mathrm{C}$ & 3.35157000 & -2.18896100 & 0.15169600 & 93 & & & \\
\hline $\mathrm{C}$ & 4.71485200 & -2.34292800 & -3.34982000 & & & & \\
\hline $\mathrm{C}$ & 3.73714200 & -0.85959600 & -0.46518400 & & & & \\
\hline $\mathrm{C}$ & 3.42562900 & -1.61277200 & -2.90315900 & & & & \\
\hline $\mathrm{C}$ & 3.74120500 & -0.57946100 & -1.84610300 & & & & \\
\hline $\mathrm{H}$ & 5.39981100 & -2.77592100 & 0.59981800 & & & & \\
\hline $\mathrm{H}$ & 2.48108900 & -2.61746200 & -0.35228100 & $\mathrm{C}$ & 5.68293500 & 0.98010700 & -1.12369800 \\
\hline $\mathrm{H}$ & 5.33550900 & -1.65608400 & -3.94309100 & $\mathrm{C}$ & 4.40311800 & 0.88586100 & -0.52378300 \\
\hline $\mathrm{H}$ & 2.96305100 & -1.14048800 & -3.77849000 & $\mathrm{C}$ & 3.40356300 & 1.85260000 & -0.76238100 \\
\hline $\mathrm{H}$ & 4.20306800 & -4.04083500 & 0.82900100 & $\mathrm{C}$ & 3.64827500 & 2.74182800 & -1.81265000 \\
\hline $\mathrm{H}$ & 3.05075600 & -2.00145200 & 1.19029200 & $\mathrm{C}$ & 4.86863800 & 2.72869200 & -2.50155200 \\
\hline $\mathrm{H}$ & 4.41975100 & -3.15315400 & -4.03136700 & $\mathrm{C}$ & 5.92300200 & 1.91175000 & -2.14858300 \\
\hline $\mathrm{H}$ & 4.45046900 & -0.30538700 & 0.14530500 & $\mathrm{C}$ & 6.53266100 & 0.04493900 & -0.43762100 \\
\hline $\mathrm{H}$ & 2.70403200 & -2.34161400 & -2.53067600 & $\mathrm{C}$ & 5.75934500 & -0.60402000 & 0.47611900 \\
\hline $\mathrm{H}$ & 4.43852600 & 0.18081400 & -2.19794900 & $\mathrm{H}$ & 2.92930200 & 3.50678400 & -2.07745100 \\
\hline $\mathrm{C}$ & 4.87531800 & -3.85288500 & -1.20507300 & $\mathrm{H}$ & 6.88631700 & 2.00571500 & -2.63819100 \\
\hline $\mathrm{H}$ & 5.53813000 & -4.70125500 & -0.98955700 & $\mathrm{H}$ & 7.59310800 & -0.10165700 & -0.59436800 \\
\hline $\mathrm{H}$ & 3.97678200 & -4.29148000 & -1.66271400 & $\mathrm{H}$ & 6.04530900 & -1.37672300 & 1.16958800 \\
\hline
\end{tabular}




\begin{tabular}{|c|c|c|c|c|c|c|c|}
\hline $\mathrm{N}$ & 4.42685900 & -0.15998700 & 0.43230200 & $\mathrm{C}$ & -0.39346300 & 4.88174900 & 0.88987000 \\
\hline $\mathrm{P}$ & 3.01456000 & -1.23683300 & 0.68770400 & $\mathrm{H}$ & 0.24332500 & 5.77081800 & 0.82672700 \\
\hline $\mathrm{C}$ & 2.98585400 & -1.43948200 & 2.59914200 & $\mathrm{H}$ & -0.91822000 & 4.81017000 & -0.07430500 \\
\hline $\mathrm{C}$ & 3.49146400 & -2.90335800 & -0.19188600 & $\mathrm{C}$ & 1.42353700 & 3.35214400 & -0.00901400 \\
\hline $\mathrm{C}$ & 4.11575000 & -2.29532300 & 3.20276700 & $\mathrm{H}$ & 0.87812400 & 3.22051400 & -0.95017600 \\
\hline $\mathrm{H}$ & 5.10816800 & -1.87278200 & 3.01655400 & $\mathrm{H}$ & 0.96523600 & 3.69947700 & 2.08160600 \\
\hline $\mathrm{H}$ & 3.98512200 & -2.33256600 & 4.29240700 & $\mathrm{Cl}$ & -0.73932200 & 0.89732000 & -1.21815800 \\
\hline $\mathrm{H}$ & 4.10120800 & -3.32719300 & 2.84189100 & $\mathrm{Rh}$ & -2.36278300 & -0.88930900 & -0.58748200 \\
\hline $\mathrm{C}$ & 3.05869200 & -0.02404400 & 3.21035200 & $\mathrm{C}$ & -4.74946200 & 2.76801400 & 0.70747300 \\
\hline $\mathrm{H}$ & 2.98436900 & -0.10719600 & 4.30223700 & $\mathrm{C}$ & -3.59765400 & 1.73844200 & 0.62722700 \\
\hline $\mathrm{H}$ & 3.99896100 & 0.48137500 & 2.97169200 & $\mathrm{C}$ & -5.04035000 & 2.05059000 & -2.82942100 \\
\hline $\mathrm{H}$ & 2.22734400 & 0.60113600 & 2.87007500 & $\mathrm{C}$ & -3.99849200 & 0.43806800 & -0.03805800 \\
\hline $\mathrm{C}$ & 1.61956700 & -2.05149400 & 2.98260900 & $\mathrm{C}$ & -3.74158500 & 1.30123000 & -2.44588900 \\
\hline $\mathrm{H}$ & 0.79428200 & -1.46649000 & 2.56493900 & $\mathrm{C}$ & -4.03327300 & 0.22199100 & -1.42918800 \\
\hline $\mathrm{H}$ & 1.50840100 & -3.08358900 & 2.64100700 & $\mathrm{H}$ & -5.63767700 & 2.29388000 & 1.15204400 \\
\hline $\mathrm{H}$ & 1.52683700 & -2.05262400 & 4.07685500 & $\mathrm{H}$ & -2.74504400 & 2.19494700 & 0.11871900 \\
\hline $\mathrm{C}$ & 2.53604100 & -4.01617700 & 0.29560700 & $\mathrm{H}$ & -5.67270500 & 1.39183800 & -3.44171900 \\
\hline $\mathrm{H}$ & 2.79396400 & -4.35667000 & 1.30368400 & $\mathrm{H}$ & -3.29927800 & 0.86827800 & -3.35152700 \\
\hline $\mathrm{H}$ & 1.48702200 & -3.72104900 & 0.28053500 & $\mathrm{H}$ & -4.42922300 & 3.54303700 & 1.41501500 \\
\hline $\mathrm{H}$ & 2.64487300 & -4.87862700 & -0.37504200 & $\mathrm{H}$ & -3.25899100 & 1.52318600 & 1.64799700 \\
\hline $\mathrm{C}$ & 3.27112000 & -2.63913400 & -1.69895500 & $\mathrm{H}$ & -4.75999600 & 2.89239300 & -3.47815900 \\
\hline $\mathrm{H}$ & 3.91085400 & -1.82654400 & -2.06109300 & $\mathrm{H}$ & -4.69732200 & -0.14596700 & 0.56136900 \\
\hline $\mathrm{H}$ & 3.53464300 & -3.54665200 & -2.25885500 & $\mathrm{H}$ & -3.01131700 & 2.01315900 & -2.05787300 \\
\hline $\mathrm{C}$ & 4.93040200 & -3.44074100 & -0.02535300 & $\mathrm{H}$ & -4.73803300 & -0.52183700 & -1.80002100 \\
\hline $\mathrm{H}$ & 4.96042100 & -4.43907700 & -0.48134200 & $\mathrm{C}$ & -5.16025100 & 3.45690400 & -0.61099100 \\
\hline $\mathrm{H}$ & 5.66675600 & -2.82570600 & -0.54416300 & $\mathrm{H}$ & -5.82293800 & 4.28944700 & -0.33982300 \\
\hline $\mathrm{H}$ & 5.23857500 & -3.55741300 & 1.01698000 & $\mathrm{H}$ & -4.27558500 & 3.92437500 & -1.06847000 \\
\hline $\mathrm{F}$ & 5.03404200 & 3.62545300 & -3.50222600 & $\mathrm{C}$ & -5.89062700 & 2.57607900 & -1.65541900 \\
\hline $\mathrm{Rh}$ & 1.17789800 & -0.22019700 & -0.17972500 & $\mathrm{H}$ & -6.71590300 & 3.15257800 & -2.09358500 \\
\hline $\mathrm{Cl}$ & -0.19771900 & -2.12709000 & -0.61038900 & $\mathrm{H}$ & -6.36509300 & 1.73074600 & -1.13965700 \\
\hline $\mathrm{H}$ & 2.23480500 & -2.38233800 & -1.92648400 & $\mathrm{C}$ & -3.84583800 & -5.14177800 & -1.27710300 \\
\hline $\mathrm{C}$ & -1.45278700 & 5.11037800 & 1.94976500 & $\mathrm{C}$ & -3.06379200 & -3.82474900 & -1.49907700 \\
\hline $\mathrm{O}$ & -2.01688500 & 4.24273300 & 2.58467200 & $\mathrm{C}$ & -3.34641900 & -4.35176900 & 2.21779200 \\
\hline $\mathrm{O}$ & -1.72410700 & 6.42716800 & 2.09311100 & $\mathrm{C}$ & -3.62047500 & -2.65429900 & -0.71164500 \\
\hline $\mathrm{C}$ & -2.75644900 & 6.74657500 & 3.03921900 & $\mathrm{C}$ & -2.48177300 & -3.28494700 & 1.50583500 \\
\hline $\mathrm{H}$ & -2.50003700 & 6.36692400 & 4.03178200 & $\mathrm{C}$ & -3.34068500 & -2.38788400 & 0.64371500 \\
\hline $\mathrm{H}$ & -2.81867500 & 7.83525500 & 3.04808700 & $\mathrm{H}$ & -4.91970000 & -4.96168400 & -1.43546200 \\
\hline $\mathrm{H}$ & -3.71099400 & 6.31136900 & 2.72989500 & $\mathrm{H}$ & -2.00833200 & -3.99401400 & -1.26852500 \\
\hline $\mathrm{C}$ & 0.42688600 & 3.60878700 & 1.12745900 & $\mathrm{H}$ & -3.94992800 & -3.86844700 & 2.99919300 \\
\hline $\mathrm{H}$ & -0.25452800 & 2.75813500 & 1.22779100 & $\mathrm{H}$ & -1.95265800 & -2.69675700 & 2.26609600 \\
\hline $\mathrm{C}$ & 2.31514500 & 2.13432900 & 0.25436600 & $\mathrm{H}$ & -3.53738200 & -5.84029200 & -2.06704200 \\
\hline $\mathrm{H}$ & 2.83467500 & 2.28638300 & 1.21215900 & $\mathrm{H}$ & -3.10496600 & -3.57278000 & -2.56679900 \\
\hline $\mathrm{H}$ & 1.61188500 & 1.29765500 & 0.60891300 & $\mathrm{H}$ & -2.66543200 & -5.03884800 & 2.73975300 \\
\hline $\mathrm{H}$ & 2.07279800 & 4.23028800 & -0.12810700 & $\mathrm{H}$ & -4.59300200 & -2.32712900 & -1.08053800 \\
\hline
\end{tabular}




\begin{tabular}{|c|c|c|c|c|c|c|c|}
\hline $\mathrm{H}$ & -1.71198400 & -3.77677000 & 0.90879100 & $\mathrm{H}$ & -2.58980300 & -3.04478300 & 0.92864700 \\
\hline $\mathrm{H}$ & -4.10740300 & -1.86920200 & 1.21707000 & $\mathrm{H}$ & -0.97013800 & -2.799999900 & 1.57074500 \\
\hline $\mathrm{C}$ & -3.65243500 & -5.84959300 & 0.08052400 & $\mathrm{H}$ & -2.34445100 & -2.98852800 & 2.68113200 \\
\hline $\mathrm{H}$ & -4.08747500 & -6.85219000 & -0.02298400 & $\mathrm{C}$ & -1.43318000 & -0.43736900 & 2.93890100 \\
\hline $\mathrm{H}$ & -2.57901800 & -6.01282000 & 0.25570000 & $\mathrm{H}$ & -1.60908700 & 0.63549100 & 3.06232500 \\
\hline $\mathrm{C}$ & -4.29321700 & -5.16843200 & 1.31558100 & $\mathrm{H}$ & -1.73742200 & -0.93828000 & 3.86697400 \\
\hline $\mathrm{H}$ & -4.75117500 & -5.93914200 & 1.94917000 & $\mathrm{C}$ & -3.75523800 & -0.75324800 & 2.05168000 \\
\hline \multirow[t]{2}{*}{$\mathrm{H}$} & -5.12383600 & -4.53212700 & 0.98222000 & $\mathrm{H}$ & -4.01055000 & -1.18849500 & 3.02597000 \\
\hline & & & & $\mathrm{H}$ & -3.98197500 & 0.31596800 & 2.10733400 \\
\hline \multirow[t]{6}{*}{94} & & & & $\mathrm{H}$ & -4.41345800 & -1.21006300 & 1.30936900 \\
\hline & & & & $\mathrm{F}$ & 2.68507500 & 4.13143600 & -1.76927900 \\
\hline & & & & $\mathrm{Rh}$ & 0.37920100 & -0.74923800 & -0.80813400 \\
\hline & & & & $\mathrm{Cl}$ & -0.10141400 & -3.10795800 & -1.25666700 \\
\hline & & & & $\mathrm{H}$ & -0.36189600 & -0.59449200 & 2.80351500 \\
\hline & & & & $\mathrm{C}$ & 2.43917100 & -0.06440900 & 1.43534400 \\
\hline $\mathrm{C}$ & -0.37733800 & 3.40942600 & 0.01882000 & $\mathrm{O}$ & 3.34141700 & 0.71778600 & 1.21754000 \\
\hline $\mathrm{C}$ & -0.32291900 & 2.00846900 & -0.11200600 & $\mathrm{O}$ & 1.80760700 & -0.13582800 & 2.63119800 \\
\hline $\mathrm{C}$ & 0.67430400 & 1.26471800 & -0.75484300 & $\mathrm{C}$ & 2.23427100 & 0.82879300 & 3.60811700 \\
\hline $\mathrm{C}$ & 1.69131800 & 2.03746200 & -1.31987800 & $\mathrm{H}$ & 2.07164100 & 1.84456800 & 3.23877200 \\
\hline $\mathrm{C}$ & 1.66353100 & 3.44234600 & -1.20327800 & $\mathrm{H}$ & 1.62538400 & 0.63785100 & 4.49236700 \\
\hline $\mathrm{C}$ & 0.67387000 & 4.15705900 & -0.54834100 & $\mathrm{H}$ & 3.29514600 & 0.69693900 & 3.83572200 \\
\hline $\mathrm{C}$ & -1.59628400 & 3.69404000 & 0.73562900 & $\mathrm{C}$ & 2.77294500 & -1.27305000 & -0.76836200 \\
\hline $\mathrm{C}$ & -2.22481000 & 2.50121100 & 0.98617600 & $\mathrm{H}$ & 3.47718500 & -0.43929100 & -0.83887200 \\
\hline $\mathrm{H}$ & 2.53711800 & 1.59687400 & -1.83907100 & $\mathrm{C}$ & 4.55173800 & -2.87506400 & 0.10859600 \\
\hline $\mathrm{H}$ & 0.72738700 & 5.23926200 & -0.49597300 & $\mathrm{H}$ & 4.13288300 & -2.89929000 & 1.12194100 \\
\hline $\mathrm{H}$ & -1.96341400 & 4.66701300 & 1.03617000 & $\mathrm{H}$ & 5.05025000 & -3.83516900 & -0.06452600 \\
\hline $\mathrm{H}$ & -3.15943100 & 2.31627400 & 1.49442000 & $\mathrm{H}$ & 5.31537500 & -2.08870000 & 0.08256800 \\
\hline $\mathrm{N}$ & -1.45735400 & 1.44666100 & 0.48049000 & $\mathrm{C}$ & 1.93284400 & -1.10712500 & 0.47919700 \\
\hline $\mathrm{P}$ & -1.64519800 & -0.24763200 & 0.16093400 & $\mathrm{H}$ & 2.11905300 & -1.14163500 & -1.69633000 \\
\hline $\mathrm{C}$ & -2.93211000 & -0.34672200 & -1.25888200 & $\mathrm{H}$ & 1.67837900 & -2.03337800 & 0.99777600 \\
\hline $\mathrm{C}$ & -2.26122000 & -1.03226800 & 1.78193100 & $\mathrm{C}$ & 3.46311900 & -2.63624500 & -0.94495600 \\
\hline $\mathrm{C}$ & -4.09497500 & 0.64954600 & -1.07956400 & $\mathrm{H}$ & 3.90857100 & -2.67349700 & -1.94719600 \\
\hline $\mathrm{H}$ & -3.74518700 & 1.68363900 & -1.01640400 & $\mathrm{H}$ & 2.70681600 & -3.42687700 & -0.90590100 \\
\hline $\mathrm{H}$ & -4.74942200 & 0.57385000 & -1.95752000 & & & & \\
\hline $\mathrm{H}$ & -4.70884600 & 0.43528000 & -0.20067300 & 95 & & & \\
\hline $\mathrm{C}$ & -2.15293400 & 0.03541800 & -2.54022100 & & & & \\
\hline $\mathrm{H}$ & -2.84328400 & 0.01796500 & -3.39373600 & & & & \\
\hline $\mathrm{H}$ & -1.71639800 & 1.03649100 & -2.48109000 & & & & \\
\hline $\mathrm{H}$ & -1.35734900 & -0.68957700 & -2.76315100 & & & & \\
\hline $\mathrm{C}$ & -3.47624700 & -1.78005900 & -1.42355900 & & & & \\
\hline $\mathrm{H}$ & -2.67089800 & -2.51227500 & -1.52759000 & $\mathrm{C}$ & 2.64051500 & 2.21022700 & 1.45941400 \\
\hline $\mathrm{H}$ & -4.12538500 & -2.07992800 & -0.59572000 & $\mathrm{C}$ & 1.78071000 & 1.34122500 & 0.75954600 \\
\hline $\mathrm{H}$ & -4.08283300 & -1.81312300 & -2.33778500 & $\mathrm{C}$ & 0.80936900 & 1.69676800 & -0.18524100 \\
\hline $\mathrm{C}$ & -2.02358500 & -2.55622900 & 1.72471600 & $\mathrm{C}$ & 0.74732400 & 3.07120100 & -0.43877400 \\
\hline
\end{tabular}




\begin{tabular}{|c|c|c|c|c|c|c|c|}
\hline $\mathrm{C}$ & 1.59328800 & 3.96655800 & 0.24966100 & $\mathrm{H}$ & -6.56057200 & -1.57509700 & 2.36725900 \\
\hline $\mathrm{C}$ & 2.52921500 & 3.59065700 & 1.19727300 & $\mathrm{H}$ & -6.14695000 & 0.15297400 & 2.62507400 \\
\hline $\mathrm{C}$ & 3.46656100 & 1.36916300 & 2.28817300 & $\mathrm{C}$ & -1.94279400 & 0.67246600 & -0.10705200 \\
\hline $\mathrm{C}$ & 3.10790400 & 0.06642900 & 2.05196100 & $\mathrm{H}$ & -1.74443500 & 1.33911900 & 0.73472400 \\
\hline $\mathrm{H}$ & 0.06616400 & 3.49877700 & -1.16732400 & $\mathrm{C}$ & -2.71865800 & 2.87056500 & -1.24129400 \\
\hline $\mathrm{H}$ & 3.14128400 & 4.33939100 & 1.68867800 & $\mathrm{H}$ & -2.04020700 & 3.44188600 & -0.60245900 \\
\hline $\mathrm{H}$ & 4.23625400 & 1.68862500 & 2.97896700 & $\mathrm{H}$ & -2.77942200 & 3.36519800 & -2.21688500 \\
\hline $\mathrm{H}$ & 3.51364200 & -0.83829200 & 2.47863600 & $\mathrm{H}$ & -3.70688100 & 2.87437200 & -0.77613700 \\
\hline $\mathrm{N}$ & 2.06764600 & 0.02340900 & 1.11882000 & $\mathrm{C}$ & -2.87428200 & -0.46557100 & 0.27503800 \\
\hline $\mathrm{P}$ & 1.23099600 & -1.16437300 & 0.16536800 & $\mathrm{H}$ & -3.05308700 & -1.11964800 & -0.58634900 \\
\hline $\mathrm{C}$ & 0.59934900 & -2.44465200 & 1.42479900 & $\mathrm{H}$ & -2.44152200 & -1.09971400 & 1.05200800 \\
\hline $\mathrm{C}$ & 2.55162100 & -1.86557400 & -1.03032500 & $\mathrm{C}$ & -2.25934700 & 1.41334200 & -1.39204000 \\
\hline $\mathrm{C}$ & 1.71214900 & -3.34162100 & 2.00511900 & $\mathrm{H}$ & -2.95025100 & 0.83193400 & -2.01004800 \\
\hline $\mathrm{H}$ & 2.50654600 & -2.77154600 & 2.49527300 & $\mathrm{H}$ & -1.32310300 & 1.44676300 & -2.05428200 \\
\hline $\mathrm{H}$ & 1.26479300 & -3.99262600 & 2.76701600 & & & & \\
\hline $\mathrm{H}$ & 2.16260200 & -3.98945000 & 1.24905500 & 96 & & & \\
\hline $\mathrm{C}$ & -0.03809600 & -1.64481400 & 2.58365800 & & & & \\
\hline $\mathrm{H}$ & -0.55587700 & -2.34449900 & 3.25132400 & & & & \\
\hline $\mathrm{H}$ & 0.71424900 & -1.10918500 & 3.16916600 & & & & \\
\hline $\mathrm{H}$ & -0.76768500 & -0.91003100 & 2.23438400 & & & & \\
\hline $\mathrm{C}$ & -0.45905600 & -3.34092300 & 0.74635500 & & & & \\
\hline $\mathrm{H}$ & -1.24027100 & -2.76614400 & 0.24777800 & $\mathrm{C}$ & 1.85405300 & 3.12346900 & 0.83597000 \\
\hline $\mathrm{H}$ & -0.01652400 & -3.99988100 & -0.00417100 & $\mathrm{C}$ & 1.31720500 & 1.87441100 & 0.45452700 \\
\hline $\mathrm{H}$ & -0.92463800 & -3.97357800 & 1.51278900 & $\mathrm{C}$ & 0.04944400 & 1.64288000 & -0.10898100 \\
\hline $\mathrm{C}$ & 2.06069100 & -3.17307300 & -1.68396900 & $\mathrm{C}$ & -0.69657500 & 2.81517000 & -0.28158100 \\
\hline $\mathrm{H}$ & 2.04012000 & -4.00815000 & -0.97684400 & $\mathrm{C}$ & -0.19257300 & 4.07072400 & 0.11257000 \\
\hline $\mathrm{H}$ & 1.07036000 & -3.06087700 & -2.13495700 & $\mathrm{C}$ & 1.05712500 & 4.27140400 & 0.66619500 \\
\hline $\mathrm{H}$ & 2.76466100 & -3.44410100 & -2.48141400 & $\mathrm{C}$ & 3.18981200 & 2.86538000 & 1.30487500 \\
\hline $\mathrm{C}$ & 2.71451200 & -0.78613500 & -2.12759300 & $\mathrm{C}$ & 3.41645400 & 1.51888800 & 1.19554100 \\
\hline $\mathrm{H}$ & 3.03783000 & 0.17624900 & -1.71794900 & $\mathrm{H}$ & -1.68301800 & 2.82454000 & -0.73026800 \\
\hline $\mathrm{H}$ & 3.47805400 & -1.11953400 & -2.84252500 & $\mathrm{H}$ & 1.38916000 & 5.26873900 & 0.93389900 \\
\hline $\mathrm{C}$ & 3.92069000 & -2.08825300 & -0.35828000 & $\mathrm{H}$ & 3.89923400 & 3.59146900 & 1.68038300 \\
\hline $\mathrm{H}$ & 4.62093200 & -2.45275400 & -1.12070300 & $\mathrm{H}$ & 4.29786800 & 0.95438700 & 1.45708500 \\
\hline $\mathrm{H}$ & 4.33224600 & -1.16236500 & 0.05273800 & $\mathrm{~N}$ & 2.27736000 & 0.88510900 & 0.69081000 \\
\hline $\mathrm{H}$ & 3.88848800 & -2.83837900 & 0.43625700 & $\mathrm{P}$ & 1.85145400 & -0.70610500 & 0.14578500 \\
\hline $\mathrm{F}$ & 1.46807500 & 5.28526700 & -0.04552500 & $\mathrm{C}$ & 1.95608800 & -1.79306900 & 1.71715400 \\
\hline $\mathrm{Rh}$ & -0.19785600 & 0.15994100 & -1.06536900 & $\mathrm{C}$ & 3.15366900 & -1.14537100 & -1.17917800 \\
\hline $\mathrm{Cl}$ & -1.14334900 & -1.58913300 & -2.50039100 & $\mathrm{C}$ & 3.14423200 & -1.42070500 & 2.62726900 \\
\hline $\mathrm{H}$ & 1.78538000 & -0.64004700 & -2.69375300 & $\mathrm{H}$ & 3.07270100 & -0.39480800 & 2.99734700 \\
\hline $\mathrm{C}$ & -4.22414600 & 0.01688600 & 0.78698600 & $\mathrm{H}$ & 3.12864500 & -2.08775700 & 3.49866000 \\
\hline $\mathrm{O}$ & -4.69193500 & 1.12774500 & 0.64676400 & $\mathrm{H}$ & 4.11386100 & -1.55095600 & 2.13861800 \\
\hline $\mathrm{O}$ & -4.87216600 & -0.98414600 & 1.42176900 & $\mathrm{C}$ & 0.64497300 & -1.51506700 & 2.48869600 \\
\hline $\mathrm{C}$ & -6.18969900 & -0.66306300 & 1.89876800 & $\mathrm{H}$ & 0.67408200 & -2.05102100 & 3.44623600 \\
\hline $\mathrm{H}$ & -6.83699000 & -0.36676200 & 1.06902000 & $\mathrm{H}$ & 0.51611500 & -0.44913900 & 2.70952400 \\
\hline
\end{tabular}




\begin{tabular}{|c|c|c|c|c|c|c|c|}
\hline $\mathrm{H}$ & -0.22630900 & -1.87557000 & 1.93258400 & & & & \\
\hline $\mathrm{C}$ & 2.02526300 & -3.29171300 & 1.36156000 & & & & \\
\hline $\mathrm{H}$ & 1.21683100 & -3.59055700 & 0.68981900 & & & & \\
\hline $\mathrm{H}$ & 2.98438300 & -3.56595600 & 0.91124100 & & & & \\
\hline $\mathrm{H}$ & 1.92694300 & -3.86943100 & 2.28982400 & & & & \\
\hline $\mathrm{C}$ & 2.67663700 & -2.37563100 & -1.97923700 & $\mathrm{C}$ & -1.07645200 & 3.23972700 & 0.86871700 \\
\hline $\mathrm{H}$ & 2.62821500 & -3.28014400 & -1.36865000 & $\mathrm{C}$ & -0.90067600 & 1.93257300 & 0.37643100 \\
\hline $\mathrm{H}$ & 1.68621300 & -2.22207800 & -2.41332500 & $\mathrm{C}$ & 0.01117500 & 1.51701900 & -0.60218500 \\
\hline $\mathrm{H}$ & 3.39189300 & -2.55705300 & -2.79185100 & $\mathrm{C}$ & 0.79783600 & 2.54994200 & -1.12171200 \\
\hline $\mathrm{C}$ & 3.24916400 & 0.06647600 & -2.13184600 & $\mathrm{C}$ & 0.64424500 & 3.87035300 & -0.64750400 \\
\hline $\mathrm{H}$ & 3.61105500 & 0.96605000 & -1.62547600 & $\mathrm{C}$ & -0.25538600 & 4.25336900 & 0.33298700 \\
\hline $\mathrm{H}$ & 3.95642300 & -0.17834700 & -2.93406500 & $\mathrm{C}$ & -2.13447800 & 3.15751200 & 1.84445400 \\
\hline $\mathrm{C}$ & 4.55215600 & -1.42257000 & -0.59103600 & $\mathrm{C}$ & -2.55919600 & 1.85383700 & 1.89417200 \\
\hline $\mathrm{H}$ & 5.24408000 & -1.61965700 & -1.41967000 & $\mathrm{H}$ & 1.55059000 & 2.37901200 & -1.88670700 \\
\hline $\mathrm{H}$ & 4.95003400 & -0.56793500 & -0.03557500 & $\mathrm{H}$ & -0.31055200 & 5.28987200 & 0.64828000 \\
\hline $\mathrm{H}$ & 4.56600700 & -2.30135200 & 0.05866000 & $\mathrm{H}$ & -2.53422200 & 3.96601600 & 2.44309700 \\
\hline $\mathrm{F}$ & -0.99681500 & 5.14638800 & -0.08350300 & $\mathrm{H}$ & -3.33960000 & 1.41586500 & 2.49809200 \\
\hline $\mathrm{Rh}$ & -0.34293300 & -0.32607100 & -0.57370700 & $\mathrm{~N}$ & -1.80980100 & 1.07670300 & 1.00584600 \\
\hline $\mathrm{Cl}$ & -0.71467900 & -2.74833300 & -0.88646900 & $\mathrm{P}$ & -1.84549800 & -0.52475800 & 0.32736500 \\
\hline $\mathrm{H}$ & 2.28673900 & 0.29821900 & -2.59775500 & $\mathrm{C}$ & -3.44149100 & -0.57844900 & -0.73088400 \\
\hline $\mathrm{C}$ & -4.72569100 & -0.49765400 & 0.53602800 & $\mathrm{C}$ & -1.88825400 & -1.72312600 & 1.80980100 \\
\hline $\mathrm{O}$ & -5.41760400 & 0.13410400 & -0.23222300 & $\mathrm{C}$ & -4.64176800 & 0.11222500 & -0.05391400 \\
\hline $\mathrm{O}$ & -5.19269100 & -1.08866900 & 1.65591000 & $\mathrm{H}$ & -4.44574400 & 1.16791100 & 0.15322900 \\
\hline $\mathrm{C}$ & -6.60460200 & -0.94499500 & 1.88450100 & $\mathrm{H}$ & -5.49810100 & 0.06176800 & -0.73857700 \\
\hline $\mathrm{H}$ & -7.17368500 & -1.38439800 & 1.06087200 & $\mathrm{H}$ & -4.94318400 & -0.37434400 & 0.87751000 \\
\hline $\mathrm{H}$ & -6.80501200 & -1.47578900 & 2.81555000 & $\mathrm{C}$ & -3.09296100 & 0.19876700 & -2.02271500 \\
\hline $\mathrm{H}$ & -6.87200900 & 0.11095600 & 1.97773900 & $\mathrm{H}$ & -3.97794900 & 0.22298100 & -2.67196800 \\
\hline $\mathrm{C}$ & -2.63351600 & 0.17853200 & -0.64719100 & $\mathrm{H}$ & -2.79975400 & 1.23312700 & -1.81768000 \\
\hline $\mathrm{H}$ & -2.73078500 & 1.23128300 & -0.40636200 & $\mathrm{H}$ & -2.29050200 & -0.29218700 & -2.58882300 \\
\hline $\mathrm{C}$ & -2.18210300 & 0.79404200 & -3.08066600 & $\mathrm{C}$ & -3.80188400 & -2.03066500 & -1.10209000 \\
\hline $\mathrm{H}$ & -1.96327100 & 1.81155000 & -2.74818300 & $\mathrm{H}$ & -2.95157700 & -2.56671000 & -1.53435700 \\
\hline $\mathrm{H}$ & -1.40179700 & 0.47691200 & -3.78014300 & $\mathrm{H}$ & -4.17980400 & -2.59340400 & -0.24298600 \\
\hline $\mathrm{H}$ & -3.12933300 & 0.81385700 & -3.63812300 & $\mathrm{H}$ & -4.60331600 & -2.00669400 & -1.85168700 \\
\hline $\mathrm{C}$ & -3.23204400 & -0.75623600 & 0.37795400 & $\mathrm{C}$ & -1.59774000 & -3.15980900 & 1.32508000 \\
\hline $\mathrm{H}$ & -3.08918600 & -1.80428900 & 0.09257000 & $\mathrm{H}$ & -2.40017300 & -3.56492200 & 0.70515400 \\
\hline $\mathrm{H}$ & -2.76681100 & -0.63610000 & 1.36501400 & $\mathrm{H}$ & -0.67521600 & -3.22141600 & 0.74350500 \\
\hline $\mathrm{C}$ & -2.32390700 & -0.17261600 & -1.93604000 & $\mathrm{H}$ & -1.50017900 & -3.80561200 & 2.20736400 \\
\hline $\mathrm{H}$ & -2.39253600 & -1.22247500 & -2.21089300 & $\mathrm{C}$ & -0.76254900 & -1.29372900 & 2.77266200 \\
\hline \multirow[t]{2}{*}{$\mathrm{H}$} & 0.24359700 & 0.13202800 & -1.89142700 & $\mathrm{H}$ & -0.89265700 & -0.27272800 & 3.14320600 \\
\hline & & & & $\mathrm{H}$ & -0.76412800 & -1.97156000 & 3.63542600 \\
\hline \multirow[t]{4}{*}{97} & & & & $\mathrm{C}$ & -3.23140600 & -1.70010400 & 2.56726200 \\
\hline & & & & $\mathrm{H}$ & -3.15304800 & -2.37642200 & 3.42785700 \\
\hline & & & & $\mathrm{H}$ & -3.47997900 & -0.70868300 & 2.95753000 \\
\hline & & & & $\mathrm{H}$ & -4.06325400 & -2.05253800 & 1.95266800 \\
\hline
\end{tabular}




\begin{tabular}{|c|c|c|c|c|c|c|c|}
\hline $\mathrm{F}$ & 1.44478300 & 4.82113900 & -1.19219700 & $\mathrm{C}$ & -2.52870300 & 1.12612600 & -1.35264100 \\
\hline $\mathrm{Rh}$ & -0.04813700 & -0.45713100 & -1.09094000 & $\mathrm{C}$ & -2.63166600 & 0.47831700 & 1.78955100 \\
\hline $\mathrm{Cl}$ & -0.28417700 & -2.71109800 & -2.01382300 & $\mathrm{C}$ & -2.86623700 & 2.63107900 & -1.35764400 \\
\hline $\mathrm{H}$ & 0.21684100 & -1.36790400 & 2.29505800 & $\mathrm{H}$ & -1.97433800 & 3.26055000 & -1.39342100 \\
\hline $\mathrm{C}$ & 4.82359300 & -0.46782800 & 0.76163600 & $\mathrm{H}$ & -3.45084900 & 2.83604200 & -2.26362000 \\
\hline $\mathrm{O}$ & 4.99856200 & 0.28200900 & -0.17670100 & $\mathrm{H}$ & -3.47905600 & 2.93833800 & -0.50550000 \\
\hline $\mathrm{O}$ & 5.77192000 & -1.31059600 & 1.22278200 & $\mathrm{C}$ & -1.61790700 & 0.83397200 & -2.56623700 \\
\hline $\mathrm{C}$ & 7.03483800 & -1.25620800 & 0.53782700 & $\mathrm{H}$ & -2.11749000 & 1.19681600 & -3.47418500 \\
\hline $\mathrm{H}$ & 7.46714100 & -0.25442800 & 0.60749900 & $\mathrm{H}$ & -0.65410300 & 1.34938900 & -2.48765000 \\
\hline $\mathrm{H}$ & 7.67112800 & -1.98507700 & 1.04046000 & $\mathrm{H}$ & -1.43189200 & -0.23673600 & -2.67824800 \\
\hline $\mathrm{H}$ & 6.91091700 & -1.51668300 & -0.51664200 & $\mathrm{C}$ & -3.84199700 & 0.32808100 & -1.50102300 \\
\hline $\mathrm{C}$ & 2.31029900 & 0.04281100 & 0.93392100 & $\mathrm{H}$ & -3.67570500 & -0.75043700 & -1.48021200 \\
\hline $\mathrm{H}$ & 2.54636600 & 1.04119300 & 0.55842100 & $\mathrm{H}$ & -4.57655900 & 0.59273000 & -0.73422900 \\
\hline $\mathrm{C}$ & 2.29148400 & -0.62497200 & -1.58436400 & $\mathrm{H}$ & -4.28509700 & 0.58167500 & -2.47289900 \\
\hline $\mathrm{H}$ & 3.02714100 & 0.17968900 & -1.63585000 & $\mathrm{C}$ & -3.37953300 & -0.87464300 & 1.81194800 \\
\hline $\mathrm{H}$ & 2.65521900 & -1.54965100 & -2.03939000 & $\mathrm{H}$ & -4.08746200 & -0.97928500 & 0.98635000 \\
\hline $\mathrm{H}$ & 1.48157900 & -0.30171600 & -2.32250600 & $\mathrm{H}$ & -2.68859800 & -1.71954400 & 1.75791700 \\
\hline $\mathrm{C}$ & 3.56081600 & -0.56447000 & 1.59900300 & $\mathrm{H}$ & -3.94396500 & -0.94369000 & 2.75092400 \\
\hline $\mathrm{H}$ & 3.77113100 & -0.03104500 & 2.53685700 & $\mathrm{C}$ & -1.66361700 & 0.51691700 & 2.99164700 \\
\hline $\mathrm{H}$ & 3.40459000 & -1.61259300 & 1.88043000 & $\mathrm{H}$ & -1.12070100 & 1.46382100 & 3.06291200 \\
\hline $\mathrm{C}$ & 1.74792100 & -0.82740000 & -0.18293400 & $\mathrm{H}$ & -2.24477800 & 0.38884200 & 3.91366100 \\
\hline $\mathrm{H}$ & 1.70095000 & -1.88150900 & 0.10633200 & $\mathrm{C}$ & -3.67067000 & 1.60887100 & 1.94260400 \\
\hline \multirow[t]{2}{*}{$\mathrm{H}$} & 1.55 & 0.17460400 & 1.71230200 & $\mathrm{H}$ & -4.23974800 & 1.42660200 & 2.86325400 \\
\hline & & & & $\mathrm{H}$ & -3.22191900 & 2.60046300 & 2.04540900 \\
\hline \multirow[t]{6}{*}{98} & & & & $\mathrm{H}$ & -4.39055600 & 1.63460400 & 1.12100700 \\
\hline & & & & $\mathrm{F}$ & 4.92906200 & 2.78066800 & -0.72508700 \\
\hline & & & & $\mathrm{Rh}$ & -0.44247700 & -1.42473600 & -0.12742500 \\
\hline & & & & $\mathrm{Cl}$ & -2.15188300 & -2.68931400 & -1.06574100 \\
\hline & & & & $\mathrm{H}$ & -0.93394800 & -0.29873200 & 2.94657200 \\
\hline & & & & $\mathrm{H}$ & 2.90361800 & -0.47482500 & 2.10733300 \\
\hline $\mathrm{C}$ & 1.44818400 & 3.14877800 & 0.18414200 & $\mathrm{C}$ & 1.59583900 & -1.62461800 & -0.89621700 \\
\hline $\mathrm{C}$ & 1.03399200 & 1.78275000 & 0.19634800 & $\mathrm{O}$ & 1.25135100 & -2.82600900 & -0.75727600 \\
\hline $\mathrm{C}$ & 1.98595700 & 0.75560500 & 0.02189300 & $\mathrm{O}$ & 2.07756500 & -1.13295400 & -2.04584600 \\
\hline $\mathrm{C}$ & 3.28897700 & 1.14095400 & -0.30760200 & $\mathrm{C}$ & 1.92963000 & -1.99309800 & -3.19514200 \\
\hline $\mathrm{C}$ & 3.65040800 & 2.48570600 & -0.39946700 & $\mathrm{H}$ & 0.87702000 & -2.25113100 & -3.33620100 \\
\hline $\mathrm{C}$ & 2.76706000 & 3.50928600 & -0.12480300 & $\mathrm{H}$ & 2.30447500 & -1.40985800 & -4.03605700 \\
\hline $\mathrm{C}$ & 0.31990900 & 3.94509700 & 0.56262900 & $\mathrm{H}$ & 2.51501100 & -2.90637800 & -3.06595500 \\
\hline $\mathrm{C}$ & -0.72093800 & 3.09245800 & 0.75316500 & $\mathrm{C}$ & 1.72483000 & -0.70589700 & 0.31563300 \\
\hline $\mathrm{H}$ & 4.05566600 & 0.39422300 & -0.47811400 & $\mathrm{H}$ & 0.74642700 & -0.75744800 & 0.98522000 \\
\hline $\mathrm{H}$ & 3.09232600 & 4.54397200 & -0.14192700 & $\mathrm{C}$ & 2.72686800 & -1.27822100 & 1.38204200 \\
\hline $\mathrm{H}$ & 0.29809100 & 5.01904400 & 0.68818900 & $\mathrm{H}$ & 3.68500100 & -1.45898900 & 0.87836200 \\
\hline $\mathrm{H}$ & -1.73419600 & 3.32849400 & 1.02657100 & $\mathrm{C}$ & 2.28703400 & -2.54521600 & 2.12583400 \\
\hline $\mathrm{N}$ & -0.35212700 & 1.75985900 & 0.51851700 & $\mathrm{H}$ & 1.31625300 & -2.36171500 & 2.60783900 \\
\hline $\mathrm{P}$ & -1.55732600 & 0.48948300 & 0.18878900 & $\mathrm{H}$ & 2.12541200 & -3.35901900 & 1.41380300 \\
\hline
\end{tabular}




\begin{tabular}{|c|c|c|c|c|c|c|c|}
\hline $\mathrm{C}$ & 3.31495200 & -2.96365900 & 3.18376100 & $\mathrm{H}$ & -1.77969100 & 1.47234000 & 2.80499200 \\
\hline $\mathrm{H}$ & 3.47248600 & -2.17327400 & 3.92837400 & $\mathrm{H}$ & -2.72625300 & 0.29290200 & 3.73109500 \\
\hline $\mathrm{H}$ & 2.98318500 & -3.86216300 & 3.71520500 & $\mathrm{C}$ & -4.17462200 & 0.89730400 & 1.51788500 \\
\hline \multirow[t]{2}{*}{$\mathrm{H}$} & 4.28709400 & -3.18832300 & 2.72780400 & $\mathrm{H}$ & -4.75913000 & 0.74679200 & 2.43505100 \\
\hline & & & & $\mathrm{H}$ & -3.94873200 & 1.96651100 & 1.45828500 \\
\hline \multirow[t]{6}{*}{99} & & & & $\mathrm{H}$ & -4.81951500 & 0.63019700 & 0.67740900 \\
\hline & & & & $\mathrm{F}$ & 4.02244900 & 3.13586700 & -1.71091700 \\
\hline & & & & $\mathrm{Rh}$ & -0.07619100 & -1.40511100 & 0.03598600 \\
\hline & & & & $\mathrm{Cl}$ & -1.20470900 & -3.19526300 & -0.94359500 \\
\hline & & & & $\mathrm{H}$ & -1.21383300 & -0.20577700 & 2.96199700 \\
\hline & & & & $\mathrm{C}$ & 2.81566200 & -1.99527500 & 0.16687400 \\
\hline $\mathrm{C}$ & 0.55709500 & 3.19049000 & -0.67600700 & $\mathrm{O}$ & 1.78489600 & -2.51120600 & -0.26198600 \\
\hline $\mathrm{C}$ & 0.45091400 & 1.89122300 & -0.11206900 & $\mathrm{O}$ & 4.02163900 & -2.40014900 & -0.21462700 \\
\hline $\mathrm{C}$ & 1.60569400 & 1.10401800 & 0.12310900 & $\mathrm{C}$ & 4.06101100 & -3.48740900 & -1.16957200 \\
\hline $\mathrm{C}$ & 2.78784600 & 1.55249100 & -0.47756900 & $\mathrm{H}$ & 3.54030500 & -3.19981100 & -2.08518000 \\
\hline $\mathrm{C}$ & 2.83863000 & 2.77291100 & -1.16478500 & $\mathrm{H}$ & 5.11980200 & -3.66010300 & -1.35846200 \\
\hline $\mathrm{C}$ & 1.76984600 & 3.64168000 & -1.22550300 & $\mathrm{H}$ & 3.58763300 & -4.37587700 & -0.74620100 \\
\hline $\mathrm{C}$ & -0.72304900 & 3.82165200 & -0.52009400 & $\mathrm{C}$ & 1.65663300 & 0.06267500 & 1.23281500 \\
\hline $\mathrm{C}$ & -1.55303800 & 2.90239000 & 0.04611000 & $\mathrm{C}$ & 2.86173700 & 1.64867500 & 2.94478700 \\
\hline $\mathrm{H}$ & 3.71903500 & 1.00888100 & -0.37054600 & $\mathrm{H}$ & 3.03638800 & 2.41752300 & 2.18625000 \\
\hline $\mathrm{H}$ & 1.87466000 & 4.62166800 & -1.67840600 & $\mathrm{H}$ & 2.71528700 & 2.15304200 & 3.90628000 \\
\hline $\mathrm{H}$ & -0.98149000 & 4.83665400 & -0.79088700 & $\mathrm{H}$ & 3.77157300 & 1.04391700 & 3.03182100 \\
\hline $\mathrm{H}$ & -2.59810900 & 3.00283500 & 0.28868600 & $\mathrm{C}$ & 2.85180300 & -0.91302900 & 1.21845700 \\
\hline $\mathrm{N}$ & -0.89879100 & 1.68430000 & 0.27915700 & $\mathrm{H}$ & 3.80819900 & -0.38957000 & 1.16649100 \\
\hline$P$ & -1.72561800 & 0.08675000 & 0.08116800 & $\mathrm{H}$ & 2.85567800 & -1.44868900 & 2.17901600 \\
\hline $\mathrm{C}$ & -2.71544000 & 0.24154300 & -1.57784600 & $\mathrm{C}$ & 1.62756800 & 0.80042500 & 2.61854000 \\
\hline $\mathrm{C}$ & -2.91055300 & 0.01960900 & 1.59452200 & $\mathrm{H}$ & 1.48859600 & 1900 & 3.39957100 \\
\hline $\mathrm{C}$ & -3.49351400 & 1.55106300 & -1.83232100 & $\mathrm{H}$ & 0.73700400 & 1.43447400 & 2.63776000 \\
\hline $\mathrm{H}$ & -2.83313900 & 2.40585200 & -1.98746300 & $\mathrm{H}$ & 0.69621600 & -0.55522100 & 1.39115900 \\
\hline $\mathrm{H}$ & -4.07076100 & 1.41314500 & -2.75599200 & & & & \\
\hline $\mathrm{H}$ & -4.20999300 & 1.79806700 & -1.04428200 & 100 & & & \\
\hline $\mathrm{C}$ & -1.66539400 & 0.09354100 & -2.70206100 & & & & \\
\hline $\mathrm{H}$ & -2.17840600 & 0.16374900 & -3.67083800 & & & & \\
\hline $\mathrm{H}$ & -0.91600300 & 0.89242100 & -2.66424500 & & & & \\
\hline $\mathrm{H}$ & -1.15120300 & -0.86864600 & -2.65044900 & & & & \\
\hline $\mathrm{C}$ & -3.72306300 & -0.92808000 & -1.65798900 & & & & \\
\hline $\mathrm{H}$ & -3.26221800 & -1.89332600 & -1.44120900 & $\mathrm{C}$ & -1.37645200 & 3.38248900 & 0.23265700 \\
\hline $\mathrm{H}$ & -4.57847600 & -0.77592100 & -0.99162700 & $\mathrm{C}$ & -1.17195600 & 2.01744000 & -0.06085700 \\
\hline $\mathrm{H}$ & -4.11548700 & -0.96771300 & -2.68270800 & $\mathrm{C}$ & -0.00948300 & 1.44190600 & -0.60278700 \\
\hline $\mathrm{C}$ & -3.33368200 & -1.45890200 & 1.75948600 & $\mathrm{C}$ & 0.98408800 & 2.38160300 & -0.90213200 \\
\hline $\mathrm{H}$ & -3.95769700 & -1.81225800 & 0.93522700 & $\mathrm{C}$ & 0.79676400 & 3.75294000 & -0.63758300 \\
\hline $\mathrm{H}$ & -2.46382400 & -2.11879500 & 1.82024700 & $\mathrm{C}$ & -0.34732600 & 4.29132100 & -0.07991900 \\
\hline $\mathrm{H}$ & -3.91627400 & -1.55582900 & 2.68537400 & $\mathrm{C}$ & -2.68098800 & 3.47607600 & 0.83351600 \\
\hline $\mathrm{C}$ & -2.09875600 & 0.42647100 & 2.84040700 & $\mathrm{C}$ & -3.20678800 & 2.21234800 & 0.88762400 \\
\hline
\end{tabular}




\begin{tabular}{|c|c|c|c|c|c|c|c|}
\hline $\mathrm{H}$ & 1.93737300 & 2.10814300 & -1.33971200 & $\mathrm{C}$ & 1.61819100 & -0.51050100 & -2.46039700 \\
\hline $\mathrm{H}$ & -0.42207600 & 5.35782900 & 0.10342900 & $\mathrm{H}$ & 1.76481400 & 0.53764400 & -2.69609000 \\
\hline $\mathrm{H}$ & -3.17699100 & 4.37512100 & 1.17601400 & $\mathrm{H}$ & 1.16707100 & -1.12347600 & -3.23489400 \\
\hline $\mathrm{H}$ & -4.16563500 & 1.89243800 & 1.26370200 & $\mathrm{H}$ & -0.81787900 & -0.21946000 & -2.08279000 \\
\hline $\mathrm{N}$ & -2.31002500 & 1.29802700 & 0.32327800 & $\mathrm{C}$ & 4.52586100 & -1.06191000 & -0.44713800 \\
\hline $\mathrm{P}$ & -2.11030100 & -0.42832900 & 0.29160200 & $\mathrm{H}$ & 4.48710100 & -2.16030200 & -0.42535000 \\
\hline $\mathrm{C}$ & -3.67050700 & -1.12288500 & -0.56250500 & $\mathrm{H}$ & 5.01573800 & -0.79363000 & -1.38906200 \\
\hline $\mathrm{C}$ & -1.97113500 & -0.91552200 & 2.14166300 & $\mathrm{C}$ & 2.19865200 & -1.10867200 & -1.37528300 \\
\hline $\mathrm{C}$ & -4.95233500 & -0.35786200 & -0.17170000 & $\mathrm{H}$ & 2.12424100 & -2.19230400 & -1.31123300 \\
\hline $\mathrm{H}$ & -4.92566100 & 0.68020100 & -0.51305700 & $\mathrm{H}$ & 2.73485900 & -0.66709800 & 0.65926100 \\
\hline $\mathrm{H}$ & -5.80312000 & -0.84442900 & -0.66481300 & & & & \\
\hline $\mathrm{H}$ & -5.15064200 & -0.37112300 & 0.90348700 & 101 & & & \\
\hline $\mathrm{C}$ & -3.46127400 & -0.96610800 & -2.08481800 & & & & \\
\hline $\mathrm{H}$ & -4.37802800 & -1.28582400 & -2.59674200 & & & & \\
\hline $\mathrm{H}$ & -3.26792100 & 0.07490500 & -2.36702300 & & & & \\
\hline $\mathrm{H}$ & -2.63555200 & -1.58812500 & -2.43863700 & & & & \\
\hline $\mathrm{C}$ & -3.84075500 & -2.62143600 & -0.23705400 & & & & \\
\hline $\mathrm{H}$ & -2.93175100 & -3.19110500 & -0.44813700 & $\mathrm{C}$ & 2.89510500 & 2.67526400 & -0.83169300 \\
\hline $\mathrm{H}$ & -4.13497200 & -2.78794300 & 0.80382500 & $\mathrm{C}$ & 2.08320700 & 1.61276500 & -0.39324400 \\
\hline $\mathrm{H}$ & -4.64531000 & -3.01940400 & -0.86859300 & $\mathrm{C}$ & 0.89093400 & 1.70634200 & 0.33409700 \\
\hline $\mathrm{C}$ & -1.52466100 & -2.38445300 & 2.29520500 & $\mathrm{C}$ & 0.51353600 & 3.01701600 & 0.64192500 \\
\hline $\mathrm{H}$ & -2.28312600 & -3.09370900 & 1.95754900 & $\mathrm{C}$ & 1.31023300 & 4.10376000 & 0.22041600 \\
\hline $\mathrm{H}$ & -0.60762700 & -2.60015600 & 1.74215200 & $\mathrm{C}$ & 2.48089600 & 3.98430700 & -0.50913600 \\
\hline $\mathrm{H}$ & -1.34112700 & -2.57529000 & 3.36057400 & $\mathrm{C}$ & 3.99897700 & 2.06644800 & -1.53092400 \\
\hline $\mathrm{C}$ & -0.87093700 & -0.00422800 & 2.73312600 & $\mathrm{C}$ & 3.82899800 & 0.70547200 & -1.48066600 \\
\hline $\mathrm{H}$ & -1.12765500 & 1.05716700 & 2.67222000 & $\mathrm{H}$ & -0.38322900 & 3.24779600 & 1.21057000 \\
\hline $\mathrm{H}$ & -0.73890500 & -0.26096000 & 3.79155700 & $\mathrm{H}$ & 3.03347700 & 4.87059200 & -0.80223400 \\
\hline $\mathrm{C}$ & -3.27823500 & -0.69093100 & 2.92655700 & $\mathrm{H}$ & 4.82370000 & 2.57302600 & -2.01563600 \\
\hline $\mathrm{H}$ & -3.08986100 & -0.91125800 & 3.98521600 & $\mathrm{H}$ & 4.45720900 & -0.07429100 & -1.88425900 \\
\hline $\mathrm{H}$ & -3.62635100 & 0.34458200 & 2.86947700 & $\mathrm{~N}$ & 2.65510400 & 0.40155100 & -0.78475400 \\
\hline $\mathrm{H}$ & -4.08327000 & -1.35431100 & 2.59767000 & $\mathrm{P}$ & 1.79877100 & -0.99850700 & -0.21065200 \\
\hline $\mathrm{F}$ & 1.81865000 & 4.58784400 & -0.95683500 & $\mathrm{C}$ & 2.94590500 & -1.77226700 & 1.10556800 \\
\hline $\mathrm{Rh}$ & -0.07354900 & -0.60118900 & -0.82262900 & $\mathrm{C}$ & 1.52648600 & -2.09703600 & -1.73947500 \\
\hline $\mathrm{Cl}$ & -0.28058300 & -3.05065500 & -0.98593000 & $\mathrm{C}$ & 4.43057100 & -1.77059600 & 0.68955400 \\
\hline $\mathrm{H}$ & 0.09454000 & -0.14968300 & 2.23632100 & $\mathrm{H}$ & 4.80607600 & -0.75916100 & 0.51177100 \\
\hline $\mathrm{C}$ & 5.39737400 & -0.61944600 & 0.71526600 & $\mathrm{H}$ & 5.01615400 & -2.20114600 & 1.51176100 \\
\hline $\mathrm{O}$ & 4.99068000 & -0.19115500 & 1.77393500 & $\mathrm{H}$ & 4.62586400 & -2.37751800 & -0.19867900 \\
\hline $\mathrm{O}$ & 6.70524100 & -0.78928700 & 0.42889500 & $\mathrm{C}$ & 2.78392100 & -0.87250000 & 2.35403900 \\
\hline $\mathrm{C}$ & 7.61759700 & -0.44504300 & 1.48551000 & $\mathrm{H}$ & 3.45947600 & -1.23214300 & 3.14086300 \\
\hline $\mathrm{H}$ & 7.42580100 & -1.05450600 & 2.37270900 & $\mathrm{H}$ & 3.03782600 & 0.17234900 & 2.14728100 \\
\hline $\mathrm{H}$ & 8.61298600 & -0.64674200 & 1.08894400 & $\mathrm{H}$ & 1.76417000 & -0.91997700 & 2.75569800 \\
\hline $\mathrm{H}$ & 7.51539200 & 0.61046600 & 1.75107300 & $\mathrm{C}$ & 2.49268400 & -3.20353100 & 1.45712900 \\
\hline $\mathrm{C}$ & 3.10701300 & -0.47283900 & -0.35537800 & $\mathrm{H}$ & 1.42947800 & -3.24559600 & 1.71223300 \\
\hline $\mathrm{H}$ & 3.14639700 & 0.61295200 & -0.46945200 & $\mathrm{H}$ & 2.69569200 & -3.91248500 & 0.64827300 \\
\hline
\end{tabular}




\begin{tabular}{|c|c|c|c|c|c|c|c|}
\hline $\mathrm{H}$ & 3.06314800 & -3.54000500 & 2.33230200 & $\mathrm{C}$ & 1.18624000 & 1.03083000 & 0.77436800 \\
\hline $\mathrm{C}$ & 0.47950700 & -3.18324000 & -1.41248200 & $\mathrm{C}$ & 2.38857200 & 1.59133200 & 0.24419300 \\
\hline $\mathrm{H}$ & 0.83585100 & -3.89766400 & -0.66665300 & $\mathrm{C}$ & 2.40837700 & 2.74434900 & -0.52082000 \\
\hline $\mathrm{H}$ & -0.45334000 & -2.75831500 & -1.03571700 & $\mathrm{C}$ & 1.27885500 & 3.52191600 & -0.75822200 \\
\hline $\mathrm{H}$ & 0.26284500 & -3.74139100 & -2.33232400 & $\mathrm{C}$ & -1.25199800 & 3.57407600 & -0.23071400 \\
\hline $\mathrm{C}$ & 0.98066600 & -1.18636500 & -2.86053200 & $\mathrm{C}$ & -2.06874400 & 2.64905700 & 0.34061300 \\
\hline $\mathrm{H}$ & 1.69818100 & -0.41425500 & -3.15241900 & $\mathrm{H}$ & 3.35127200 & 1.17311600 & 0.51244500 \\
\hline $\mathrm{H}$ & 0.77301700 & -1.80771700 & -3.74053500 & $\mathrm{H}$ & 1.36296200 & 4.49407300 & -1.23159700 \\
\hline $\mathrm{C}$ & 2.82398900 & -2.76881000 & -2.23491300 & $\mathrm{H}$ & -1.53358900 & 4.57003600 & -0.54678100 \\
\hline $\mathrm{H}$ & 2.59305600 & -3.33092800 & -3.14855000 & $\mathrm{H}$ & -3.12946600 & 2.71228800 & 0.53644600 \\
\hline $\mathrm{H}$ & 3.60283100 & -2.04407000 & -2.49161600 & $\mathrm{~N}$ & -1.37698400 & 1.47305700 & 0.66806600 \\
\hline $\mathrm{H}$ & 3.23152600 & -3.47943000 & -1.51130800 & $\mathrm{P}$ & -1.83350200 & -0.13397300 & 0.01743400 \\
\hline $\mathrm{F}$ & 0.88411500 & 5.34817200 & 0.55474000 & $\mathrm{C}$ & -2.89606500 & 0.09306300 & -1.58526100 \\
\hline $\mathrm{Rh}$ & 0.00187100 & -0.05720900 & 0.81188600 & $\mathrm{C}$ & -2.90085600 & -0.87199000 & 1.42313400 \\
\hline $\mathrm{Cl}$ & -0.99989100 & -2.05962000 & 1.76645800 & $\mathrm{C}$ & -4.30854700 & 0.68159100 & -1.41144400 \\
\hline $\mathrm{H}$ & 0.04871200 & -0.69726200 & -2.56970000 & $\mathrm{H}$ & -4.30568900 & 1.67806400 & -0.96064400 \\
\hline $\mathrm{C}$ & -6.00310500 & 0.11764500 & -0.22860600 & $\mathrm{H}$ & -4.76711100 & 0.78051900 & -2.40420500 \\
\hline $\mathrm{O}$ & -6.50803400 & 0.01080400 & 0.86770700 & $\mathrm{H}$ & -4.96272900 & 0.03369800 & -0.82137400 \\
\hline $\mathrm{O}$ & -6.66776000 & -0.11953800 & -1.38252000 & $\mathrm{C}$ & -2.08152200 & 0.98321600 & -2.55136800 \\
\hline $\mathrm{C}$ & -8.04604800 & -0.49567900 & -1.23053400 & $\mathrm{H}$ & -2.59451300 & 0.99709500 & -3.52230800 \\
\hline $\mathrm{H}$ & -8.60828500 & 0.29570000 & -0.72712800 & $\mathrm{H}$ & -1.99514700 & 2.01462600 & -2.20429400 \\
\hline $\mathrm{H}$ & -8.42152300 & -0.64777300 & -2.24302500 & $\mathrm{H}$ & -1.07497200 & 0.57978500 & -2.70272100 \\
\hline $\mathrm{H}$ & -8.13100600 & -1.41587100 & -0.64633600 & $\mathrm{C}$ & -3.01441400 & -1.29971700 & -2.25235400 \\
\hline $\mathrm{C}$ & -3.65949400 & 0.36443300 & 0.70738700 & $\mathrm{H}$ & -2.04053200 & -1.78052200 & -2.36935200 \\
\hline $\mathrm{H}$ & -3.55709800 & -0.70090300 & 0.94093000 & $\mathrm{H}$ & -3.66442900 & -1.98426500 & -1.70462800 \\
\hline $\mathrm{C}$ & -1.41551900 & 0.28618000 & -0.58776000 & $\mathrm{H}$ & -3.45328500 & -1.15743300 & -3.24884600 \\
\hline $\mathrm{H}$ & -1.80701000 & -0.67255400 & -0.93574200 & $\mathrm{C}$ & -3.63399700 & -2.14067700 & 0.94078100 \\
\hline $\mathrm{H}$ & -1.10672500 & 0.92791000 & -1.41307300 & $\mathrm{H}$ & -4.44550500 & -1.91474200 & 0.24422800 \\
\hline $\mathrm{H}$ & -1.73893900 & 0.88822000 & 1.47801600 & $\mathrm{H}$ & -2.94768800 & -2.85053700 & 0.46802900 \\
\hline $\mathrm{C}$ & -4.57697200 & 0.55777000 & -0.50506200 & $\mathrm{H}$ & -4.08427800 & -2.63505100 & 1.81131200 \\
\hline $\mathrm{H}$ & -4.61946200 & 1.62001700 & -0.78940500 & $\mathrm{C}$ & -1.95064500 & -1.28278100 & 2.56557300 \\
\hline $\mathrm{H}$ & -4.20894900 & 0.02395200 & -1.38803700 & $\mathrm{H}$ & -1.44914100 & -0.41530800 & 3.00434200 \\
\hline $\mathrm{C}$ & -2.26390400 & 0.96328100 & 0.47437000 & $\mathrm{H}$ & -2.54074700 & -1.75698400 & 3.36084400 \\
\hline $\mathrm{H}$ & -2.30701200 & 2.04435800 & 0.29901400 & $\mathrm{C}$ & -3.90913000 & 0.15110900 & 1.98568800 \\
\hline \multirow[t]{2}{*}{$\mathrm{H}$} & -4.12317900 & 0.83361800 & 1.58115900 & $\mathrm{H}$ & -4.45832700 & -0.31408400 & 2.81482500 \\
\hline & & & & $\mathrm{H}$ & -3.40064300 & 1.03475200 & 2.38438300 \\
\hline \multirow[t]{6}{*}{102} & & & & $\mathrm{H}$ & -4.64797500 & 0.47436800 & 1.24851700 \\
\hline & & & & $\mathrm{F}$ & 3.60550600 & 3.19314600 & -0.96575800 \\
\hline & & & & $\mathrm{Rh}$ & 0.25093400 & -0.70894700 & -0.48481800 \\
\hline & & & & $\mathrm{Cl}$ & -0.18407700 & -3.01276100 & -0.78857300 \\
\hline & & & & $\mathrm{H}$ & -1.19924500 & -1.99936600 & 2.22131000 \\
\hline & & & & $\mathrm{C}$ & 3.08654400 & -1.57812900 & -0.08847700 \\
\hline $\mathrm{C}$ & 800 & 3.00 & -0.30942600 & $\mathrm{O}$ & 2.33627900 & -1.16001900 & -0.97032000 \\
\hline $\mathrm{C}$ & 200 & 1.709 & 0.31396500 & $\mathrm{O}$ & 4.37613100 & -1.81348000 & -0.33483200 \\
\hline
\end{tabular}




\begin{tabular}{|c|c|c|c|c|c|c|c|}
\hline $\mathrm{C}$ & 4.82754400 & -1.57818000 & -1.68572100 & $\mathrm{H}$ & -4.70292500 & 1.46713800 & -0.59565800 \\
\hline $\mathrm{H}$ & 4.68547500 & -0.52907100 & -1.95557600 & $\mathrm{H}$ & -3.59816400 & 0.77483200 & 0.60448200 \\
\hline $\mathrm{H}$ & 5.88581500 & -1.83803500 & -1.68222000 & $\mathrm{H}$ & -2.95838800 & 1.78798400 & -0.70650200 \\
\hline $\mathrm{H}$ & 4.27288700 & -2.21091300 & -2.38224200 & $\mathrm{C}$ & -3.66007500 & 0.20074500 & -2.84444300 \\
\hline $\mathrm{C}$ & 2.55871000 & -0.62481400 & 2.26070500 & $\mathrm{H}$ & -2.81217900 & 0.80549400 & -3.16623900 \\
\hline $\mathrm{H}$ & 2.53057300 & -1.01809600 & 3.28423300 & $\mathrm{H}$ & -3.75892500 & -0.66932900 & -3.50194700 \\
\hline $\mathrm{C}$ & 1.28872900 & 1.30132600 & 3.27020500 & $\mathrm{H}$ & -4.56746200 & 0.80558400 & -2.97263700 \\
\hline $\mathrm{H}$ & 2.15171900 & 1.97564600 & 3.21268800 & $\mathrm{C}$ & -0.82298300 & -1.57638800 & -3.54420500 \\
\hline $\mathrm{H}$ & 0.38133200 & 1.91308400 & 3.23216500 & $\mathrm{H}$ & -1.64414000 & -1.10061100 & -4.08392600 \\
\hline $\mathrm{H}$ & 1.31612000 & 0.79399700 & 4.24249600 & $\mathrm{H}$ & -0.06855300 & -0.80915600 & -3.34762300 \\
\hline $\mathrm{C}$ & 2.69309900 & -1.86662500 & 1.33981800 & $\mathrm{H}$ & -0.38360000 & -2.33373600 & -4.20680900 \\
\hline $\mathrm{H}$ & 3.45505900 & -2.52687300 & 1.76527800 & $\mathrm{C}$ & -0.07123200 & -3.00765800 & -1.64247100 \\
\hline $\mathrm{H}$ & 1.74137800 & -2.40664100 & 1.29794100 & $\mathrm{H}$ & -0.31318000 & -3.50601900 & -0.69912100 \\
\hline $\mathrm{C}$ & 1.30778400 & 0.27437300 & 2.11401600 & $\mathrm{H}$ & 0.26802700 & -3.77199400 & -2.35320500 \\
\hline $\mathrm{H}$ & 0.44114800 & -0.37409500 & 2.22717300 & $\mathrm{C}$ & -2.37372000 & -3.31120900 & -2.58444100 \\
\hline $\mathrm{H}$ & 3.47694300 & -0.02579100 & 2.21025000 & $\mathrm{H}$ & -1.96991100 & -4.01305600 & -3.32611700 \\
\hline & & & & $\mathrm{H}$ & -2.67346300 & -3.90595800 & -1.71605100 \\
\hline 103 & & & & $\mathrm{H}$ & -3.26545800 & -2.85641500 & -3.02353800 \\
\hline & & & & $\mathrm{F}$ & -0.96832800 & -0.24457000 & 5.72295700 \\
\hline & & & & $\mathrm{Rh}$ & -0.17519500 & 0.84557200 & -0.66614900 \\
\hline & & & & $\mathrm{Cl}$ & -0.78763000 & 2.03866200 & -2.58496400 \\
\hline & $C$ & & & $\mathrm{H}$ & 0.76503400 & -2.32030300 & -1.47596300 \\
\hline & & & & $\mathrm{C}$ & 4.96451000 & -2.59033500 & -0.13232300 \\
\hline $\mathrm{C}$ & -2.45138300 & -1.76150400 & 2.79390600 & $\mathrm{O}$ & 5.86131900 & -1.78937700 & -0.28422400 \\
\hline $\mathrm{C}$ & -1.51753200 & -1.34952500 & 1.80800300 & $\mathrm{O}$ & 4.98496300 & -3.84048800 & -0.64709100 \\
\hline $\mathrm{C}$ & -0.28339800 & -0.77194200 & 2.17782600 & $\mathrm{C}$ & 6.16608800 & -4.17953200 & -1.39221200 \\
\hline $\mathrm{C}$ & -0.15720600 & -0.37796600 & 3.51373900 & $\mathrm{H}$ & 7.05495200 & -4.10590700 & -0.75974200 \\
\hline $\mathrm{C}$ & -1.16458500 & -0.65142100 & 4.44770200 & $\mathrm{H}$ & 6.01692400 & -5.20693700 & -1.72578000 \\
\hline $\mathrm{C}$ & -2.28794100 & -1.39005500 & 4.13874800 & $\mathrm{H}$ & 6.28490700 & -3.51053400 & -2.24874500 \\
\hline $\mathrm{C}$ & -3.40928200 & -2.60542600 & 2.13584700 & $\mathrm{C}$ & 3.49238400 & -0.87493300 & 1.01365800 \\
\hline $\mathrm{C}$ & -3.07919200 & -2.62930600 & 0.81638400 & $\mathrm{H}$ & 3.40041200 & -0.29196900 & 0.08845500 \\
\hline $\mathrm{H}$ & 0.75343900 & 0.08032200 & 3.87973000 & $\mathrm{C}$ & 0.92394900 & -0.84499600 & 1.26823100 \\
\hline $\mathrm{H}$ & -2.99113000 & -1.68061700 & 4.91184000 & $\mathrm{H}$ & 0.94634800 & -0.17138600 & 0.35747200 \\
\hline $\mathrm{H}$ & -4.22414200 & -3.14441200 & 2.60039900 & $\mathrm{H}$ & 0.91208700 & -1.83855600 & 0.80903800 \\
\hline $\mathrm{H}$ & -3.57415100 & -3.14657100 & 0.01289400 & $\mathrm{H}$ & 2.34703000 & 0.42997000 & 2.29211300 \\
\hline $\mathrm{N}$ & -1.95409300 & -1.83094000 & 0.54482200 & $\mathrm{C}$ & 3.69064700 & -2.35230700 & 0.65861400 \\
\hline $\mathrm{P}$ & -1.79122100 & -0.90715600 & -0.99284400 & $\mathrm{H}$ & 3.75101700 & -2.96453100 & 1.57029000 \\
\hline $\mathrm{C}$ & -3.57541400 & -0.21608400 & -1.35872000 & $\mathrm{H}$ & 2.85388400 & -2.76171500 & 0.08159600 \\
\hline $\mathrm{C}$ & -1.28731000 & -2.27302600 & -2.24459100 & $\mathrm{C}$ & 2.28974200 & -0.60504000 & 1.93261800 \\
\hline $\mathrm{C}$ & -4.79403600 & -1.12168200 & -1.07721600 & $\mathrm{H}$ & 2.36135000 & -1.24394200 & 2.82314700 \\
\hline $\mathrm{H}$ & -4.94815000 & -1.30443600 & -0.01302300 & $\mathrm{H}$ & 4.40274500 & -0.51438800 & 1.50401800 \\
\hline $\mathrm{H}$ & -5.68117500 & -0.59268100 & -1.44906300 & $\mathrm{C}$ & 2.91581100 & 4.37084700 & -0.83023500 \\
\hline $\mathrm{H}$ & -4.75587300 & -2.08141500 & -1.60041700 & $\mathrm{C}$ & 2.05294500 & 3.18956800 & -1.33314900 \\
\hline $\mathrm{C}$ & -3.70446800 & 1.03136100 & -0.45617300 & $\mathrm{C}$ & 0.02352200 & 5.02828200 & 1.23311700 \\
\hline
\end{tabular}




\begin{tabular}{|c|c|c|c|c|c|c|c|}
\hline $\mathrm{C}$ & 1.48935200 & 2.33322500 & -0.21921800 & $\mathrm{H}$ & 3.61543000 & -2.82244200 & -2.30265500 \\
\hline $\mathrm{C}$ & -0.47541600 & 3.87985600 & 0.32338900 & $\mathrm{H}$ & 3.42035500 & -3.07957800 & -0.57175000 \\
\hline $\mathrm{C}$ & 0.34274900 & 2.62850300 & 0.52149500 & $\mathrm{C}$ & 1.93146200 & -0.70308500 & -2.63650200 \\
\hline $\mathrm{H}$ & 3.67013500 & 4.00299300 & -0.11830600 & $\mathrm{H}$ & 2.52355400 & -1.01297300 & -3.50781800 \\
\hline $\mathrm{H}$ & 1.25505000 & 3.56911800 & -1.97442500 & $\mathrm{H}$ & 0.94476900 & -1.17290100 & -2.72038500 \\
\hline $\mathrm{H}$ & -0.26085900 & 4.81550200 & 2.27357400 & $\mathrm{H}$ & 1.80264900 & 0.38151800 & -2.67291600 \\
\hline $\mathrm{H}$ & -1.52934300 & 3.68216000 & 0.55384400 & $\mathrm{C}$ & 4.03180500 & -0.42625200 & -1.27059900 \\
\hline $\mathrm{H}$ & 3.47755200 & 4.75296200 & -1.69372800 & $\mathrm{H}$ & 3.92811000 & 0.65601700 & -1.17430200 \\
\hline $\mathrm{H}$ & 2.67727500 & 2.55360800 & -1.97382500 & $\mathrm{H}$ & 4.64583400 & -0.80058600 & -0.44525100 \\
\hline $\mathrm{H}$ & -0.52176600 & 5.93901200 & 0.94853300 & $\mathrm{H}$ & 4.58096700 & -0.62857500 & -2.19941200 \\
\hline $\mathrm{H}$ & 2.23522200 & 1.69268600 & 0.25170000 & $\mathrm{C}$ & 3.25587200 & 0.49497900 & 2.06816900 \\
\hline $\mathrm{H}$ & -0.44101600 & 4.18749400 & -0.72378100 & $\mathrm{H}$ & 4.07590900 & 0.59598800 & 1.35254900 \\
\hline $\mathrm{H}$ & 0.25151500 & 2.18852100 & 1.51884300 & $\mathrm{H}$ & 2.65077800 & 1.40203600 & 2.00273400 \\
\hline $\mathrm{C}$ & 2.16698000 & 5.56012600 & -0.19289600 & $\mathrm{H}$ & 3.69328700 & 0.43387100 & 3.07330000 \\
\hline $\mathrm{H}$ & 2.89332800 & 6.37836500 & -0.10178400 & $\mathrm{C}$ & 1.28933200 & -0.82287100 & 2.89200100 \\
\hline $\mathrm{H}$ & 1.40029500 & 5.92481800 & -0.89211900 & $\mathrm{H}$ & 0.68725600 & -1.73353000 & 2.82142700 \\
\hline $\mathrm{C}$ & 1.53759200 & 5.31327000 & 1.20034700 & $\mathrm{H}$ & 1.75189800 & -0.79881400 & 3.88716200 \\
\hline $\mathrm{H}$ & 1.70757600 & 6.19728200 & 1.82897300 & $\mathrm{C}$ & 3.32417900 & -2.00459700 & 2.00459900 \\
\hline \multirow[t]{2}{*}{$\mathrm{H}$} & 2.07640000 & 4.49403900 & 1.69505300 & $\mathrm{H}$ & 3.78392100 & -1.94654600 & 2.99954700 \\
\hline & & & & $\mathrm{H}$ & 2.78748700 & -2.95615200 & 1.96573400 \\
\hline \multirow[t]{6}{*}{104} & & & & $\mathrm{H}$ & 4.13893800 & -2.03142400 & 1.27672300 \\
\hline & & & & $\mathrm{F}$ & -4.98508200 & -2.33350200 & -1.53399800 \\
\hline & & & & $\mathrm{Rh}$ & 0.69692800 & 1.43635000 & -0.17499400 \\
\hline & & & & $\mathrm{Cl}$ & 2.55714400 & 2.69489600 & -0.72016200 \\
\hline & & & & $\mathrm{H}$ & 0.61885400 & 0.03861000 & 2.81227200 \\
\hline & & & & $\mathrm{C}$ & -2.65102300 & 1.17272600 & 1.22011700 \\
\hline $\mathrm{C}$ & -1.60355900 & -3.00415100 & -0.44089500 & $\mathrm{O}$ & -3.72756500 & 1.71337000 & 1.09886500 \\
\hline $\mathrm{C}$ & -1.11254800 & -1.67548800 & -0.25353200 & $\mathrm{O}$ & -2.15062900 & 0.74598000 & 2.39337200 \\
\hline $\mathrm{C}$ & -1.99316500 & -0.58012000 & -0.39079000 & $\mathrm{C}$ & -3.00069300 & 0.93986300 & 3.54051600 \\
\hline $\mathrm{C}$ & -3.29052700 & -0.84885100 & -0.84182200 & $\mathrm{H}$ & -3.94133300 & 0.39947100 & 3.40939500 \\
\hline $\mathrm{C}$ & -3.71747400 & -2.15238500 & -1.10162600 & $\mathrm{H}$ & -2.44101600 & 0.54196300 & 4.38646200 \\
\hline $\mathrm{C}$ & -2.91202500 & -3.24920200 & -0.88131600 & $\mathrm{H}$ & -3.21078700 & 2.00291800 & 3.68092700 \\
\hline $\mathrm{C}$ & -0.56060100 & -3.90786300 & -0.06349200 & $\mathrm{C}$ & -1.90680800 & 1.90543700 & -1.06997500 \\
\hline $\mathrm{C}$ & 0.50681700 & -3.14870100 & 0.29885300 & $\mathrm{H}$ & -2.98349700 & 1.95202700 & -1.27407800 \\
\hline $\mathrm{H}$ & -4.01269900 & -0.04637300 & -0.93903100 & $\mathrm{C}$ & -1.63869000 & 4.31158700 & -1.86398000 \\
\hline $\mathrm{H}$ & -3.28886300 & -4.25531400 & -1.03129400 & $\mathrm{H}$ & -2.69991100 & 4.36975900 & -2.13451800 \\
\hline $\mathrm{H}$ & -0.61226900 & -4.98799500 & -0.05058200 & $\mathrm{H}$ & -1.30436600 & 5.31629100 & -1.58602400 \\
\hline $\mathrm{H}$ & 1.47538900 & -3.47678300 & 0.63092200 & $\mathrm{H}$ & -1.07712100 & 4.00430100 & -2.75349800 \\
\hline $\mathrm{N}$ & 0.23869700 & -1.77922300 & 0.17329800 & $\mathrm{C}$ & -1.69594200 & 0.84954000 & 0.04513500 \\
\hline $\mathrm{P}$ & 1.54906000 & -0.56881500 & 0.11428000 & $\mathrm{H}$ & -1.41614200 & 1.55912500 & -1.98532700 \\
\hline $\mathrm{C}$ & 2.66918400 & -1.14717200 & -1.35352600 & $\mathrm{H}$ & -0.71869800 & 0.84650200 & 0.66742100 \\
\hline $\mathrm{C}$ & 2.40768500 & -0.77555500 & 1.82955700 & $\mathrm{C}$ & -1.42945100 & 3.32375700 & -0.71235100 \\
\hline $\mathrm{C}$ & 2.93266200 & -2.66394100 & -1.45805900 & $\mathrm{H}$ & -0.35149300 & 3.35194600 & -0.44366300 \\
\hline $\mathrm{H}$ & 2.02460500 & -3.23570400 & -1.66101100 & $\mathrm{H}$ & -1.95944600 & 3.67195000 & 0.18234500 \\
\hline
\end{tabular}


105
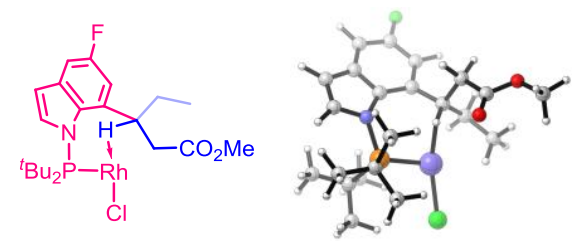

C $\quad 0.09740300$

C $\quad 0.23551000$

C 1.41656500

C 2.33356800

C 2.12865400

C 1.04705200

C $\quad-1.08791100$

C $\quad-1.64433900$

H 3.25972100

H 0.94974700

H $\quad-1.46488100$

$\mathrm{H} \quad-2.55202500$

$\mathrm{N} \quad-0.89663100$

P $\quad-1.62890700$

C $\quad-3.14662200$

C $\quad-2.14482300$

C $\quad-3.99578500$

$\mathrm{H} \quad-3.44349700$

H $\quad-4.84421600$

H $\quad-4.41205200$

C $\quad-2.55401800$

H $\quad-3.37438200$

H $\quad-1.86946500$

H $\quad-2.01329500$

C $\quad-4.07162000$

$\mathrm{H} \quad-3.53344400$

$\mathrm{H} \quad-4.61426800$

H $\quad-4.82137300$

C $\quad-2.34585600$

H $\quad-3.16031000$

H -1.44095200

$\mathrm{H} \quad-2.59261400$

C $\quad-0.95868000$

H $\quad-0.79848800$

H $\quad-1.17907400$

C $\quad-3.42670700$

H $\quad-3.61600800$

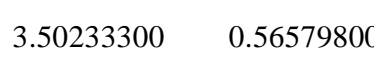

2.11261200

1.63067000

2.59026500

3.95593300

4.44023300

3.65695400

2.42214800

2.29136300

5.49949700

4.57494800

2.14698800

1.44515600

$-0.09280400$

0.44491900

$-0.93650500$

1.60794200

2.54927400

1.75040900

1.40756600

0.88690600

1.20552300

1.73525200

0.07130500

$-0.77198800$

$-1.64737700$

$-1.04410700$

$-0.49947200$

$-2.43875600$

$-2.62072300$

$-2.88628100$

$-2.95271900$

$-0.79477500$

0.24256400

$-1.38357400$

$-0.40471200$

$-1.01060000$
0.26834700

$-0.33714100$

$-0.78187700$

$-0.56969100$

0.13464800

1.35371800

1.47695200

$-1.25997600$

0.34705600

1.78364800

1.98508200

0.80722400

0.25923600

$-0.81224300$

1.91378100

$-0.25943000$

$-0.22405600$

$-0.94103800$

0.73155600

$-2.16872900$

$-2.82559300$

$-2.05474200$

$-2.65501600$

$-1.02831500$

$-1.39662100$

$-0.11734600$

$-1.78240900$

1.60726300

0.90132700

1.18984100

2.54551500

2.89192400

3.20159200

3.79170800

2.58844700

3.48392000
H $\quad-3.34170900$

0.63148200

2.92715900

H $\quad-4.30997700$

$-0.49217500$

1.95017500

$\mathrm{Rh}$

$\mathrm{Cl}$

$\mathrm{C}$

$\mathrm{H}$

\section{C}

O

$\mathrm{O}$

3.06595400

4.81692000

$-1.02735600$

$-0.14119600$

$-1.26201100$

$-0.84358600$

$-1.40841600$

$-2.86316700$

$-1.92091500$

$-0.02872700$

$-1.18215000$

2.46386400

3.18688900

$-1.41524200$

1.21257500

$-2.10353200$

1.70699900

O

2.31753800

$-1.82252100$

1.05789500

C $\quad 4.75182700$

$-3.15117200$

1.53422600

H 4.16659300

$-3.88972600$

0.98023600

H $\quad 5.81816400$

$-3.29611800$

1.36104000

H 4.51720500

$-3.23399200$

2.59828100

C $\quad 1.85612500$

0.17408100

$-0.35520900$

H 1.06371400

$-0.47860100$

0.17657800

C 2.55522700

$-1.79909300$

$-1.94631600$

H 1.65206500

$-2.40447500$

$-1.75526200$

H 2.83611900

$-1.99995600$

$-2.98560500$

H 3.34529000

$-2.19469800$

$-1.30171900$

C 2.98009600

0.00772500

0.72850000

H 3.91756900

0.41913500

0.34558600

H 2.69495500

0.59092100

1.61095400

C $\quad 2.35197600$

$-0.28703800$

$-1.75293300$

H $\quad 3.31370400$

0.20924400

$-1.94671800$

$\mathrm{H} \quad 1.65510400$

0.08710000

$-2.50991500$

106
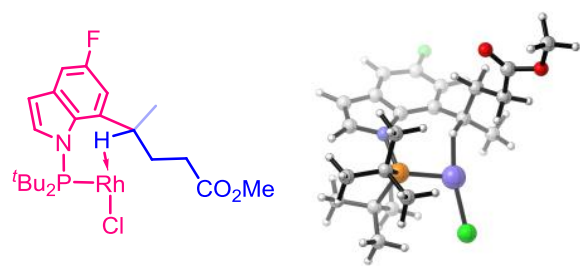

C

$-0.64614400$

3.35739700

0.89179800

C $\quad-0.31057800$

2.08165200

0.34945700

C $\quad 0.74399400$

1.95861500

$-0.58026100$

C $\quad 1.30841000$

3.14692600

$-1.05590200$

C $\quad 0.89841400$

4.39464000

$-0.57905400$

C $\quad-0.04409800$

4.53232800

0.41805200

C -1.60122200

3.14244600

1.93735400

C $\quad-1.84713000$

1.80602600

1.98161200

H 2.11844500

3.12405600

$-1.77667600$

H $\quad-0.29380700$

5.50991900

0.81612200

$\mathrm{H} \quad-2.03349700$

3.89380700

2.58409500

$\mathrm{H}$

$-2.51997200$

2.63023400 


\begin{tabular}{|c|c|c|c|c|c|c|c|}
\hline $\mathrm{N}$ & -1.10969500 & 1.10973000 & 1.01331700 & $\mathrm{C}$ & 3.47878000 & -0.81037400 & -0.34592400 \\
\hline $\mathrm{P}$ & -1.61435600 & -0.49138900 & 0.40964200 & $\mathrm{H}$ & 2.74548500 & -1.62764600 & -0.25652400 \\
\hline $\mathrm{C}$ & -3.44206300 & -0.25232100 & -0.17570300 & $\mathrm{H}$ & 3.94559100 & -0.92219700 & -1.32863700 \\
\hline $\mathrm{C}$ & -1.47235400 & -1.60686100 & 1.97425100 & $\mathrm{C}$ & 1.42254800 & 0.65328400 & -0.94970600 \\
\hline $\mathrm{C}$ & -4.35106400 & 0.58407700 & 0.74944200 & $\mathrm{H}$ & 0.90296400 & -0.21057700 & -0.37470100 \\
\hline $\mathrm{H}$ & -4.03188400 & 1.62612200 & 0.81723100 & $\mathrm{H}$ & 2.59230300 & 0.70913500 & 0.89024700 \\
\hline $\mathrm{H}$ & -5.35891200 & 0.57734000 & 0.31489100 & \multirow{7}{*}{107} & \multirow{7}{*}{$\underbrace{F}_{\substack{H \\
1 \\
-R h \\
1 \\
C l}}$} & \multirow[b]{7}{*}{$-k$} & \\
\hline $\mathrm{H}$ & -4.43648400 & 0.17575300 & 1.76005800 & & & & \\
\hline $\mathrm{C}$ & -3.34738500 & 0.47608300 & -1.53495500 & & & & \\
\hline $\mathrm{H}$ & -4.36386200 & 0.65367600 & -1.91030600 & & & & \\
\hline $\mathrm{H}$ & -2.85205400 & 1.44913600 & -1.43985400 & & & & \\
\hline $\mathrm{H}$ & -2.80342600 & -0.11809700 & -2.27337800 & & & & \\
\hline $\mathrm{C}$ & -4.09498800 & -1.63607200 & -0.37803400 & & & & \\
\hline $\mathrm{H}$ & -3.49424900 & -2.29664400 & -1.00647000 & $\mathrm{C}$ & -2.87821300 & 2.71893000 & 0.32266000 \\
\hline $\mathrm{H}$ & -4.30113700 & -2.13790600 & 0.57289400 & $\mathrm{C}$ & -1.76650100 & 1.82591200 & 0.22928600 \\
\hline $\mathrm{H}$ & -5.05989700 & -1.48691700 & -0.87964500 & $\mathrm{C}$ & -0.46687200 & 2.33704600 & 0.03173200 \\
\hline $\mathrm{C}$ & -1.42944500 & -3.07320000 & 1.48607600 & $\mathrm{C}$ & -0.35041400 & 3.71582200 & -0.17727200 \\
\hline $\mathrm{H}$ & -2.35351300 & -3.38060700 & 0.99003500 & $\mathrm{C}$ & -1.46190600 & 4.55970400 & -0.15679100 \\
\hline $\mathrm{H}$ & -0.60928600 & -3.23757800 & 0.78298500 & $\mathrm{C}$ & -2.73219500 & 4.09765600 & 0.11454600 \\
\hline $\mathrm{H}$ & -1.28337100 & -3.72462400 & 2.35759800 & $\mathrm{C}$ & -4.02502000 & 1.94569400 & 0.68706500 \\
\hline $\mathrm{C}$ & -0.12624500 & -1.28103800 & 2.65560800 & $\mathrm{C}$ & -3.61673900 & 0.65271800 & 0.78075500 \\
\hline $\mathrm{H}$ & -0.09542200 & -0.26494000 & 3.05885600 & $\mathrm{H}$ & 0.62562400 & 4.16782300 & -0.32071000 \\
\hline $\mathrm{H}$ & 0.02309700 & -1.98033300 & 3.48787200 & $\mathrm{H}$ & -3.57177000 & 4.78143100 & 0.17884900 \\
\hline $\mathrm{C}$ & -2.60540600 & -1.47581700 & 3.01396100 & $\mathrm{H}$ & -5.02449500 & 2.31547700 & 0.87098100 \\
\hline $\mathrm{H}$ & -2.41739100 & -2.20007800 & 3.81687200 & $\mathrm{H}$ & -4.20522800 & -0.21245500 & 1.02755500 \\
\hline $\mathrm{H}$ & -2.64800600 & -0.49074700 & 3.48651100 & $\mathrm{~N}$ & -2.25377800 & 0.51491700 & 0.48569300 \\
\hline $\mathrm{H}$ & -3.58892900 & -1.70691200 & 2.59731000 & $\mathrm{P}$ & -1.50629000 & -1.06834100 & 0.15877900 \\
\hline $\mathrm{F}$ & 1.49591100 & 5.49615100 & -1.08702400 & $\mathrm{C}$ & -2.55313900 & -1.80644300 & -1.29127200 \\
\hline $\mathrm{Rh}$ & -0.29666400 & -1.13798900 & -1.21266800 & $\mathrm{C}$ & -1.75842600 & -2.01155000 & 1.82135200 \\
\hline $\mathrm{Cl}$ & -1.37215800 & -2.88289400 & -2.24942700 & $\mathrm{C}$ & -4.07793800 & -1.57842100 & -1.22498300 \\
\hline $\mathrm{H}$ & 0.71557800 & -1.40517800 & 1.96675400 & $\mathrm{H}$ & -4.34886100 & -0.52273600 & -1.29601700 \\
\hline $\mathrm{C}$ & 4.52622500 & -1.04580400 & 0.72852300 & $\mathrm{H}$ & -4.52339800 & -2.08946300 & -2.08800600 \\
\hline $\mathrm{O}$ & 4.49412700 & -0.58791000 & 1.85115100 & $\mathrm{H}$ & -4.54285500 & -2.00237800 & -0.33003700 \\
\hline $\mathrm{O}$ & 5.49909700 & -1.86945600 & 0.28553700 & $\mathrm{C}$ & -2.02697300 & -1.11448300 & -2.56904600 \\
\hline $\mathrm{C}$ & 6.50886800 & -2.20688700 & 1.25177400 & $\mathrm{H}$ & -2.60119200 & -1.48180700 & -3.42982700 \\
\hline $\mathrm{H}$ & 6.06384700 & -2.71792000 & 2.10981500 & $\mathrm{H}$ & -2.15511200 & -0.02686800 & -2.52308300 \\
\hline $\mathrm{H}$ & 7.20318100 & -2.86621100 & 0.73038600 & $\mathrm{H}$ & -0.96985700 & -1.33266200 & -2.73889200 \\
\hline $\mathrm{H}$ & 7.02076700 & -1.30646700 & 1.60141400 & $\mathrm{C}$ & -2.29925100 & -3.32618100 & -1.38855600 \\
\hline $\mathrm{C}$ & 2.77387600 & 0.54052500 & -0.17554000 & $\mathrm{H}$ & -1.23654200 & -3.57439200 & -1.40647500 \\
\hline $\mathrm{H}$ & 3.42911000 & 1.35302300 & -0.51364400 & $\mathrm{H}$ & -2.78256700 & -3.87796600 & -0.57561100 \\
\hline $\mathrm{C}$ & 1.63934600 & 0.46318500 & -2.46253100 & $\mathrm{H}$ & -2.74034900 & -3.68441100 & -2.32780200 \\
\hline $\mathrm{H}$ & 2.45465400 & 1.11380300 & -2.80875100 & $\mathrm{C}$ & -0.81900000 & -3.23899700 & 1.80686900 \\
\hline $\mathrm{H}$ & 1.94275100 & -0.56383800 & -2.70333000 & $\mathrm{H}$ & -1.09740400 & -3.97357300 & 1.04735000 \\
\hline $\mathrm{H}$ & 0.74406500 & 0.70930500 & -3.03986800 & $\mathrm{H}$ & 0.21816700 & -2.95049400 & 1.62166500 \\
\hline
\end{tabular}




\begin{tabular}{|c|c|c|c|c|c|c|c|}
\hline $\mathrm{H}$ & -0.87941100 & -3.73081600 & 2.78642600 & $\mathrm{C}$ & 3.90123700 & 1.15705500 & 1.32579500 \\
\hline $\mathrm{C}$ & -1.30192100 & -1.06319400 & 2.95042200 & $\mathrm{C}$ & 2.99600900 & 0.28523700 & 0.77958200 \\
\hline $\mathrm{H}$ & -1.94341700 & -0.18156200 & 3.03996800 & $\mathrm{H}$ & 7.92157200 & 0.32162600 & -2.04928000 \\
\hline $\mathrm{H}$ & -1.33924300 & -1.60691700 & 3.90297200 & $\mathrm{H}$ & 6.55074700 & 2.47211400 & 1.39921800 \\
\hline $\mathrm{C}$ & -3.19076800 & -2.49967000 & 2.12039900 & $\mathrm{H}$ & 3.72189300 & 1.80917800 & 2.16708300 \\
\hline $\mathrm{H}$ & -3.16511600 & -3.09327100 & 3.04315800 & $\mathrm{~N}$ & 3.61315900 & -0.37714500 & -0.31608200 \\
\hline $\mathrm{H}$ & -3.89489500 & -1.68214700 & 2.29505700 & $\mathrm{P}$ & 2.31897000 & -1.26626000 & -1.01762000 \\
\hline $\mathrm{H}$ & -3.58836100 & -3.14580800 & 1.33394300 & $\mathrm{C}$ & 2.79988700 & -3.11900100 & -1.06106500 \\
\hline $\mathrm{F}$ & -1.26349400 & 5.88067400 & -0.36738900 & $\mathrm{C}$ & 1.99399000 & -0.57085600 & -2.77399100 \\
\hline $\mathrm{Rh}$ & 0.61724200 & -0.82425800 & -0.34651500 & $\mathrm{C}$ & 4.18607500 & -3.34804200 & -1.69966000 \\
\hline $\mathrm{Cl}$ & 1.17162400 & -2.87557500 & -1.24919700 & $\mathrm{H}$ & 4.97297000 & -2.84618800 & -1.12897200 \\
\hline $\mathrm{H}$ & -0.26959500 & -0.72994800 & 2.79860600 & $\mathrm{H}$ & 4.40284600 & -4.42381600 & -1.68508400 \\
\hline $\mathrm{C}$ & 5.30970200 & 0.42912100 & 0.10560300 & $\mathrm{H}$ & 4.24506100 & -3.01936100 & -2.73770200 \\
\hline $\mathrm{O}$ & 5.82493200 & 0.04763100 & -0.92270100 & $\mathrm{C}$ & 2.86984900 & -3.65991800 & 0.38245100 \\
\hline $\mathrm{O}$ & 5.88293300 & 0.30589500 & 1.32179600 & $\mathrm{H}$ & 3.22937300 & -4.69652600 & 0.34337500 \\
\hline $\mathrm{C}$ & 7.18050800 & -0.31388200 & 1.32985200 & $\mathrm{H}$ & 3.56866600 & -3.08826000 & 1.00194000 \\
\hline $\mathrm{H}$ & 7.88810700 & 0.26823100 & 0.73323400 & $\mathrm{H}$ & 1.88965000 & -3.66262000 & 0.86626900 \\
\hline $\mathrm{H}$ & 7.48705700 & -0.33594400 & 2.37596100 & $\mathrm{C}$ & 1.70491800 & -3.90203900 & -1.81588500 \\
\hline $\mathrm{H}$ & 7.12234900 & -1.32697700 & 0.92364100 & $\mathrm{H}$ & 0.71060600 & -3.71538300 & -1.39490400 \\
\hline $\mathrm{C}$ & 3.03653000 & 0.76142100 & -0.95778500 & $\mathrm{H}$ & 1.67949000 & -3.67071300 & -2.88401600 \\
\hline $\mathrm{H}$ & 2.79432100 & -0.31718000 & -0.88526200 & $\mathrm{H}$ & 1.91011300 & -4.97582300 & -1.72015500 \\
\hline $\mathrm{C}$ & 0.82127900 & 1.55531600 & 0.16154300 & $\mathrm{C}$ & 2.23250400 & 0.95395900 & -2.70057800 \\
\hline $\mathrm{H}$ & 0.62442400 & 0.54787400 & 0.71848900 & $\mathrm{H}$ & 1.62332800 & 1.43374000 & -1.93349800 \\
\hline $\mathrm{H}$ & 1.37240700 & 1.98684900 & 1.01191200 & $\mathrm{H}$ & 3.28421400 & 1.19098100 & -2.51536600 \\
\hline $\mathrm{H}$ & 1.22015900 & 1.37065100 & -1.97116300 & $\mathrm{H}$ & 1.95352500 & 1.38810100 & -3.66945600 \\
\hline $\mathrm{C}$ & 3.96524500 & 1.13042100 & 0.20448400 & $\mathrm{C}$ & 2.90220200 & -1.13800500 & -3.88417900 \\
\hline $\mathrm{H}$ & 4.16546000 & 2.212 & 0.19286000 & $\mathrm{H}$ & 2.75111100 & -2.20608900 & -4.06062800 \\
\hline $\mathrm{H}$ & 3.52419800 & 0.90671100 & 1.18083800 & $\mathrm{H}$ & 2.65312000 & -0.61927400 & -4.81892900 \\
\hline $\mathrm{C}$ & 1.76968400 & 1.63021400 & -1.06057900 & $\mathrm{C}$ & 0.51681000 & -0.84880000 & -3.13391200 \\
\hline $\mathrm{H}$ & 2.08124400 & 2.67887000 & -1.17511000 & $\mathrm{H}$ & 0.30234000 & -0.37767700 & -4.10231300 \\
\hline \multirow[t]{2}{*}{$\mathrm{H}$} & 3.5 & 0.843 & 400 & $\mathrm{H}$ & 0.30353500 & -1.91718600 & -3.23645700 \\
\hline & & & & $\mathrm{H}$ & -0.16745200 & -0.42818100 & -2.39391000 \\
\hline \multirow[t]{7}{*}{108} & & & & $\mathrm{~F}$ & 8.51086400 & 2.06825300 & -0.25011200 \\
\hline & & & & $\mathrm{Cl}$ & -1.11021600 & -1.68001100 & 0.62470900 \\
\hline & & & & $\mathrm{H}$ & 3.96372300 & -0.95709300 & -3.69028800 \\
\hline & & & & $\mathrm{C}$ & 5.90013600 & -0.16695800 & -1.44024500 \\
\hline & & & & $\mathrm{H}$ & 5.74461100 & -0.89311300 & -2.23113600 \\
\hline & & & & $\mathrm{Rh}$ & 1.12365700 & -0.46123800 & 0.87375000 \\
\hline & & & & $\mathrm{H}$ & 1.85660500 & -1.66003100 & 1.47889400 \\
\hline $\mathrm{C}$ & 5.11011000 & 1.07450500 & 0.54165400 & $\mathrm{Cl}$ & -0.37513700 & 1.34114600 & -0.28034000 \\
\hline $\mathrm{C}$ & 4.90 & 0.11718000 & -0.49874800 & $\mathrm{Rh}$ & -2.56111900 & 0.14450400 & -0.27870200 \\
\hline $\mathrm{C}$ & 7.11889100 & 0.50552000 & -1.34333300 & $\mathrm{C}$ & -4.93727800 & -3.55833000 & -1.49671400 \\
\hline $\mathrm{C}$ & 5000 & 1.43545300 & -0.31642600 & $\mathrm{C}$ & -3.75568000 & -2.56649300 & -1.37663200 \\
\hline $\mathrm{C}$ & 6.345 & 1.73789400 & 0.62691500 & $\mathrm{C}$ & -5.60367300 & -2.49817300 & 1.89771700 \\
\hline
\end{tabular}




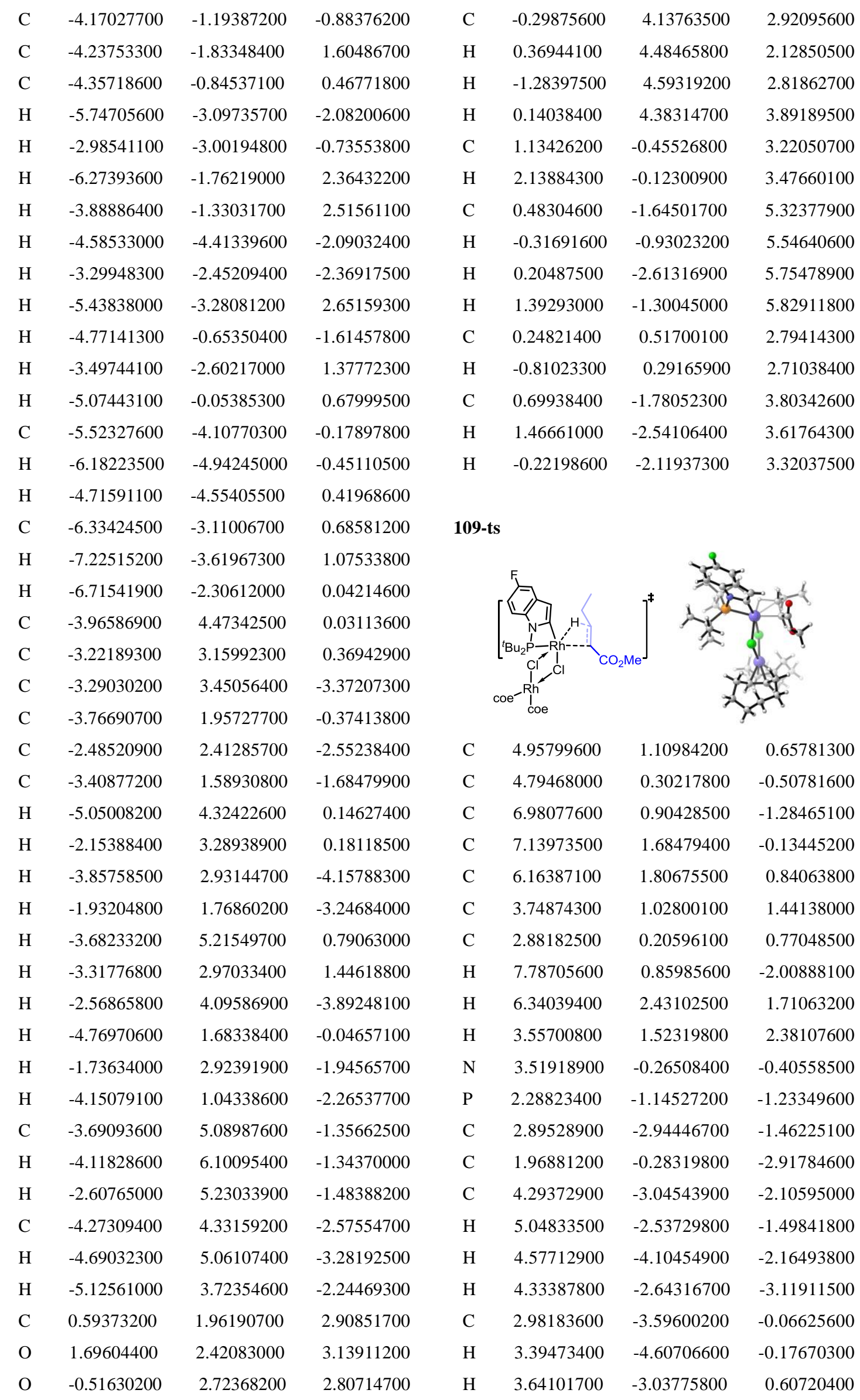




\begin{tabular}{|c|c|c|c|c|c|c|c|}
\hline $\mathrm{H}$ & 1.99669700 & -3.68451000 & 0.39934900 & $\mathrm{C}$ & -6.53073500 & -2.81637100 & 0.27991500 \\
\hline $\mathrm{C}$ & 1.84774700 & -3.71790600 & -2.29025700 & $\mathrm{H}$ & -7.45643100 & -3.31024000 & 0.60285300 \\
\hline $\mathrm{H}$ & 0.84432200 & -3.62766600 & -1.85915900 & $\mathrm{H}$ & -6.85034300 & -1.89097900 & -0.21733700 \\
\hline $\mathrm{H}$ & 1.80729900 & -3.38923900 & -3.33218600 & $\mathrm{C}$ & -3.73204400 & 4.59837700 & 0.61792800 \\
\hline $\mathrm{H}$ & 2.11301300 & -4.78289400 & -2.29429800 & $\mathrm{C}$ & -3.07788100 & 3.20741300 & 0.80340400 \\
\hline $\mathrm{C}$ & 2.17227200 & 1.23191500 & -2.69682800 & $\mathrm{C}$ & -2.99875500 & 4.00060400 & -2.87263400 \\
\hline $\mathrm{H}$ & 1.56732200 & 1.61690600 & -1.87447800 & $\mathrm{C}$ & -3.65659200 & 2.14714000 & -0.11319400 \\
\hline $\mathrm{H}$ & 3.22079500 & 1.47561700 & -2.50252900 & $\mathrm{C}$ & -2.27756200 & 2.82476300 & -2.17280100 \\
\hline $\mathrm{H}$ & 1.86755200 & 1.75549000 & -3.61255000 & $\mathrm{C}$ & -3.27390800 & 1.94538400 & -1.45340400 \\
\hline $\mathrm{C}$ & 2.88185000 & -0.71977100 & -4.08041900 & $\mathrm{H}$ & -4.82629200 & 4.50022300 & 0.68127100 \\
\hline $\mathrm{H}$ & 2.75352200 & -1.76918000 & -4.35845400 & $\mathrm{H}$ & -1.99602300 & 3.29522100 & 0.67490300 \\
\hline $\mathrm{H}$ & 2.62089700 & -0.11914200 & -4.96160700 & $\mathrm{H}$ & -3.56806200 & 3.61923500 & -3.73244100 \\
\hline $\mathrm{C}$ & 0.49523700 & -0.55638900 & -3.29595900 & $\mathrm{H}$ & -1.72817200 & 2.24766600 & -2.92680100 \\
\hline $\mathrm{H}$ & 0.26648400 & -0.02148100 & -4.22738600 & $\mathrm{H}$ & -3.43510600 & 5.21461000 & 1.47789700 \\
\hline $\mathrm{H}$ & 0.29777000 & -1.61876600 & -3.47191200 & $\mathrm{H}$ & -3.23504600 & 2.88834300 & 1.84201500 \\
\hline $\mathrm{H}$ & -0.19163900 & -0.20364800 & -2.52301500 & $\mathrm{H}$ & -2.22721000 & 4.66423300 & -3.28759600 \\
\hline $\mathrm{F}$ & 8.30899500 & 2.35356100 & 0.02377400 & $\mathrm{H}$ & -4.68391900 & 1.88731800 & 0.14230800 \\
\hline $\mathrm{Cl}$ & -1.21284000 & -1.75598400 & 0.31280400 & $\mathrm{H}$ & -1.52939200 & 3.21620400 & -1.48154400 \\
\hline $\mathrm{H}$ & 3.93957900 & -0.53773700 & -3.86925400 & $\mathrm{H}$ & -4.02229600 & 1.53090300 & -2.12800600 \\
\hline $\mathrm{C}$ & 5.79220300 & 0.19992600 & -1.47899800 & $\mathrm{C}$ & -3.37450700 & 5.37693700 & -0.66560600 \\
\hline $\mathrm{H}$ & 5.66396900 & -0.40992200 & -2.36711100 & $\mathrm{H}$ & -3.74533900 & 6.40064100 & -0.52584500 \\
\hline $\mathrm{Rh}$ & 1.03308800 & -0.60976000 & 0.76660300 & $\mathrm{H}$ & -2.28136900 & 5.46902800 & -0.74297600 \\
\hline $\mathrm{H}$ & 1.74453100 & -1.83507600 & 1.51182500 & $\mathrm{C}$ & -3.95627800 & 4.82711000 & -1.99178100 \\
\hline $\mathrm{Cl}$ & -0.29828400 & 1.32949000 & -0.08244700 & $\mathrm{H}$ & -4.30420400 & 5.66955800 & -2.60368600 \\
\hline $\mathrm{Rh}$ & -2.55414000 & 0.27482800 & -0.25322600 & $\mathrm{H}$ & -4.85505500 & 4.23621500 & -1.77031900 \\
\hline $\mathrm{C}$ & -5.20606800 & -3.01338500 & -1.98129300 & $\mathrm{C}$ & 0.77027000 & 0.99666700 & 3.43863000 \\
\hline $\mathrm{C}$ & -3.95478200 & -2.13620700 & -1.73574300 & $\mathrm{O}$ & 1.85948200 & 1.24760800 & 3.92456100 \\
\hline $\mathrm{C}$ & -5.74001000 & -2.46833800 & 1.55635000 & $\mathrm{O}$ & -0.25493000 & 1.87860100 & 3.43614000 \\
\hline $\mathrm{C}$ & -4.26168600 & -0.83705500 & -1.01663600 & $\mathrm{C}$ & 0.04270000 & 3.16563900 & 3.99471900 \\
\hline $\mathrm{C}$ & -4.33196200 & -1.86344100 & 1.33912900 & $\mathrm{H}$ & 0.84158600 & 3.65301800 & 3.42945900 \\
\hline $\mathrm{C}$ & -4.39970600 & -0.69841700 & 0.37843300 & $\mathrm{H}$ & -0.88251700 & 3.73751200 & 3.91832800 \\
\hline $\mathrm{H}$ & -5.98839500 & -2.41002400 & -2.46587100 & $\mathrm{H}$ & 0.35162300 & 3.07172700 & 5.03975100 \\
\hline $\mathrm{H}$ & -3.20652200 & -2.72060500 & -1.19447200 & $\mathrm{C}$ & 1.22405700 & -1.42406500 & 2.90797600 \\
\hline $\mathrm{H}$ & -6.34566800 & -1.77328500 & 2.15525100 & $\mathrm{H}$ & 2.21820000 & -1.20314000 & 3.29891700 \\
\hline $\mathrm{H}$ & -3.93386000 & -1.53995700 & 2.30917700 & $\mathrm{C}$ & 0.44818800 & -2.90793400 & 4.77984800 \\
\hline $\mathrm{H}$ & -4.92514500 & -3.78559400 & -2.71065500 & $\mathrm{H}$ & -0.29762200 & -2.17946200 & 5.11609900 \\
\hline $\mathrm{H}$ & -3.50715700 & -1.89324600 & -2.70880900 & $\mathrm{H}$ & 0.08677400 & -3.90929800 & 5.03831400 \\
\hline $\mathrm{H}$ & -5.62306200 & -3.37474700 & 2.16686700 & $\mathrm{H}$ & 1.37226400 & -2.72761500 & 5.34143600 \\
\hline $\mathrm{H}$ & -4.83666200 & -0.14505200 & -1.63232900 & $\mathrm{C}$ & 0.36038600 & -0.29249300 & 2.83030400 \\
\hline $\mathrm{H}$ & -3.65373300 & -2.63447800 & 0.97079000 & $\mathrm{H}$ & -0.71245800 & -0.45713000 & 2.78768900 \\
\hline $\mathrm{H}$ & -5.05379600 & 0.09547700 & 0.73630100 & $\mathrm{C}$ & 0.68147700 & -2.80252600 & 3.26345700 \\
\hline $\mathrm{C}$ & -5.81168200 & -3.72139300 & -0.75124500 & $\mathrm{H}$ & 1.39262100 & -3.57714500 & 2.95127300 \\
\hline $\mathrm{H}$ & -6.53571900 & -4.45304300 & -1.13271300 & $\mathrm{H}$ & -0.25299300 & -2.97933200 & 2.72199700 \\
\hline $\mathrm{H}$ & -5.03109600 & -4.31383900 & -0.25202900 & & & & \\
\hline
\end{tabular}




\begin{tabular}{|c|c|c|c|}
\hline $\mathrm{H}$ & -0.22115000 & -0.16763000 & -2.59941500 \\
\hline $\mathrm{F}$ & 8.22282800 & 2.40411900 & 0.10315500 \\
\hline $\mathrm{Cl}$ & -1.27403700 & -1.77495000 & 0.26069400 \\
\hline $\mathrm{H}$ & 3.91232000 & -0.40547000 & -3.95441900 \\
\hline $\mathrm{C}$ & 5.71719400 & 0.31328200 & -1.50342000 \\
\hline $\mathrm{H}$ & 5.59168100 & -0.25133800 & -2.42122900 \\
\hline $\mathrm{Rh}$ & 0.97647800 & -0.65551600 & 0.73634600 \\
\hline $\mathrm{H}$ & 1.67291000 & -1.99209000 & 1.67300600 \\
\hline $\mathrm{Cl}$ & -0.25372200 & 1.29222300 & 0.02538100 \\
\hline $\mathrm{Rh}$ & -2.54370400 & 0.31621700 & -0.24168200 \\
\hline $\mathrm{C}$ & -5.25379200 & -2.81675900 & -2.15512700 \\
\hline $\mathrm{C}$ & -3.98222800 & -1.98659700 & -1.85441600 \\
\hline $\mathrm{C}$ & -5.83765100 & -2.40124000 & 1.39283400 \\
\hline $\mathrm{C}$ & -4.26557100 & -0.70976600 & -1.08750200 \\
\hline $\mathrm{C}$ & -4.40964900 & -1.82786200 & 1.22446400 \\
\hline $\mathrm{C}$ & -4.42925800 & -0.62562100 & 0.30908700 \\
\hline $\mathrm{H}$ & -6.01007500 & -2.17255700 & -2.62832800 \\
\hline $\mathrm{H}$ & -3.25983300 & -2.61360000 & -1.32608000 \\
\hline $\mathrm{H}$ & -6.43473900 & -1.71429200 & 2.00938300 \\
\hline $\mathrm{H}$ & -4.02136700 & -1.55181700 & 2.21279500 \\
\hline $\mathrm{H}$ & -4.98120200 & -3.56678400 & -2.91026200 \\
\hline $\mathrm{H}$ & -3.51244700 & -1.71739800 & -2.80987800 \\
\hline $\mathrm{H}$ & -5.75640400 & -3.33453800 & 1.96746400 \\
\hline $\mathrm{H}$ & -4.80652800 & 0.02471500 & -1.68478300 \\
\hline $\mathrm{H}$ & -3.74503200 & -2.60270300 & 0.83957800 \\
\hline $\mathrm{H}$ & -5.06908700 & 0.17081700 & 0.68619800 \\
\hline $\mathrm{C}$ & -5.90094600 & -3.55621500 & -0.96542500 \\
\hline $\mathrm{H}$ & -6.63746700 & -4.25125700 & -1.38899300 \\
\hline $\mathrm{H}$ & -5.14637800 & -4.18986900 & -0.47708500 \\
\hline $\mathrm{C}$ & -6.61407800 & -2.67427700 & 0.08971700 \\
\hline $\mathrm{H}$ & -7.55872500 & -3.15474200 & 0.37573300 \\
\hline $\mathrm{H}$ & -6.89917800 & -1.72066200 & -0.37399400 \\
\hline $\mathrm{C}$ & -3.60959200 & 4.62069300 & 0.83051500 \\
\hline $\mathrm{C}$ & -3.00589800 & 3.20017100 & 0.95132600 \\
\hline $\mathrm{C}$ & -2.80405500 & 4.18908400 & -2.66924100 \\
\hline $\mathrm{C}$ & -3.59530200 & 2.21260400 & -0.03698200 \\
\hline $\mathrm{C}$ & -2.14119300 & 2.95062400 & -2.02116100 \\
\hline $\mathrm{C}$ & -3.18342300 & 2.06659500 & -1.37567500 \\
\hline $\mathrm{H}$ & -4.70758700 & 4.55604500 & 0.86279900 \\
\hline $\mathrm{H}$ & -1.91870000 & 3.26090500 & 0.85375900 \\
\hline $\mathrm{H}$ & -3.36187500 & 3.87647400 & -3.56346200 \\
\hline $\mathrm{H}$ & -1.59127000 & 2.39945600 & -2.79382900 \\
\hline $\mathrm{H}$ & -3.31403900 & 5.17736100 & 1.73046000 \\
\hline $\mathrm{H}$ & -3.19832900 & 2.82766700 & 1.96 \\
\hline
\end{tabular}




\begin{tabular}{|c|c|c|c|c|c|c|c|}
\hline $\mathrm{H}$ & -1.99952800 & 4.84739400 & -3.02581400 & $\mathrm{H}$ & 5.07994600 & 4.21320500 & -0.21352700 \\
\hline $\mathrm{H}$ & -4.63641700 & 1.97338600 & 0.17922600 & $\mathrm{H}$ & 2.44028000 & 3.02088500 & 0.61180700 \\
\hline $\mathrm{H}$ & -1.39943100 & 3.27817000 & -1.29076000 & $\mathrm{~N}$ & 3.45487800 & 0.09426800 & -0.55849300 \\
\hline $\mathrm{H}$ & -3.92408600 & 1.70698600 & -2.08838500 & $\mathrm{P}$ & 2.59896500 & -1.37867300 & -0.83394800 \\
\hline $\mathrm{C}$ & -3.19412200 & 5.45548100 & -0.39883100 & $\mathrm{C}$ & 3.69527900 & -2.86509700 & -0.35654600 \\
\hline $\mathrm{H}$ & -3.53670700 & 6.48155000 & -0.21253000 & $\mathrm{C}$ & 2.11778100 & -1.38549700 & -2.68603700 \\
\hline $\mathrm{H}$ & -2.09695800 & 5.51684100 & -0.44361400 & $\mathrm{C}$ & 4.52922400 & -2.44659100 & 0.87279700 \\
\hline $\mathrm{C}$ & -3.75735800 & 4.99732000 & -1.76736100 & $\mathrm{H}$ & 3.93729800 & -2.00907800 & 1.67922800 \\
\hline $\mathrm{H}$ & -4.06228000 & 5.88257600 & -2.34035600 & $\mathrm{H}$ & 5.03911600 & -3.33767900 & 1.26163600 \\
\hline $\mathrm{H}$ & -4.67953800 & 4.42466800 & -1.60107800 & $\mathrm{H}$ & 5.29484500 & -1.71328100 & 0.60127500 \\
\hline $\mathrm{C}$ & 0.85655500 & 0.64247300 & 3.56078700 & $\mathrm{C}$ & 2.75539900 & -4.03861500 & 0.00209300 \\
\hline $\mathrm{O}$ & 1.94689400 & 0.80653900 & 4.08261500 & $\mathrm{H}$ & 3.36399900 & -4.88082800 & 0.35491500 \\
\hline $\mathrm{O}$ & -0.14191100 & 1.55412500 & 3.65255700 & $\mathrm{H}$ & 2.05286300 & -3.77631000 & 0.79448900 \\
\hline $\mathrm{C}$ & 0.19127300 & 2.75857700 & 4.35485900 & $\mathrm{H}$ & 2.17898600 & -4.38818900 & -0.86016600 \\
\hline $\mathrm{H}$ & 1.00756200 & 3.28388300 & 3.85136000 & $\mathrm{C}$ & 4.66143300 & -3.32895000 & -1.46401900 \\
\hline $\mathrm{H}$ & -0.71559700 & 3.36413200 & 4.34292900 & $\mathrm{H}$ & 4.14311000 & -3.73596900 & -2.33615200 \\
\hline $\mathrm{H}$ & 0.49197800 & 2.53739600 & 5.38294600 & $\mathrm{H}$ & 5.33431700 & -2.53334000 & -1.79579900 \\
\hline $\mathrm{C}$ & 1.28111700 & -1.74558000 & 2.80158400 & $\mathrm{H}$ & 5.28710000 & -4.13190500 & -1.05331800 \\
\hline $\mathrm{H}$ & 2.24066900 & -1.54531100 & 3.28827300 & $\mathrm{C}$ & 1.15533100 & -0.18908700 & -2.87302000 \\
\hline $\mathrm{C}$ & 0.37585800 & -3.12726900 & 4.72604100 & $\mathrm{H}$ & 0.24750400 & -0.28543000 & -2.26237200 \\
\hline $\mathrm{H}$ & -0.32971800 & -2.34374700 & 5.02416400 & $\mathrm{H}$ & 1.63394700 & 0.76852400 & -2.64765600 \\
\hline $\mathrm{H}$ & -0.05412500 & -4.09443200 & 5.00911400 & $\mathrm{H}$ & 0.82667000 & -0.16026200 & -3.92043100 \\
\hline $\mathrm{H}$ & 1.29716800 & -2.98594700 & 5.30331400 & $\mathrm{C}$ & 3.30869000 & -1.18764200 & -3.64552100 \\
\hline $\mathrm{C}$ & 0.42412800 & -0.54517400 & 2.78754400 & $\mathrm{H}$ & 3.99664600 & -2.03490400 & -3.65713200 \\
\hline $\mathrm{H}$ & -0.65212000 & -0.70307400 & 2.80925500 & $\mathrm{H}$ & 2.91826500 & -1.07301100 & -4.66551500 \\
\hline $\mathrm{C}$ & 0.64943600 & -3.08213200 & 3.21711900 & $\mathrm{C}$ & 1.35742100 & -2.68631400 & -3.01499700 \\
\hline $\mathrm{H}$ & 1.32315100 & -3.90410900 & 2.94253000 & $\mathrm{H}$ & 0.95726700 & -2.61486900 & -4.03461500 \\
\hline \multirow[t]{2}{*}{$\mathrm{H}$} & 700 & -3.2 & 2.6 & $\mathrm{H}$ & 2.00322000 & -3.56913100 & -2.98268200 \\
\hline & & & & $\mathrm{H}$ & 0.51053000 & -2.84570400 & -2.33840800 \\
\hline \multirow[t]{7}{*}{111} & & & & $\mathrm{~F}$ & 7.38982800 & 3.82045500 & -1.31954300 \\
\hline & & & & $\mathrm{Cl}$ & -1.28494200 & -1.60940200 & 0.01656400 \\
\hline & & & & $\mathrm{H}$ & 3.87585500 & -0.28266900 & -3.40797700 \\
\hline & & & & $\mathrm{C}$ & 5.79279200 & 0.55726200 & -1.47323500 \\
\hline & & & & $\mathrm{H}$ & 6.01315300 & -0.44625400 & -1.82301900 \\
\hline & & & & $\mathrm{Rh}$ & 0.84262900 & -0.24205700 & 0.24985800 \\
\hline & & & & $\mathrm{H}$ & 0.28702200 & -2.63613700 & 2.06697700 \\
\hline $\mathrm{C}$ & 4.28166800 & 2.18815600 & -0.39713400 & $\mathrm{Cl}$ & -0.78950800 & 1.52245600 & 0.67108800 \\
\hline $\mathrm{C}$ & 4.57436600 & 0.87072900 & -0.86892500 & $\mathrm{Rh}$ & -2.85301600 & 0.34048400 & -0.09195500 \\
\hline $\mathrm{C}$ & 6.73874900 & 1.57091100 & -1.62100600 & $\mathrm{C}$ & -5.09049200 & -2.68496900 & -2.68642700 \\
\hline $\mathrm{C}$ & 6.44654800 & 2.86020900 & -1.16044900 & $\mathrm{C}$ & -3.94646300 & -1.76635100 & -2.19328400 \\
\hline $\mathrm{C}$ & 5.24865700 & 3.19620700 & -0.55289100 & $\mathrm{C}$ & -5.72247700 & -3.07664800 & 0.85392700 \\
\hline $\mathrm{C}$ & 2.95920700 & 2.17748500 & 0.17765500 & $\mathrm{C}$ & -4.40321100 & -0.71597100 & -1.20000200 \\
\hline $\mathrm{C}$ & 2.47005400 & 0.89898700 & 0.06655400 & $\mathrm{C}$ & -4.39372100 & -2.28354200 & 0.83862200 \\
\hline $\mathrm{H}$ & 7.70118800 & 1.38111600 & -2.08371400 & $\mathrm{C}$ & -4.58795400 & -0.93840900 & 0.17824100 \\
\hline
\end{tabular}




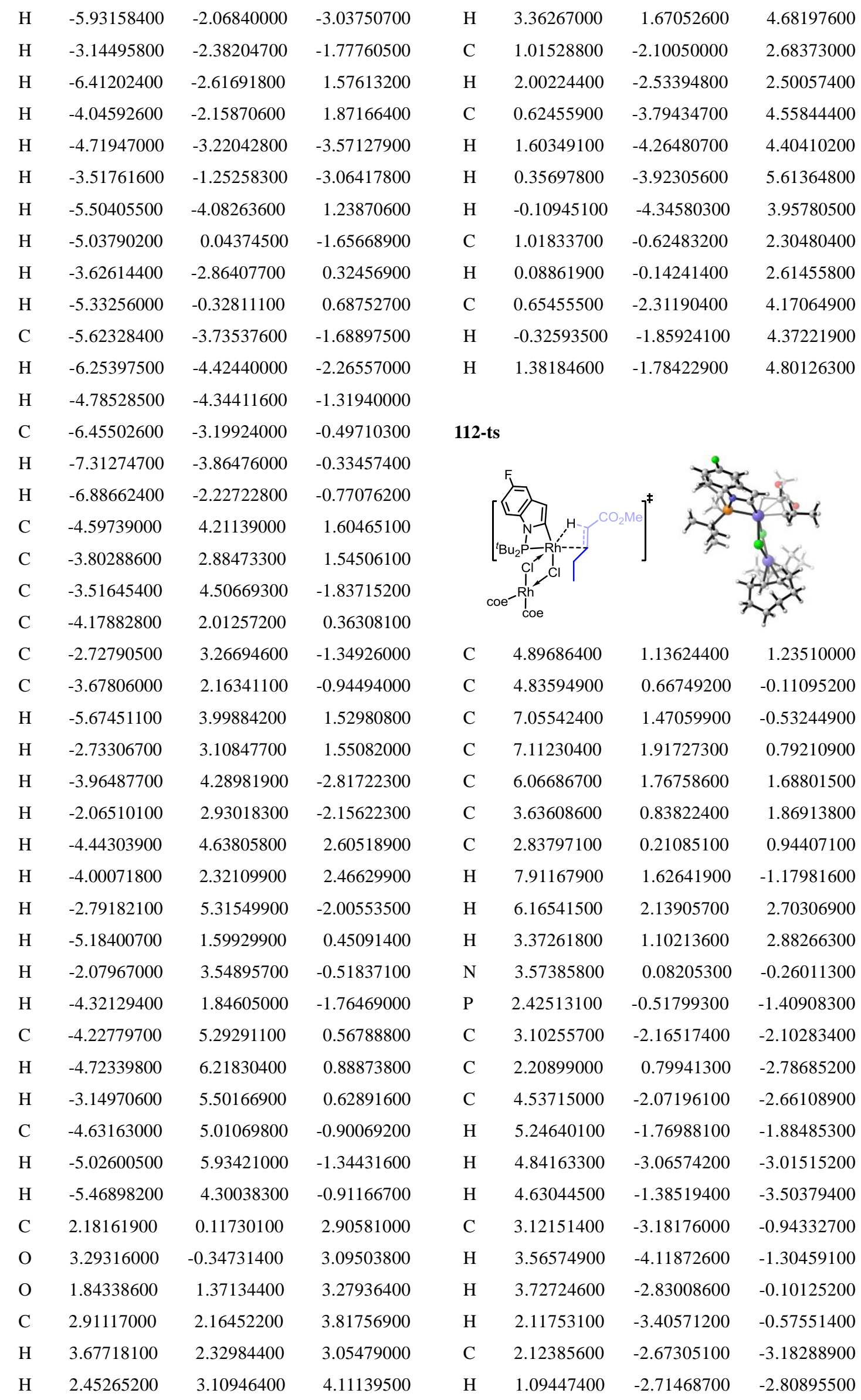




\begin{tabular}{|c|c|c|c|c|c|c|c|}
\hline $\mathrm{H}$ & 2.13691400 & -2.05666000 & -4.08637000 & $\mathrm{C}$ & -3.75653800 & 4.32330000 & 2.07673100 \\
\hline $\mathrm{H}$ & 2.41323200 & -3.69015700 & -3.47710500 & $\mathrm{C}$ & -3.07772800 & 2.96279900 & 1.78980500 \\
\hline$C$ & 2.34887900 & 2.18413900 & -2.11781000 & $\mathrm{C}$ & -3.14215800 & 4.88458700 & -1.44383400 \\
\hline $\mathrm{H}$ & 1.67067200 & 2.30671200 & -1.27129700 & $\mathrm{C}$ & -3.67542500 & 2.23217700 & 0.60316300 \\
\hline $\mathrm{H}$ & 3.37161100 & 2.36821400 & -1.77586300 & $\mathrm{C}$ & -2.38649300 & 3.56008000 & -1.18092300 \\
\hline $\mathrm{H}$ & 2.09851700 & 2.95207100 & -2.86160800 & $\mathrm{C}$ & -3.34625600 & 2.47761400 & -0.74395800 \\
\hline $\mathrm{C}$ & 3.20420800 & 0.73048700 & -3.96051600 & $\mathrm{H}$ & -4.84639300 & 4.18350600 & 2.13681500 \\
\hline $\mathrm{H}$ & 3.12552500 & -0.19657900 & -4.53470100 & $\mathrm{H}$ & -2.00297800 & 3.11463100 & 1.66119100 \\
\hline $\mathrm{H}$ & 2.97909300 & 1.55556800 & -4.64914200 & $\mathrm{H}$ & -3.73683100 & 4.78762600 & -2.36346300 \\
\hline $\mathrm{C}$ & 0.76924400 & 0.63609700 & -3.32668100 & $\mathrm{H}$ & -1.86442700 & 3.26287800 & -2.09882100 \\
\hline $\mathrm{H}$ & 0.57689500 & 1.42592100 & -4.06493700 & $\mathrm{H}$ & -3.43753300 & 4.64129800 & 3.07886400 \\
\hline $\mathrm{H}$ & 0.61781200 & -0.32558700 & -3.82770600 & $\mathrm{H}$ & -3.19222100 & 2.32702100 & 2.67794800 \\
\hline $\mathrm{H}$ & 0.02763500 & 0.72561700 & -2.52892700 & $\mathrm{H}$ & -2.39155500 & 5.66145500 & -1.64614700 \\
\hline $\mathrm{F}$ & 8.24895800 & 2.52710300 & 1.21052000 & $\mathrm{H}$ & -4.68716600 & 1.87621800 & 0.79847000 \\
\hline $\mathrm{Cl}$ & -1.14944200 & -1.51982200 & -0.36471000 & $\mathrm{H}$ & -1.61322300 & 3.72438700 & -0.42853400 \\
\hline $\mathrm{H}$ & 4.24137600 & 0.85648000 & -3.63583300 & $\mathrm{H}$ & -4.11632500 & 2.27845200 & -1.48859100 \\
\hline $\mathrm{C}$ & 5.90291900 & 0.83562900 & -0.99579600 & $\mathrm{C}$ & -3.45424900 & 5.47697600 & 1.09754000 \\
\hline $\mathrm{H}$ & 5.85364900 & 0.48480800 & -2.02106700 & $\mathrm{H}$ & -3.83061100 & 6.39486800 & 1.56745500 \\
\hline $\mathrm{Rh}$ & 1.01003500 & -0.57228800 & 0.57188400 & $\mathrm{H}$ & -2.36573600 & 5.61251500 & 1.01965900 \\
\hline $\mathrm{H}$ & 1.63828400 & -1.99779200 & 1.06894300 & $\mathrm{C}$ & -4.07719000 & 5.36544000 & -0.31661800 \\
\hline $\mathrm{Cl}$ & -0.29841900 & 1.53564200 & 0.29206600 & $\mathrm{H}$ & -4.45693100 & 6.35083100 & -0.61656200 \\
\hline $\mathrm{Rh}$ & -2.54858500 & 0.53480500 & -0.16953800 & $\mathrm{H}$ & -4.95963100 & 4.71359800 & -0.26905400 \\
\hline $\mathrm{C}$ & -5.25091800 & -2.12287000 & -2.71478900 & $\mathrm{C}$ & 0.31451800 & -3.29350800 & 2.29424900 \\
\hline $\mathrm{C}$ & -4.00224400 & -1.32095300 & -2.27504900 & $\mathrm{O}$ & -0.83253700 & -3.49501600 & 2.61713200 \\
\hline $\mathrm{C}$ & -5.56586400 & -2.75113400 & 0.83250100 & $\mathrm{O}$ & 1.15964500 & -4.25752600 & 1.87171900 \\
\hline $\mathrm{C}$ & -4.28306600 & -0.32766500 & -1.16388000 & $\mathrm{C}$ & 0.60037200 & -5.58184400 & 1.80764400 \\
\hline $\mathrm{C}$ & -4.18672900 & -2.05758200 & 0.73438400 & $\mathrm{H}$ & -0.23581400 & -5.60645700 & 1.10439600 \\
\hline $\mathrm{C}$ & -4.34062900 & -0.65052000 & 0.20553400 & $\mathrm{H}$ & 1.41191100 & -6.22424600 & 1.46599700 \\
\hline $\mathrm{H}$ & -6.07419000 & -1.42696500 & -2.93592400 & $\mathrm{H}$ & 0.24933900 & -5.89504300 & 2.79430100 \\
\hline $\mathrm{H}$ & -3.20877200 & -2.01667800 & -1.99035800 & $\mathrm{C}$ & 0.23772200 & -0.77371600 & 2.60084400 \\
\hline $\mathrm{H}$ & -6.14344800 & -2.30194100 & 1.65309500 & $\mathrm{H}$ & -0.83495500 & -0.90481500 & 2.46721200 \\
\hline $\mathrm{H}$ & -3.72088400 & -2.05361600 & 1.72684900 & $\mathrm{C}$ & -0.12723600 & 0.07468200 & 4.94782400 \\
\hline $\mathrm{H}$ & -5.00464100 & -2.61045400 & -3.66824200 & $\mathrm{H}$ & 0.11038500 & -0.89774700 & 5.39540900 \\
\hline $\mathrm{H}$ & -3.62540200 & -0.76700300 & -3.14529600 & $\mathrm{H}$ & 0.14642600 & 0.85563300 & 5.66673400 \\
\hline $\mathrm{H}$ & -5.39033700 & -3.79705000 & 1.12030300 & $\mathrm{H}$ & -1.21304500 & 0.11619100 & 4.80201400 \\
\hline $\mathrm{H}$ & -4.90069300 & 0.50848900 & -1.49208400 & $\mathrm{C}$ & 1.02204200 & -1.94910300 & 2.37434700 \\
\hline $\mathrm{H}$ & -3.52748000 & -2.64460800 & 0.09424100 & $\mathrm{H}$ & 1.98388000 & -2.01096000 & 2.88872900 \\
\hline $\mathrm{H}$ & -4.98116500 & -0.03517200 & 0.83590700 & $\mathrm{C}$ & 0.62017400 & 0.27886800 & 3.61608100 \\
\hline $\mathrm{C}$ & -5.76267500 & -3.21081700 & -1.74692000 & $\mathrm{H}$ & 0.38394400 & 1.27165100 & 3.21686900 \\
\hline $\mathrm{H}$ & -6.49492900 & -3.80862400 & -2.30516900 & $\mathrm{H}$ & 1.69915700 & 0.25257200 & 3.79752800 \\
\hline $\mathrm{H}$ & -4.94038600 & -3.90120300 & -1.50852900 & & & & \\
\hline $\mathrm{C}$ & -6.43355400 & -2.71538600 & -0.44145100 & 113 & & & \\
\hline $\mathrm{H}$ & -7.32016000 & -3.33121600 & -0.24140200 & & & & \\
\hline $\mathrm{H}$ & -6.81456700 & -1.69758800 & -0.59985200 & & & & \\
\hline
\end{tabular}



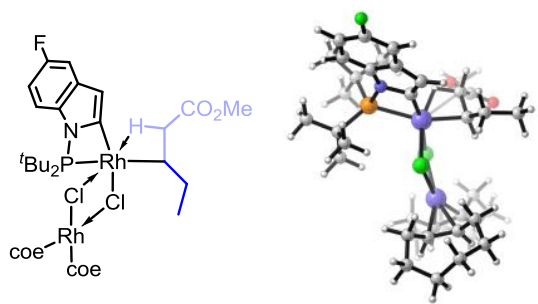$$
\text { C }
$$

4.77082700

C $\quad 4.78708000$

C $\quad 7.00367200$

C $\quad 6.98472400$

C $\quad 5.90098900$

C $\quad 3.48829000$

C 2.75454300

$\mathrm{H} \quad 7.88578500$

H 5.93939900

H 3.16403300

$\mathrm{N} \quad 3.54752600$

P $\quad 2.51328800$

C $\quad 3.32436300$

C 2.37132700

C $\quad 4.79116400$

H 5.42990000

H 5.15841700

H 4.92043700

C $\quad 3.29341100$

H 3.79939500

H 3.82096700

H 2.27309400

C 2.45272800

H 1.40047400

$\mathrm{H} \quad 2.50748200$

H 2.80516000

C 2.41688000

H 1.66456800

H 3.39926500

H 2.21322600

C $\quad 3.44058800$

H 3.43750600

$\mathrm{H} \quad 3.22834000$

C $\quad 0.97475400$

H 0.79766800

H 0.88972900

H 0.18186400

F 8.08383600

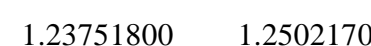

0.65963800

1.49298100

2.04528900

1.93692500

0.95708500

0.23011700

1.62270900

2.39141900

0.03143400

$-0.73255700$

$-2.40689700$

$-2.31891300$

$-1.89419700$

$-3.33293400$

$-1.73361500$

$-3.26543200$

$-4.21871500$

$-2.78010000$

$-3.49341100$

$-3.09943200$

$-3.14677000$

$-2.60394000$

$-4.12979300$

1.87208300

2.06290400

2.13087800

2.54362100

0.25841300

$-0.73430500$

0.98265200

0.14024000

0.84298200

$-0.87247700$

0.27412300

2.71995900
1.25021700

$-0.05412700$

$-0.43512000$

0.85033500

1.70521100

1.84453200

0.93876300

$-1.05291000$

2.69011600

2.81726900

$-0.21931700$

$-1.38678700$

$-1.82525800$

$-2.91541600$

$-2.28978100$

$-1.51009300$

$-2.49815800$

$-3.20148800$

$-0.54269200$

$-0.74418700$

0.28608100

$-0.22356000$

$-2.89431400$

$-2.59051400$

$-3.86813600$

$-3.03312600$

$-2.40067400$

$-1.63268900$

$-1.99474700$

$-3.24543500$

$-4.01265100$

$-4.47102600$

$-4.81048900$

$-3.52052800$

$-4.34542700$

$-3.92886500$

$-2.77945000$

1.27017200
1.29551100

0.41726700
Cl -1.23604500

$-1.54055000$

$-0.32310500$

H 4.44874000

0.47112500

$-3.64474300$

C $\quad 5.89203900$

0.78932500

$-0.89858700$

H $\quad 5.90208200$

0.35883700

$-1.89396200$

Rh $\quad 0.95116300$

$-0.59025400$

0.57032400

H 1.48684100

$-2.14565000$

1.38476700

$\mathrm{Cl}-0.18784100$

1.48547000

0.15538200

$\mathrm{Rh} \quad-2.50914000$

0.60387700

$-0.22633600$

C $\quad-5.34132200$

$-1.93372300$

$-2.74102500$

C $\quad-4.05096600$

$-1.19907900$

$-2.30353100$

C $\quad-5.73738100$

$-2.46509000$

0.81510300

C $\quad-4.28408200$

$-0.17087800$

$-1.21343200$

C $\quad-4.31577800$

$-1.86384100$

0.72014900

C $\quad-4.37231100$

$-0.46078800$

0.16203800

$\mathrm{H}$

$-6.11874300$

$-1.19431000$

$-2.98523200$

$\mathrm{H}$

$-3.30109300$

$-1.93411100$

$-2.00028800$

$\mathrm{H}$

$-6.29457000$

$-1.96141300$

1.61783600

$\mathrm{H}$

$-3.86283600$

$-1.86610400$

1.71881300

$\mathrm{H}$

$-5.11502600$

$-2.45498900$

$-3.68137800$

H

$-3.63587100$

$-0.68302000$

$-3.17953900$

H $\quad-5.63350600$

$-3.51330100$

1.12847900

$\mathrm{H}$

$-4.84791900$

0.69303900

$-1.56548800$

H $\quad-3.68896900$

$-2.50503200$

0.09907600

H $\quad-4.97984200$

0.20553000

0.77290000

C $\quad-5.92804700$

$-2.96755600$

$-1.75663800$

$\mathrm{H}$

$-6.68762100$

$-3.53366700$

$-2.31130500$

$\mathrm{H}$

$-5.15128500$

$-3.69981200$

$-1.49225200$

C

$\mathrm{H}$

$-6.58448100$

$-2.40326100$

$-0.47161900$

$-0.27151000$

$\mathrm{H}$

C

$$
\text { C }
$$

$\mathrm{C}$
$\mathrm{C}$

C

C

$-2.95821900$

$-0.27151000$

$-6.89913300$

$-1.36750300$

$-0.65660300$

$-3.46128800$

4.47732900

1.98480100

3.07590100

1.70028300

$-1.54696200$

4.97046300

0.52620300

C $\quad-2.17343600$

2.37666800

$-1.28064700$

C -3.19288700

3.60412800

$-0.82574900$

H $\quad-4.55680400$

2.58566100

2.05847400

1.55842400

3.16295700

$-2.45984800$

4.90269100

$-2.20017500$

H $\quad-1.67802900$

3.26877500

2.98065600

4.78208100

2.59359100

2.45318700

$-1.76335700$

H $\quad-2.05456400$

5.69779100

0.73460800 


\begin{tabular}{|c|c|c|c|c|c|c|c|}
\hline $\mathrm{H}$ & -1.38542600 & 3.73062400 & -0.53574200 & $\mathrm{~N}$ & 3.59980700 & -0.04395700 & -0.62705600 \\
\hline $\mathrm{H}$ & -3.97898600 & 2.42469900 & -1.56259600 & $\mathrm{P}$ & 2.81256600 & -1.56680900 & -0.41187200 \\
\hline $\mathrm{C}$ & -3.10071600 & 5.60323900 & 0.99299700 & $\mathrm{C}$ & 3.88456700 & -2.65142200 & 0.74060900 \\
\hline $\mathrm{H}$ & -3.41930500 & 6.54495500 & 1.45832200 & $\mathrm{C}$ & 2.60234800 & -2.32845000 & -2.15306900 \\
\hline $\mathrm{H}$ & -2.00680500 & 5.67519900 & 0.90574100 & $\mathrm{C}$ & 5.05899600 & -3.36530200 & 0.04297700 \\
\hline $\mathrm{C}$ & -3.74156100 & 5.51774200 & -0.41504700 & $\mathrm{H}$ & 5.72872800 & -2.67247100 & -0.47317200 \\
\hline $\mathrm{H}$ & -4.06153100 & 6.52232100 & -0.72083600 & $\mathrm{H}$ & 5.65200000 & -3.88064800 & 0.80958700 \\
\hline $\mathrm{H}$ & -4.66315800 & 4.92354600 & -0.35395700 & $\mathrm{H}$ & 4.72569700 & -4.12396900 & -0.67038900 \\
\hline $\mathrm{C}$ & 0.04708300 & -3.30329700 & 2.47623000 & $\mathrm{C}$ & 4.45381100 & -1.72195300 & 1.83280700 \\
\hline $\mathrm{O}$ & -0.88999000 & -3.44863300 & 3.22724900 & $\mathrm{H}$ & 4.99627100 & -2.33284700 & 2.56543900 \\
\hline $\mathrm{O}$ & 0.49370600 & -4.25588700 & 1.63359100 & $\mathrm{H}$ & 5.15054500 & -0.98642000 & 1.42036000 \\
\hline $\mathrm{C}$ & -0.24819900 & -5.48889000 & 1.64768000 & $\mathrm{H}$ & 3.66972000 & -1.18215600 & 2.36824600 \\
\hline $\mathrm{H}$ & -1.28378900 & -5.30834400 & 1.34964400 & $\mathrm{C}$ & 2.97422700 & -3.71827100 & 1.38726700 \\
\hline $\mathrm{H}$ & 0.25184500 & -6.13755000 & 0.92860200 & $\mathrm{H}$ & 2.14408400 & -3.27021300 & 1.93496500 \\
\hline $\mathrm{H}$ & -0.23150000 & -5.93247700 & 2.64650500 & $\mathrm{H}$ & 2.56068400 & -4.41531500 & 0.65265500 \\
\hline $\mathrm{C}$ & 0.18628600 & -0.74683000 & 2.52189200 & $\mathrm{H}$ & 3.56895200 & -4.30512100 & 2.09894400 \\
\hline $\mathrm{H}$ & -0.89515500 & -0.84029300 & 2.41440300 & $\mathrm{C}$ & 1.94736600 & -3.71853400 & -2.02563400 \\
\hline $\mathrm{C}$ & -0.17554500 & -0.04338500 & 4.92878700 & $\mathrm{H}$ & 2.62310900 & -4.45967800 & -1.58741200 \\
\hline $\mathrm{H}$ & 0.02707800 & -1.05475500 & 5.30014200 & $\mathrm{H}$ & 1.02812300 & -3.68372500 & -1.43010200 \\
\hline $\mathrm{H}$ & 0.12609400 & 0.67158600 & 5.70342100 & $\mathrm{H}$ & 1.67786600 & -4.07764000 & -3.02710300 \\
\hline $\mathrm{H}$ & -1.25983000 & 0.04346100 & 4.79194200 & $\mathrm{C}$ & 1.62878300 & -1.38606400 & -2.90002400 \\
\hline $\mathrm{C}$ & 0.90325300 & -2.04450000 & 2.41517500 & $\mathrm{H}$ & 2.03692700 & -0.37698900 & -3.01350400 \\
\hline $\mathrm{H}$ & 1.74666600 & -2.126 & 3.11260400 & $\mathrm{H}$ & 1.45025000 & -1.78803100 & -3.90616600 \\
\hline $\mathrm{C}$ & 0.57296800 & 0.232 & 3.61081500 & $\mathrm{C}$ & 3.90533300 & -2.40825200 & -2.97191600 \\
\hline $\mathrm{H}$ & 0.34727900 & 1.25122900 & 3.27214800 & $\mathrm{H}$ & 3.65874700 & -2.74938400 & -3.98600400 \\
\hline \multirow[t]{2}{*}{$\mathrm{H}$} & 1.65247000 & 0.18862600 & 3.79063900 & $\mathrm{H}$ & 4.38545400 & -1.42953500 & -3.06252800 \\
\hline & & & & $\mathrm{H}$ & 4.62941900 & -3.11413400 & -2.56079700 \\
\hline \multirow[t]{7}{*}{114} & \multirow{7}{*}{ 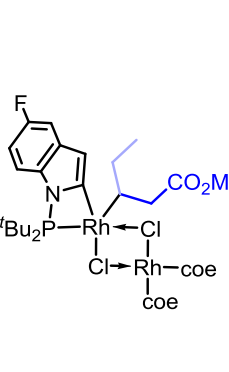 } & \multirow{7}{*}{ कर } & & $\mathrm{F}$ & 7.53314400 & 3.40363900 & -2.24707800 \\
\hline & & & & $\mathrm{Cl}$ & -1.18515800 & -1.67494900 & -0.06619300 \\
\hline & & & & $\mathrm{H}$ & 0.65120900 & -1.32034300 & -2.40493700 \\
\hline & & & & $\mathrm{C}$ & 6.02859000 & 0.20725300 & -1.38623800 \\
\hline & & & & $\mathrm{H}$ & 6.31096800 & -0.83887200 & -1.33031000 \\
\hline & & & & $\mathrm{Rh}$ & 0.90655700 & -0.21884100 & -0.05398400 \\
\hline & & & & $\mathrm{Cl}$ & -0.74105100 & 1.51268700 & -0.50390500 \\
\hline $\mathrm{C}$ & 4.36369200 & 2.01535700 & -1.14968300 & $\mathrm{Rh}$ & -2.85356600 & 0.16796000 & -0.41717700 \\
\hline $\mathrm{C}$ & 4.73732100 & 0.63686200 & -1.07514700 & $\mathrm{C}$ & -5.23111800 & -3.40853600 & -1.93864000 \\
\hline $\mathrm{C}$ & 6.96689900 & 1.15866500 & -1.78561300 & $\mathrm{C}$ & -4.08909300 & -2.37197400 & -1.80956400 \\
\hline $\mathrm{C}$ & 6.59510900 & 2.50599000 & -1.85817200 & $\mathrm{C}$ & -5.38449900 & -3.03005400 & 1.65606400 \\
\hline $\mathrm{C}$ & 5.32360500 & 2.959 & -1.55137600 & $\mathrm{C}$ & -4.47983000 & -1.14515900 & -1.00918000 \\
\hline $\mathrm{C}$ & 2.97962000 & 2.12953900 & -0.76513400 & $\mathrm{C}$ & -4.11372100 & -2.22306000 & 1.29538100 \\
\hline $\mathrm{C}$ & 2.52857600 & 0.87061700 & -0.45379200 & $\mathrm{C}$ & -4.46133500 & -1.05951100 & 0.39660500 \\
\hline $\mathrm{H}$ & 7.98253200 & 0.87630400 & -2.04088800 & $\mathrm{H}$ & -6.14287600 & -2.90803900 & -2.29762400 \\
\hline $\mathrm{H}$ & 5.09348800 & 4.01805800 & -1.62368400 & $\mathrm{H}$ & -3.20830200 & -2.85879400 & -1.38358700 \\
\hline $\mathrm{H}$ & 2.39810100 & 3.04123400 & -0.73538500 & $\mathrm{H}$ & -6.00199600 & -2.44375400 & 2.35137800 \\
\hline
\end{tabular}




\begin{tabular}{|c|c|c|c|c|c|c|c|}
\hline $\mathrm{H}$ & -3.64798900 & -1.86697500 & 2.22291100 & $\mathrm{H}$ & 1.14977100 & 1.19259500 & 6.52582200 \\
\hline $\mathrm{H}$ & -4.94253300 & -4.11390500 & -2.73007200 & $\mathrm{H}$ & 2.93238700 & 1.15778600 & 6.73420500 \\
\hline $\mathrm{H}$ & -3.80166700 & -2.04759300 & -2.81864500 & $\mathrm{H}$ & 2.09498800 & 2.67744300 & 6.27229800 \\
\hline $\mathrm{H}$ & -5.06454000 & -3.92305000 & 2.21117800 & $\mathrm{C}$ & 0.72815700 & -1.12710400 & 2.84191900 \\
\hline $\mathrm{H}$ & -5.21429600 & -0.52603200 & -1.52460900 & $\mathrm{C}$ & -0.36448400 & -1.11378900 & 3.92946400 \\
\hline $\mathrm{H}$ & -3.38386500 & -2.87998400 & 0.82012300 & $\mathrm{H}$ & 1.70756300 & -1.29682800 & 3.30698100 \\
\hline $\mathrm{H}$ & -5.16687300 & -0.37223900 & 0.86120900 & $\mathrm{H}$ & 0.52471000 & -1.98493300 & 2.19560900 \\
\hline $\mathrm{C}$ & -5.57455700 & -4.22759900 & -0.67684900 & $\mathrm{H}$ & -0.33190800 & -2.04127600 & 4.51413100 \\
\hline $\mathrm{H}$ & -6.23527300 & -5.04238600 & -1.00042400 & $\mathrm{H}$ & -0.25877600 & -0.27323100 & 4.62167600 \\
\hline $\mathrm{H}$ & -4.66431300 & -4.71871200 & -0.30288100 & $\mathrm{H}$ & -1.35604800 & -1.04110800 & 3.46840000 \\
\hline $\mathrm{C}$ & -6.27465800 & -3.46194000 & 0.47372300 & & & & \\
\hline $\mathrm{H}$ & -7.07007300 & -4.09443300 & 0.88897900 & 115 & & & \\
\hline $\mathrm{H}$ & -6.78644400 & -2.58354800 & 0.05846900 & & & & \\
\hline $\mathrm{C}$ & -4.41859400 & 4.26378200 & 0.83432000 & & & & \\
\hline $\mathrm{C}$ & -3.57229800 & 2.96828700 & 0.84377700 & & & & \\
\hline $\mathrm{C}$ & -4.13031800 & 3.89026200 & -2.75332900 & & & & \\
\hline $\mathrm{C}$ & -4.14716300 & 1.87521400 & -0.03579200 & & & & \\
\hline $\mathrm{C}$ & -3.17759100 & 2.78708900 & -2.23298500 & & & & \\
\hline $\mathrm{C}$ & -3.94380100 & 1.76523700 & -1.42519000 & $\mathrm{C}$ & -4.84175600 & 1.24195900 & 0.52326500 \\
\hline $\mathrm{H}$ & -5.46946100 & 4.01744900 & 1.04872700 & $\mathrm{C}$ & -4.77394000 & -0.18190600 & 0.57587200 \\
\hline $\mathrm{H}$ & -2.54413000 & 3.20923100 & 0.56275400 & $\mathrm{C}$ & -6.97513000 & -0.28634400 & 1.52334500 \\
\hline $\mathrm{H}$ & -4.76499300 & 3.47579200 & -3.54959900 & $\mathrm{C}$ & -7.03833900 & 1.10962100 & 1.45603000 \\
\hline $\mathrm{H}$ & -2.68350300 & 2.31185000 & -3.08949000 & $\mathrm{C}$ & -6.00502300 & 1.89153000 & 0.96797600 \\
\hline $\mathrm{H}$ & -4.07541500 & 4.88166300 & 1.67508300 & $\mathrm{C}$ & -3.59351600 & 1.72621400 & -0.01093800 \\
\hline $\mathrm{H}$ & -3.52507100 & 2.59433300 & 1.87490100 & $\mathrm{C}$ & -2.80001600 & 0.63820500 & -0.28147700 \\
\hline $\mathrm{H}$ & -3.51252200 & 4.66516900 & -3.22828500 & $\mathrm{H}$ & -7.82131900 & -0.83493400 & 1.92285000 \\
\hline $\mathrm{H}$ & -5.08550400 & 1.47809200 & 0.35092500 & $\mathrm{H}$ & -6.10701500 & 2.97183600 & 0.94377800 \\
\hline $\mathrm{H}$ & -2.38590300 & 3.24311800 & -1.63658600 & $\mathrm{H}$ & -3.33463900 & 2.76790900 & -0.13435800 \\
\hline $\mathrm{H}$ & -4.72688600 & 1.27858400 & -2.00498300 & $\mathrm{~N}$ & -3.52194000 & -0.53461100 & 0.05670300 \\
\hline $\mathrm{C}$ & -4.35911400 & 5.12776900 & -0.44321700 & $\mathrm{P}$ & -2.37388900 & -1.83631900 & -0.05011500 \\
\hline $\mathrm{H}$ & -4.83980700 & 6.08383900 & -0.19861200 & $\mathrm{C}$ & -3.07868700 & -3.11796500 & -1.28053000 \\
\hline $\mathrm{H}$ & -3.31128100 & 5.37554700 & -0.66706700 & $\mathrm{C}$ & -2.15473700 & -2.57994700 & 1.70387900 \\
\hline $\mathrm{C}$ & -5.04821300 & 4.54722200 & -1.70364300 & $\mathrm{C}$ & -4.51475800 & -3.58860700 & -0.97974800 \\
\hline $\mathrm{H}$ & -5.58807200 & 5.35424400 & -2.21607000 & $\mathrm{H}$ & -5.22122600 & -2.75407400 & -1.01042900 \\
\hline $\mathrm{H}$ & -5.82037800 & 3.83148800 & -1.39129700 & $\mathrm{H}$ & -4.82159200 & -4.30692100 & -1.75186700 \\
\hline $\mathrm{C}$ & 0.75553900 & 0.15764200 & 2.00303100 & $\mathrm{H}$ & -4.60989800 & -4.08923800 & -0.01454100 \\
\hline $\mathrm{H}$ & -0.24828100 & 0.59049200 & 1.97565900 & $\mathrm{C}$ & -3.08812400 & -2.44376400 & -2.66891300 \\
\hline $\mathrm{C}$ & 1.73691500 & 1.23076400 & 2.49642600 & $\mathrm{H}$ & -3.51800300 & -3.14062800 & -3.40015800 \\
\hline $\mathrm{H}$ & 1.65624900 & 2.11621000 & 1.86243300 & $\mathrm{H}$ & -3.69868500 & -1.53426700 & -2.67567200 \\
\hline $\mathrm{H}$ & 2.76754500 & 0.87330800 & 2.45594400 & $\mathrm{H}$ & -2.07711800 & -2.19064200 & -3.00094000 \\
\hline $\mathrm{C}$ & 1.41703500 & 1.67648900 & 3.91451000 & $\mathrm{C}$ & -2.10972600 & -4.31831700 & -1.33354400 \\
\hline $\mathrm{O}$ & 0.46446900 & 2.35320300 & 4.24032000 & $\mathrm{H}$ & -1.07841700 & -3.99918700 & -1.52362800 \\
\hline $\mathrm{O}$ & 2.33210400 & 1.21298800 & 4.80233000 & $\mathrm{H}$ & -2.12264200 & -4.91009000 & -0.41361700 \\
\hline $\mathrm{C}$ & 2.10487300 & 1.58901200 & 6.16966300 & $\mathrm{H}$ & -2.40808700 & -4.98331400 & -2.15423200 \\
\hline
\end{tabular}




\begin{tabular}{|c|c|c|c|c|c|c|c|}
\hline $\mathrm{C}$ & -2.27056700 & -1.41416400 & 2.70932300 & $\mathrm{C}$ & 2.90283300 & 0.59364200 & 4.97464600 \\
\hline $\mathrm{H}$ & -1.58984200 & -0.59425200 & 2.47116500 & $\mathrm{C}$ & 3.59682400 & 1.28207000 & 1.73199300 \\
\hline $\mathrm{H}$ & -3.28888300 & -1.01782100 & 2.75800200 & $\mathrm{C}$ & 2.21216200 & 0.24686300 & 3.63362400 \\
\hline $\mathrm{H}$ & -2.00786900 & -1.79124300 & 3.70665100 & $\mathrm{C}$ & 3.22693900 & 0.17200000 & 2.51578600 \\
\hline $\mathrm{C}$ & -3.14717600 & -3.68083600 & 2.12257600 & $\mathrm{H}$ & 4.69665000 & 3.52311600 & 2.87299500 \\
\hline $\mathrm{H}$ & -3.08498800 & -4.57475700 & 1.49573000 & $\mathrm{H}$ & 1.90128000 & 2.61152000 & 1.97711200 \\
\hline $\mathrm{H}$ & -2.90839400 & -3.99034600 & 3.14889200 & $\mathrm{H}$ & 3.48725600 & -0.27224400 & 5.31716800 \\
\hline $\mathrm{C}$ & -0.71398800 & -3.14049700 & 1.74917300 & $\mathrm{H}$ & 1.68796200 & -0.71053800 & 3.74369500 \\
\hline $\mathrm{H}$ & -0.50266300 & -3.49910000 & 2.76547200 & $\mathrm{H}$ & 3.28187600 & 4.56063500 & 2.79937600 \\
\hline $\mathrm{H}$ & -0.57118900 & -3.98544800 & 1.06737700 & $\mathrm{H}$ & 3.14336700 & 3.18570300 & 0.88363000 \\
\hline $\mathrm{H}$ & 0.02209400 & -2.37176900 & 1.49721700 & $\mathrm{H}$ & 2.11419700 & 0.74566500 & 5.72482900 \\
\hline $\mathrm{F}$ & -8.16826700 & 1.71831700 & 1.89385000 & $\mathrm{H}$ & 4.62888200 & 1.30996600 & 1.38275000 \\
\hline $\mathrm{Cl}$ & 1.24512500 & -1.09066400 & -1.44143000 & $\mathrm{H}$ & 1.44556800 & 0.99338200 & 3.41833100 \\
\hline $\mathrm{H}$ & -4.18202100 & -3.32599800 & 2.12583600 & $\mathrm{H}$ & 3.99246000 & -0.58087000 & 2.69998900 \\
\hline $\mathrm{C}$ & -5.82990400 & -0.94621200 & 1.07740800 & $\mathrm{C}$ & 3.22460300 & 3.14135200 & 4.41497500 \\
\hline $\mathrm{H}$ & -5.77813800 & -2.02832000 & 1.12500100 & $\mathrm{H}$ & 3.56123400 & 3.96642000 & 5.05595300 \\
\hline $\mathrm{Rh}$ & -0.97974700 & 0.04454300 & -0.91516400 & $\mathrm{H}$ & 2.12948800 & 3.12175200 & 4.51360800 \\
\hline $\mathrm{H}$ & -1.64831800 & -0.01618800 & -2.55795600 & $\mathrm{C}$ & 3.83109100 & 1.82411300 & 4.95989700 \\
\hline $\mathrm{Cl}$ & 0.25409300 & 0.69996700 & 1.06722900 & $\mathrm{H}$ & 4.16124300 & 1.98578100 & 5.99427800 \\
\hline $\mathrm{Rh}$ & 2.53729900 & -0.14441300 & 0.47092500 & $\mathrm{H}$ & 4.74323700 & 1.58948900 & 4.39509300 \\
\hline $\mathrm{C}$ & 5.15062600 & -3.70602400 & -0.66212500 & $\mathrm{C}$ & -0.37103200 & 1.70797800 & -2.04019800 \\
\hline $\mathrm{C}$ & 3.90045300 & -2.90286200 & -0.23089400 & $\mathrm{H}$ & 0.70040900 & 1.58698600 & -2.20891000 \\
\hline $\mathrm{C}$ & 5.81532700 & -0.76479600 & -2.66252800 & $\mathrm{C}$ & -1.21382600 & 1.02151400 & -3.04069700 \\
\hline $\mathrm{C}$ & 4.22451500 & -1.49830400 & 0.23880700 & $\mathrm{H}$ & -2.16417900 & 1.53531200 & -3.21720300 \\
\hline $\mathrm{C}$ & 4.39563200 & -0.48162800 & -2.11590300 & $\mathrm{C}$ & -0.72385700 & 3.10425100 & -1.58137200 \\
\hline $\mathrm{C}$ & 4.41744800 & -0.38952700 & -0.60782700 & $\mathrm{H}$ & -0.25520700 & 3.33651900 & -0.62029700 \\
\hline $\mathrm{H}$ & 5.90813500 & -3.66218200 & 0.13474900 & $\mathrm{H}$ & -1.80476100 & 3.21126600 & -1.43680900 \\
\hline $\mathrm{H}$ & 3.17809500 & -2.89030200 & -1.05063300 & $\mathrm{C}$ & -0.54760200 & 0.53471600 & -4.32914900 \\
\hline $\mathrm{H}$ & 6.43781500 & 0.13321800 & -2.54153900 & $\mathrm{H}$ & -1.22201700 & -0.09364000 & -4.92147700 \\
\hline $\mathrm{H}$ & 4.02779300 & 0.45483800 & -2.55343500 & $\mathrm{H}$ & -0.27258700 & 1.41380000 & -4.92007800 \\
\hline $\mathrm{H}$ & 4.85032100 & -4.76039200 & -0.73477400 & $\mathrm{H}$ & 0.35899700 & -0.03484900 & -4.10538700 \\
\hline $\mathrm{H}$ & 3.41521400 & -3.43876800 & 0.59577600 & $\mathrm{C}$ & -0.31672900 & 4.15793000 & -2.60222200 \\
\hline $\mathrm{H}$ & 5.72749200 & -0.93173100 & -3.74519500 & $\mathrm{O}$ & -0.14661200 & 3.96340200 & -3.78875900 \\
\hline $\mathrm{H}$ & 4.76724800 & -1.48916000 & 1.18392800 & $\mathrm{O}$ & -0.19294300 & 5.37365200 & -2.02581100 \\
\hline $\mathrm{H}$ & 3.71205400 & -1.26407800 & -2.44824100 & $\mathrm{C}$ & 0.13446700 & 6.45048000 & -2.91885700 \\
\hline $\mathrm{H}$ & 5.07765900 & 0.39824300 & -0.24830100 & $\mathrm{H}$ & -0.63813600 & 6.56492800 & -3.68421400 \\
\hline $\mathrm{C}$ & 5.80917900 & -3.30884400 & -2.00036300 & $\mathrm{H}$ & 0.18960400 & 7.34233900 & -2.29382900 \\
\hline $\mathrm{H}$ & 6.52431800 & -4.10421300 & -2.24721400 & $\mathrm{H}$ & 1.09390000 & 6.26463500 & -3.40912500 \\
\hline $\mathrm{H}$ & 5.05371300 & -3.33496900 & -2.79916900 & & & & \\
\hline $\mathrm{C}$ & 6.56101500 & -1.95436400 & -2.02560000 & 116-ts & & & \\
\hline $\mathrm{H}$ & 7.49819600 & -2.07852500 & -2.58365400 & & & & \\
\hline $\mathrm{H}$ & 6.86285400 & -1.69368800 & -1.00232800 & & & & \\
\hline $\mathrm{C}$ & 3.60042800 & 3.52293500 & 2.96812100 & & & & \\
\hline $\mathrm{C}$ & 2.98378600 & 2.66507800 & 1.83733800 & & & & \\
\hline
\end{tabular}



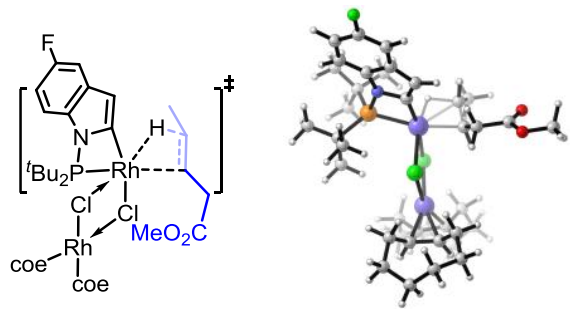

C $\quad-4.98626700$

1.12844300

0.39087000

C $\quad-4.80705100$

C $\quad-6.99431000$

C $\quad-7.16762900$

$-0.27025600$

0.60676900

$-0.44071000$

1.57413900

0.92896000

1.34780500

C $\quad-6.19803400$

C $\quad-3.77737200$

1.73003600

1.64551600

0.76799000

C $\quad-2.89231700$

0.60272200

$-0.20144400$

$-0.33454700$

H $\quad-7.79556300$

$-1.00508000$

2.03888900

$-6.38539400$

2.78934500

0.62382600

2.68144400

$-0.46233900$

$-3.61402900$

$-3.52620700$

$-0.57403400$

0.13330700

$-1.80229400$

0.03389400

$-3.16996600$

$-1.13315800$

C $\quad-2.96249600$

C $\quad-1.98864300$

C $\quad-4.36161900$

$-2.46824800$

1.80298800

$-3.69933100$

$-0.75997500$

$-2.90453700$

$-0.80077200$

$-4.46578300$

$-1.48965700$

$-4.16085600$

0.22775700

$-2.56498300$

$-2.54970600$

$-3.31751700$

$-3.22501200$

$-1.68503600$

$-2.57473300$

$-2.28271700$

$-2.93685800$

$-4.31819600$

$-1.16655600$

$-3.95291900$

$-1.39988500$

$-4.87309200$

$-0.22500000$

$-5.03029900$

$-1.95155800$

$-1.27722200$

2.77095800

$-0.42567100$

2.49261900

2.82613700

$-0.94432800$

3.77444500

2.28266100

1.68387400

3.31158200

1.83123900

2.85448600

1.17712000

1.53538600

1.71983900
$\mathrm{Cl} \quad 1.19825400$

$-1.05680600$

$-1.46369200$

H $\quad-3.95686000$

$-3.32196500$

2.30291900

C $\quad-5.79805700$

$-1.05380800$

1.20130300

$\mathrm{H} \quad-5.65718500$

$-2.11529600$

1.37401400

Rh $\quad-1.04237800$

0.04764800

$-0.93480600$

H $\quad-1.73393300$

$-0.16790900$

$-2.37763300$

Cl 0.31148300

0.77582300

1.05411300

$\mathrm{Rh} \quad 2.55232700$

$-0.12140500$

0.41499400

C $\quad 5.06974900$

$-3.73904600$

$-0.76929200$

C $\quad 3.84543600$

$-2.91093700$

$-0.31188200$

C $\quad 5.74696800$

$-0.81527400$

$-2.79069300$

C $\quad 4.20742500$

$-1.51248000$

0.14877600

C $\quad 4.34696200$

$-0.50240900$

$-2.21053800$

C 4.40477300

$-0.40900800$

$-0.70331900$

H 5.84655900

$-3.70777300$

0.00938300

$\mathrm{H}$

3.10657200

$-2.88517200$

$-1.11656800$

H $\quad 6.39058600$

0.06988500

$-2.68644900$

H 3.98846100

0.44097600

$-2.64125100$

H 4.74809900

$-4.78779500$

$-0.83222200$

H 3.36725800

$-3.43653700$

0.52553900

H 5.63003800

$-0.98196600$

$-3.87071600$

H 4.77047600

$-1.51386800$

1.08187000

$-1.27115200$

$-2.52549500$

H $\quad 5.09073700$

0.36423800

$-0.36051500$

C $\quad 5.70387400$

$-3.35738600$

$-2.12366000$

H 6.39602500

$-4.16783300$

$-2.38659100$

H 4.92869900

$-3.36844300$

$-2.90377300$

C $\quad 6.48316500$

$-2.01903100$

$-2.16968800$

H 7.40362700

$-2.16378200$

$-2.75016300$

$-1.76313800$

$-1.15455400$

3.52402500

2.88807700

2.68095300

1.77170400

0.60393700

4.90832700

C $\quad 3.04812200$

1.28653000

1.64744400

C $\quad 2.31599600$

0.27271700

3.58574600

C $\quad 3.29874900$

0.18261800

2.44081500

H $\quad 4.83859400$

3.50364000

2.76582500

2.64754600

1.93862700

$-0.27226300$

5.23302900

$-0.67627700$

3.70788300

4.56807800

2.72923500

3.20105900

0.81543100

0.76655000

5.68001800

1.29578200

1.27033400 


\begin{tabular}{|c|c|c|c|c|c|c|c|}
\hline $\mathrm{H}$ & 1.55591500 & 1.03083900 & 3.39033100 & $\mathrm{H}$ & -3.54080400 & 2.46596400 & -1.58329900 \\
\hline $\mathrm{H}$ & 4.05655400 & -0.58276700 & 2.60427400 & $\mathrm{~N}$ & -3.72998800 & -0.41788300 & 0.03138000 \\
\hline $\mathrm{C}$ & 3.39823800 & 3.14682700 & 4.34330700 & $P$ & -2.55536400 & -1.60572100 & 0.47001200 \\
\hline $\mathrm{H}$ & 3.76404900 & 3.96519000 & 4.97691000 & $\mathrm{C}$ & -3.13578500 & -3.30934400 & -0.18408100 \\
\hline $\mathrm{H}$ & 2.30566300 & 3.14511300 & 4.46822300 & $\mathrm{C}$ & -2.39031900 & -1.59325300 & 2.37990600 \\
\hline $\mathrm{C}$ & 3.99584300 & 1.81895800 & 4.87180000 & $\mathrm{C}$ & -4.58625200 & -3.64316000 & 0.22407800 \\
\hline $\mathrm{H}$ & 4.35472500 & 1.97370200 & 5.89768800 & $\mathrm{H}$ & -5.29212200 & -2.91266400 & -0.18231000 \\
\hline $\mathrm{H}$ & 4.88951900 & 1.57012500 & 4.28397500 & $\mathrm{H}$ & -4.84875500 & -4.62279700 & -0.19567900 \\
\hline $\mathrm{C}$ & -0.38966800 & 1.78826900 & -2.02958700 & $\mathrm{H}$ & -4.73090900 & -3.70356300 & 1.30332800 \\
\hline $\mathrm{H}$ & 0.67966400 & 1.64201900 & -2.17975000 & $\mathrm{C}$ & -3.08723600 & -3.29794600 & -1.72662200 \\
\hline $\mathrm{C}$ & -1.23571900 & 1.11619200 & -2.96543200 & $\mathrm{H}$ & -3.49496900 & -4.25033900 & -2.08966600 \\
\hline $\mathrm{H}$ & -2.23694900 & 1.53515700 & -3.07840400 & $\mathrm{H}$ & -3.69406200 & -2.49172800 & -2.15143700 \\
\hline $\mathrm{C}$ & -0.74069400 & 3.13675300 & -1.45067200 & $\mathrm{H}$ & -2.06552800 & -3.20335600 & -2.10296900 \\
\hline $\mathrm{H}$ & -0.20453400 & 3.31786900 & -0.51507600 & $\mathrm{C}$ & -2.15546400 & -4.38968200 & 0.32091300 \\
\hline $\mathrm{H}$ & -1.80722900 & 3.20596400 & -1.21313900 & $\mathrm{H}$ & -1.11614700 & -4.13619000 & 0.08340300 \\
\hline $\mathrm{C}$ & -0.65686900 & 0.50968700 & -4.23302800 & $\mathrm{H}$ & -2.23352700 & -4.56148300 & 1.39792000 \\
\hline $\mathrm{H}$ & -1.34996200 & -0.19002100 & -4.71182500 & $\mathrm{H}$ & -2.38844300 & -5.33884500 & -0.17827400 \\
\hline $\mathrm{H}$ & -0.45880400 & 1.33378100 & -4.92861600 & $\mathrm{C}$ & -2.54861700 & -0.12460000 & 2.83199800 \\
\hline $\mathrm{H}$ & 0.28405200 & -0.00679600 & -4.02677000 & $\mathrm{H}$ & -1.85021200 & 0.54377900 & 2.32642000 \\
\hline $\mathrm{C}$ & -0.43620200 & 4.25830900 & -2.43572300 & $\mathrm{H}$ & -3.56686800 & 0.24114800 & 2.67036700 \\
\hline $\mathrm{O}$ & -0.36621700 & 4.13727200 & -3.64156900 & $\mathrm{H}$ & -2.33824400 & -0.07473500 & 3.90825600 \\
\hline $\mathrm{O}$ & -0.28010600 & 5.43768800 & -1.79672900 & $\mathrm{C}$ & -3.43554300 & -2.43746800 & 3.13593300 \\
\hline $\mathrm{C}$ & -0.04484100 & 6.57354300 & -2.64477700 & $\mathrm{H}$ & -3.34136600 & -3.50962800 & 2.94449600 \\
\hline $\mathrm{H}$ & -0.88047200 & 6.71869600 & -3.33494000 & $\mathrm{H}$ & -3.27620300 & -2.28738400 & 4.21154200 \\
\hline $\mathrm{H}$ & 0.04910000 & 7.42638200 & -1.97182900 & $\mathrm{C}$ & -0.97385700 & -2.09770500 & 2.73773500 \\
\hline \multirow[t]{2}{*}{$\mathrm{H}$} & 0.87289600 & 6.43709300 & \multirow[t]{2}{*}{-3.22314800} & $\mathrm{H}$ & -0.84540000 & -2.02686700 & 3.82593900 \\
\hline & \multirow{9}{*}{ 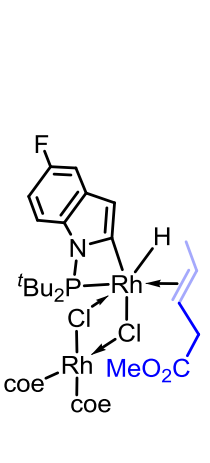 } & \multirow{9}{*}{ ? } & & $\mathrm{H}$ & -0.81889600 & -3.14388600 & 2.45705900 \\
\hline \multirow[t]{8}{*}{117} & & & & $\mathrm{H}$ & -0.19751200 & -1.49304400 & 2.26477900 \\
\hline & & & & $\mathrm{F}$ & -8.50878100 & 2.23212600 & 0.38749900 \\
\hline & & & & $\mathrm{Cl}$ & 0.99792400 & -1.67104700 & -0.94560200 \\
\hline & & & & $\mathrm{H}$ & -4.46119000 & -2.12728000 & 2.91618900 \\
\hline & & & & $\mathrm{C}$ & -6.11073700 & -0.44097200 & 0.96466400 \\
\hline & & & & $\mathrm{H}$ & -6.06121600 & -1.39755000 & 1.47367800 \\
\hline & & & & $\mathrm{Rh}$ & -1.14567600 & -0.28737000 & -0.91785000 \\
\hline & & & & $\mathrm{H}$ & -1.85389100 & -1.10684500 & -1.99608400 \\
\hline $\mathrm{C}$ & 0100 & 1.33258300 & -0.40849700 & $\mathrm{Cl}$ & 0.32665300 & 0.85395700 & 0.94849400 \\
\hline $\mathrm{C}$ & -5.02091600 & 0.07251800 & 0.25973500 & $\mathrm{Rh}$ & 2.51163100 & -0.21911700 & 0.40933300 \\
\hline $\mathrm{C}$ & 4000 & 0.30500700 & 1.00000600 & $\mathrm{C}$ & 5.03643300 & -4.00456200 & 0.03012200 \\
\hline $\mathrm{C}$ & 000 & 1.53 & 0.33541000 & $\mathrm{C}$ & 3.83724000 & -3.08842400 & 0.37304200 \\
\hline $\mathrm{C}$ & -6.27972600 & 2.06831500 & -0.36690900 & $\mathrm{C}$ & 5.36965300 & -1.78259200 & -2.80619300 \\
\hline $\mathrm{C}$ & -3.79988000 & 1.58332100 & -1.01538300 & $\mathrm{C}$ & 4.18669100 & -1.61313600 & 0.36812600 \\
\hline $\mathrm{C}$ & -2.98065700 & 0.52093000 & -0.72249700 & $\mathrm{C}$ & 4.02869900 & -1.32706300 & -2.18247600 \\
\hline $\mathrm{H}$ & -8.16443400 & -0.04955400 & 1.53363200 & $\mathrm{C}$ & 4.24457100 & -0.80195600 & -0.78158800 \\
\hline $\mathrm{H}$ & -6.38170600 & 3.02974100 & -0.86001600 & $\mathrm{H}$ & 5.89115000 & -3.74564000 & 0.67296500 \\
\hline
\end{tabular}




\begin{tabular}{|c|c|c|c|c|c|c|c|}
\hline $\mathrm{H}$ & 3.01321400 & -3.30098000 & -0.31263800 & $\mathrm{C}$ & -0.26480700 & -0.39509700 & -4.02159900 \\
\hline $\mathrm{H}$ & 5.98283900 & -0.89934500 & -3.03467800 & $\mathrm{H}$ & -0.98900100 & -1.18222100 & -4.25360700 \\
\hline $\mathrm{H}$ & 3.59136800 & -0.54886500 & -2.82055500 & $\mathrm{H}$ & -0.00840500 & 0.10686100 & -4.96575200 \\
\hline $\mathrm{H}$ & 4.75213900 & -5.02970800 & 0.30525900 & $\mathrm{H}$ & 0.63911700 & -0.85787800 & -3.62010000 \\
\hline $\mathrm{H}$ & 3.47707400 & -3.35395700 & 1.37597000 & $\mathrm{C}$ & 0.54121100 & 3.78917000 & -2.26564100 \\
\hline $\mathrm{H}$ & 5.14340100 & -2.25500200 & -3.77252700 & $\mathrm{O}$ & 1.69940900 & 3.57720900 & -2.56112300 \\
\hline $\mathrm{H}$ & 4.84533600 & -1.33633900 & 1.19185800 & $\mathrm{O}$ & -0.03142100 & 5.01010000 & -2.33347400 \\
\hline $\mathrm{H}$ & 3.32286900 & -2.15894200 & -2.18225200 & $\mathrm{C}$ & 0.83487200 & 6.07671900 & -2.75453100 \\
\hline $\mathrm{H}$ & 4.92698800 & 0.04582300 & -0.75695700 & $\mathrm{H}$ & 1.22365400 & 5.88231400 & -3.75765300 \\
\hline $\mathrm{C}$ & 5.50443800 & -4.02364400 & -1.44027600 & $\mathrm{H}$ & 0.21611100 & 6.97448900 & -2.74959600 \\
\hline $\mathrm{H}$ & 6.19735600 & -4.86951500 & -1.53849200 & $\mathrm{H}$ & 07500 & 6.18502200 & 2.06324800 \\
\hline $\mathrm{H}$ & 4.65119500 & -4.26738300 & -2.09011300 & & & & \\
\hline $\mathrm{C}$ & 6.21792700 & -2.74947700 & -1.95680500 & 118 & & & \\
\hline $\mathrm{H}$ & 7.07532800 & -3.04779600 & -2.57416400 & & & & \\
\hline $\mathrm{H}$ & 6.64640600 & -2.20810400 & -1.10291000 & & & & \\
\hline $\mathrm{C}$ & 3.90195400 & 3.95519000 & 1.63936300 & & & & \\
\hline $\mathrm{C}$ & 3.13989700 & 2.84907400 & 0.87099000 & & & & \\
\hline $\mathrm{C}$ & 3.39159300 & 1.73654900 & 4.45165100 & & & & \\
\hline $\mathrm{C}$ & 3.71951700 & 1.46584400 & 1.09006900 & & & & \\
\hline $\mathrm{C}$ & 2.55002200 & 1.06176900 & 3.34174700 & $\mathrm{C}$ & 5.16701200 & -0.31689300 & 1.29520100 \\
\hline $\mathrm{C}$ & 3.43247800 & 0.63676000 & 2.19102100 & $\mathrm{C}$ & 4.95020200 & -0.67377600 & 0.06991400 \\
\hline $\mathrm{H}$ & 4.97883700 & 3.87711400 & 1.42678900 & $\mathrm{C}$ & 7.17612000 & -1.54879700 & 0.23641400 \\
\hline $\mathrm{H}$ & 2.08441800 & 2.88143200 & 1.15254300 & $\mathrm{C}$ & 7.38507300 & -1.18767500 & -1.09872100 \\
\hline $\mathrm{H}$ & 3.99609000 & 0.97425200 & 4.96392000 & $\mathrm{C}$ & 6.41643100 & -0.57991100 & -1.88013200 \\
\hline $\mathrm{H}$ & 2.03104100 & 0.19496500 & 3.76959100 & $\mathrm{C}$ & 3.95218100 & 0.27013500 & -1.80427400 \\
\hline $\mathrm{H}$ & 3.58272300 & 4.92037400 & 1.22229600 & $\mathrm{C}$ & 3.02438100 & 0.26094700 & -0.79223800 \\
\hline $\mathrm{H}$ & 3.17686300 & 3.07787500 & -0.20123600 & $\mathrm{H}$ & 7.97974900 & -2.02556300 & 0.78716500 \\
\hline $\mathrm{H}$ & 2.69517000 & 2.13034800 & 5.20536700 & $\mathrm{H}$ & 6.63338300 & -0.32653700 & -2.91288800 \\
\hline $\mathrm{H}$ & 4.70292100 & 1.34832600 & 0.63539900 & $\mathrm{H}$ & 3.81128900 & 0.63451600 & -2.81199500 \\
\hline $\mathrm{H}$ & 1.77291500 & 1.74800600 & 3.00227700 & $\mathrm{~N}$ & 3.63722300 & -0.28650400 & 0.36279200 \\
\hline $\mathrm{H}$ & 4.20377600 & -0.06878200 & 2.49632600 & $\mathrm{P}$ & 2.35160900 & -0.35453000 & 1.51336500 \\
\hline $\mathrm{C}$ & 3.69650900 & 4.01113600 & 3.16794200 & $\mathrm{C}$ & 2.85317100 & 0.67905400 & 3.04645300 \\
\hline $\mathrm{H}$ & 4.12526600 & 4.96225300 & 3.50982200 & $\mathrm{C}$ & 2.06568700 & -2.19787300 & 1.96131800 \\
\hline $\mathrm{H}$ & 2.62084900 & 4.07851200 & 3.38759300 & $\mathrm{C}$ & 4.24873900 & 0.30415600 & 3.58762200 \\
\hline $\mathrm{C}$ & 4.33483400 & 2.86685600 & 3.99474400 & $\mathrm{H}$ & 5.02690300 & 0.48890300 & 2.84143700 \\
\hline $\mathrm{H}$ & 4.78377800 & 3.29203700 & 4.90199900 & $\mathrm{H}$ & 4.46813900 & 0.93862700 & 4.45614000 \\
\hline $\mathrm{H}$ & 5.17193600 & 2.43943100 & 3.42705200 & $\mathrm{H}$ & 4.32238900 & -0.73269800 & 3.91697200 \\
\hline $\mathrm{C}$ & -0.07542100 & 1.37168300 & -2.19273600 & $\mathrm{C}$ & 2.90249100 & 2.16948800 & 2.64937600 \\
\hline $\mathrm{H}$ & 0.97100100 & 1.10761000 & -2.06313500 & $\mathrm{H}$ & 3.26341200 & 2.74367800 & 3.51265600 \\
\hline $\mathrm{C}$ & -0.83286200 & 0.63460100 & -3.07513100 & $\mathrm{H}$ & 3.59001100 & 2.35015600 & 1.81673700 \\
\hline $\mathrm{H}$ & -1.82260000 & 1.01135500 & -3.33140200 & $\mathrm{H}$ & 1.91555200 & 2.55270900 & 2.37801000 \\
\hline $\mathrm{C}$ & -0.44350300 & 2.76086600 & -1.73051800 & $\mathrm{C}$ & 1.77350100 & 0.50668300 & 4.13570400 \\
\hline $\mathrm{H}$ & -0.39602200 & 2.83357500 & -0.63677100 & $\mathrm{H}$ & 0.77272300 & 0.73851200 & 3.75442400 \\
\hline $\mathrm{H}$ & -1.45567400 & 3.04176200 & -2.02924200 & $\mathrm{H}$ & 1.75848000 & -0.49997900 & 4.56202600 \\
\hline
\end{tabular}




\begin{tabular}{|c|c|c|c|c|c|c|c|}
\hline $\mathrm{H}$ & 1.98564800 & 1.20452700 & 4.95575600 & $\mathrm{C}$ & -3.19328100 & -1.86966300 & -2.86340700 \\
\hline $\mathrm{C}$ & 2.29968200 & -3.01313300 & 0.67024900 & $\mathrm{C}$ & -3.23527200 & -5.06216400 & -0.87114000 \\
\hline $\mathrm{H}$ & 1.68185300 & -2.66515900 & -0.15897400 & $\mathrm{C}$ & -3.74108800 & -1.76051600 & -1.45347600 \\
\hline $\mathrm{H}$ & 3.34915700 & -2.99048000 & 0.36201000 & $\mathrm{C}$ & -2.44747400 & -3.77764700 & -0.51995600 \\
\hline $\mathrm{H}$ & 2.02981200 & -4.05779500 & 0.87258300 & $\mathrm{C}$ & -3.38215200 & -2.59813900 & -0.37973900 \\
\hline $\mathrm{C}$ & 2.99239400 & -2.76669200 & 3.05442800 & $\mathrm{H}$ & -5.00404600 & -2.77472300 & -3.66962500 \\
\hline $\mathrm{H}$ & 2.84056400 & -2.30325500 & 4.03277300 & $\mathrm{H}$ & -2.12122900 & -2.07980400 & -2.85725100 \\
\hline $\mathrm{H}$ & 2.76453900 & -3.83469400 & 3.16611400 & $\mathrm{H}$ & -3.80937100 & -5.38922500 & 0.00770400 \\
\hline $\mathrm{C}$ & 0.59579900 & -2.35136100 & 2.41202100 & $\mathrm{H}$ & -1.89828200 & -3.94570600 & 0.41491300 \\
\hline $\mathrm{H}$ & 0.40117100 & -3.41427500 & 2.60702600 & $\mathrm{H}$ & -3.63282200 & -2.76028900 & -4.76603600 \\
\hline $\mathrm{H}$ & 0.38163300 & -1.80490300 & 3.33557500 & $\mathrm{H}$ & -3.31267500 & -0.89514700 & -3.35562400 \\
\hline $\mathrm{H}$ & -0.09978800 & -2.01523700 & 1.64048200 & $\mathrm{H}$ & -2.50283800 & -5.85725800 & -1.06988800 \\
\hline $\mathrm{F}$ & 8.59628200 & -1.45102300 & -1.64766000 & $\mathrm{H}$ & -4.74609900 & -1.34088700 & -1.42938500 \\
\hline $\mathrm{Cl}$ & -1.11195500 & 1.22333600 & 0.86394900 & $\mathrm{H}$ & -1.69462200 & -3.58833700 & -1.28653400 \\
\hline $\mathrm{H}$ & 4.05056600 & -2.69095400 & 2.78778500 & $\mathrm{H}$ & -4.13129700 & -2.75179400 & 0.39623900 \\
\hline $\mathrm{C}$ & 5.94213300 & -1.29069800 & 0.83408300 & $\mathrm{C}$ & -3.61598400 & -4.41382500 & -3.39041400 \\
\hline $\mathrm{H}$ & 5.77623100 & -1.56520700 & 1.87019700 & $\mathrm{H}$ & -4.02199100 & -5.01021100 & -4.21775300 \\
\hline $\mathrm{Rh}$ & 1.14536600 & 0.72902100 & -0.24312300 & $\mathrm{H}$ & -2.52894800 & -4.57788300 & -3.41621000 \\
\hline $\mathrm{H}$ & 1.85319400 & 1.94924300 & 0.34606000 & $\mathrm{C}$ & -4.20473200 & -4.95825500 & -2.06494600 \\
\hline $\mathrm{Cl}$ & -0.36432900 & -1.29957900 & -1.00352400 & $\mathrm{H}$ & -4.60285000 & -5.96622900 & -2.24025600 \\
\hline $\mathrm{Rh}$ & -2.54925900 & -0.61006100 & -0.03977200 & $\mathrm{H}$ & -5.07225000 & -4.34701200 & -1.78189300 \\
\hline $\mathrm{C}$ & -4.85222500 & 0.54310700 & 3.72473600 & $\mathrm{C}$ & 1.05144900 & 2.53465100 & -1.71363800 \\
\hline $\mathrm{C}$ & -3.68237400 & 0.08681300 & 2.82075700 & $\mathrm{H}$ & 2.07745100 & 2.65526700 & -2.05633300 \\
\hline $\mathrm{C}$ & -5.65023400 & 2.63456600 & 0.88092600 & $\mathrm{C}$ & 0.29098800 & 1.54678000 & -2.29759400 \\
\hline $\mathrm{C}$ & -4.12810600 & -0.34067300 & 1.43585400 & $\mathrm{H}$ & -0.77648800 & 1.53856800 & -2.08402800 \\
\hline $\mathrm{C}$ & -4.28147900 & 2.03755500 & 0.47256100 & $\mathrm{C}$ & 0.45245400 & 3.74016400 & -1.02552800 \\
\hline $\mathrm{C}$ & -4.36751500 & 0.53281500 & 0.35775600 & $\mathrm{H}$ & 1.12503900 & 4.16451900 & -0.27399200 \\
\hline $\mathrm{H}$ & -5.64180700 & -0.22322100 & 3.72110300 & $\mathrm{H}$ & -0.47080100 & 3.46796400 & -0.50045500 \\
\hline $\mathrm{H}$ & -2.93623300 & 0.88355400 & 2.77105200 & $\mathrm{C}$ & 0.72185100 & 0.74113900 & -3.49177800 \\
\hline $\mathrm{H}$ & -6.34498700 & 2.56478800 & 0.03174200 & $\mathrm{H}$ & 0.38263300 & -0.29508700 & -3.42672000 \\
\hline $\mathrm{H}$ & -3.97662100 & 2.47891900 & -0.48487000 & $\mathrm{H}$ & 0.26562500 & 1.19222200 & -4.38470400 \\
\hline $\mathrm{H}$ & -4.47273300 & 0.58034000 & 4.75524500 & $\mathrm{H}$ & 1.80540200 & 0.75205300 & -3.62784300 \\
\hline $\mathrm{H}$ & -3.18697900 & -0.76745700 & 3.30145500 & $\mathrm{C}$ & 0.10328500 & 4.82858100 & -2.03314400 \\
\hline $\mathrm{H}$ & -5.50374600 & 3.70788900 & 1.06707900 & $\mathrm{O}$ & -0.06611600 & 4.66995800 & -3.22273700 \\
\hline $\mathrm{H}$ & -4.70441500 & -1.26521600 & 1.45261700 & $\mathrm{O}$ & -0.02487400 & 6.02287500 & -1.41356500 \\
\hline $\mathrm{H}$ & -3.52191400 & 2.32905200 & 1.19960000 & $\mathrm{C}$ & -0.40889700 & 7.11754300 & -2.26133000 \\
\hline $\mathrm{H}$ & -5.10226400 & 0.21381000 & -0.38066200 & $\mathrm{H}$ & -1.37610500 & 6.91949600 & -2.73129400 \\
\hline $\mathrm{C}$ & -5.48368300 & 1.91363200 & 3.40317400 & $\mathrm{H}$ & -0.47171300 & 7.98682800 & -1.60601800 \\
\hline $\mathrm{H}$ & -6.12865900 & 2.17101000 & 4.25339500 & $\mathrm{H}$ & 0.33853900 & 7.27834100 & -3.04291700 \\
\hline $\mathrm{H}$ & -4.69677400 & 2.68183900 & 3.38833800 & & & & \\
\hline $\mathrm{C}$ & -6.33019700 & 1.99689600 & 2.10827100 & 119-ts & & & \\
\hline $\mathrm{H}$ & -7.23432000 & 2.58518800 & 2.31261300 & & & & \\
\hline $\mathrm{H}$ & -6.68792300 & 0.99156600 & 1.84918100 & & & & \\
\hline $\mathrm{C}$ & -3.91680200 & -2.93683700 & -3.71957600 & & & & \\
\hline
\end{tabular}



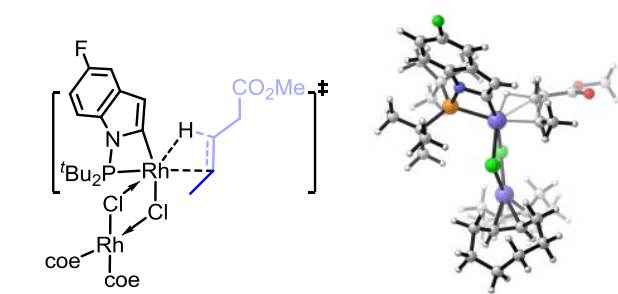

C $\quad 4.94836500$

C $\quad 4.87771400$

C $\quad 7.11084200$

C $\quad 7.17684900$

C $\quad 6.12961100$

C 3.68425700

C 2.87459500

H 7.96916900

H $\quad 6.23551900$

H $\quad 3.42677400$

N 3.60540100

P 2.44785300

C $\quad 3.10657100$

C 2.25779800

C $\quad 4.55157700$

H $\quad 5.25169200$

H 4.84799100

H 4.66861600

C $\quad 3.09037600$

H 3.51957600

H 3.68978200

H 2.07247000

C 2.13905000

H 1.10251400

H 2.17661100

H 2.41731000

C 2.39962200

H 1.71167500

H 3.41919900

H 2.16402100

C $\quad 3.26433400$

H $\quad 3.18836900$

H 3.05101600

C $\quad 0.82118500$

H $\quad 0.64128700$

H $\quad 0.66426200$

H $\quad 0.07482000$

F
$-0.68957100-1.44146700$

$-0.66691100 \quad-0.01645000$

$-1.52509700$

0.14651600

$-1.53372100 \quad-1.25081400$

$-1.12869600-2.06154500$

$-0.22659000-1.95909000$

$0.06568900-0.88935800$

$-1.86123200 \quad 0.71822700$

$-1.16180900 \quad-3.14122100$

$-0.16005600 \quad-3.00584300$

$-0.17742500$

0.30031300

0.02933700

1.57260300

1.40089900

$-1.64351400$

2.73273200

2.49025100

1.17284300

3.22045200

1.13147200

2.01848000

2.38101700

3.85517100

0.26533500

3.81477900

2.72485600

1.94052700

3.51695800

2.56773200

2.65944900

1.02611100

3.02061900

1.67243200

1.54209000

3.92692700

1.67414600

0.68546500

3.59633300

4.60571500

2.43025700

4.50899700

$-2.75664100$

1.42958400

0.59302500

1.03742600

$-2.81582400$

$-3.71813400$

1.90470800

$-1.92387200$

3.62164400

$-1.21240200$

$-2.91958600$

4.44824600

4.03243900

$-1.66553700$

3.06193400

$-2.64257200$

3.52980300

$-0.90024400$

3.82917000

$-1.51994500$

2.27657800

$-1.96273300$
$\mathrm{Cl}-1.18242600$

H 4.29859900

C $\quad 5.94672500$

H $\quad 5.89126500$

Rh $\quad 1.03705700$

H 1.66623800

$\mathrm{Cl}$

Rh

$\mathrm{Rh}-250980800$

C $\quad-5.10984800$

C $\quad-3.86979200$

C $\quad-5.73217900$

C $\quad-4.20052700$

C $\quad-4.32269200$

C $\quad-4.37057000$

$\mathrm{H} \quad-5.88631100$

H $\quad-3.12409700$

H $\quad-6.35467000$

$\mathrm{H}$

$\mathrm{H}$

H

$\mathrm{H}$

$\mathrm{H}$

$\mathrm{H}$
$\mathrm{H}$

$\mathrm{H}$
$\mathrm{H}$
$\mathrm{C}$

C

$$
\mathrm{H}
$$

$-3.94227100$

$-4.80806000$

$-3.40920800$

$-5.62590700$

$-4.75970200$

$-3.63770700$

$-5.03502800$

$-5.73635600$

$-6.44358100$

$-4.96175300$

C -6.49029800

$\mathrm{H}$

$\mathrm{H}$$$
\text { C }
$$

C

$\mathrm{C}$
$\mathrm{C}$

C

$$
\text { C }
$$$$
\text { C }
$$

C

$\mathrm{H}$

H

$\mathrm{H}$
$\mathrm{H}$

$\mathrm{H}$

$\mathrm{H}$
$\mathrm{H}$

$\mathrm{H}$

$\mathrm{H}$
$\mathrm{H}$
1.23251500

$-1.93842500$

$-1.08618300$

$-1.07429600$

0.66841800

2.15711500

$-1.45250600$

$-0.73774300$

0.72540900

0.19855900

2.49543500

$-0.37038500$

1.90188100

0.39145200

$-0.05394900$

0.99432600

2.31192500

2.25970200

0.87645400

$-0.59650900$

3.58669200

$-1.30388100$

2.28109200

$-0.01493400$

2.04146700

2.37109700

2.82103900

1.97159500

2.55509500

0.93628200

$-3.47568500$

$-2.29930100$

$-5.27681500$

$-2.07728000$

$-3.93273300$

$-2.78632100$

$-3.35290000$

$-2.45934600$

$-5.53764300$

$-3.98379400$

$-3.39267200$

$-1.38326200$

$-6.05747600$

$-1.70693100$
0.83499500

3.26486800

0.77788900

1.86115300

$-0.31271200$

$-0.33229700$

$-0.71024900$

0.04530800

3.49424600

2.73159400

0.40006800

1.36583100

0.16978400

0.19358700

3.51352300

2.65885800

$-0.48730600$

$-0.79540900$

4.53986500

3.33300800

0.47789000

1.42520100

0.92946100

$-0.56779700$

2.98783000

3.75998300

2.94707700

1.63599200

1.71370000

1.45832100

$-3.43191600$

$-2.65378700$

$-0.35469600$

$-1.28850500$

$-0.10191000$

$-0.12058000$

$-3.45307800$

$-2.57767600$

0.52777000

0.86735900

$-4.47547400$

$-3.24475300$

$-0.44132700$

$-1.35103800$ 


\begin{tabular}{|c|c|c|c|c|c|c|c|}
\hline $\mathrm{H}$ & -1.48075500 & -3.79188800 & -0.85207400 & $\mathrm{~N}$ & 3.57544600 & -0.12150600 & 0.28701600 \\
\hline $\mathrm{H}$ & -4.02101500 & -2.89292600 & 0.63680000 & $\mathrm{P}$ & 2.47709500 & 0.08121400 & 1.61818700 \\
\hline $\mathrm{C}$ & -3.26959200 & -4.89943200 & -2.94005700 & $\mathrm{C}$ & 3.18696700 & 1.47165900 & 2.72436700 \\
\hline $\mathrm{H}$ & -3.61504800 & -5.59104400 & -3.71938100 & $\mathrm{C}$ & 2.36575500 & -1.58623300 & 2.55950800 \\
\hline $\mathrm{H}$ & -2.17764300 & -5.02356900 & -2.90094800 & $\mathrm{C}$ & 4.65931500 & 1.28402300 & 3.13830200 \\
\hline $\mathrm{C}$ & -3.90160700 & -5.33022000 & -1.59291100 & $\mathrm{H}$ & 5.31604300 & 1.24408200 & 2.26445900 \\
\hline $\mathrm{H}$ & -4.25692200 & -6.36516600 & -1.68140400 & $\mathrm{H}$ & 4.97151800 & 2.14420400 & 3.74580200 \\
\hline $\mathrm{H}$ & -4.80087000 & -4.72697300 & -1.41045700 & $\mathrm{H}$ & 4.82494100 & 0.38690200 & 3.73750500 \\
\hline $\mathrm{C}$ & 1.04615600 & 2.54883800 & -1.61571200 & $\mathrm{C}$ & 3.09372900 & 2.77953900 & 1.90957600 \\
\hline $\mathrm{H}$ & 2.00529800 & 2.72186300 & -2.10641800 & $\mathrm{H}$ & 3.53687700 & 3.59641600 & 2.49388000 \\
\hline $\mathrm{C}$ & 0.25690400 & 1.49575000 & -2.17284400 & $\mathrm{H}$ & 3.64156100 & 2.71080700 & 0.96322700 \\
\hline $\mathrm{H}$ & -0.81513800 & 1.56129000 & -1.98768300 & $\mathrm{H}$ & 2.05431300 & 3.04680200 & 1.69602200 \\
\hline $\mathrm{C}$ & 0.37801800 & 3.81143300 & -1.08612700 & $\mathrm{C}$ & 2.28278700 & 1.61539600 & 3.96654500 \\
\hline $\mathrm{H}$ & 1.03582800 & 4.38261800 & -0.42124100 & $\mathrm{H}$ & 1.22709400 & 1.71810700 & 3.68974900 \\
\hline $\mathrm{H}$ & -0.50985900 & 3.55152000 & -0.49826400 & $\mathrm{H}$ & 2.37636000 & 0.77224900 & 4.65681300 \\
\hline $\mathrm{C}$ & 0.62178000 & 0.80519400 & -3.46265700 & $\mathrm{H}$ & 2.57030800 & 2.52006800 & 4.51805200 \\
\hline $\mathrm{H}$ & 0.30057600 & -0.24075300 & -3.45860600 & $\mathrm{C}$ & 2.45884600 & -2.70687300 & 1.50161300 \\
\hline $\mathrm{H}$ & 0.11820100 & 1.31486200 & -4.29629400 & $\mathrm{H}$ & 1.72401800 & -2.58274700 & 0.70331700 \\
\hline $\mathrm{H}$ & 1.69653000 & 0.84147600 & -3.65607300 & $\mathrm{H}$ & 3.45440600 & -2.75944900 & 1.05130100 \\
\hline $\mathrm{C}$ & -0.05221300 & 4.71748300 & -2.22920500 & $\mathrm{H}$ & 2.26066800 & -3.66750700 & 1.99549900 \\
\hline $\mathrm{O}$ & -0.06018300 & 4.42253600 & -3.40482500 & $\mathrm{C}$ & 3.42990100 & -1.84892400 & 3.64124900 \\
\hline $\mathrm{O}$ & -0.44382000 & 5.91873900 & -1.75484500 & $\mathrm{H}$ & 3.38959200 & -1.12881100 & 4.46304200 \\
\hline $\mathrm{C}$ & -0.90505100 & 6.85301100 & -2.74562900 & $\mathrm{H}$ & 3.24771400 & -2.84202500 & 4.07325300 \\
\hline $\mathrm{H}$ & -1.77183100 & 6.45161100 & -3.27743900 & $\mathrm{C}$ & 0.95991400 & -1.61861100 & 3.20404100 \\
\hline $\mathrm{H}$ & -1.17662000 & 7.75310500 & -2.19359000 & $\mathrm{H}$ & 0.81376500 & -2.59208700 & 3.69079300 \\
\hline \multirow[t]{2}{*}{$\mathrm{H}$} & -0.11319700 & 7.06833500 & -3.46793300 & $\mathrm{H}$ & 0.83256600 & -0.84656200 & 3.97010100 \\
\hline & & & & $\mathrm{H}$ & 0.17221600 & -1.48869600 & 2.45644100 \\
\hline \multirow[t]{7}{*}{120} & \multirow{7}{*}{ 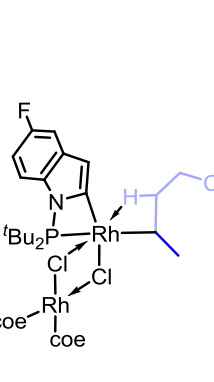 } & \multirow{7}{*}{3} & \multirow{7}{*}{ s } & $\mathrm{F}$ & 8.22065400 & -1.95220600 & -1.97373600 \\
\hline & & & & $\mathrm{Cl}$ & -1.24413700 & 1.22794800 & 0.86741400 \\
\hline & & & & $\mathrm{H}$ & 4.44441500 & -1.85712800 & 3.23223600 \\
\hline & & & & $\mathrm{C}$ & 5.94046200 & -1.00325800 & 0.69749500 \\
\hline & & & & $\mathrm{H}$ & 5.92866400 & -0.95614400 & 1.78085900 \\
\hline & & & & $\mathrm{Rh}$ & 0.97705200 & 0.69293000 & -0.28587800 \\
\hline & & & & $\mathrm{Cl}$ & -0.17828100 & -1.39595700 & -0.70673900 \\
\hline $\mathrm{C}$ & 4.85418200 & -0.6836 & -1.49147500 & $\mathrm{Rh}$ & -2.48599100 & -0.77966300 & 0.04511900 \\
\hline $\mathrm{C}$ & 36900 & -0.61550700 & -0.06634100 & $\mathrm{C}$ & -5.15752700 & 0.52503400 & 3.50060500 \\
\hline $\mathrm{C}$ & 7.08151500 & -1.45695600 & 0.03541700 & $\mathrm{C}$ & -3.89230300 & 0.06559900 & 2.73670100 \\
\hline $\mathrm{C}$ & 53200 & -1.50997300 & -1.36249400 & $\mathrm{C}$ & -5.84073300 & 2.31081900 & 0.42732300 \\
\hline $\mathrm{C}$ & 6.01344100 & -1.13529400 & -2.14369500 & $\mathrm{C}$ & -4.19361200 & -0.49891200 & 1.36226700 \\
\hline $\mathrm{C}$ & 3.56906600 & -0.24377200 & -1.97352600 & $\mathrm{C}$ & -4.40616300 & 1.78079500 & 0.19403900 \\
\hline $\mathrm{C}$ & 2.80191600 & 0.08613800 & -0.88361200 & $\mathrm{C}$ & -4.39131500 & 0.26982600 & 0.19857300 \\
\hline $\mathrm{H}$ & 7.96329500 & -1.77061500 & 0.58379800 & $\mathrm{H}$ & -5.89873800 & -0.28812400 & 3.50486200 \\
\hline $\mathrm{H}$ & 6.07713900 & -1.20100100 & -3.22520200 & $\mathrm{H}$ & -3.18358100 & 0.89535200 & 2.67813800 \\
\hline $\mathrm{H}$ & 3.26814400 & -0.21314800 & -3.01019600 & $\mathrm{H}$ & -6.45164700 & 2.11524600 & -0.46550600 \\
\hline
\end{tabular}




\begin{tabular}{|c|c|c|c|c|c|c|c|}
\hline $\mathrm{H}$ & -4.03752500 & 2.16596600 & -0.76505200 & $\mathrm{H}$ & 0.16377500 & 1.70455700 & -4.18403700 \\
\hline $\mathrm{H}$ & -4.86738800 & 0.67437800 & 4.54970200 & $\mathrm{H}$ & 1.69681100 & 1.06338000 & -3.58112400 \\
\hline $\mathrm{H}$ & -3.39775200 & -0.71624400 & 3.32839700 & $\mathrm{C}$ & -0.18722200 & 4.78903000 & -2.20384800 \\
\hline $\mathrm{H}$ & -5.78062600 & 3.40416600 & 0.52240500 & $\mathrm{O}$ & -0.06585700 & 4.47998900 & -3.36998100 \\
\hline $\mathrm{H}$ & -4.71454000 & -1.45506000 & 1.40811600 & $\mathrm{O}$ & -0.77633900 & 5.93091300 & -1.79020900 \\
\hline $\mathrm{H}$ & -3.74049900 & 2.17871000 & 0.96119500 & $\mathrm{C}$ & -1.27754700 & 6.78066600 & -2.83580000 \\
\hline $\mathrm{H}$ & -5.03525300 & -0.15453100 & -0.57075100 & $\mathrm{H}$ & -2.03344900 & 6.25674700 & -3.42684600 \\
\hline $\mathrm{C}$ & -5.83906400 & 1.81963900 & 3.00901500 & $\mathrm{H}$ & -1.71533000 & 7.64059600 & -2.32825800 \\
\hline $\mathrm{H}$ & -6.56454800 & 2.10638200 & 3.78128500 & $\mathrm{H}$ & -0.46562200 & 7.09598800 & -3.49656800 \\
\hline $\mathrm{H}$ & -5.09935100 & 2.63314100 & 2.98438300 & $\mathrm{H}$ & 1.56366800 & 93300 & -0.41720700 \\
\hline $\mathrm{C}$ & -6.58116400 & 1.73616900 & 1.65136400 & & & & \\
\hline $\mathrm{H}$ & -7.53298900 & 2.27719400 & 1.73170100 & 121 & & & \\
\hline $\mathrm{H}$ & -6.85460700 & 0.69056100 & 1.45721800 & & & & \\
\hline $\mathrm{C}$ & -3.43751900 & -3.52489300 & -3.46806500 & & & & \\
\hline $\mathrm{C}$ & -2.86173300 & -2.32616200 & -2.67607900 & & & & \\
\hline $\mathrm{C}$ & -2.74802900 & -5.32911000 & -0.40841600 & & & & \\
\hline $\mathrm{C}$ & -3.49957300 & -2.14805200 & -1.31228900 & & & & \\
\hline $\mathrm{C}$ & -2.09430300 & -3.95291500 & -0.13786400 & $\mathrm{C}$ & -4.69632600 & -2.05839900 & 0.67939700 \\
\hline $\mathrm{C}$ & -3.13319500 & -2.85553100 & -0.15113900 & $\mathrm{C}$ & -5.08102500 & -0.80076600 & 0.11717400 \\
\hline $\mathrm{H}$ & -4.53560800 & -3.45711600 & -3.48979800 & $\mathrm{C}$ & -7.33958700 & -1.57219300 & -0.14129700 \\
\hline $\mathrm{H}$ & -1.77729100 & -2.43459100 & -2.59620100 & $\mathrm{C}$ & -6.95767200 & -2.79409300 & 0.42203300 \\
\hline $\mathrm{H}$ & -3.34250000 & -5.62836200 & 0.46662700 & $\mathrm{C}$ & -5.66511500 & -3.06392700 & 0.83749800 \\
\hline $\mathrm{H}$ & -1.58532800 & -3.98810700 & 0.83340500 & $\mathrm{C}$ & -3.28481400 & -2.01593400 & 0.96304400 \\
\hline $\mathrm{H}$ & -3.10725700 & -3.41358200 & -4.50984200 & $\mathrm{C}$ & -2.82422100 & -0.77635200 & 0.60177900 \\
\hline $\mathrm{H}$ & -3.03515100 & -1.41203400 & -3.25919400 & $\mathrm{H}$ & -8.36955800 & -1.43131400 & -0.45077300 \\
\hline $\mathrm{H}$ & -1.93977500 & -6.06859300 & -0.49546200 & $\mathrm{H}$ & -5.42263800 & -4.03215400 & 1.26356900 \\
\hline $\mathrm{H}$ & -4.53740700 & -1.82141100 & -1.37568300 & $\mathrm{H}$ & -2.68783100 & -2.82489200 & 1.36190900 \\
\hline $\mathrm{H}$ & -1.31874700 & -3.76882400 & -0.88354600 & $\mathrm{~N}$ & -3.92261600 & -0.00411200 & 0.12467100 \\
\hline $\mathrm{H}$ & -3.90702000 & -3.00661300 & 0.60044200 & $\mathrm{P}$ & -3.09145800 & 1.31336900 & -0.63926800 \\
\hline $\mathrm{C}$ & -3.03281400 & -4.93572300 & -2.99185300 & $\mathrm{C}$ & -3.96960800 & 2.94868100 & -0.17350100 \\
\hline $\mathrm{H}$ & -3.33759000 & -5.63365700 & -3.78237300 & $\mathrm{C}$ & -3.14793800 & 0.98592200 & -2.52769400 \\
\hline $\mathrm{H}$ & -1.93617300 & -5.00412300 & -2.94756900 & $\mathrm{C}$ & -5.50459000 & 2.84188200 & -0.26186500 \\
\hline $\mathrm{C}$ & -3.65024200 & -5.41737400 & -1.65525200 & $\mathrm{H}$ & -5.89413300 & 2.11518200 & 0.45601900 \\
\hline $\mathrm{H}$ & -3.94652300 & -6.46929500 & -1.75922200 & $\mathrm{H}$ & -5.93623800 & 3.81913700 & -0.00858200 \\
\hline $\mathrm{H}$ & -4.58371700 & -4.86790500 & -1.47359200 & $\mathrm{H}$ & -5.86195600 & 2.57600200 & -1.25895000 \\
\hline $\mathrm{C}$ & 1.04542300 & 2.72008200 & -1.45898800 & $\mathrm{C}$ & -3.60788400 & 3.29089700 & 1.28548700 \\
\hline $\mathrm{H}$ & 1.94778500 & 2.94985900 & -2.03352300 & $\mathrm{H}$ & -4.19154000 & 4.16774400 & 1.59397200 \\
\hline $\mathrm{C}$ & 0.26289500 & 1.61092300 & -2.04645400 & $\mathrm{H}$ & -3.84545400 & 2.47475800 & 1.97256300 \\
\hline $\mathrm{H}$ & -0.81493700 & 1.70244700 & -1.89810300 & $\mathrm{H}$ & -2.55032300 & 3.54303900 & 1.39410800 \\
\hline $\mathrm{C}$ & 0.28700000 & 3.97348900 & -1.01421700 & $\mathrm{C}$ & -3.46240900 & 4.09134500 & -1.07647300 \\
\hline $\mathrm{H}$ & 0.90482800 & 4.62113300 & -0.37984600 & $\mathrm{H}$ & -2.36825100 & 4.14557200 & -1.10151000 \\
\hline $\mathrm{H}$ & -0.58533300 & 3.69278800 & -0.41260700 & $\mathrm{H}$ & -3.82998000 & 4.00920200 & -2.10260400 \\
\hline $\mathrm{C}$ & 0.61735500 & 1.06908700 & -3.41034500 & $\mathrm{H}$ & -3.83001400 & 5.04359600 & -0.67372800 \\
\hline $\mathrm{H}$ & 0.23795700 & 0.05014400 & -3.53830400 & $\mathrm{C}$ & -2.73029500 & -0.49259200 & -2.70693000 \\
\hline
\end{tabular}




\begin{tabular}{|c|c|c|c|c|c|c|c|}
\hline $\mathrm{H}$ & -1.72598100 & -0.69197300 & -2.31287600 & $\mathrm{C}$ & 2.23763100 & -3.70154600 & -1.17093200 \\
\hline $\mathrm{H}$ & -3.42908300 & -1.18576000 & -2.23098900 & $\mathrm{C}$ & 3.20218100 & -2.53926300 & -1.12956900 \\
\hline $\mathrm{H}$ & -2.70535600 & -0.71871100 & -3.78063300 & $\mathrm{H}$ & 5.44909100 & -3.76899800 & 1.49684900 \\
\hline $\mathrm{C}$ & -4.53501200 & 1.18665000 & -3.16557500 & $\mathrm{H}$ & 2.50956000 & -2.87486000 & 1.59260500 \\
\hline $\mathrm{H}$ & -4.85595500 & 2.23236300 & -3.14971200 & $\mathrm{H}$ & 3.34493800 & -5.03688400 & -2.48264500 \\
\hline $\mathrm{H}$ & -4.48305000 & 0.88283900 & -4.21926000 & $\mathrm{H}$ & 1.48769800 & -3.55471200 & -1.95821100 \\
\hline $\mathrm{C}$ & -2.10304500 & 1.86848400 & -3.24649600 & $\mathrm{H}$ & 4.33526300 & -4.13565500 & 2.80299800 \\
\hline $\mathrm{H}$ & -2.04798600 & 1.55115200 & -4.29604500 & $\mathrm{H}$ & 3.84810200 & -1.90896000 & 2.17807200 \\
\hline $\mathrm{H}$ & -2.36742700 & 2.92837900 & -3.24301400 & $\mathrm{H}$ & 2.26902800 & -5.84496900 & -1.35257100 \\
\hline $\mathrm{H}$ & -1.10499700 & 1.75869000 & -2.81213300 & $\mathrm{H}$ & 4.82151500 & -1.67345600 & -0.05037400 \\
\hline $\mathrm{F}$ & -7.90357500 & -3.75484700 & 0.56111000 & $\mathrm{H}$ & 1.68582500 & -3.78458700 & -0.23354200 \\
\hline $\mathrm{Cl}$ & 0.83724200 & 1.36186500 & -0.90569900 & $\mathrm{H}$ & 3.75623600 & -2.42217700 & -2.06030900 \\
\hline $\mathrm{H}$ & -5.30351000 & 0.57299000 & -2.68611500 & $\mathrm{C}$ & 3.94045900 & -5.24225900 & 1.00211900 \\
\hline $\mathrm{C}$ & -6.39361200 & -0.55799800 & -0.29371600 & $\mathrm{H}$ & 4.47982400 & -6.07069800 & 1.47961500 \\
\hline $\mathrm{H}$ & -6.69065200 & 0.38957500 & -0.72721300 & $\mathrm{H}$ & 2.87660800 & -5.42620900 & 1.21165600 \\
\hline $\mathrm{Rh}$ & -1.16977200 & 0.24998200 & 0.19317200 & $\mathrm{C}$ & 4.19985100 & -5.31144700 & -0.52403500 \\
\hline $\mathrm{Cl}$ & 0.42623300 & -1.56043100 & 0.52017300 & $\mathrm{H}$ & 4.56026600 & -6.31680200 & -0.77811700 \\
\hline $\mathrm{Rh}$ & 2.42984900 & -0.57113000 & -0.64406400 & $\mathrm{H}$ & 5.02424400 & -4.63171100 & -0.77769900 \\
\hline $\mathrm{C}$ & 4.47337300 & 1.79880300 & -3.97216700 & $\mathrm{C}$ & -0.97124800 & 1.12620100 & 2.10042800 \\
\hline $\mathrm{C}$ & 3.37476200 & 1.01167300 & -3.21843500 & $\mathrm{H}$ & -1.18462900 & 2.17536200 & 1.87795200 \\
\hline $\mathrm{C}$ & 5.29980600 & 2.97035000 & -0.64982900 & $\mathrm{C}$ & 0.48758900 & 1.00919700 & 2.56227000 \\
\hline $\mathrm{C}$ & 3.90251900 & 0.21006100 & -2.04497000 & $\mathrm{H}$ & 0.67917700 & 0.00314900 & 2.95014000 \\
\hline $\mathrm{C}$ & 3.98101400 & 2.19607600 & -0.40874900 & $\mathrm{H}$ & 1.17704400 & 1.16434200 & 1.73321500 \\
\hline $\mathrm{C}$ & 4.16138900 & 0.73991300 & -0.76575200 & $\mathrm{C}$ & -1.94116500 & 0.58724100 & 3.14629100 \\
\hline $\mathrm{H}$ & 5.29956900 & 1.11937700 & -4.23055500 & $\mathrm{H}$ & -1.81353300 & 1.13738700 & 4.09232600 \\
\hline $\mathrm{H}$ & 2.59110400 & 1.70356800 & -2.89927800 & $\mathrm{H}$ & -2.98677700 & 0.68310800 & 2.84865400 \\
\hline $\mathrm{H}$ & 6.03346700 & 2.68572200 & 0.11754600 & $\mathrm{H}$ & -1.75140800 & -0.47108600 & 3.35013600 \\
\hline $\mathrm{H}$ & 3.69567100 & 2.29625500 & 0.64443200 & $\mathrm{C}$ & 0.83254500 & 2.05309800 & 3.64579800 \\
\hline $\mathrm{H}$ & 4.04201300 & 2.12572900 & -4.92854500 & $\mathrm{H}$ & 0.60673300 & 3.06336100 & 3.27243400 \\
\hline $\mathrm{H}$ & 2.90094600 & 0.31763200 & -3.92557000 & $\mathrm{H}$ & 0.25344100 & 1.91969600 & 4.56372400 \\
\hline $\mathrm{H}$ & 5.09132900 & 4.03777500 & -0.49103300 & $\mathrm{C}$ & 2.30894200 & 2.03901900 & 3.99541900 \\
\hline $\mathrm{H}$ & 4.51826700 & -0.63606100 & -2.35273200 & $\mathrm{O}$ & 3.22080600 & 1.83252400 & 3.22061700 \\
\hline $\mathrm{H}$ & 3.18192100 & 2.64696700 & -0.99908800 & $\mathrm{O}$ & 2.50486100 & 2.31536800 & 5.30485200 \\
\hline $\mathrm{H}$ & 4.93517500 & 0.25992800 & -0.16888400 & $\mathrm{C}$ & 3.87681500 & 2.37302900 & 5.72589100 \\
\hline $\mathrm{C}$ & 5.05291600 & 3.04321600 & -3.26625300 & $\mathrm{H}$ & 4.41827600 & 3.14478600 & 5.17183900 \\
\hline $\mathrm{H}$ & 5.64463000 & 3.58294100 & -4.01720700 & $\mathrm{H}$ & 3.84668900 & 2.61410500 & 6.78904600 \\
\hline $\mathrm{H}$ & 4.23183300 & 3.72305500 & -2.99532400 & $\mathrm{H}$ & 4.36946000 & 1.41032100 & 5.56447100 \\
\hline $\mathrm{C}$ & 5.95371600 & 2.78256800 & -2.03316200 & & & & \\
\hline $\mathrm{H}$ & 6.81629700 & 3.46075500 & -2.07391600 & 122-ts & & & \\
\hline $\mathrm{H}$ & 6.37393100 & 1.77059000 & -2.10602900 & & & & \\
\hline $\mathrm{C}$ & 4.38731900 & -3.95177100 & 1.72096500 & & & & \\
\hline $\mathrm{C}$ & 3.57140600 & -2.66803600 & 1.43487200 & & & & \\
\hline $\mathrm{C}$ & 2.99396000 & -5.02370700 & -1.44086300 & & & & \\
\hline $\mathrm{C}$ & 3.82030100 & -2.09193300 & 0.05489200 & & & & \\
\hline
\end{tabular}




\begin{tabular}{|c|c|c|c|c|c|c|c|}
\hline $\mathrm{C}$ & 4.78094500 & 1.21226300 & -1.08026200 & $\mathrm{Cl}$ & -1.12270600 & 1.52774500 & -0.80208300 \\
\hline $\mathrm{C}$ & 4.82776200 & -0.02532100 & -0.37578300 & $\mathrm{Rh}$ & -2.97493700 & -0.02085500 & -0.32653800 \\
\hline $\mathrm{C}$ & 7.18868600 & -0.18749900 & -0.76321000 & $\mathrm{C}$ & -5.15260300 & -3.86024700 & -1.46548100 \\
\hline $\mathrm{C}$ & 7.12998400 & 1.02090300 & -1.46662800 & $\mathrm{C}$ & -4.16570200 & -2.67129100 & -1.54464900 \\
\hline $\mathrm{C}$ & 5.95963000 & 1.74113000 & -1.63472600 & $\mathrm{C}$ & -4.51380200 & -3.58821400 & 2.08511500 \\
\hline $\mathrm{C}$ & 3.43393200 & 1.69921700 & -1.02911900 & $\mathrm{C}$ & -4.51933000 & -1.53007700 & -0.61094200 \\
\hline $\mathrm{C}$ & 2.66743300 & 0.80465600 & -0.29864500 & $\mathrm{C}$ & -3.48712800 & -2.59705000 & 1.48600900 \\
\hline $\mathrm{H}$ & 8.14481900 & -0.68764200 & -0.65352100 & $\mathrm{C}$ & -4.18917900 & -1.47462600 & 0.75586000 \\
\hline $\mathrm{H}$ & 5.97196300 & 2.68327500 & -2.17273200 & $\mathrm{H}$ & -6.18308300 & -3.49109600 & -1.57693500 \\
\hline $\mathrm{H}$ & 3.08770500 & 2.63290700 & -1.44503000 & $\mathrm{H}$ & -3.15277900 & -3.03822700 & -1.35958400 \\
\hline $\mathrm{N}$ & 3.52763400 & -0.28144500 & 0.10173700 & $\mathrm{H}$ & -5.02655100 & -3.11148100 & 2.93269900 \\
\hline $\mathrm{P}$ & 2.37986000 & -1.60000500 & 0.04165200 & $\mathrm{H}$ & -2.86733500 & -2.19819700 & 2.29928900 \\
\hline $\mathrm{C}$ & 2.55626400 & -2.70990600 & 1.60300800 & $\mathrm{H}$ & -4.96440900 & -4.49862500 & -2.33966900 \\
\hline $\mathrm{C}$ & 2.75319700 & -2.58109600 & -1.57443600 & $\mathrm{H}$ & -4.16636400 & -2.28766900 & -2.57338700 \\
\hline $\mathrm{C}$ & 4.01177000 & -2.72827300 & 2.10800100 & $\mathrm{H}$ & -3.95369100 & -4.43557600 & 2.50532000 \\
\hline $\mathrm{H}$ & 4.38623300 & -1.72130500 & 2.31465000 & $\mathrm{H}$ & -5.42454400 & -1.00507900 & -0.91618000 \\
\hline $\mathrm{H}$ & 4.05889400 & -3.30154300 & 3.04308000 & $\mathrm{H}$ & -2.81026100 & -3.13231300 & 0.81761900 \\
\hline $\mathrm{H}$ & 4.68968900 & -3.21017400 & 1.39585800 & $\mathrm{H}$ & -4.85300100 & -0.90538200 & 1.40510500 \\
\hline $\mathrm{C}$ & 1.62728600 & -2.13282000 & 2.69461100 & $\mathrm{C}$ & -5.07323100 & -4.74871900 & -0.20590200 \\
\hline $\mathrm{H}$ & 1.69372800 & -2.77269900 & 3.58453500 & $\mathrm{H}$ & -5.66897600 & -5.64661100 & -0.41564400 \\
\hline $\mathrm{H}$ & 1.90157600 & -1.12139100 & 2.99355400 & $\mathrm{H}$ & -4.04076600 & -5.10552300 & -0.07731000 \\
\hline $\mathrm{H}$ & 0.58576600 & -2.11236400 & 2.36103300 & $\mathrm{C}$ & -5.58625500 & -4.12286900 & 1.11519100 \\
\hline $\mathrm{C}$ & 2.07766500 & -4.15310200 & 1.33909800 & $\mathrm{H}$ & -6.16482500 & -4.87682000 & 1.66481400 \\
\hline $\mathrm{H}$ & 1.04327200 & -4.18221700 & 0.98337300 & $\mathrm{H}$ & -6.29938900 & -3.32240300 & 0.87776000 \\
\hline $\mathrm{H}$ & 2.70804200 & -4.69549200 & 0.63162800 & $\mathrm{C}$ & -4.64863800 & 3.81701600 & 1.47331700 \\
\hline $\mathrm{H}$ & 2.11163200 & -4.70016700 & 2.28977900 & $\mathrm{C}$ & -3.68690600 & 2.63160600 & 1.22072900 \\
\hline $\mathrm{C}$ & 2.82459900 & -1.54263400 & -2.71680500 & $\mathrm{C}$ & -5.19694800 & 3.55688500 & -2.09518500 \\
\hline $\mathrm{H}$ & 1.89928600 & -0.96543500 & -2.79864800 & $\mathrm{C}$ & -4.32581200 & 1.49415300 & 0.44790000 \\
\hline $\mathrm{H}$ & 3.65541500 & -0.84339500 & -2.59782900 & $\mathrm{C}$ & -4.02682200 & 2.56977800 & -1.86904400 \\
\hline $\mathrm{H}$ & 2.97662100 & -2.08353400 & -3.65989600 & $\mathrm{C}$ & -4.45253200 & 1.44392800 & -0.95499500 \\
\hline $\mathrm{C}$ & 4.08416000 & -3.36014700 & -1.52730900 & $\mathrm{H}$ & -5.58383900 & 3.44466400 & 1.91788600 \\
\hline $\mathrm{H}$ & 4.11120600 & -4.11499400 & -0.73664200 & $\mathrm{H}$ & -2.78884000 & 2.99620100 & 0.71613500 \\
\hline $\mathrm{H}$ & 4.20847300 & -3.88906700 & -2.48127800 & $\mathrm{H}$ & -5.95437500 & 3.08219900 & -2.73522500 \\
\hline $\mathrm{C}$ & 1.59742900 & -3.55202300 & -1.90478200 & $\mathrm{H}$ & -3.70272900 & 2.17507200 & -2.83990600 \\
\hline $\mathrm{H}$ & 1.80081900 & -3.99007200 & -2.89069800 & $\mathrm{H}$ & -4.18489400 & 4.45555900 & 2.23774000 \\
\hline $\mathrm{H}$ & 1.50980000 & -4.37756500 & -1.19688800 & $\mathrm{H}$ & -3.35478600 & 2.24263600 & 2.19265500 \\
\hline $\mathrm{H}$ & 0.63449100 & -3.03976700 & -1.95940700 & $\mathrm{H}$ & -4.80723500 & 4.41088100 & -2.66694200 \\
\hline $\mathrm{F}$ & 8.27774300 & 1.50684400 & -1.99347700 & $\mathrm{H}$ & -5.09186000 & 0.97556500 & 1.02450300 \\
\hline $\mathrm{Cl}$ & -1.05187900 & -1.60413900 & -0.54173100 & $\mathrm{H}$ & -3.17077200 & 3.10411900 & -1.45403800 \\
\hline $\mathrm{H}$ & 4.94396400 & -2.69795500 & -1.40749600 & $\mathrm{H}$ & -5.29237500 & 0.87737200 & -1.35613800 \\
\hline $\mathrm{C}$ & 6.02607100 & -0.71757200 & -0.20070900 & $\mathrm{C}$ & -4.99148500 & 4.70645400 & 0.25956900 \\
\hline $\mathrm{H}$ & 6.07237200 & -1.63874400 & 0.36916800 & $\mathrm{H}$ & -5.49426200 & 5.59869800 & 0.65479900 \\
\hline $\mathrm{Rh}$ & 0.76907500 & -0.01946400 & -0.16155600 & $\mathrm{H}$ & -4.06001900 & 5.07237600 & -0.19665500 \\
\hline $\mathrm{H}$ & 2.97275800 & 0.34233200 & 2.41818900 & $\mathrm{C}$ & -5.90047100 & 4.07619900 & -0.82529600 \\
\hline
\end{tabular}




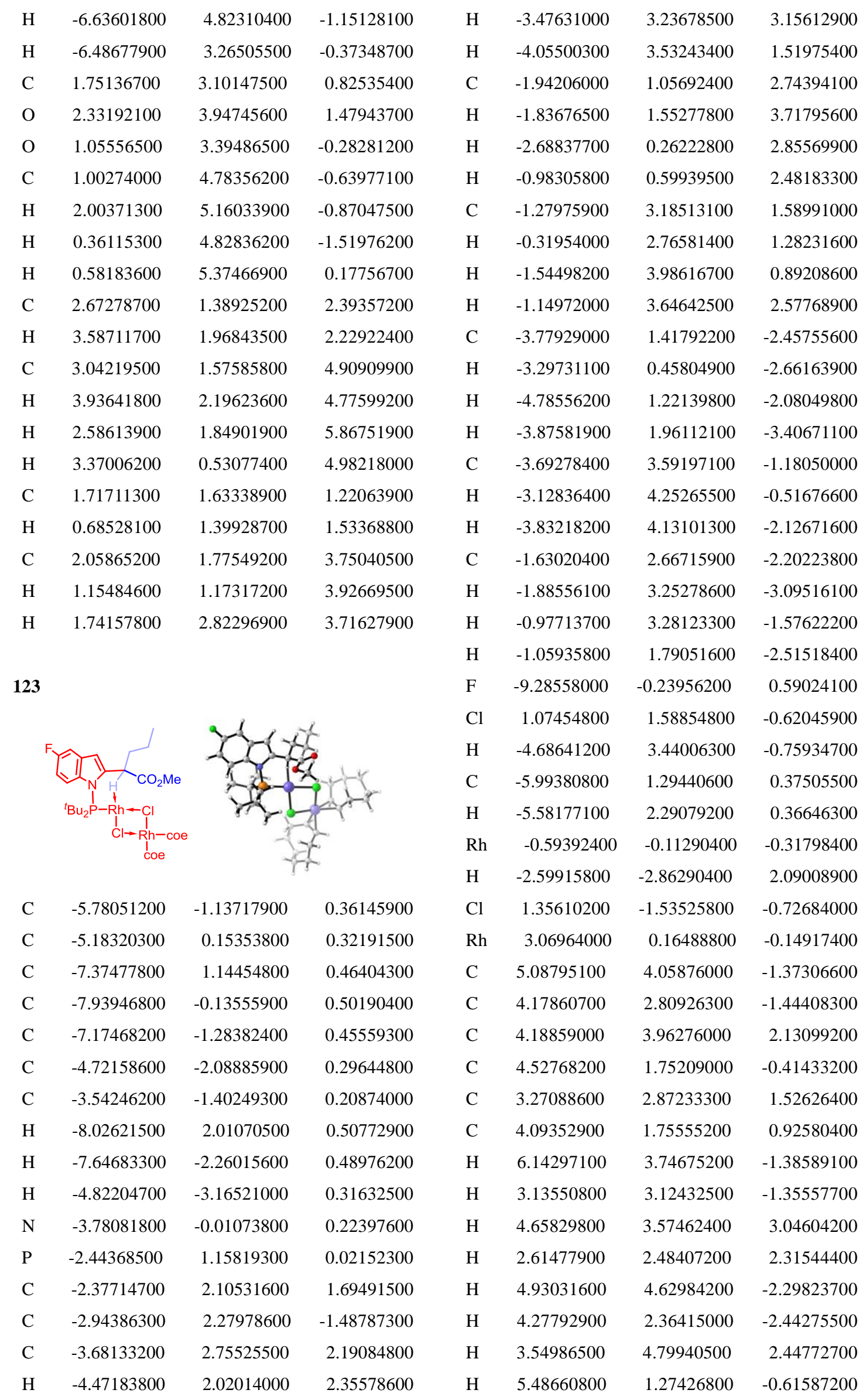




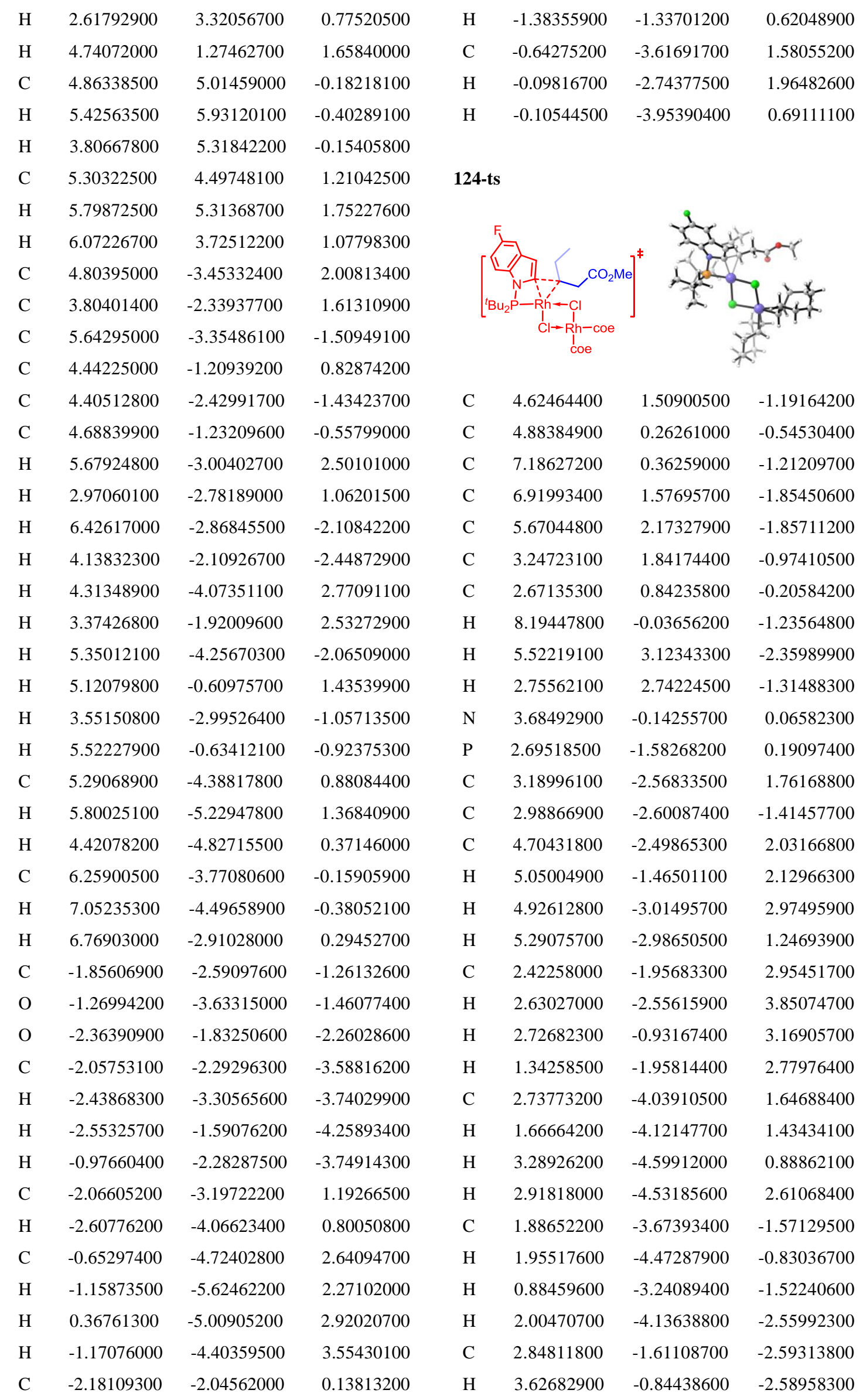




\begin{tabular}{|c|c|c|c|c|c|c|c|}
\hline $\mathrm{H}$ & 2.94045300 & -2.17736500 & -3.52903300 & $\mathrm{H}$ & -3.14633300 & 1.49090100 & -3.32950100 \\
\hline $\mathrm{C}$ & 4.37522600 & -3.27134700 & -1.49100200 & $\mathrm{H}$ & -4.45733400 & 4.54723700 & 1.16037600 \\
\hline $\mathrm{H}$ & 4.44194400 & -3.82088400 & -2.43919200 & $\mathrm{H}$ & -3.60214700 & 2.38645700 & 1.60847900 \\
\hline $\mathrm{H}$ & 5.18690600 & -2.54040000 & -1.48213000 & $\mathrm{H}$ & -4.26666200 & 3.69196400 & -3.70837400 \\
\hline $\mathrm{H}$ & 4.54555700 & -3.99495800 & -0.68916000 & $\mathrm{H}$ & -5.12919000 & 0.88737000 & 0.41300700 \\
\hline $\mathrm{F}$ & 7.94118600 & 2.19696600 & -2.49167300 & $\mathrm{H}$ & -2.84932100 & 2.64871100 & -2.04993800 \\
\hline $\mathrm{Cl}$ & -0.88232800 & -1.80500300 & -0.14722800 & $\mathrm{H}$ & -4.95248900 & 0.40466000 & -1.92344400 \\
\hline $\mathrm{H}$ & 1.87478500 & -1.11237800 & -2.58805100 & $\mathrm{C}$ & -4.92521800 & 4.45336900 & -0.93628100 \\
\hline $\mathrm{C}$ & 6.15929000 & -0.30323500 & -0.54060600 & $\mathrm{H}$ & -5.48735200 & 5.38286100 & -0.77700300 \\
\hline $\mathrm{H}$ & 6.36672800 & -1.22932100 & -0.01622600 & $\mathrm{H}$ & -3.93254300 & 4.76722500 & -1.29076900 \\
\hline $\mathrm{Rh}$ & 0.92024600 & -0.17252800 & 0.07244800 & $\mathrm{C}$ & -5.64486500 & 3.63365400 & -2.03645300 \\
\hline $\mathrm{Cl}$ & -0.90187000 & 1.29905700 & -0.82183300 & $\mathrm{H}$ & -6.31439000 & 4.30043800 & -2.59532900 \\
\hline $\mathrm{Rh}$ & -2.81715400 & -0.23987800 & -0.42032000 & $\mathrm{H}$ & -6.30038500 & 2.89340400 & -1.55881100 \\
\hline $\mathrm{C}$ & -4.81056600 & -4.26713900 & -1.19989300 & $\mathrm{C}$ & 0.14107000 & 3.36596300 & 1.99306200 \\
\hline $\mathrm{C}$ & -3.82231000 & -3.08554900 & -1.34569400 & $\mathrm{O}$ & -0.45751700 & 2.69279600 & 2.80543800 \\
\hline $\mathrm{C}$ & -4.63980900 & -3.40385100 & 2.31243800 & $\mathrm{O}$ & -0.11916200 & 4.67625300 & 1.78109500 \\
\hline $\mathrm{C}$ & -4.29712100 & -1.81598200 & -0.66633400 & $\mathrm{C}$ & -1.17268800 & 5.23556100 & 2.58031500 \\
\hline $\mathrm{C}$ & -3.54626100 & -2.49764900 & 1.69673600 & $\mathrm{H}$ & -0.94390400 & 5.13433400 & 3.64484300 \\
\hline $\mathrm{C}$ & -4.15346800 & -1.53117200 & 0.70591800 & $\mathrm{H}$ & -1.22644500 & 6.28769600 & 2.29826900 \\
\hline $\mathrm{H}$ & -5.81755800 & -3.94499500 & -1.50466400 & $\mathrm{H}$ & -2.12155600 & 4.73403500 & 2.37221000 \\
\hline $\mathrm{H}$ & -2.84253300 & -3.39360100 & -0.97201500 & $\mathrm{C}$ & 1.79680200 & 1.51807000 & 1.44482200 \\
\hline $\mathrm{H}$ & -5.25909200 & -2.81023900 & 2.99988300 & $\mathrm{H}$ & 1.03089600 & 0.96948400 & 2.00937300 \\
\hline $\mathrm{H}$ & -3.04026500 & -1.95263700 & 2.50341400 & $\mathrm{C}$ & 2.76919200 & 2.17859800 & 3.71800600 \\
\hline $\mathrm{H}$ & -4.50798200 & -5.03640900 & -1.92371100 & $\mathrm{H}$ & 2.02505600 & 1.57272400 & 4.24655700 \\
\hline $\mathrm{H}$ & -3.68778800 & -2.87415800 & -2.41470600 & $\mathrm{H}$ & 3.69107500 & 2.18621200 & 4.31172500 \\
\hline $\mathrm{H}$ & -4.13712500 & -4.16051400 & 2.93120600 & $\mathrm{H}$ & 2.39272000 & 3.20672500 & 3.68646100 \\
\hline $\mathrm{H}$ & -5.15674100 & -1.37052300 & -1.16731500 & $\mathrm{C}$ & 1.25344600 & 2.89645300 & 1.06855300 \\
\hline $\mathrm{H}$ & -2.78482700 & -3.11639500 & 1.21915000 & $\mathrm{H}$ & 2.05233700 & 3.64694600 & 1.07758300 \\
\hline $\mathrm{H}$ & -4.90181800 & -0.88304600 & 1.15977200 & $\mathrm{H}$ & 0.82870300 & 2.91708400 & 0.06200900 \\
\hline $\mathrm{C}$ & -4.89700500 & -4.93337700 & 0.18953800 & $\mathrm{C}$ & 3.05937500 & 1.62162300 & 2.31367300 \\
\hline $\mathrm{H}$ & -5.46092000 & -5.86597200 & 0.05758300 & $\mathrm{H}$ & 3.79353200 & 2.26222700 & 1.80922300 \\
\hline $\mathrm{H}$ & -3.89016000 & -5.24191400 & 0.50703100 & $\mathrm{H}$ & 3.53426700 & 0.64532700 & 2.41189100 \\
\hline $\mathrm{C}$ & -5.57681900 & -4.10883200 & 1.31128500 & & & & \\
\hline $\mathrm{H}$ & -6.22688500 & -4.77251800 & 1.89626200 & 125 & & & \\
\hline $\mathrm{H}$ & -6.24759700 & -3.36930100 & 0.85435300 & & & & \\
\hline $\mathrm{C}$ & -4.78298200 & 3.77893300 & 0.44469800 & & & & \\
\hline $\mathrm{C}$ & -3.78545100 & 2.60056000 & 0.54766400 & & & & \\
\hline $\mathrm{C}$ & -4.74400600 & 2.93269100 & -3.07313400 & & & & \\
\hline $\mathrm{C}$ & -4.28703500 & 1.33350000 & -0.11609700 & & & & \\
\hline $\mathrm{C}$ & -3.62508500 & 2.02865400 & -2.50184300 & & & & \\
\hline $\mathrm{C}$ & -4.19185400 & 1.05547600 & -1.49418900 & $\mathrm{C}$ & 3.75952100 & 5900 & -0.44673200 \\
\hline $\mathrm{H}$ & -5.77653300 & 3.45263800 & 0.78707200 & $\mathrm{C}$ & 4.44878500 & 0.84028700 & 0.18263600 \\
\hline $\mathrm{H}$ & -2.82249400 & 2.90631700 & 0.13172900 & $\mathrm{C}$ & 6.54608600 & 1.70990400 & -0.58313200 \\
\hline $\mathrm{H}$ & -5.38713000 & 2.33909300 & -3.73840100 & $\mathrm{C}$ & 5.85257900 & 2.74311300 & -1.21045300 \\
\hline
\end{tabular}




\begin{tabular}{|c|c|c|c|c|c|c|c|}
\hline C & 4.46506900 & 2.87102600 & -1.15719900 & $\mathrm{C}$ & -3.93993600 & -1.75300300 & 445700 \\
\hline $\mathrm{C}$ & 2.34067300 & 1.77619100 & -0.09471700 & $\mathrm{C}$ & -2.50034400 & -2.40141400 & 1.36903500 \\
\hline C & 2.23144300 & 0.76817600 & 0.91228800 & $\mathrm{C}$ & -3.41785300 & -1.46949200 & 0.61303600 \\
\hline $\mathrm{H}$ & 7.62911100 & 1.68863100 & -0.64105900 & $\mathrm{H}$ & -5.59602700 & -3.91333900 & -1.00003600 \\
\hline $\mathrm{H}$ & 3.97724400 & 3.70548600 & -1.65091500 & $\mathrm{H}$ & -2.61091400 & -3.30368400 & -1.38912400 \\
\hline $\mathrm{H}$ & 1.68195200 & 2.63729700 & -0.13694400 & $\mathrm{H}$ & -3.71544900 & -2.78422700 & 3.12834900 \\
\hline $\mathrm{N}$ & 3.52423500 & -0.03702300 & 0.84181300 & $\mathrm{H}$ & -1.81396800 & -1.82641500 & 2.00215600 \\
\hline $\mathrm{P}$ & 2.85767900 & -1.31378000 & -0.24710600 & $\mathrm{H}$ & -4.46016100 & -4.97833500 & -1.81102200 \\
\hline $\mathrm{C}$ & 2.87035900 & -2.84493700 & 0.91386600 & $\mathrm{H}$ & -3.85694100 & -2.81051100 & -2.51567700 \\
\hline $\mathrm{C}$ & 3.84774100 & -1.70238600 & -1.86681400 & $\mathrm{H}$ & -2.62849600 & -4.09301700 & 2.69483500 \\
\hline $\mathrm{C}$ & 4.25624500 & -3.01587300 & 1.57154400 & $\mathrm{H}$ & -4.91653000 & -1.32249700 & -0.89003700 \\
\hline $\mathrm{H}$ & 4.56967700 & -2.09359000 & 2.06910100 & $\mathrm{H}$ & -1.88690400 & -2.98295400 & 0.67793300 \\
\hline $\mathrm{H}$ & 4.18833600 & -3.80439900 & 2.33259200 & $\mathrm{H}$ & -4.01736000 & -0.84434700 & 1.27350400 \\
\hline $\mathrm{H}$ & 5.03285100 & -3.31288100 & 0.86306700 & $\mathrm{C}$ & -4.18214900 & -4.89012800 & 0.31972800 \\
\hline $\mathrm{C}$ & 1.84222600 & -2.58952600 & 2.03500200 & $\mathrm{H}$ & -4.74145000 & -5.83405900 & 0.35686600 \\
\hline $\mathrm{H}$ & 1.75047200 & -3.50188100 & 2.63848400 & $\mathrm{H}$ & -3.12057500 & -5.17786500 & 0.31096200 \\
\hline $\mathrm{H}$ & 2.17125200 & -1.79230700 & 2.70363700 & $\mathrm{C}$ & -4.50166300 & -4.08620000 & 1.60456800 \\
\hline $\mathrm{H}$ & 0.85238200 & -2.33661900 & 1.64445600 & $\mathrm{H}$ & -4.91659000 & -4.77045900 & 2.35641400 \\
\hline $\mathrm{C}$ & 2.45777200 & -4.14075400 & 0.18801800 & $\mathrm{H}$ & -5.30523000 & -3.37012100 & 1.38673700 \\
\hline $\mathrm{H}$ & 1.49148000 & -4.03932100 & -0.31672300 & $\mathrm{C}$ & -4.58286900 & 3.74831200 & 0.44276600 \\
\hline $\mathrm{H}$ & 3.20223800 & -4.47797600 & -0.53751900 & $\mathrm{C}$ & -3.48569600 & 2.65869700 & 0.38127900 \\
\hline $\mathrm{H}$ & 2.35736100 & -4.93701500 & 0.93659000 & $\mathrm{C}$ & -5.01191400 & 2.89578300 & -3.04532700 \\
\hline $\mathrm{C}$ & 2.88837500 & -2.57013900 & -2.72371500 & $\mathrm{C}$ & -3.97223400 & 1.34344700 & -0.19740500 \\
\hline $\mathrm{H}$ & 2.62959400 & -3.52591800 & -2.26919700 & $\mathrm{C}$ & -3.75771100 & 2.07726100 & -2.65451800 \\
\hline $\mathrm{H}$ & 1.95959500 & -2.03709300 & -2.94540400 & $\mathrm{C}$ & -4.09726200 & 1.07376800 & -1.57688800 \\
\hline $\mathrm{H}$ & 3.39210100 & -2.78501800 & -3.67507100 & $\mathrm{H}$ & -5.48227200 & 3.34006500 & 0.92833200 \\
\hline C & 4.09729900 & -0.41728000 & -2.68777800 & $\mathrm{H}$ & -2.63167000 & 3.04504700 & -0.18059800 \\
\hline $\mathrm{H}$ & 4.91315500 & 0.18766100 & -2.29433300 & $\mathrm{H}$ & -5.69908500 & 2.25524200 & -3.61626600 \\
\hline $\mathrm{H}$ & 4.37354600 & -0.72264400 & -3.70565700 & $\mathrm{H}$ & -3.37557600 & 1.56643200 & -3.54714700 \\
\hline $\mathrm{C}$ & 5.18432800 & -2.43951000 & -1.66725500 & $\mathrm{H}$ & -4.21502500 & 4.54290100 & 1.10653800 \\
\hline $\mathrm{H}$ & 5.67041200 & -2.54876400 & -2.64567200 & $\mathrm{H}$ & -3.12092600 & 2.47966300 & 1.40106400 \\
\hline $\mathrm{H}$ & 5.87222600 & -1.88593900 & -1.02162300 & $\mathrm{H}$ & -4.69087900 & 3.68941400 & -3.73431400 \\
\hline $\mathrm{H}$ & 5.05511800 & -3.44535600 & -1.25941700 & $\mathrm{H}$ & -4.67912400 & 0.83374600 & 0.45921300 \\
\hline F & 6.56132200 & 3.67727700 & -1.88346500 & $\mathrm{H}$ & -2.96531800 & 2.75083000 & -2.32348100 \\
\hline $\mathrm{Cl}$ & -0.55143100 & -1.65601400 & -1.32853700 & $\mathrm{H}$ & -4.87610700 & 0.37615400 & -1.88483600 \\
\hline $\mathrm{H}$ & 3.19785700 & 0.20137300 & -2.75613000 & $\mathrm{C}$ & -4.99079000 & 4.40399500 & -0.89359700 \\
\hline C & 5.83205100 & 0.73596900 & 0.12971500 & $\mathrm{H}$ & -5.59723000 & 5.28357700 & -0.64102000 \\
\hline $\mathrm{H}$ & 6.35326300 & -0.06639000 & 0.64319300 & $\mathrm{H}$ & -4.09388500 & 4.79946500 & -1.39217200 \\
\hline $\mathrm{Rh}$ & 1.09346900 & 0.00880800 & -0.65487900 & $\mathrm{C}$ & -5.79951400 & 3.52375600 & -1.87878800 \\
\hline $\mathrm{Cl}$ & -0.72499100 & 1.56349300 & -1.33551200 & $\mathrm{H}$ & -6.59846100 & 4.13073700 & -2.32429900 \\
\hline $\mathrm{Rh}$ & -2.48747600 & -0.12995700 & -0.80674300 & $\mathrm{H}$ & -6.31174600 & 2.73350100 & -1.31394800 \\
\hline C & -4.53857000 & -4.21696900 & -1.02265800 & $\mathrm{C}$ & -0.68333000 & 1.42300100 & 3.29630700 \\
\hline $\mathrm{C}$ & -3.66342200 & -3.01703900 & -1.45479200 & $\mathrm{O}$ & -0.92842900 & 0.28693400 & 3.65139600 \\
\hline $\mathrm{C}$ & -3.32272200 & -3.35247400 & 2.27359200 & $\mathrm{O}$ & -1.35369700 & 2.50062000 & 3.76709300 \\
\hline
\end{tabular}




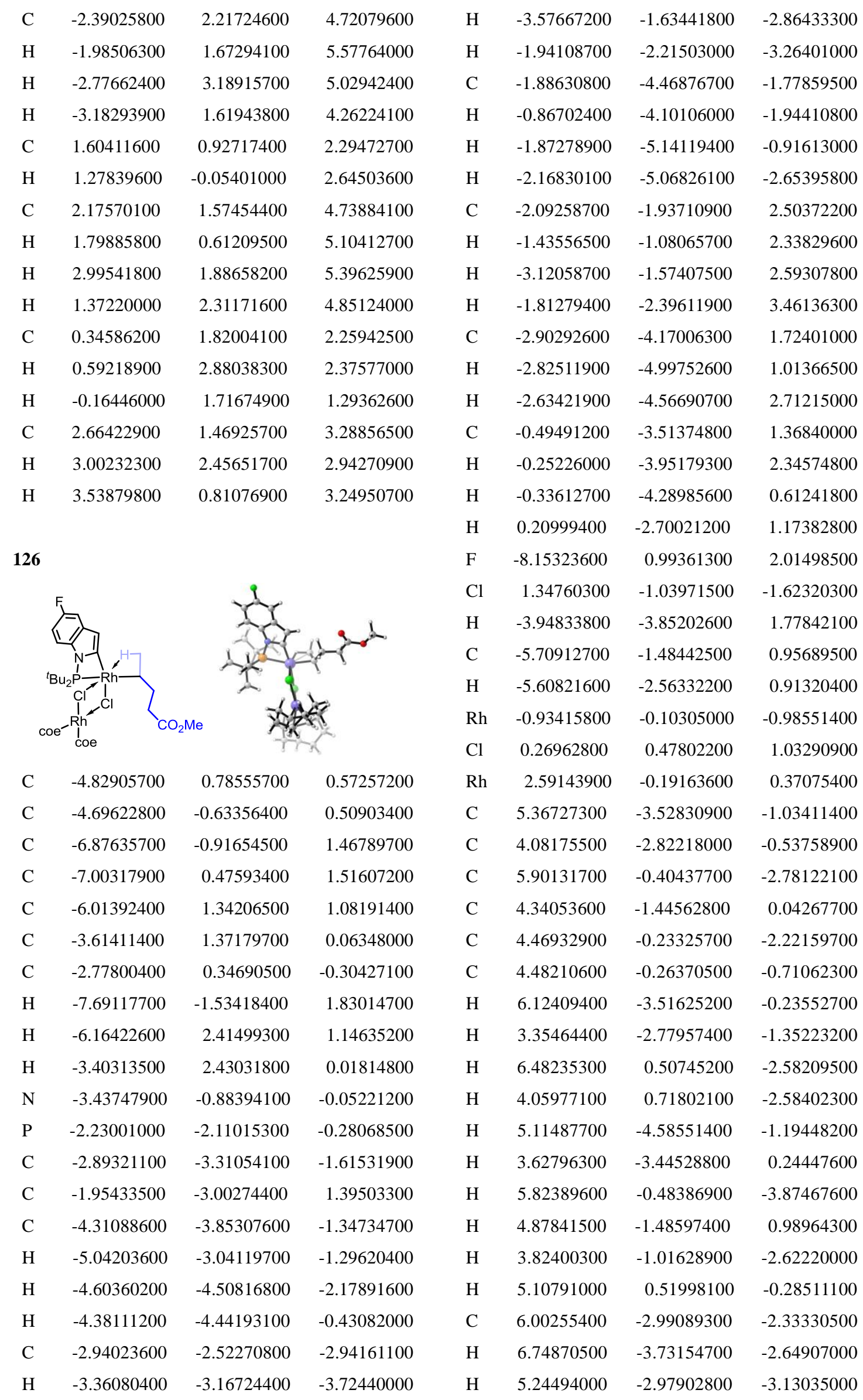




\begin{tabular}{|c|c|c|c|c|c|c|c|}
\hline $\mathrm{C}$ & 6.69733900 & -1.60905500 & -2.24152400 & \multirow{2}{*}{\multicolumn{2}{|c|}{127 -ts }} & & \\
\hline $\mathrm{H}$ & 7.64022600 & -1.64603300 & -2.80247300 & & & & \\
\hline $\mathrm{H}$ & 6.98477800 & -1.42178900 & -1.19836500 & & & & \\
\hline $\mathrm{C}$ & 3.45998200 & 3.33269300 & 3.13247300 & & & & \\
\hline $\mathrm{C}$ & 2.89108100 & 2.52800100 & 1.93887800 & & & & \\
\hline $\mathrm{C}$ & 2.93119500 & 0.23029200 & 4.91832800 & & & & \\
\hline $\mathrm{C}$ & 3.57818800 & 1.19244400 & 1.73384000 & & & & \\
\hline $\mathrm{C}$ & 2.25775600 & -0.05364400 & 3.55427200 & $\mathrm{C}$ & -4.96648900 & 0.73371000 & 0.36055300 \\
\hline $\mathrm{C}$ & 3.27194100 & 0.01067900 & 2.43553700 & $\mathrm{C}$ & -4.76240300 & -0.67518400 & 0.45530100 \\
\hline $\mathrm{H}$ & 4.55435900 & 3.40174300 & 3.04025400 & $\mathrm{C}$ & -6.94586200 & -0.96804600 & 1.40120400 \\
\hline $\mathrm{H}$ & 1.81340600 & 2.40408100 & 2.07015500 & $\mathrm{C}$ & -7.14347400 & 0.41279000 & 1.29426000 \\
\hline $\mathrm{H}$ & 3.56614200 & -0.62347900 & 5.19506900 & $\mathrm{C}$ & -6.18913800 & 1.27833800 & 0.78610600 \\
\hline $\mathrm{H}$ & 1.78898000 & -1.04478100 & 3.59099900 & $\mathrm{C}$ & -3.76978400 & 1.32520900 & -0.18660500 \\
\hline $\mathrm{H}$ & 3.08358500 & 4.36050500 & 3.03885100 & $\mathrm{C}$ & -2.87213400 & 0.31254000 & -0.41844500 \\
\hline $\mathrm{H}$ & 3.02132700 & 3.12567700 & 1.02686800 & $\mathrm{H}$ & -7.73691600 & -1.58468800 & 1.81418300 \\
\hline $\mathrm{H}$ & 2.13735100 & 0.27861700 & 5.67669300 & $\mathrm{H}$ & -6.39496400 & 2.34256900 & 0.73206000 \\
\hline $\mathrm{H}$ & 4.60567200 & 1.29968100 & 1.38697400 & $\mathrm{H}$ & -3.61432900 & 2.38288800 & -0.34227500 \\
\hline $\mathrm{H}$ & 1.44906100 & 0.66177500 & 3.39406500 & $\mathrm{~N}$ & -3.48019800 & -0.91538600 & -0.05257300 \\
\hline $\mathrm{H}$ & 4.07812800 & -0.71058300 & 2.56305100 & $\mathrm{P}$ & -2.19498700 & -2.07242400 & -0.14139200 \\
\hline $\mathrm{C}$ & 3.10747200 & 2.82714400 & 4.54699000 & $\mathrm{C}$ & -2.73598600 & -3.44477200 & -1.35737800 \\
\hline $\mathrm{H}$ & 3.39892500 & 3.62175200 & 5.24598100 & $\mathrm{C}$ & -1.88363800 & -2.76112900 & 1.61920400 \\
\hline $\mathrm{H}$ & 2.01520500 & 2.74112700 & 4.64084900 & $\mathrm{C}$ & -4.12075200 & -4.04598700 & -1.04370600 \\
\hline $\mathrm{C}$ & 3.78640500 & 1.51033000 & 4.99923200 & $\mathrm{H}$ & -4.9041 & -3.283 & -1.08994500 \\
\hline $\mathrm{H}$ & 4.10316800 & 1.61499200 & 6.04508300 & $\mathrm{H}$ & -4.35620400 & -4.80549600 & -1.80104000 \\
\hline $\mathrm{H}$ & 4.71283100 & 1.37111400 & 4.42617400 & $\mathrm{H}$ & -4.16825600 & -4.53423900 & -0.06897900 \\
\hline $\mathrm{C}$ & -1.15056200 & 0.95524000 & -3.04052800 & $\mathrm{C}$ & -2.81144300 & -2.81494300 & -2.76413900 \\
\hline $\mathrm{H}$ & -2.08471900 & 1.41974600 & -3.36958600 & $\mathrm{H}$ & -3.18107800 & -3.57069500 & -3.46924900 \\
\hline $\mathrm{C}$ & -0.42702000 & 1.68640400 & -1.97789900 & $\mathrm{H}$ & -3.50140900 & -1.96477000 & -2.79335700 \\
\hline $\mathrm{H}$ & 0.65948000 & 1.66572900 & -2.07679100 & $\mathrm{H}$ & -1.82874700 & -2.48266300 & -3.11037100 \\
\hline $\mathrm{C}$ & -0.96068100 & 3.00969000 & -1.47438500 & $\mathrm{C}$ & -1.65079800 & -4.54182700 & -1.37723400 \\
\hline $\mathrm{H}$ & -0.66249200 & 3.15386300 & -0.42991100 & $\mathrm{H}$ & -0.65510600 & -4.12303600 & -1.56279700 \\
\hline $\mathrm{H}$ & -2.05283700 & 3.01312600 & -1.49356600 & $\mathrm{H}$ & -1.61530100 & -5.11748400 & -0.44800200 \\
\hline $\mathrm{H}$ & -1.58229800 & -0.10565000 & -2.65080500 & $\mathrm{H}$ & -1.87123400 & -5.24682100 & -2.18908000 \\
\hline $\mathrm{H}$ & -0.53930300 & 0.60174700 & -3.87508500 & $\mathrm{C}$ & -2.11982500 & -1.59533900 & 2.60403100 \\
\hline $\mathrm{C}$ & -0.44775700 & 4.19614500 & -2.30806300 & $\mathrm{H}$ & -1.52857400 & -0.71349500 & 2.35032800 \\
\hline $\mathrm{H}$ & 0.64682800 & 4.26431600 & -2.28133500 & $\mathrm{H}$ & -3.17437600 & -1.30820900 & 2.64709800 \\
\hline $\mathrm{H}$ & -0.71071700 & 4.07444800 & -3.36875400 & $\mathrm{H}$ & -1.81845700 & -1.92530600 & 3.60701100 \\
\hline $\mathrm{C}$ & -1.02443500 & 5.52182900 & -1.84648500 & $\mathrm{C}$ & -2.76201800 & -3.94852900 & 2.05829700 \\
\hline $\mathrm{O}$ & -1.92773900 & 5.66242700 & -1.05039400 & $\mathrm{H}$ & -2.60868500 & -4.84344500 & 1.44898000 \\
\hline $\mathrm{O}$ & -0.40416300 & 6.55763800 & -2.45811800 & $\mathrm{H}$ & -2.49357400 & -4.21135600 & 3.09005600 \\
\hline $\mathrm{C}$ & -0.89871500 & 7.86093300 & -2.11205800 & $\mathrm{C}$ & -0.39523500 & -3.17642500 & 1.67036600 \\
\hline $\mathrm{H}$ & -0.29783900 & 8.56513300 & -2.68863500 & $\mathrm{H}$ & -0.15072100 & -3.48938300 & 2.69414700 \\
\hline $\mathrm{H}$ & -1.95634000 & 7.95431800 & -2.37383100 & $\mathrm{H}$ & -0.17309900 & -4.01927700 & 1.00807600 \\
\hline $\mathrm{H}$ & -0.78300400 & 8.04457500 & -1.04040500 & $\mathrm{H}$ & 0.26323800 & -2.34659600 & 1.40033100 \\
\hline
\end{tabular}




\begin{tabular}{|c|c|c|c|c|c|c|c|}
\hline $\mathrm{F}$ & -8.32946700 & 0.92038500 & 1.71262800 & $\mathrm{H}$ & 1.51660200 & 0.78766800 & 3.35047700 \\
\hline $\mathrm{Cl}$ & 1.29598800 & -1.08680100 & -1.60062900 & $\mathrm{H}$ & 4.08774500 & -0.68310300 & 2.51123500 \\
\hline $\mathrm{H}$ & -3.82763900 & -3.70049100 & 2.05635500 & $\mathrm{C}$ & 3.26708200 & 2.92014100 & 4.43710200 \\
\hline $\mathrm{C}$ & -5.73951500 & -1.52546000 & 0.97631000 & $\mathrm{H}$ & 3.59610400 & 3.71739900 & 5.11616300 \\
\hline $\mathrm{H}$ & -5.58093800 & -2.59574400 & 1.05322600 & $\mathrm{H}$ & 2.17437800 & 2.87091500 & 4.55062700 \\
\hline $\mathrm{Rh}$ & -0.99732800 & -0.14323900 & -1.01876100 & $\mathrm{C}$ & 3.91026000 & 1.58999300 & 4.90221600 \\
\hline $\mathrm{Cl}$ & 0.30608600 & 0.60208900 & 0.98939300 & $\mathrm{H}$ & 4.25115100 & 1.70334400 & 5.93958000 \\
\hline $\mathrm{Rh}$ & 2.58994600 & -0.16852900 & 0.33044200 & $\mathrm{H}$ & 4.81946700 & 1.40732800 & 4.31405400 \\
\hline $\mathrm{C}$ & 5.31329800 & -3.57554500 & -1.02497600 & $\mathrm{C}$ & -1.12622400 & 0.88314800 & -3.02561300 \\
\hline $\mathrm{C}$ & 4.04411300 & -2.84478400 & -0.52282200 & $\mathrm{H}$ & -2.11161300 & 1.21378100 & -3.35550200 \\
\hline $\mathrm{C}$ & 5.82269800 & -0.51677000 & -2.88935400 & $\mathrm{C}$ & -0.43970300 & 1.68766300 & -2.06408000 \\
\hline $\mathrm{C}$ & 4.32208900 & -1.45101000 & 0.00555400 & $\mathrm{H}$ & 0.64800800 & 1.63468200 & -2.08269100 \\
\hline $\mathrm{C}$ & 4.40573200 & -0.31894100 & -2.30141100 & $\mathrm{C}$ & -1.01050000 & 2.98525200 & -1.54197300 \\
\hline $\mathrm{C}$ & 4.45513000 & -0.29724600 & -0.79107100 & $\mathrm{H}$ & -0.79711000 & 3.08333700 & -0.47187400 \\
\hline $\mathrm{H}$ & 6.08893600 & -3.54145600 & -0.24528700 & $\mathrm{H}$ & -2.09681300 & 2.99589100 & -1.65317900 \\
\hline $\mathrm{H}$ & 3.29887600 & -2.82442000 & -1.32189300 & $\mathrm{H}$ & -1.62906500 & -0.38340500 & -2.49109600 \\
\hline $\mathrm{H}$ & 6.41258300 & 0.39792700 & -2.73449500 & $\mathrm{H}$ & -0.53666700 & 0.37787500 & -3.79031100 \\
\hline $\mathrm{H}$ & 3.99163800 & 0.62141000 & -2.68681100 & $\mathrm{C}$ & -0.42610500 & 4.20284600 & -2.27880800 \\
\hline $\mathrm{H}$ & 5.05148800 & -4.63606100 & -1.14345200 & $\mathrm{H}$ & 0.65989500 & 4.27311100 & -2.14763100 \\
\hline $\mathrm{H}$ & 3.60476100 & -3.43922500 & 0.28931400 & $\mathrm{H}$ & -0.59346200 & 4.11869300 & -3.36245500 \\
\hline $\mathrm{H}$ & 5.71864700 & -0.63232200 & -3.97739300 & $\mathrm{C}$ & -1.05942900 & 5.50481400 & -1.82368300 \\
\hline $\mathrm{H}$ & 4.88204100 & -1.46298100 & 0.94050400 & $\mathrm{O}$ & -2.10660600 & 5.60730300 & -1.22132500 \\
\hline $\mathrm{H}$ & 3.74729900 & -1.11224300 & -2.65802000 & $\mathrm{O}$ & -0.30999700 & 900 & -2.19910400 \\
\hline $\mathrm{H}$ & 5.09333600 & 0.49771000 & -0.40748100 & $\mathrm{C}$ & -0.84733500 & 7.85213000 & -1.84972400 \\
\hline $\mathrm{C}$ & 5.92113100 & -3.08705500 & -2.35642600 & $\mathrm{H}$ & -0.12903100 & 8.58172200 & -2.22527300 \\
\hline $\mathrm{H}$ & 6.65560200 & -3.84282100 & -2.66367800 & $\mathrm{H}$ & -1.82549300 & 8.00104400 & -2.31533700 \\
\hline $\mathrm{H}$ & 5.14514900 & -3.09777900 & -3.13568500 & $\mathrm{H}$ & -0.95515600 & 7.94183600 & -0.76539200 \\
\hline $\mathrm{C}$ & 6.62542100 & -1.70731000 & -2.32860200 & & & & \\
\hline $\mathrm{H}$ & 7.55416200 & -1.76899800 & -2.91060600 & 128 & & & \\
\hline $\mathrm{H}$ & 6.93895300 & -1.48643100 & -1.29963800 & & & & \\
\hline $\mathrm{C}$ & 3.61110900 & 3.38695500 & 3.00730500 & & & & \\
\hline $\mathrm{C}$ & 2.99465200 & 2.57891700 & 1.84007700 & & & & \\
\hline $\mathrm{C}$ & 3.01005300 & 0.33931700 & 4.86200800 & & & & \\
\hline $\mathrm{C}$ & 3.63451800 & 1.21788300 & 1.64914400 & & & & \\
\hline $\mathrm{C}$ & 2.30510000 & 0.05140000 & 3.51473200 & & & & \\
\hline $\mathrm{C}$ & 3.30294200 & 0.06067400 & 2.37976400 & $\mathrm{C}$ & -5.110 & 0.92621500 & -0.22345700 \\
\hline $\mathrm{H}$ & 4.70547800 & 3.41732600 & 2.89555400 & $\mathrm{C}$ & -4.95493300 & -0.40505700 & 0.26772500 \\
\hline $\mathrm{H}$ & 1.91607300 & 2.49236100 & 1.99217900 & $\mathrm{C}$ & -7.20116500 & -0.40348400 & 1.10465500 \\
\hline $\mathrm{H}$ & 3.61967000 & -0.53015900 & 5.14694900 & $\mathrm{C}$ & -7.35011300 & 0.89747000 & 0.61253000 \\
\hline $\mathrm{H}$ & 1.80619800 & -0.92339900 & 3.57892600 & $\mathrm{C}$ & -6.34058500 & 1.57986400 & -0.04598400 \\
\hline $\mathrm{H}$ & 3.26772800 & 4.42505800 & 2.90038100 & $\mathrm{C}$ & -3.86543200 & 1.33183500 & -0.82957900 \\
\hline $\mathrm{H}$ & 3.12792100 & 3.15445100 & 0.91426000 & $\mathrm{C}$ & -2.98073800 & 0600 & -0.70867900 \\
\hline $\mathrm{H}$ & 2.23093700 & 0.43110900 & 5.63165700 & $\mathrm{H}$ & -8.03475700 & -0.87480400 & 1.61400900 \\
\hline $\mathrm{H}$ & 4.66002300 & 1.28609000 & 1.28569300 & $\mathrm{H}$ & -6.51146000 & 4800 & -0.40163700 \\
\hline
\end{tabular}




\begin{tabular}{|c|c|c|c|c|c|c|c|}
\hline $\mathrm{H}$ & -3.67354700 & 2.29975400 & -1.26979100 & $\mathrm{H}$ & 6.11508200 & -0.26446900 & -3.15439400 \\
\hline $\mathrm{N}$ & -3.65010700 & -0.78555500 & -0.06667400 & $\mathrm{H}$ & 3.70434600 & -0.03966400 & -2.93222000 \\
\hline $\mathrm{P}$ & -2.38678800 & -1.92692700 & 0.20694100 & $\mathrm{H}$ & 4.98991400 & -4.94682700 & -0.59472600 \\
\hline $\mathrm{C}$ & -2.87150400 & -3.57788000 & -0.63025000 & $\mathrm{H}$ & 3.63664800 & -3.52412000 & 0.71935000 \\
\hline $\mathrm{C}$ & -2.15622900 & -2.12845000 & 2.09836700 & $\mathrm{H}$ & 5.33610500 & -1.50129600 & -4.12910800 \\
\hline $\mathrm{C}$ & -4.29072500 & -4.04478600 & -0.24166600 & $\mathrm{H}$ & 4.93189500 & -1.46170300 & 0.90944600 \\
\hline $\mathrm{H}$ & -5.04554200 & -3.31616700 & -0.55170400 & $\mathrm{H}$ & 3.49137800 & -1.74190300 & -2.58481300 \\
\hline $\mathrm{H}$ & -4.50460800 & -4.98392800 & -0.76817400 & $\mathrm{H}$ & 4.99567100 & 0.23718300 & -0.77109600 \\
\hline $\mathrm{H}$ & -4.41027600 & -4.23823200 & 0.82520100 & $\mathrm{C}$ & 5.72889100 & -3.63100300 & -2.12745500 \\
\hline $\mathrm{C}$ & -2.86134400 & -3.38440500 & -2.16135000 & $\mathrm{H}$ & 6.45497200 & -4.42261200 & -2.35381200 \\
\hline $\mathrm{H}$ & -3.21844600 & -4.31200200 & -2.62748000 & $\mathrm{H}$ & 4.89572000 & -3.78857200 & -2.82767300 \\
\hline $\mathrm{H}$ & -3.52703300 & -2.57435800 & -2.47642400 & $\mathrm{C}$ & 6.40156900 & -2.26242900 & -2.40237400 \\
\hline $\mathrm{H}$ & -1.85710400 & -3.18131500 & -2.54200300 & $\mathrm{H}$ & 7.27886300 & -2.42011800 & -3.04322200 \\
\hline $\mathrm{C}$ & -1.82075400 & -4.65056000 & -0.27280400 & $\mathrm{H}$ & 6.79649300 & -1.86231800 & -1.45909900 \\
\hline $\mathrm{H}$ & -0.80310500 & -4.31638200 & -0.50388000 & $\mathrm{C}$ & 3.88595800 & 3.57211600 & 2.34100900 \\
\hline $\mathrm{H}$ & -1.85846600 & -4.94544900 & 0.77929300 & $\mathrm{C}$ & 3.17351300 & 2.63552900 & 1.33550100 \\
\hline $\mathrm{H}$ & -2.01799300 & -5.54959900 & -0.87037500 & $\mathrm{C}$ & 3.24962300 & 0.86557500 & 4.65268200 \\
\hline $\mathrm{C}$ & -2.38655500 & -0.73773900 & 2.72983700 & $\mathrm{C}$ & 3.74262700 & 1.23022900 & 1.31385600 \\
\hline $\mathrm{H}$ & -1.73589200 & 0.02625800 & 2.30148700 & $\mathrm{C}$ & 2.46680100 & 0.42438100 & 3.39334900 \\
\hline $\mathrm{H}$ & -3.42635600 & -0.41399500 & 2.62656000 & $\mathrm{C}$ & 3.40673700 & 0.21503100 & 2.22911100 \\
\hline $\mathrm{H}$ & -2.15921900 & -0.80832600 & 3.80143300 & $\mathrm{H}$ & 4.97312400 & 3.52815200 & 2.17679100 \\
\hline $\mathrm{C}$ & -3.11773200 & -3.12443600 & 2.77688500 & $\mathrm{H}$ & 2.10190700 & 2.62339500 & 1.54968000 \\
\hline $\mathrm{H}$ & -2.98325900 & -4.15316200 & 2.43297900 & $\mathrm{H}$ & 3.83423400 & 0.01666500 & 5.03527900 \\
\hline $\mathrm{H}$ & -2.90855900 & -3.11500100 & 3.85447800 & $\mathrm{H}$ & 1.92452800 & -0.50175400 & 3.62047200 \\
\hline $\mathrm{C}$ & -0.69793600 & -2.57632200 & 2.34648400 & $\mathrm{H}$ & 3.58534600 & 4.60022600 & 2.09645200 \\
\hline $\mathrm{H}$ & -0.52395100 & -2.61102300 & 3.43007800 & $\mathrm{H}$ & 3.28159000 & 3.06695400 & 0.33096700 \\
\hline $\mathrm{H}$ & -0.49334300 & -3.57567600 & 1.95049900 & $\mathrm{H}$ & 2.51408500 & 1.10589700 & 5.43316500 \\
\hline $\mathrm{H}$ & 0.01842200 & -1.87720500 & 1.90983800 & $\mathrm{H}$ & 4.74616900 & 1.18927200 & 0.89080200 \\
\hline $\mathrm{F}$ & -8.54355900 & 1.51425200 & 0.79536200 & $\mathrm{H}$ & 1.70981000 & 1.17199800 & 3.15301000 \\
\hline $\mathrm{Cl}$ & 1.13996200 & -1.53006200 & -1.34261100 & $\mathrm{H}$ & 4.16296600 & -0.54148500 & 2.43565600 \\
\hline $\mathrm{H}$ & -4.16750700 & -2.84330800 & 2.65099000 & $\mathrm{C}$ & 3.59751300 & 3.34095000 & 3.83952000 \\
\hline $\mathrm{C}$ & -5.98745600 & -1.06958800 & 0.93155400 & $\mathrm{H}$ & 3.99793300 & 4.21203100 & 4.37423600 \\
\hline $\mathrm{H}$ & -5.86772300 & -2.08081800 & 1.30561400 & $\mathrm{H}$ & 2.51131400 & 3.36659100 & 4.00887700 \\
\hline $\mathrm{Rh}$ & -1.10442700 & -0.36642200 & -1.03644800 & $\mathrm{C}$ & 4.20365700 & 2.06309400 & 4.47224200 \\
\hline $\mathrm{Cl}$ & 0.36279700 & 0.67412700 & 0.90163300 & $\mathrm{H}$ & 4.59521800 & 2.31091500 & 5.46745800 \\
\hline $\mathrm{Rh}$ & 2.57358300 & -0.29613600 & 0.28222100 & $\mathrm{H}$ & 5.07736800 & 1.75285400 & 3.88356800 \\
\hline $\mathrm{C}$ & 5.23831300 & -3.88033200 & -0.68541100 & $\mathrm{C}$ & -0.77558200 & 0.74253600 & -3.04121500 \\
\hline $\mathrm{C}$ & 4.00034000 & -3.07918400 & -0.21673300 & $\mathrm{H}$ & -1.75030900 & 0.99914700 & -3.44807900 \\
\hline $\mathrm{C}$ & 5.53060900 & -1.19361700 & -3.09207100 & $\mathrm{C}$ & -0.17133800 & 1.54661900 & -2.10612400 \\
\hline $\mathrm{C}$ & 4.29387800 & -1.61348600 & 0.03787900 & $\mathrm{H}$ & 0.87169500 & 1.35461600 & -1.86517900 \\
\hline $\mathrm{C}$ & 4.16483700 & -0.89854200 & -2.42767200 & $\mathrm{C}$ & -0.72580100 & 2.86408000 & -1.62897000 \\
\hline $\mathrm{C}$ & 4.34214100 & -0.61536100 & -0.95385200 & $\mathrm{H}$ & -0.74392200 & 2.89411100 & -0.53504500 \\
\hline $\mathrm{H}$ & 6.07329600 & -3.70455500 & 0.00939800 & $\mathrm{H}$ & -1.75198000 & 3.00105800 & -1.97775500 \\
\hline $\mathrm{H}$ & 3.19359900 & -3.20198100 & -0.94385300 & $\mathrm{H}$ & -1.77844600 & -1.10344300 & -2.19537600 \\
\hline
\end{tabular}




\begin{tabular}{|c|c|c|c|c|c|c|c|}
\hline $\mathrm{H}$ & .20801600 & -0.01195800 & -3.57687600 & $\mathrm{H}$ & 2.25026700 & 1.17957100 & 4.94788400 \\
\hline $\mathrm{C}$ & 0.13798700 & 4.02903300 & -2.14271100 & $\mathrm{C}$ & 2.66294400 & -2.96732100 & 0.59591400 \\
\hline $\mathrm{H}$ & 1.16055400 & 3.97502700 & -1.75246000 & $\mathrm{H}$ & 1.98341100 & -2.66353100 & -0.20165000 \\
\hline $\mathrm{H}$ & 0.22787600 & 3.99175300 & -3.23787300 & $\mathrm{H}$ & 3.69274800 & -2.85934300 & 0.24294600 \\
\hline $\mathrm{C}$ & -0.45383700 & 5.37997300 & -1.78535100 & $\mathrm{H}$ & 2.48578700 & -4.03176000 & 0.79754900 \\
\hline $\mathrm{O}$ & -1.60857600 & 5.58616600 & -1.47992400 & $\mathrm{C}$ & 3.43782700 & -2.68730900 & 2.95062500 \\
\hline $\mathrm{O}$ & 0.47830400 & 6.35545700 & -1.87681100 & $\mathrm{H}$ & 3.29614400 & -2.24092400 & 3.93821900 \\
\hline $\mathrm{C}$ & 0.00513200 & 7.68450900 & -1.60444400 & $\mathrm{H}$ & 3.29708800 & -3.77005800 & 3.06372900 \\
\hline $\mathrm{H}$ & 0.87345900 & 8.33390900 & -1.72059700 & $\mathrm{C}$ & 0.98783600 & -2.45332600 & 2.41349000 \\
\hline $\mathrm{H}$ & -0.78191000 & 7.96558600 & -2.30966500 & $\mathrm{H}$ & 0.88060100 & -3.52944500 & 2.60353800 \\
\hline \multirow[t]{2}{*}{$\mathrm{H}$} & -0.39148600 & 7.75040700 & -0.58777700 & $\mathrm{H}$ & 0.77016900 & -1.93188800 & 3.35094400 \\
\hline & & \multirow{8}{*}{\multicolumn{2}{|c|}{ 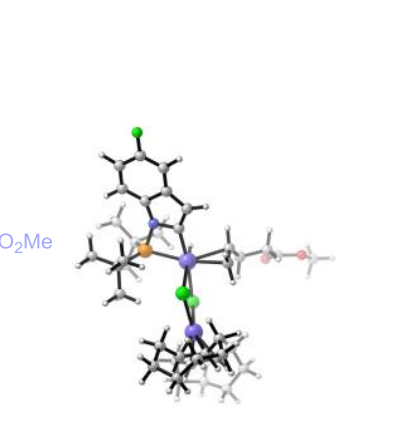 }} & $\mathrm{H}$ & 0.23881300 & -2.16264400 & 1.67363900 \\
\hline \multirow[t]{7}{*}{129} & \multirow{7}{*}{ 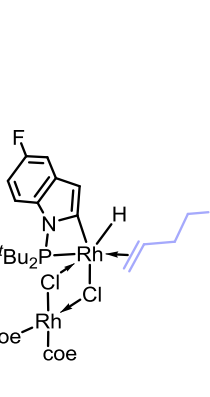 } & & & $\mathrm{F}$ & 8.56187000 & -0.96367600 & -2.19415000 \\
\hline & & & & $\mathrm{Cl}$ & -1.13397300 & 0.88530900 & 0.96924000 \\
\hline & & & & $\mathrm{H}$ & 4.47365100 & -2.52894900 & 2.63571700 \\
\hline & & & & $\mathrm{C}$ & 6.13392300 & -0.94872700 & 0.51366200 \\
\hline & & & & $\mathrm{H}$ & 6.07884800 & -1.21440400 & 1.56399500 \\
\hline & & & & $\mathrm{Rh}$ & 1.11737500 & 0.64888400 & -0.18400100 \\
\hline & & & & $\mathrm{Cl}$ & -0.17824500 & -1.49212400 & -1.00354200 \\
\hline $\mathrm{C}$ & 5.10195300 & -0.07638100 & -1.55161200 & $\mathrm{Rh}$ & -2.42493900 & -0.97853200 & -0.05997400 \\
\hline $\mathrm{C}$ & 5.03572000 & -0.42119000 & -0.16671500 & $\mathrm{C}$ & -5.02805700 & 0.04358700 & 3.55280600 \\
\hline $\mathrm{C}$ & 7.32587300 & -1.12885100 & -0.18886200 & $\mathrm{C}$ & -3.77896400 & -0.35863900 & 2.73363000 \\
\hline $\mathrm{C}$ & 7.38787500 & -0.77912900 & -1.54176600 & $\mathrm{C}$ & -5.70700000 & 2.12726200 & 0.67337600 \\
\hline $\mathrm{C}$ & 6.31151200 & -0.25871600 & -2.24147100 & $\mathrm{C}$ & -4.10449400 & -0.79095100 & 1.31719300 \\
\hline $\mathrm{C}$ & 3.80448000 & 0.40933400 & -1.95605200 & $\mathrm{C}$ & -4.28864600 & 1.58997900 & 0.36566800 \\
\hline $\mathrm{C}$ & 2.98184300 & 0.35708600 & -0.85901300 & $\mathrm{C}$ & -4.30710900 & 0.08440300 & 0.23325200 \\
\hline $\mathrm{H}$ & 8.20825400 & -1.53550200 & 0.29326100 & $\mathrm{H}$ & -5.78318500 & -0.75416900 & 3.48745100 \\
\hline $\mathrm{H}$ & 6.41803300 & -0.01086200 & -3.29271600 & $\mathrm{H}$ & -3.06161200 & 0.46544000 & 2.74229700 \\
\hline $\mathrm{H}$ & 3.53343200 & 0.73058000 & -2.95337200 & $\mathrm{H}$ & -6.33430600 & 2.03298900 & -0.22462900 \\
\hline $\mathrm{N}$ & 3.73115400 & -0.11983700 & 0.24253900 & $\mathrm{H}$ & -3.93074700 & 2.05270700 & -0.56300000 \\
\hline $\mathrm{P}$ & 2.53270700 & -0.31924800 & 1.47349100 & $\mathrm{H}$ & -4.72551400 & 0.08652700 & 4.60825400 \\
\hline $\mathrm{C}$ & 3.04432700 & 0.73656700 & 2.98599600 & $\mathrm{H}$ & -3.28695700 & -1.19717900 & 3.24461200 \\
\hline $\mathrm{C}$ & 2.42233500 & -2.18476600 & 1.90545500 & $\mathrm{H}$ & -5.61888100 & 3.20440700 & 0.87305100 \\
\hline $\mathrm{C}$ & .08000 & 0.46243100 & 3.43835900 & $\mathrm{H}$ & -4.64451400 & -1.73732800 & 1.28487000 \\
\hline $\mathrm{H}$ & 5.20969200 & 0.70922200 & 2.64852600 & $\mathrm{H}$ & -3.59833700 & 1.90426300 & 1.14971900 \\
\hline $\mathrm{H}$ & 4.71875100 & 1.10622200 & 4.29850400 & $\mathrm{H}$ & -4.97218100 & -0.25480400 & -0.55941000 \\
\hline $\mathrm{H}$ & 4.66342000 & -0.56826300 & 3.75164100 & $\mathrm{C}$ & -5.69185200 & 1.39079900 & 3.19729400 \\
\hline $\mathrm{C}$ & 2.95796100 & 2.22912900 & 2.60348300 & $\mathrm{H}$ & -6.40604400 & 1.61165600 & 4.00120400 \\
\hline $\mathrm{H}$ & 3.33352800 & 2.82318000 & 3.44665400 & $\mathrm{H}$ & -4.93908000 & 2.19107700 & 3.24559000 \\
\hline $\mathrm{H}$ & 3.57209500 & 2.46523200 & 1.72837000 & $\mathrm{C}$ & -6.44722600 & 1.45413500 & 1.84620000 \\
\hline $\mathrm{H}$ & 1.92972000 & 2.54103800 & 2.40381500 & $\mathrm{H}$ & -7.38494200 & 2.00685900 & 1.98946300 \\
\hline $\mathrm{C}$ & 2.04654400 & 0.47278600 & 4.13353800 & $\mathrm{H}$ & -6.74802700 & 0.43845200 & 1.55650000 \\
\hline $\mathrm{H}$ & 1.00997100 & 0.62712000 & 3.81346200 & $\mathrm{C}$ & -3.54843600 & -3.41088300 & -3.76410600 \\
\hline $\mathrm{H}$ & 2.13476800 & -0.53514400 & 4.54802500 & $\mathrm{C}$ & -2.92346200 & -2.28987400 & -2.89961400 \\
\hline
\end{tabular}




\begin{tabular}{|c|c|c|c|c|c|c|c|}
\hline $\mathrm{C}$ & -2.76020700 & -5.46679900 & -0.89292600 & & & & \\
\hline $\mathrm{C}$ & -3.50271700 & -2.21676400 & -1.49993300 & & & & \\
\hline $\mathrm{C}$ & -2.07624900 & -4.12511600 & -0.53643100 & & & & \\
\hline $\mathrm{C}$ & -3.10155200 & -3.02112500 & -0.41624300 & & & & \\
\hline $\mathrm{H}$ & -4.64546500 & -3.32900200 & -3.73689700 & & & & \\
\hline $\mathrm{H}$ & -1.83861600 & -2.41632500 & -2.87364900 & & & & \\
\hline $\mathrm{H}$ & -3.32056600 & -5.83300200 & -0.02067800 & $\mathrm{C}$ & 4.93635600 & -0.44012500 & -1.63546100 \\
\hline $\mathrm{H}$ & -1.52991800 & -4.24767600 & 0.40719600 & $\mathrm{C}$ & 4.93966700 & -0.55495400 & -0.21135700 \\
\hline $\mathrm{H}$ & -3.25685000 & -3.21903700 & -4.80585300 & $\mathrm{C}$ & 7.22765500 & -1.27206200 & -0.23431600 \\
\hline $\mathrm{H}$ & -3.10734100 & -1.32880000 & -3.39887200 & $\mathrm{C}$ & 7.22095500 & -1.14638100 & -1.62743500 \\
\hline $\mathrm{H}$ & -1.96771100 & -6.20550100 & -1.07846500 & $\mathrm{C}$ & 6.10948000 & -0.73988500 & -2.34710900 \\
\hline $\mathrm{H}$ & -4.53795100 & -1.87580400 & -1.49503300 & $\mathrm{C}$ & 3.61858900 & -0.01925500 & -2.04664100 \\
\hline $\mathrm{H}$ & -1.32958500 & -3.88137500 & -1.29354100 & $\mathrm{C}$ & 2.85655200 & 0.11052100 & -0.91312300 \\
\hline $\mathrm{H}$ & -3.84808400 & -3.22843800 & 0.34976800 & $\mathrm{H}$ & 8.13398900 & -1.60121500 & 0.26241300 \\
\hline $\mathrm{C}$ & -3.14759200 & -4.86053700 & -3.42027600 & $\mathrm{H}$ & 6.16146100 & -0.66700200 & -3.42877200 \\
\hline $\mathrm{H}$ & -3.49543600 & -5.48940500 & -4.25011700 & $\mathrm{H}$ & 3.29104400 & 0.13135800 & -3.06693400 \\
\hline $\mathrm{H}$ & -2.05124900 & -4.94602500 & -3.42691800 & $\mathrm{~N}$ & 3.65655900 & -0.18738300 & 0.21166000 \\
\hline $\mathrm{C}$ & -3.71751600 & -5.43836200 & -2.10063900 & $\mathrm{P}$ & 2.55173000 & -0.13901700 & 1.55107700 \\
\hline $\mathrm{H}$ & -4.04082700 & -6.47286000 & -2.27544300 & $\mathrm{C}$ & 3.19507600 & 1.17724800 & 2.77943300 \\
\hline $\mathrm{H}$ & -4.62946600 & -4.88783100 & -1.83383700 & $\mathrm{C}$ & 2.50153200 & -1.88639200 & 2.33849800 \\
\hline $\mathrm{C}$ & 0.24404600 & 1.36072200 & -2.17953200 & $\mathrm{C}$ & 4.66980800 & 0.99162900 & 3.19077500 \\
\hline $\mathrm{H}$ & -0.82719300 & 1.20022100 & -2.10031500 & $\mathrm{H}$ & 5.33459300 & 1.04744100 & 2.32384600 \\
\hline $\mathrm{C}$ & 0.82855100 & 2.48605300 & -1.64720000 & $\mathrm{H}$ & 4.95061100 & 1.80521700 & 3.87268200 \\
\hline $\mathrm{H}$ & 1.86215800 & 2.70258900 & -1.91166700 & $\mathrm{H}$ & 4.85795100 & 0.05241200 & 3.71305100 \\
\hline $\mathrm{C}$ & 0.05961300 & 3.63322500 & -1.03547500 & $\mathrm{C}$ & 3.07783300 & 2.55244400 & 2.08898800 \\
\hline $\mathrm{H}$ & 0.61671800 & 4.07531500 & -0.20397500 & $\mathrm{H}$ & 3.48875100 & 3.31894400 & 2.75864700 \\
\hline $\mathrm{H}$ & -0.89080800 & 3.27856600 & -0.62739800 & $\mathrm{H}$ & 3.64526400 & 2.58736900 & 1.15261900 \\
\hline $\mathrm{H}$ & 1.70641300 & 1.93087500 & 0.41281900 & $\mathrm{H}$ & 2.03699400 & 2.81292100 & 1.87861300 \\
\hline $\mathrm{H}$ & 0.77642200 & 0.71914600 & -2.87375400 & $\mathrm{C}$ & 2.27497600 & 1.17780800 & 4.01890800 \\
\hline $\mathrm{C}$ & -0.19317900 & 4.72343400 & -2.09118800 & $\mathrm{H}$ & 1.22025400 & 1.28526500 & 3.74088700 \\
\hline $\mathrm{H}$ & 0.74933500 & 5.02993100 & -2.56796200 & $\mathrm{H}$ & 2.38213400 & 0.27263800 & 4.62348600 \\
\hline $\mathrm{H}$ & -0.83348800 & 4.35860500 & -2.90188200 & $\mathrm{H}$ & 2.53787900 & 2.03065400 & 4.65767800 \\
\hline $\mathrm{C}$ & -0.81835200 & 5.97096300 & -1.49315200 & $\mathrm{C}$ & 2.66508100 & -2.90713600 & 1.19110000 \\
\hline $\mathrm{O}$ & -0.84720100 & 6.25273500 & -0.31471300 & $\mathrm{H}$ & 1.93691600 & -2.75388400 & 0.39243200 \\
\hline $\mathrm{O}$ & -1.33255500 & 6.76091000 & -2.46227500 & $\mathrm{H}$ & 3.66960300 & -2.87556700 & 0.75901200 \\
\hline $\mathrm{C}$ & -1.91192200 & 7.99476400 & -2.00814300 & $\mathrm{H}$ & 2.50546200 & -3.91311800 & 1.60121700 \\
\hline $\mathrm{H}$ & -2.26958400 & 8.49825800 & -2.90685200 & $\mathrm{C}$ & 3.56942500 & -2.18996500 & 3.40590200 \\
\hline $\mathrm{H}$ & -2.73977700 & 7.80078500 & -1.32082800 & $\mathrm{H}$ & 3.48423900 & -1.55440300 & 4.29137400 \\
\hline \multirow[t]{2}{*}{$\mathrm{H}$} & -1.16362900 & 8.60661700 & -1.49689100 & $\mathrm{H}$ & 3.43542100 & -3.22725900 & 3.74021900 \\
\hline & & & & $\mathrm{C}$ & 1.09351800 & -2.04264200 & 2.95878700 \\
\hline \multirow[t]{4}{*}{ 130-ts } & & & & $\mathrm{H}$ & 0.99260900 & -3.06106000 & 3.35665500 \\
\hline & & & & $\mathrm{H}$ & 0.92162100 & -1.34845400 & 3.78797100 \\
\hline & & & & $\mathrm{H}$ & 0.30812200 & -1.88791400 & 2.21415800 \\
\hline & & & & $\mathrm{F}$ & 8.36153500 & -1.44115500 & -2.29925400 \\
\hline
\end{tabular}




\begin{tabular}{|c|c|c|c|c|c|c|c|}
\hline $\mathrm{Cl}$ & -1.20892300 & 0.94890100 & 0.91801600 & $\mathrm{H}$ & -3.95894000 & -3.22769600 & 0.26057900 \\
\hline $\mathrm{H}$ & 4.58695600 & -2.10817200 & 3.01189900 & $\mathrm{C}$ & -2.98349700 & -4.83508100 & -3.45661800 \\
\hline $\mathrm{C}$ & 6.07240800 & -0.97361000 & 0.48869700 & $\mathrm{H}$ & -3.28099000 & -5.44154600 & -4.32195200 \\
\hline $\mathrm{H}$ & 6.07061900 & -1.06757600 & 1.56937400 & $\mathrm{H}$ & -1.89361000 & -4.95625100 & -3.37463100 \\
\hline $\mathrm{Rh}$ & 1.02412200 & 0.55688900 & -0.23615700 & $\mathrm{C}$ & -3.67737000 & -5.41238500 & -2.19745400 \\
\hline $\mathrm{Cl}$ & -0.12272400 & -1.60012300 & -0.75156500 & $\mathrm{H}$ & -4.01325000 & -6.43484800 & -2.41415600 \\
\hline $\mathrm{Rh}$ & -2.44024900 & -1.00945200 & -0.01261000 & $\mathrm{H}$ & -4.59303100 & -4.83979100 & -1.99806900 \\
\hline $\mathrm{C}$ & -5.01589800 & 0.01954400 & 3.60220200 & $\mathrm{C}$ & 0.31642200 & 1.40841300 & -2.06659400 \\
\hline $\mathrm{C}$ & -3.76581100 & -0.35208400 & 2.76913800 & $\mathrm{H}$ & -0.77133100 & 1.39778300 & -2.06461000 \\
\hline $\mathrm{C}$ & -5.83730000 & 1.98601600 & 0.67750800 & $\mathrm{C}$ & 0.99530200 & 2.50467100 & -1.44873000 \\
\hline $\mathrm{C}$ & -4.10502100 & -0.84109200 & 1.37440500 & $\mathrm{H}$ & 1.99105000 & 2.72246400 & -1.84065300 \\
\hline $\mathrm{C}$ & -4.40499500 & 1.49840500 & 0.35239100 & $\mathrm{C}$ & 0.22979800 & 3.72965700 & -0.96171600 \\
\hline $\mathrm{C}$ & -4.35951400 & -0.00983600 & 0.26576000 & $\mathrm{H}$ & 0.81814800 & 4.29275500 & -0.22997900 \\
\hline $\mathrm{H}$ & -5.73717400 & -0.81096800 & 3.57468400 & $\mathrm{H}$ & -0.68794700 & 3.41399400 & -0.45766800 \\
\hline $\mathrm{H}$ & -3.08720100 & 0.50339500 & 2.73884900 & $\mathrm{H}$ & 1.53941600 & 2.08979600 & -0.13215200 \\
\hline $\mathrm{H}$ & -6.47918200 & 1.84029500 & -0.20319300 & $\mathrm{H}$ & 0.78828100 & 0.88044000 & -2.89032100 \\
\hline $\mathrm{H}$ & -4.08676900 & 1.94634300 & -0.59760400 & $\mathrm{C}$ & -0.10684300 & 4.64927700 & -2.14353300 \\
\hline $\mathrm{H}$ & -4.69465400 & 0.10553800 & 4.64935100 & $\mathrm{H}$ & 0.79988600 & 4.91683900 & -2.70495900 \\
\hline $\mathrm{H}$ & -3.22676700 & -1.15232200 & 3.29360300 & $\mathrm{H}$ & -0.76694200 & 4.15189900 & -2.86308400 \\
\hline $\mathrm{H}$ & -5.79042500 & 3.07128800 & 0.84472600 & $\mathrm{C}$ & -0.76037400 & 5.94408600 & -1.69481100 \\
\hline $\mathrm{H}$ & -4.60471600 & -1.80920800 & 1.38557800 & $\mathrm{O}$ & -0.82220100 & 6.34557500 & -0.55294600 \\
\hline $\mathrm{H}$ & -3.71098400 & 1.86528500 & 1.10992700 & $\mathrm{O}$ & -1.26132300 & 6.61856200 & -2.75301200 \\
\hline $\mathrm{H}$ & -5.02990300 & -0.40324700 & -0.49732900 & $\mathrm{C}$ & -1.87626900 & 7.88026900 & -2.44457500 \\
\hline $\mathrm{C}$ & -5.74272200 & 1.32657700 & 3.22167600 & $\mathrm{H}$ & -2.21586500 & 8.28129000 & -3.39998800 \\
\hline $\mathrm{H}$ & -6.45071000 & 1.54078500 & 4.03282100 & $\mathrm{H}$ & -2.72066600 & 7.73993800 & -1.76440600 \\
\hline $\mathrm{H}$ & -5.02389400 & 2.15874400 & 3.23208100 & $\mathrm{H}$ & -1.15455500 & 8.55651000 & -1.97834700 \\
\hline $\mathrm{C}$ & -6.52484000 & 1.31740300 & 1.88440600 & & & & \\
\hline $\mathrm{H}$ & -7.48291700 & 1.83330700 & 2.03028700 & 131 & & & \\
\hline $\mathrm{H}$ & -6.78684300 & 0.28157300 & 1.63048400 & & & & \\
\hline $\mathrm{C}$ & -3.30897600 & -3.36908200 & -3.81166600 & & & & \\
\hline $\mathrm{C}$ & -2.72680000 & -2.27890500 & -2.87999400 & & & & \\
\hline $\mathrm{C}$ & -2.82586200 & -5.48418900 & -0.91428400 & & & & \\
\hline $\mathrm{C}$ & -3.42717000 & -2.20984700 & -1.53727600 & & & & \\
\hline $\mathrm{C}$ & -2.14101600 & -4.16689100 & -0.47847700 & & & & \\
\hline $\mathrm{C}$ & -3.13967000 & -3.03319500 & -0.43132400 & $\mathrm{C}$ & 4.85698300 & -0.296 & -1.71538500 \\
\hline $\mathrm{H}$ & -4.40122600 & -3.25217700 & -3.87715100 & $\mathrm{C}$ & 4.92172900 & -0.40893600 & -0.29269900 \\
\hline $\mathrm{H}$ & -1.65292400 & -2.44024100 & -2.76168900 & $\mathrm{C}$ & 7.23025300 & -1.04933200 & -0.41148000 \\
\hline $\mathrm{H}$ & -3.46733200 & -5.84792700 & -0.09877000 & $\mathrm{C}$ & 7.16226100 & -0.92457500 & -1.80315300 \\
\hline $\mathrm{H}$ & -1.68235100 & -4.31613600 & 0.50698800 & $\mathrm{C}$ & 6.00917000 & -0.555 & -2.47563900 \\
\hline $\mathrm{H}$ & -2.92291900 & -3.17360600 & -4.82142000 & $\mathrm{C}$ & 3.51039300 & 0.07793200 & -2.07141800 \\
\hline $\mathrm{H}$ & -2.83738500 & -1.30570300 & -3.37700500 & $\mathrm{C}$ & 2.79266300 & 0.18740700 & -0.90743000 \\
\hline $\mathrm{H}$ & -2.04003500 & -6.24144500 & -1.04487800 & $\mathrm{H}$ & 8.16661900 & -1.34825600 & 0.04724500 \\
\hline $\mathrm{H}$ & -4.44865500 & -1.84061100 & -1.62206700 & $\mathrm{H}$ & 6.01341100 & -0.48163100 & -3.55845900 \\
\hline $\mathrm{H}$ & -1.32386700 & -3.93859900 & -1.16488700 & $\mathrm{H}$ & 3.13442300 & 0.21660100 & -3.07642000 \\
\hline
\end{tabular}




\begin{tabular}{|c|c|c|c|c|c|c|c|}
\hline $\mathrm{N}$ & 3.64532100 & -0.08599300 & 0.18569700 & $\mathrm{H}$ & -4.11781900 & 1.82226200 & -0.55647000 \\
\hline $\mathrm{P}$ & 2.62046500 & -0.06947000 & 1.59273000 & $\mathrm{H}$ & -4.82357900 & -0.16797100 & 4.62267900 \\
\hline $\mathrm{C}$ & 3.30087200 & 1.27671800 & 2.76808200 & $\mathrm{H}$ & -3.27320800 & -1.34347800 & 3.28591100 \\
\hline $\mathrm{C}$ & 2.68816200 & -1.81198400 & 2.39062800 & $\mathrm{H}$ & -5.90590200 & 2.84853700 & 0.85842500 \\
\hline $\mathrm{C}$ & 4.80779600 & 1.16916200 & 3.07320800 & $\mathrm{H}$ & -4.56324000 & -1.99810500 & 1.31707700 \\
\hline $\mathrm{H}$ & 5.40442200 & 1.24850600 & 2.15992400 & $\mathrm{H}$ & -3.79265600 & 1.71302300 & 1.15946300 \\
\hline $\mathrm{H}$ & 5.09781300 & 2.00099700 & 3.72909400 & $\mathrm{H}$ & -4.97903400 & -0.55878400 & -0.54366200 \\
\hline $\mathrm{H}$ & 5.07831400 & 0.24364500 & 3.58503100 & $\mathrm{C}$ & -5.87303900 & 1.04709500 & 3.19120200 \\
\hline $\mathrm{C}$ & 3.05888000 & 2.63467300 & 2.07558200 & $\mathrm{H}$ & -6.61559400 & 1.21293800 & 3.98253100 \\
\hline $\mathrm{H}$ & 3.46722800 & 3.43455000 & 2.70676400 & $\mathrm{H}$ & -5.18819100 & 1.90547700 & 3.24731200 \\
\hline $\mathrm{H}$ & 3.55931000 & 2.68975100 & 1.10254900 & $\mathrm{C}$ & -6.60905000 & 1.04266800 & 1.82808500 \\
\hline $\mathrm{H}$ & 1.99181100 & 2.83102800 & 1.93492900 & $\mathrm{H}$ & -7.59048400 & 1.51850200 & 1.95330700 \\
\hline $\mathrm{C}$ & 2.47471200 & 1.24619800 & 4.07148400 & $\mathrm{H}$ & -6.82255900 & 0.00448500 & 1.54066500 \\
\hline $\mathrm{H}$ & 1.39795300 & 1.29857300 & 3.87208500 & $\mathrm{C}$ & -3.11194600 & -3.42423700 & -3.83198200 \\
\hline $\mathrm{H}$ & 2.67020300 & 0.35344500 & 4.67241600 & $\mathrm{C}$ & -2.59193600 & -2.32227400 & -2.87754900 \\
\hline $\mathrm{H}$ & 2.73999900 & 2.11723400 & 4.68442400 & $\mathrm{C}$ & -2.51961500 & -5.55729300 & -0.96962100 \\
\hline $\mathrm{C}$ & 2.80504000 & -2.83472800 & 1.23978000 & $\mathrm{C}$ & -3.30158700 & -2.31344600 & -1.53746400 \\
\hline $\mathrm{H}$ & 2.01643400 & -2.71204800 & 0.49454800 & $\mathrm{C}$ & -1.91130900 & -4.21171800 & -0.50619400 \\
\hline $\mathrm{H}$ & 3.77278900 & -2.76916000 & 0.73388600 & $\mathrm{C}$ & -2.97320000 & -3.13799300 & -0.44380900 \\
\hline $\mathrm{H}$ & 2.71318600 & -3.84359900 & 1.66354900 & $\mathrm{H}$ & -4.20855000 & -3.36411600 & -3.89992200 \\
\hline $\mathrm{C}$ & 3.83190000 & -2.07478200 & 3.38738300 & $\mathrm{H}$ & -1.51137400 & -2.43100600 & -2.75746700 \\
\hline $\mathrm{H}$ & 3.79106600 & -1.42565800 & 4.26600900 & $\mathrm{H}$ & -3.13953600 & -5.97213700 & -0.16197400 \\
\hline $\mathrm{H}$ & 3.74889600 & -3.10937000 & 3.74620200 & $\mathrm{H}$ & -1.44870100 & -4.35413000 & 0.47836200 \\
\hline $\mathrm{C}$ & 1.32766600 & -2.00602000 & 3.10039400 & $\mathrm{H}$ & -2.73331300 & -3.19002200 & -4.83620700 \\
\hline $\mathrm{H}$ & 1.27392600 & -3.02885900 & 3.49612400 & $\mathrm{H}$ & -2.74948800 & -1.34726600 & -3.35784300 \\
\hline $\mathrm{H}$ & 1.19413500 & -1.32095300 & 3.94425500 & $\mathrm{H}$ & -1.69173500 & -6.26567600 & -1.11317500 \\
\hline $\mathrm{H}$ & 0.49178200 & -1.86273300 & 2.40937700 & $\mathrm{H}$ & -4.34247400 & -2.00276700 & -1.62255900 \\
\hline $\mathrm{F}$ & 8.28361100 & -1.18142500 & -2.52153200 & $\mathrm{H}$ & -1.10637400 & -3.92504000 & -1.18542200 \\
\hline $\mathrm{Cl}$ & -1.25620300 & 0.90155900 & 1.00791400 & $\mathrm{H}$ & -3.78070300 & -3.39008500 & 0.24278500 \\
\hline $\mathrm{H}$ & 4.81722500 & -1.97291900 & 2.92271600 & $\mathrm{C}$ & -2.71039300 & -4.87700700 & -3.50157800 \\
\hline $\mathrm{C}$ & 6.09665600 & -0.78915500 & 0.35882000 & $\mathrm{H}$ & -2.97592400 & -5.48373400 & -4.37699700 \\
\hline $\mathrm{H}$ & 6.14284300 & -0.88297600 & 1.43829800 & $\mathrm{H}$ & -1.61565100 & -4.94234500 & -3.42077000 \\
\hline $\mathrm{Rh}$ & 0.97103700 & 0.58593700 & -0.18282000 & $\mathrm{C}$ & -3.37324200 & -5.51005100 & -2.25237500 \\
\hline $\mathrm{Cl}$ & -0.02612300 & -1.55277900 & -0.70599500 & $\mathrm{H}$ & -3.65373800 & -6.54547900 & -2.48549100 \\
\hline $\mathrm{Rh}$ & -2.38891500 & -1.08503400 & 0.00919200 & $\mathrm{H}$ & -4.31834700 & -4.99040800 & -2.04531200 \\
\hline $\mathrm{C}$ & -5.10976300 & -0.24087300 & 3.56447900 & $\mathrm{C}$ & 0.27138300 & 1.51351500 & -1.90902400 \\
\hline $\mathrm{C}$ & -3.82172400 & -0.54852200 & 2.76332800 & $\mathrm{H}$ & -0.81825500 & 1.51697100 & -1.93801000 \\
\hline $\mathrm{C}$ & -5.90798400 & 1.76724000 & 0.66195800 & $\mathrm{C}$ & 0.92944900 & 2.66537100 & -1.26298400 \\
\hline $\mathrm{C}$ & -4.09823400 & -1.01300500 & 1.34652500 & $\mathrm{H}$ & 1.86566700 & 2.95616400 & -1.75204500 \\
\hline $\mathrm{C}$ & -4.44904200 & 1.34025000 & 0.37196300 & $\mathrm{C}$ & 0.06996600 & 3.86411700 & -0.84404000 \\
\hline $\mathrm{C}$ & -4.34811200 & -0.16287400 & 0.25100600 & $\mathrm{H}$ & 0.62543000 & 4.51162000 & -0.15691000 \\
\hline $\mathrm{H}$ & -5.79872100 & -1.09618600 & 3.49626700 & $\mathrm{H}$ & -0.80885100 & 3.50553200 & -0.29949400 \\
\hline $\mathrm{H}$ & -3.17192100 & 0.32959700 & 2.77614900 & $\mathrm{H}$ & 0.73239000 & 1.10105400 & -2.80428000 \\
\hline $\mathrm{H}$ & -6.51560300 & 1.62252600 & -0.24288000 & $\mathrm{C}$ & -0.36586600 & 4.68299800 & -2.06333600 \\
\hline
\end{tabular}




\begin{tabular}{|c|c|c|c|c|c|c|c|}
\hline $\mathrm{H}$ & 0.50438100 & 5.01290500 & -2.64855300 & $\mathrm{C}$ & -0.31768400 & -4.05236100 & -1.44653500 \\
\hline $\mathrm{H}$ & -0.97480700 & 4.08383500 & -2.75056300 & $\mathrm{H}$ & -0.68122400 & -4.27471600 & -2.45267000 \\
\hline $\mathrm{C}$ & -1.15291500 & 5.92175700 & -1.67702500 & $\mathrm{H}$ & 0.41338000 & -3.24124000 & -1.51480000 \\
\hline $\mathrm{O}$ & -1.30481300 & 6.34052400 & -0.54984100 & $\mathrm{H}$ & 0.20397200 & -4.94739300 & -1.08311700 \\
\hline $\mathrm{O}$ & -1.66653600 & 6.52361500 & -2.77312500 & $\mathrm{C}$ & -0.84748600 & -3.52842700 & 0.94547800 \\
\hline $\mathrm{C}$ & -2.41366800 & 7.72501300 & -2.52316400 & $\mathrm{H}$ & -1.60206300 & -3.28964700 & 1.70019700 \\
\hline $\mathrm{H}$ & -2.74860800 & 8.07030800 & -3.50176600 & $\mathrm{H}$ & -0.35313300 & -4.46273200 & 1.24061200 \\
\hline $\mathrm{H}$ & -3.26951800 & 7.51803000 & -1.87494400 & $\mathrm{C}$ & -2.46731400 & -4.88048100 & -0.41071900 \\
\hline $\mathrm{H}$ & -1.78220000 & 8.47896200 & -2.04515700 & $\mathrm{H}$ & -1.95021700 & -5.77638800 & -0.04252300 \\
\hline \multirow[t]{2}{*}{$\mathrm{H}$} & 1.40324600 & 2.30636700 & -0.18965100 & $\mathrm{H}$ & -3.29825900 & -4.68014300 & 0.27256400 \\
\hline & & & & $\mathrm{H}$ & -2.87220500 & -5.12764800 & -1.39652300 \\
\hline \multirow[t]{7}{*}{132} & & & & $\mathrm{~F}$ & -6.59145000 & -1.85506200 & 4.65770100 \\
\hline & & & & $\mathrm{Cl}$ & 1.45113600 & -0.61529200 & -1.61433700 \\
\hline & & & & $\mathrm{H}$ & -0.08368600 & -2.74068000 & 0.96200600 \\
\hline & & & & $\mathrm{C}$ & -5.11591900 & -2.69835000 & 1.44201700 \\
\hline & & & & $\mathrm{H}$ & -5.27006900 & -3.40949900 & 0.63866300 \\
\hline & & & & $\mathrm{Rh}$ & -0.68669500 & -0.35180100 & -0.28217000 \\
\hline & & & & $\mathrm{Cl}$ & 0.74993900 & 1.08560900 & 1.05638500 \\
\hline $\mathrm{C}$ & -3.88682000 & -0.82116800 & 2.46785000 & $\mathrm{Rh}$ & 2.94914900 & 0.26836400 & 0.18795400 \\
\hline $\mathrm{C}$ & -4.09338900 & -1.74756800 & 1.39736000 & $\mathrm{C}$ & 5.60905700 & -2.98293000 & -1.60459500 \\
\hline $\mathrm{C}$ & -5.95351500 & -2.72445800 & 2.55708100 & $\mathrm{C}$ & 4.38389900 & -2.32008000 & -0.93113600 \\
\hline $\mathrm{C}$ & -5.75509200 & -1.80451100 & 3.59239000 & $\mathrm{C}$ & 5.76869000 & 0.15366100 & -3.40222500 \\
\hline $\mathrm{C}$ & -4.74766300 & -0.85543600 & 3.57824000 & $\mathrm{C}$ & 4.67277300 & -0.93691700 & -0.38194900 \\
\hline $\mathrm{C}$ & -2.73892400 & -0.01105000 & 2.15148300 & $\mathrm{C}$ & 4.43012700 & 0.28023900 & -2.63488500 \\
\hline $\mathrm{C}$ & -2.26268100 & -0.42030400 & 0.93124400 & $\mathrm{C}$ & 4.66995200 & 0.24796700 & -1.14338300 \\
\hline $\mathrm{H}$ & -6.75914300 & -3.44617400 & 2.63832300 & $\mathrm{H}$ & 6.47098000 & -2.94199900 & -0.92175700 \\
\hline $\mathrm{H}$ & 33800 & -0.17081500 & 4.41279800 & $\mathrm{H}$ & 3.55279200 & -2.29523100 & -1.64033700 \\
\hline $\mathrm{H}$ & -2.31044600 & 0.76107900 & 2.77603700 & $\mathrm{H}$ & 6.34061600 & 1.08593400 & -3.29198900 \\
\hline $\mathrm{N}$ & -3.11032800 & -1.45324200 & 0.44127100 & $\mathrm{H}$ & 3.94204900 & 1.21876500 & -2.92530800 \\
\hline $\mathrm{P}$ & -2.20625500 & -2.01694400 & -0.92347000 & $\mathrm{H}$ & 5.37454100 & -4.04903200 & -1.73039600 \\
\hline $\mathrm{C}$ & -3.39773400 & -2.12264200 & -2.41181600 & $\mathrm{H}$ & 4.05914800 & -2.95997300 & -0.09962300 \\
\hline $\mathrm{C}$ & -1.45963300 & -3.71735700 & -0.46194300 & $\mathrm{H}$ & 5.53128700 & 0.06628000 & -4.47168900 \\
\hline $\mathrm{C}$ & -4.71986100 & -2.83469300 & -2.05954800 & $\mathrm{H}$ & 5.34012100 & -0.95791000 & 0.48000500 \\
\hline $\mathrm{H}$ & -5.28111000 & -2.27944200 & -1.30359800 & $\mathrm{H}$ & 3.75628100 & -0.52180200 & -2.93934500 \\
\hline $\mathrm{H}$ & -5.34032100 & -2.87891300 & -2.96412800 & $\mathrm{H}$ & 5.31747300 & 1.05731000 & -0.80822400 \\
\hline $\mathrm{H}$ & -4.58041400 & -3.85991700 & -1.71063900 & $\mathrm{C}$ & 6.03478900 & -2.42773300 & -2.98028000 \\
\hline $\mathrm{C}$ & -3.74507300 & -0.68291100 & -2.84209200 & $\mathrm{H}$ & 6.75963800 & -3.13962500 & -3.39606600 \\
\hline $\mathrm{H}$ & -4.55151400 & -0.72468200 & -3.58517300 & $\mathrm{H}$ & 5.17414400 & -2.45186500 & -3.66466100 \\
\hline $\mathrm{H}$ & -4.09422200 & -0.07402500 & -2.00286000 & $\mathrm{C}$ & 6.67924400 & -1.01893300 & -2.98778500 \\
\hline $\mathrm{H}$ & -2.89429300 & -0.18126900 & -3.30682100 & $\mathrm{H}$ & 7.53025300 & -1.02161100 & -3.68135700 \\
\hline $\mathrm{C}$ & -2.70551500 & -2.83796000 & -3.58927800 & $\mathrm{H}$ & 7.10976900 & -0.81780500 & -1.99779300 \\
\hline $\mathrm{H}$ & -1.72424700 & -2.40673300 & -3.81699700 & $\mathrm{C}$ & 4.29396500 & 3.62695100 & 2.97451300 \\
\hline $\mathrm{H}$ & -2.58149900 & -3.90982700 & -3.41100100 & $\mathrm{C}$ & 3.55731200 & 2.91223900 & 1.81625200 \\
\hline $\mathrm{H}$ & -3.33015100 & -2.72608700 & -4.48440900 & $\mathrm{C}$ & 3.76025900 & 0.47781200 & 4.67904200 \\
\hline
\end{tabular}




\begin{tabular}{|c|c|c|c|c|c|c|c|}
\hline C & 4.13655600 & 1.55086100 & 1.48503100 & & & & \\
\hline $\mathrm{C}$ & 2.93783500 & 0.29444900 & 3.38072700 & & & & \\
\hline $\mathrm{C}$ & 3.84164400 & 0.35659300 & 2.17108900 & & & & \\
\hline $\mathrm{H}$ & 5.37491600 & 3.64063500 & 2.76943800 & & & & \\
\hline $\mathrm{H}$ & 2.49323300 & 2.84549600 & 2.05792900 & & & & \\
\hline $\mathrm{H}$ & 4.36304600 & -0.42399600 & 4.85823300 & $\mathrm{C}$ & 4.58240300 & 1.75844100 & -1.19903000 \\
\hline $\mathrm{H}$ & 2.41772500 & -0.67070300 & 3.42486500 & $\mathrm{C}$ & 4.96358000 & 0.69939200 & -0.32035000 \\
\hline $\mathrm{H}$ & 3.97184800 & 4.67721000 & 2.96538700 & $\mathrm{C}$ & 7.28005900 & 1.09125600 & -0.81005000 \\
\hline $\mathrm{H}$ & 3.62691400 & 3.54318400 & 0.92032600 & $\mathrm{C}$ & 6.89599600 & 2.12102100 & -1.67594200 \\
\hline $\mathrm{H}$ & 3.05084200 & 0.54201000 & 5.51579500 & $\mathrm{C}$ & 5.57541000 & 2.48051400 & -1.88435800 \\
\hline $\mathrm{H}$ & 5.12743900 & 1.61716200 & 1.03526400 & $\mathrm{C}$ & 3.15404400 & 1.87053100 & -1.16499100 \\
\hline $\mathrm{H}$ & 2.16048000 & 1.05787800 & 3.32740200 & $\mathrm{C}$ & 2.66587600 & 0.92161800 & -0.28386200 \\
\hline $\mathrm{H}$ & 4.61423100 & -0.41098800 & 2.18948300 & $\mathrm{H}$ & 8.33518900 & 0.87469400 & -0.68279800 \\
\hline $\mathrm{C}$ & 4.06042900 & 3.07270700 & 4.39569400 & $\mathrm{H}$ & 5.33309800 & 3.29528100 & -2.55882600 \\
\hline $\mathrm{H}$ & 4.46883600 & 3.81437300 & 5.09436100 & $\mathrm{H}$ & 2.56287500 & 2.59816800 & -1.70266300 \\
\hline $\mathrm{H}$ & 2.98044000 & 3.04093600 & 4.60017600 & $\mathrm{~N}$ & 3.78480300 & 0.19087100 & 0.25181800 \\
\hline $\mathrm{C}$ & 4.70059500 & 1.69862800 & 4.71657700 & $\mathrm{P}$ & 3.04070800 & -1.36791600 & 0.54675200 \\
\hline $\mathrm{H}$ & 5.13524700 & 1.73494200 & 5.72398900 & $\mathrm{C}$ & 3.49445600 & -1.85826500 & 2.33746700 \\
\hline $\mathrm{H}$ & 5.54738100 & 1.53055500 & 4.03801700 & $\mathrm{C}$ & 3.72238500 & -2.62192800 & -0.73665800 \\
\hline $\mathrm{C}$ & -3.84821300 & 5.59447200 & -2.02134100 & $\mathrm{C}$ & 4.97897100 & -1.60342900 & 2.66204400 \\
\hline $\mathrm{O}$ & -3.55049800 & 5.69389800 & -3.19245300 & $\mathrm{H}$ & 5.23938100 & -0.54852300 & 2.53149700 \\
\hline $\mathrm{O}$ & -4.53132700 & 6.54893500 & -1.34550900 & $\mathrm{H}$ & 5.16363000 & -1.86184800 & 3.71308700 \\
\hline $\mathrm{C}$ & -4.90367300 & 7.70103400 & -2.11623300 & $\mathrm{H}$ & 5.65651000 & -2.20742700 & 2.05342700 \\
\hline $\mathrm{H}$ & -5.55272400 & 7.41580700 & -2.94901500 & $\mathrm{C}$ & 2.63508900 & -0.98586400 & 3.27535100 \\
\hline $\mathrm{H}$ & -5.43441000 & 8.35729900 & -1.42540800 & $\mathrm{H}$ & 2.84829900 & -1.26612500 & 4.31506000 \\
\hline $\mathrm{H}$ & -4.01706900 & 8.20101800 & -2.51590000 & $\mathrm{H}$ & 2.86724200 & 0.07669800 & 3.16578400 \\
\hline $\mathrm{C}$ & -2.52306700 & 3.44204900 & -1.72879600 & $\mathrm{H}$ & 1.56535800 & -1.12607600 & 3.09319000 \\
\hline $\mathrm{H}$ & -1.57272500 & 3.96719200 & -1.88884500 & $\mathrm{C}$ & 3.13265900 & -3.33543200 & 2.59108800 \\
\hline $\mathrm{C}$ & -1.24685200 & 1.28003700 & -1.42308800 & $\mathrm{H}$ & 2.09387800 & -3.55582500 & 2.32096000 \\
\hline $\mathrm{H}$ & -1.53573900 & 0.89345200 & -2.40149400 & $\mathrm{H}$ & 3.78563200 & -4.02625200 & 2.05137600 \\
\hline $\mathrm{H}$ & -0.28341800 & 1.78883300 & -1.52190900 & $\mathrm{H}$ & 3.24736600 & -3.54758100 & 3.66175800 \\
\hline $\mathrm{H}$ & -3.24819100 & 1.68255500 & -0.69184400 & $\mathrm{C}$ & 2.79829700 & -3.86047300 & -0.77086200 \\
\hline $\mathrm{C}$ & -3.54085900 & 4.41244500 & -1.12259400 & $\mathrm{H}$ & 2.83096800 & -4.44331000 & 0.15281600 \\
\hline $\mathrm{H}$ & -4.49240000 & 3.89894700 & -0.92136400 & $\mathrm{H}$ & 1.75961400 & -3.58953600 & -0.97212800 \\
\hline $\mathrm{H}$ & -3.20255900 & 4.79326700 & -0.15119600 & $\mathrm{H}$ & 3.13814200 & -4.51615100 & -1.58325200 \\
\hline $\mathrm{C}$ & -2.29597200 & 2.20872000 & -0.83197600 & $\mathrm{C}$ & 3.63691400 & -1.92795100 & -2.11487300 \\
\hline $\mathrm{H}$ & -1.97633200 & 2.53693600 & 0.16426500 & $\mathrm{H}$ & 4.29804200 & -1.06023200 & -2.18733900 \\
\hline \multirow[t]{2}{*}{$\mathrm{H}$} & -2.86996800 & 3.13290400 & -2.72214200 & $\mathrm{H}$ & 3.94452100 & -2.64919600 & -2.88309600 \\
\hline & & & & $\mathrm{C}$ & 5.17368300 & -3.07954100 & -0.49094900 \\
\hline \multirow[t]{5}{*}{ 133-ts } & & & & $\mathrm{H}$ & 5.46662600 & -3.75043600 & -1.30914800 \\
\hline & & & & $\mathrm{H}$ & 5.87887300 & -2.24512900 & -0.48765100 \\
\hline & & & & $\mathrm{H}$ & 5.28777200 & -3.64314200 & 0.43991800 \\
\hline & & & & $\mathrm{F}$ & 7.86871200 & 2.80007300 & -2.32875900 \\
\hline & & & & $\mathrm{Cl}$ & -0.41876700 & -2.26001500 & -0.33682200 \\
\hline
\end{tabular}




\begin{tabular}{|c|c|c|c|c|c|c|c|}
\hline $\mathrm{H}$ & 2.61687300 & -1.60324100 & -2.34069600 & $\mathrm{C}$ & -5.11474300 & 3.45291400 & -1.67705700 \\
\hline $\mathrm{C}$ & 6.30494600 & 0.37312100 & -0.11547100 & $\mathrm{H}$ & -5.76978300 & 4.33297000 & -1.63228200 \\
\hline $\mathrm{H}$ & 6.59980800 & -0.40654000 & 0.57750400 & $\mathrm{H}$ & -4.17230000 & 3.81083800 & -2.11757700 \\
\hline $\mathrm{Rh}$ & 1.08634600 & -0.35699900 & -0.07475100 & $\mathrm{C}$ & -5.77052000 & 2.40733200 & -2.61380200 \\
\hline $\mathrm{Cl}$ & -0.76237700 & 0.75555800 & -1.28871800 & $\mathrm{H}$ & -6.52430600 & 2.90839000 & -3.23521600 \\
\hline $\mathrm{Rh}$ & -2.50321100 & -0.88643100 & -0.59547100 & $\mathrm{H}$ & -6.32709100 & 1.68293900 & -2.00454500 \\
\hline $\mathrm{C}$ & -4.22308500 & -5.11460100 & -0.55830400 & $\mathrm{C}$ & -1.75157700 & 5.30239500 & 2.03548600 \\
\hline $\mathrm{C}$ & -3.35496900 & -3.90724800 & -0.98548600 & $\mathrm{O}$ & -2.65705900 & 4.57673100 & 2.39394500 \\
\hline $\mathrm{C}$ & -3.78122100 & -3.70497000 & 2.74527700 & $\mathrm{O}$ & -1.72621800 & 6.63027600 & 2.28676600 \\
\hline $\mathrm{C}$ & -3.86376300 & -2.57944300 & -0.45809700 & $\mathrm{C}$ & -2.86475500 & 7.14505600 & 2.99581400 \\
\hline $\mathrm{C}$ & -2.83682400 & -2.82944200 & 1.88589700 & $\mathrm{H}$ & -2.95724200 & 6.66570500 & 3.97411400 \\
\hline $\mathrm{C}$ & -3.61849800 & -2.07564900 & 0.83483600 & $\mathrm{H}$ & -2.68099500 & 8.21406900 & 3.10802300 \\
\hline $\mathrm{H}$ & -5.27829000 & -4.91069000 & -0.79425300 & $\mathrm{H}$ & -3.78321500 & 6.97018700 & 2.42872300 \\
\hline $\mathrm{H}$ & -2.32091300 & -4.08589300 & -0.67985200 & $\mathrm{C}$ & -0.04850400 & 3.46431400 & 1.63415800 \\
\hline $\mathrm{H}$ & -4.37746300 & -3.05704200 & 3.40349600 & $\mathrm{H}$ & -0.90902100 & 2.78733800 & 1.65892300 \\
\hline $\mathrm{H}$ & -2.30733500 & -2.12964400 & 2.54498600 & $\mathrm{C}$ & 1.51168100 & 1.54314100 & 1.09633000 \\
\hline $\mathrm{H}$ & -3.93172600 & -5.96534200 & -1.18967400 & $\mathrm{H}$ & 2.34196500 & 1.61839400 & 1.79683100 \\
\hline $\mathrm{H}$ & -3.34362800 & -3.86048000 & -2.08239800 & $\mathrm{H}$ & 0.70991800 & 0.99668400 & 1.62216000 \\
\hline $\mathrm{H}$ & -3.15556000 & -4.31953600 & 3.40792800 & $\mathrm{H}$ & 1.85550000 & 3.60836300 & 0.58567100 \\
\hline $\mathrm{H}$ & -4.79977200 & -2.27290600 & -0.92621900 & $\mathrm{C}$ & -0.53928700 & 4.86347300 & 1.23732900 \\
\hline $\mathrm{H}$ & -2.07138400 & -3.45532500 & 1.42471500 & $\mathrm{H}$ & 0.24974800 & 5.61639600 & 1.32987600 \\
\hline $\mathrm{H}$ & -4.37081300 & -1.41624000 & 1.26540800 & $\mathrm{H}$ & -0.84566200 & 4.85609300 & 0.18082200 \\
\hline $\mathrm{C}$ & -4.11743200 & -5.56185800 & 0.91480800 & $\mathrm{C}$ & 1.00552700 & 2.91823900 & 0.65892500 \\
\hline $\mathrm{H}$ & -4.61336400 & -6.53876400 & 0.98433100 & $\mathrm{H}$ & 0.55425200 & 2.85946900 & -0.33580000 \\
\hline $\mathrm{H}$ & -3.06239300 & -5.75011900 & 1.16245500 & $\mathrm{H}$ & 0.35803800 & 3.49763500 & 2.65481800 \\
\hline $\mathrm{C}$ & -4.74940900 & -4.62012100 & 1.97038200 & & & & \\
\hline $\mathrm{H}$ & -5.27901800 & -5.22714400 & 2.71626400 & 134 & & & \\
\hline $\mathrm{H}$ & -5.52306300 & -4.00961200 & 1.48649800 & & & & \\
\hline $\mathrm{C}$ & -4.86361600 & 3.01089000 & -0.22020200 & & & & \\
\hline $\mathrm{C}$ & -3.74507600 & 1.96734100 & 0.01193400 & & & & \\
\hline $\mathrm{C}$ & -4.82764100 & 1.65586300 & -3.57432300 & & & & \\
\hline $\mathrm{C}$ & -4.12013400 & 0.56857700 & -0.43581200 & & & & \\
\hline $\mathrm{C}$ & -3.60648600 & 0.96218300 & -2.92396300 & $\mathrm{C}$ & 5.67650000 & 1.67087700 & 0.69983600 \\
\hline $\mathrm{C}$ & -4.04218700 & 0.09965400 & -1.76221000 & $\mathrm{C}$ & 5.29032900 & 0.31805600 & 0.48506700 \\
\hline $\mathrm{H}$ & -5.80778800 & 2.63892600 & 0.20572500 & $\mathrm{C}$ & 7.61167500 & -0.32662000 & 0.58853500 \\
\hline $\mathrm{H}$ & -2.83169700 & 2.30591500 & -0.48338100 & $\mathrm{C}$ & 7.96607500 & 1.01222400 & 0.78629500 \\
\hline $\mathrm{H}$ & -5.42057700 & 0.90917300 & -4.12168400 & $\mathrm{C}$ & 7.02807500 & 2.02278700 & 0.85188200 \\
\hline $\mathrm{H}$ & -3.09675900 & 0.35637900 & -3.68328200 & $\mathrm{C}$ & 4.48043200 & 2.44314200 & 0.73713700 \\
\hline $\mathrm{H}$ & -4.59442300 & 3.89752200 & 0.36815400 & $\mathrm{C}$ & 3.42141000 & 1.60042900 & 0.54246800 \\
\hline $\mathrm{H}$ & -3.51919800 & 1.94751800 & 1.08505800 & $\mathrm{H}$ & 8.39064300 & -1.08095800 & 0.56179800 \\
\hline $\mathrm{H}$ & -4.44803100 & 2.35740900 & -4.33050800 & $\mathrm{H}$ & 7.33650700 & 3.04941300 & 1.01992700 \\
\hline $\mathrm{H}$ & -4.88925000 & 0.11770800 & 0.19153000 & $\mathrm{H}$ & 4.40681500 & 3.50672800 & 0.92276200 \\
\hline $\mathrm{H}$ & -2.88756000 & 1.71593900 & -2.59902000 & $\mathrm{~N}$ & 3.87614600 & 0.27196800 & 0.37714600 \\
\hline $\mathrm{H}$ & -4.74105100 & -0.68177200 & -2.05915800 & $\mathrm{P}$ & 2.76382600 & -1.02217600 & -0.17320900 \\
\hline
\end{tabular}




\begin{tabular}{|c|c|c|c|c|c|c|c|}
\hline C & 2.79560300 & -2.33490800 & 23213800 & $\mathrm{H}$ & -3.97963900 & -2.95559400 & -2.48746000 \\
\hline $\mathrm{C}$ & 3.50131700 & -1.65502000 & -1.85469300 & $\mathrm{H}$ & -2.06164300 & -5.73174900 & 1.87805800 \\
\hline $\mathrm{C}$ & 4.16825300 & -2.79922400 & 1.74899000 & $\mathrm{H}$ & -4.96645600 & -2.24191900 & -0.36533000 \\
\hline $\mathrm{H}$ & 4.75203800 & -1.97893500 & 2.17284500 & $\mathrm{H}$ & -1.66135200 & -3.97585900 & 0.26133600 \\
\hline $\mathrm{H}$ & 4.00075300 & -3.52915600 & 2.55202600 & $\mathrm{H}$ & -3.83254800 & -2.37409000 & 1.73893100 \\
\hline $\mathrm{H}$ & 4.76432700 & -3.30157300 & 0.98215500 & $\mathrm{C}$ & -3.79058800 & -5.87697200 & -0.50649700 \\
\hline $\mathrm{C}$ & 2.03934000 & -1.68178000 & 2.41071900 & $\mathrm{H}$ & -4.25925800 & -6.83986800 & -0.74780700 \\
\hline $\mathrm{H}$ & 1.96429400 & -2.40743700 & 3.23143100 & $\mathrm{H}$ & -2.71580000 & -6.01953300 & -0.69149100 \\
\hline $\mathrm{H}$ & 2.57080000 & -0.80237800 & 2.79227700 & $\mathrm{C}$ & -4.03481400 & -5.58094900 & 0.99443900 \\
\hline $\mathrm{H}$ & 1.03030000 & -1.37471100 & 2.11960300 & $\mathrm{H}$ & -4.30690100 & -6.51554700 & 1.50202800 \\
\hline C & 2.00391800 & -3.56860800 & 0.74821100 & $\mathrm{H}$ & -4.91237300 & -4.92806400 & 1.09233800 \\
\hline $\mathrm{H}$ & 1.00152100 & -3.30765500 & 0.40234100 & $\mathrm{C}$ & -4.28729900 & 2.21248300 & 2.80475600 \\
\hline $\mathrm{H}$ & 2.51991600 & -4.10345700 & -0.05618200 & $\mathrm{C}$ & -3.27933600 & 1.32282900 & 2.03860600 \\
\hline $\mathrm{H}$ & 1.90104600 & -4.26765800 & 1.58857500 & $\mathrm{C}$ & -5.88630100 & 2.40586400 & -0.43384100 \\
\hline $\mathrm{C}$ & 2.33097100 & -2.25228100 & -2.66759400 & $\mathrm{C}$ & -3.94531900 & 0.20982500 & 1.25414000 \\
\hline $\mathrm{H}$ & 1.88289300 & -3.12700000 & -2.18775300 & $\mathrm{C}$ & -4.56355000 & 1.67334000 & -0.76367100 \\
\hline $\mathrm{H}$ & 1.53879000 & -1.52132800 & -2.83785100 & $\mathrm{C}$ & -4.49384800 & 0.35053200 & -0.03511800 \\
\hline $\mathrm{H}$ & 2.72193200 & -2.57109200 & -3.64271800 & $\mathrm{H}$ & -4.96198700 & 1.57779300 & 3.39946200 \\
\hline $\mathrm{C}$ & 4.01427400 & -0.41102900 & -2.61093100 & $\mathrm{H}$ & -2.66749100 & 1.95545900 & 1.39190100 \\
\hline $\mathrm{H}$ & 4.89398900 & 0.03446700 & -2.13915400 & $\mathrm{H}$ & -6.72279100 & 1.88538700 & -0.92234200 \\
\hline $\mathrm{H}$ & 4.29346200 & -0.71114400 & -3.62921300 & $\mathrm{H}$ & -4.50557900 & 1.51805300 & -1.84843700 \\
\hline $\mathrm{C}$ & 4.61163600 & -2.72252400 & -1.79532900 & $\mathrm{H}$ & -3.71084400 & 2.80429900 & 3.52858400 \\
\hline $\mathrm{H}$ & 4.81310100 & -3.06202600 & -2.81960800 & $\mathrm{H}$ & -2.59427800 & 0.86741100 & 2.76661000 \\
\hline $\mathrm{H}$ & 5.55188800 & -2.33869000 & -1.40219800 & $\mathrm{H}$ & -5.83567600 & 3.40231500 & -0.89500200 \\
\hline $\mathrm{H}$ & 4.31845600 & -3.60541600 & -1.21980100 & $\mathrm{H}$ & -4.39611400 & -0.54418300 & 1.89883200 \\
\hline $\mathrm{F}$ & 9.27718700 & 1.31500800 & 0.92744900 & $\mathrm{H}$ & -3.72072200 & 2.31243200 & -0.49571900 \\
\hline $\mathrm{Cl}$ & -0.59972600 & -1.88096800 & -1.12395000 & $\mathrm{H}$ & -5.31215700 & -0.31854900 & -0.30051400 \\
\hline $\mathrm{H}$ & 3.23568600 & 0.35544000 & -2.69299500 & $\mathrm{C}$ & -5.12864400 & 3.19164000 & 1.95921500 \\
\hline $\mathrm{C}$ & 6.27279800 & -0.67953500 & 0.44177800 & $\mathrm{H}$ & -5.61868200 & 3.87362000 & 2.66676700 \\
\hline $\mathrm{H}$ & 6.02798400 & -1.72172400 & 0.32249300 & $\mathrm{H}$ & -4.45655000 & 3.81990100 & 1.35943300 \\
\hline $\mathrm{Rh}$ & 0.74951500 & -0.03040300 & -0.42885300 & $\mathrm{C}$ & -6.22342300 & 2.55908900 & 1.06299000 \\
\hline $\mathrm{Cl}$ & -1.35519300 & 1.13582300 & -0.86877300 & $\mathrm{H}$ & -7.13135500 & 3.17388200 & 1.12181200 \\
\hline $\mathrm{Rh}$ & -2.66991600 & -0.84272400 & -0.16217400 & $\mathrm{H}$ & -6.50637600 & 1.58147200 & 1.47670600 \\
\hline $\mathrm{C}$ & -4.34977800 & -4.84888300 & -1.51290500 & $\mathrm{C}$ & -1.56665300 & 5.44766300 & -0.57261700 \\
\hline $\mathrm{C}$ & -3.64086000 & -3.47473900 & -1.58148700 & $\mathrm{O}$ & -2.27740100 & 5.05481500 & 0.33017000 \\
\hline $\mathrm{C}$ & -2.85203700 & -4.97458200 & 1.77592800 & $\mathrm{O}$ & -1.87104300 & 6.51682100 & -1.34146700 \\
\hline C & -3.93354900 & -2.58931300 & -0.38492800 & $\mathrm{C}$ & -3.12395700 & 7.15358600 & -1.04654200 \\
\hline $\mathrm{C}$ & -2.21381200 & -3.70654800 & 1.16307700 & $\mathrm{H}$ & -3.14020300 & 7.51250700 & -0.01393400 \\
\hline $\mathrm{C}$ & -3.26778400 & -2.66789700 & 0.85494000 & $\mathrm{H}$ & -3.19973800 & 7.98830600 & -1.74420500 \\
\hline $\mathrm{H}$ & -5.42069700 & -4.69843400 & -1.31019500 & $\mathrm{H}$ & -3.95402400 & 6.45645100 & -1.19182600 \\
\hline $\mathrm{H}$ & -2.56604400 & -3.63284200 & -1.70612500 & $\mathrm{C}$ & 0.37252200 & 3.95230200 & 0.08191200 \\
\hline $\mathrm{H}$ & -3.19467400 & -4.75226700 & 2.79669500 & $\mathrm{H}$ & -0.39417000 & 3.27852400 & 0.47074500 \\
\hline $\mathrm{H}$ & -1.47636400 & -3.30627800 & 1.86997300 & $\mathrm{C}$ & 1.98857300 & 2.05540400 & 0.60973500 \\
\hline $\mathrm{H}$ & -4.29214800 & -5.29486700 & -2.51531500 & $\mathrm{H}$ & 1.85633900 & 2.49699400 & 1.61056200 \\
\hline
\end{tabular}




\begin{tabular}{|c|c|c|c|c|c|c|c|}
\hline $\mathrm{H}$ & 1.22496400 & 1.21820600 & 0.76046100 & $\mathrm{H}$ & -4.03476800 & -0.15547500 & -2.50055600 \\
\hline $\mathrm{H}$ & 2.43707800 & 3.80694800 & -0.56835600 & $\mathrm{H}$ & -3.58026500 & 0.85412600 & -3.88107100 \\
\hline $\mathrm{C}$ & -0.26379600 & 4.80926800 & -1.01436400 & $\mathrm{C}$ & -4.81745800 & 2.48424000 & -2.10709800 \\
\hline $\mathrm{H}$ & 0.41095500 & 5.59099400 & -1.38147900 & $\mathrm{H}$ & -4.93512400 & 3.42271000 & -1.55827200 \\
\hline $\mathrm{H}$ & -0.50530700 & 4.17695600 & -1.88097200 & $\mathrm{H}$ & -5.02757700 & 2.70049500 & -3.16284500 \\
\hline $\mathrm{C}$ & 1.57881100 & 3.14086600 & -0.41371900 & $\mathrm{C}$ & -2.37604300 & 2.89695700 & -2.60193000 \\
\hline $\mathrm{H}$ & 1.36136900 & 2.67959400 & -1.38254700 & $\mathrm{H}$ & -2.61403500 & 3.02823300 & -3.66558100 \\
\hline \multirow[t]{2}{*}{$\mathrm{H}$} & 0.68325200 & 4.59249400 & 68000 & $\mathrm{H}$ & -2.41740700 & 3.88398000 & -2.13666100 \\
\hline & & & & $\mathrm{H}$ & -1.35082100 & 2.52429600 & -2.53252500 \\
\hline \multirow[t]{7}{*}{ 135-ts } & & & & $\mathrm{F}$ & -8.03038400 & -3.25601500 & -0.90471400 \\
\hline & & & & $\mathrm{Cl}$ & 0.70307400 & 1.68107800 & -0.97895700 \\
\hline & & & & $\mathrm{H}$ & -5.57761800 & 1.77898700 & -1.76275400 \\
\hline & & & & $\mathrm{C}$ & -6.26569700 & -0.17744400 & -0.10220100 \\
\hline & & & & $\mathrm{H}$ & -6.49228300 & 0.86065200 & 0.11384500 \\
\hline & & & & $\mathrm{Rh}$ & -1.01149700 & 0.12574600 & -0.16324200 \\
\hline & & & & $\mathrm{Cl}$ & 0.71258100 & -1.50272200 & -1.01577400 \\
\hline $\mathrm{C}$ & -4.66413700 & -2.03716600 & -0.31410800 & $\mathrm{Rh}$ & 2.60625600 & 0.06815600 & -0.72207400 \\
\hline $\mathrm{C}$ & -4.95581600 & -0.65847800 & -0.07685900 & $\mathrm{C}$ & 4.81823200 & 4.04174100 & -1.24074900 \\
\hline $\mathrm{C}$ & -7.29844900 & -1.07099700 & -0.39128000 & $\mathrm{C}$ & 3.83940600 & 2.88333200 & -1.54723900 \\
\hline $\mathrm{C}$ & -7.00237800 & -2.41740600 & -0.63295400 & $\mathrm{C}$ & 3.99248300 & 3.24856400 & 2.19159400 \\
\hline $\mathrm{C}$ & -5.71608800 & -2.92750700 & -0.59558200 & $\mathrm{C}$ & 4.15194100 & 1.60877300 & -0.78689300 \\
\hline $\mathrm{C}$ & -3.24917200 & -2.21912300 & -0.17868600 & $\mathrm{C}$ & 3.01795700 & 2.34006400 & 1.40434200 \\
\hline $\mathrm{C}$ & -2.68004400 & -0.99841700 & 0.14827900 & $\mathrm{C}$ & 3.78126700 & 1.35484800 & 0.54885100 \\
\hline $\mathrm{H}$ & -8.33255900 & -0.74512300 & -0.42043500 & $\mathrm{H}$ & 5.85424200 & 3.68789200 & -1.35220900 \\
\hline $\mathrm{H}$ & -5.54387000 & -3.98302100 & -0.77952200 & $\mathrm{H}$ & 2.81779100 & 3.21944400 & -1.35278200 \\
\hline $\mathrm{H}$ & -2.71711200 & -3.15424800 & -0.28619800 & $\mathrm{H}$ & 4.46364600 & 2.66499600 & 2.99579900 \\
\hline $\mathrm{N}$ & -3.73879200 & -0.02432400 & 0.21855200 & $\mathrm{H}$ & 2.37339700 & 1.80998900 & 2.11682000 \\
\hline $\mathrm{P}$ & -2.86045500 & 1.42809300 & -0.21846800 & $\mathrm{H}$ & 4.67490900 & 4.80444400 & -2.01873200 \\
\hline $\mathrm{C}$ & -3.27325100 & 2.81528800 & 1.03811600 & $\mathrm{H}$ & 3.88790000 & 2.66443400 & -2.62214700 \\
\hline $\mathrm{C}$ & -3.39431900 & 1.89966400 & -2.00368700 & $\mathrm{H}$ & 3.39553600 & 4.02501600 & 2.69020300 \\
\hline $\mathrm{C}$ & -4.75286100 & 2.78618300 & 1.46520800 & $\mathrm{H}$ & 5.06848200 & 1.12742900 & -1.13183600 \\
\hline $\mathrm{H}$ & -5.02873800 & 1.82018700 & 1.89939900 & $\mathrm{H}$ & 2.35743100 & 2.95368000 & 0.78926100 \\
\hline $\mathrm{H}$ & -4.92171100 & 3.55524200 & 2.23044600 & $\mathrm{H}$ & 4.43113300 & 0.70306400 & 1.13354900 \\
\hline $\mathrm{H}$ & -5.43263600 & 2.99800400 & 0.63416800 & $\mathrm{C}$ & 4.67148100 & 4.73485700 & 0.13027400 \\
\hline $\mathrm{C}$ & -2.37527100 & 2.60013100 & 2.27545000 & $\mathrm{H}$ & 5.27838700 & 5.64873700 & 0.08798600 \\
\hline $\mathrm{H}$ & -2.55270800 & 3.41901700 & 2.98514700 & $\mathrm{H}$ & 3.63387900 & 5.07764000 & 0.25537300 \\
\hline $\mathrm{H}$ & -2.59128300 & 1.66521000 & 2.79375800 & $\mathrm{C}$ & 5.11232800 & 3.91823400 & 1.37075700 \\
\hline $\mathrm{H}$ & -1.31441000 & 2.59966600 & 2.00845200 & $\mathrm{H}$ & 5.65572300 & 4.58136200 & 2.05674500 \\
\hline $\mathrm{C}$ & -2.91382700 & 4.19766800 & 0.45415100 & $\mathrm{H}$ & 5.84198100 & 3.15946500 & 1.05779000 \\
\hline $\mathrm{H}$ & -1.87317100 & 4.23999600 & 0.11536500 & $\mathrm{C}$ & 4.61234500 & -3.92996200 & 0.27307800 \\
\hline $\mathrm{H}$ & -3.56240600 & 4.49498900 & -0.37300200 & $\mathrm{C}$ & 3.58957300 & -2.77155100 & 0.34962300 \\
\hline $\mathrm{H}$ & -3.03101500 & 4.94839900 & 1.24591400 & $\mathrm{C}$ & 4.63527400 & -3.10797400 & -3.24639200 \\
\hline $\mathrm{C}$ & -3.32632000 & 0.60316800 & -2.84298400 & $\mathrm{C}$ & 4.07544500 & -1.48950900 & -0.29869600 \\
\hline $\mathrm{H}$ & -2.32372200 & 0.16652300 & -2.83187500 & $\mathrm{C}$ & 3.49705500 & -2.21327400 & -2.69929200 \\
\hline
\end{tabular}




\begin{tabular}{|c|c|c|c|c|c|c|c|}
\hline $\mathrm{C}$ & 4.03397100 & -1.23064800 & -1.68442700 & $\mathrm{C}$ & -3.26170600 & 0.25444500 & 1.96019200 \\
\hline $\mathrm{H}$ & 5.59315800 & -3.58042700 & 0.63001800 & $\mathrm{C}$ & -4.12565900 & -0.58435500 & 1.22168100 \\
\hline $\mathrm{H}$ & 2.64388700 & -3.09813800 & -0.08990000 & $\mathrm{C}$ & -5.35858600 & -1.10768600 & 3.20901500 \\
\hline $\mathrm{H}$ & 5.28303800 & -2.51044100 & -3.90348500 & $\mathrm{C}$ & -4.48758800 & -0.29103900 & 3.92746200 \\
\hline $\mathrm{H}$ & 3.03156100 & -1.68163500 & -3.53851400 & $\mathrm{C}$ & -3.43651200 & 0.40767100 & 3.33445200 \\
\hline $\mathrm{H}$ & 4.28743800 & -4.69795800 & 0.98867800 & $\mathrm{C}$ & -2.36590900 & 0.91501000 & 1.00442800 \\
\hline $\mathrm{H}$ & 3.37663500 & -2.57100800 & 1.40690600 & $\mathrm{C}$ & -2.84074800 & 0.62395000 & -0.31029300 \\
\hline $\mathrm{H}$ & 4.17651600 & -3.87496700 & -3.88591600 & $\mathrm{H}$ & -6.17527400 & -1.59627100 & 3.72967000 \\
\hline $\mathrm{H}$ & 4.88957100 & -1.01897600 & 0.25457400 & $\mathrm{H}$ & -2.80144400 & 1.04898900 & 3.93730800 \\
\hline $\mathrm{H}$ & 2.71668700 & -2.83766700 & -2.26107800 & $\mathrm{H}$ & -1.87730000 & 1.84891300 & 1.25863300 \\
\hline $\mathrm{H}$ & 4.80107400 & -0.57738900 & -2.09828800 & $\mathrm{~N}$ & -3.75045800 & -0.59494600 & -0.16357600 \\
\hline $\mathrm{C}$ & 4.79500800 & -4.61418800 & -1.09823000 & $\mathrm{P}$ & -2.46776400 & -1.81007300 & -0.53218100 \\
\hline $\mathrm{H}$ & 5.36829200 & -5.53286600 & -0.91744800 & $\mathrm{C}$ & -3.06944100 & -2.47902100 & -2.23284300 \\
\hline $\mathrm{H}$ & 3.81527800 & -4.94783900 & -1.47029400 & $\mathrm{C}$ & -2.26771600 & -3.27549700 & 0.72150700 \\
\hline $\mathrm{C}$ & 5.52512000 & -3.79511600 & -2.19131600 & $\mathrm{C}$ & -4.56091800 & -2.86909600 & -2.17070300 \\
\hline $\mathrm{H}$ & 6.20872400 & -4.45957500 & -2.73578400 & $\mathrm{H}$ & -5.16713500 & -2.04156700 & -1.79182400 \\
\hline $\mathrm{H}$ & 6.16748200 & -3.04716600 & -1.70745900 & $\mathrm{H}$ & -4.90587000 & -3.10631300 & -3.18578700 \\
\hline $\mathrm{C}$ & -1.59852800 & -1.02386500 & 1.83288200 & $\mathrm{H}$ & -4.74529500 & -3.74892900 & -1.54998600 \\
\hline $\mathrm{H}$ & -0.91536300 & -0.20778100 & 2.08585900 & $\mathrm{C}$ & -2.91717300 & -1.33581300 & -3.25737900 \\
\hline $\mathrm{C}$ & -0.86077800 & -2.36252500 & 1.89944100 & $\mathrm{H}$ & -3.16160000 & -1.72548200 & -4.25422500 \\
\hline $\mathrm{H}$ & -1.57932900 & -3.18099100 & 1.77254100 & $\mathrm{H}$ & -3.61087400 & -0.51938700 & -3.04880200 \\
\hline $\mathrm{H}$ & -0.13177200 & -2.44867600 & 1.09455600 & $\mathrm{H}$ & -1.89846700 & -0.93671700 & -3.28516900 \\
\hline $\mathrm{C}$ & -2.78065000 & -0.99372500 & 2.80279500 & $\mathrm{C}$ & -2.22782700 & -3.66929400 & -2.73175500 \\
\hline $\mathrm{H}$ & -2.39394800 & -0.93304000 & 3.82890500 & $\mathrm{H}$ & -1.15678600 & -3.44185300 & -2.74397900 \\
\hline $\mathrm{H}$ & -3.44167700 & -0.14185600 & 2.65325500 & $\mathrm{H}$ & -2.39026300 & -4.57643500 & -2.14430800 \\
\hline $\mathrm{H}$ & -3.38821900 & -1.90071400 & 2.71080600 & $\mathrm{H}$ & -2.52841000 & -3.89680000 & -3.76252900 \\
\hline $\mathrm{C}$ & -0.13291800 & -2.61093800 & 3.24813400 & $\mathrm{C}$ & -2.01976700 & -2.73811100 & 2.14905100 \\
\hline $\mathrm{H}$ & -0.85125500 & -2.58610100 & 4.07705300 & $\mathrm{H}$ & -1.27948700 & -1.93274900 & 2.15026000 \\
\hline $\mathrm{H}$ & 0.30705100 & -3.61176600 & 3.23347500 & $\mathrm{H}$ & -2.92682800 & -2.38467500 & 2.63706600 \\
\hline $\mathrm{C}$ & 0.95117600 & -1.60420400 & 3.58270100 & $\mathrm{H}$ & -1.62163700 & -3.56499400 & 2.75189400 \\
\hline $\mathrm{O}$ & 0.80291600 & -0.39865600 & 3.64681300 & $\mathrm{C}$ & -3.42864000 & -4.28576700 & 0.75313700 \\
\hline $\mathrm{O}$ & 2.12996800 & -2.21053900 & 3.84935200 & $\mathrm{H}$ & -3.53548400 & -4.83444100 & -0.18638800 \\
\hline $\mathrm{C}$ & 3.20092500 & -1.33401400 & 4.23989100 & $\mathrm{H}$ & -3.22474600 & -5.02706100 & 1.53728500 \\
\hline $\mathrm{H}$ & 2.91356200 & -0.74497200 & 5.11480100 & $\mathrm{C}$ & -0.95673700 & -3.99780000 & 0.31229300 \\
\hline $\mathrm{H}$ & 4.04166800 & -1.98757600 & 4.47427100 & $\mathrm{H}$ & -0.76305100 & -4.78076800 & 1.05686200 \\
\hline \multirow[t]{2}{*}{$\mathrm{H}$} & 3.45731900 & -0.65607600 & 3.42160200 & $\mathrm{H}$ & -1.00388100 & -4.48308900 & -0.66221300 \\
\hline & & & & $\mathrm{H}$ & -0.10421300 & -3.31322800 & 0.31191300 \\
\hline \multirow[t]{7}{*}{136} & & & & $\mathrm{~F}$ & -4.68683400 & -0.15096600 & 5.25733100 \\
\hline & & & & $\mathrm{Cl}$ & 0.97939400 & -1.21453500 & -1.42307800 \\
\hline & & & & $\mathrm{H}$ & -4.38484900 & -3.81265500 & 0.99303300 \\
\hline & & & & $\mathrm{C}$ & -5.17740900 & -1.25924400 & 1.82652200 \\
\hline & & & & $\mathrm{H}$ & -5.85793500 & -1.87316700 & 1.24430600 \\
\hline & & & & $\mathrm{Rh}$ & -0.92300500 & -0.20132500 & -0.27894700 \\
\hline & & & & $\mathrm{H}$ & -5.04094400 & 2.31803600 & -0.36063000 \\
\hline
\end{tabular}




\begin{tabular}{|c|c|c|c|c|c|c|c|}
\hline $\mathrm{Cl}$ & 0.75613800 & 1.57369200 & 0.14744300 & $\mathrm{H}$ & 4.99564700 & 3.11335200 & 4.74910200 \\
\hline $\mathrm{Rh}$ & 2.65801800 & -0.01264200 & 0.01827700 & $\mathrm{H}$ & 5.39925700 & 2.12482600 & 3.36585700 \\
\hline $\mathrm{C}$ & 4.40959300 & -4.23253100 & 0.03815600 & $\mathrm{C}$ & -3.22302900 & 1.65154100 & -1.37860000 \\
\hline $\mathrm{C}$ & 3.39082900 & -3.08511500 & 0.23895100 & $\mathrm{H}$ & -2.97538700 & 1.24135400 & -2.36340100 \\
\hline $\mathrm{C}$ & 5.38393700 & -2.27952800 & -2.84930000 & $\mathrm{C}$ & -2.50033400 & 3.87081600 & -2.44532900 \\
\hline $\mathrm{C}$ & 4.01930300 & -1.70555000 & 0.20485300 & $\mathrm{H}$ & -3.53772500 & 4.08438100 & -2.73752200 \\
\hline $\mathrm{C}$ & 4.11621000 & -1.53794000 & -2.36059400 & $\mathrm{H}$ & -2.03622600 & 3.39313500 & -3.31662300 \\
\hline $\mathrm{C}$ & 4.32767300 & -0.98920700 & -0.96812900 & $\mathrm{C}$ & -4.74620000 & 1.90345100 & -1.33236300 \\
\hline $\mathrm{H}$ & 5.24856200 & -4.10501400 & 0.73853600 & $\mathrm{H}$ & -5.04775000 & 2.61686100 & -2.10707500 \\
\hline $\mathrm{H}$ & 2.59703500 & -3.17452900 & -0.50721500 & $\mathrm{C}$ & -2.43257700 & 2.96643300 & -1.21067800 \\
\hline $\mathrm{H}$ & 6.17476400 & -1.54595500 & -3.06125200 & $\mathrm{H}$ & -1.38033800 & 2.74684000 & -1.00891600 \\
\hline $\mathrm{H}$ & 3.88664400 & -0.72752800 & -3.06347800 & $\mathrm{H}$ & -2.80921800 & 3.51970300 & -0.34252600 \\
\hline $\mathrm{H}$ & 3.91116800 & -5.16688500 & 0.33144700 & $\mathrm{H}$ & -5.30596800 & 0.97756100 & -1.48754100 \\
\hline $\mathrm{H}$ & 2.90907300 & -3.21960200 & 1.21663600 & $\mathrm{C}$ & -1.82700000 & 5.21141300 & -2.21875400 \\
\hline $\mathrm{H}$ & 5.14276300 & -2.75382700 & -3.81097600 & $\mathrm{O}$ & -1.53417100 & 5.68749300 & -1.14319000 \\
\hline $\mathrm{H}$ & 4.65329800 & -1.51272500 & 1.07124800 & $\mathrm{O}$ & -1.60877500 & 5.84582800 & -3.39519900 \\
\hline $\mathrm{H}$ & 3.26353100 & -2.21853200 & -2.38675200 & $\mathrm{C}$ & -1.00514200 & 7.14378300 & -3.28881100 \\
\hline $\mathrm{H}$ & 5.15896800 & -0.28621300 & -0.91962000 & $\mathrm{H}$ & -0.90669800 & 7.50653600 & -4.31270000 \\
\hline $\mathrm{C}$ & 4.97187300 & -4.42393600 & -1.38620700 & $\mathrm{H}$ & -1.63649200 & 7.81714100 & -2.70214900 \\
\hline $\mathrm{H}$ & 5.49010200 & -5.39164900 & -1.39346800 & $\mathrm{H}$ & -0.02428600 & 3000 & -2.81042200 \\
\hline $\mathrm{H}$ & 4.13713900 & -4.53284700 & -2.09421500 & & & & \\
\hline $\mathrm{C}$ & 5.95821600 & -3.34073700 & -1.89049000 & 137 & & & \\
\hline $\mathrm{H}$ & 6.78631500 & -3.83198400 & -2.41797800 & & & & \\
\hline $\mathrm{H}$ & 6.41618000 & -2.84424400 & -1.02490400 & & & & \\
\hline $\mathrm{C}$ & 4.78478600 & 3.79011800 & 1.37990200 & & & & \\
\hline $\mathrm{C}$ & 3.91606700 & 2.84746100 & 0.51314300 & & & & \\
\hline $\mathrm{C}$ & 3.37405600 & 1.87814100 & 4.10996500 & & & & \\
\hline $\mathrm{C}$ & 4.11614200 & 1.37972600 & 0.83500100 & $\mathrm{C}$ & -2.41056100 & 39000 & -0.30936300 \\
\hline $\mathrm{C}$ & 2.57270800 & 1.35971000 & 2.89159900 & $\mathrm{C}$ & -2.84782100 & 1.08834200 & -0.23497400 \\
\hline $\mathrm{C}$ & 3.49447500 & 0.69342100 & 1.89695600 & $\mathrm{C}$ & -5.11664000 & 1.81451800 & 0.03890700 \\
\hline $\mathrm{H}$ & 5.83427700 & 3.46090600 & 1.34330300 & $\mathrm{C}$ & -4.67410100 & 3.14429600 & -0.01656700 \\
\hline $\mathrm{H}$ & 2.86670100 & 3.13608800 & 0.61156500 & $\mathrm{C}$ & -3.34804800 & 3.48908200 & -0.19463800 \\
\hline $\mathrm{H}$ & 3.70985100 & 1.02462700 & 4.71624900 & $\mathrm{C}$ & -0.99741100 & 2.43709400 & -0.54734900 \\
\hline $\mathrm{H}$ & 1.81054200 & 0.65305900 & 3.24367500 & $\mathrm{C}$ & -0.59888200 & 1.11907300 & -0.59456100 \\
\hline $\mathrm{H}$ & 4.76200500 & 4.78053400 & 0.90504400 & $\mathrm{H}$ & -6.17689100 & 1.61973200 & 0.15945400 \\
\hline $\mathrm{H}$ & 4.17780600 & 3.00701400 & -0.54122900 & $\mathrm{H}$ & -3.05791500 & 4.53312700 & -0.24903000 \\
\hline $\mathrm{H}$ & 2.68125900 & 2.44891800 & 4.74395000 & $\mathrm{H}$ & -0.36768100 & 3.30107000 & -0.71264700 \\
\hline $\mathrm{H}$ & 5.09811700 & 1.01375500 & 0.53334400 & $\mathrm{H}$ & 0.21896700 & 0.74912400 & -1.35538100 \\
\hline $\mathrm{H}$ & 2.03254900 & 2.18599700 & 2.42795000 & $\mathrm{~N}$ & -1.72674900 & 0.27751300 & -0.41947900 \\
\hline $\mathrm{H}$ & 4.02966300 & -0.15309900 & 2.32469700 & $\mathrm{P}$ & -1.07761800 & -1.31430200 & -0.02016000 \\
\hline $\mathrm{C}$ & 4.36623500 & 3.96469100 & 2.85519100 & $\mathrm{C}$ & -1.67893400 & -2.49237600 & -1.39085700 \\
\hline $\mathrm{H}$ & 4.93824800 & 4.81628800 & 3.24587400 & $\mathrm{C}$ & -1.64188200 & -1.78756000 & 1.73826300 \\
\hline $\mathrm{H}$ & 3.31207700 & 4.27435400 & 2.89935300 & $\mathrm{C}$ & -3.13492800 & -2.96996200 & -1.25167800 \\
\hline $\mathrm{C}$ & 4.60340800 & 2.75190100 & 3.78953400 & $\mathrm{H}$ & -3.84474600 & -2.13643500 & -1.21695900 \\
\hline
\end{tabular}




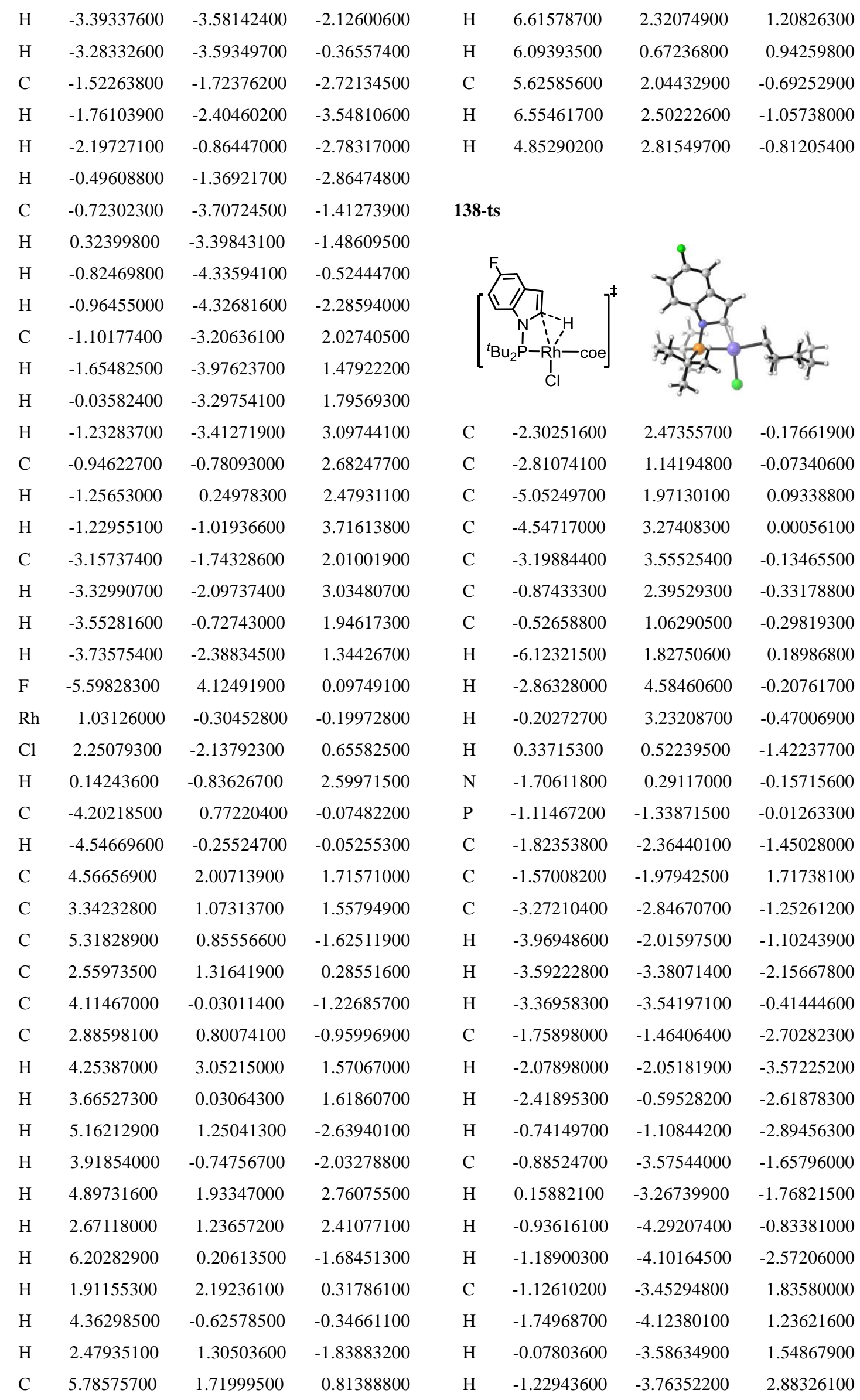




\begin{tabular}{|c|c|c|c|c|c|c|c|}
\hline $\mathrm{C}$ & -0.75003500 & -1.12842100 & 2.71387100 & $\mathrm{C}$ & -5.05785100 & 2.01019200 & 0.08466100 \\
\hline $\mathrm{H}$ & -0.99254200 & -0.06326200 & 2.63773200 & $\mathrm{C}$ & -4.53253300 & 3.30745800 & 0.05272600 \\
\hline $\mathrm{H}$ & -0.99339400 & -1.45617200 & 3.73295300 & $\mathrm{C}$ & -3.17483500 & 3.57635700 & 0.00943800 \\
\hline $\mathrm{C}$ & -3.06393300 & -1.83721600 & 2.06931900 & $\mathrm{C}$ & -0.84867700 & 2.39284300 & -0.06005100 \\
\hline $\mathrm{H}$ & -3.21887400 & -2.23201300 & 3.08189400 & $\mathrm{C}$ & -0.50912600 & 1.06062000 & -0.04672700 \\
\hline $\mathrm{H}$ & -3.38134700 & -0.79092700 & 2.07409000 & $\mathrm{H}$ & -6.13385300 & 1.87727400 & 0.11547300 \\
\hline $\mathrm{H}$ & -3.71943300 & -2.39856400 & 1.39902100 & $\mathrm{H}$ & -2.82695400 & 4.60408300 & -0.01487300 \\
\hline $\mathrm{F}$ & -5.42802600 & 4.30141800 & 0.04217800 & $\mathrm{H}$ & -0.16500800 & 3.23058900 & -0.11116000 \\
\hline $\mathrm{Rh}$ & 0.98838600 & -0.31402300 & -0.24358900 & $\mathrm{H}$ & 0.78389500 & -0.22091600 & -1.58383400 \\
\hline $\mathrm{Cl}$ & 2.26735500 & -2.21925800 & 0.44472400 & $\mathrm{~N}$ & -1.71345500 & 0.30703100 & 0.00987100 \\
\hline $\mathrm{H}$ & 0.32539800 & -1.25382300 & 2.56018800 & $\mathrm{P}$ & -1.14257800 & -1.32091600 & -0.03641700 \\
\hline $\mathrm{C}$ & -4.17797100 & 0.88680300 & 0.05583500 & $\mathrm{C}$ & -1.84415300 & -2.19694400 & -1.56942900 \\
\hline $\mathrm{H}$ & -4.56350300 & -0.12473800 & 0.12479100 & $\mathrm{C}$ & -1.57520200 & -2.14643800 & 1.61687700 \\
\hline $\mathrm{C}$ & 4.56181200 & 1.86098700 & 1.78477000 & $\mathrm{C}$ & -3.26775400 & -2.75482300 & -1.37734800 \\
\hline $\mathrm{C}$ & 3.32548900 & 0.95731300 & 1.55681200 & $\mathrm{H}$ & -3.98899100 & -1.97442400 & -1.11429600 \\
\hline $\mathrm{C}$ & 5.28420800 & 0.97250100 & -1.64338800 & $\mathrm{H}$ & -3.59909000 & -3.19875900 & -2.32467800 \\
\hline $\mathrm{C}$ & 2.54841600 & 1.30966800 & 0.30772900 & $\mathrm{H}$ & -3.31007600 & -3.54122000 & -0.61904100 \\
\hline $\mathrm{C}$ & 4.07646300 & 0.06373800 & -1.30973600 & $\mathrm{C}$ & -1.86664200 & -1.16193600 & -2.71457800 \\
\hline $\mathrm{C}$ & 2.86022800 & 0.88456700 & -0.96903400 & $\mathrm{H}$ & -2.20438700 & -1.66555500 & -3.62890200 \\
\hline $\mathrm{H}$ & 4.26253400 & 2.91804700 & 1.72695400 & $\mathrm{H}$ & -2.55120500 & -0.33349200 & -2.50992100 \\
\hline $\mathrm{H}$ & 3.63676300 & -0.09020500 & 1.53582900 & $\mathrm{H}$ & -0.87433300 & -0.74545600 & -2.91168200 \\
\hline $\mathrm{H}$ & 5.12411600 & 1.44996400 & -2.62059700 & $\mathrm{C}$ & -0.87372300 & -3.34181400 & -1.93857000 \\
\hline $\mathrm{H}$ & 3.86889600 & -0.58228000 & -2.17097600 & $\mathrm{H}$ & 0.15379200 & -2.98490000 & -2.05212500 \\
\hline $\mathrm{H}$ & 4.89384900 & 1.69694200 & 2.81878300 & $\mathrm{H}$ & -0.86674100 & -4.13954200 & -1.19098800 \\
\hline $\mathrm{H}$ & 2.65539900 & 1.06353900 & 2.41932700 & $\mathrm{H}$ & -1.19754700 & -3.78126100 & -2.89060000 \\
\hline $\mathrm{H}$ & 6.16185300 & 0.32226700 & -1.76219700 & $\mathrm{C}$ & -1.14487500 & -3.62693500 & 1.57584300 \\
\hline $\mathrm{H}$ & 1.89042800 & 2.17085900 & 0.40897700 & $\mathrm{H}$ & -1.77976200 & -4.22787400 & 0.91709700 \\
\hline $\mathrm{H}$ & 4.32652400 & -0.60396700 & -0.48346200 & $\mathrm{H}$ & -0.10162000 & -3.73560900 & 1.26030800 \\
\hline $\mathrm{H}$ & 2.42937000 & 1.43902400 & -1.80303700 & $\mathrm{H}$ & -1.23566900 & -4.04711900 & 2.58560200 \\
\hline $\mathrm{C}$ & 5.77192200 & 1.62973400 & 0.85581000 & $\mathrm{C}$ & -0.72377900 & -1.40942500 & 2.67687500 \\
\hline $\mathrm{H}$ & 6.61243100 & 2.18496400 & 1.29207800 & $\mathrm{H}$ & -0.94888500 & -0.33823100 & 2.71238500 \\
\hline $\mathrm{H}$ & 6.06525600 & 0.57094000 & 0.89796300 & $\mathrm{H}$ & -0.94759700 & -1.83109300 & 3.66522600 \\
\hline $\mathrm{C}$ & 5.60776500 & 2.07753400 & -0.61815800 & $\mathrm{C}$ & -3.06175900 & -2.00934700 & 1.99705200 \\
\hline $\mathrm{H}$ & 6.53901100 & 2.55483500 & -0.94988800 & $\mathrm{H}$ & -3.21399400 & -2.46351800 & 2.98483500 \\
\hline \multirow[t]{2}{*}{$\mathrm{H}$} & 4.84257600 & 2.86378000 & -0.66967500 & $\mathrm{H}$ & -3.36552900 & -0.96059500 & 2.06658400 \\
\hline & & & & $\mathrm{H}$ & -3.72895000 & -2.51975100 & 1.29818900 \\
\hline \multirow[t]{6}{*}{139} & & & & $\mathrm{~F}$ & -5.40331000 & 4.34552800 & 0.06431500 \\
\hline & & & & $\mathrm{Rh}$ & 0.95438300 & -0.33583000 & -0.08177500 \\
\hline & & & & $\mathrm{Cl}$ & 2.34099400 & -2.32351600 & 0.17166300 \\
\hline & & & & $\mathrm{H}$ & 0.34837400 & -1.54420000 & 2.49531300 \\
\hline & & & & $\mathrm{C}$ & -4.19198200 & 0.91730700 & 0.07586100 \\
\hline & & & & $\mathrm{H}$ & -4.58989100 & -0.09207100 & 0.10541800 \\
\hline $\mathrm{C}$ & 7600 & & -0.00524400 & $\mathrm{C}$ & 4.83804200 & 1.53234600 & 1.79083300 \\
\hline $\mathrm{C}$ & 7900 & 1.16 & 0.03246500 & $\mathrm{C}$ & 3.57228200 & 0.66610300 & 1.57519500 \\
\hline
\end{tabular}




\begin{tabular}{|c|c|c|c|c|c|c|c|}
\hline $\mathrm{C}$ & 5.03604900 & 1.35071400 & -1.81098500 & $\mathrm{H}$ & 3.27912100 & 2.77482900 & -0.78303500 \\
\hline $\mathrm{C}$ & 2.62065700 & 1.23949900 & 0.54863000 & $\mathrm{H}$ & 2.68537600 & 4.13506200 & -1.73931300 \\
\hline $\mathrm{C}$ & 3.87677700 & 0.37651300 & -1.48688700 & $\mathrm{H}$ & 2.37902700 & 4.09460800 & -0.00295300 \\
\hline $\mathrm{C}$ & 2.73827300 & 1.09313900 & -0.81203000 & $\mathrm{C}$ & 1.27749800 & 1.96995200 & -2.53354700 \\
\hline $\mathrm{H}$ & 4.54505400 & 2.57466000 & 1.98424600 & $\mathrm{H}$ & 1.53119900 & 2.68374400 & -3.32700000 \\
\hline $\mathrm{H}$ & 3.86603200 & -0.35145800 & 1.30353200 & $\mathrm{H}$ & 2.07464800 & 1.22274500 & -2.47924800 \\
\hline $\mathrm{H}$ & 4.73916200 & 2.00860700 & -2.63988700 & $\mathrm{H}$ & 0.35303200 & 1.46337700 & -2.82661400 \\
\hline $\mathrm{H}$ & 3.53284200 & -0.08577300 & -2.41947400 & $\mathrm{C}$ & -0.02250400 & 3.78303000 & -1.36390900 \\
\hline $\mathrm{H}$ & 5.31579400 & 1.17551200 & 2.71296400 & $\mathrm{H}$ & -0.98615500 & 3.30217000 & -1.55428100 \\
\hline $\mathrm{H}$ & 3.04312700 & 0.58927900 & 2.53393400 & $\mathrm{H}$ & -0.13545500 & 4.41334100 & -0.47773900 \\
\hline $\mathrm{H}$ & 5.87421800 & 0.74748600 & -2.18547500 & $\mathrm{H}$ & 0.21260500 & 4.43959700 & -2.21114200 \\
\hline $\mathrm{H}$ & 1.94106000 & 2.00806700 & 0.91480800 & $\mathrm{C}$ & 0.25799700 & 3.44239000 & 2.13673200 \\
\hline $\mathrm{H}$ & 4.23381100 & -0.43970600 & -0.85553700 & $\mathrm{H}$ & 0.78145900 & 4.24439200 & 1.60681500 \\
\hline $\mathrm{H}$ & 2.14860100 & 1.75785500 & -1.44179500 & $\mathrm{H}$ & -0.79590300 & 3.44769500 & 1.83889100 \\
\hline $\mathrm{C}$ & 5.89534500 & 1.50559500 & 0.66791500 & $\mathrm{H}$ & 0.30545200 & 3.67587200 & 3.20782900 \\
\hline $\mathrm{H}$ & 6.79934100 & 0400 & 1.07871500 & $\mathrm{C}$ & 0.19908400 & 1.01789200 & 2.79649300 \\
\hline $\mathrm{H}$ & 6.17382300 & 0.46346700 & 0.45573300 & $\mathrm{H}$ & 0.58956500 & 0.00811900 & 2.63301700 \\
\hline $\mathrm{C}$ & 5.52700300 & 2.23506100 & -0.64787200 & $\mathrm{H}$ & 0.37139700 & 1.27753200 & 3.84891800 \\
\hline $\mathrm{H}$ & 6.40938000 & 2.77641800 & -1.01285100 & $\mathrm{C}$ & 2.40582000 & 2.08913000 & 2.27421600 \\
\hline \multirow[t]{2}{*}{$\mathrm{H}$} & 7901200 & 8800 & -0.43120700 & $\mathrm{H}$ & 2.49903600 & 2.36803300 & 3.33180500 \\
\hline & & & & $\mathrm{H}$ & 2.87005700 & 1.10616800 & 2.15095600 \\
\hline \multirow[t]{7}{*}{140} & & & & $\mathrm{H}$ & 2.97593900 & 2.81927200 & 1.69433100 \\
\hline & & & & $\mathrm{F}$ & 5.67778400 & -3.36165800 & -0.72085300 \\
\hline & & & & $\mathrm{Rh}$ & -1.32726300 & 0.24202000 & -0.13653200 \\
\hline & & & & $\mathrm{Cl}$ & -2.99260900 & 1.90414400 & 0.48883600 \\
\hline & & & & $\mathrm{H}$ & -0.88384000 & 1.01261200 & 2.63053600 \\
\hline & & & & $\mathrm{C}$ & 3.95466700 & -0.21305600 & -0.14084400 \\
\hline & & & & $\mathrm{H}$ & 4.19419100 & 0.82582800 & 0.06332600 \\
\hline $\mathrm{C}$ & 2.31154500 & -2.02190400 & -0.50743600 & $\mathrm{C}$ & -2.40446600 & -2.49505400 & 0.83170400 \\
\hline $\mathrm{C}$ & 2.63430100 & -0.65616300 & -0.23970000 & $\mathrm{O}$ & -1.91473500 & -3.48890900 & 0.33376800 \\
\hline $\mathrm{C}$ & 4.97760300 & 700 & -0.30855100 & $\mathrm{O}$ & -2.50814900 & -2.29967300 & 2.16530200 \\
\hline $\mathrm{C}$ & 4.65724700 & -2.48420300 & -0.56528300 & $\mathrm{C}$ & -1.98094300 & -3.35685700 & 2.98393200 \\
\hline $\mathrm{C}$ & 3.35668100 & -2.94781700 & -0.66738100 & $\mathrm{H}$ & -0.91170400 & -3.48601500 & 2.79679800 \\
\hline $\mathrm{C}$ & 0.87592900 & -2.1 & -0.56124700 & $\mathrm{H}$ & -2.15825700 & -3.04413100 & 4.01295400 \\
\hline $\mathrm{C}$ & 0.33551200 & -0.90693300 & -0.32522400 & $\mathrm{H}$ & -2.49705000 & -4.29716100 & 2.77227400 \\
\hline $\mathrm{H}$ & 6.02083700 & -0.85553500 & -0.24496800 & $\mathrm{C}$ & -2.84490100 & -1.24262300 & -1.27583000 \\
\hline $\mathrm{H}$ & 3.16893400 & -3.99739400 & -0.86944900 & $\mathrm{H}$ & -2.19148300 & -1.97211100 & -1.75199300 \\
\hline $\mathrm{H}$ & 0.32116400 & -3.05921100 & -0.73060100 & $\mathrm{C}$ & -4.80600300 & -1.36580600 & -2.82317700 \\
\hline $\mathrm{H}$ & -1.10483600 & 0.43271300 & -1.62561700 & $\mathrm{H}$ & -5.45327900 & -1.80897900 & -2.05862300 \\
\hline $\mathrm{N}$ & 1.41067400 & 0.00811000 & -0.13052800 & $\mathrm{H}$ & -5.43550500 & -0.79069600 & -3.51072500 \\
\hline $\mathrm{P}$ & 0.59708200 & 1.50111000 & 0.12039000 & $\mathrm{H}$ & -4.34511500 & -2.18270600 & -3.39072200 \\
\hline $\mathrm{C}$ & 1.11736600 & 2.75046400 & -1.21156400 & $\mathrm{C}$ & -3.00267000 & -1.34934400 & 0.08489100 \\
\hline $\mathrm{C}$ & 0.90928900 & 2.06360900 & 1.90578500 & $\mathrm{H}$ & -3.74651700 & -0.75783400 & 0.61131800 \\
\hline $\mathrm{C}$ & 2.44326400 & 3.47210100 & -0.89920300 & $\mathrm{C}$ & -3.74353100 & -0.44677600 & -2.18561800 \\
\hline
\end{tabular}




\begin{tabular}{|c|c|c|c|c|c|c|c|}
\hline $\mathrm{H}$ & -3.14660900 & 0.01375700 & -2.98234400 & $\mathrm{H}$ & 2.57372300 & 2.35647100 & 3.36970100 \\
\hline \multirow[t]{2}{*}{$\mathrm{H}$} & -4.22077700 & 0.36382600 & -1.62813500 & $\mathrm{H}$ & 2.90640800 & 1.04132500 & 2.23753300 \\
\hline & & & & $\mathrm{H}$ & 3.10601200 & 2.73222100 & 1.72975700 \\
\hline \multirow[t]{6}{*}{ 141-ts } & & & & $\mathrm{F}$ & 5.53838400 & -3.55206900 & -0.74856100 \\
\hline & & & & $\mathrm{Rh}$ & -1.36177300 & 0.27865000 & -0.26761200 \\
\hline & & $7^{\ddagger}$ & & $\mathrm{Cl}$ & -2.95837700 & 2.06808100 & 0.14980100 \\
\hline & & & & $\mathrm{H}$ & -0.85363500 & 1.11998300 & 2.60529700 \\
\hline & & & & $\mathrm{C}$ & 3.92891000 & -0.37439500 & -0.02164900 \\
\hline & & & & $\mathrm{H}$ & 4.20415500 & 0.63560200 & 0.26377100 \\
\hline $\mathrm{C}$ & 2.22688400 & -2.07697400 & -0.57618100 & $\mathrm{C}$ & -2.37381400 & -2.30013400 & 0.94989900 \\
\hline $\mathrm{C}$ & 2.59489900 & -0.74852500 & -0.20071800 & $\mathrm{O}$ & -2.00948700 & -3.35286600 & 0.46289500 \\
\hline $\mathrm{C}$ & 4.91683800 & -1.33822600 & -0.21451000 & $\mathrm{O}$ & -2.39409600 & -2.05293400 & 2.27565700 \\
\hline $\mathrm{C}$ & 4.55034400 & -2.64190000 & -0.57345300 & $\mathrm{C}$ & -1.90474700 & -3.11886200 & 3.10673100 \\
\hline $\mathrm{C}$ & 3.23706300 & -3.03805500 & -0.75655400 & $\mathrm{H}$ & -0.86058300 & -3.33761000 & 2.86797100 \\
\hline $\mathrm{C}$ & 0.79279400 & -2.13122700 & -0.69334300 & $\mathrm{H}$ & -1.99939800 & -2.75654400 & 4.13035100 \\
\hline $\mathrm{C}$ & 0.29448400 & -0.88629400 & -0.38897100 & $\mathrm{H}$ & -2.50236100 & -4.02258400 & 2.96054200 \\
\hline $\mathrm{H}$ & 5.96802100 & -1.10093300 & -0.09156800 & $\mathrm{C}$ & -2.84674600 & -1.15942300 & -1.26112600 \\
\hline $\mathrm{H}$ & 3.01286900 & -4.06242900 & -1.03594000 & $\mathrm{H}$ & -2.41986500 & -2.09111200 & -1.64426200 \\
\hline $\mathrm{H}$ & 0.20886500 & -3.00731500 & -0.93861600 & $\mathrm{C}$ & -5.19639400 & -1.59583700 & -2.07583500 \\
\hline $\mathrm{H}$ & -1.85043700 & -0.40840200 & -1.68567100 & $\mathrm{H}$ & -5.57652600 & -1.76271300 & -1.06158400 \\
\hline $\mathrm{N}$ & 1.39759400 & -0.04014900 & -0.07940600 & $\mathrm{H}$ & -6.01877200 & -1.20306400 & -2.68330100 \\
\hline $\mathrm{P}$ & 0.68459100 & 1.51220700 & 0.13522300 & $\mathrm{H}$ & -4.90597400 & -2.56827100 & -2.49091900 \\
\hline $\mathrm{C}$ & 1.31246000 & 2.69139700 & -1.21917900 & $\mathrm{C}$ & -2.88542400 & -1.12672000 & 0.18170700 \\
\hline $\mathrm{C}$ & 1.00200800 & 2.08225600 & 1.91538700 & $\mathrm{H}$ & -3.66275300 & -0.54898500 & 0.67672300 \\
\hline $\mathrm{C}$ & 2.70031600 & 3.29766700 & -0.94039100 & $\mathrm{C}$ & -4.02141900 & -0.60770800 & -2.07210000 \\
\hline $\mathrm{H}$ & 3.47057700 & 2.53077900 & -0.81282000 & $\mathrm{H}$ & -3.69380500 & -0.42116700 & -3.10239300 \\
\hline $\mathrm{H}$ & 2.99311900 & 3.91665700 & -1.79829300 & $\mathrm{H}$ & -4.32295800 & 0.35512400 & -1.64972700 \\
\hline $\mathrm{H}$ & 2.69960900 & 3.94537100 & -0.05882300 & & & & \\
\hline $\mathrm{C}$ & 1.36600500 & 1.86137200 & -2.51939000 & 142 & & & \\
\hline $\mathrm{H}$ & 1.63849900 & 2.52785800 & -3.34710100 & & & & \\
\hline $\mathrm{H}$ & 2.10929700 & 1.06045600 & -2.46726500 & & & & \\
\hline $\mathrm{H}$ & 0.39476300 & 1.41322200 & -2.75381500 & & & & \\
\hline $\mathrm{C}$ & 0.26704900 & 3.81751800 & -1.38544400 & & & & \\
\hline $\mathrm{H}$ & -0.73876600 & 3.42074200 & -1.55241900 & & & & \\
\hline $\mathrm{H}$ & 0.22435000 & 4.48053800 & -0.51719500 & & & & \\
\hline $\mathrm{H}$ & 0.54754500 & 4.42823500 & -2.25321100 & $\mathrm{C}$ & 2.51940900 & -1.47223600 & -0.94743600 \\
\hline $\mathrm{C}$ & 0.41216900 & 3.49538900 & 2.10081400 & $\mathrm{C}$ & 2.55473700 & -0.32055500 & -0.10304400 \\
\hline $\mathrm{H}$ & 0.99050900 & 4.25910600 & 1.57120700 & $\mathrm{C}$ & 4.92418300 & -0.54884300 & 0.17782200 \\
\hline $\mathrm{H}$ & -0.63076400 & 3.54766500 & 1.76925700 & $\mathrm{C}$ & 4.88304100 & -1.67809400 & -0.64797900 \\
\hline $\mathrm{H}$ & 0.43766800 & 3.74915100 & 3.16801000 & $\mathrm{C}$ & 3.71531900 & -2.16026000 & -1.21460700 \\
\hline $\mathrm{C}$ & 0.22215800 & 1.09175200 & 2.80949900 & $\mathrm{C}$ & 1.15599200 & -1.68616400 & -1.36749300 \\
\hline $\mathrm{H}$ & 0.57385000 & 0.06322000 & 2.68004200 & $\mathrm{C}$ & 0.38331100 & -0.70344800 & -0.80386500 \\
\hline $\mathrm{H}$ & 0.37327400 & 1.37069300 & 3.86030300 & $\mathrm{H}$ & 5.87595800 & -0.22944800 & 0.58830800 \\
\hline $\mathrm{C}$ & 2.48863800 & 2.04910000 & 2.31923200 & $\mathrm{H}$ & 3.74203400 & -3.04221500 & -1.84632400 \\
\hline
\end{tabular}




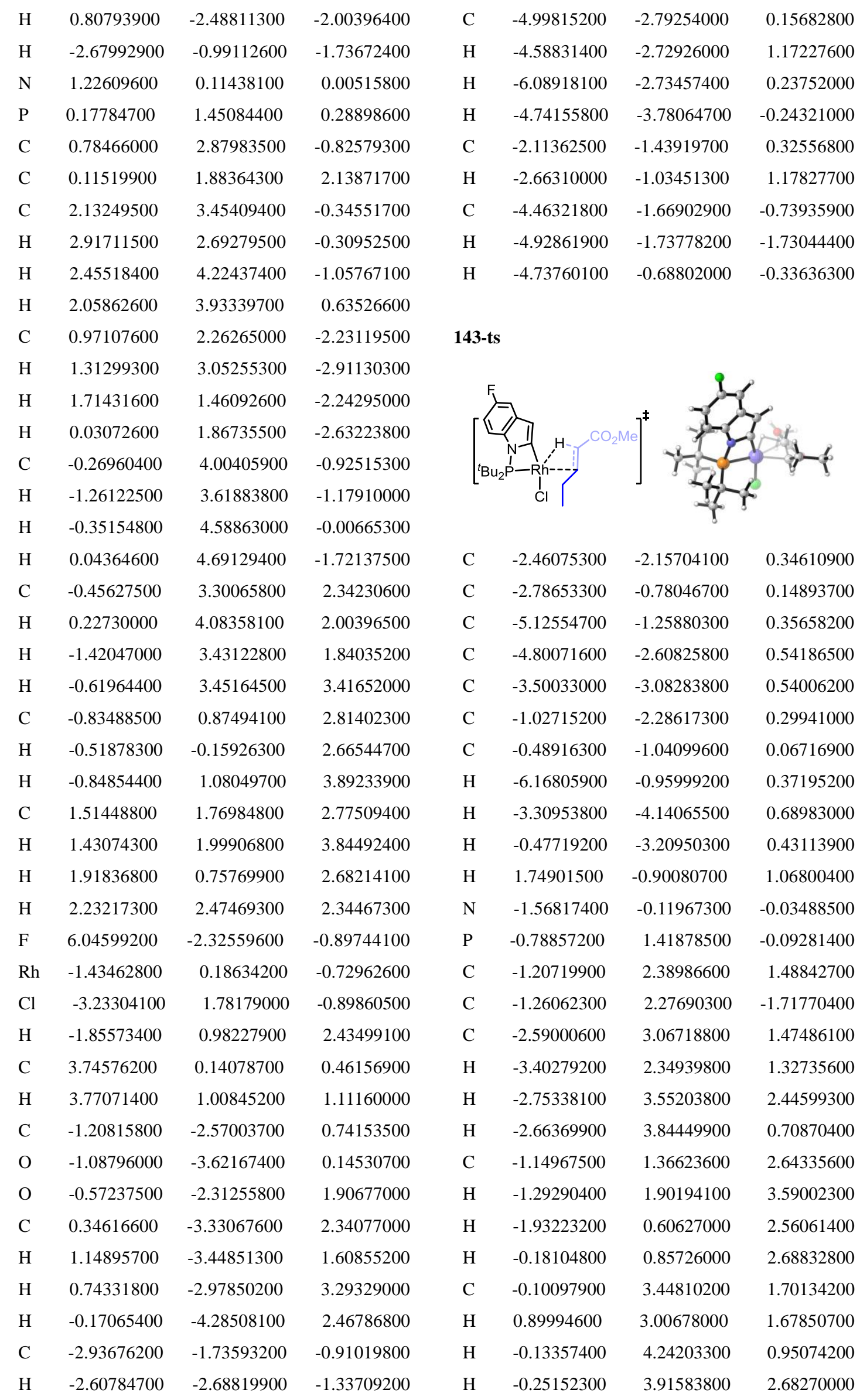




\begin{tabular}{|c|c|c|c|c|c|c|c|}
\hline $\mathrm{C}$ & -0.62248700 & 3.68058400 & -1.74463600 & $\mathrm{C}$ & 2.87631800 & -0.75483300 & -0.02033300 \\
\hline $\mathrm{H}$ & -1.10753600 & 4.37112900 & -1.04773000 & $\mathrm{C}$ & 5.23464400 & -1.17180800 & 0.08114800 \\
\hline $\mathrm{H}$ & 0.44891800 & 3.64466500 & -1.51867200 & $\mathrm{C}$ & 5.00269300 & -2.43898900 & -0.46544400 \\
\hline $\mathrm{H}$ & -0.73821400 & 4.09997900 & -2.75200700 & $\mathrm{C}$ & 3.74111100 & -2.90027700 & -0.80190800 \\
\hline $\mathrm{C}$ & -0.63118900 & 1.41966900 & -2.83869600 & $\mathrm{C}$ & 1.23178500 & -2.18776800 & -0.82869700 \\
\hline $\mathrm{H}$ & -1.01335100 & 0.39359100 & -2.82902700 & $\mathrm{C}$ & 0.60920300 & -1.03167700 & -0.42499700 \\
\hline $\mathrm{H}$ & -0.88542800 & 1.86524400 & -3.80897000 & $\mathrm{H}$ & 6.25090600 & -0.87687600 & 0.31896100 \\
\hline $\mathrm{C}$ & -2.78099800 & 2.35647100 & -1.95152200 & $\mathrm{H}$ & 3.62185900 & -3.89319100 & -1.22340100 \\
\hline $\mathrm{H}$ & -2.96339800 & 2.83190800 & -2.92407600 & $\mathrm{H}$ & 0.75365500 & -3.05065400 & -1.27446000 \\
\hline $\mathrm{H}$ & -3.23551800 & 1.36176100 & -1.98229800 & $\mathrm{H}$ & -2.39355200 & -1.25765500 & -1.15448100 \\
\hline $\mathrm{H}$ & -3.29713600 & 2.95352000 & -1.19515100 & $\mathrm{~N}$ & 1.61117600 & -0.16438400 & 0.10910700 \\
\hline $\mathrm{F}$ & -5.81558900 & -3.48510500 & 0.73169300 & $\mathrm{P}$ & 0.72819900 & 1.31328500 & 0.19198300 \\
\hline $\mathrm{Rh}$ & 1.21083000 & 0.05722000 & -0.07895500 & $\mathrm{C}$ & 1.30444600 & 2.41987000 & -1.25493000 \\
\hline $\mathrm{Cl}$ & 2.87532100 & 1.79638600 & -0.33075100 & $\mathrm{C}$ & 0.92531400 & 2.10250200 & 1.90666400 \\
\hline $\mathrm{H}$ & 0.46030700 & 1.38876400 & -2.75690300 & $\mathrm{C}$ & 2.73202200 & 2.96754800 & -1.06685800 \\
\hline $\mathrm{C}$ & -4.10796000 & -0.32791800 & 0.15395400 & $\mathrm{H}$ & 3.46482700 & 2.17172600 & -0.90308100 \\
\hline $\mathrm{H}$ & -4.35280400 & 0.71782900 & 0.00055500 & $\mathrm{H}$ & 3.02405500 & 3.49981100 & -1.98125200 \\
\hline $\mathrm{C}$ & 4.00792800 & -1.27375800 & 0.75360400 & $\mathrm{H}$ & 2.79622600 & 3.68535500 & -0.24388100 \\
\hline $\mathrm{O}$ & 5.03911600 & -1.16132400 & 0.13404600 & $\mathrm{C}$ & 1.27559600 & 1.50554500 & -2.50231500 \\
\hline $\mathrm{O}$ & 3.90592700 & -1.17760400 & 2.09312900 & $\mathrm{H}$ & 1.58101700 & 2.09929800 & -3.37274700 \\
\hline $\mathrm{C}$ & 5.12609500 & -0.84423300 & 2.78052100 & $\mathrm{H}$ & 1.95666500 & 0.65462200 & -2.41449000 \\
\hline $\mathrm{H}$ & 5.49192600 & 0.12730200 & 2.44018200 & $\mathrm{H}$ & 0.26886900 & 1.12087000 & -2.70687300 \\
\hline $\mathrm{H}$ & 4.86339300 & -0.81029500 & 3.83770200 & $\mathrm{C}$ & 0.31348400 & 3.58338100 & -1.47854600 \\
\hline $\mathrm{H}$ & 5.88851300 & -1.60530700 & 2.59504000 & $\mathrm{H}$ & -0.72421900 & 3.24098600 & -1.53560500 \\
\hline $\mathrm{C}$ & 2.43640200 & -1.26870400 & -1.23796700 & $\mathrm{H}$ & 0.38065100 & 4.34690600 & -0.70026100 \\
\hline $\mathrm{H}$ & 3.18838500 & -0.61514400 & -1.67864900 & $\mathrm{H}$ & 0.56625100 & 4.06703800 & -2.43087300 \\
\hline $\mathrm{C}$ & 2.71006300 & -3.06162700 & -2.97285900 & $\mathrm{C}$ & 0.29777400 & 3.51076900 & 1.90204600 \\
\hline $\mathrm{H}$ & 3.24351200 & -3.72750900 & -2.28526800 & $\mathrm{H}$ & 0.90048200 & 4.23017200 & 1.34123100 \\
\hline $\mathrm{H}$ & 2.17847600 & -3.68139300 & -3.70320900 & $\mathrm{H}$ & -0.71887500 & 3.50900000 & 1.49421600 \\
\hline $\mathrm{H}$ & 3.45773300 & -2.46792100 & -3.51019300 & $\mathrm{H}$ & 0.24168600 & 3.86893900 & 2.93744000 \\
\hline $\mathrm{C}$ & 2.66508200 & -1.61167900 & 0.13280700 & $\mathrm{C}$ & 0.15844000 & 1.21709900 & 2.90670000 \\
\hline $\mathrm{H}$ & 2.25821300 & -2.56558800 & 0.47864900 & $\mathrm{H}$ & 0.56058900 & 0.20088100 & 2.93044700 \\
\hline $\mathrm{C}$ & 1.71413700 & -2.15828100 & -2.21585600 & $\mathrm{H}$ & 0.27017100 & 1.64587900 & 3.91048200 \\
\hline $\mathrm{H}$ & 1.17854500 & -1.52959900 & -2.93840000 & $\mathrm{C}$ & 2.40247400 & 2.16220000 & 2.34442300 \\
\hline \multirow[t]{2}{*}{$\mathrm{H}$} & 0.95998000 & -2.76366300 & -1.70454300 & $\mathrm{H}$ & 2.45018400 & 2.61422800 & 3.34326800 \\
\hline & & & & $\mathrm{H}$ & 2.83915100 & 1.16177500 & 2.41464200 \\
\hline \multirow[t]{7}{*}{144} & & & & $\mathrm{H}$ & 3.02265800 & 2.77039700 & 1.68124300 \\
\hline & & & & $\mathrm{F}$ & 6.06970700 & -3.24717600 & -0.67210500 \\
\hline & & & & $\mathrm{Rh}$ & -1.08352000 & 0.08250100 & -0.44183600 \\
\hline & & & & $\mathrm{Cl}$ & -2.83388100 & 1.73441600 & -0.68029100 \\
\hline & & & & $\mathrm{H}$ & -0.90793100 & 1.16912400 & 2.67165000 \\
\hline & & & & $\mathrm{C}$ & 4.15887000 & -0.31513800 & 0.31331200 \\
\hline & & & & $\mathrm{H}$ & 4.33225700 & 0.66499000 & 0.74433700 \\
\hline $\mathrm{C}$ & 2.64635600 & -2.046 & 38600 & $\mathrm{C}$ & -4.26034600 & -1.37613400 & -0.13802200 \\
\hline
\end{tabular}




$\begin{array}{lrrr}\mathrm{O} & -4.92251600 & -1.22931100 & 0.86406400 \\ \mathrm{O} & -4.75841400 & -1.39623700 & -1.38624800 \\ \mathrm{C} & -6.17087900 & -1.14222800 & -1.49138400 \\ \mathrm{H} & -6.39957300 & -0.14543600 & -1.10707900 \\ \mathrm{H} & -6.39880900 & -1.20603900 & -2.55522100 \\ \mathrm{H} & -6.73675200 & -1.88862000 & -0.92774600 \\ \mathrm{C} & -1.99768900 & -1.02493000 & 1.02036500 \\ \mathrm{H} & -2.61032500 & -0.31243500 & 1.57706700 \\ \mathrm{C} & -2.30689700 & -2.74709100 & 2.84065000 \\ \mathrm{H} & -3.04582100 & -3.30322000 & 2.25304400 \\ \mathrm{H} & -1.78596600 & -3.45932100 & 3.49059400 \\ \mathrm{H} & -2.85929600 & -2.04507000 & 3.47482700 \\ \mathrm{C} & -2.75776100 & -1.63318000 & -0.14833300 \\ \mathrm{H} & -2.56811300 & -2.71141300 & -0.22306300 \\ \mathrm{C} & -1.29707300 & -2.01067900 & 1.93773900 \\ \mathrm{H} & -0.57913200 & -1.48282900 & 2.57165400 \\ \mathrm{H} & -0.71985700 & -2.73076800 & 1.34852300\end{array}$




\section{Reference}

1. (a) Chen, P.-P.; Zhang, H.; Cheng, B.; Chen, X.; Cheng, F.; Zhang, S.-Q.; Lu, Z.; Meng, F.; Hong, X. How Solvents Control the Stereospecificity of Ni-Catalyzed Miyaura Borylation of Allylic Pivalates. ACS Catal. 2019, 9, 9589-9598. (b) Grimme, S.; Antony, J.; Ehrlich, S.; Krieg, H. A consistent and accurate ab initio parametrization of density functional dispersion correction (DFT-D) for the 94 elements H-Pu. J. Chem. Phys. 2010, 132, 154104. (c) Lam, Y.-H.; Houk, K. N. Origins of stereoselectivity in intramolecular aldol reactions catalyzed by cinchona amines. J. Am. Chem. Soc. 2015, 137, 2116-2127.

2. Zhao, Y.; Truhlar, D. G. The M06 suite of density functionals for main group thermochemistry, thermochemical kinetics, noncovalent interactions, excited states, and transition elements: two new func-tionals and systematic testing of four M06-class functionals and 12 other functionals. Theor. Chem. Acc. 2008, 120, 215-241.

3. Peverati, R.; Truhlar, D. G. Improving the Accuracy of Hybrid Meta-GGA Density Functionals by Range Separation. J. Phys. Chem. Lett. 2011, 2, 2810-2817.

4. Peverati, R.; Truhlar, D. G. M11-L: A Local Density Functional That Provides Improved Accuracy for Electronic Structure Calculations in Chemistry and Physics. J. Phys. Chem. Lett. 2012, 3, 117-124.

5. Lin, Y.-S.; Li, G.-D.; Mao, S.-P.; Chai, J.-D. Long-Range Corrected Hybrid Density Functionals with Improved Dispersion Corrections. J. Chem. Theory Comput. 2013, 9, 263-272.

6. (a) Green, A. G.; Liu, P.; Merlic, C. A.; Houk, K. N. Distortion/Interaction analysis reveals the origins of selectivities in iridium-catalyzed $\mathrm{C}-\mathrm{H}$ borylation of substituted arenes and 5-membered heterocycles. J. Am. Chem. Soc. 2014, 136, 4575-4583. (b) Liu, F.; Liang, Y.; Houk, K. N. Bioorthogonal Cycloadditions: Computational Analysis with the Distortion/Interaction Model and Predictions of Reactivities. Acc. Chem. Res. 2017, 50, 2297-2308. (c) Liu, P.; McCarren, P.; Cheong, P. H.-Y.; Jamison, T. F.; Houk, K. N. Origins of regioselectivity and alkene-directing effects in nickel-catalyzed reductive couplings of alkynes and aldehydes. J. Am. Chem. Soc. 2010, 132, 2050-2057. (d) Liu, S.; Lei, Y.; Qi, X.; Lan, Y. Reactivity for the Diels-Alder reaction of cumulenes: a distortion-interaction analysis along the reaction pathway. J. Phys. Chem. A 2014, 118, 2638-2645.

7. Glendening, E. D.; Reed, A. E.; Carpenter, J. E.; Weinhold, F. NBO Version 3.1. 